\title{
Structural characterization of human spliceosome activation by cryo-EM
}

\author{
DISSERTATION \\ for the award of the degree \\ "Doctor of Philosophy" (Ph.D.) \\ of the Georg-August-Universität Göttingen
}

within the doctoral program

International Max Planck Research School for Molecular BIOLOGY

of the Göttingen Graduate School for Neurosciences,

Biophysics, and Molecular Biosciences (GGNB)

submitted by

Cole Townsend

from Ada, Oklahoma, USA

Göttingen 2021 


\section{Thesis Committee}

Prof. Dr. Holger Stark

Department of Structural Dynamics, Max Planck Institute for Biophysical Chemistry

Prof. Dr. Henning Urlaub

Bioanalytical Mass Spectrometry, Max Planck Institute for Biophysical Chemistry

Dr. Alexander Stein

Membrane Protein Biochemistry, Max Planck Institute for Biophysical Chemistry

\section{Members of the Examination Board}

$1^{\text {st }}$ Referee: Prof. Dr. Holger Stark

Department of Structural Dynamics, Max Planck Institute for Biophysical Chemistry

$2^{\text {nd }}$ Referee: Prof. Dr. Henning Urlaub

Bioanalytical Mass Spectrometry, Max Planck Institute for Biophysical Chemistry

Dr. Alexander Stein

Membrane Protein Biochemistry, Max Planck Institute for Biophysical Chemistry

Prof. Dr. Reinhard Lührmann

Department of Cellular Biochemistry, Max Planck Institute for Biophysical Chemistry

Prof. Dr. Kai Tittmann

Department of Molecular Enzymology, Georg-August-Universität Göttingen

Dr. Alex Faesen

Biochemistry of Signal Dynamics, Max Planck Institute for Biophysical Chemistry

Date of oral examination: December $8^{\text {th }}, 2021$ 


\section{AFFIDAVIT}

I hereby declare that this dissertation with the title "Structural characterization of human spliceosome activation by cryo-EM" has been written independently and with no other aids or sources than quoted. This thesis (wholly or in part) has not been submitted elsewhere for any academic award or qualification.

Cole Townsend 


\section{Abstract}

Eukaryotic genes are transcribed as precursor mRNA (pre-mRNA), in which coding regions (exons) are interrupted by non-coding regions (introns). Introns are excised and exons are ligated together in a two-step process termed "splicing" to produce mature mRNA. Both steps of pre-mRNA splicing are catalyzed by RNA within a complex molecular machine consisting of 5 small nuclear ribonucleoproteins (U1, U2, U4/U6. U5 snRNPs) and over 150 proteins: the spliceosome. For each intron to be spliced, the spliceosome is assembled de novo on its pre-mRNA substrate in a stepwise manner catalyzed by DExD/H-box ATPases, which remodel RNA-RNA and RNA-protein interactions of each complex. Spliceosome assembly begins with consecutive association of U1 and U2 snRNPs with the pre-mRNA, followed by the integration of the U4/U6.U5 tri-snRNP and subsequent release of U1 snRNP to form a pre-catalytic spliceosome, or B complex. The B complex lacks a catalytic center, and must therefore be extensively remodeled in a process called activation, to form an activated complex ( $\mathrm{B}^{\text {act }}$ complex). Spliceosome activation constitutes the largest flux in the composition of the spliceosome, with over 30 proteins being dissociated and more than 25 being integrated to form the activated complex. The activation phase is catalyzed by the $\mathrm{DExD} / \mathrm{H}$-box ATPase BRR2, which unwinds the base-pairing between U4/U6 snRNAs, leading to the dissociation of U4 snRNP and numerous proteins, and allowing for the reorganization of U6 snRNA to form intramolecular base-pairing interactions as well as intermolecular base-pairs with U2 snRNA. The resulting U2/U6 RNA-RNA network results in a triple-helix of RNA that coordinates two divalent metal cations $\left(\mathrm{Mg}^{2+}\right)$ which are involved in splicing catalysis. While structural and biochemical insights have been gleaned about both the pre-catalytic and activated states of the spliceosome, it is unknown whether structurally and compositionally distinct intermediates during the activation phase may exist. Moreover, the role of proteins in facilitating the formation of the RNA-based catalytic center at the core of the spliceosome is unclear. Using a previously identified small molecule chemical inhibitor of pre-mRNA splicing, we isolated spliceosomes stalled at intermediate stages of activation. By employing single particle cryo-EM and image classifications, we identified two novel and distinct states of the spliceosome following the release of U4 snRNP but prior to the formation of an activated complex, which we termed pre- $\mathrm{B}^{\text {act- } 1}$ and pre- $\mathrm{B}^{\text {act- } 2}$. The pre- $\mathrm{B}^{\text {act }}$ structures offer new insights into the massive exchange of proteins during activation as well as the role of these proteins in guiding the formation of the RNA-base catalytic network formed by base-pairing interactions between U2/U6 snRNAs. 


\section{Acknowledgment}

It has been a privilege to carry out my doctoral work in the Department of Structural Dynamics over the past three and a half years and I would like to convey my thanks to the people who made it a joy to come to the lab every day.

First, I thank Holger Stark for providing an excellent environment in which to carry out scientific research. From the unparalleled microscope facilities to the freedom to pursue many fascinating projects, I am truly happy to have been in such a creative and exciting lab for the past few years. I am grateful for his guidance, future-oriented thinking, and availability to discuss scientific topics at length.

I am extremely thankful to Reinhard Lührmann for allowing me the chance to work on cutting-edge topics in spliceosome structural biology. From the lectures he held during my master's curriculum, I became fascinated by the spliceosome and the possibility of studying its assembly with cryo-EM. I feel fortunate to have had countless inspiring meetings with him, during which I learned something new at each one.

I would also like to sincerely thank the members of my Thesis Advisory Committee, Henning Urlaub and Alex Stein, for their support and advice. It was always very helpful to receive feedback about ongoing projects, and to see aspects of the work from different perspectives. To Henning Urlaub I am also particularly thankful for the mass spectrometry analyses he masterfully oversees for many spliceosome projects, including the one detailed in this thesis.

In addition, I would like to extend my gratitude to members of Reinhard Lührmann's lab, the Department of Cellular Biochemistry, for their longstanding efforts to characterize difficult samples that extend our knowledge of the spliceosome. In particular, I am grateful to Dmitry Agafonov for many long and entertaining discussions about technical and theoretical aspects of spliceosome biochemistry. Furthermore, I thank Dmitry Agafonov, Majety Leelaram, and Olex Dybkov for establishing the biochemical protocol to purify and analyze the samples described in this work. Additionally, my sincere thanks go to Cindy Will and Berthold Kastner for their collaboration and guidance in completing the manuscript related to this work, as well as for deep discussions that helped me view problems from new vantage points. I am also thankful to Klaus Hartmuth, for his enthusiasm about science in general and about RNA in particular. 
Next, I thank Karl Bertram for supervising my master's thesis work, including theoretical and practical aspects of single particle cryo-EM. In addition, I am thankful to him for cryo-EM data collection of the sample detailed within in this work, and for entrusting the project to me early on in my master's thesis. It was an exciting time and I appreciate his always-upbeat attitude.

I would like to express my thanks to David Haselbach, who took the time to introduce me to cryo-EM during my lab rotation in the Department of Structural Dynamics. I was always impressed by how he took care to explain things with enthusiasm, all while driving forward his own projects and having time left over to prepare delicious cakes that the whole lab frequently enjoyed.

Very importantly, I thank my fellow officemates and friends, Zhenwei Zhang and Ka Man Yip, for contributing to a superb "feng shui" that included a constant flow of stimulating conversations about topics ranging from the day-to-day to the bizarre, as well as to a fun and energetic ecosystem in which we exchanged ideas about the finer points of cryo-EM on a daily basis. It has been an outstanding time sharing our fishtank office!

My gratitude also goes out to the original members of the (in)famous Office 113: Fabian Henneberg, Karl Bertram, Kashish Singh, and Lukas Schulte. Being a visitor in their very entertaining office during my lab rotation and master's thesis was a humorous and welcoming way to be introduced to the lab, providing me with a collection of stories I remember fondly.

I sincerely thank Dietmar Riedel for his constant willingness to share anecdotes and solutions to practical problems learned from his extensive experience in electron microscopy. It was a great time working with him, whether at the CM200, Talos, or Krios 3. Additionally, I thank Erik Schliep for practical advice in cryo-EM sample preparation and for many stimulating discussions, as well as Niels Fischer for ensuring smooth running of the microscopes.

Finally, I thank my family for their steadfast support, and especially my parents for traveling across the Atlantic multiple times to visit me. I would also like to thank my wife, Grace, for her constant companionship and encouragement. In summary, I am thankful to the many people who have enriched and made possible my time in Göttingen. 



\section{Contents}

Abstract II

Acknowledgment III

List of Tables $\quad$ IX

\begin{tabular}{|l|l}
\hline List of Figures & XI
\end{tabular}

$\begin{array}{lll}1 & \text { Introduction } & 1\end{array}$

1.1 Central Dogma of Molecular Biology . . . . . . . . . . . . . . . . . . 1

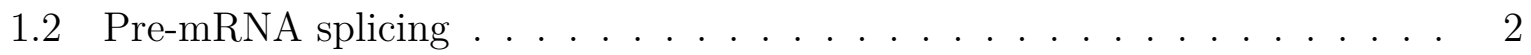

1.3 Spliceosome $\ldots \ldots \ldots \ldots \ldots \ldots \ldots$

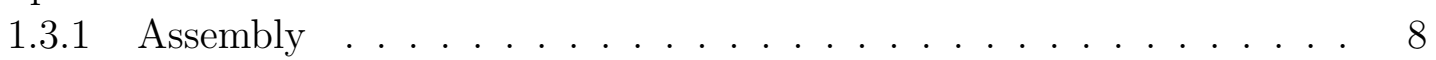

1.3 .2 Activation . . . . . . . . . . . . . . . . . . . . . 9

1.3 .3 Splicing . . . . . . . . . . . . . . . . . . . . . . 10

1.3 .4 Disassembly $\ldots \ldots \ldots \ldots$. . . . . . . . . . . . . . . . . . . . 10

1.3.5 Remodeling of the spliceosome by ATP-dependent DExD/H-box RNA helicases $\ldots \ldots \ldots \ldots \ldots \ldots \ldots \ldots$

1.4 Spliceosome activation $\ldots \ldots \ldots \ldots \ldots \ldots$

$1.4 .1 \quad$ Architecture of PRP8 and overview of its dynamics . . . . . . . . 24

1.4.2 Cryo-EM structure of the pre-catalytic spliceosome (B complex) . . 26

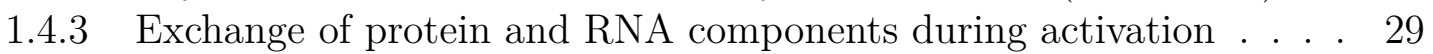

1.4.4 Cryo-EM structure of the activated spliceosome (B ${ }^{\text {act }}$ complex) . . . 30

1.4.5 Proteins surrounding the active site RNA in $\mathrm{B}^{\mathrm{act}} \ldots \ldots$. . . . . . 36

1.5 Small molecule chemical inhibitors of pre-mRNA splicing . . . . . . . . . . 38

1.6 Aim of this study $\ldots \ldots \ldots \ldots \ldots \ldots$. . . . . . . . . . . . . . . 41

2 Materials and Methods $\quad 43$

2.1 Materials . . . . . . . . . . . . . . . . . . . . . . . . 43

$2.1 .1 \quad$ Software . . . . . . . . . . . . . . . . . . . . 43

2.1.2 Equipment. . . . . . . . . . . . . . . . . . . . 44

2.2 Methods . . . . . . . . . . . . . . . . . . . . . . . . . . 44

2.2 .1 In vitro splicing . . . . . . . . . . . . . . . . . . . . . . . . . . . . . 44

$2.2 .2 \quad$ MS2 affinity purification of spliceosomes . . . . . . . . . . . . . . . 45

$2.2 .3 \quad 2 D$ gel electrophoresis and mass spectrometry. . . . . . . . . . . . . 46

2.2.4 Chase of pre- $B^{\text {act }}$ complexes with micrococcal nuclease-treated extract 46

$2.2 .5 \quad$ Western blotting . . . . . . . . . . . . . . 47

2.2.6 Cross-linking of pre-B ${ }^{\text {act }}$ complexes and cross-link identification . . 47

$2.2 .7 \quad$ Purification and buffer exchange . . . . . . . . . . . . . . . 48

2.2 .8 Cryo-EM sample preparation and data acquisition . . . . . . . . . . 48

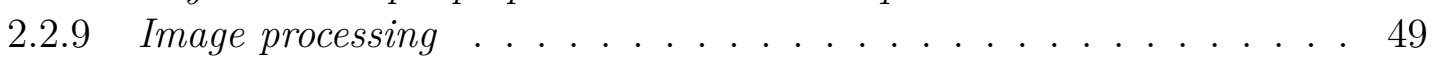

2.2 .10 Model building and refinement . . . . . . . . . . . . . . . . . 51 
3.1 Biochemical characterization of spliceosomes stalled during activation . . . 53

3.2 Cryo-EM structures of two novel activation intermediates $\ldots \ldots \ldots \ldots 56$

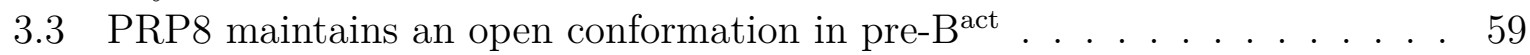

3.4 Novel repositioning of BRR2 and U2 snRNP . . . . . . . . . . . . . . 61

3.5 Recruitment of proteins at distinct stages of activation in pre- $\mathrm{B}^{\mathrm{act}} \ldots \ldots 63$

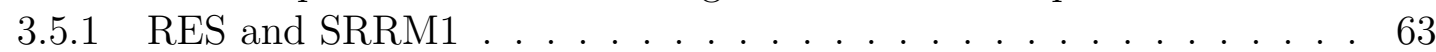

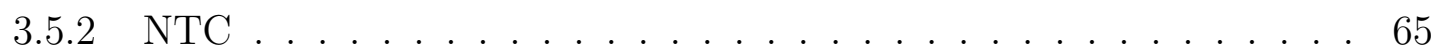

$3.5 .3 \quad$ NTR proteins $\ldots \ldots \ldots \ldots \ldots \ldots$

$3.5 .4 \quad \mathrm{IBC}$ and SYF3 $\ldots \ldots \ldots \ldots \ldots \ldots$

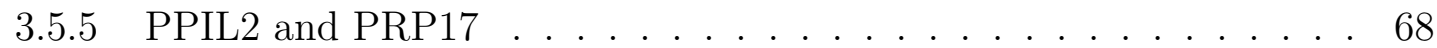

$3.6 \quad$ Identification of previously unobserved factors $\ldots \ldots \ldots \ldots \ldots \ldots$

3.6 .1 Stabilization of pre-B ${ }^{\text {act-1 }}$ by transiently interacting factors . . . . . 69

3.6.2 Coordination of a network of protein-protein interactions in pre-

$\mathrm{B}^{\text {act-2 }}$ by the DNA/RNA-binding protein KIN17 $\ldots \ldots \ldots . .72$

3.7 Early steps in the stepwise folding of the U2/U6 active site RNA $\ldots \ldots 73$

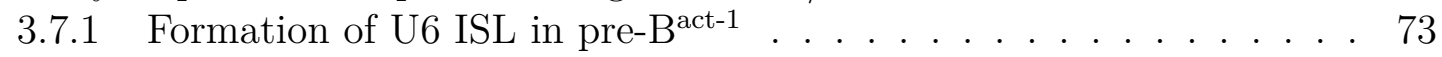

3.7 .2 Stable formation of $\mathrm{U} 2 / \mathrm{U} 6$ helix Ib in pre- $\mathrm{B}^{\text {act-2 }} \ldots \ldots \ldots \ldots .75$

$\begin{array}{lll}4 & \text { Discussion } & \mathbf{7 9}\end{array}$

$4.1 \quad$ Functional implications of large-scale dynamics of BRR2 and PRP8 $\ldots 79$

4.2 TCERG-1 and potential links to co-transcriptional RNA splicing . . . . . . 82

4.3 Mutually exclusive interactions during spliceosome activation $\ldots . . . .884$

4.3 .1 Dissociation of B-specific proteins is a prerequisite for PRP8 conformational change and integration of $\mathrm{B}^{\text {act }}$ proteins $\ldots \ldots \ldots 85$

4.3.2 $\quad$ Displacement of WBP11 from pre- $\mathrm{B}^{\text {act-1 }}$ allows for rearrangements of U6 ISL en route to a mature catalytic center . . . . . . . . . . . 88

4.3 .3 Exchange of U6 LSm for IBC, SYF3, and PPIL2 . . . . . . . . . 88

$4.3 .4 \quad$ Destabilization of KIN17 liberates PRP2 binding site . . . . . . . 90

4.4 Stabilization of proteins within the spliceosome during activation. . . . . . 92

$4.4 .1 \quad$ RES proteins: BUD13, SNU17, SNIP1 $\ldots \ldots \ldots \ldots . \ldots . \ldots 92$

$4.4 .2 \quad$ NTC binding and stabilization of U6 snRNA $\ldots \ldots \ldots \ldots . \ldots 93$

4.4 .3 NTR proteins (SKIP, RBM22) and PRP17. . . . . . . . . . . 95 95

4.4.4 PRP8 $^{\mathrm{NTDL}}$, SF3B2, SF3A2, and CWC15 closely interact with U2/U6 RNA at catalytic center $\ldots \ldots \ldots \ldots \ldots . \ldots \ldots$

$4.5 \quad$ Protein-guided folding of the U2/U6 RNA-based catalytic center . . . . . . 100

4.5 .1 Protein-RNA interactions may contribute to rearrangements of the U6 ISL stem region during activation . . . . . . . . . . . . . . . 100

4.5 .2 Conformational change of PRP8 allows for the formation of U2/U6 helix la and subsequently the triple helix $\ldots \ldots \ldots \ldots$

$4.6 \quad$ Further considerations on spliceosome activation $\ldots \ldots \ldots \ldots$. . . . . 106

4.6 .1 Energy sources governing pre-B ${ }^{\text {act }}$ transitions . . . . . . . . . . 106

4.6 .2 Directionality of spliceosome activation phase . . . . . . . . 107

4.6 .3 Alternative spliceosome assembly pathways? . . . . . . . . . . . . 108

4.6.4 Possible mechanisms by which the small molecule NSC95397 may stall spliceosome assembly . . . . . . . . . . . . . . . . . . . . 109

$4.7 \quad$ Classification of dynamic complexes in single particle cryo-EM $\ldots \ldots \ldots .112$ 
\begin{tabular}{ll}
\hline A Abbreviations & 119
\end{tabular}

\begin{tabular}{ll}
\hline B Supplementary information & 121
\end{tabular}

\begin{tabular}{lll}
\hline C & References & 187
\end{tabular} 


\section{List of Tables}

B.1 Intermolecular crosslinks. . . . . . . . . . . . . . . . . . . . . . . 121

B.2 Intramolecular crosslinks. . . . . . . . . . . . . . . . . . . . 137 


\section{List of Figures}

1.1 Pre-mRNA splicing reactions. . . . . . . . . . . . . . . . . . . . . . . . . . . . 3

1.2 Alternative splicing patterns. . . . . . . . . . . . . . . . . . . . . . 4

1.3 Composition of human spliceosomal snRNPs. . . . . . . . . . . . . . . . . . . . 6

1.4 Splicing cycle. . . . . . . . . . . . . . . . . . . . . . . . . . 12

$1.5 \quad$ RNA remodeling events during assembly and activation. . . . . . . . . . . . . . 14

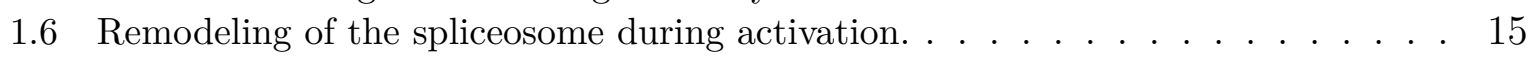

1.7 Folding of catalytic RNA in the spliceosome. . . . . . . . . . . . . . . . 17

1.8 Comparison of triple helix in Group IIB introns and spliceosomal snRNA. . . . . 18

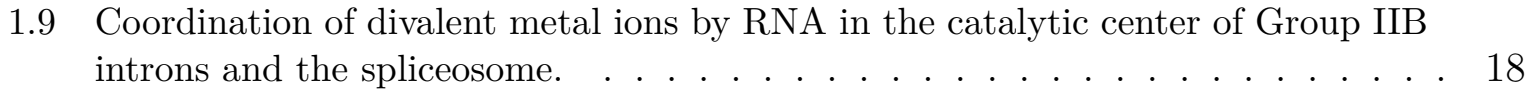

1.10 Domain architecture of PRP $8 . \ldots \ldots \ldots \ldots \ldots$

1.11 PRP8 conformational change during spliceosome assembly, activation, and splic-

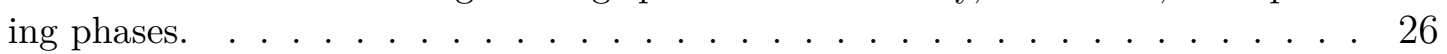

1.12 Protein and RNA exchanges during activation. . . . . . . . . . . . . . . . . 29

1.13 Structural changes involved in B-to-B ${ }^{\text {act }}$ transition. . . . . . . . . . . . . . 30

1.14 Retention and Splicing (RES) complex. . . . . . . . . . . . . . . . . . . . 31

1.15 NineTeen Complex (NTC) architecture. . . . . . . . . . . . . . . . . . . . . 32

1.16 Intron Binding Complex (IBC) and SYF3. . . . . . . . . . . . . . . . . 35

3.1 Biochemical characterization of pre-B ${ }^{\text {act }} . \ldots \ldots \ldots \ldots \ldots$. . . . . . . . 55

3.2 Image processing and reconstruction of cryo-EM data. . . . . . . . . . . . . . . 57

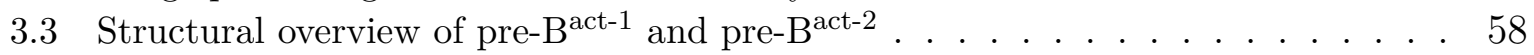

$3.4 \quad$ PRP8 is held in an open conformation by B-specific proteins in pre- ${ }^{\text {act }}$. . . . . . 59

$3.5 \quad \mathrm{PRP}^{\mathrm{RH}}$ and $\mathrm{PRP} 8^{\mathrm{Jab} 1}$ occupying structurally unique positions in both states of

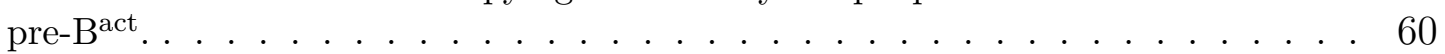

$3.6 \quad$ BRR2 is repositioned to a novel orientation in pre-B ${ }^{\text {act }} . \ldots$. . . . . . . . . . 61

3.7 Repositioning of BRR2 and docking of U2 snRNP during activation. . . . . . . 62

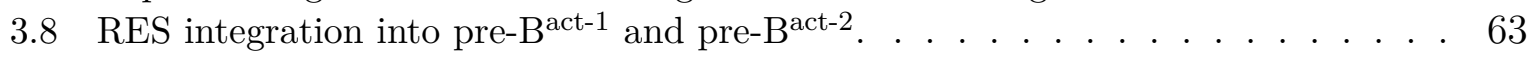

$3.9 \quad$ NTC integration into pre-B ${ }^{\text {act-1 }}$ and pre-B ${ }^{\text {act-2 }}$. . . . . . . . . . . . . . . 65

3.10 NineTeen complex Related (NTR) proteins. . . . . . . . . . . . . . . 66

3.11 Mutually exclusive interactions of LSm proteins and the IBC/SYF3. . . . . . . 68

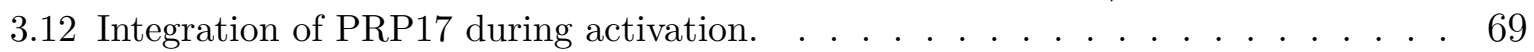

3.13 Localization of TCERG-1, CBP80/20, SRSF1 ${ }^{\text {RRM2 }}$ in pre-B ${ }^{\text {act-1 }}$. . . . . . . . . 70

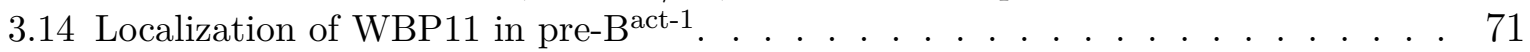

3.15 KIN17 binds transiently in pre-B ${ }^{\text {act-2 }}$. . . . . . . . . . . . . 73

3.16 Reorganization of U2/U6 snRNA upon unwinding of U4 snRNA by BRR2. . . . 74 \begin{tabular}{|l}
\hline 3.17 Unique conformations of U6 ISL in pre-B ${ }^{\text {act }}$ and proposed alternative base-pairing \\
\hline scheme. $\ldots \ldots \ldots \ldots \ldots \ldots$
\end{tabular}

3.18 Establishment of catalytic center through interaction of U2/U6 snRNAs. . . . . 76

3.19 EM densities of RNA structures involved in formation of the active site. . . . . . 77

$4.1 \quad$ Structuring of proteins surrounding catalytic center during activation. . . . . . . 98

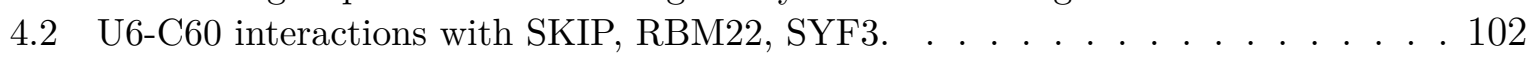

$4.3 \quad$ Assembly pathway of pre- $\mathrm{B}^{\text {act-1 }}$ and pre-B ${ }^{\text {act-2 }}$ during activation. . . . . . . . . . 105 
B.1 Data collection and model refinement statistics. . . . . . . . . . . . . . . . 185

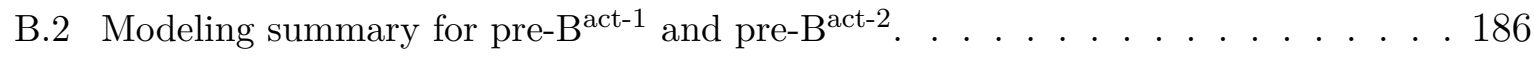





\section{Introduction}

\subsection{Central Dogma of Molecular Biology}

In all domains of life, genetic information is encoded in DNA, which forms the set of molecular instructions required for an organism to function. DNA is transcribed into RNA, which can then be translated into proteins that carry out specific roles in the cell. Gene expression was long thought to be a unidirectional flow of information that could be described as DNA $->$ RNA $->$ protein. This basic schema describing the conversion of DNA instructions into functional proteins was formulated in the mid-20th century and formed what Francis Crick termed the "Central Dogma of Molecular Biology" [1]. Towards the latter half of the 20th century, it was discovered that large segments of transcribed RNA served no clear functional role in eukaryotes. Genetic and biochemical studies indicated that much more RNA was present than was strictly required for the synthesis of the encoded proteins. Surprisingly, it was found that in many organisms, large segments of noncoding RNA are removed from the newly transcribed precursor messenger RNA (premRNA), before mature mRNA is translated into protein by the ribosome. Genes bearing this characteristic were termed "split genes," to describe how coding regions are initially separated from one another by noncoding regions [2]. Coding regions were termed "exons" and remain in the RNA template to form mRNA, whereas noncoding regions were termed "introns," and are excised prior to the formation of the mature mRNA [3]. The molecular process by which exons are ligated and introns are removed was termed "splicing" [4, 5]. 


\subsection{Pre-mRNA splicing}

All nuclear pre-mRNAs have characteristic sequence features: a $5^{\prime}$ and $3^{\prime}$ splice site (ss) and a branch site (BS) (Fig. 1.1). The 5'ss and 3'ss separate the intron from the $5^{\prime}$ and $3^{\prime}$ exons and consist of a conserved sequence of $\mathrm{GU}$ and $\mathrm{AG}$ in $>95 \%$ of introns [6] 8 ]. In a minor class of introns in higher eukaryotes, but not in simpler organisms such as $S$. cerevisiae, the $5^{\prime}$ and $3^{\prime}$ splice sites are demarcated by $\mathrm{AU}$ and $\mathrm{AC}[9$, 10]. In all types of pre-mRNAs, an adenosine nucleotide at the branch site (BS-A) is located within the intron and is the nucleophile in the first step of splicing (Fig. 1.1). Nuclear pre-mRNA splicing is carried out in two $\mathrm{S}_{\mathrm{N}} 2$-type transesterification reactions: Step 1, or branching; and Step 2, or exon ligation (Fig. 1.1). In Step 1, a 2'OH group of the BS-A carries out an $\mathrm{S}_{\mathrm{N}} 2$ nucleophilic attack at the $5^{\prime}$ end of the intron, looping the intron into an "intron lariat" [11-14. In Step 2, the $3^{\prime} \mathrm{OH}$ of the terminal $3^{\prime}$ nt of the $5^{\prime}$ exon carries out an $\mathrm{S}_{\mathrm{N}} 2$ attack the $5^{\prime}$ end of the $3^{\prime}$ exon, ligating the two exons while leading to the dissociation of the intron [15, 16]. The ligated exons form a messenger RNA (mRNA) that can exported to the cytoplasm to be translated into polypeptides by the ribosome [17].

After a gene is transcribed into pre-mRNA, it can be cleaved into numerous potential mRNA products [18, 19]. For example, a single pre-mRNA sequence can be processed to generate thousands of protein isoforms in some cases; for example, one gene in Drosophila melanogaster can code for up to 38,000 mRNAs [20, 21]. This phenomenon is known as "alternative splicing" and explains how relatively simple genomes can give rise to complex proteomes in eukaryotes [22]. In humans, over $90 \%$ of genes containing more than one exon are alternatively spliced [23]. Alternative splicing in lower eukaryotes (e.g., S. cerevisiae) has been documented but does not occur to the same extent as in higher eukaryotes [24 27]. Thus, alternative splicing is a means by which cells in higher eukaryotes such as humans can produce hundreds of thousands of unique isoforms while containing a genome of approximately 20,000 protein-coding genes [28]. The principal patterns of alternative splicing are intron retention, exon skipping, variable splice site usage, and mutual exclusion of exons (Fig. 1.2] [22]. 

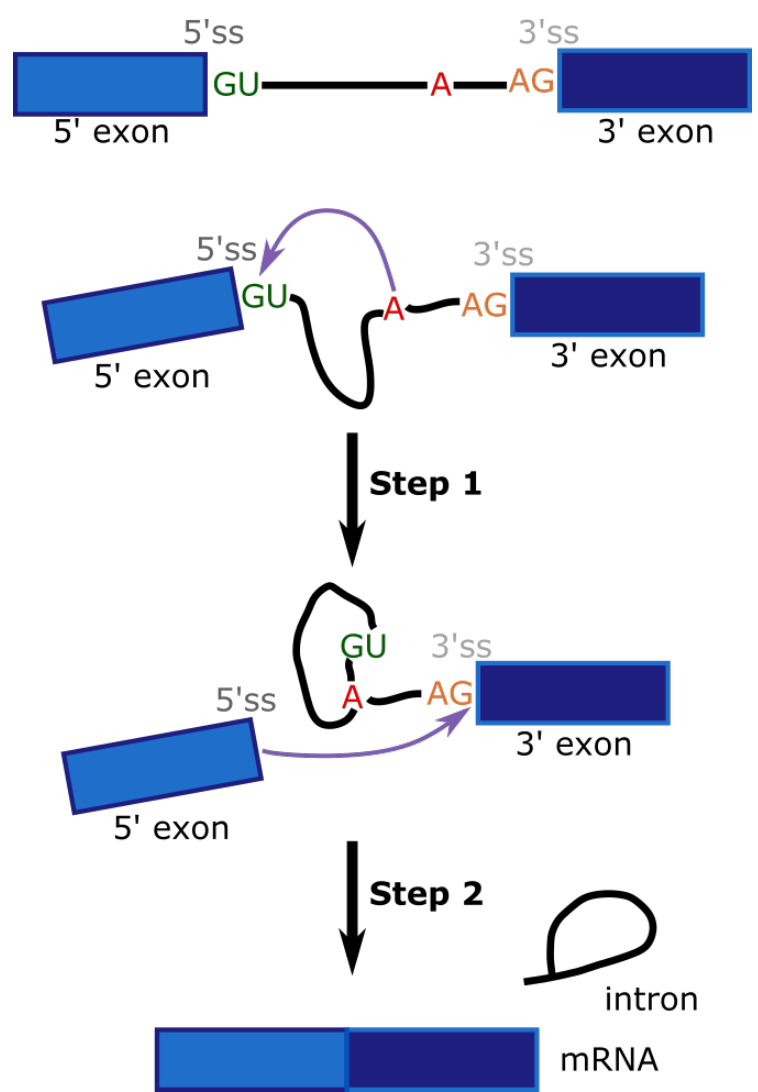

Fig. 1.1: Pre-mRNA splicing reactions. The top panel depicts the basic structure of a nuclear pre-mRNA, where the $5^{\prime}$ and $3^{\prime}$ exons are depicted as colored rectangles while the intron is shown as a solid line. Constitutive sequence elements at the 5'ss (GU) and $3^{\prime}$ ss (AG) are colored green and orange. The BS-A nucleotide is colored red. In Step 1 of splicing, the $2^{\prime} \mathrm{OH}$ of the BS-A attacks the $5^{\prime}$ ss in an $\mathrm{S}_{\mathrm{N}} 2$-type reaction, resulting in the formation of an intron lariat. In Step 2 of splicing, the $3^{\prime}$ end of the $5^{\prime}$ exon attacks the $3^{\prime}$ ss, ligating the two exons and allowing for the removal of the intron lariat.

Intron retention occurs when an intron is not excised from the pre-mRNA and thus becomes part of the final mRNA template. Exon skipping is the excision of exons from the pre-mRNA that could otherwise be translated into protein. Variable splice site usage is the recognition of a non-consensus intron-exon boundaries. Mutual exclusion of exons is removal of certain exons and the retention of corresponding ones, and vice versa. Alternative splicing patterns are regulated by multiple mechanisms, including binding of proteins to the pre-mRNA which negatively or positively effect the expression of the gene on which they act [22]. Alternative splicing is regulated in a tissue-specific manner, facilitating the development of highly differentiated cell types in multicellular organisms [30]. Aberrant alternative splicing is observed in many diseases, including several cancers, and can result from mutations within the splicing machinery or the gene to be expressed [31]. 
Alternative 5' Splice Sites

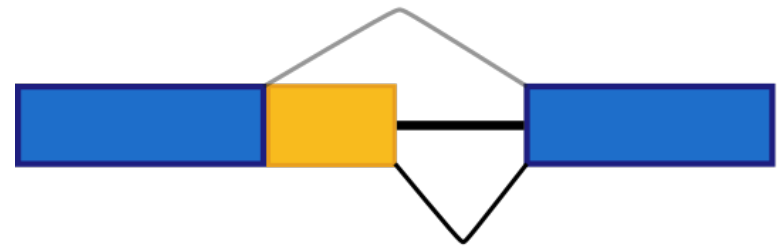

Alternative 3' Splice Sites

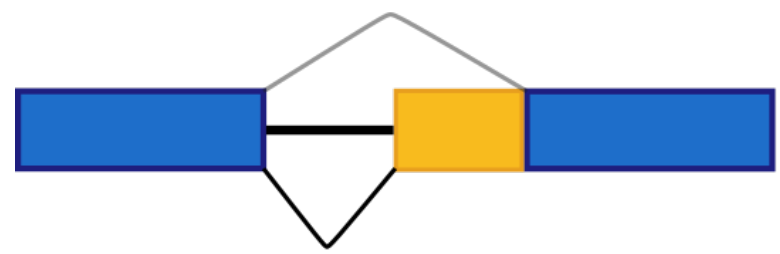

\section{Exon Exclusion}

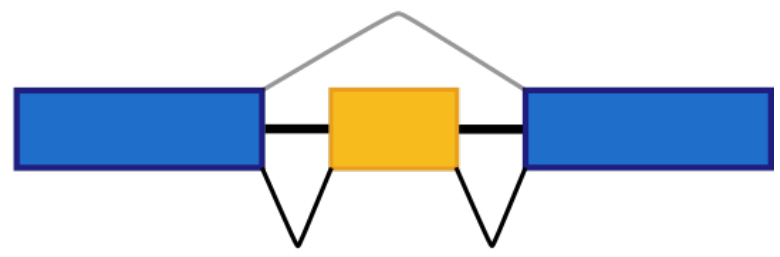

Intron Retention

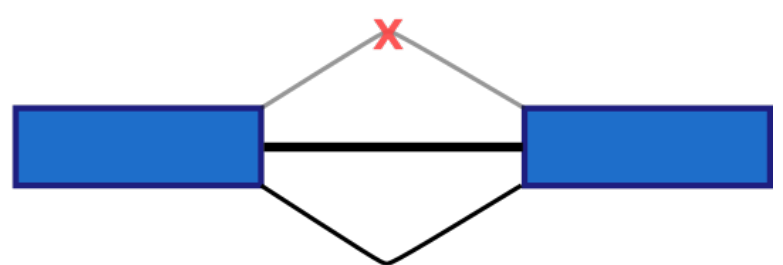

\section{Mutually Exclusive Exons}

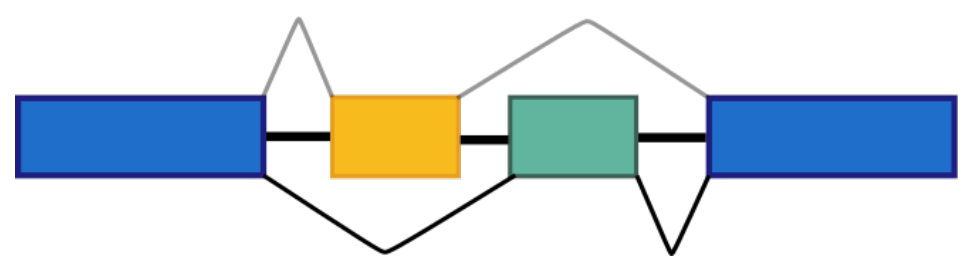

Fig. 1.2: Alternative splicing patterns. Exons are depicted as rectangles and introns as thin black lines. Blue rectangles indicate constitutively spliced exons whereas yellow or teal rectangles demonstrate exons that are included in the mRNA as a result of alternative splicing. Bent gray or black lines indicate the region that is excised. Adapted from [29].

Pre-mRNA splicing is heavily regulated by serine-arginine (SR) rich proteins and heterogeneous nuclear ribonucleoproteins (hnRNPs) that influence where splicing machinery associates to the pre-mRNA [32, 33]. SR proteins typically enhance while hnRNPs generally repress splicing of a specific transcript, although both classes of proteins can bind to the pre-mRNA at splicing enhancers or splicing silencers [34, 35]. Introns have been shown to be coated with hnRNPs, but whether hnRNPs always bind in a sequence-specific manner or as preformed complexes is not well understood [36 38].

Sequence elements of the pre-mRNA called enhancers and silencers provide binding sites for proteins that positively or negatively regulate splicing by influencing the recognition of splice sites and the assembly of the splicing machinery at specific positions [39]. An enhancer or silencer is found within the intron or the exon, and is thus termed an "intronic splicing enhancer/silencer" (ISE/ISS) or "exonic splicing enhancer/silencer" (ESE/ESS) [23]. 


\subsection{Spliceosome}

Both steps of pre-mRNA splicing are catalyzed by RNA within a multi-megadalton (up to ca. $4 \mathrm{MDa}$, with it longest axis spanning approximately $400 \AA$ ) ribonucleoprotein (RNP) complex comprised of five small nuclear RNAs (snRNAs) and over 150 proteins, the spliceosome. The spliceosome is a massive molecular machine consisting of 5 small nuclear RNPs (snRNPS): U1, U2, U4, U5, and U6 (Fig. 1.3.). In addition, some higher eukaryotes have a minor spliceosome in which $\mathrm{U} 1, \mathrm{U} 2, \mathrm{U} 4$, and $\mathrm{U} 6$ snRNPs are replaced for U11, U12, U4atac, and U6atac snRNPs [9, 10]. All spliceosomal snRNPs contain several shared characteristics: uridine-rich small nuclear RNA (U snRNA), a heptameric beta-propeller Sm core, and associated proteins [40].

Biogenesis of spliceosomal snRNPs begins with the transcription of snRNA by RNA polymerase II (Pol II) for all snRNPs except U6 snRNA, which is transcribed by RNA polymerase III [42]. Pol II-transcribed snRNAs are modified by the addition of a $5^{\prime}$ 7-methylguanosine $\left(\mathrm{m}^{7} \mathrm{G}\right)$ cap and $3^{\prime}$ polyadenylation before being exported to the cytoplasm through the nuclear pore complex [43, 44]. In contrast, U6 and U6atac snRNAs are not exported to the cytoplasm, but are instead assembled into functional snRNPs entirely within the nucleus [45, 46].

Pol II-transcribed snRNPs associate with a heptameric complex consisting of B/B', D1, D2, D3, E, F, G Sm proteins [47, 48]. This complex is called an Sm core and forms a doughnut-shaped beta-propeller that binds to snRNAs at specific RNA sequences consisting of PuAU4-6GPu [49]. Sm-bound snRNAs are subsequently bound by additional proteins and their $\mathrm{m}^{7} \mathrm{G}$ cap is hypermethylated to form 2,2,7-tri-methyl guanosine $\left(\mathrm{m}^{3} \mathrm{G}\right)$ and the $3^{\prime}$ end of each snRNA is shortened by ribonucleases [50 52]. The Sm core and $\mathrm{m}^{3} \mathrm{G}$ cap together form a nuclear localization signal which is recognized by protein factors that aid in the import of the particle into the nucleus [53, 54]. Additional snRNP proteins are imported separately into the nucleus and join the Sm core-bound snRNA to form a mature snRNP [55, 56]. U6 and U6atac snRNAs are not bound by Sm proteins, 

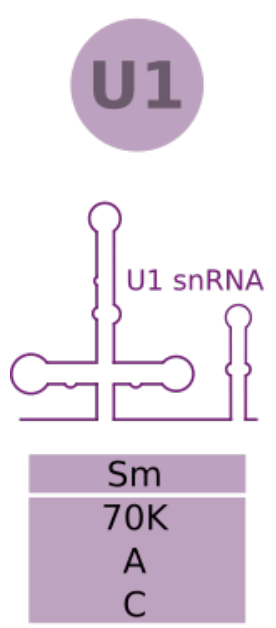

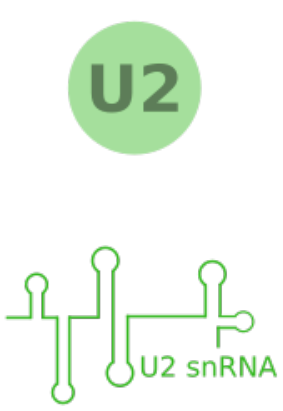

\begin{tabular}{|c|}
\hline Sm \\
\hline$A^{\prime}$ \\
$B^{\prime \prime}$ \\
SF3A1 \\
SF3A2 \\
SF3A3 \\
SF3B1 \\
SF3B2 \\
SF3B3 \\
SF3B4 \\
SF3B5 \\
SF3B6 \\
PHF5A \\
\hline
\end{tabular}

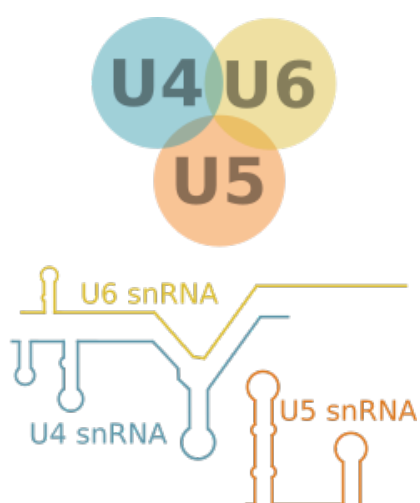

\begin{tabular}{|c|}
\hline Sm \\
\hline PRP3 \\
PRP4 \\
PPIH \\
PRP31 \\
SNU13 \\
\hline LSm \\
\hline Sm \\
\hline PRP8 \\
BRR2 \\
SNU114 \\
\hline U5-40K \\
PRP6 \\
DIM1 \\
\hline SNU66 \\
SAD1 \\
\hline
\end{tabular}

Fig. 1.3: Composition of spliceosomal snRNPs. The protein and snRNA inventory of spliceosomal snRNPs is depicted. Small nuclear ribonucleoproteins (snRNPS) consist of one or more small nuclear RNA (snRNA) bound by numerous proteins. U1 and U2 snRNPs exist as individual particles and associate to the spliceosome in a stepwise manner, yet the U4, U5, and U6 snRNPs are packaged into a triple-snRNP (U4/U6.U5 tri-snRNP) in which U4 and U6 snRNAs are base-paired and associated to U5 snRNP via protein-protein interactions. U2 snRNP contains the SF3a and SF3b complexes, which account for the majority of the molecular weight of the particle. Each snRNA is bound to an Sm core, with the exception of U6 snRNA, which binds a similar heptameric, doughnut-shaped LSm core. U1 snRNP is the smallest snRNP of the spliceosome and is responsible for recognition of the $5^{\prime}$ ss to form the E complex. U2 snRNP recognizes and the BS-A and base-pairs with the pre-mRNA to form the U2/BS helix, in which the BS-A is flipped out. U2 and U6 snRNPs form the catalytic RNA at the core of the spliceosome, while U5 contributes to proper positioning of the pre-mRNA ( $5^{\prime}$ ss). The snRNPs shown here are joined by many additional proteins during the assembly and function of the spliceosome, but snRNPs form the core structure of the complex. Adapted from [41].

but instead associate with Sm-like proteins that also form a heptameric ring structure:

LSm2-8 (or "LSm proteins") [57].

Spliceosomal snRNAs undergo extensive post-transcriptional modifications, including pseudouridylation and methylation [58, 59]. In addition to its constitutive $5^{\prime}$ tri-methylguanosine cap, U2 snRNA contains at least 13 pseudouridines and 10 methyl groups [60]. In U2 snRNA, a pseudouridine near the branch site was shown to affect positioning of the BS-A 
for Step 1 of splicing [61]. All snRNAs of the major spliceosome have been reported to undergo pseudouridylation and methylation [59].

Pre-mRNAs are also post-transcriptionally modified, which influences gene expression by altering the usage of splice sites. RNA modifications have been documented to control pre-mRNA splicing by altering how the spliceosome recognizes splice sites [62]. The enzyme double-stranded RNA-specific editase 1 (ADAR2), which converts adenosine to inosine, was shown to convert the sequence AA (adenosine-adenosine) to AI (adenosineinosine) within the intronic region of its own pre-mRNA [62, 63]. AI can be substituted for the conserved AG dinucleotide of the 3 'ss, and therefore caused the newly substituted inosine to demarcate an alternative $3^{\prime}$ ss $[62,64]$. This results in alternative splicing of the ADAR2 pre-mRNA, generating multiple isoforms of the enzyme [62, 65]. It was recently demonstrated that pre-mRNA is chemically modified in vivo in eukaryotes; adenosine methylation $\left(\mathrm{m}^{6} \mathrm{~A}\right)$ of the AG dinucleotide at the $3^{\prime}$ ss prevents binding of the protein U2AF35, which is involved in recognition of the 3 'ss and early assembly of the spliceosome [66]. Thus modification of the pre-mRNA can alter splice site choice, thereby regulating alternative splicing.

Assembly of the spliceosome and splicing of some pre-mRNAs occurs during transcription, and is thus a co-transcriptional process [67]. The spliceosome begins to assemble and splice pre-mRNAs as they are synthesized; that is, before they are released from Pol II and the rest of the transcription machinery [68]. U1 snRNP recognizes the 5'ss on the pre-mRNA, followed by binding of U2AF, U2 snRNP, and other splicing factors that identify the BS and the 3'ss [69, 70]. U1 snRNP also has been shown to play a role in regulating transcription by preventing premature transcription termination in a process termed "telescripting", in which U1 bound to the pre-mRNA blocks cleavage and polyadenylation at cryptic sites [71, 72]. Early insights into the structural basis of U1 snRNP's interactions with Pol II have recently been reported, outlining the molecular architecture of portions of the co-transcriptional machinery [73]. Transcription factors are also known to associate with the spliceosome, suggesting that parts of the transcription apparatus may also regulate constitutive and alternative splicing [74 76$]$. 
Splicing is also heavily regulated by post-translational modifications of core spliceosomal proteins or proteins that bind to enhancer/silencer regions in the pre-mRNA. Posttranslational modifications of spliceosomal proteins regulate spliceosome function at all stages of assembly and function [77]. Protein phosphorylation is perhaps the best understood post-translation modification in the spliceosome, and it can promote or repress splicing, while also influencing which splice sites are utilized (i.e., regulation of alternative splicing) [78, 79]. The best documented class of post-translational modifications in the spliceosome are those of SR proteins [80], although the SF3B1 protein of the spliceosomal $\mathrm{U} 2$ snRNP is also known to undergo phosphorylation [81]. Acetylation has been proposed to be a regulator of pre-mRNA splicing, although understanding of the role of this modification is still in its early stages [82, 83]. Finally, ubiquitination was demonstrated to play a role in spliceosome assembly by binding to PRP8 (the core scaffold protein of the spliceosome) and preventing the premature dissociation of U4 snRNP [84].

\subsubsection{Assembly}

For each round of splicing, the spliceosome is assembled anew on its pre-mRNA substrate (Fig. 1.4), a process entailing major rearrangements in the molecular architecture and composition of the complex. The functional cycle of the spliceosome can be divided into four main phases: assembly, activation, splicing, and disassembly (Fig. 1.4). During the assembly phase, U1 snRNP base-pairs to the 5'ss to form the E complex (where "E" stands for "Early") [85, 86]. Base-pairing of U2 snRNA with a region of the intron containing the branch site (BS) forms the pre-spliceosome, or "A complex" [87, 88]. The interaction between U2 snRNA and the pre-mRNA forms a helix known as the U2/BS helix, which remains intact for both steps of splicing. The U4/U6.U5 tri-snRNP is integrated into the A complex to form the pre-B complex, which contains all 5 snRNPs of the spliceosome [89]. In the pre-B complex, a connection is established between the $\mathrm{U} 2$ snRNP and the U4/U6.U5 tri-snRNP via base-pairing of the $5^{\prime}$ end of U2 snRNA and the $3^{\prime}$ end of U6 snRNA, which forms a duplex, U2/U6 helix II. This short helix is bound by the U6 LSm 
proteins and anchors the highly flexible U2 snRNP to the body of the tri-snRNP. Once established, helix II remains intact throughout the remainder of the splicing cycle.

At this stage the $5^{\prime}$ 'ss of the intron, which is still base-paired to U1 snRNA, must be handed off to the U5 and U6 snRNAs, allowing for the formation new base-pairing interactions that anchor the $5^{\prime}$ ss in place for Step 1 of splicing (Figs. 1.1] and 1.5] [90]. An invariant sequence of ACAGA(GA) in U6 snRNA base-pairs to the region immediately downstream of the $5^{\prime}$ ss, forming the U6/5'ss helix. The hand-off of the $5^{\prime}$ ss results in the dissociation of U1 snRNP and the formation of the B complex, or precatalytic spliceosome (Fig. 1.4.

\subsubsection{Activation}

The B complex, while containing all of the snRNPs and pre-mRNA elements needed for splicing to occur, does not yet contain an RNA-based catalytic center (Fig. 1.5). For this to occur, U4 snRNP must be dissociated from the complex, allowing for large-scale structural rearrangements to take place (Fig. 1.5 and 1.6.).

$\mathrm{U} 4$ and $\mathrm{U} 6$ are extensively base-paired in the B complex and this interaction must be therefore disrupted for an activated complex to be formed. An ATP-dependent RNA helicase, BRR2, is involved in mediating the dissociation of U4 from U6 snRNA, freeing U6 snRNA to base-pair with itself and with U2 snRNA to form an internal stem-loop (ISL) and two short helices that are essential for pre-mRNA splicing (Figs. 1.7, 1.8, 1.9) [91 93].

This transition from a precatalytic to an activated spliceosome is termed the "activation" phase and constitutes the largest flux in protein and RNA composition of the entire splicing cycle, with approximately 60 proteins being exchanged in humans (32 proteins dissociated 1 , 28 incorporated) [94, 95]. Many of the proteins that are exchanged are not part of snRNPs, but are rather individual proteins or pre-organized subcomplexes that are dissociated from or integrated to the spliceosome. Activation results in the $\mathrm{B}^{\text {act }}$

\footnotetext{
${ }^{1}$ U4 Sm and U6 LSm complexes contain seven individual polypeptides each
} 
complex - containing U2, U5, and U6 snRNPs, all of which remain in the spliceosome until splicing is complete - which contains a fully formed catalytic center (Figs. 1.7 1.8, 1.9. However, the $\mathrm{B}^{\text {act }}$ is not catalytically active, and must be further remodelled to liberate the BS-A from the U2 snRNP-associated SF3a and SF3b proteins (Fig. 1.4] [96]. Structural rearrangements lead to the formation of a catalytically active complex, "B*," which is poised to carry out Step 1 of splicing (Fig. 1.4] [96, 97].

Rearrangements in base-pairing during activation also bring the BS-A and 5'ss progressively into much closer spatial proximity en route to intermolecular distances at which splicing can be catalyzed. In the B complex, the BS-A is separated from the $5^{\prime}$ ss by approximately $150 \AA$ [98]. In contrast, this distance is shortened to about $50 \AA$ in the $\mathrm{B}^{\text {act }}$ complex [99], although the BS-A remains sequestered within the SF3B1 protein of the U2 snRNP and is therefore inhibited. The distance between the BS-A and 5"ss is subsequently shortened to approximately $4 \AA$ in the catalytically activated B* complex (as documented in S. cerevisiae, which has a highly similar catalytic center to that of humans) immediately prior to Step 1 of splicing [100].

\subsubsection{Splicing}

Step 1 of splicing results in the C complex, in which an intron loop or "lariat" is formed (Figs. 1.1 and 1.4] [101]. Exon 1 and 2 are positioned into closer proximity to prepare for exon ligation, or Step 2 of splicing, in a complex termed $\mathrm{C}^{*}$, which is catalytically active (Figs. 1.1] and 1.4] [15, 102].

\subsubsection{Disassembly}

Completion of Step 2 of splicing results in a post-catalytic spliceosome, or P complex, in which Exon 1 and 2 have been ligated, but the splicing machinery and intron are still present [103]. The P complex is dissociated into a messenger RNA particle (mRNP) and a complex comprised of $\mathrm{U} 2, \mathrm{U} 5$ and $\mathrm{U} 6$ snRNPs plus the intron lariat, which together 
form an intron lariat spliceosome (ILS) [104]. The ILS is subsequently disassembled and recycled whereas the mRNA can be exported to the cytoplasm to be translated into protein by the ribosome.

\subsubsection{Remodeling of the spliceosome by ATP-dependent DExD/H-box RNA helicases}

Transitions throughout the splicing cycle are driven by eight ATP-dependent RNA helicases known as DExD/H-box ATPases [106], named after a conserved sequence of AspGlu-x-Asp/His ("D-E-x-D/H") residues [107]. Several DExD/H-box helicases utilize the energy from ATP hydrolysis to disrupt RNA basepairing (Fig. 1.5) [108]. DExD/H-box helicases have both remodeling and proofreading functions in pre-mRNA splicing and are required for assembly and disassembly of the spliceosome [109].

Two DEAD-box helicases, UAP56 and PRP5, are involved in the integration of the U2 snRNP onto the pre-mRNA, at which step a branch-site stem loop (BSL) of U2 snRNA is unwound to allow for base-pairing with the BS of the intron, forming the A complex 110 112.

Following the binding of the U4/U6.U5 tri-snRNP to form the pre-B complex, the DEADbox helicase PRP28 facilitates dissociation of base-pairing between U1 snRNA and the $5^{\prime}$ ss, permitting the destabilization of U1 snRNP as well as the transfer of the $5^{\prime}$ ss to loop I of U5 snRNA [90, 113, 114]. This results in the pre-catalytic (B complex), which still does not yet contain a catalytic center.

The DExD/H-box helicase BRR2 is involved in unwinding the U4/U6 duplex, resulting in the dissociation of U4 snRNP and many associated proteins, while freeing the U6 snRNA to form the internal stem-loop (ISL) as well as helix Ia and helix Ib with U2 snRNA, resulting in a mature catalytic center of the activated spliceosome, or B ${ }^{\text {act }}$ (Fig. 1.6 and 1.7] [91, 115]. PRP2 is involved in proofreading the assembly of the RNP complex, 


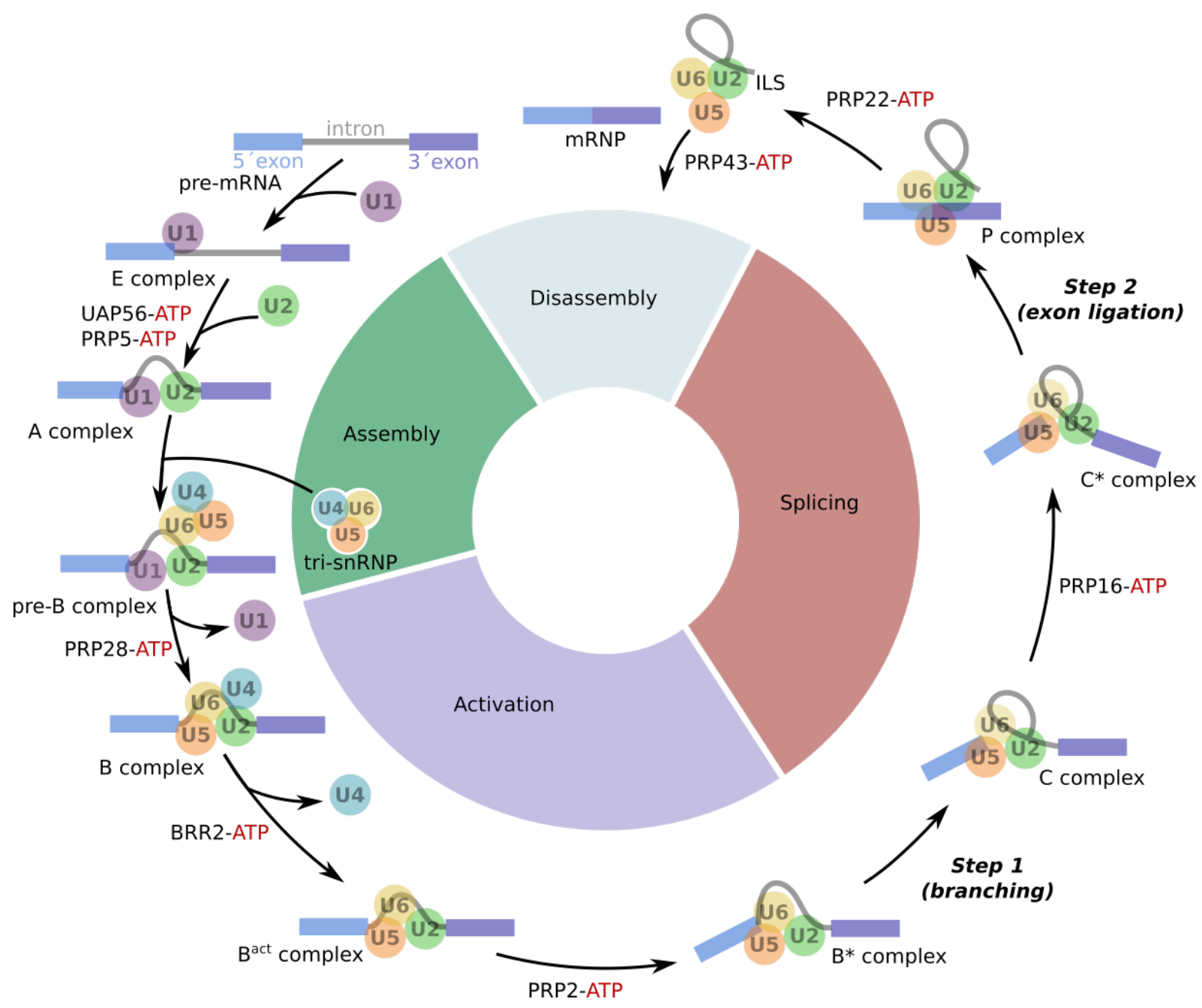

Fig. 1.4: Splicing cycle. The 4 phases of the spliceosome's cycle - assembly, activation, splicing, and disassembly - are shown by the colored wheel. ATP-dependent steps involving DExD/H-box helicases are indicated. ATP hydrolysis provides the "driving force" that catalyzes the forward progression of the splicing cycle. Some DExD/H-box helicases exert their function in the spliceosome by rearranging protein-RNA interactions instead of unwinding RNA helices. During the assembly phase, U1 snRNP recognizes the 5'ss, and U1 snRNA base-pairs to the pre-mRNA to form the E complex. Two DEAD-box helicases, UAP56 and PRP5, facilitate the binding of U2 snRNP to the pre-mRNA, where U2 snRNA recognizes and base-pairs with the region surrounding the BS-A to form the A complex, sequestering the BS-A within the HEAT repeats of the SF3B1 protein of the U2 snRNP. In the final stage of assembly, the U4/U6.U5 tri-snRNP is loosely docked to the A complex to generate the pre-B complex, containing all 5 spliceosomal snRNPs. Unwinding of base-pairing between the U1/5'ss interaction (assisted by the DEAD-box helicase PRP28) allows for the release of U1 snRNP and the transfer of the 5 'ss to U5 snRNA. At this point, the invariant ACAGA(GA) sequence of U6 snRNA base-pairs to the intron downstream of the $5^{\prime} \mathrm{ss}$, forming the $\mathrm{U} 6 / 5^{\prime} \mathrm{ss}$ helix, resulting in the $\mathrm{B}$ complex, or pre-catalytic spliceosome. The activation phase begins with the unwinding of the U4/U6 snRNA duplex (mediated by DExD/H-box helicase BRR2), triggering the removal of U4 snRNP as well as a massive change in the RNA and protein architecture of the spliceosome to generate a $\mathrm{B}^{\text {act }}$ complex, or activated spliceosome, which contains a fully formed, RNA-based catalytic center. Activity of the DEAH-box helicase PRP2 is involved in the removal of the SF3a and SF3b complexes of the U2 snRNP, freeing the BS-A to carry out Step 1 (branching) of splicing in the $\mathrm{B}^{*}$ complex. The resulting $\mathrm{C}$ complex is catalytically activated following the action of the DEAH-box helicase PRP16, generating the $\mathrm{C}^{*}$ complex, which carries out Step 2 (exon ligation) of splicing. The postcatalytic spliceosome (P complex), containing the ligated exons as well as the intron lariat, is dissociated following the action of DEAH-box helicase PRP22, freeing the mRNA and associated proteins (mRNP) and intron lariat spliceosome (ILS). The ILS is broken down into individual snRNPs and the intron lariat in a process mediated by PRP43 (a DEAH-box helicase), completing the disassembly phase. The mRNA is exported to the cytoplasm to be translated by the ribosome. Adapted from [41, 105]. 
leading to rearrangements that bring about a catalytically activated spliceosome $\left(\mathrm{B}^{*}\right)$ which carries out Step 1 of splicing, resulting in the C complex [116].

Following Step 1 of splicing, PRP16 is involved facilitating dissociation of the proteins YJU2 and CWC25, allowing for structural rearrangements of the reactants within the catalytic center [117, 118]. Following the action of PRP16, the catalytically activated Step 2 spliceosome ( ${ }^{*}$ complex) is formed, poised to carry out exon ligation [15, 102].

PRP22 is implicated in dissociating the postcatalytic spliceosome ( $\mathrm{P}$ complex) into an $\mathrm{mRNP}$ and intron lariat spliceosome (ILS), preparing the former to be exported from the nucleus and the latter to be further processed [119, 120]. Finally, PRP43 catalyzes the disassembly of the ILS, allowing U2/U6 and U5 snRNPs and associated components to be recycled [121]. Thus the major rearrangements in the RNA-RNA and RNA-protein interactions of the spliceosome are driven by DExD/H-box helicases, which use the energy of ATP hydrolysis to drive the splicing cycle forward, albeit through mechanisms that remain largely unclear.

Nearly all DExD/H-box proteins involved in splicing associate transiently with the spliceosome, although the U5 snRNP-associated helicase, BRR2, remains bound to the core spliceosomal machinery throughout the entire splicing cycle [122]. Moreover, BRR2 is repositioned throughout the entire splicing cycle [122]. Prior to activation, BRR2 docks onto U4 snRNA (see also 1.4.2). As BRR2 is responsible for the activation step, it must be tightly regulated to prevent premature unwinding of the U4/U6 duplex, involving many protein-protein interactions that either physically separate BRR2 from premature loading onto its substrate or that block the RNA binding channel of the complex [123-126]. Disruption of U4/U6 base-pairing triggers the most drastic change in RNA-RNA network of the spliceosome, paving the way for activation to occur (Fig. 1.5. 

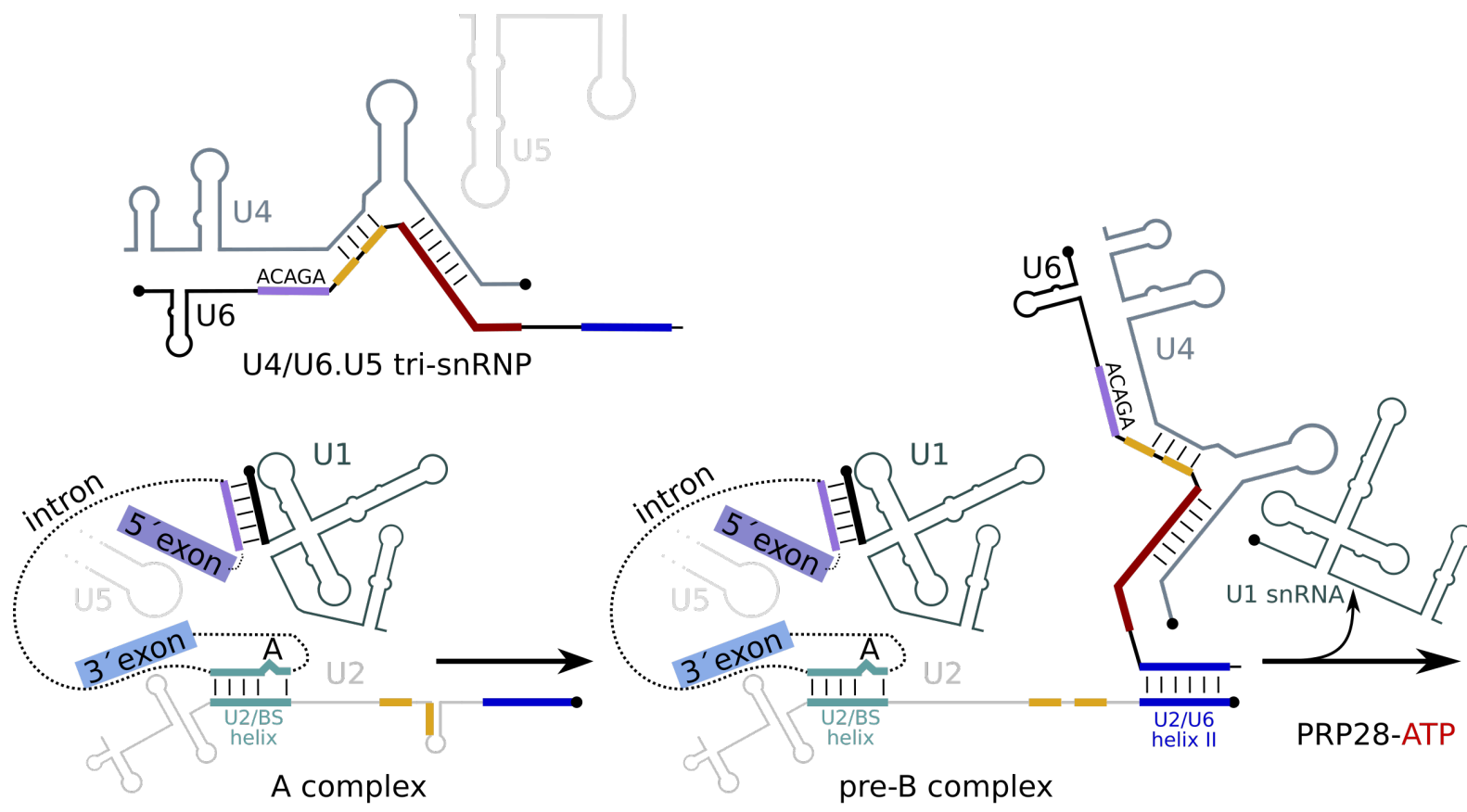

assembly
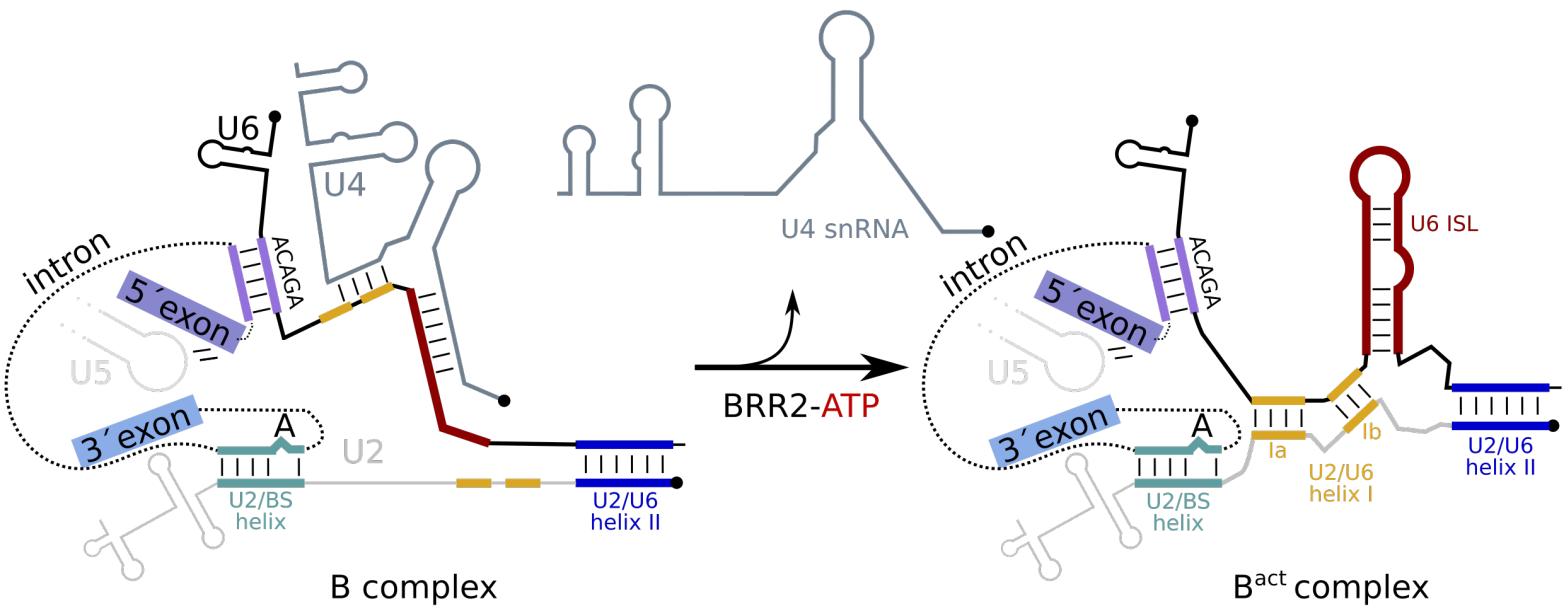

\section{activation}

Fig. 1.5: RNA remodeling events during assembly and activation. The major events in the remodeling of the RNA-RNA interactions during assembly (upper panel) and activation (lower panel) are depicted. In the upper panel, the stippled green line indicates the continuation of assembly as the E complex, containing only the U1 snRNP, is not included. Base-pairing interactions that are either disrupted or formed are indicated as colored lines. The U2/BS helix, established in the A complex, is colored teal. The BS-A is labeled and depicted as a bulged nt in the U2/BS helix. U2/U6 helix II, first formed by the integration of the tri-snRNP to the A complex to form the pre-B complex, is dark blue. The sequences of the intron and the U6 snRNA that base-pair to form the U6/5'ss helix are indicated in light purple. New base-pairing interactions formed upon activation, which comprise the catalytic center, are indicated in dark yellow (U2/U6 helix I, consisting of helix Ia and Ib) and crimson (U6 ISL). Adapted from [41, 113 . 


\subsection{Spliceosome activation}

Catalytic transesterification steps 1 and 2 of pre-mRNA splicing are catalyzed by RNA (Figs. 1.1, 1.8, 1.9 [127]. More specifically, the catalytic center of the spliceosome is constructed from the base-pairing and tertiary interactions between U2 and U6 snRNAs. However, these interactions are not found in the B complex, as the U6 nucleotides relevant for formation of the catalytic center (A50-U74) are base-paired to U4 snRNA. Thus, the spliceosome's catalytic center is not a preformed entity, and is instead formed for the first time during activation of the spliceosome on its pre-mRNA substrate.

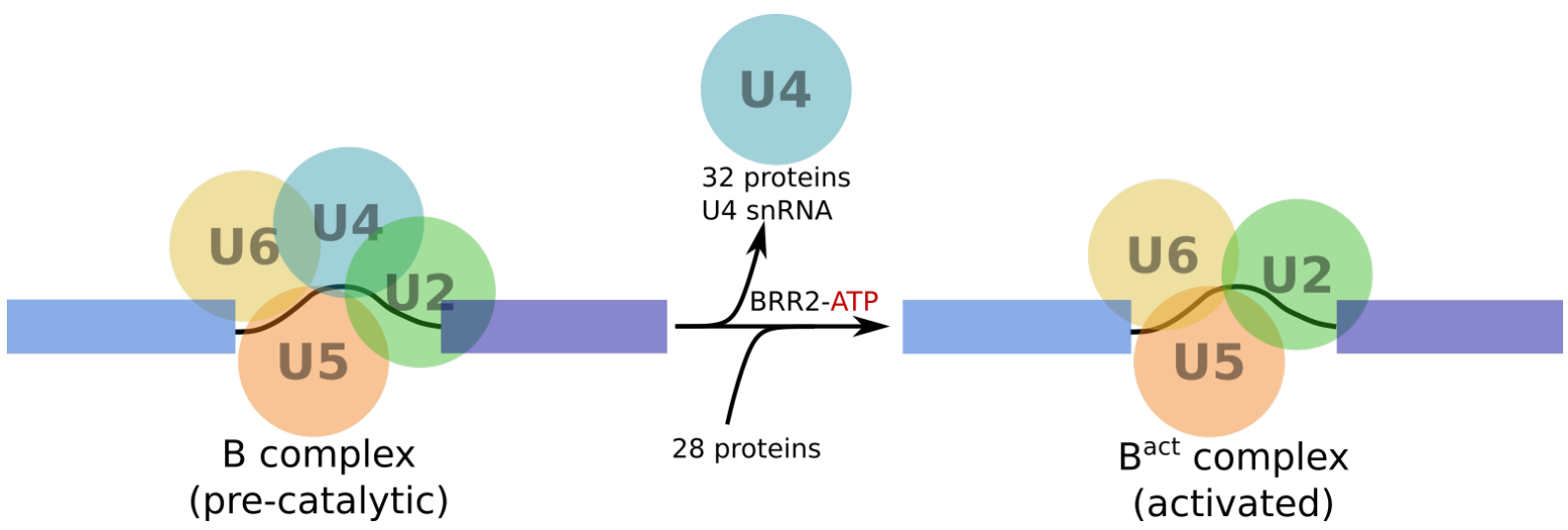

Fig. 1.6: Remodeling of the spliceosome during activation. The snRNP inventory of the $B$ complex (pre-catalytic spliceosome) and $\mathrm{B}^{\text {act }}$ complex (activated spliceosome) are shown as colored circles assembled on the pre-mRNA substrate, with exons represented as rectangles and the intron depicted as a black line. BRR2 uses the energy of ATP hydrolysis to catalyze spliceosome activation, resulting in the dissociation of U4 snRNP, including approximately 32 proteins and U4 snRNA. Approximately 28 new proteins are subsequently integrated into the spliceosome, facilitating structural rearrangements and the formation of the catalytic center in $\mathrm{B}^{\text {act }}$.

In order for activation to proceed, the U4/U6 duplex must be unwound by BRR2, allowing for the release of U4 snRNA and multiple proteins (Fig. 1.6). This frees U6 snRNA to establish new intramolecular base-pairing interactions as well as intermolecular base-pairing interactions with U2 snRNA. The result of this reorganization of RNA-RNA interactions is the formation of three conserved secondary structures that are the key components of the catalytic center: the U6 internal stem-loop (ISL), U2/U6 helix Ia, and U2/U6 helix Ib (Fig. 1.7) [91, 115]. The U6 nucleotides A53, G54 and C55 of helix Ib comprise the invariant sequence AGC, known as the catalytic triad. Together with the U6/5'ss, these secondary structures are positioned in a compact arrangement such that a catalytically 
essential triple helix is formed (Fig. 1.7] [128]. The first two strands of the triple helix are contributed by the Watson-Crick base-pairing of U2/U6 helix Ib, consisting of the catalytic triad (U6 nts A53, G54, C55) and three nts of U2 (G20, C21, and U22) (Fig. 1.8. The third and final strand of the triple helix is contributed by the U6 nts G46, A47, and U74, which engage in Hoogsteen interactions with U6 nts G54, A53, and C55 (i.e., the catalytic triad), respectively [129]. U6 nts G46 and A47 form the $3^{\prime}$ end of the conserved ACAGA(GA) sequence of the U6/5'ss helix, while U74 is bulged out of the U6 ISL. U2/U6 helix Ia connects these secondary structures to allow for the triple helix to be formed, bringing the $\mathrm{U} 6 / 5^{\prime}$ ss, helix Ib, and the ISL into close proximity. Nucleotides of the triple helix coordinate divalent metal ions that stabilize reaction intermediates in Steps 1 and 2 of pre-mRNA splicing (Fig. 1.9] [128, 130].

Another type of intron - known as Group II - is excised by a two-step mechanism that is very similar to that found in the spliceosome [132]. Group II introns have not been detected in the human genome, but are found in the genomes of bacteria, archaea, and eukaryotic organelles (mitochondria and chloroplasts) of several eukaryotic genomes [133]. In addition, Group II introns and nuclear pre-mRNAs exhibit similar sequences at the $5^{\prime}$ and $3^{\prime}$ termini of their introns: GUGYC and AY in Group II, GU and AG in nuclear pre-mRNA introns (Fig. 1.8] [134]. Group II introns fold into catalytically active RNA structures that facilitate their own removal [106]. A Group II intron forms six conserved secondary structure domains: D1-D6 [135, 136]. At the core of a Group II intron lies an RNA triple-helix comprised of tertiary interactions between three of these domains: D2, D3, and D5 (Fig. 1.8) [132]. The nucleotides of D2 and D3 involved in this interaction are referred to as "J2/J3," as they are situated at the junction ("J") between these two domains [137]. D5 forms an internal stem-loop structure existing in all Group II introns [138].

As in the spliceosome, the Group II catalytic center contains an internal stem-loop (D5) and an invariant AGC catalytic triad. These structures interact with an upstream GA sequence (i.e., analogous to the U6 nts G46 and A47 of the ACAGA(GA) sequence in the spliceosome) to form a catalytically essential triple helix of RNA, which coordinates 


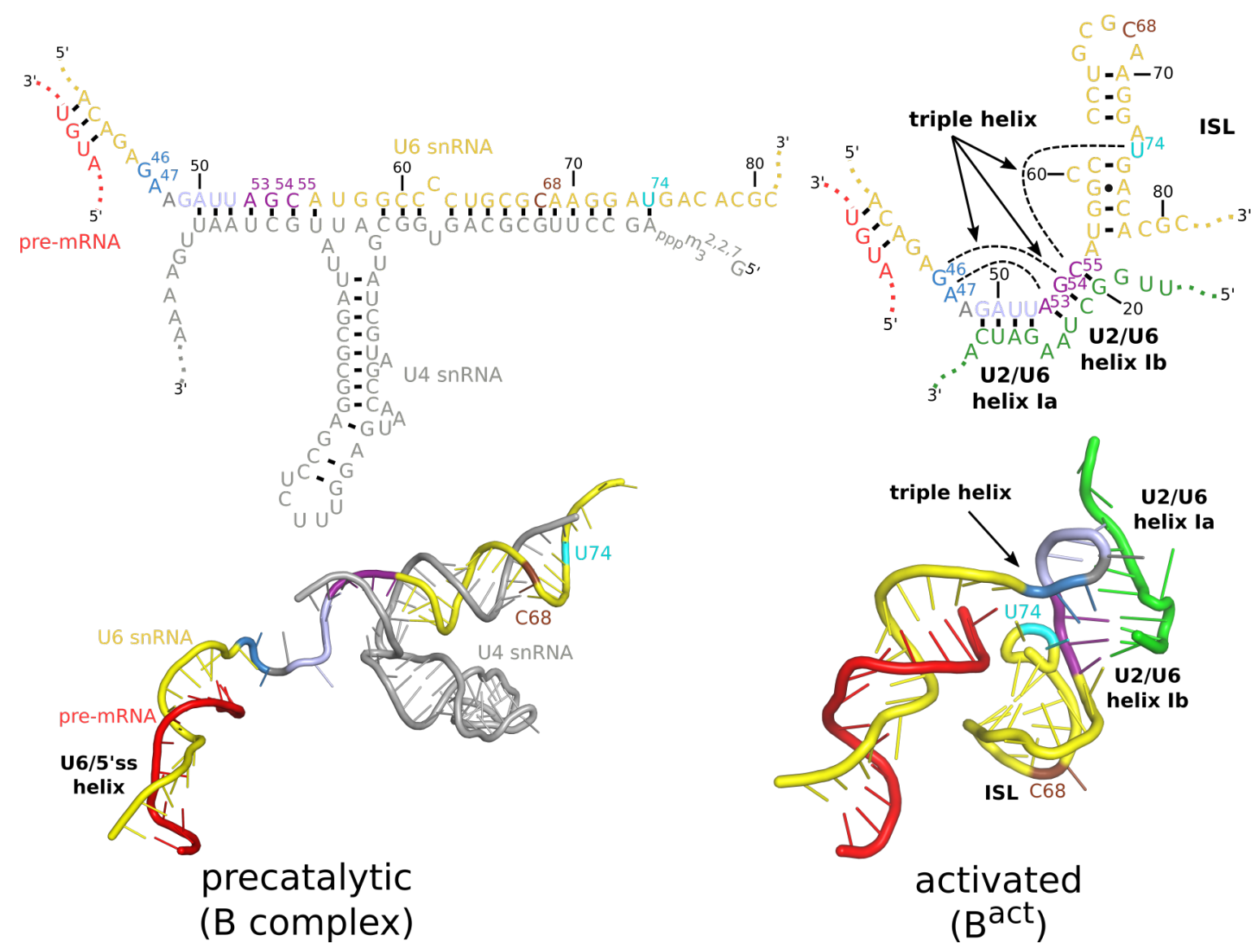

Fig. 1.7: Folding of catalytic RNA in the spliceosome. The U4/U6 and U6/5'ss RNA structures of the B complex are depicted as a 2D diagram (top-left panel) and 3D structure (bottom-left panel). U4 snRNA is depicted in gray, pre-mRNA in red, and U6 snRNA in yellow. U6 nucleotides that later contribute to the catalytic center are indicated in blue (G46, A47, which are the $3^{\prime}$ terminal nts of the U6 ACAGA(GA) sequence), purple (U6 nts of U2/U6 helix Ib; also called the catalytic triad: A53, G54, C55), and U74 (cyan). U6 nts (G49, A50, U51, U52) that later base-pair with U2 snRNA to form helix Ia are indicated in lavender. With the exception of G46 and A47, U6 nts that participate in catalytic center formation are base-paired to U4 snRNA. C68 is indicated in brown for orientation purposes. Following BRR2 unwinding of U4/U6 snRNA, U4 snRNA is released during activation. The mature catalytic center of the $\mathrm{B}^{\text {act }}$ is depicted as a $2 \mathrm{D}$ diagram (top-right panel) as well as a 3D structure (bottom-right panel). U2 snRNA is colored green. The nucleotides that participate in Hoogsteen interactions to form the triple helix are indicated by stippled lines in the 2D diagram (top-right panel) and by an arrow in the 3D depiction (bottom-right panel). Model for B ${ }^{\text {act }}$ : PDB 6FF4. Adapted from [131.

divalent metal cations required for splicing catalysis [128, 141, 142]. The 3D architecture of the RNA at the catalytic center in Group II introns is thus highly similar to that of the spliceosome (Fig. 1.9 [128]. Taken together, these lines of evidence suggest that Group II introns and nuclear pre-mRNAs may share a common evolutionary lineage, resulting in the conservation of the RNA core of the spliceosome [143].

Group II intron splicing occurs in an autocatalytic manner that does not require proteins [142]. Instead, a network of RNA-RNA interactions not found in the spliceosome stabilizes 

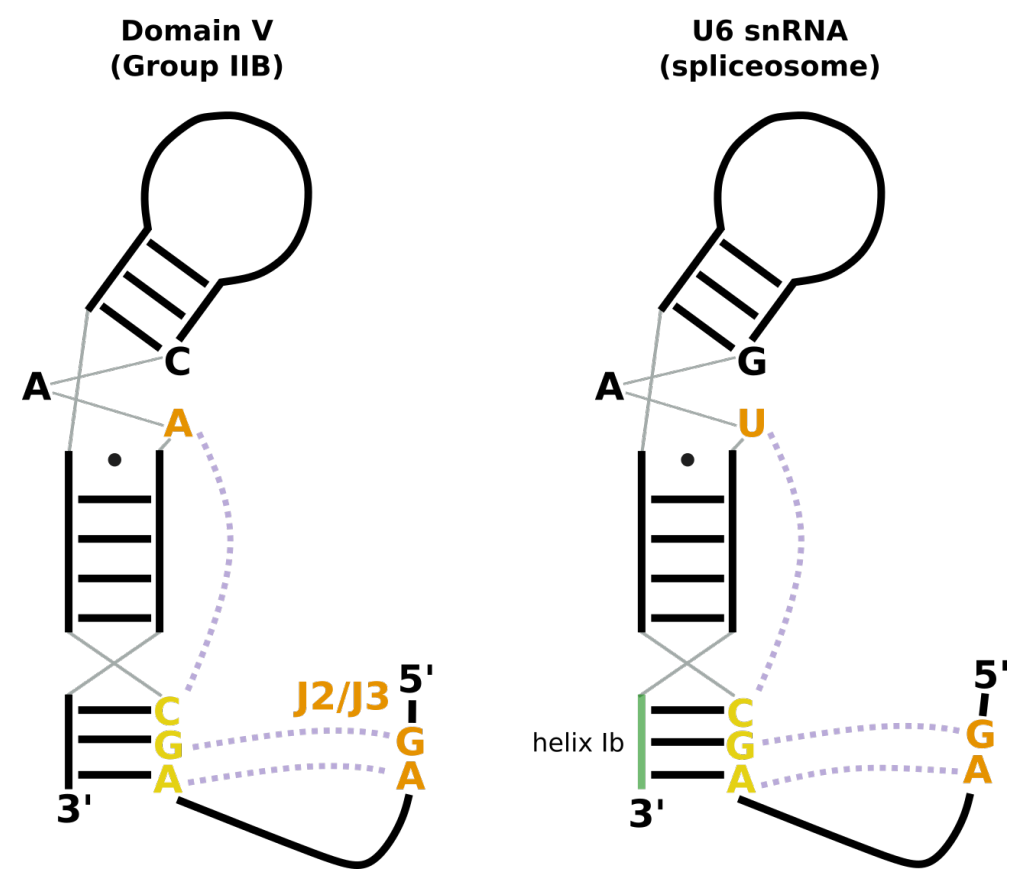

Fig. 1.8: Comparison of triple helix in Group IIB introns and spliceosomal snRNA. Nucleotides (orange) that form Hoogsteen interactions (indicated by stippled lavender line) with the ACG catalytic triad (yellow) are depicted. In Group IIB introns, an adenosine (A) nucleotide of Domain V is present instead of a uracil (U) as in the spliceosome. U2 nts of U2/U6 helix Ib (green) are indicated. The interactions of these nucleotides form a triple helix in Group IIB introns and spliceosomal snRNA. Adapted from [128, 136, 139, 140]
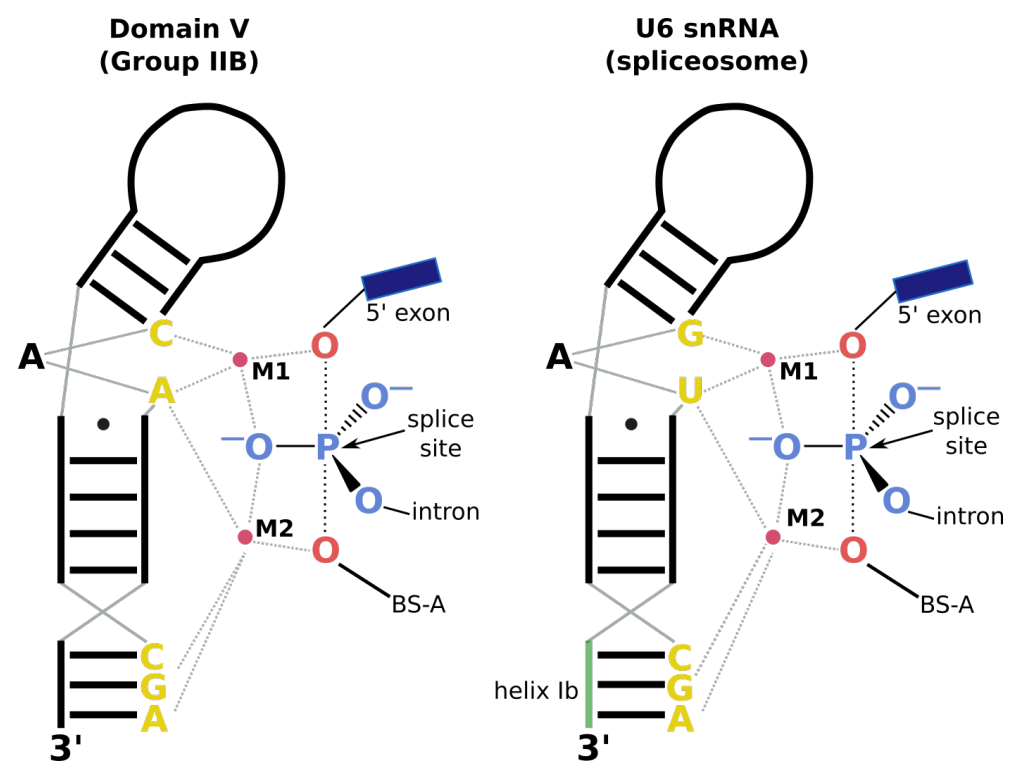

Fig. 1.9: Coordination of divalent metal ions by RNA in the catalytic center of Group IIB introns and the spliceosome. Divalent metal cations (magnesium, in the spliceosome) within the catalytic center are designated M1 and M2 (pink) and coordination thereof is indicated with stippled grey lines. Nucleotides of Domain V (Group IIB) and U6 ISL (spliceosome) that coordinate M1 and M2 are indicated in yellow. The phosphate group of the 5'ss is designated with a black arrow ("splice site"). Breakage of the covalent bond between the phosphate at the $5^{\prime}$ ss nt and the $5^{\prime}$ exon, and consequent formation of a covalent bond between the $2^{\prime} \mathrm{OH}$ of the BS-A and the phosphate at the $5^{\prime}$ ss nt of the $5^{\prime}$ end of the intron is indicated with a stippled black line. Adapted from [128, 136, 139, 140] 
the Group II catalytic center [144]. In stark contrast to the spliceosome, the spliceosome's catalytic center is completely surrounded by numerous proteins. The largest of these is the most conserved and largest single component of the spliceosome by molecular weight, PRP8 [145, 146].

\section{Structural studies of spliceosome complexes by cryo-EM}

Spliceosome structure and function is highly conserved throughout eukaryotes [147], with almost all S. cerevisiae splicing components having homologs in humans [41]. In contrast to those of yeast, spliceosomes of higher eukaryotes such as humans are estimated to contain as many as 80 additional splicing factors, many of which are required for regulation of splicing in more complex genomes [41].

Single particle cryo electron microscopy (cryo-EM) has emerged as a standard method for reconstructing the 3D structures of dynamic macromolecular complexes, such as the spliceosome. Compared to X-ray crystallography or nuclear magnetic resonance (NMR), a major advantage of cryo-EM is that different conformations and species within a single biochemical purification can be extensively sorted through image classification techniques. Moreover, cryo-EM does not require crystallization, therefore removing a time-intensive search for crystallization conditions.

In cryo-EM, biological samples are vitrified within a thin (typically $<500 \AA$ ) layer of buffer and are imaged within an electron microscope operated at liquid nitrogen temperature $\left(-196^{\circ} \mathrm{C}\right)$ [148, 149]. Images in cryo-EM are formed as a result of the interaction of the electron beam with the sample and are collected on a direct electron detector [150]. The resulting image, called a micrograph, contains projections of the macromolecules within the sample. Due to optical imperfections (i.e., aberrations) in electron microscopes, micrographs are not perfect representations of the projected macromolecules, but are systematically blurred. This blurring is described by a point spread function (PSF), which quantitatively evaluates how distortions in the optical system influence the quality of the resulting micrograph [151]. Micrographs are recorded in real space, but determination of their PSF and corresponding ideal image is more easily achieved in Fourier space, in which 
the PSF is described by the Contrast Transfer Function (CTF) [152]. The CTF takes into account the parameters of the optical system, such as spherical aberration, defocus, and electron wavelength [153]. The CTF describes the phases and amplitudes recovered in a micrograph as a function of spatial frequency (i.e., resolution). CTF correction is a method in which CTF parameters for every micrograph are estimated and applied to recover an approximation of a perfect image. Thus, it is crucial that CTF parameters are as accurate as possible to avoid introduction of artefacts in the data processing pipeline. CTF correction is typically carried out following motion correction and dose-weighting (see below).

Irradiation of thin vitreous films by the electron beam in cryo-EM results in beam-induced motion and radiation damage of the biological sample. Motion and radiation damage both limit the amount of information in a micrograph and thus reduce image quality [154, 155]. To mitigate the motion in an image, the thin film of vitreous buffer can be suspended in grids with small holes and/or on top of a thin layer of amorphous carbon film [149, 156]. Moreover, direct electron detectors with fast frame rates and are used to collect "movies" of the sample over a few seconds, allowing the individual frames to be aligned to one another to account for intra- and interframe motions during acquisition (i.e., "motion correction") [157].

Biological samples are sensitive to radiation damage, limiting the "dose" or fluence that can be used to record a micrograph (number of electrons per unit area per unit time). Radiation damage is unavoidable, but can be mitigated by low temperatures, which are thought to limit the diffusion of free radicals produced by ionizing radiation [152, 158]. High frame rates of most modern direct electron detectors used to collect micrographs also allow for the fractionation of the dose across many individual frames. Initial frames are exposed to the least radiation and therefore contain the highest resolution information [158]. Thus, weights can be assigned that take into account the relative contribution of high and low resolution signals within subframes of a micrograph, with earlier frames having higher weights for high resolution information than later frames. This approach is known as "dose weighting" [159, 160]. 
As a result of low electron fluence required in cryo-EM to mitigate radiation damage, micrographs have very low signal-to-noise ratios (SNR), complicating direct interpretation of single projection images of macromolecules. To overcome this low SNR, thousands of single particle images in similar orientations are averaged. Practically, this is achieved by first identifying individual individual macromolecules (i.e., "particles") within each micrograph and subsequently cropping them out as individual images. Particle images are subjected to reference-free alignment and classification, in which particles with similar orientations and properties are sorted iteratively into groups or "classes" [161].

Well aligned 2D classes often reveal ordered domain structures and often finer secondary structure features of the sample. In ideal cases, the random orientation of the sample in the vitreous buffer leads to many different orientations of particles, thus resulting in distinct "views" of the macromolecular complex. Poorly defined classes often represent damaged particles or debris that are excluded from further processing. Particle images belonging to well defined 2D classes are retained for structure determination.

The next step of image processing is to determine how 2D projection images of a macromolecule are related to the 3D object from which they are derived. Once particle images are annotated with these angular assignments (Euler angles), they are used to generate a 3D model $a b$ initio from the data [162, 163]. Starting models generated in this manner are free from model bias and therefore do not introduce parameters which could lead to spurious results from an external reference. The ab initio starting model is then used as a reference to carry out $3 \mathrm{D}$ classifications, in which the $3 \mathrm{D}$ volume is reprojected and matched to single particle images ("projection matching") belonging to distinct classes [164]. Angular assignments of particle images are iteratively refined to result in higher resolution reconstructions, as the angular assignment becomes progressively more accurate.

3D classification is a powerful tool for sorting out conformational differences in biochemical samples in which multiple distinct macromolecular complexes may exist. In some cases, 3D classification is also used to focus only on smaller regions of a large complex that may 
be flexible in relation to a large, well aligned portion of a macromolecular complex. If left unclassified, such regions are often poorly resolved due to structural heterogeneity in the overall reconstruction of the complex. Several approaches have been adopted to address this challenge, including in silico subtraction of stable regions directly from particle images followed by classification of the region of interest [165]. One of these approaches, known as multi-body refinement, involves signal subtraction and 3D refinement (see below) of two or more rigid bodies within a macromolecular assembly [166], allowing simultaneous refinement of several regions of a single complex. In addition, structural heterogeneity in the sample has been described by 3D principal component analysis (PCA), yielding energy landscapes that provide quantitative representations of distinct sub-populations of a macromolecular complex [99, 167].

Following computational sorting to obtain more homogeneous populations of 3D reconstructions, a map is refined against a 3D reference. All particle images contributing to a map chosen for refinement are split into 2 random subsets and independently reconstructed. The resolution of an EM map is estimated by comparing the two subsets that are divided during map refinement [168]. The overall resolution is estimated to be the spatial resolution at which the correlation of the maps is 0.143 [169]. This method is called Fourier Shell Correlation (FSC) and is the primary method of estimating resolution of EM maps [170]. FSC estimations report a single resolution value, but resolution in EM maps is often highly variable. Better aligned or more stable portions of a macromolecular complex are generally better resolved, while poorly aligned or flexible regions are less well resolved. Local resolution estimates differ substantially from global resolution estimates in many cases [171]. In some cases, the local resolution can be improved with focused classifications or multi-body refinements.

After reconstructions of the macromolecular complexes are determined and classified using 3D classification and refinement, molecular models of the complexes can be built. It is through model building and interpretation of the resulting structures that biological insights from the cryo-EM reconstructions begin to emerge. In most cases, model building begins by consulting the biochemical composition of a sample, which is either known in ad- 
vance (e.g., for in vitro reconstitutions) or determined experimentally (e.g., endogenously purified complexes). Once the biochemical composition of the sample is well characterized, this information can be used to begin building a model of the 3D reconstructions from single particle cryo-EM. Model building is carried out iteratively, often beginning by docking the largest subunits into well defined density elements followed by docking of progressively smaller units. Experimentally determined or predicted structures of individual components often differ in conformation from those within a macromolecular complex, necessitating adjustments of inter-domain or flexible regions of reference structures within the 3D reconstruction [172]. For large assemblies containing many (e.g., $\geq 10$ ) proteins, such as the spliceosome, it is helpful to have cross-linking mass spectrometry data to guide modeling, as protein-protein and protein-RNA crosslinks assist in determining which components are likely to interact within a macromolecular complex [173, 174]. In practice, model building is often a very time consuming step of structure determination, especially in parts of the map where regions of multiple components converge. After all components have been identified and docked within the density maps, the model is subjected to a refinement (or fitting) step to minimize geometric errors and atomic clashes such that the model is accommodated within the density, but is not overfitted [175, 176].

Cryo-EM is the method of choice for structural biology studies of spliceosomes, as it allows for classification of conformational and compositional heterogeneity of highly complex macromolecules. Structures for many individual components of the spliceosome have previously been determined by X-ray crystallography or NMR, including the pioneering achievements of structures for U1 snRNP and the core spliceosomal proteins PRP8 and BRR2 [177 181]. However, crystal structures of functional splicing complexes proved elusive due to the dynamic nature of the spliceosome. The "Resolution Revolution", resulting from major advancements in software and hardware used for single particle cryo-EM, has made it possible to determine the molecular architecture of highly dynamic complexes to sub-nanometer resolution [182], and it is reasonable to expect that further improvements will lead to resolutions better than $2 \AA$ even for large assemblies [183, 184]. Aided by a wealth of previous biochemical studies as well as protein-protein cross-linking 
mass spectrometry (XL-MS) data, cryo-EM structures for all major yeast and human intermediates (with the exception of the human E, A, and $\mathrm{B}^{*}$ complexes) have been determined in the last 6 years, uncovering deep insights into assembly, activation, and function of the spliceosome [122, 185, 186].

\subsubsection{Architecture of PRP8 and overview of its dynamics}

PRP8 is a $220 \mathrm{kDa}$, multidomain protein that forms the central scaffold of the spliceosome, is an integral component of U5 snRNP, and joins the complex as part of the U4/U6.U5 tri-snRNP during conversion of the pre-spliceosomal A complex to the pre-catalytic B complex (Fig. 1.4 [187]. PRP8 consists of a large N-terminal domain (NTD) connected to a helical bundle (HB) via a NTD Linker (NTDL) region, a reverse transcriptaselike/endonuclease-like (RT/En) domain, an RNaseH-like (RH) domain, and a Jab1 domain connected by short linker regions (Fig. 1.10] [122]. PRP8 ${ }^{\mathrm{RH}}$ contains an RNase H-like active site but is not known to unwind RNA duplexes [188]. The RT/En domain contains several smaller domains, including the Thumb/X, a Switch-Loop (SWL), Linker, $\alpha$-finger [122]. This multidomain organization affords the spliceosome a massive amount of flexibility in organizing the molecular architecture of the complex.

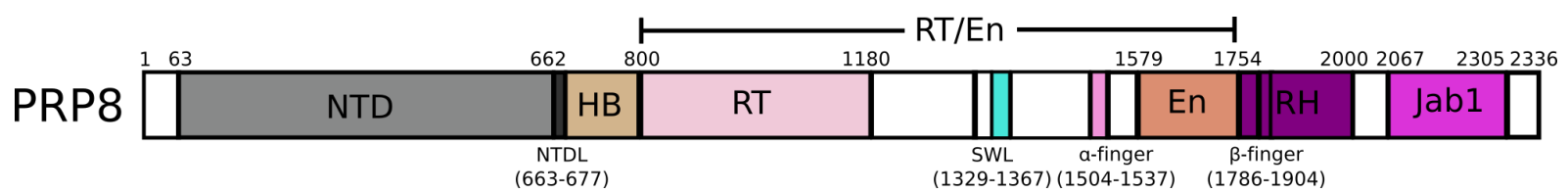

Fig. 1.10: Domain architecture of PRP8. The multidomain architecture of PRP8 is depicted. $\mathrm{PRP}^{\mathrm{RT} / \mathrm{EN}}$ is indicated, containing the $\mathrm{PRP} 8^{\mathrm{SWL}}$ and $\mathrm{PRP} 8^{a \text {-finger }} . \mathrm{PRP}^{\mathrm{NTD}}, \mathrm{PRP} 8^{\mathrm{NTDL}}, \mathrm{PRP} 8^{\mathrm{HB}}$, and $\mathrm{PRP} 8^{\mathrm{RT} / \mathrm{En}}$ surround the active site, while the C-terminal $\mathrm{PRP} 8^{\mathrm{RH}}$ and $\mathrm{PRP} 8^{\mathrm{Jab}}{ }^{\mathrm{J}}$ play regulatory roles.

Early crosslinking and structural investigations determined that pre-mRNA as well as spliceosomal snRNAs associate with PRP8 and that several important secondary structures of U2 and U6 snRNAs, later found to be the catalytic center, were likely situated within a large cavity of PRP8 [145, 180, 189]. This large cavity is formed by the arrangement of the NTD, RT/En and the HB domains of PRP8, which form a compact region in which the active site $\mathrm{U} 2 / \mathrm{U} 6$ is situated [180]. 
The molecular architecture of PRP8 is very comparable to the 3D structure of Group II introns, suggesting that the environment surrounding the catalytic RNA structures of the spliceosome and Group II introns is conserved, despite the fact that one compartment is composed of RNA and the other of protein and RNA, respectively [180]. This finding suggested that the protein "mold" of the spliceosome may have emerged throughout evolution to replace the RNA-only scaffold of Group II introns or a similar common ancestor [134]. However, PRP8 alone is not likely to be sufficient for the formation and stabilization of the active site, which is surrounded by additional proteins in the activated complex (see 1.4 .5 .

Several domains of PRP8 are highly dynamic throughout the splicing cycle, regulating the progression of spliceosome assembly and activation. The C-terminal region of PRP8 is involved in regulating the $\mathrm{DExD} / \mathrm{H}$-box helicase BRR2 to prevent premature unwinding of the U4/U6 snRNA duplex, which could lead to premature activation [124, 190, 191]. The RNase $\mathrm{H}$ domain was found to have multiple regulatory functions, including regulation of BRR2 activity as well as control of splicing catalysis, and has been reported to toggle between different conformations to regulate splicing fidelity [188, 192-194]. Cryo-EM structures documented the dynamic nature of PRP8, showing that the RNase H and Jab domains are highly mobile and occupy distinctly different positions in nearly every stage of the splicing cycle [195 199]. The switch-loop (SWL) domain of PRP8 (PRP8 ${ }^{\mathrm{SWL}}$ ) is repositioned during spliceosome activation, and is returned to its original position after splicing is complete [200].

The overall conformation of PRP8 gradually transitions from an open to a closed conformation as the spliceosome is assembled (Fig. 1.11). In the tri-snRNP as well as the pre-B complex, PRP8 is in an open conformation, with the En domain separated from the NTD domain. Following the formation of the pre-catalytic B complex, the RT/En domain pivots on the NTD such that the En repositioned closer to NTD, yet remains in an overall open conformation. These movements are accompanied by slight rotation of the HB domain, which is connected to the NTD via the NTDL. 

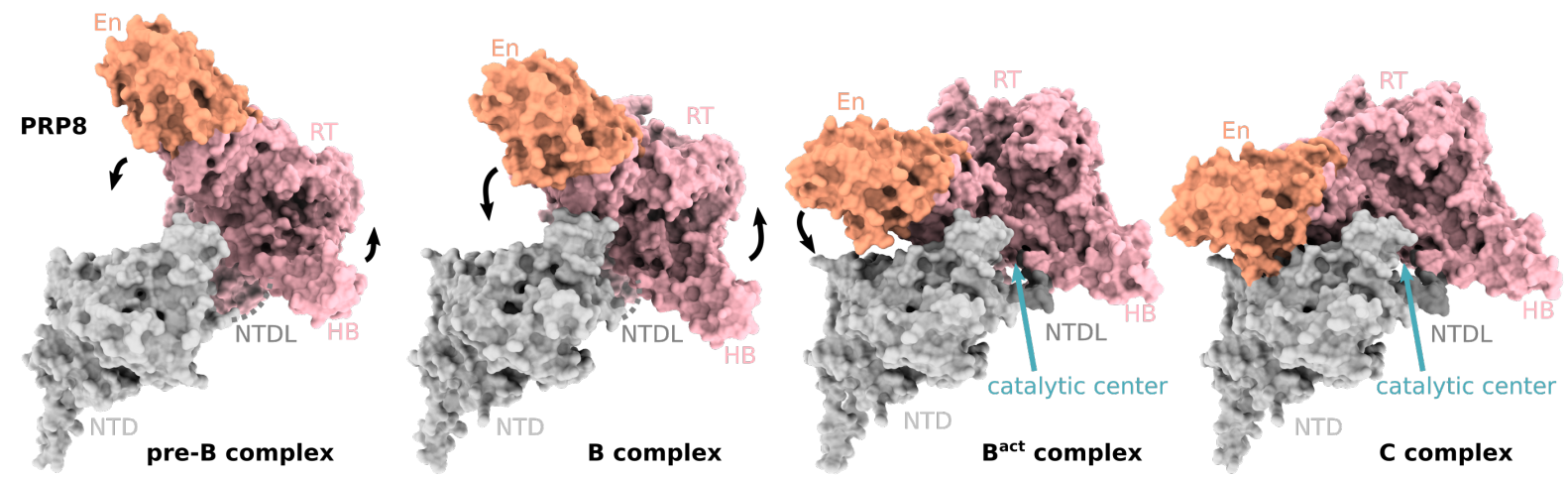

Fig. 1.11: PRP8 conformational change. $\mathrm{PRP}^{\mathrm{NTD}}$ (light grey), $\mathrm{PRP} 8^{\mathrm{HB}}$ (light pink), $\mathrm{PRP} 8^{\mathrm{RT}}$ (light pink), and $\mathrm{PRP} 8^{\mathrm{En}}$ domains (salmon) are depicted in their conformations as found in the pre-B, $\mathrm{B}, \mathrm{B}^{\text {act }}$, and $\mathrm{C}$ complexes. The $\mathrm{PRP} 8^{\mathrm{NTDL}}$ (dark grey), which surrounds catalytic center (dark teal) is indicated by a stippled line (unstructured) or a surface representation (structured). In the tri-snRNP (not shown) and pre-B complex, PRP8 is in an open conformation. Release of U1 snRNP and PRP28 results in a slight pivoting of the PRP8 $8^{\mathrm{RT} / \mathrm{En}}$, narrowing the gap between $\mathrm{PRP} 8^{\mathrm{NTD}}$ and $\mathrm{PRP} 8^{\mathrm{En}}$ to generate a partially open conformation in the B complex. Following release of the B-specific proteins, $\mathrm{PRP} 8^{\mathrm{En}}$ is clamped onto $\mathrm{PRP} 8^{\mathrm{NTD}}$ to form a closed conformation of PRP8. The catalytic center is nested within the cavity formed by the closed conformation of PRP8 in $B^{\text {act }}$. Following Step 1 of splicing, the remaining proteins wedged between $\mathrm{PRP} 8^{\mathrm{En}}$ and $\mathrm{PRP} 8^{\mathrm{NTD}}$ are destabilized, allowing for the distance between the domains to be even more restricted in the $\mathrm{C}$ complex. PDBs 6QX9 (pre-B complex), 6AHD (B complex) complex, 5Z58 ( $\mathrm{B}^{\text {act }}$ complex), and 5YZG (C complex) shown.

The largest conformational change of PRP8 occurs in the transition from the B to the $\mathrm{B}^{\text {act }}$ complex during activation: the En domain clamps down onto the NTD, while the RT domain is concomitantly shifted upwards relative to the NTD (Fig. 1.11). This closed conformation thus forms a compact "chamber" that harbors the mature catalytic center of the $\mathrm{B}^{\text {act }}$. Following Step 1 of splicing (i.e., in the $\mathrm{C}$ complex) proteins that reside within the gap between the NTD and En domains have been displaced, allowing the En domain to clamp even more tightly onto the NTD. The fully closed conformation of PRP8 is retained through Step 2 of splicing and is intact in the post-catalytic spliceosome (P complex). PRP8 is thus a dynamic scaffold that facilitates the organization of the entire spliceosome, in particular the active site U2/U6 RNA network.

\subsubsection{Cryo-EM structure of the pre-catalytic spliceosome (B complex)}

Comparison of the cryo-EM structures of the pre-B and $\mathrm{B}$ complexes reveals drastic rearrangements in the molecular architecture of the spliceosome leading up to activation 
[196, 201]. In both complexes, the U5 snRNP proteins PRP8, BRR2, SNU114, U5 Sm, and $\mathrm{U} 5-40 \mathrm{~K}$ as well as the U5 snRNA form the rigid core of the complex. U4/U6 components are positioned upon the U5 snRNP scaffold, held in place by the U5 protein PRP6 as well as the tri-snRNP proteins DIM1, SNU66, and SAD1 [195].

U2 snRNP is loosely associated to the complex by U2/U6 helix II. In the pre-B complex, all 5 snRNPs are still present and the $5^{\prime}$ ss is not yet base-paired to U6 and U5 snRNAs to form the U6/5'ss helix and the exon-U5 interaction found in the activated spliceosome. Moreover, BRR2 is kept in its tri-snRNP orientation, separated from its U4/U6 snRNA substrate. Unwinding of the U1/5'ss interaction allows for the release of DExD-box helicase PRP28 and for the integration of the 5'ss and U6 ACAGA sequence into the core of the spliceosome. The release of PRP28 and U1 snRNP likely allows for the large-scale repositioning of BRR2 onto U4 snRNA, where it is primed to unwind the U4/U6 base-pairing that keeps this interaction intact. Repositioning of BRR2 is also accompanied by the translocation of U4 Sm, including the $3^{\prime}$ domain of U4 snRNA, as well as the repositioning of the entire U2 snRNP and U4/U6 di-snRNP. The tri-snRNP proteins RBM42 and PRPF4B are also dissociated at this stage. The remaining building blocks of the complex are thus the U2 snRNP and almost all components from a heavily remodeled U4/U6.U5 tri-snRNP.

These major rearrangements make way for the integration of a set of proteins that were first identified as co-purifiying with the B complex, the B-specific proteins, which consist of PRP38, SNU23, MFAP1, UBL5, FBP21, SMU1, RED, WBP11 and PQBP1 [202]. Intriguingly, only PRP38, SNU23, MFAP1 and UBL5 have known homologs in S. cerevisiae (Prp38, Snu23, and Spp381, Hub1), suggesting that the remaining five proteins may have evolved as regulatory factors to handle the more complex genomes of higher eukaryotes [122]. In the $\mathrm{B}$ complex, the $\mathrm{PRP}^{\mathrm{En}}$ domain is rotated toward PRP8 ${ }^{\mathrm{NTD}}$, but still displays an open conformation. PRP38, SNU23, MFAP1, and UBL5 - all of which have homologs in yeast - bind to this large cleft separating the PRP8 ${ }^{\mathrm{NTD}}$ and PRP8 ${ }^{\text {En }}$ domains, which was previously occupied by the RecA domains of PRP28 in the pre-B complex. This is a mutually exclusive interaction observed between the pre-B and 
B complex, one of many in the assembly of a functional spliceosome. UBL5 is situated very close to the 5 'ss, possibly stabilizing this interaction leading up to Step 1 of splicing [203]. PRP38 makes contacts to SNU23, MFAP1, and UBL5, likely acting as a platform upon which these factors are organized [204]. SNU23 passes alongside the U6/5'ss helix. FBP21 is positioned at the interface between the helicase BRR2 and its U4/U6 substrate, appearing to prevent premature unwinding of this duplex. The U2 snRNP, although still loosely associated to the body of the spliceosome by U2/U6 helix II, is partially stabilized by contacts to SMU1 and RED, which form a heterodimer that bridges the U2 protein SF3B3 to BRR2. SMU1 and RED were shown to be important for the splicing of short introns (e.g., 200 nts or fewer) [205]. The remaining B-specific proteins, WBP11 and PQBP1 (also known as NPW38BP and NPW38), were also detected in the biochemical preparation of the B complex but were not observed in the cryo-EM structure, hinting that they may act at a distance or bind transiently to the spliceosome during B complex formation [196].

Despite major conformational and compositional remodeling, the catalytic center of the spliceosome is still not formed in the B complex. For this to occur, the U4/U6 snRNA duplex must be unwound by BRR2, leading to the dissociation of the U4 snRNP; the U5 proteins PRP6, DIM1, SNU66, and SAD1; as well as the U6 LSm proteins from U2/U6 helix II [99]. In particular, PRP6, DIM1, and PRP31 occlude the large cavity between $\mathrm{PRP}^{\mathrm{NTD}}$ and $\mathrm{PRP} 8^{\mathrm{HB}}$ in which the catalytic center will be formed. Thus, removal of these factors is a clear prerequisite for activation. The U2 snRNP must subsequently and dock onto PRP8 ${ }^{\mathrm{RT} / \mathrm{En}}$, to allow for new base-pairing interactions between U2 and U6 snRNA to form within the nascent catalytic center (see 1.4.4). The B-specific proteins must be dissociated from the complex, allowing for a conformational change in PRP8 from an open to a closed conformation (Fig. 1.11). This drastic loss of approximately 32 proteins and U4 snRNA from the B complex allows for the incorporation of approximately 28 additional proteins, leading to massive rearrangements in the molecular architecture of the spliceosome en route to an activated complex containing a fully formed catalytic center (Fig. 1.12). 


\subsubsection{Exchange of protein and RNA components during activation}

The activation phase comprises the largest change in composition and molecular architecture of the spliceosome (Fig. 1.12] [97]. Formation of the catalytic center - including the U6 ISL and U2/U6 helix Ia and Ib - requires the removal of the U4/U6 proteins and the integration of numerous non-snRNP proteins into the spliceosome. Many of these proteins are organized into subcomplexes that join the spliceosome as preformed units, while certain other proteins are reported to join the spliceosome as independent entities (Fig. 1.12. The major subcomplexes that join during activation to form the $\mathrm{B}^{\text {act }}$ complex are the Retention and Splicing complex (RES), NineTeen Complex (NTC), and the Intron Binding Complex (IBC) (Fig. 1.13). Additional proteins that join the spliceosome during activation are the NineTeen Complex Related (NTR) proteins, SRRM1, SYF3, PPIL2, PRP17, CWC22/CWC27, RNF113A, SRRM2, PRP2, and GPKOW.
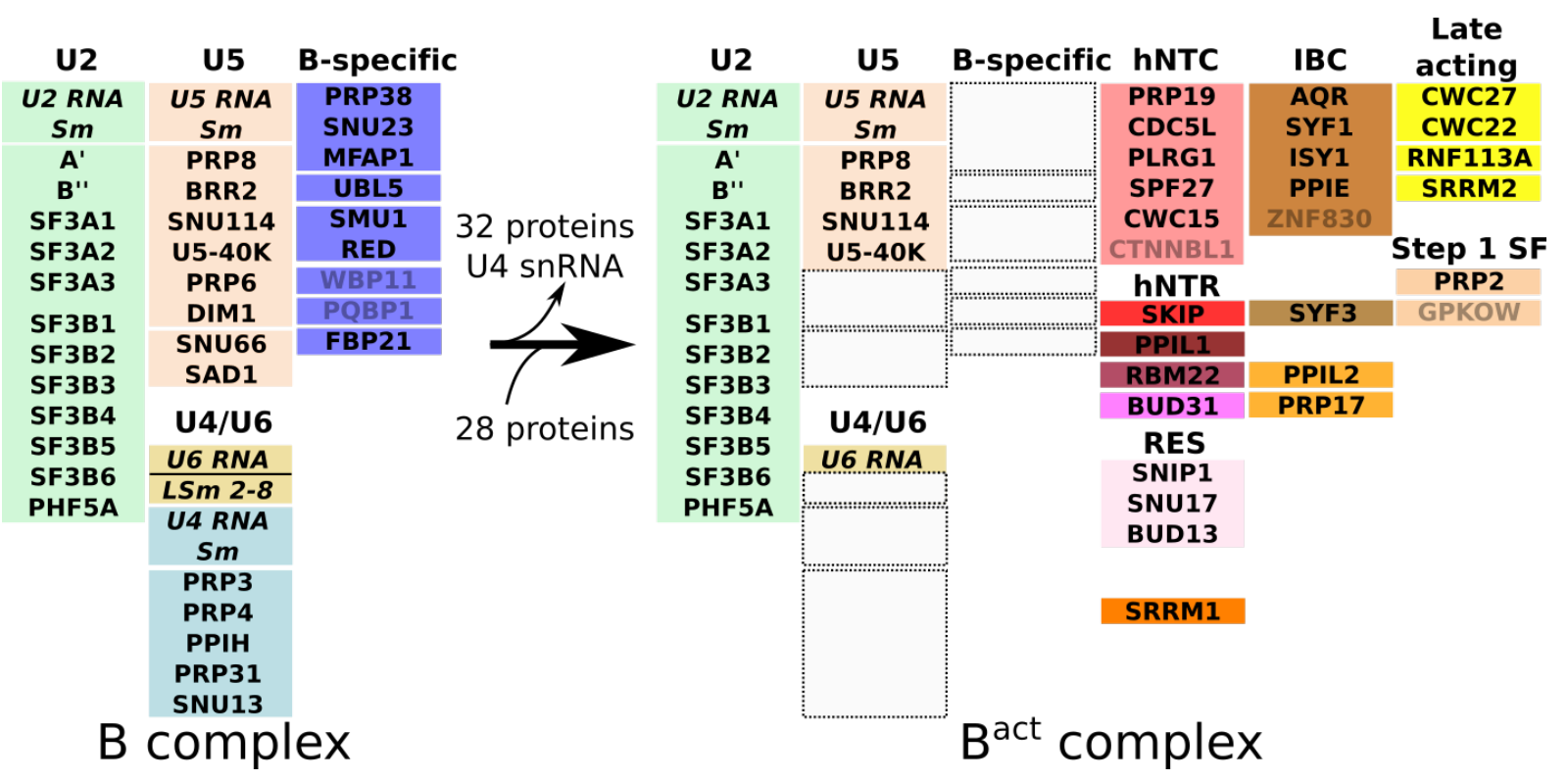

Fig. 1.12: Protein and RNA exchanges during activation. The protein and RNA composition of the B complex (left) and B ${ }^{\text {act }}$ complex (right) are shown, with the snRNPs or protein groups labeled above the colored rectangles. Protein components that are organized into a complex are grouped together within a single box. Components that are dissociated from the spliceosome following activation are indicated by a gray box with a stippled border. Lighter colored text within in individual box (i.e., WBP11, PQBP1, CTNNBL1, ZNF830, GPKOW) indicates that the protein has been identified in biochemical preparations of the complex but has not been localized in structures of the spliceosome. Adapted from [131. 


\subsubsection{Cryo-EM structure of the activated spliceosome ( $B^{\text {act }}$ complex)}

The cryo-EM structure of the human $\mathrm{B}^{\text {act }}$ complex revealed for the first time the 3D organization of the catalytic center of the human spliceosome prior to splicing [99]. In the activated spliceosome, removal of the B-specific proteins allows PRP8 to adopt a closed conformation, in which the catalytic center is formed (Figs. 1.11 and 1.12). The overall architecture of the $\mathrm{B}^{\text {act }}$ complex is strikingly distinct from that of the $\mathrm{B}$ complex: following unwinding of the U4/U6 snRNA duplex by BRR2, leading to the removal of U4 snRNA and associated proteins (Fig. 1.12 and 1.13), U2 snRNP is shifted towards the body of the U5 snRNP, docking to the PRP8 ${ }^{\mathrm{RT} / \mathrm{En}}$ domain in $\mathrm{B}^{\text {act }}$ (Fig. 1.12 and 1.13 . This major repositioning of U2 snRNP moves the U2/BS helix in closer proximity to the 5'ss, setting the stage for Step 1 of splicing. U2/U6 helix II is drastically repositioned and the U6 LSm proteins, which were previously bound to helix II, are dissociated. Following its unwinding of U4/U6 duplex, BRR2 rotates on its anchor at the PRP8 ${ }^{\mathrm{Jab} 1}$ domain, such that it comes into contact with the SF3B3 protein of U2 snRNP. Thus the bridge between BRR2 and U2 snRNP which was formed by SMU1/RED in the B complex is broken, to be replaced by direct protein-protein interactions between SF3B3 and BRR2 [99, 196].

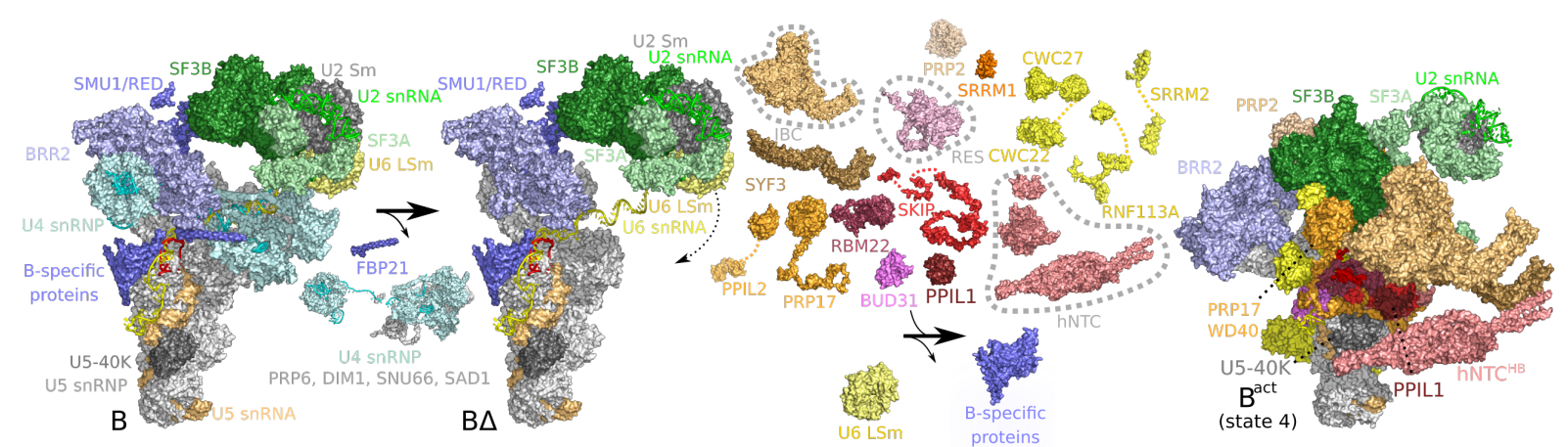

Fig. 1.13: Structural changes involved in B-to-B ${ }^{\text {act }}$ transition. Change in the ribonucleoprotein inventory and the molecular architecture of the spliceosome during activation are shown. In the B complex (left; PDB 5O9Z), U4 snRNP (light blue) is still associated and U2 snRNP (green, with SF3b, SF3a, U2 Sm, and U2 snRNA highlighted) is loosely bridged to BRR2 by SMU1/RED. Unwinding of U4/U6 base-pairing allows U4 snRNP, FBP21, and several U4/U6 proteins to be dissociated. The hypothetical intermediate, $\mathrm{B} \Delta$ is shown (middle structure) to emphasize the translocation of U2 snRNP, U6 LSm, and U6 snRNA downwards towards the body of the spliceosome. Proteins that join the spliceosome at this stage are indicated above the arrow, while proteins that dissociate (B-specific proteins and U6 LSm) are shown below the arrow. Subcomplexes that join the spliceosome (IBC, RES, NTC) are surrounded by a stippled gray line. The $\mathrm{B}^{\text {act }}$ complex (state 4, unpublished PDB) is shown on the far right. Adapted from 131 


\section{Retention and Splicing Complex}

The RES complex is comprised of the proteins SNU17, PML1, and BUD13, and is important for the formation of the activated spliceosome (Fig. 1.14] [116, 206]. The RES complex was shown to bind cooperatively at the pre-catalytic (B complex) stage of assembly and to be stably integrated in the activated spliceosome, prior to becoming more loosely bound to the spliceosome during the conversion to the catalytically activated complex $\left(\mathrm{B}^{*}\right)$ [207, 208]. In the $\mathrm{B}^{\text {act }}$ complex, the RES complex binds to the intron within a region spanning from the BS to the 3'ss [207, 209]. SNU17 interacts with the pre-mRNA via its RNA Recognition Motif (RRM), but the mechanism of action of SNU17 and the RES complex as a whole is not well understood [207, 210]. However, spliceosomes lacking RES are disassembled prematurely by the DEAH-box helicase PRP2, which joins the complex during activation and catalyzes the progression from an activated $\left(\mathrm{B}^{\text {act }}\right)$ to a catalytically activated $\left(\mathrm{B}^{*}\right)$ complex [211]. Therefore, binding of the RES proteins to the intron appears to be a critical checkpoint in formation of the $\mathrm{B}^{\text {act }}$ complex.

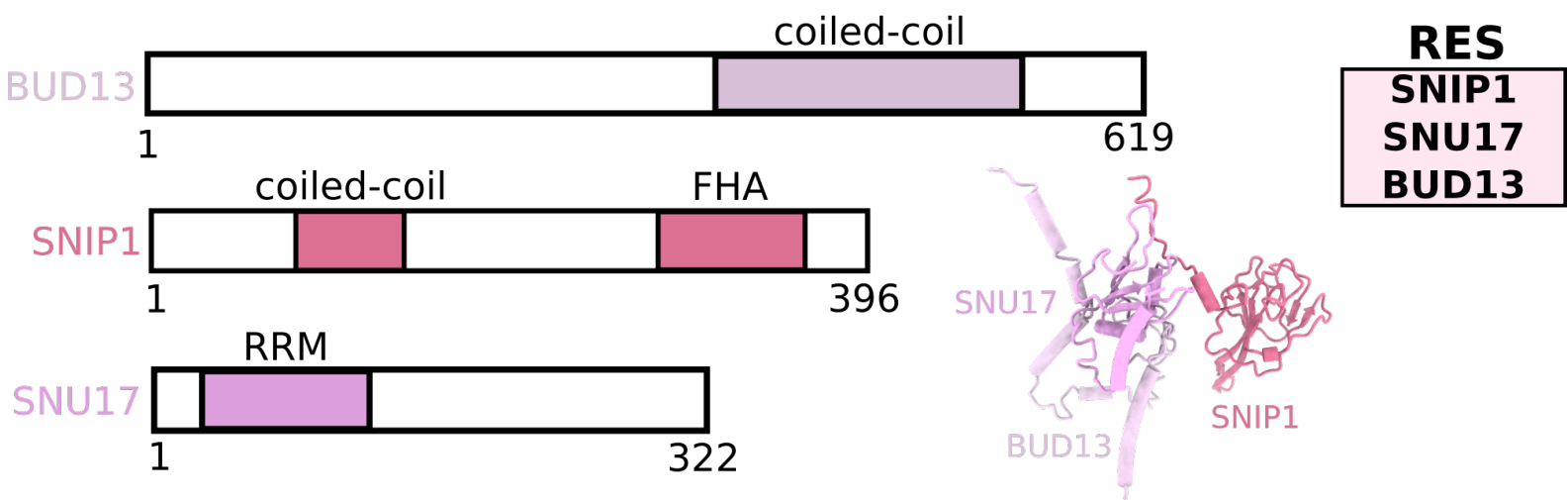

Fig. 1.14: Retention and Splicing (RES) complex. Names of the RES proteins (right), their domain architectures (left) and 3D organization in $\mathrm{B}^{\text {act }}$ (middle; from PDB 6FF7) are shown. Both BUD13 and SNIP1 contain coiled-coil domains. SNIP1 has a forkhead-associated (FH) domain in its C-terminal. The RNA recognition motif RRM) of SNU17, which associates the RES complex to the pre-mRNA, is indicated.

NineTeen Complex

The NineTeen Complex (NTC) is a preformed subcomplex consisting of PRP19, CDC5L, PLRG1, SPF27, CWC15, and CTNNBL1 (Fig. 1.15] [212, 213]. The NTC associates with the spliceosome during activation and is required for splicing [214]. Association of the 
NTC with the spliceosome is implicated in removal of U6 LSm proteins and subsequent stabilization of U6 snRNP within the spliceosome [214]. The structure of the activated complex is consistent with this evidence, as the U6 LSm proteins are not present and the $\mathrm{U} 6$ snRNA is tightly integrated into the mature U2/U6 catalytic center. Components of the NTC form extensive contacts throughout the spliceosome in $\mathrm{B}^{\text {act }}$, facilitated by the complex domain structures of its constituent proteins [99]. The WD40 domain of PLRG1 $\left(\mathrm{PLRG} 1^{\mathrm{WD} 40}\right)$ docks to PRP8 ${ }^{\mathrm{HB}}$, near the catalytic center. PLRG1 ${ }^{\mathrm{WD} 40}$ contacts many additional proteins, acting as an organizer of protein-protein interactions. CWC15 (also called Ad002) is wound around PLRG $1^{\mathrm{WD} 40}$, threading along the $\mathrm{PRP} 8^{\mathrm{NTDL}}, \mathrm{PRP} 8^{\mathrm{HB}}$, and PRP8 ${ }^{\mathrm{RT}}$. The low complexity regions of CWC15 present one of many examples of an intrinsically disordered protein that is structured upon integration into the spliceosome [197, 215].

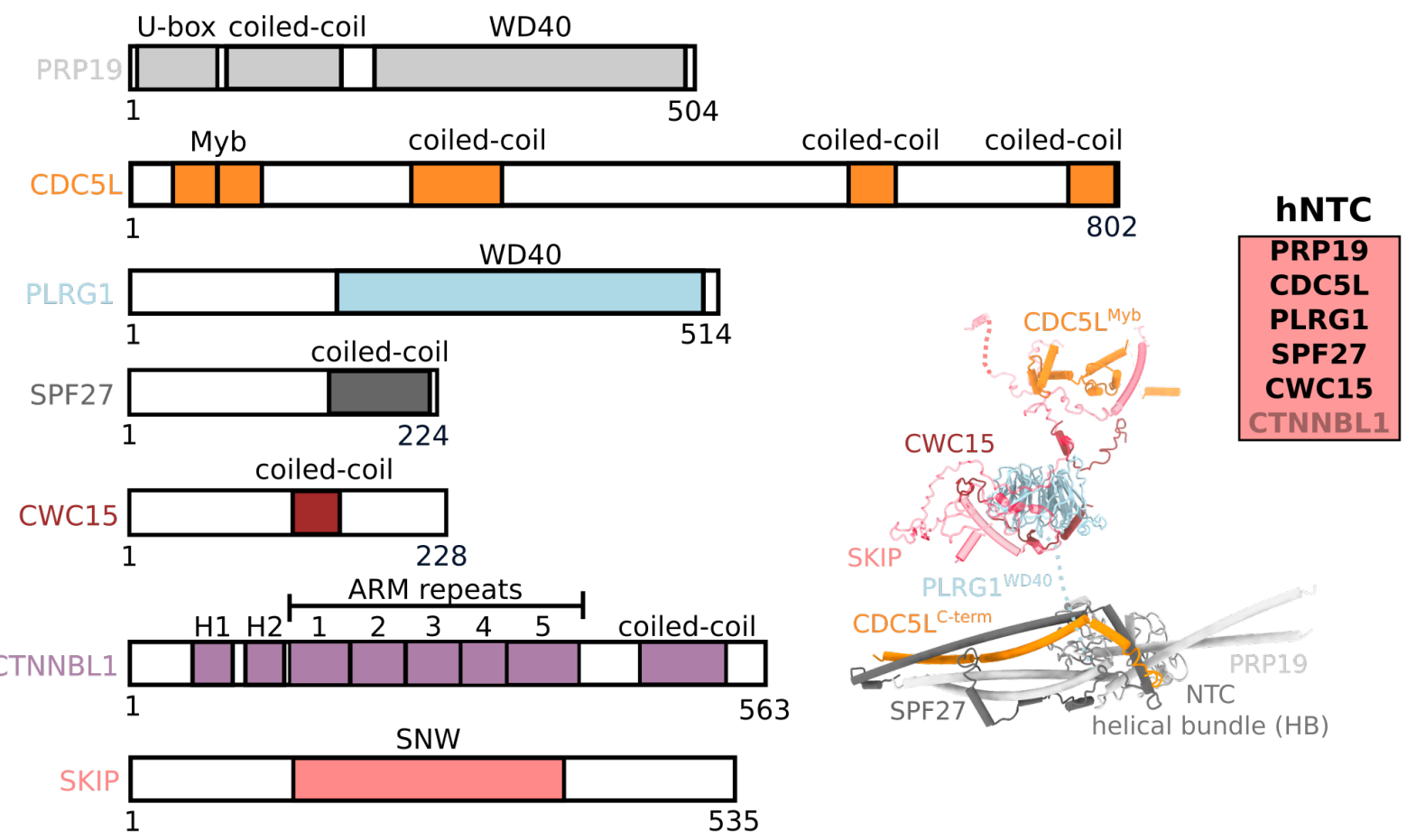

Fig. 1.15: NineTeen Complex (NTC) architecture. Names of proteins making up the NTC (right), their domain architectures (left) and 3D structure (middle; from PDB 6FF7) of NTC proteins identified in $\mathrm{B}^{\text {act }}$. Stippled lines indicate flexible linker regions that are not modelled in the structure. The NTR protein, SKIP, associates closely with the core NTC proteins and is therefore also shown. CTNNBL1 is not shown as a structural representation as it not been identified in a structure of the spliceosome. The NTC helical bundle is found only in later stages of B $\mathrm{B}^{\text {act }}$ (e.g., state 4).

CDC5L is a protein that interacts with both the core as well as the periphery of the spliceosome. The N-terminal region of CDC5L contains two domains comprised of short 
$\alpha$-helices, known as Myb domains $\left(\mathrm{CDC} 5 \mathrm{~L}^{\mathrm{Myb}}\right)$, which have nucleic acid binding activity [216]. CDC5L $\mathrm{L}^{\mathrm{Myb}}$ domains are positioned very close to U2/U6 helix Ib of the active site, and are proposed to facilitate formation of $\mathrm{U} 2 / \mathrm{U} 6$ snRNA interactions of the catalytic center [95, 99]. The C-terminal region of CDC5L contains coiled-coil regions that together with PRP19 and SPF27 form the NTC Helical Bundle (NTC $\left.{ }^{\mathrm{HB}}\right)$, which is stably integrated into the spliceosome during late stages of $\mathrm{B}^{\text {act }}$ formation [99].

CTNNBL1 contains two short helices, $\mathrm{H} 1$ and $\mathrm{H} 2$, in its N-terminal region, followed by $\alpha$-helical armadillo repeats (ARM) and a coiled-coil region [217, 218. CTNNBL1 and CWC15 are the least stably bound components of the NTC and are known to readily dissociate under high ionic strength conditions [215].

\section{NineTeen Complex-Related proteins}

Several proteins associate with the NTC and are therefore termed NTC-related (NTR) proteins, which include BUD31, RBM22, SKIP, and PPIL1 [219]. The NTR proteins are thought to not be part of a preformed complex and are thus expected to associate individually to the spliceosome. BUD31 binds near the $5^{\prime}$ stem-loop (SL) of U6 snRNA and is likely involved in stabilization thereof, while RBM22 latches onto the $3^{\prime}$ end of the U6/5'ss helix [220]. RBM22 (Cwc2 in yeast) contains a zinc finger (Znf) domain in its N-terminal region involved in protein-protein and protein-RNA interactions, as well as an RRM domain in its C-terminal region that associates with surrounding proteins and the intron [220, 221].

RBM $22^{\text {RRM }}$ forms a bridge between the U5 snRNP core of the spliceosome and a large (ca. $400 \mathrm{kDa}$ ) protein subcomplex, the Intron Binding Complex, into which the intron is threaded from the U6/5'ss across RBM22 $2^{\mathrm{Znf} / \mathrm{RRM}}$ [99]. SKIP contains an N-terminal region that mediates its interaction with PPIL1 and a C-terminal SNW domain that tethers SKIP to the spliceosome [222 224]. PPIL1 binds to the spliceosome in later stages of activation, prior to the first catalytic step, and interacts with SKIP and RBM22 [99, 220]. 


\section{Intron Binding Complex}

The Intron Binding Complex (IBC), comprised of AQR, SYF1, PPIE, ISY1 and ZNF830 (Fig. 1.16] 225], bridges the $3^{\prime}$ end of the intron at the U6/5'ss helix to the U2/BS helix within U2 snRNP [225]. Connections between RBM22 and AQR stabilize IBC association with the spliceosome [99]. AQR is a large (171 kDa), ATP-dependent RNA helicase that exhibits a multi-domain architecture [225]. ATPase activity of AQR is required for splicing, although the target of AQR's activity is not known [225]. SYF1 contains 14 Half-A-Tetricopeptide (HAT) repeats which form a curved structure of short $\alpha$-helices [99, 226, 227]. PPIE (also known as CypE) consists of an N-terminal RRM domain as well as a peptidyl-prolyl cis-trans-isomerase (PPIase) domain in its C-terminal region [228]. PPIE is situated at a juncture between the U2 snRNP-associated proteins SF3B2/SF3B4 and SYF1/AQR, forming an additional bridging interaction between the IBC and the body of the spliceosome. PPIE ${ }^{\mathrm{RRM}}$ interacts with the intron [120, 199]. As for all PPIases of the spliceosome, the role of PPIE ${ }^{\text {PPIase }}$ is unclear.

ISY1 contains at least two $\alpha$-helices in its N-terminal region, which bind to the IBC via interfaces to PPIE ${ }^{\text {PPIase }}$ [199]. ISY1 was suggested to regulate Step 2 of splicing by modulating activity of the DExD/H-box helicase, PRP16 [229]. ZNF830 is a small protein (106 aa) containing a zinc finger motif and a predicted coiled-coil region, but has not been visualized in reconstructions of the spliceosome and the function of ZNF830 in the spliceosome is unknown [225].

$B^{\text {act }}$ proteins

Numerous additional proteins join the spliceosome during activation, including SYF3, PPIL2, and PRP17 but are not known to join as part of preformed subcomplexes. SYF3 (also known as CRNKL1 or CRN) contains 17 HAT repeats and binds to the spliceosome between the IBC and PLRG ${ }^{\mathrm{WD}} 40$ of the NTC, and has been reported to interact with PPIL2 (also known as CYC4) [230]. SYF3 also interacts with U6 snRNA at the catalytic center, potentially contributing to stabilization of the activated complex [99]. PPIL2 has been reported to have E3 ubiquitin ligase activity but its role in the spliceosome is unclear, 


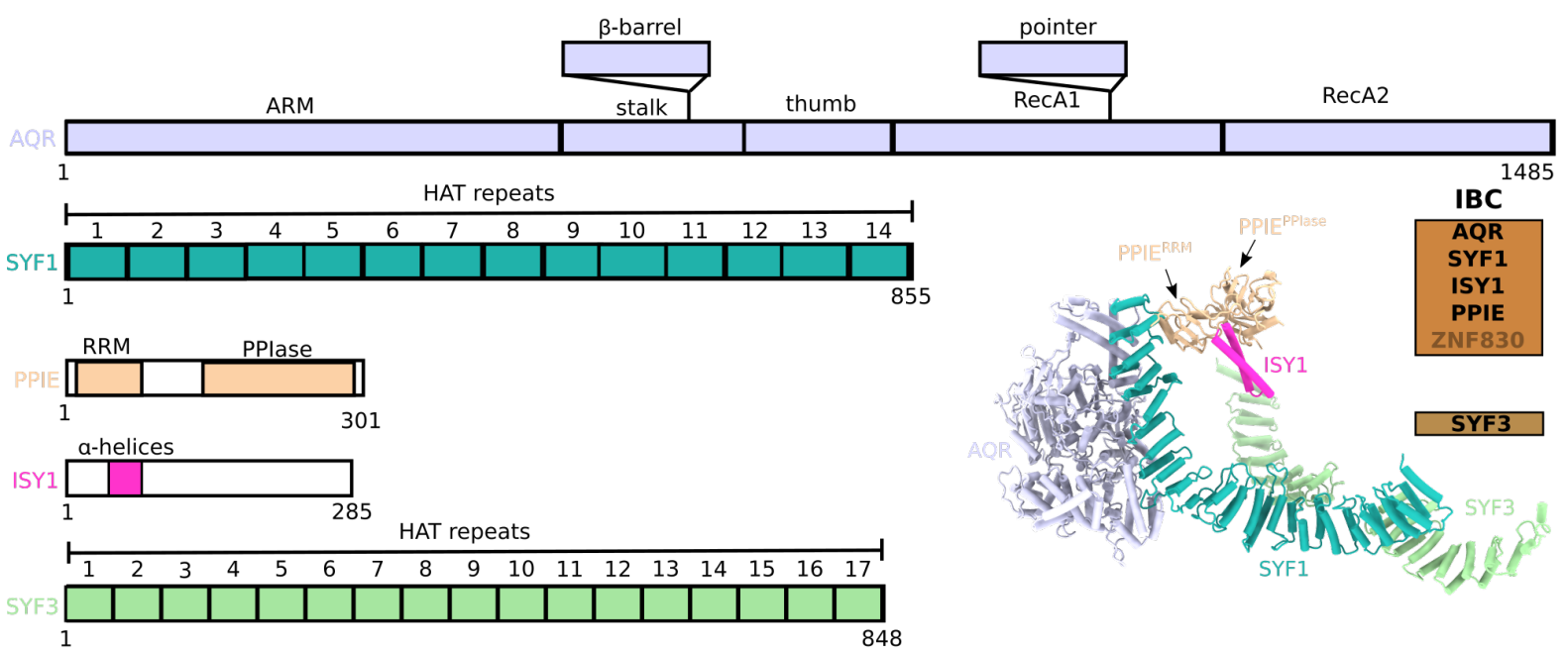

Fig. 1.16: Intron Binding Complex (IBC) and SYF3. Names of IBC proteins and the closely associated SYF3 (right), their domain architectures (left) and 3D representation as identified in $\mathrm{B}^{\text {act }}$ (PDB 6FF7).

and it has not been identified in any published spliceosome structures as of this writing [231]. PRP17 contains a WD40 domain $\left(\mathrm{PRP} 17^{\mathrm{WD} 40}\right)$ that binds near the catalytic center of the activated spliceosome, and is known to join the spliceosome during late stages of activation [99]. Disordered regions of PRP17 interact tightly with NTR proteins, forming a complex web of interactions.

Several factors associate with the spliceosome during the final stages of activation, including CWC27, CWC22, RNF113A, SRRM1, SRRM2, PRP2, and GPKOW. CWC27 and CWC22 are splicing factors that together form a heterodimer which is reported to stabilize the interaction between the exon junction complex (EJC) and the spliceosome following Step 1 of splicing [232]. In addition, CWC27 contains a PPIase domain that binds between PRP8 ${ }^{\mathrm{En}}$ and $\mathrm{PRP} 8^{\mathrm{NTD}}$ domains following the dissociation of the B-specific proteins from their binding site in the B complex. RING finger protein 113A (RNF113A), a ubiquitin ligase proposed to polyubiquitinate BRR2 [233], is integrated into the spliceosome during activation and closely associated to the catalytic RNA network [99]. Along with $\mathrm{CWC} 27^{\text {PPIase }}, \mathrm{RNF} 113 \mathrm{~A}$ binds to the narrow gap between the PRP8 ${ }^{\mathrm{En}}$ and PRP8 $8^{\text {NTD }}$ domains, preventing complete closure of PRP8 (Fig. 1.11).

Serine/arginine repetitive matrix protein 1 (SRRM1, also known as SRm160) binds premRNA and associates with SR proteins involved in splicing [234]. SRRM1 was localized 
in the activated spliceosome, but has not been observed in the pre-catalytic spliceosome [98, 99]. It is unclear whether SRRM1 joins the spliceosome during activation, or merely becomes stabilized due to protein-protein interactions as the spliceosome assembles. Serine/arginine repetitive matrix protein 2 (SRRM2, also known as SRm300) forms a heterodimer with SRRM1 (SRm160/300) that is known to be involved in the control of splicing by exonic splicing enhancers (ESEs) by forming contacts to SR proteins and U2 snRNP [235], and was reported to be involved in modulation of alternative splicing [236]. SRMM2 interacts with PRP8 ${ }^{\mathrm{SWL}}$, and was proposed to contribute to stabilization of $\mathrm{PRP} 8^{\mathrm{SWL}}$, which appears to be initially flexible after repositioning from the B-complex configuration (Fig. 1.10] [99, 237].

PRP2 and GPKOW (Spp2) are also recruited in the formation of $\mathrm{B}^{\text {act }}$. PRP2, the $\mathrm{DExD} / \mathrm{H}$-box helicase responsible for promoting catalytic activation of the spliceosome (i.e., B* formation), docks to the U2 snRNP component SF3B1 (HEAT domain, repeats 7-8), where it binds the pre-mRNA [238]. Association of PRP2 to the spliceosome is mediated by GPKOW (Spp2), which co-activates PRP2 and is required for its activity [238. With PRP2 in place, the activated spliceosome can be converted to the $\mathrm{B}^{*}$ complex to carry out Step 1 of splicing (Fig. 1.4] [211].

\subsubsection{Proteins surrounding the active site RNA in $B^{\text {act }}$}

The catalytic center of the spliceosome is surrounded not only by PRP8, but also by many other factors that are repositioned and/or join the spliceosome during the formation of an activated complex [99]. The loop of U6 ISL is docked to PRP8 ${ }^{\text {NTD }}$ and is contacted by the flexible strand of $\mathrm{PRP} 8^{\mathrm{NTDL}}$, which connects the $\mathrm{PRP} 8^{\mathrm{NTD}}$ to the $\mathrm{PRP} 8^{\mathrm{HB}}$ domain. SYF $3^{\text {N-term }}$ HAT repeats, as well as SKIP and RBM22 (NTR proteins), contact a flippedout nucleotide of the stem region of the ISL (C60), while CWC15 and PLRG1 (NTC proteins) interact with C68 of the ISL loop region [99].

Helix $\mathrm{Ib}$ is fit into a groove of $\mathrm{PRP} 8^{\mathrm{HB}}$, while helix Ia is surrounded by $\mathrm{PRP} 8^{a-f i n g e r}$, the CDC5L ${ }^{\mathrm{Myb}}$ (an NTC protein), as well as SF3A2 and SF3B2 (U2 snRNP proteins). 
The 5'ss is bound by RNF113A, following the dissociation of the B-specific proteins. This ensemble of proteins forms a complex network in which protein-RNA interactions stabilize the mature catalytic center within the closed conformation of PRP8. With the exception of SF3A2, SF3B2, and PRP8, all of these proteins become integrated as part of spliceosome activation, implying a role in facilitating formation of the U2/U6 active site RNA.

Surprisingly, both steps of pre-mRNA splicing can be carried out in the absence of proteins by $\mathrm{U} 2 / \mathrm{U} 6$ snRNAs alone in vitro, although it is unclear whether such a minimal system is as tightly controlled as found in vivo in the spliceosome [239 242]. In a protein-free system, Step 1 occurred by hydrolysis as opposed to branching; that is, the BS-A did not act as the nucleophile in the reaction, indicating mechanistic differences in the proteinfree, in vitro system. Such discrepancies appear to suggest that proteins are likely involved in mediating the proper positioning of the BS-A in the spliceosome [239]. Furthermore, splicing efficiency is increased by NTR, IBC, and RES proteins [225, 243, 244], while the NTC is essential for Step 1 of splicing [245]. The observed regulatory roles for these factors is perhaps unsurprising, as proteins make up approximately $90 \%$ of the molecular weight of the spliceosome [246]. All of these events strongly suggest that although protein-free systems in vitro can technically carry out reactions that are similar - but not identical to pre-mRNA splicing, proteins are required for coordinating the formation of the activated spliceosome and for tightly regulating splicing fidelity and efficiency. While the formation of a catalytically essential, RNA triple helix in the spliceosome is well documented, the potential role of proteins in facilitating folding of U2/U6 active site RNA has not been fully determined. Moreover, the temporal ordering of active site formation - that is, the order in which the ISL, helix Ia, helix Ib, and the resulting triple helix are formed - is unclear.

Structural and biochemical studies have been successful at characterizing snapshots of spliceosomes stalled before and after the catalytic center is formed (i.e., the pre-catalytic $\mathrm{B}$ complex and the activated $\mathrm{B}^{\text {act }}$ complex), but have so far not been able to shed light onto intermediate states during the formation of the catalytic RNA network at the core of the 
spliceosome [99, 196, 247, 248]. The molecular architecture of such intermediates would provide insight into the critical steps during activation of the spliceosome, while possibly revealing assembly checkpoints that contribute to accurate folding of the catalytic center. In particular, it would be of interest to isolate complexes trapped at intermediate stages of activation, following the dissociation of U4 snRNP but prior to the stable association of the non-snRNP proteins that become integrated in $\mathrm{B}^{\text {act }}$ (i.e., RES, NTC/NTR, IBC and later stage assembly proteins). It would also be necessary to analyze the conformation and arrangement of PRP8, and to determine whether its conformational change occurs before or after the binding of the RES, NTC/NTR, IBC and additional factors, and how this may be coordinated by the B-specific proteins. The identification of activation intermediates may also yield new insights into the roles of proteins that co-purify with the spliceosome but have not yet been identified in cryo-EM structures of spliceosome assembly. However, the biochemical preparation of novel structural "snapshots" has been hindered by a lack of available means by which to stall spliceosome assembly at intermediate stages of activation.

\subsection{Small molecule chemical inhibitors of pre-mRNA splicing}

Small molecule chemical inhibitors provide a method by which to trap spliceosomal complexes at intermediate stages of assembly. A major focus of spliceosome inhibition has been on SF3B1, a core component of the U2 snRNP that accommodates the U2/BS helix and is frequently mutated in numerous cancers [249]. Targeting of SF3B1 has therefore been the subject of many studies due to its relevance in disease. A number of natural products or synthetic derivatives target SF3B1, stalling spliceosome assembly at the A complex stage at low nanomolar or micromolar concentrations [250]. Many of these compounds share a similar structural backbone consisting of a conjugated diene [251]. These compounds are relatively well characterized through structure-activity relationships, in 
some cases detailing the mechanism of how these inhibitors work by blocking a functionally relevant conformational change within SF3B1 [252].

Several examples exist of spliceosome inhibitors that block assembly by interfering with post-translational modifications of proteins involved in splicing [250]. It has been shown that inhibition of Ser/Thr protein phosphatase-1 (PP1) and protein phosphatase 2A (PP2A) by the natural products okadaic acid, tautomycin, and microcrystin-LR block splicing reactions [253]. Use of a phosphatase inhibitor cocktail (PhosSTOP, Roche) was shown to arrest spliceosome assembly at a pre-catalytic stage, resulting in a mixture of pre-B and B complexes [203]. Blockage of histone acetyltransferases, histone deacetylases and SR protein kinases all results in arrest of splicing activity [83, 254]. Thus, interruption of the post-translational modifications of proteins involved in splicing has profound effects on the assembly and function of the spliceosome, and presents an additional way to study assembly intermediates. A large-scale screen of small molecules identified a distinct class of quinone derivatives that stall splicing at low micromolar concentrations [255]. The most potent of these compounds (measured by the in vitro $50 \%$ inhibitory concentration $\left(\mathrm{IC}_{50}\right)$ in the assay) were BN82685 $(7 \mu \mathrm{M})$, NSC663284 $(10 \mu \mathrm{M})$, and NSC95397 (21 $\mu \mathrm{M})$. BN82685 was shown to inhibit exon ligation (Step 2), thus stalling the spliceosome at approximately the $\mathrm{C}$ complex stage [255]. However, the accumulation of spliceosome assembly intermediates was not reported. A later high throughput screen of over 160,000 compounds identified eight small molecules that inhibited in vitro pre-mRNA splicing [256]. Fortuitously, one of these eight compounds was NSC95397. Further investigation of NSC95397 (also referred to as compound 297) revealed that at concentrations at or above $75 \mu \mathrm{M}$, a mixture of what appeared to be A and B complexes resulted, indicating that the compound stalls splicing at a pre-catalytic stage [256]. Deeper analysis of the resulting affinity purified complexes revealed stoichiometric amounts of U2, U5 and U6 snRNA and a decrease in U1 and U4 snRNAs relative to the B complex [256]. Additionally, mass spectrometry analysis indicated the presence of the NTC/NTR proteins as well as the B-specific proteins, but underrepresentation of later stage assembly factors (i.e., RNF113A and PRP17) [256]. Taken together, these findings indicated that NSC95397 
could potentially stall spliceosome assembly at an intermediate stage of activation, following the assembly of the $\mathrm{B}$ complex yet prior to the formation of a mature $\mathrm{B}^{\text {act }}$ complex, providing a putative tool to study elusive states of the human spliceosome during the formation of its catalytic center. In summary, the two key questions of such an investigation are as follows:

- Are there structurally distinct intermediate stages of activation at which formation of the catalytic center can be observed?

- How do proteins facilitate the folding of active site U2/U6 snRNAs in the spliceosome? 


\subsection{Aim of this study}

In eukaryotes, DNA is transcribed into precursor mRNA (pre-mRNA), which contains protein-coding exons separated by non-coding introns. Prior to export of mRNA from the nucleus to the cytoplasm, introns are excised from the pre-mRNA by the spliceosome: a highly dynamic, multimegadalton machine that is assembled de novo for each intron to be spliced. The spliceosome is constructed of small nuclear ribonucleoprotein (snRNP) building blocks - U1, U2, and the U4/U6.U5 tri-snRNP, in which U4 and U6 snRNAs are base-paired and U5 is associated by protein-protein interactions - which bind to their pre-mRNA substrate in a stepwise manner, proceeding through a series of complexes until an catalytically competent complex is formed. Progression through the spliceosome assembly pathway is driven by the action of ATP-dependent RNA helicases that remodel the RNA-protein and RNA-RNA network of the complex. Over 150 proteins are associated with the spliceosome, making it one of the most complicated molecular machines in the nucleus. While over $90 \%$ of the molecular mass of the spliceosome is contributed by proteins, the remaining $10 \%$ consists of RNA. This relatively minor contribution by RNA is responsible for the catalysis of RNA splicing, making the spliceosome a ribozyme. Unlike many enzymes, the catalytic center of the spliceosome is not a preformed entity, but must be actively sculpted by remodeling base-pairing interactions in a step known as activation. Activation is the single largest change in the molecular architecture and composition of the spliceosome. During activation, the pre-catalytic spliceosome - which contains $\mathrm{U} 2, \mathrm{U} 4, \mathrm{U} 5$ and $\mathrm{U} 6 \mathrm{snRNPs}$ - is drastically altered by the action of an ATPdependent helicase, BRR2, which unwinds the base-pairing of the U4/U6 snRNA duplex, allowing for the liberation of the U4 snRNP and associated proteins, as well as for largescale rearrangements in the molecular architecture of the protein and RNA content of the spliceosome. It is as this step that at least 28 additional factors, some of which are organized into salt-stable subcomplexes, join the spliceosome. At some point during activation, a group of proteins known as the B-specific proteins is destabilized from the complex, allowing for the central scaffold protein of the spliceosome, PRP8, to undergo 
a conformational change from an open to a closed conformation. It is within a compact cavity of PRP8 that the U2/U6 RNA network of the active site is formed, resulting in an activated spliceosome.

Structural studies by cryo-EM have elucidated the molecular architecture of complexes prior to and following activation, providing major insights into the conformational and compositional rearrangements that must occur for an activated complex to be formed. However, the temporal ordering in the exchange of proteins is not well understood. Moreover, the potential role of proteins in mediating the folding of U2/U6 RNA of the catalytic center is unclear. Thus, it would be advantageous to capture "snapshots" of the spliceosome at discrete points along its activation pathway, which would likely reveal new insights into the integration and destabilization of proteins that may assist in the formation of an active complex. Despite this clear need for finer sampling of the spliceosome activation step, no such structures have been reported. Several approaches to stall the splicing using chemical inhibitors have been introduced, suggesting possibly viable new methods to trap assembly at biochemical and structural stages of activation. High throughput screening of small molecule libraries successfully identified quinone derivatives as compounds that inhibited splicing, blocking assembly at distinct stages. Further investigation of this phenomenon suggested that one of these compounds, NSC95397, appeared to stall spliceosome activation. We therefore set out to determine the structure of spliceosomes stalled by NSC95397, with the goal of identifying previously uncharacterized structural stages of activation. The aim of this thesis is to unravel the spliceosome activation step using a small molecule chemical inhibitor, NSC95397, and to determine the 3D molecular architectures of the resulting complexes with cryo-EM. 


\section{Materials and Methods}

\subsection{Materials}

\subsubsection{Software}

\begin{tabular}{ll}
\hline Software & Reference \\
\hline COW & unpublished \\
Coot & {$[257,258]$} \\
cryoSPARC & {$[259]$} \\
Gautomatch & unpublished \\
Gctf & {$[260]$} \\
Mascot & {$[261]$} \\
MolProbity & {$[262]$} \\
MotionCor2 & {$[160]$} \\
PHENIX & {$[258,263]$} \\
pLink2.3.5 & {$[264$,} \\
Protein Data Bank & {$[266]$} \\
PyMOL & unpublished \\
RELION 2.0-1 and 3.0 & {$[267,[268]$} \\
SpliProt3D & {$[269]$} \\
SWISS-MODEL & {$[270]$} \\
UCSF Chimera & {$[271]$} \\
UCSF ChimeraX & {$[272]$} \\
UniProt & {$[273]$} \\
\hline
\end{tabular}




\subsubsection{Equipment}

\begin{tabular}{ll}
\hline Equipment & Manufacturer \\
\hline Amicon 50 kDa filters & Merck \\
C $_{\text {s }}$ corrector & CEOS GmbH \\
Falcon 3EC Direct Electron Detector & Thermo Fisher Scientific \\
LTQ Orbitrap XL mass spectrometer & Thermo Fisher Scientific \\
MBP Trap HP column & GE Healthcare \\
Q Exactive HF-X mass spectrometer & Thermo Fisher Scientific \\
Quantifoil 3.5/1 Cu grids & Quantifoil \\
Superdex Peptide PC3.2/30 column & GE Healthcare \\
Surespin TLS-630 rotor & Thermo Fisher Scientific \\
Titan Krios electron microscope & Thermo Fisher Scientific \\
TST41.14 rotor & Kontron \\
Typhoon phosphoimager & GE Healthcare \\
Vitrobot Mark IV & Thermo Fisher Scientific \\
\hline
\end{tabular}

\subsection{Methods}

Biochemical experiments (see 2.2.1, 2.2.2, 2.2.3, 2.2.4, 2.2.5, 2.2.7 were carried out by Dr. Majety Leelaram and Dr. Dmitry Agafonov (Department of Cellular Biochemistry, MPIBPC), and crosslink analysis was performed by Dr. Olex Dybkov (Department of Cellular Biochemistry, MPI-BPC) in collaboration with Prof. Dr. Henning Urlaub (Bioanalytical Mass Spectrometry, MPI-BPC) (see 2.2.6).

\subsubsection{In vitro splicing}

HeLa S3 cells (Helmholtz Zentrum für Infektionsforschung, Braunschweig) were obtained, tested negative for mycoplasma, and used to prepare splicing extracts [274]. MINX-MS2 pre-mRNA was prepared in vitro transcription by T7 RNA polymerase, with $\alpha^{32} \mathrm{P}-\mathrm{UTP}$ (for radiolabeling of pre-mRNA) and $\mathrm{m}^{7} \mathrm{G}\left(5^{\prime}\right) \mathrm{ppp}\left(5^{\prime}\right) \mathrm{G}$-cap present. To carry out in vitro splicing reactions, the resulting ${ }^{32}$ P-labeled MINX-MS2 pre-mRNA (5.0 nM) was incubated at $30^{\circ} \mathrm{C}$ for specified time points (Fig. 3.1) in the presence of $40 \%(\mathrm{v} / \mathrm{v})$ HeLa 
nuclear extract, $20 \mathrm{mM}$ HEPES-KOH pH 7.9, $3 \mathrm{mM} \mathrm{MgCl}_{2}, 65 \mathrm{mM} \mathrm{KCl,} 2 \mathrm{mM}$ adenosine triphosphate (ATP), and $20 \mathrm{mM}$ creatine phosphate as well as increasing amounts (Fig. 3.1 of the splicing inhibitor splicing inhibitor NSC95397 (Sigma Aldrich), which was solubilized in dimethyl sulfoxide (DMSO). Following the splicing reaction, NSC95397 was quenched with a twofold molar excess of cysteine for 2 min at $30^{\circ} \mathrm{C}$ and then put on ice. RNA isolation was carried out using phenol-chloroform extraction and a subsequent ethanol precipitation step, and the purified RNAs were examined using a $14 \%$ denaturing polyacrylamide gel. Splicing products, including intermediates and unspliced pre-mRNA, were visualized with a Typhoon phosphoimager (GE Healthcare). Formation of spliceosomal complexes was monitored using $1.5 \%$ low-melting agarose gel electrophoresis containing $0.4 \mathrm{mg} / \mathrm{ml}$ heparin [275].

\subsubsection{MS2 affinity purification of spliceosomes}

To prepare for affinity purification of spliceosomes stalled by the action of NSC95397, a 10-fold molar excess of MS2-MBP fusion protein was incubated with $\alpha^{32} \mathrm{P}$-labeled MINXMS2 pre-mRNA (5 nM) for $30 \mathrm{~min}$ on ice. The pre-mRNA, bound by MS2-MBP, was used as the substrate in a splicing reaction in the presence of $160 \mu \mathrm{M}$ of NSC95397, incubated for $60 \mathrm{~min}$ at $30^{\circ} \mathrm{C}$, and subsequently quenched with $320 \mu \mathrm{M}$ cysteine for 2 min. Centrifugation was used to remove aggregates and the salt concentration was increased to $165 \mathrm{mM}$ by addition of sodium chloride $(\mathrm{NaCl})$. For affinity purification, the resulting splicing reaction was loaded on an MBP Trap HP column (GE Healthcare) preequilibrated with G-150 buffer (20 mM HEPES-KOH pH 7.9, $1.5 \mathrm{mM} \mathrm{MgCl}, 150 \mathrm{mM}$ $\mathrm{NaCl}$ ). G-150 buffer was used to wash the column and $10 \mathrm{mM}$ maltose (in G-150 buffer) was used to elute the spliceosomes. Following isolation of the affinity purified complexes, a further gradient purification step was used to separate complexes by their sedimentation coefficients. Samples were loaded onto a 36-ml linear 10 to $30 \%$ (v/v) glycerol gradient (G-150 buffer) and centrifuged for 15 hours at $4^{\circ} \mathrm{C}$ and 25,000 rotations per minute (rpm) using a Surespin TLS-630 rotor (Thermo Fisher Scientific). A peristaltic pump was used 
to fractionate the gradients from the bottom of each tube, and the radioactivity of each fraction was measured with Cherenkov counting. Peak gradient fractions were analyzed for RNA composition using denaturing 4 to 12\% NuPAGE gels (Life Technologies), which were subsequently stained with SYBR Gold for detection and visualization.

\subsubsection{D gel electrophoresis and mass spectrometry}

2D gel electrophoresis, as described in [276], was used to separate all proteins in the purified sample larger than $25 \mathrm{kDa}$. Following staining of the gel with Coomassie, individual proteins spots were excised, digested with trypsin, and extracted. The extracted peptides were subjected to a liquid chromatography-coupled electrospray ionization mass spectrometer (LTQ Orbitrap XL). Protein identities were determined by comparison of fragment spectra against the UniProt database [273], with Mascot used as a search engine [261].

\subsubsection{Chase of pre- $B^{\text {act }}$ complexes with micrococcal nuclease-treated extract}

To digest RNAs in the sample, micrococcal nuclease (MN) was added to HeLa nuclear extract (as described in [197]). B and pre- $\mathrm{B}^{\text {act }}$ complexes were formed separately on $\alpha^{32}$ P-labeled MINX-MS2 pre-mRNA, affinity purified, and incubated under the following two conditions: 1) in splicing buffer alone (20 mM HEPES-KOH pH 7.9, $65 \mathrm{mM} \mathrm{KCl,} 3$ $\mathrm{mM} \mathrm{MgCl}_{2}, 2 \mathrm{mM}$ ATP, $20 \mathrm{mM}$ creatine phosphate); 2) in the presence of HeLa nuclear extract that had been treated with $20 \%$ (v/v) MN. Unlabeled MINX-MS2 pre-mRNA was added in 20-fold excess to block the reassembly of spliceosomes on radiolabeled premRNA. Reactions were kept at $30^{\circ} \mathrm{C}$ for timepoints from 0 to $60 \mathrm{~min}$. RNA from the resulting samples was isolated, analyzed on a $14 \%$ denaturing polyacrylamide gel, and visualized with a Typhoon phosphoimager (GE Healthcare). 


\subsubsection{Western blotting}

Proteins from the purified complexes were separated on denaturing 4 to $12 \%$ NuPAGE gels and transferred onto nitrocellulose membranes (Amersham Protran 0.2, GE Healthcare). Antibodies against the following human proteins were used to probe the membrane: hSnu114 [277], LSm4 [57], hPrp19 and AQR [213], hCwc22 (Proteintech Europe, Manchester, UK catalog number 26898-1-AP), hRNF113A (Sigma Aldrich, catalog number HPA000160, RRID: AB_1079821), and hPPIL2 (ThermoFisher Scientific, catalog number PA5-29239, RRID: AB_2546715). An ECL detection kit (GE Healthcare) was used to detect bound antibody.

\subsubsection{Cross-linking of pre- $B^{\text {act }}$ complexes and cross-link identification}

Following MS2 affinity purification, an additional step was used to further purify spliceosomes, by introducing a 10 to $30 \%$ (v/v) glycerol gradient centrifuged at 23,300 rpm for 16 hours $40 \mathrm{~min}$ at $4^{\circ} \mathrm{C}$ in a TST41.14 rotor (Kontron). Peak fractions having approximately $15 \mathrm{pmol}$ of the pre-B $\mathrm{B}^{\text {act }}$ complexes were combined and cross-linked with $150 \mu \mathrm{M}$ $\mathrm{BS} 3$ at $25^{\circ} \mathrm{C}$ for $30 \mathrm{~min}$. Samples were subsequently quenched using $1.5 \mathrm{mM}$ Tris- $\mathrm{HCl}$ $\mathrm{pH} 8.0$ at $25^{\circ} \mathrm{C}$ for $10 \mathrm{~min}$, placed on ice for $20 \mathrm{~min}$, and pelleted using ultracentrifugation and a S100AT4 rotor (Thermo Fisher Scientific) and analyzed as previously reported [197]. Peptide extraction was carried out in a reverse-phase manner with Sep-Pak Vac tC18 1-cc cartridges (Waters) and peptides were fractionated using gel filtration by the use of a Superdex Peptide PC3.2/30 column (GE Healthcare). A Thermo Fisher Scientific Q Exactive HF-X mass spectrometer was used to analyze fifty- $\mu$ l fractions in triplicate, corresponding to an elution volume of 1.2 to $1.8 \mathrm{ml}$. The pLink2.3.5 search engine (pfind.ict.ac.cn/software/pLink) was used to identify protein-protein crosslinks, and the results were filtered by false discovery rates of 1,3 , and $5 \%$ [264, 265]. The score for crosslinks is shown as the negative value of the common logarithm of the original pLink score: Score $=-\log 10(" p L i n k$ Score" $)$. 


\subsubsection{Purification and buffer exchange}

To prepare samples for cryo-EM, peak fractions of samples purified by MS2 affinity selection and glycerol gradient centrifugation were combined. These combined samples were concentrated and buffer exchanged in G-150 buffer (without glycerol) using an Amicon $50-\mathrm{kD}$ cut-off filter as reported in [98]. For further purification, buffer-exchanged samples were loaded on a 36-ml linear 10 to $30 \%$ (v/v) glycerol gradient in G150 buffer in the presence of 0 to $0.15 \%$ glutaraldehyde (for fixation) [278], centrifuged at 25,000 rpm at $4^{\circ} \mathrm{C}$ for 15 hours using a Surespin TLS-630 rotor (Thermo Fisher Scientific). The gradient was fractionated and quenched immediately using $100 \mathrm{mM}$ aspartate (pH 7.0). Peak fractions were combined and subjected to one more round of concentration and buffer exchange as before. Control experiments were carried out by following the same protocol as described, only without addition of glutaraldehyde to the gradient, thus allowing the protein and RNA content of the final samples to be analyzed using 2D gel electrophoresis and denaturing polyacrylamide gel electrophoresis.

\subsubsection{Cryo-EM sample preparation and data acquisition}

Approximately $30 \mu \mathrm{l}$ of purified sample was added to a well of a custom Teflon block kept on ice. A piece of continuous carbon film (prepared in-house) was placed directly on top of the sample and incubated for 1 minute, allowing the purified samples to adsorb to the carbon film. The carbon film was adhered to the evaporated carbon-coated face of a QUANTIFOIL 3.5/1 grid (Quantifoil, Jena, Germany) and $4 \mu$ l of distilled-deionized water was added to the same side as the sample to prevent dehydration. Grids were blotted and plunge-frozen in liquid ethane at ambient conditions of $100 \%$ humidity and $4^{\circ} \mathrm{C}$ using a Vitrobot Mark IV (Thermo Fisher Scientific). For data acquisition, a Titan Krios electron microscope (Thermo Fisher Scientific) equipped with a $\mathrm{C}_{\mathrm{s}}$ corrector (CEOS, Heidelberg) and Falcon III direct electron detector (Thermo Fisher Scientific) operated in integration mode was used. Images were acquired at a nominal magnification of 59,000x, resulting in a pixel size of $1.16 \AA$. Each individual micrograph was comprised of 40 individual frames 
and a total fluence of $45 \mathrm{e} / \AA^{2}$ acquired over an exposure time of $1 \mathrm{~s}$, divided into 20 fractions (2.25 e/ $\AA^{2}$ per fraction). 10,000 micrographs were collected in an automated acquisition, carried out using spot-scanning in a 3x3 array for each $3.5 \mu \mathrm{m}$ hole. Grid preparation and data acquisition were carried out by Dr. Karl Bertram (Department of Structural Dynamics, MPI-BPC).

\subsubsection{Image processing}

Motion correction and dose-weighting of micrographs was carried out with MotionCor2 [160], followed by calculation of CTF parameters with Gctf [260]. Each micrograph was inspected manually using the Micrograph Quality Checker (MQC) of COW (www.cow-em.de/) and micrographs were judged by optical contrast, nominal resolution, and astigmatism. Micrographs showing predominantly crystalline ice, ethane contamination, or particle aggregates were exluded from further processing, resulting in 4676 out of 10,000 micrographs retained. Gautomatch (https://www2.mrc-lmb.cam.ac. uk/research/locally-developed-software/zhang-software/\#gauto) was used for reference-free identification of 503,208 particle images, which were extracted from micrographs with $2 \mathrm{x}$ binning and split into three equally proportioned subgroups prior to five rounds of 2D classification with RELION 2.0 [267]. 2D class averages showing clear features were chosen (72,193 particle images) for the determination of an initial model, which was calculated using ab initio reconstruction within cryoSPARC [259]. The best resolved initial model was low-pass filtered to $60 \AA$ and used for an initial reference in RELION 2.1 in a 3D classification using 15 classes (regularization parameter, "T", set to 4). Two of the resulting classes exhibited distinguishable features and were better resolved (13 to $15 \AA$ ) than the other 13 classes (>24 $\AA$ ). The two well defined 3D classes consisted of of 108,932 (26\% of particle images) and 56,177 (14\% of particle images) particles. Each class was refined using RELION 2.1 without masks to nominal resolutions of of 6.3 and $6.4 \AA$ (data not shown). Angular orientations were adopted from these refinements and used to re-extract unbinned particle images from dose-weighted micrographs (box size 
384 pixels). The extracted particles were refined against up-scaled references without masking using RELION 3.0 [268], resulting in an improvement in resolution to 5.6 and $6.5 \AA$ A. At this point, a soft mask encompassing the entirety of each particle was generated from each reconstruction and applied to carry out a masked refinement step, improving the average resolutions to $4.4 \AA$ (not shown) and $4.6 \AA$. It was suspected at this point that there may be further structural heterogeneity in the reconstructions. Therefore, soft masks were generated to include the most stable core of each complex, and applied to each reconstruction in a $3 \mathrm{D}$ classification without re-alignment, instead using refined particle coordinates obtained in the previous refinement step. For this step, particle images from each of the two populations were divided into four classes and the regularization parameter $(\mathrm{T})$ was set to 20 . For both populations, this classification step resulted in a single class having a large majority of particle images, containing containing $84,539(77 \%$ of particles classified) and 39,336 (70\% of particles classified). These classes also displayed higher resolution features than the less populated subsets and were thus chosen for further structure determination. As previously, each class was refined initially without a mask, this time resulting in average resolutions of 5.1 and $6.0 \AA$. The reconstructions were then refined with a soft mask encompassing the entire particle, resulting in average resolutions of 4.3 and $4.6 \AA$. To further improve two large regions of the map, multibody refinement was carried out using the refined coordinates of the previous refinement step [166]. Two "bodies" were defined: body 1, consisting of the core of each complex; and body 2, which encompasses the $5^{\prime}$ domain of the $\mathrm{U} 2$ snRNP, particularly the SF3b proteins. For the first group of particles, body 1 was determined to an average resolution of $3.9 \AA$, whereas the body 2 resolution did not improve. For the second group of particles, body 1 was resolved to $4.2 \AA$ and body 2 was resolved to $4.5 \AA$. Although CTF refinement was attempted, it did not improve the resolution. To test for the possible effect of the box size on the resolution of the reconstruction, refinements of both complexes were also carried out in a larger box (512 pixels), but this also failed to result in resolution improvements. The Fourier shell correlation (FSC) 0.143 criterion was used for resolution determination 
[169]. Sharpening of maps using automatically determined B-factors and local resolution variability were carried out in RELION 3.0.

\subsubsection{Model building and refinement}

The protein and RNA components used for building integrative structural models are detailed in Figure B.2 Based on prior knowledge and our protein-protein crosslinking dataset (Table B.1], model files from the Protein Data Bank (PDB) [266] for individual proteins of the spliceosomal $\mathrm{B}$ [98, 203] and $\mathrm{B}^{\text {act }}$ [99, 237], as well as X-ray crystal structures and NMR structures of various single components, were docked as rigid-bodies into the cryo-EM maps using UCSF Chimera v1.12 [271]. A model of the B-specific protein WBP11/NPW38BP was generated using SWISS-MODEL [270] and placed within a helical density within the pre-B ${ }^{\text {act-1 }}$ (see Results) reconstruction, consistent with numerous crosslinks. Models of CTNNBL1, PPIL2, and TCERG-1 (coiled-doil domain and FF4 to 6 domains) were downloaded from the SpliProt3D predicted structure database [269] and docked into densities consistent with intermolecular crosslinks. The remining protein components incorporated into the models were taken from previously published structures (Figure B.2). De novo modeling of several regions of MFAP1, PRP8, and SF3B2 were guided by well-defined EM densities continuing from previously identified, well documented portions of each protein as observed in the B or $\mathrm{B}^{\text {act }}$ complexes. Chains were truncated to polyalanine/polyproline traces and subsequently docked as rigid bodies (for regions with secondary structure) or were docked and manually adjusted (disordered regions) using Coot [257, 258]. U5 snRNA was docked as a rigid-body and its fit was further improved using the jiggle-fit and model morphing scripts in Coot (https://www2.mrc-lmb.cam.ac.uk/groups/murshudov/content/em_ fitting/em_fitting.html), For modeling of the U2/U6 RNA network, the U6/5'ss helix and U6 5'SL regions (nucleotides 1 to 46) from a $\mathrm{B}^{\text {act }}$ complex [237] were fit into both maps, followed by manual adjustment of the 5'ss and a single-stranded region of U6 snRNA (nucleotides C25 to A30). U6 RNA nucleotides A47 to U51 were rebuilt into 
the unmasked and unsharpened EM map of the pre-B ${ }^{\text {act-2 }}$ complex. U2/U6 helix Ib was docked as a rigid body into the pre- $\mathrm{B}^{\text {act-2 }}$ map, guided by clear map features consistent with the phosphate backbone of several nucleotides within the helix. U6 ISL was taken from a published hB ${ }^{\text {act }}$ cryo-EM structure [99] and docked into both maps, followed by manual rebuilding of nucleotides C60 to C61 and A73 to G75 (G58 to C61 and A73 to A76 for pre- $\left.\mathrm{B}^{\text {act-1}}\right)$ in Coot.

U2/U6 helix II [99] was rigid-body fit into the pre-B $\mathrm{B}^{\text {act-2 }}$ reconstruction, based on the proximity of this density to U6 ISL and U2/U6 helix Ib. Based on comparison to previous structures [99, 237], U2 nucleotides of the stem-loop II (U46-U65) and several of stem-loop IIb (G68 to C73 and G79 to A85) were placed in well-defined densities. Individual chains and then the composite models of both complexes were processed by real-space refinement using PHENIX [263]. The resulting models were subjected to MolProbity for analysis of model geometry [262], and FSCs between the map and the model were calculated using phenix.mtriage [258]. Statistics from model refinement are shown in Figure B.1. Model building was overseen by Dr. Berthold Kastner (Department of Cellular Biochemistry, MPI-BPC). 


\section{Results}

\subsection{Biochemical characterization of spliceosomes stalled during activation}

A small molecule chemical inhibitor was used to stall spliceosome assembly at an intermediate stage of activation (Fig. 3.1A). The inhibitor, NSC95397, had been previously identified as a splicing inhibitor in a high-throughput screen [71] and further characterized as a compound that likely stalled spliceosome assembly during activation but prior to the formation of a mature $\mathrm{B}^{\text {act }}$ complex [256]. At a concentration of $150 \mu \mathrm{M}$, splicing was completely blocked, as shown by the RNA products (Fig. 3.1B). Formation of spliceosomal complexes in the presence of NSC95397 was analyzed with agarose gels (Fig. 3.1C). The RNA composition of the purified spliceosomes suggested a complex with decreased amounts of U4 snRNA and stoichiometric amounts of U2, U6 and U5 snRNAs (Fig. 3.1D). Comparison of the RNA profiles of the purified spliceosomes to pre-catalytic spliceosomes (B complex) and activated spliceosomes ( $\mathrm{B}^{\text {act }}$ complex) indicated that the stalled complex contained decreased amounts of U4 snRNA relative to the B complex, while containing stoichiometric amounts of U2, U6 and U5 snRNAs, as in the B ${ }^{\text {act }}$ complex (Fig. 3.1D). U1 snRNA was detected in the B complex and stalled complex, but was strongly decreased in $\mathrm{B}^{\text {act }}$ (Fig. 3.1D). Analysis of the protein composition of the complex of interest was carried out with 2D gel electrophoresis followed by mass spectrometry for all proteins larger than $25 \mathrm{kDa}$ in the sample (Fig. 3.1E). Western blotting for complex-specific markers revealed that the stalled complex contained increased levels PRP19, PPIL2, and AQR relative to the B complex (Fig. 3.1F). The stalled complexes were purified and subjected to chase experiments in nuclear extract in which snRNAs have been digested by micrococcal nuclease treatment. Chase experiments demonstrated 
that the pre- $\mathrm{B}^{\text {act }}$ complexes were still functional and not dead-end intermediates (Fig. $3.1 \mathrm{G}$ ). Biochemical experiments (see 2.2.1, 2.2.2, 2.2.3, 2.2.4, 2.2.5, 2.2.7 and 3.1 were carried out by Dr. Majety Leelaram and Dr. Dmitry Agafonov (Department of Cellular Biochemistry, MPI-BPC), and crosslink analysis was performed by Dr. Olex Dybkov (Department of Cellular Biochemistry, MPI-BPC) in collaboration with Prof. Dr. Henning Urlaub (Bioanalytical Mass Spectrometry, MPI-BPC) (see 2.2.6, B.1, and B.2. 


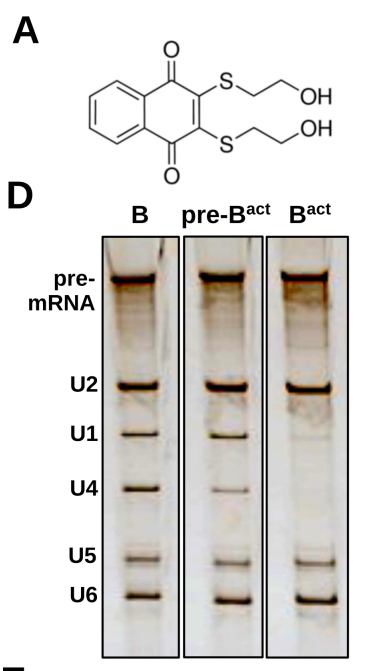

E

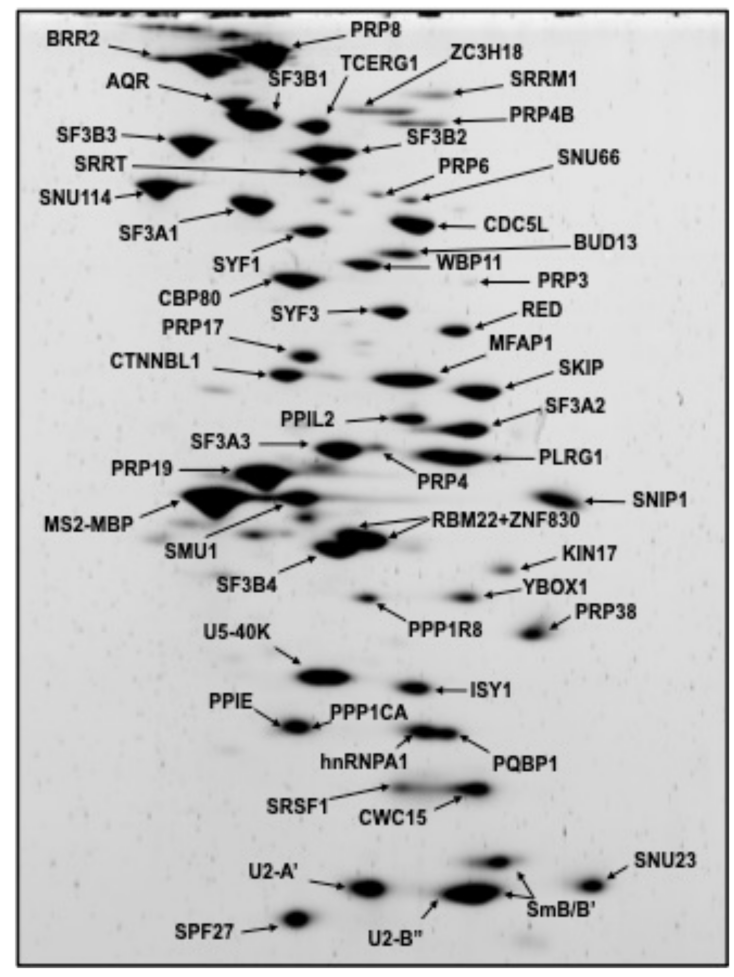

B

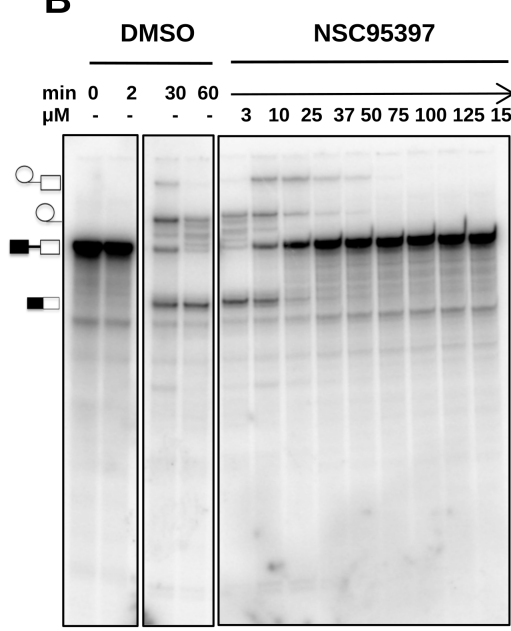

C
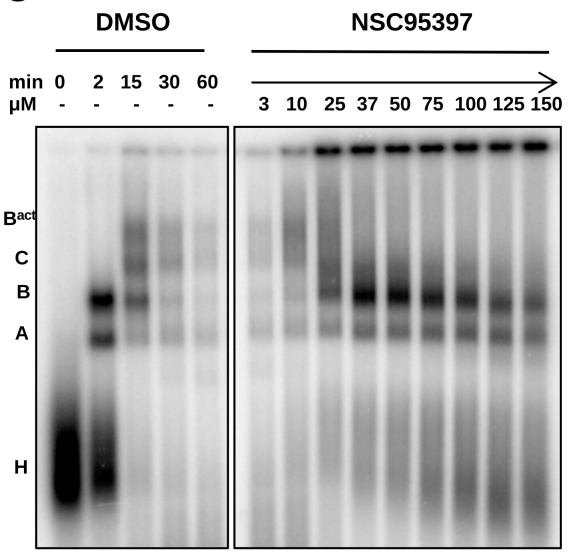

$\mathbf{F}$

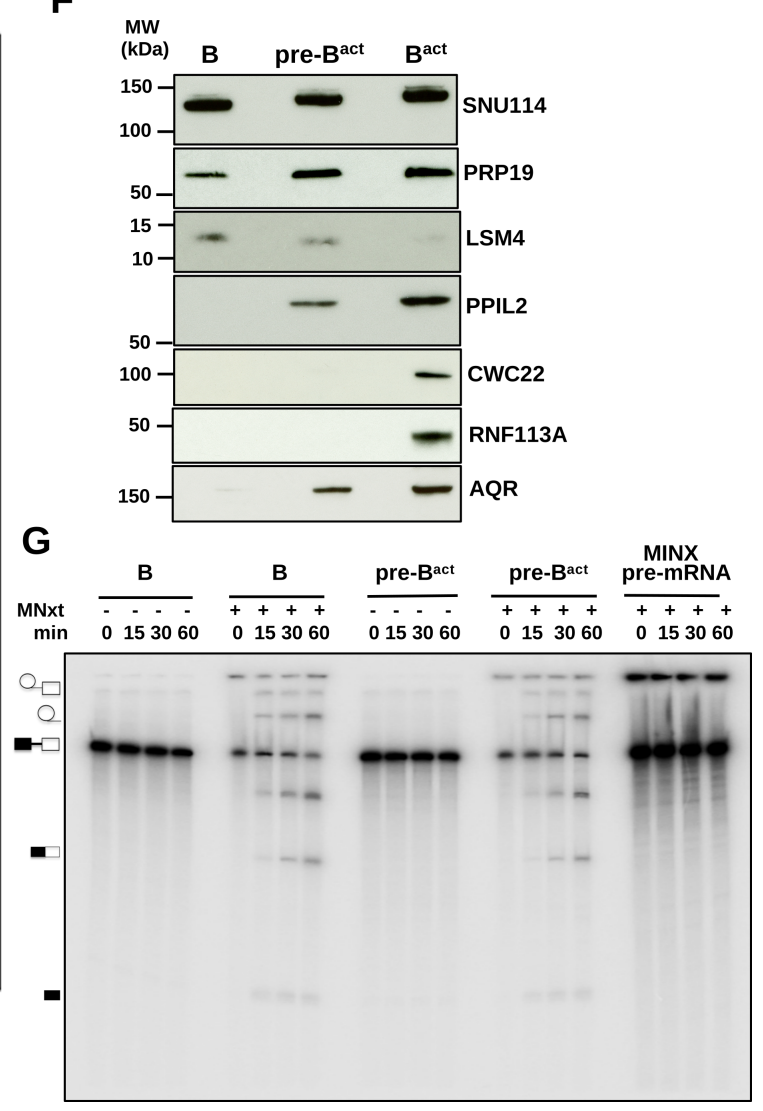

Fig. 3.1: Biochemical characterization of pre- act $^{\text {at }}$ (A) Chemical structure of NSC95397. (B) RNA products assessed by denaturing PAGE after 60 min incubation with 3-150 $\mathrm{\mu M}$ of NSC95397 in HeLa nuclear extract. In the diagrams on the far-left hand side of the figure, the $5^{\prime}$ exon is represented as a dark rectangle, the intron is represented as a thin black line, and the $3^{\prime}$ exon is shown as a white rectangle. From top to bottom are an intron lariat- $3^{\prime}$ exon, intron lariat only, unspliced pre-mRNA, and mRNA. (C) Formation of spliceosomal complexes in the presence of 3-150 $\mu \mathrm{M}$ of NSC95397 in HeLa nuclear extract after $60 \mathrm{~min}$ monitored by agarose gel electrophoresis. The far-left panel indicates labels of distinct, well characterized spliceosome intermediates for comparison. (D) RNA composition of B, pre- $\mathrm{B}^{\text {act }}$ and $\mathrm{B}^{\text {act }}$ complexes following affinity purification and gradient ultracentifigation to purify each complex assembled for $60 \mathrm{~min}$ in HeLa nuclear extract containing $150 \mu \mathrm{M}$ NSC95397. (E) Protein composition of purified pre- $\mathrm{B}^{\text {act }}$ complexes assessed by $2 \mathrm{D}$ gel electrophoresis followed by mass spectrometry to identify the individual protein spots. Proteins larger $25 \mathrm{kDa}$ are separated on the gel. (F) Western blotting of several proteins contained in $\mathrm{B}$, pre- $\mathrm{B}^{\text {act }}$ and $\mathrm{B}^{\text {act }}$. Dissociation of LSm proteins and recruitment of PRP19 (NTC component), PPIL2, CWC22, RNF113A, and AQR (IBC component) occurs during activation [276]. SNU114 (integral component of U5 snRNP) is included as a loading control. (G) Stalled pre-B ${ }^{\text {act }}$ complexes can be rescued in micrococcal-nuclease treated HeLa nuclear extract (MNxt). The B complex and unspliced MINX pre-mRNA are included for comparison. Affinity purified pre- $\mathrm{B}^{\text {act }}$ complexes splice MINX pre-mRNA in MNxt. From [131]. Reprinted with permission from AAAS. 


\subsection{Cryo-EM structures of two novel activation intermediates}

The purified pre-B ${ }^{\text {act }}$ sample was analyzed by cryo-EM, and computational sorting of 503,208 particle images revealed two distinct spliceosomal structures at intermediate stages of activation (Fig. 3.1A-C). Neither reconstruction suffered from strongly preferred orientation, indicating sufficient angular sampling (Fig. 3.1D,E). The average resolution of the overall complexes was 4.3 and $4.6 \AA$ (Fig. $3.1 \mathrm{~F}, \mathrm{G}$ ) and the map-model Fourier Shell Correlation (FSC) for the core and SF3B-focused maps was 4.2 and $4.6 \AA$ (Fig. $3.1 \mathrm{H}$ ). Using focused classifications, the core structures were resolved to 3.9 and $4.2 \AA$ (Fig. 3.1A,F,G). The local resolution varies widely in each reconstruction, ranging from approximately $3.5 \AA$ at the cores to $>20 \AA$ at the peripheries (Fig. 3.1, J). Grid preparation and data acquisition were carried out by Dr. Karl Bertram (Department of Structural Dynamics, MPI-BPC) (see 2.2.8 and $3.2 \mathrm{~B}$ ).

A model of each complex was built using an integrative approach. Published cryo-EM and crystallographic structures were docked into moderately resolved (ca. 4-10 A) map regions and XL-MS data was used to guide placement of less well resolved regions at flexible interfaces or the periphery of the complexes (Table B.2. Each spliceosomal intermediate has a relatively similar core structure, comprised of an approximately triangular body (Fig. 3.3). The resulting models allow for inspection of previously uncharacterized intermediates of spliceosome activation. The resulting reconstructions are denoted pre- $\mathrm{B}^{\text {act-1 }}$ and pre-B ${ }^{\text {act-2}}$, as they represent pre-activated complexes which lack U4 snRNP but do not yet have a mature catalytic center (see sections 3.3, 3.4, 3.5, 3.6, 3.7). Model building was overseen by Dr. Berthold Kastner (Department of Cellular Biochemistry, MPI-BPC) (see 2.2.10 
A

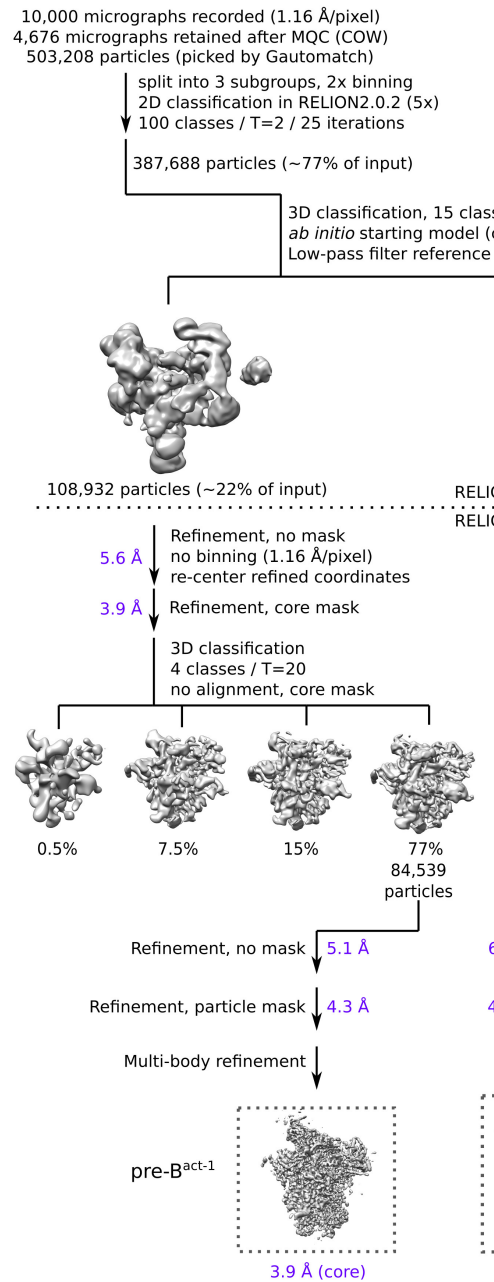

B

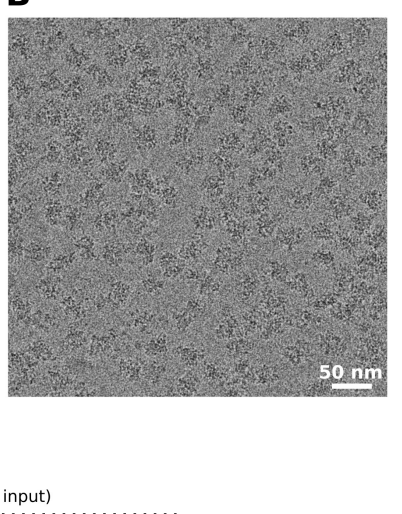

C

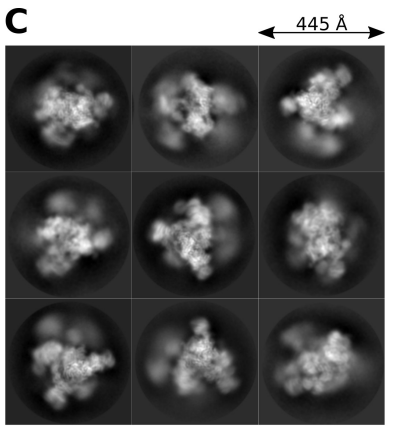

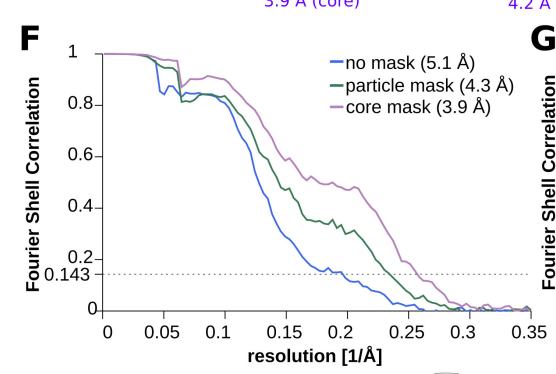

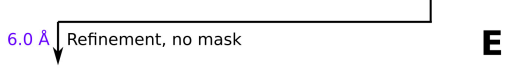

E

$6.5 \AA \quad \begin{aligned} & \text { Refinement, no mask } \\ & \text { no binning (1.16 } \AA / \text { pixel) } \\ & \text { re-center refined coordinate }\end{aligned}$

$4.6 \AA \downarrow$ Refinement, particle mask
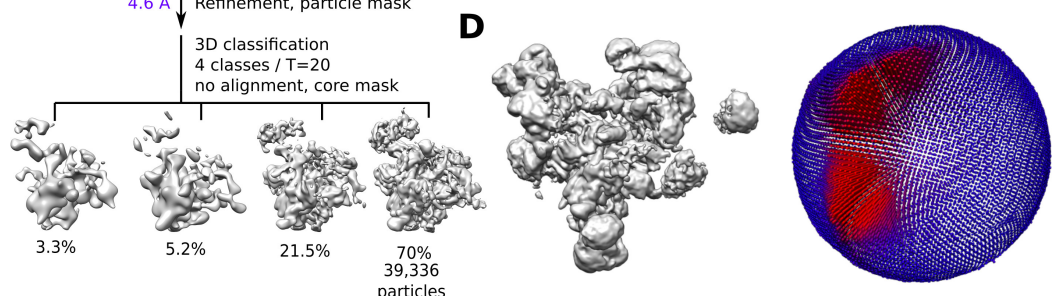

$4.6 \AA$ Refinement, particle mask

$\downarrow$ Multi-body refinement

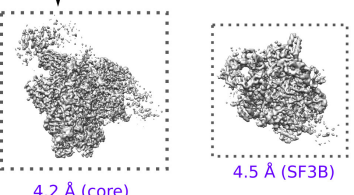

pre- $\mathrm{B}^{\text {act-2 }}$
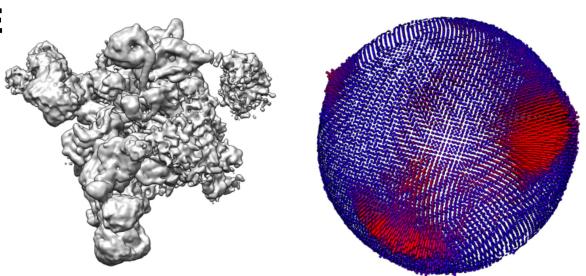

G
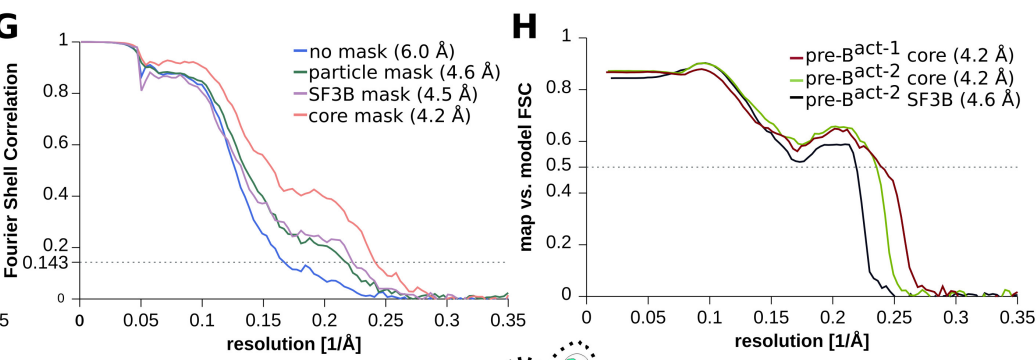

I

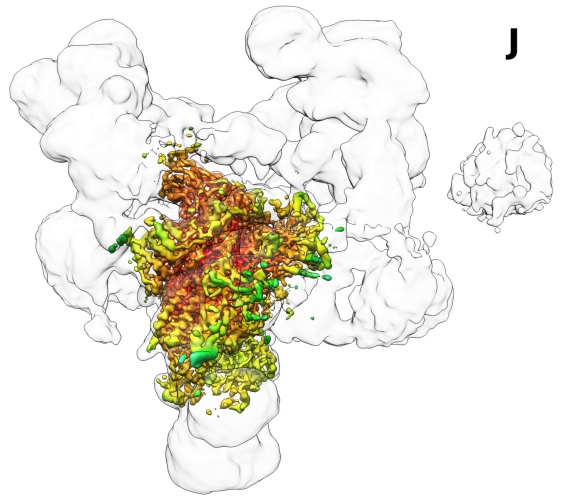

J
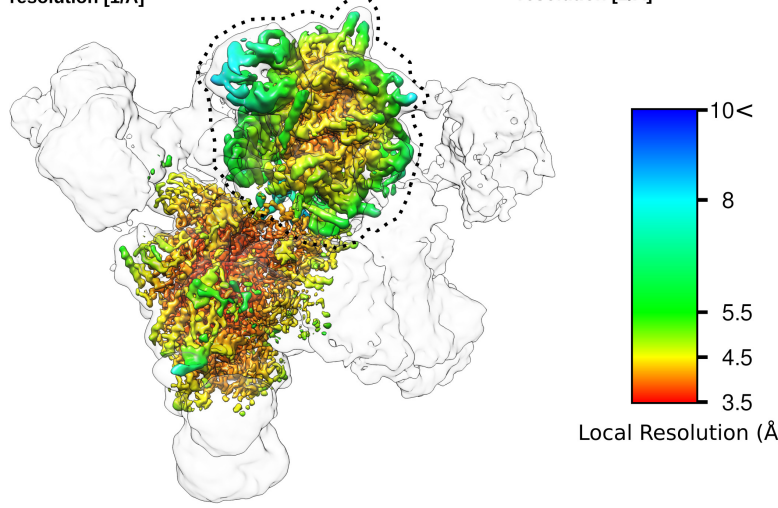

Fig. 3.2: Image processing and reconstruction of cryo-EM data. (A) Computational sorting scheme of cryo-EM data. For a detailed explanation of data acquisition and image processing, please refer to sections 2.2.8 and 2.2.9 of "Methods." (B) Cryo-EM micrograph and (C) representative 2D class averages. (D) Overall cryo-EM reconstruction (left) and angular distribution (right) of pre-Bact-1 and (E) overall cryo-EM reconstruction (left) and angular distribution (right) of pre- $\mathrm{B}^{\text {act-2 }}$, where red shading indicates relative number of particle images assigned to a particular orientation. Resolution estimates of pre-B $^{\text {act-1 }}(\mathrm{F})$, pre-B ${ }^{\text {act-2 }}(\mathrm{G})$ at the 0.143 Fourier Shell Correlation (FSC) threshold. Map-to-model FSC (0.5 threshold) of pre- $\mathrm{B}^{\text {act-1 }}$ and pre- $\mathrm{B}^{\text {act-2 }}$. Local resolution estimates of pre- $\mathrm{B}^{\text {act- }}$ (I) and pre- $\mathrm{B}^{\text {act-2 }}$ (J) masked reconstructions (colored densities) and overall reconstructions (gray overlays). From [131. Reprinted with permission from AAAS. 
A
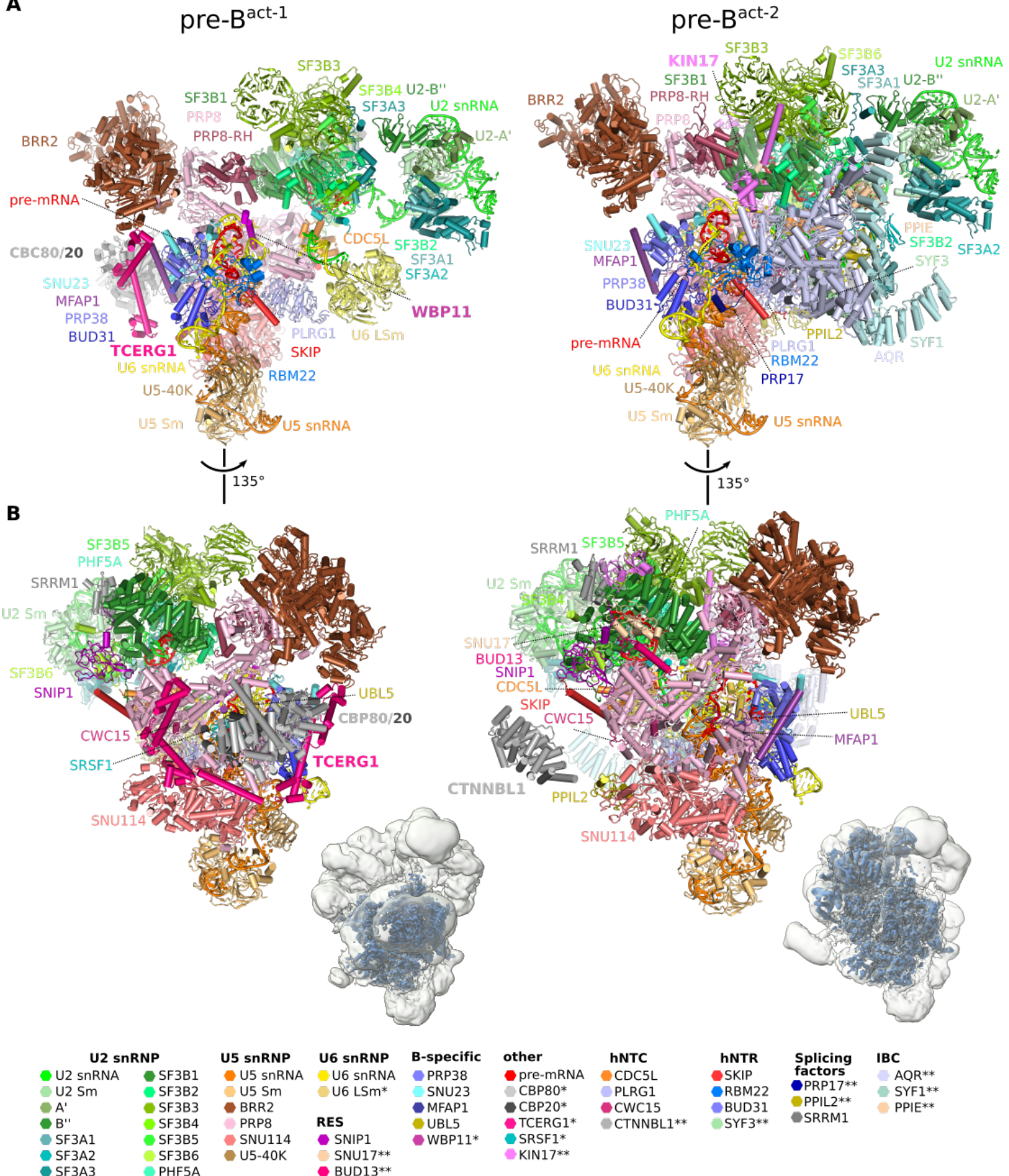

Splicing IBC

factors $\quad \mathrm{AQR}^{* *}$

SYF1**

STRM1

SYF1**
PPIE**

SF3A3

BUD13**

Fig. 3.3: Structural overview of pre- $B^{\text {act-1 }}$ and pre-B ${ }^{\text {act-2 }}$ Model composition of pre- $B^{\text {act-1 }}$ (A,B; left panel) and pre-B ${ }^{\text {act-2 }}$ (A,B; right panel). All modeled components are labeled within the 3D models and indicated in the table at the bottom of the figure. Components belonging to snRNPs or specific subcomplexes or groups (RES, B-specific, hNTC, hNTR, splicing factors, IBC) are indicated in bold text. Cryo-EM maps are shown as insets (B; left and right), with the higher resolved masked reconstructions shown in dark blue and the overall reconstructions shown in light gray overlay. From [131. Reprinted with permission from AAAS. 


\subsection{PRP8 maintains an open conformation in pre- $\mathrm{B}^{\text {act }}$}

Analysis of the pre- $\mathrm{B}^{\text {act }}$ structures indicates that the central scaffold protein of the spliceosome, PRP8, is in an open conformation (Fig. 3.4). At some point of spliceosome activation, PRP8 undergoes a large-scale conformational change from open to closed, resulting in a compact arrangement found in the activated spliceosome ( $\mathrm{B}^{\text {act }}$ ) (Fig. 1.11). During this conformational change, a large portion of PRP8's C-terminal region (PRP8RT/En) clamps down onto its N-terminal domain $\left(\mathrm{PRP} 8^{\mathrm{NTD}}\right)$. Conversion from the open to the closed conformation of PRP8 requires loss of U4 snRNP and dissociation of the B-specific proteins (see section 1.4.1). The closed conformation forms a chamber that accommodates the active site, and is therefore a critical event during spliceosome activation.

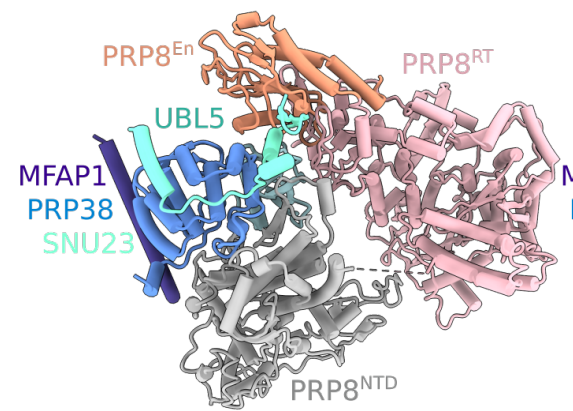

B

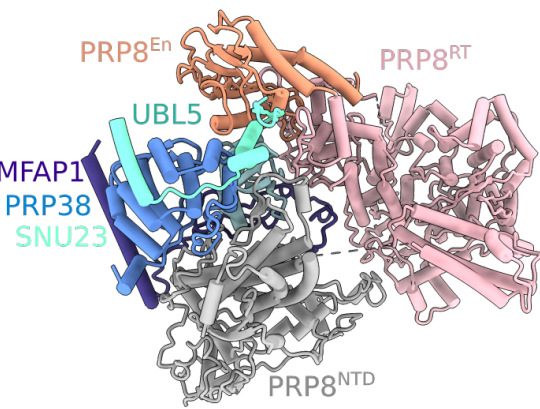

pre-B $\mathrm{B}^{\text {act }}$

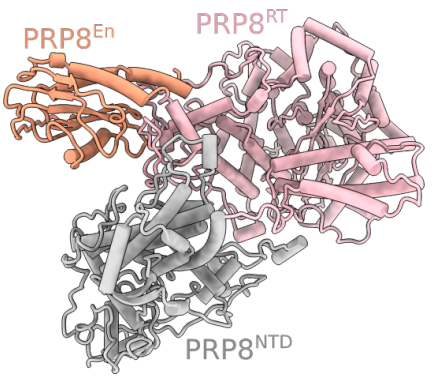

$B^{\text {act }}$

Fig. 3.4: PRP8 is held in an open conformation by B-specific proteins in pre- ${ }^{\text {act }}$. The B-specific proteins MFAP1, PRP38, SNU23, and UBL5 are still bound between PRP8 ${ }^{\mathrm{En}}$ and $\mathrm{PRP} 8^{\mathrm{NTD}}$ in both pre- $\mathrm{B}^{\text {act-1 }}$ and pre- $\mathrm{B}^{\text {act-2 }}$ (middle panel), as in the B complex (left; PDBs 5O9Z and 6AHD - from $6 \mathrm{AHD}$, only UBL5, as UBL5 is not modeled in PDB 5O9Z). This prevents open-to-closed conformational change of PRP8. All complexes are aligned to the PRP8 ${ }^{\mathrm{NTD}}$ of $\mathrm{B}^{\text {act }}$ (PDB 6FF7).

The B-specific proteins MFAP1, PRP38, SNU23, and UBL5 must be dissociated from their binding site between the $\mathrm{PRP} 8^{\mathrm{En}}$ and $\mathrm{PRP} 8^{\mathrm{NTD}}$ domains in order for PRP8 to be converted from an open to a closed conformation during activation (Fig. 3.4). In pre-B ${ }^{\text {act }}$, the B-specific proteins UBL5, PRP38, MFAP1, and SNU23 are still present, holding PRP8 in an open conformation (Fig. 3.4. UBL5 is situated between PRP8 ${ }^{\text {En }}$ and PRP8 $8^{\text {NTD }}$ domains, preventing closure of PRP8. SNU23 and PRP38 remain bound alongside the U6/5'ss helix. MFAP1 contains an $\alpha$-helix that is associated with PRP38 as well as a C-terminal region of 80 amino acids that forms multiple interactions with UBL5, loop I of U5 snRNA, PRP8 ${ }^{\mathrm{SWL}}$, and the $5^{\prime}$ exon. Thus, the B-specific proteins MFAP1, PRP38, 
SNU23, and UBL5 remain bound even after the dissociation of U4 snRNP, holding PRP8 in the same open conformation as in the pre-catalytic spliceosome (B complex).

PRP8 ${ }^{\mathrm{SWL}}$ points upwards (i.e., away from $\mathrm{U} 5 \mathrm{Sm}$ ) in the B complex and downwards in $\mathrm{B}^{\text {act}}$, indicating dynamic repositioning of this domain following activation [122]. In pre-B ${ }^{\text {act }}, \mathrm{PRP} 8^{\mathrm{SWL}}$ remains in its $\mathrm{B}$ complex position and forms contacts with the Cterminal region of MFAP1, which likely clamps $\mathrm{PRP} 8^{\mathrm{SWL}}$ in place (Fig. 3.5). PRP8 $8^{\mathrm{SWL}}$ repositioning appears to coincide with the closed conformation of PRP8, which can only occur following the displacement of B-specific proteins.

The distal C-terminal domains of $\mathrm{PRP}^{\mathrm{RH}}$ and $\mathrm{PRP} 8^{\mathrm{Jab} 1}$ domains were localized in both pre- $\mathrm{B}^{\text {act-1 }}$ and pre- $\mathrm{B}^{\text {act-2}}$, and in largely different positions as found in the $\mathrm{B}$ or $\mathrm{B}^{\text {act }}$ complex, further underscoring the drastic repositioning of these domains at every stage of spliceosome assembly (Fig. 3.5). $\mathrm{PRP}^{\mathrm{RH}}$ is rotated between pre-B ${ }^{\text {act-1 }}$ and pre-B $\mathrm{B}^{\text {act-2}}$, occupying a gap between BRR2 and the U2 snRNP proteins SF3B1/SF3B3 (Fig. 3.5), replacing the bridge of SMU1/RED that previously bridged BRR2 and U2 snRNP in the B complex. The PRP8 $8^{\mathrm{Jab} 1}$ domain is anchored to BRR2, maintaining the association of BRR2 to the spliceosome following its unwinding of the U4/U6 snRNA duplex.
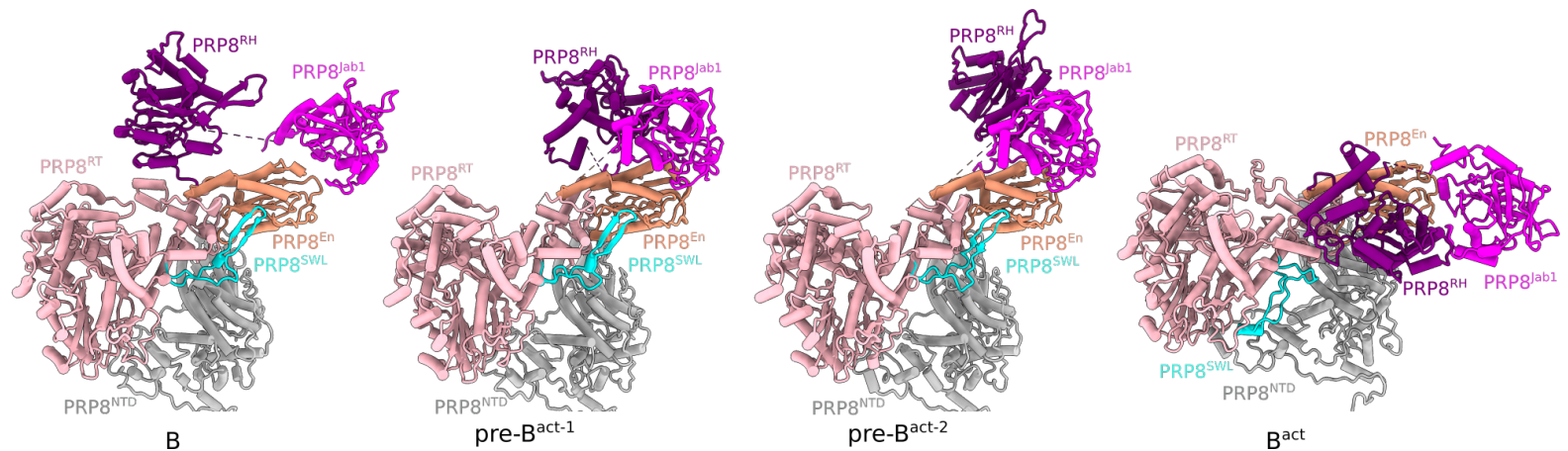
$B^{\text {act }}$

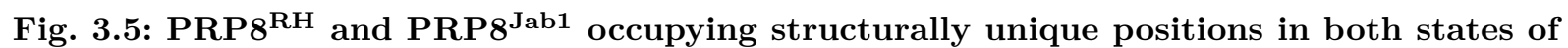
pre- $\mathrm{B}^{\text {act }}$. In both pre- $\mathrm{B}^{\text {act-1 }}$ and pre- $\mathrm{B}^{\text {act-2 }}, \mathrm{PRP}^{\mathrm{RH}}$ and $\mathrm{PRP} 8^{\mathrm{Jab} 1}$ occupy positions and orientations previously not observed in the spliceosome. $\mathrm{PRP} 8^{\mathrm{SWL}}$ is also shown for comparison among the $\mathrm{B}$ (PDB

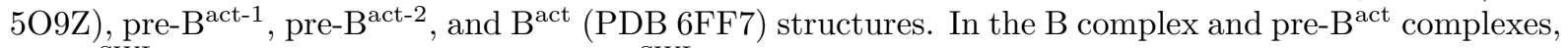
$\mathrm{PRP}^{\mathrm{SWL}}$ points upwards. In $\mathrm{B}^{\text {act }}, \mathrm{PRP}^{\mathrm{SWL}}$ is repositioned and points downwards. All structures are aligned to $\mathrm{PRP}^{\mathrm{En}}$ (aa $\left.1579-1754\right)$ of $\mathrm{B}^{\text {act }}$. 


\subsection{Novel repositioning of BRR2 and U2 snRNP}

BRR2, the ATP-dependent RNA helicase responsible for unwinding the U4/U6 RNA duplex to allow for release of U4 snRNP during activation, has undergone a complex rotational movement following release of U4 snRNP, yet has not assumed its final position corresponding as in $\mathrm{B}^{\text {act }}$ (Fig. 3.6). BRR2 has not been documented in this orientation relative to the rest of the spliceosomal body, and exhibits a similar orientation in both pre- $\mathrm{B}^{\text {act-1 }}$ and pre- $\mathrm{B}^{\text {act-2 }}$. The catalytically active region of BRR2 (BRR2 $\left.2^{\mathrm{N} \text {-term }}\right)$ is facing away from $\mathrm{PRP} 8^{\mathrm{SWL}}$, while BRR2 $2^{\mathrm{C}-\text { term }}$ points toward $\mathrm{PRP} 8^{\mathrm{SWL}}$. In $\mathrm{B}^{\text {act }}, \mathrm{BRR} 2^{\mathrm{N} \text {-term }}$ contacts the U2 snRNP $5^{\prime}$ domain! in pre-B ${ }^{\text {act }}$, BRR2 is not directly contacting the U2 snRNP $5^{\prime}$ domain, but is loosely bridged to $\mathrm{U} 2$ snRNP by $\mathrm{PRP} 8^{\mathrm{RH}}$.

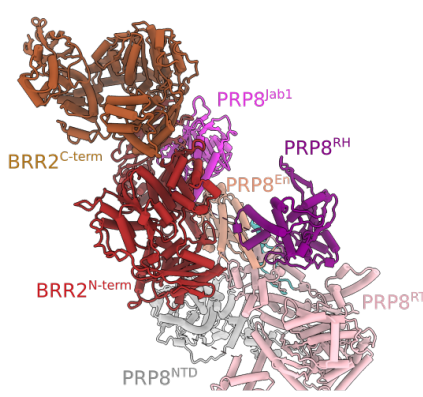

B

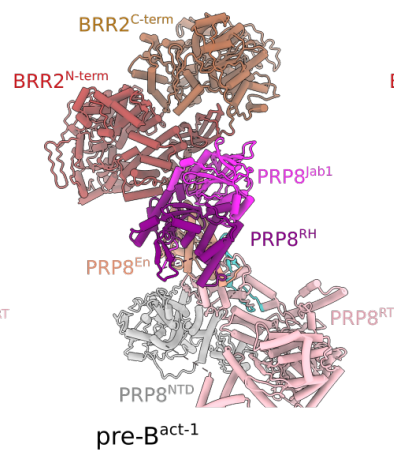

pre- $\mathrm{B}^{\text {act-1 }}$
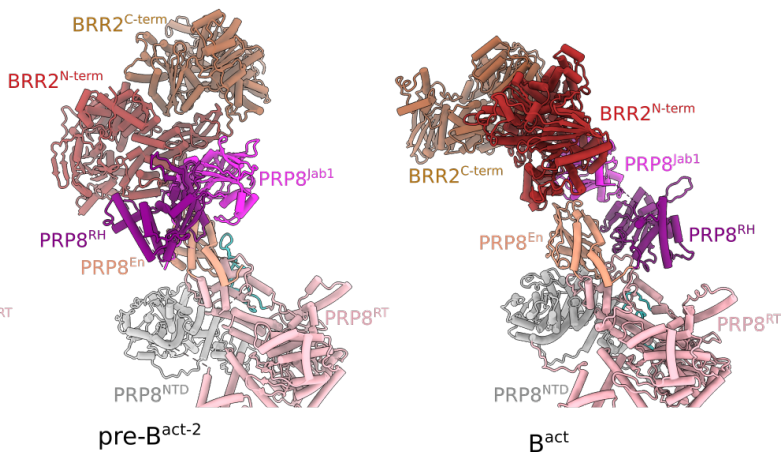

Fig. 3.6: BRR2 is repositioned to a novel orientation in pre- $\mathrm{B}^{\text {act }}$. In both pre- $\mathrm{B}^{\text {act }}$ structures, BRR2 has been rotated upon its contact with the PRP8, PRP8 ${ }^{\mathrm{Jab} 1}$. BRR2 occupies a similar position in both pre- $\mathrm{B}^{\text {act-1 }}$ and pre- $\mathrm{B}^{\text {act-2 }}$, distinct from its position in the $\mathrm{B}$ complex (PDB 5O9Z) and $\mathrm{B}^{\text {act }}$ (PDB 6FF7). All structures are aligned to $\mathrm{PRP} 8^{\mathrm{En}}$ (aa 1579-1754) of $\mathrm{B}^{\text {act }}$.

U2 snRNP, essential for the construction of the spliceosome's catalytic center, is initially separated from the core of the spliceosome by U4 snRNP in the B complex (Fig. 3.7. upper left panel). Following unwinding of the U4/U6 RNA duplex by BRR2 to result in dissociation of U4 snRNP, U2 snRNP has begun to dock with the U5 snRNP at the core of the spliceosome, but has not yet been tightly integrated into the complex as in $\mathrm{B}^{\text {act }}$ (Fig. 3.7, right panels). U2 snRNP is thus flexible in both pre-B ${ }^{\text {act }}$ structures, but especially so in pre- $\mathrm{B}^{\text {act- } 1}$. Many proteins of the U2 snRNP $5^{\prime}$ domain in pre- $\mathrm{B}^{\text {act-2 }}$ can be clearly mapped to the density based on clearly resolved secondary structure elements,

\footnotetext{
1"U2 snRNP 5' domain" refers to the SF3b and PHF5A proteins and $5^{\prime}$ half of the U2 snRNA, including portions contributing to helix I, helix II, the U2/BS helix; "U2 snRNP $3^{\prime}$ domain" encompasses the Sm, A', B", SF3a proteins and the $3^{\prime}$ half of the U2 snRNA; see Figs. 1.3 and 3.3
} 
including those of the larger components SF3B1 and SF3B3. The U2 snRNP $3^{\prime}$ domain is likely not yet stably docked and therefore not as well resolved as in the $\mathrm{B}^{\text {act }}$ complex. $\mathrm{PRP}^{\mathrm{RH}}$ separates SF3B1/SF3B3 and BRR2 in both pre-B ${ }^{\text {act }}$ structures (Fig. 3.7, right panel). The U2/BS helix is also distinguishable in both pre-B ${ }^{\text {act }}$ reconstructions, although is better resolved in pre- $\mathrm{B}^{\text {act-2 }}$.

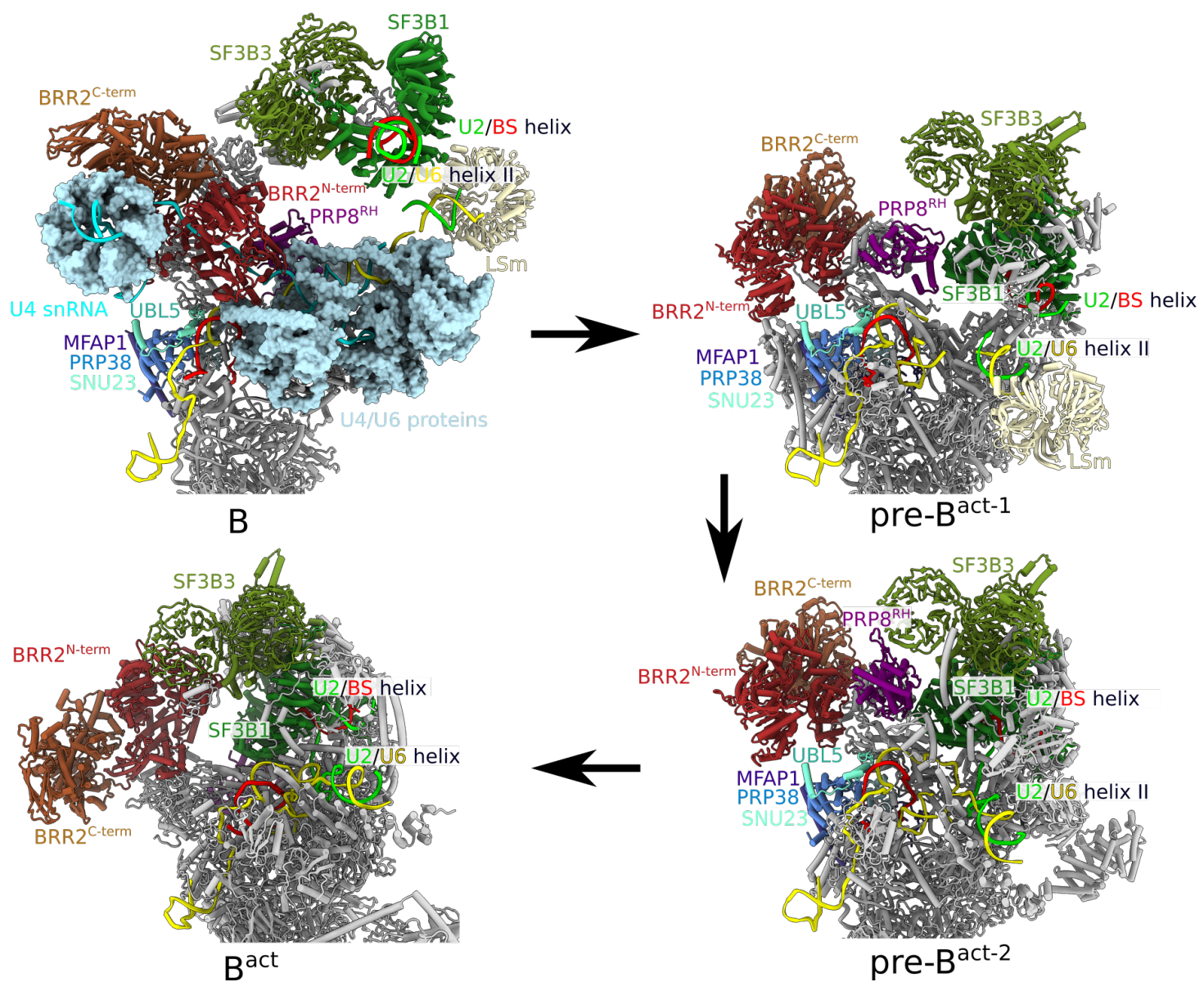

Fig. 3.7: Repositioning of BRR2 and docking of U2 snRNP during activation. The $3^{\prime}$ domain of U2 snRNP and several proteins are omitted from the figure for clarity. Release of U4 snRNA allows for rearrangement of BRR2 and components of the U2 snRNP. Relevant protein and RNA components from the B complex (PDB 5O9Z), pre- $\mathrm{B}^{\text {act-1 }}$, pre- $\mathrm{B}^{\text {act-2 }}$, and $\mathrm{B}^{\text {act }}$ (PDB 6FF7) are highlighted (clockwise from upper left to bottom right quadrant). The U4/U6 proteins, bound to U4 snRNA, occlude a large cavity and prevent docking of U2 snRNP onto PRP8 and the rest of the spliceosome. Following U4/U6 snRNA unwinding, BRR2 becomes repositioned and U2 snRNP as well as U2/U6 helix II and the LSm proteins are moved toward the body of the spliceosome (grey). U2 snRNP becomes progressively more tightly associated with the spliceosome in pre- $\mathrm{B}^{\text {act-2 }}$ and finally in $\mathrm{B}^{\text {act }}$, in which the B-specific proteins are dissociated and PRP8 is in a closed conformation, and BRR2 directly contacts SF3B3 of U2 snRNP. 


\subsection{Recruitment of proteins at distinct stages of activation in pre- $\mathrm{B}^{\text {act }}$}

\subsubsection{RES and SRRM1}

The Retention and Splicing complex (RES) is required for splicing and consists of three proteins: SNIP1, SNU17, and BUD13 (Fig. 1.14] [116, 206]. RES is not present in the $\mathrm{B}$ complex, but joins the spliceosome at a yet undetermined stage of activation. The FH

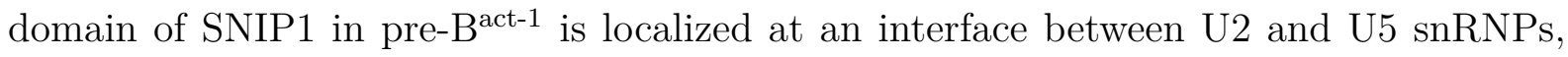
where it interacts with PRP8 ${ }^{\mathrm{RT}}$, SKIP, and SF3B6 (Fig. 3.8. left panel). Densities for BUD13 and SNU17 were not detected in pre-B ${ }^{\text {act-1 }}$, but become apparent in pre-B ${ }^{\text {act-2 }}$. BUD13 and SNU17 are positioned adjacent to one another, with SNU17 forming contacts to SF3B1 and BUD13 binding within a gap between SNU17 and PRP8 ${ }^{\text {RT }}$ (Fig. 3.8, middle panel). The position of RES complex proteins agrees closely with their binding sites in the $\mathrm{B}^{\text {act }}$ (Fig. 3.8, right panel) [99].
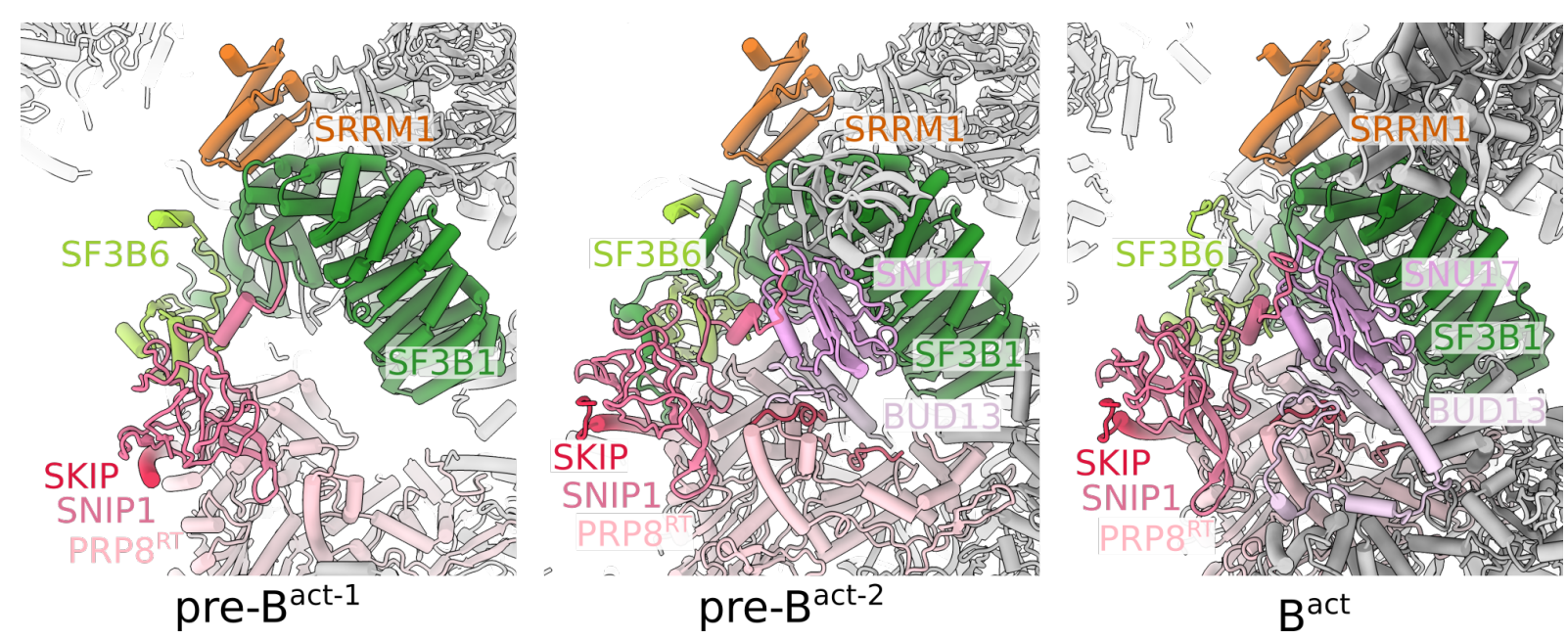

Fig. 3.8: RES integration into pre- $\mathbf{B}^{\text {act-1 }}$ and pre-B $\mathbf{B}^{\text {act-2 }}$. RES proteins are gradually integrated into pre- $\mathrm{B}^{\text {act-2}}$, leading to $\mathrm{B}^{\text {act }}$. In pre- $\mathrm{B}^{\text {act-1}}$, only SNIP1 is observed. SNU17 and several fragments of BUD13 are stabilized in pre- $\mathrm{B}^{\text {act-2 }}$. In $\mathrm{B}^{\text {act }}$, additional regions of BUD13 become stabilized. SKIP (NTR protein) and SRRM1 are shown for orientation. All structures are aligned on SF3B1 of B ${ }^{\text {act }}$ (PDB 6FF7).

Nearby the RES complex proteins, SRRM1 is bound. SRRM1 is a protein involved in regulation of splicing (see section 1.4.3) that is associated with $\mathrm{B}^{\text {act }}$. SRRM1 interacts 
with the U2 snRNP by binding to the SF3B1 ${ }^{\mathrm{HEAT}}$ domain adjacent to SF3B6, consistent with the positioning of this protein relative to neighboring proteins in $\mathrm{B}^{\text {act }}$ (Fig. 3.8). 


\subsubsection{NTC}

The NineTeen Complex (NTC) is a preformed unit consisting of PRP19, CDC5L, PLRG1, SPF27, CWC15, and CTNNBL1 that joins the spliceosome during activation (Fig. 1.15). The NTC is involved in the removal of the U6 LSm proteins and the stabilization of newly formed U6 snRNA interactions within the spliceosome during activation [214]. Of these proteins, parts of CDC5L, PLRG1, CWC15, and CTNNBL1 can be localized in both or at least one of the pre- $\mathrm{B}^{\text {act }}$ reconstructions (CTNNBL1 is only localized in pre-B ${ }^{\text {act-2}}$ ) (Fig. 3.9. CDC5 $\mathrm{L}^{\mathrm{Myb}}$ domains bridge SF3B1 of $\mathrm{U} 2$ snRNP to $\mathrm{PRP} 8^{\mathrm{HB}}$, likely contributing to docking of U2 snRNP to the U5 snRNP. PLRG1 $1^{\mathrm{WD} 40}$ is stably docked near the core of the spliceosome, lodged beneath $\mathrm{PRP} 8^{\mathrm{HB}}$, providing an interface for other proteins such as SKIP (see section 3.5.3) to bind. A short $\alpha$-helix of CWC15 could be docked into a density near $\mathrm{PRP} 8^{\mathrm{HB}}$, based on crosslinks as well as the position of this protein in the published $\mathrm{B}^{\text {act }}$ cryo-EM structure [99]. The ARM repeats of CTNNBL1, a protein previously unmapped in any spliceosomal structure, could be docked based on crosslinks into a large curved-cylindrical density at the outer periphery of pre-B ${ }^{\text {act-2 }}$. CTNNBL1 is linked to the body of the complex by a short $\alpha$-helix of CDC5L as well as the U-box domain of PPIL2. All components of the NTC are docked to positions that are highly similar to their previously observed positions in $\mathrm{B}^{\text {act }}$.
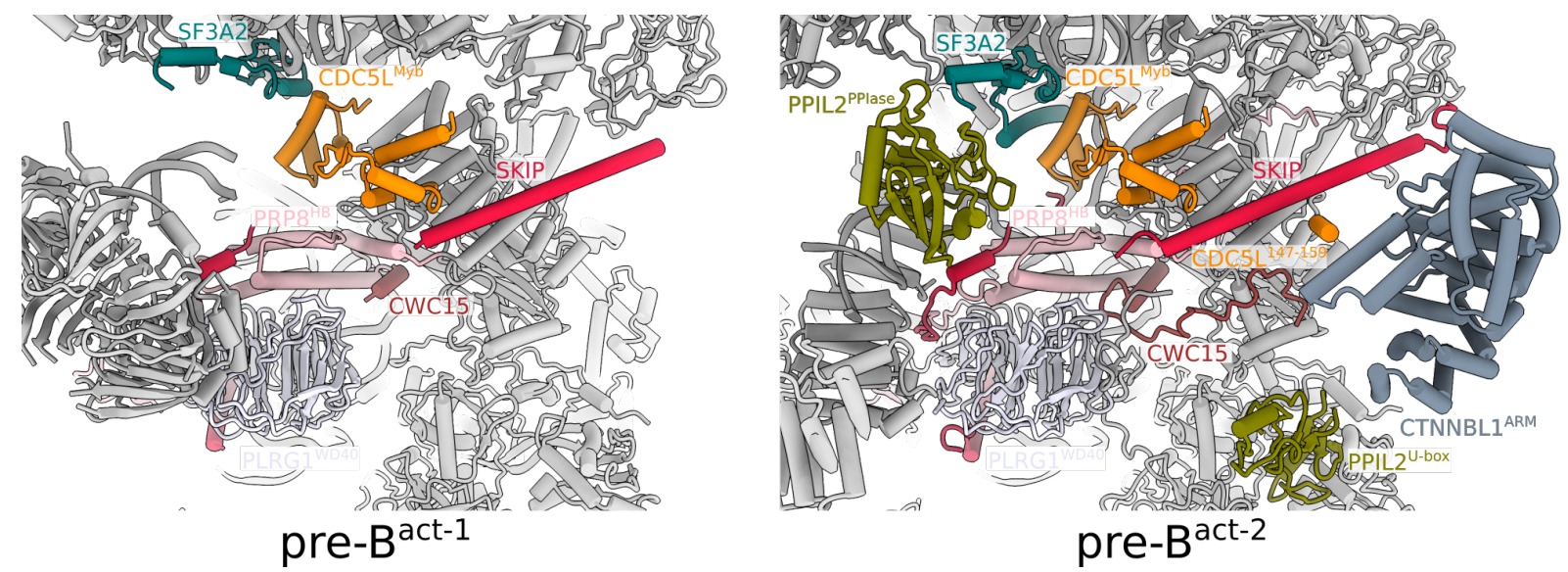

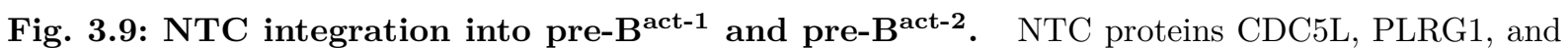
CWC15 binding to pre- ${ }^{\text {act }}$ are shown. $\mathrm{CDC}^{\mathrm{Myb}}$ binds above PRP8 ${ }^{\mathrm{HB}}$, between SF3A2 (U2 snRNP protein) and SKIP (NTR protein). In pre-B ${ }^{\text {act-2 }}$ (right panel), CTTNBL1 is attached to the periphery of the complex and additional domains of CWC15 are structured. PPIL2 is shown for orientation. Structures are aligned on $\mathrm{PRP} 8^{\mathrm{NTD}}$. 


\subsubsection{NTR proteins}

NineTeen Complex Related (NTR) proteins SKIP, RBM22, BUD31, and PPIL1 all bind during spliceosome activation. Of these four proteins, only SKIP, RBM22, and BUD31 were detectable (Fig. 3.10). PPIL1 joins in late stages activation (mature $\mathrm{B}^{\text {act }}$ and is stabilized by surrounding protein-protein interactions. Several portions of SKIP could be docked throughout the core of each complex (Fig. 3.10); its N-terminal domain interacts with the NTC proteins PLRG1 and CDC5L and the NTR proteins RBM22 and BUD31, as well as $\mathrm{PRP} 8^{\mathrm{HB}}$, while its long helical region spans the outer edge of $\mathrm{PRP} 8^{\mathrm{RT}}$, ending at the RES protein SNIP1. In pre-B ${ }^{\text {act-2 }}$, additional regions of SKIP are stably integrated; in particular, a C-terminal strand that is sandwiched between PRP8 ${ }^{\mathrm{RT}}$ and the RES protein BUD13. SKIP thus forms an extensive "wiring" to connect far-reaching regions of the spliceosome. RBM22 and BUD31 bind near the 5' SL of U6 snRNA, nestled into an interface on PRP8 ${ }^{\mathrm{NTD}}$ (Fig. 3.10, left and middle panel). Moreover, RBM22 and BUD31 are bound immediately adjacent to the B-specific proteins MFAP1, PRP38, and SNU23, which are engaged in maintaining the open conformation of PRP8. RBM22 contains a Zinc-finger (Znf) domain and an RNA recognition motif (RRM). RBM22 ${ }^{\text {Znf }}$ is stably bound in both pre- $\mathrm{B}^{\text {act-1 }}$ and pre- $\mathrm{B}^{\text {act-2}}$, yet RBM $22^{\mathrm{RRM}}$ is clearly resolved only in pre$\mathrm{B}^{\text {act-2 }}$. RBM22 binds the intron distal to the U6/5'ss helix, guiding the intron into the five-membered Intron Binding Complex (IBC).

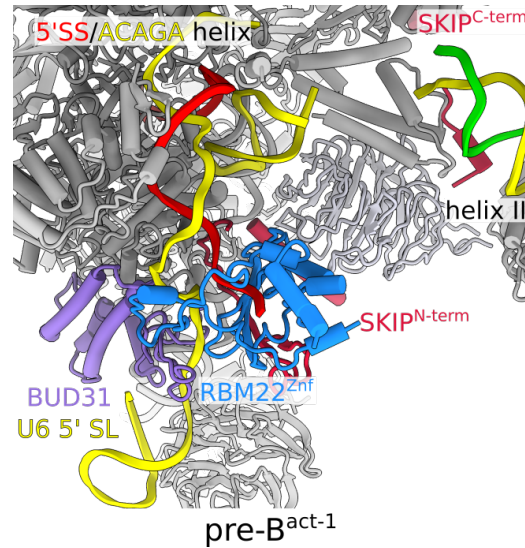

Fig. 3.10: NineTeen complex Related (NTR) proteins. NTR proteins gradually integrated from pre-B ${ }^{\text {act-1 }}$ to $\mathrm{B}^{\text {act }}$. In all structures, BUD31 and RBM $22^{\text {Znf }}$ bind to the U6/5'ss helix (i.e., $5^{\prime}$ SS $/$ ACAGA helix). RBM22 $2^{\text {RRM }}$ and several portions of SKIP are first observed in pre- $\mathrm{B}^{\text {act-2 }}$ and retained in $\mathrm{B}^{\text {act }}$. PPIL1 joins at a later stage of $\mathrm{B}^{\text {act }}$. All structures are aligned on PRP8 ${ }^{\mathrm{NTD}}$ of $\mathrm{B}^{\text {act }}$ (PDB $\left.6 \mathrm{FF} 7\right)$. 


\subsubsection{IBC and SYF3}

In the B complex, the $3^{\prime}$ end of $\mathrm{U} 6$ and the $5^{\prime}$ end of $\mathrm{U} 2$ snRNA are base-paired to form U2/U6 helix II, which remains intact also in the activated spliceosome ( $\mathrm{B}^{\text {act }}$; see 1.3.5. Helix II is bound by the U6 LSm proteins in the B complex [98]. At some point during spliceosome activation, U6 LSm proteins are replaced by SYF3 and the intron-binding complex (IBC) (see section 3.5.4, [225]). Helix II appears to be flexible in pre-B ${ }^{\text {act-1 }}$, as evidenced by a cylindrical-shaped density distal to the U6 ISL and the U2/U6 helix Ib, where helix II is located in $\mathrm{B}^{\text {act }}$. Crosslinking data allowed for the placement of the U6 LSm into a globular density near the expected position of helix II (Fig. 3.11), revealing that U6 LSm remains associated with helix II even after loss of U4 snRNP, integration of the NTC, and the large-scale remodeling of the spliceosome's molecular architecture that occurs during spliceosome activation. U6 LSm is replaced by SYF3 and the IBC in pre- $\mathrm{B}^{\text {act-2}}$, as its binding site on helix II would otherwise clash with this protein ensemble (Fig. 3.11). The HAT repeats of SYF3 provide a platform on which helix II appears to be stabilized in pre-B ${ }^{\text {act-2 }}$ (Fig. 3.19).

The IBC, consisting of five proteins (AQR, SYF1, ISY1, PPIE, and ZNF830; Fig. 1.16) [225], is absent from pre- $\mathrm{B}^{\text {act-1 }}$, but is stably integrated in pre- $\mathrm{B}^{\text {act-2 }}$ (Fig. 3.11). IBC components interact with SYF3, upon which U2/U6 helix II is accommodated. In pre-B ${ }^{\text {act-1, }}$ helix II is bound by LSm proteins, abrogating binding of SYF3 (Fig. 3.11). Stabilization of the IBC onto the spliceosome is apparently mediated not only by RBM22, but also by SYF3 and SF3B2 and SF3B4. The N-terminal HAT repeats of SYF3 bridge the IBC to the spliceosome by contacting SYF1 (Fig. 3.11). PPIE also bridges the IBC to the spliceosome by providing a stable binding interface to channel the intron from RBM22 to SF3B2/SF3B4 and finally to the SF3B1 ${ }^{\mathrm{HEAT}}$ domain, where the U2/BS helix is sequestered. 

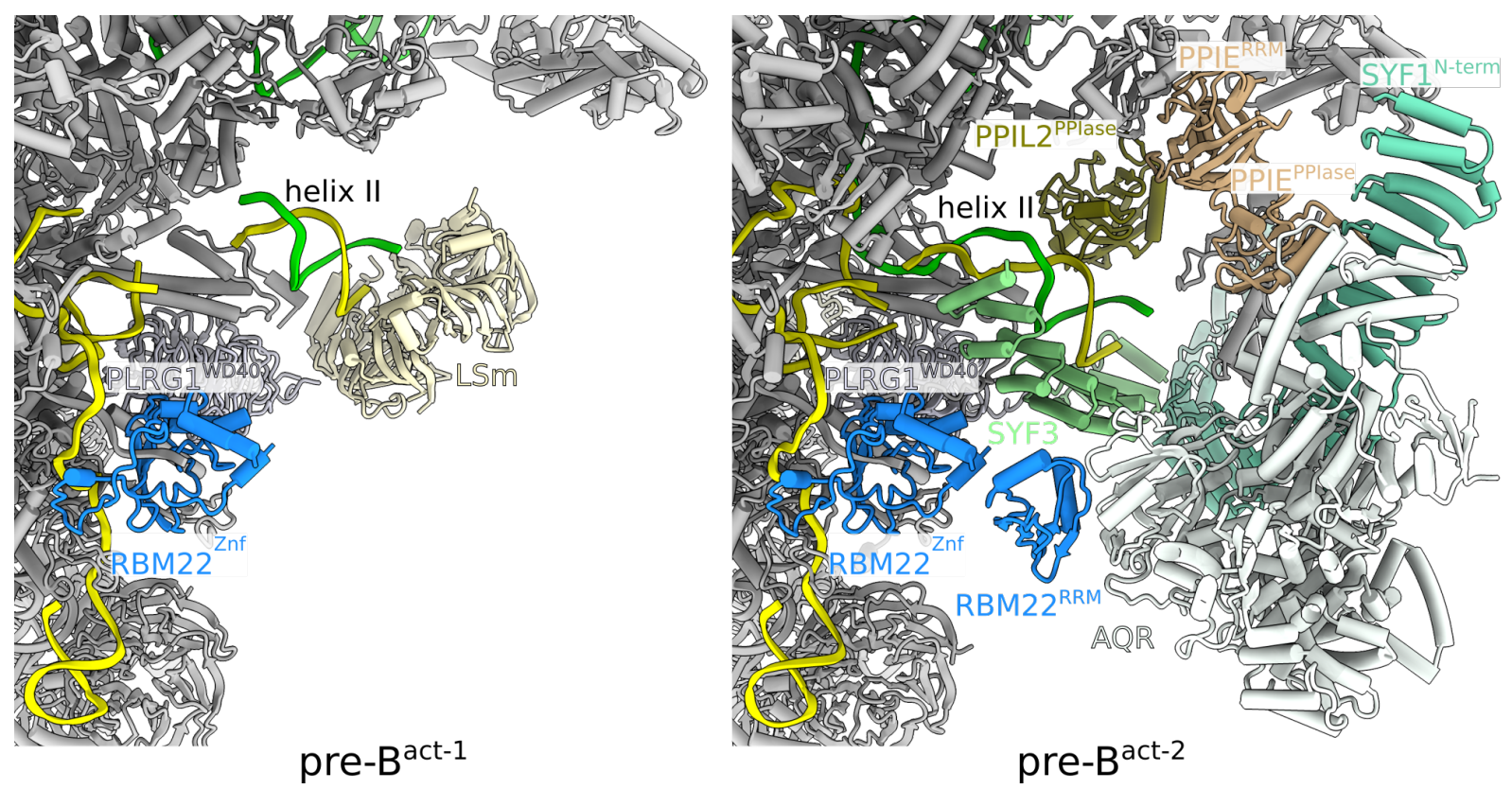

Fig. 3.11: Mutually exclusive interactions of LSm proteins and the IBC/SYF3. In pre-B ${ }^{\text {act-1 }}$, LSm proteins are still bound to helix II and interact with PLRG1. LSm proteins are replaced by SYF3 and the IBC (AQR, PPIE, SYF1) in pre-B act-2 $^{\text {. PPIL2 }} 2^{\text {PPIase }}$ also appears first in pre-B ${ }^{\text {act-2}}$. Structures are aligned on $\mathrm{PRP} 8^{\mathrm{NTD}}$.

\subsubsection{PPIL2 and PRP17}

Several proteins involved in generation of the $\mathrm{B}^{\text {act }}$ join during spliceosome activation, including PPIL2 and PRP17. The function of PPIL2 is not well understood. PPIL2 contains a PPIase and a U-box domain [231]. The U-box of PPIL2 binds to SNU114 and crosslinks to the nearby CTNNBL1 ${ }^{\mathrm{ARM}}$ domain (Fig. 3.9. The PPIase domain of PPIL2 docks near U2/U6 helix II and forms numerous crosslinks to SYF3 (Fig. 3.11 and Table B.1). Densities corresponding to the PPIL2 U-box and PPIase domains are evident in pre- $\mathrm{B}^{\text {act-2}}$, yet absent in pre-B ${ }^{\text {act-1 }}$, suggesting that PPIL2 may be recruited only at intermediate stages of spliceosome activation. PRP17 is a protein that becomes structured during the formation of the $\mathrm{B}^{\text {act }}$. Several short $\alpha$-helices of PRP17 can be localized in early states of $\mathrm{B}^{\text {act }}$ while its WD40 domain is stably integrated near the catalytic center at the latter stages of $\mathrm{B}^{\text {act }}$ formation [99]. In pre-B ${ }^{\text {act-2}}$, a short fragment of PRP17 can be docked alongside SKIP, similar as in $\mathrm{B}^{\text {act }}$ (Fig. 3.12. Absence of density for PRP17 in pre- $\mathrm{B}^{\text {act-1 }}$ may indicate either a gradual structuring of PRP17 during the transition from pre- $\mathrm{B}^{\text {act-1 }}$ to pre- $\mathrm{B}^{\text {act-2 }}$ or a recruitment of this protein in the same transition. 

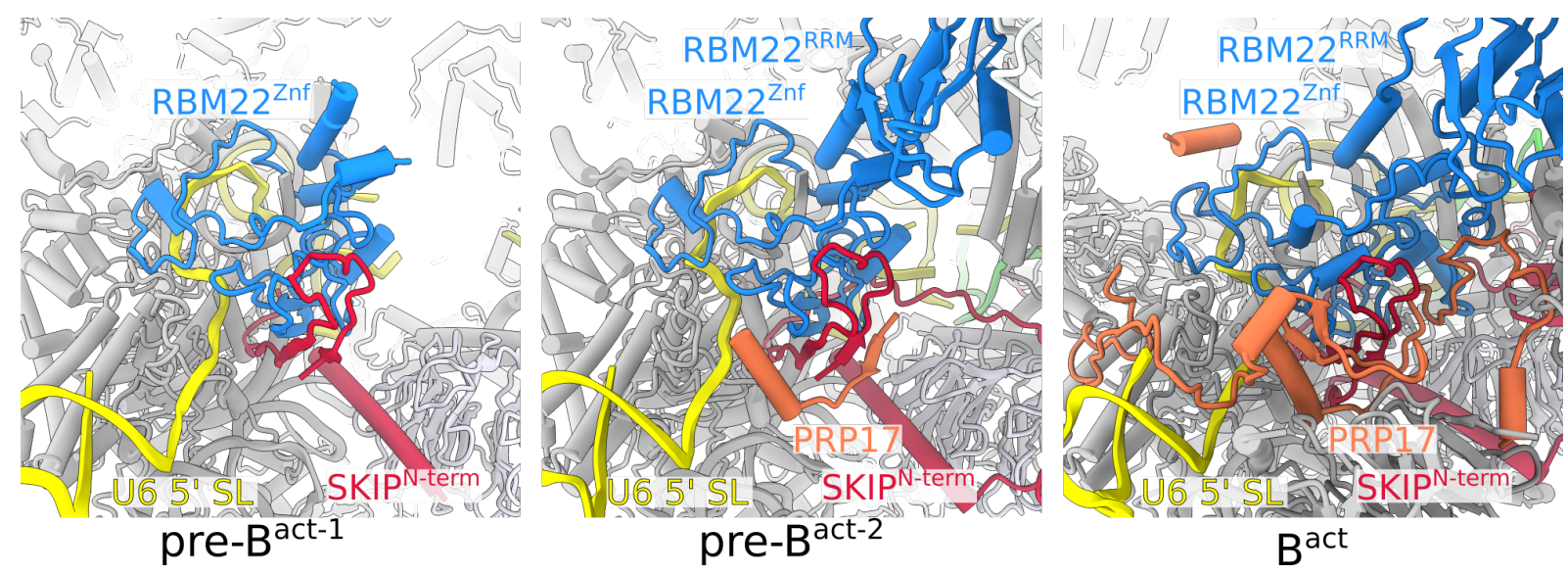

Fig. 3.12: Integration of PRP17 during activation. PRP17 is observed in pre- $\mathrm{B}^{\text {act-2 }}$ but not in pre$\mathrm{B}^{\text {act-1 }}$, interacting with the NTR proteins SKIP and RBM22. Additional regions of PRP17 are stabilized in $\mathrm{B}^{\text {act }}$ (right panel). All structures are aligned to PRP8 ${ }^{\mathrm{NTD}}$ of $\mathrm{B}^{\text {act }}$ (PDB 6FF7).

\subsection{Identification of previously unobserved factors}

\subsubsection{Stabilization of pre- $B^{\text {act-1 }}$ by transiently interacting factors}

TCERG-1 is a transcription factor that binds to the C-terminal domain (CTD) of Pol II and has also been identified by multiple studies as a component of the spliceosome (see section 4.2 , although no structural evidence detailing the presence of TCERG-1 in the spliceosome has been reported. TCERG-1 contains three WW domains, six FF domains (FF1-FF6) and three coiled-coil domains [279]. TCERG-1 is abundant in the pre-B ${ }^{\text {act }}$ sample, as shown by 2D gel electrophoresis followed by tandem mass spectrometry as well as by XL-MS (Fig. 3.1 and Table B.2. In pre-B ${ }^{\text {act-1 }}$, two long $\alpha$-helical densities stretch along the side of the spliceosome, extending from PRP8 ${ }^{\mathrm{RT}}$ to the RecA2 domain of $\mathrm{BRR} 2^{\mathrm{N}-\text { term }}$ (Fig. 3.13). These long helical densities connect compact bundles of short $\alpha$ helices comprising FF domain 1-6. Based on crosslinks, all $6 \mathrm{FF}$ domains of TCERG-1 can be docked onto pre-B ${ }^{\text {act-1}}$. TCERG-1 forms crosslinks to CBP80 as well as to SRSF1, MFAP1, and multiple other proteins (Table B.1. Density for TCERG1 is completely absent from pre-B ${ }^{\text {act-2 }}$, suggesting that it binds only transiently to the pre- $\mathrm{B}^{\text {act- } 1}$, potentially playing a stabilizing role. TCERG-1 is to our knowledge the first transcription factor that has been observed in the cryo-EM structure of a spliceosome. 
The cap-binding complex $(\mathrm{CBC})$ is a bipartite structure composed of two proteins, CBP20 and CBP80, that associates with the 7 -methyl guanosine $\left(\mathrm{m}^{7} \mathrm{G}\right)$ cap at the $5^{\prime}$ end of pre-mRNAs during early spliceosome assembly [280]. The CBC mediates spliceosome assembly in a co-transcriptional manner [281] and facilitates binding of U1 and U5 snRNPs to the pre-mRNA [282]. Although the cap-binding complex is expected to remain bound to the $\mathrm{m}^{7} \mathrm{G}$ cap structure and is present in all spliceosome purifications, it has not been localized in any human spliceosome structure to date. In pre- $\mathrm{B}^{\text {act-1 }}$, but not pre-B ${ }^{\text {act-2}}$, a large density is evident near the exon-binding channel, from which the $5^{\prime}$ end of the pre-mRNA emerges (Fig. 3.13). The crystal structure of the cap-binding complex can be docked into this density based on crosslinks as well as on proximity to the $5^{\prime}$ end of the pre-mRNA. After docking of the cap-binding complex, a low-resolution density between the CBP20 and the $5^{\prime}$ end of the $5^{\prime}$ exon remained. Inspection of crosslinks permitted docking of an RNA binding protein, SRSF1 (Table B.1. Fig. 3.13. SRSF1 is a serineand arginine-rich (SR) protein containing two RRMs and is known to be implicated in 5'ss recognition by the $\mathrm{U} 1 \mathrm{snRNP}$ (see section 4.2). This is the first report of an SR protein being localized in a spliceosome structure.
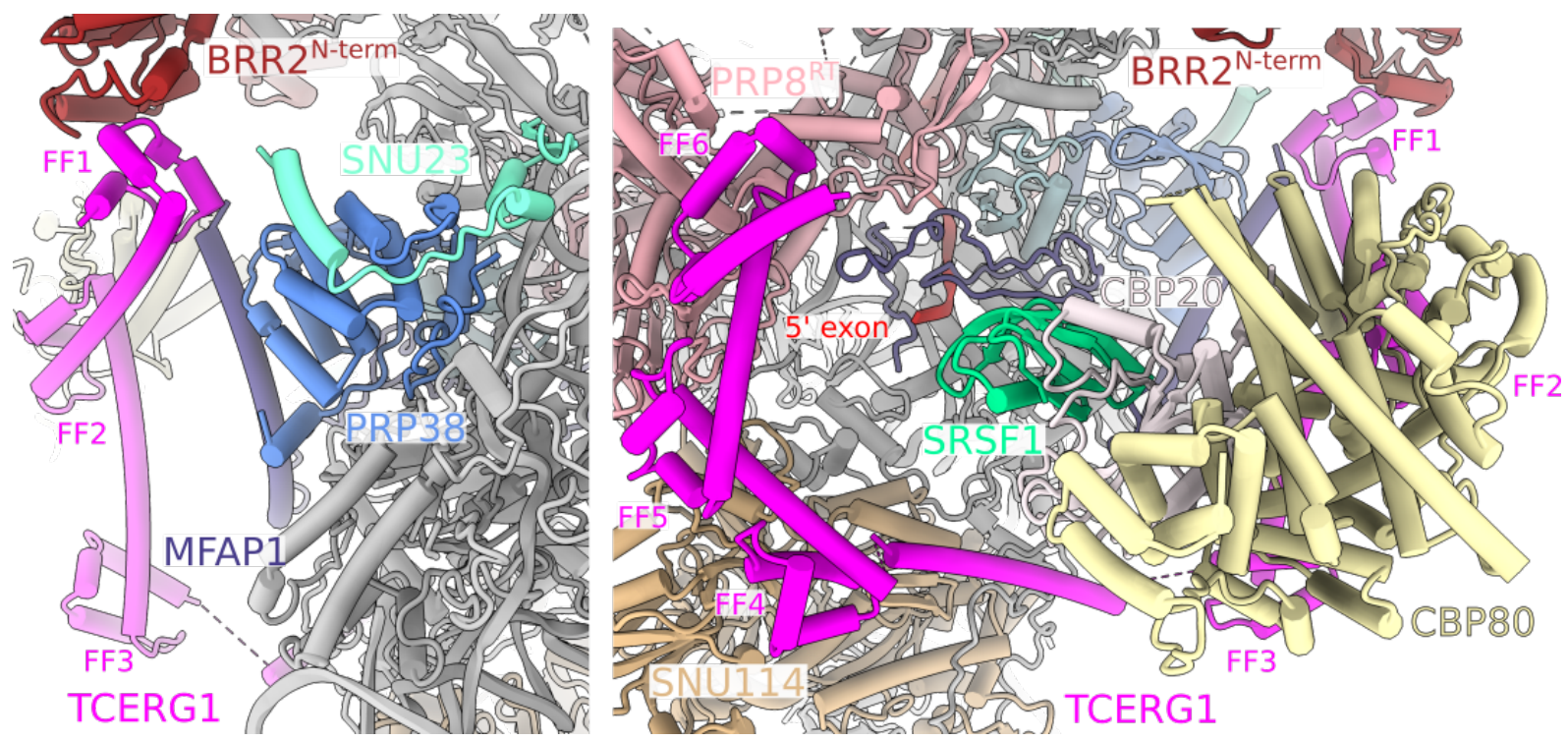

Fig. 3.13: Localization of TCERG-1, CBP80/20, SRSF1 $1^{\text {RRM2 }}$ in pre-B ${ }^{\text {act-1 }}$. The pre- Bct-1 $^{\text {act }}$ structure is shown in two orientations. TCERG-1 wraps around pre- $\mathrm{B}^{\text {act-1 }}$ and contains 6 FF domains (FF1-6). FF1 interacts with BRR2 ${ }^{\text {N-term }}$ (left panel). In addition to TCERG-1, several other proteins bind to the periphery of pre-B ${ }^{\text {act-1 }}$, including the cap-binding complex (CBP80/20), SRSF1 RRM2 . 
WBP11 (also known as SIPP1/NPW38BP) is a component of the spliceosome [283]. WBP11 binds to PQBP1 in vitro and protein phosphatase 1 (PP1) in vivo [283, 284]. The positioning and mechanism by which WBP11 exerts its activity in a biological context is not well understood. Structural evidence of WBP11 has not been reported, although secondary structure predictions of WBP11 exist. In pre-B ${ }^{\text {act-1 }}$, an $\alpha$-helical density intersects the core of the spliceosome, near the nascent catalytic center, situated between the U6/5'ss helix and the U6 ISL. Multiple crosslinks to PRP8, PLRG1, and SNU23 allow for the placement of WBP11 (aa 24-59), a predicted $\alpha$-helix, at this position (Fig. 3.14) (Table B.2). WBP11 passes along the U6 ISL immediately above U74, a nucleotide that is part of the catalytic center and must be flipped out of the stem-loop in order to participate in triple helix formation. Although crosslinks to the binding partner of WBP11, PQBP1, are also present in the XL-MS dataset (Table B.1), no corresponding density was observed in pre-B ${ }^{\text {act-1 }}$. In pre-B ${ }^{\text {act-2 }}$, density for WBP11 between the U6/5'ss helix is absent, indicating that WBP11 is displaced from this position during catalytic center formation (see 4.3 .2 for detailed discussion).

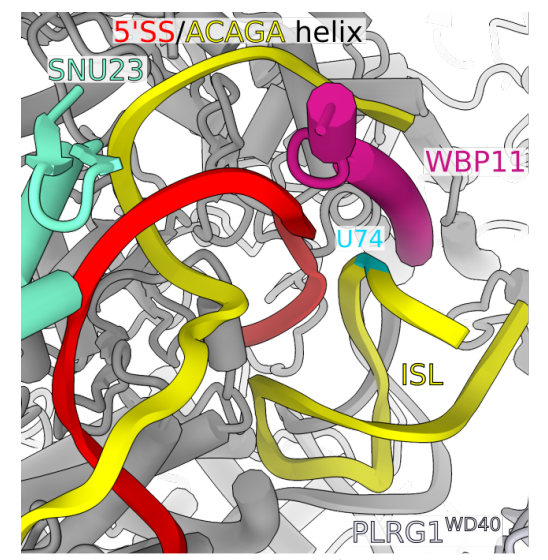

pre-B ${ }^{\text {act-1 }}$

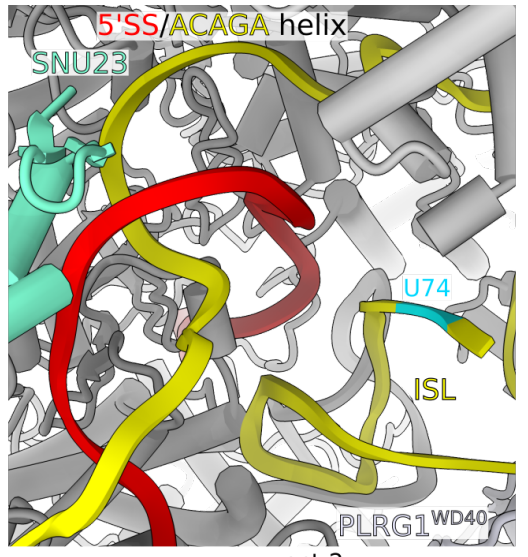

pre-B ${ }^{\text {act-2 }}$

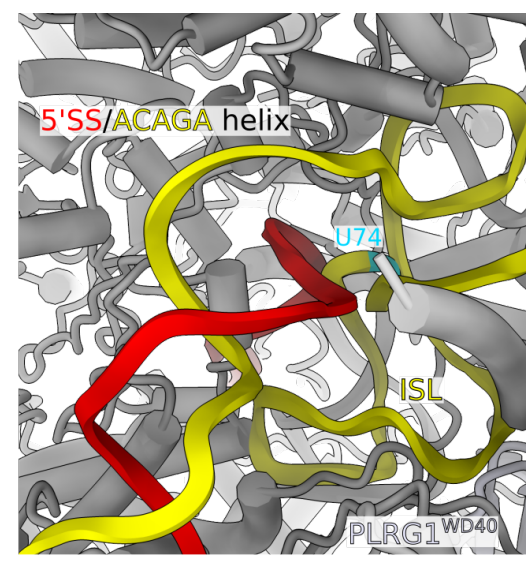

$B^{\text {act }}$

Fig. 3.14: Localization of WBP11 in pre-B ${ }^{\text {act-1 }}$. An $\alpha$-helix of the B-specific protein WBP11 intersects the core of pre- $\mathrm{B}^{\text {act-1 }}$ but is displaced in pre-B ${ }^{\text {act-2 }}$ and $\mathrm{B}^{\text {act }}$ (PDB 6FF7). WBP11 passes between the U6/5'ss helix (i.e., 5'SS/ACAGA helix) and the U6 ISL, in particular immediately above the phosphate backbone of U6 nt U74. 


\subsubsection{Coordination of a network of protein-protein interactions in pre- $^{\text {act-2 }}$ by the DNA/RNA-binding protein KIN17}

KIN17 is an RNA/DNA-binding protein implicated in UV-induced DNA damage repair, although an exact function for this protein has yet to be documented [285, 286]. The solution structure of a KIN17 fragment demonstrated that it forms a winged-helix (WH) motif [287]. KIN17 has not been observed in spliceosomal structures, yet it appears in mass spectrometry data of spliceosomal complexes [75]. Mass spectrometry analysis of the pre-B ${ }^{\text {act }}$ sample indicated the presence of KIN17 (Fig. 3.1). In pre-B ${ }^{\text {act-2 }}$ only, the WH motif can be docked into a globular density between the U6 ISL and PRP8 ${ }^{\mathrm{RH}}$, based on crosslinks to PRP8 and SNU23 (Fig. 3.15) (Table B.2. An $\alpha$-helix of KIN17 is connected by a short linker to the WH domain, and is located in a density running between the WD40 domains of SF3B3-BPB and SF3B3-BPC. The SH3 domain of KIN17 was docked into a low-resolution density at the periphery of the complex, guided by crosslinks proceeding C-terminally from the SF3B3-bound $\alpha$-helix of KIN17 as well as a crosslink between the SH3 domain and SF3B1 (Fig. 3.15. right panel). Binding of KIN17 ${ }^{\mathrm{SH} 3}$ is in a mutually exclusive position compared to the DEAH box helicase PRP2 binding site in $\mathrm{B}^{\text {act }}$, suggesting that KIN17 transiently binds and must be displaced to allow for PRP2 to be integrated to the complex en route to catalytic activation. Positioning of the KIN17 WH and $\alpha$-helix may imply a possible role of KIN17 in organizing protein-protein and/or protein-RNA interactions surrounding the active site, as it binds close to the catalytic core of the complex. 

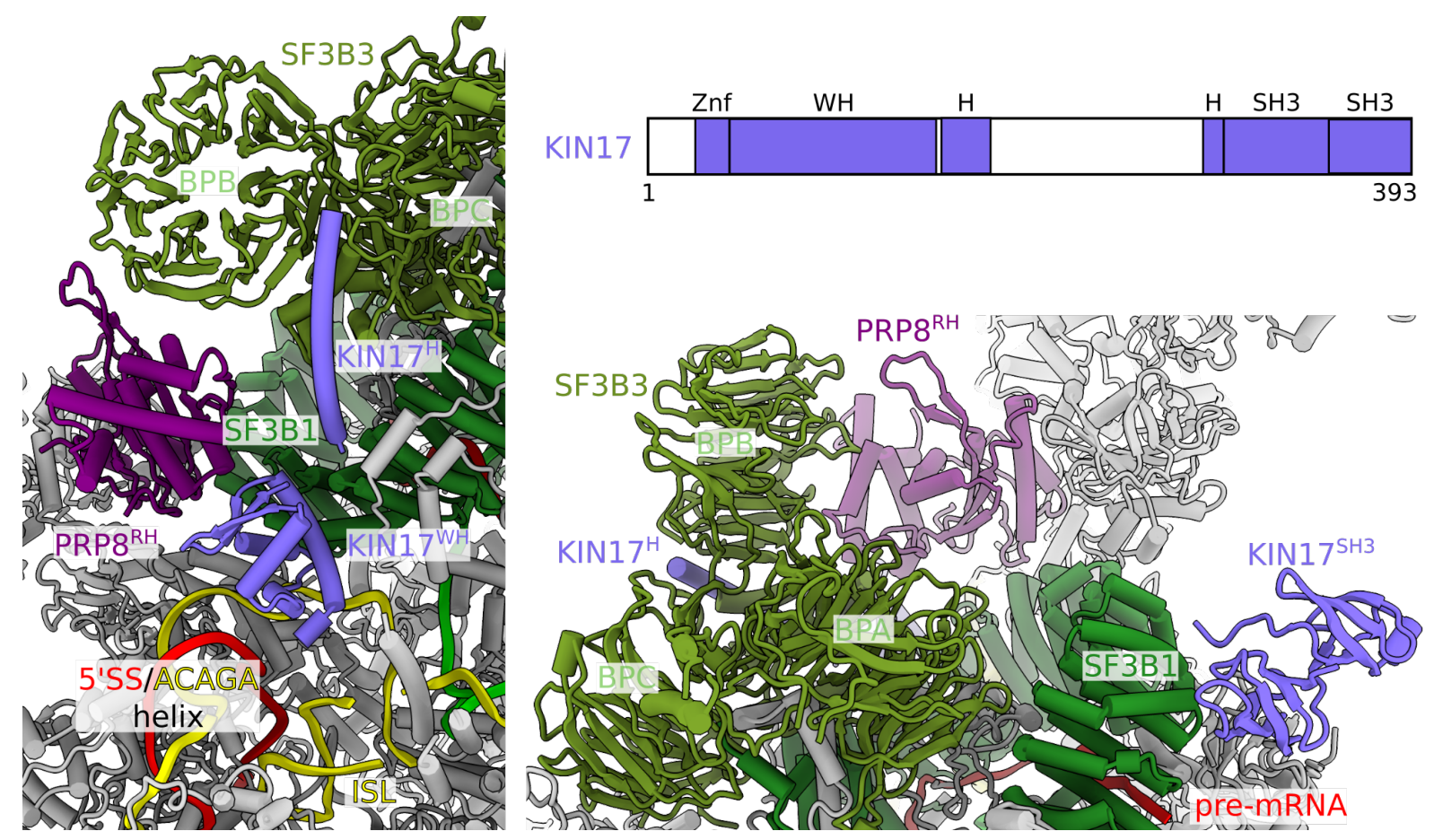

Fig. 3.15: KIN17 binds transiently in pre-B ${ }^{\text {act-2 }}$. KIN17 interacts with SF3B3 and PRP $8^{\mathrm{RH}}$, and SF3B1 in pre-B ${ }^{\text {act-2 }}$. The domain structure of KIN17 is depicted for orientation (upper right panel). Znf - zinc finger, H - helix. 5'SS/ACAGA helix label indicates U6/5'ss helix.

\subsection{Early steps in the stepwise folding of the U2/U6 active site RNA}

The spliceosome's catalytic center - comprised of the U6 ISL and U2/U6 helix Ia and Ib as well as interactions with the U6/5'ss helix - begins to form during spliceosome activation. Pre- $\mathrm{B}^{\text {act }}$ structures reveal new insights into the order in which these secondary structures form en route to the fully formed catalytic center of $\mathrm{B}^{\text {act }}$ (Fig. 3.16). Pre-B ${ }^{\text {act-1 }}$ and pre- $\mathrm{B}^{\text {act-2 }}$ contain similar features, yet also exhibit stark differences in the maturity of the formation of their RNA networks (Fig. 3.18).

\subsubsection{Formation of U6 ISL in pre- $B^{\text {act-1 }}$}

Following liberation of U4 snRNA from the spliceosome, a single-stranded region (nts A56 to A78) of U6 snRNA refolds into the catalytically essential internal stem-loop (ISL). 


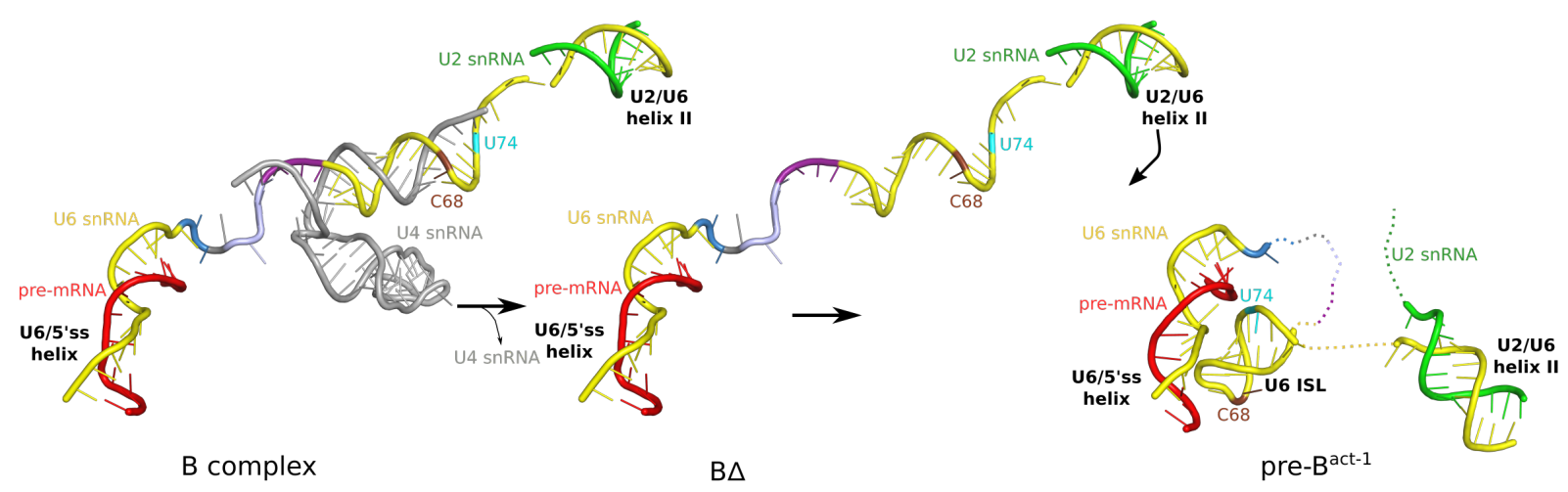

Fig. 3.16: Reorganization of U2/U6 snRNA upon unwinding of U4 snRNA by BRR2. 3D representation of changes in the RNA network that occur during activation. B complex (left; from PDB 5O9Z) contains U4 snRNA, which is dissociated by BRR2 unwindase activity to allow U6 snRNA to engage in new base-pairing interactions. A hypothetical intermediate, $\mathrm{B} \Delta$, is shown to illustrate how the absence of U4 snRNA liberates U6 the nucleotide sequence that are rearranged. In pre-B ${ }^{\text {act- }} 1$, U6 ISL is formed and U2/U6 helix II has been repositioned. Unstable parts of the catalytic center in pre-B ${ }^{\text {act- } 1}$ are indicated with stippled lines corresponding to functionally relevant sequences.

The loop of U6 ISL is formed in both pre- ${ }^{\text {act }}$ structures and is nested within the core of the spliceosome. The loop region of the ISL (nts G65 to A69) in pre-B ${ }^{\text {act-2 }}$ exhibits a similar arrangement to that of $\mathrm{B}^{\text {act }}$. The loop region (nts G65 to A69) of the ISL is docked to its final position on $\mathrm{PRP} 8^{\mathrm{NTD}}$, as in $\mathrm{B}^{\text {act }}$. PRP8 $8^{\mathrm{NTD}}$, a flexible strand of basic residues that meanders around the loop of the ISL in the activated spliceosome, is partially structured in pre- $\mathrm{B}^{\text {act-1 }}$ and pre- $\mathrm{B}^{\text {act-2 }}$ (Fig. 1.11). The residues of PRP8 $8^{\mathrm{NTDL}}$ (aa 659 to 676 ) connecting $\mathrm{PRP} 8^{\mathrm{NTD}}$ to $\mathrm{PRP} 8^{\mathrm{HB}}$ appear to be flexible in both pre-B ${ }^{\text {act }}$ reconstructions and was therefore not observed. Several other regions of proteins that encompass the U6 ISL in the fully formed catalytic network of B ${ }^{\text {act }}$, such as SF3A2 and SF3B2 are also not observed. The mid-stem region (nts G75 to A73 and C60 through C61) of the ISL appears to be distorted in both pre- $\mathrm{B}^{\text {act }}$ complexes relative to $\mathrm{B}^{\text {act }}$, with pre$\mathrm{B}^{\text {act-1 }}$ and pre- $\mathrm{B}^{\text {act-2 }}$ each exhibiting unique conformations in this region 3.17. Nucleotides (A73 and U74) of the mid-stem region important for the construction of the triple helix as well as for metal ion coordination appear to be stacked into the ISL, instead of flipped out as in $\mathrm{B}^{\text {act }}$, although the resolution of the mid-stem region does not permit detailed comparison of the configuration of these nucleotides. The lower-stem of the ISL (nts A76 to A78 and U57 to G59), distal to the loop region, is not well resolved and therefore could not be modeled. 


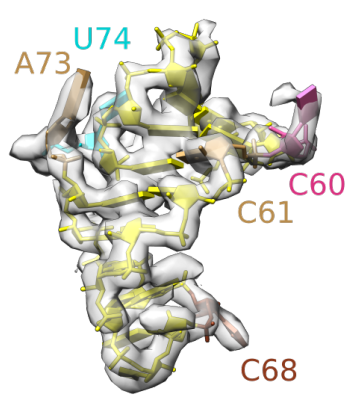

$\mathrm{B}^{\text {act }}$

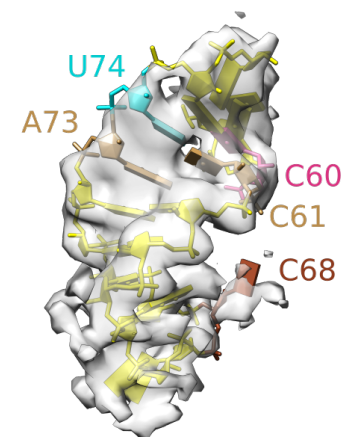

pre-B $B^{\text {act- } 1}$

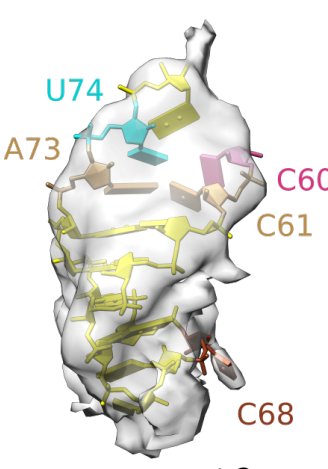

pre-B ${ }^{\text {act-2 }}$

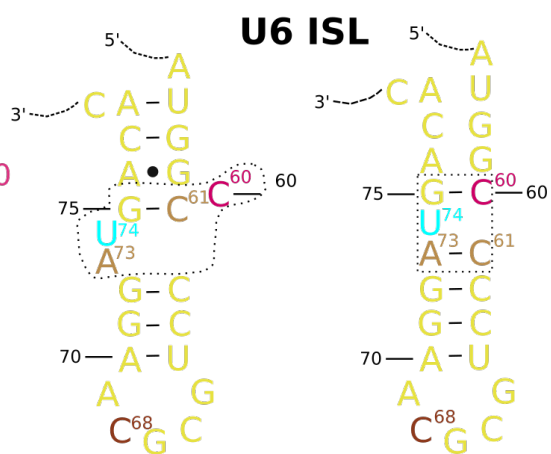

$\mathrm{B}^{\text {act }}$
pre-B $\mathrm{B}^{\text {act-1 }}$

Fig. 3.17: Unique conformations of U6 ISL in pre- ${ }^{\text {act }}$ and proposed alternative base-pairing scheme. Density fits of the U6 ISL in $\mathrm{B}^{\text {act }}$ (EMD 4255 and PDB 6FF4), pre-B ${ }^{\text {act-1 }}$, and pre- ${ }^{\text {act-2 }}$ suggest rearrangement of U6 ISL base-pairing, leading to unique conformations of the ISL. The base-pairing scheme of $\mathrm{B}^{\text {act }}$ and a proposed base-pairing scheme of pre- $\mathrm{B}^{\text {act-1 }}$ (right panel) are indicated. Alternative base-pairing of the mid-stem region (nts $\mathrm{C} 60, \mathrm{C} 61, \mathrm{~A} 73$, and U74) may lead to a more compact ( $\mathrm{B}^{\text {act }}$ ) or elongated (pre-B ${ }^{\text {act-1}}$ ) ISL conformation. Adapted from [131. Reprinted with permission from AAAS.

\subsubsection{Stable formation of $\mathrm{U} 2 / \mathrm{U} 6$ helix $\mathrm{Ib}$ in pre- $\mathrm{B}^{\text {act-2 }}$}

In the fully formed catalytic center of $\mathrm{B}^{\text {act }}$, the U6 ISL is tightly packed against two short RNA duplexes, U2/U6 helix Ia and Ib (Fig. 3.18, right panel). U6 (nt A56) connects the ISL to helix Ib. The U6 nucleotides (A53, G54, and C55) of helix Ib form the AGC catalytic triad and are essential for splicing catalysis, forming part of the RNA triple helix of the active site (see Fig. 3.18). In addition, the U6/5'ss helix is situated close to helix Ib and the ISL, allowing G46 and A47 of the U6 ACAGA(GA) sequence to form Hoogsteen interactions making up the third strand of the triple helix.

Pre- $\mathrm{B}^{\text {act-1 }}$ does not exhibit a stably formed helix Ib, whereas pre- $\mathrm{B}^{\text {act-2 }}$ shows clear density for helix Ib (Fig. 3.19A,C,E). In pre-B ${ }^{\text {act-2 }}$, helix Ib is docked to its final position on $\mathrm{PRP}^{\mathrm{HB}}$ and CDC5L $\mathrm{L}^{\mathrm{Myb}}$ as in $\mathrm{B}^{\text {act }}$ (Fig. 3.19G,F). Basic residues of PRP8 (K774, R781, K1020) surround the flipped-out U2 nts A23 and A24 separating helix Ia and helix Ib nucleotides, clamping helix Ib into place in pre-B ${ }^{\text {act-2 }}$ (Fig. $3.19 \mathrm{G}$ ). The second RNA duplex, U2/U6 helix Ia, could not be detected in either pre-B ${ }^{\text {act }}$ reconstruction. At low thresholds in an unmasked, unsharpened map of pre-B ${ }^{\text {act-2 }}$, a strand-like density extends from the U6 nts of helix Ib to the U6/5'ss helix (Fig. 3.19D). The U6 nucleotides that will later contribute to U2/U6 helix Ia are thus initially in an extended conformation, connecting the U6/5'ss helix and helix Ib prior to base-pairing with U2 snRNA (nts G25, 


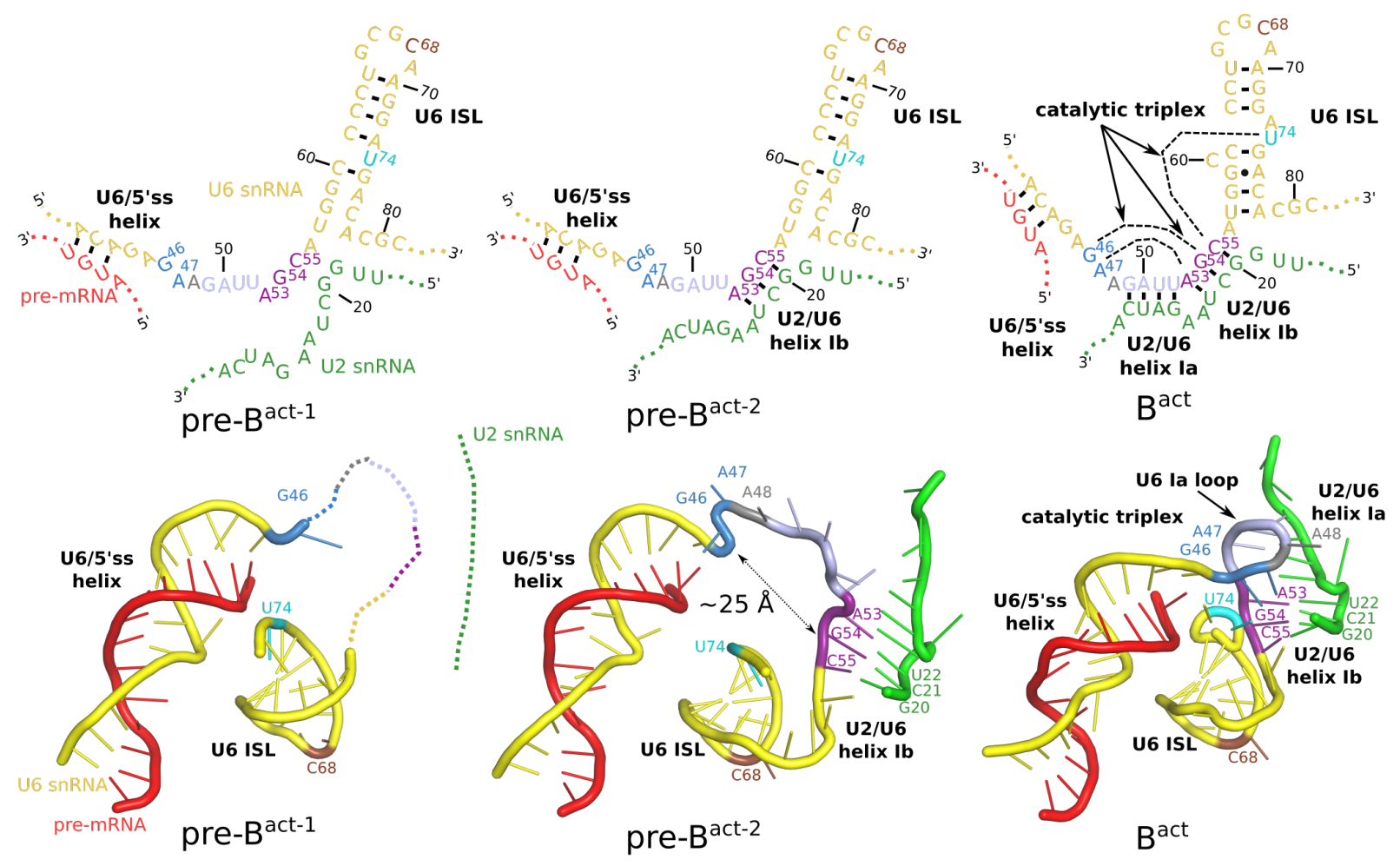

Fig. 3.18: Establishment of catalytic center through interaction of U2/U6 snRNAs. The stepwise formation of the catalytic center is shown as a 2D diagram (upper row) and 3D models (bottom row) from left-to-right for pre-B ${ }^{\text {act-1}}$, pre-B ${ }^{\text {act-2}}$, and $\mathrm{B}^{\text {act }}$ (from PDB 6FF4). Unstable regions of RNA are indicated with colored stippled lines. The U6 nts C68 and U74 are labeled for orientation. Nucleotides that form the triple helix are indicated in the $2 \mathrm{D}$ diagram of $\mathrm{B}^{\text {act }}$ (upper-right panel). Adapted from [131. Reprinted with permission from AAAS.

A26, U27, C28) to form helix Ia (Fig. 3.18, right panel). This extended conformation must later be changed to a more compact arrangement to allow for the triple helix to form (Fig. 3.18). 
A
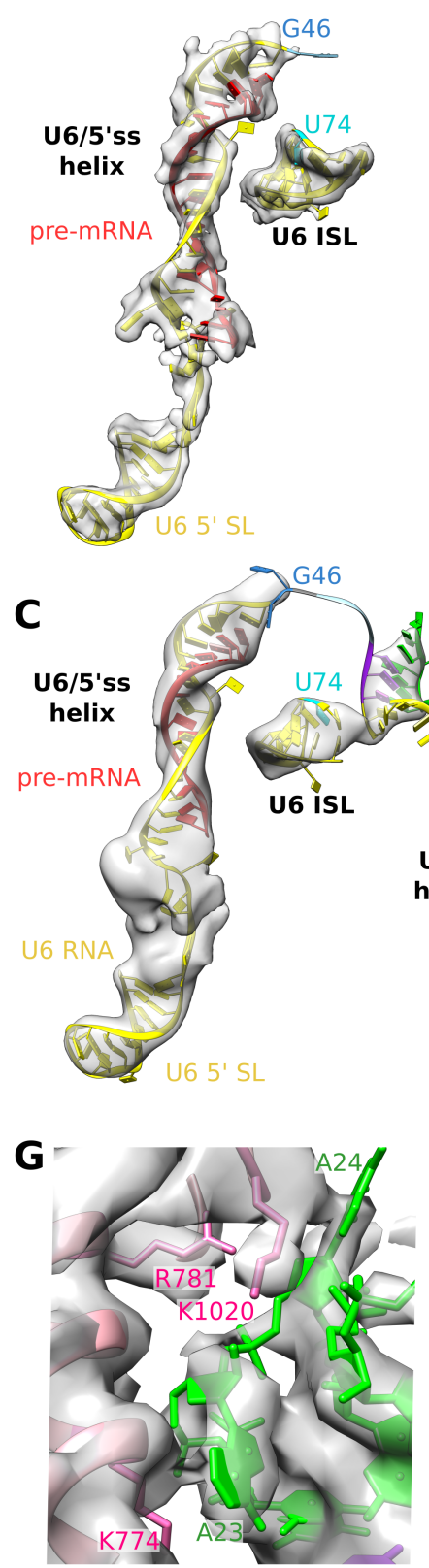

pre-Bact-2
B
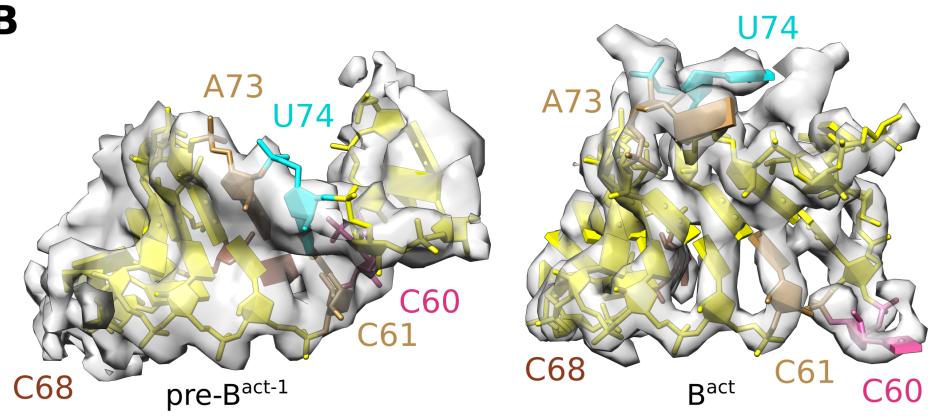

D

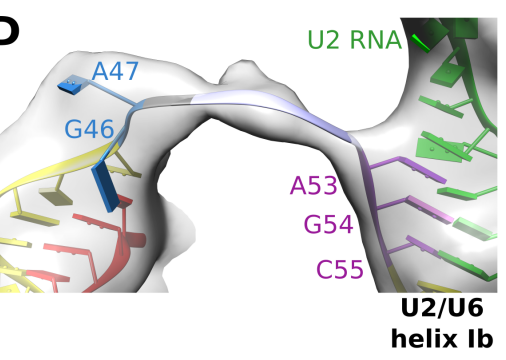

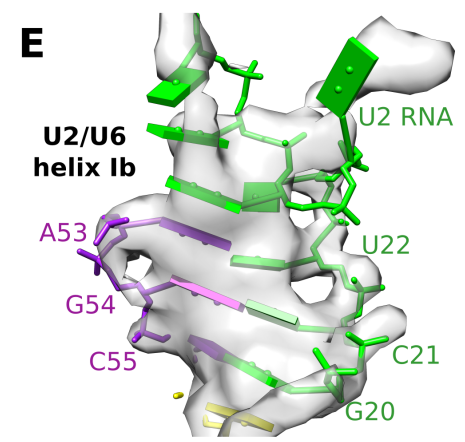

$\mathbf{F}$ helix II

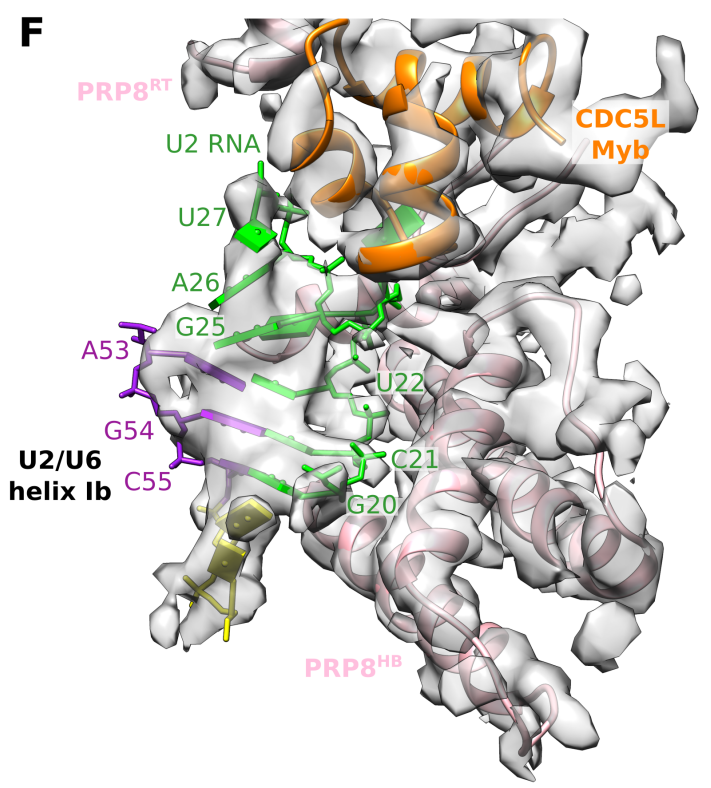

Fig. 3.19: EM densities of RNA structures involved in formation of the active site. Cryo-EM

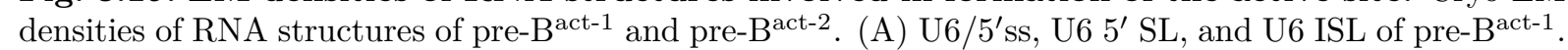
(B) Comparison of U6 ISL model and density from pre-B ${ }^{\text {act-1 }}$ (left) and $\mathrm{B}^{\text {act }}$ (right; EMD 4255 and PDB $6 \mathrm{FF} 4$ ). (C) U6/5'ss, U6 $5^{\prime} \mathrm{SL}$, and U6 ISL, U2/U6 helix Ib, helix II of pre-B ${ }^{\text {act-2 }}$. Note that the linker region (U6 loop Ia) between $\mathrm{U} 6 / 5^{\prime}$ ss and helix Ib is not visible at this threshold but is shown in (D) at a lower map threshold. (E) Fit of U2/U6 helix Ib in pre-B ${ }^{\text {act-2 }}$. (F) Docking of U2/U6 helix Ib to PRP8 $8^{\mathrm{HB}}$ and organization of NTC protein CDC5 $\mathrm{L}^{\mathrm{Myb}}$ in pre- $\mathrm{B}^{\text {act-2 }}$. (G) Comparison of basic residues of PRP8 (R774, R781, K1020) that surround the flipped-out U2 nts A23 and A24, which separate U2 helix Ib and Ia, in pre-B ${ }^{\text {act-2 }}$ and $\mathrm{B}^{\text {act }}$ (EMD 4255 and PDB 6FF4). From 131. Reprinted with permission from AAAS. 



\section{Discussion}

The pre- $\mathrm{B}^{\text {act }}$ structures reported here provide new insights into the complex assembly pathway of the spliceosome during activation. By stalling the spliceosome during the formation of its catalytic center, we were able to observe novel aspects of several strategies the spliceosome uses to form the U2/U6 RNA network at intermediate stages of activation, ensuring accurate folding of the active site. These strategies (discussed in detail in the following sections) include large-scale dynamics in the organization of protein-protein interactions; potential links to co-transcriptional splicing; mutually exclusive interactions; and gradual stabilization of proteins surrounding the active site. All of these modalities appear to contribute to the protein-guided folding of U2/U6 RNA, beginning with the U6 ISL, followed by the U2/U6 helix Ib, and finally the U2/U6 helix Ia and resulting catalytic triplex. In addition to highlighting details of the human spliceosome's activation step, our structures bring up questions for further studies into the necessity and specific roles of various proteins or protein domains in productive formation of activated spliceosomes.

\subsection{Functional implications of large-scale dynamics of BRR2 and PRP8}

BRR2 is the only DExD/H-box ATP-dependent helicase that remains bound to the spliceosome following its unwindase action and occupies distinct positions in the trisnRNP, B complex, and $\mathrm{B}^{\text {act }}$, as well as in later stage complexes) [99, 122, 195, 196]. BRR2 is kept tethered to the spliceosome by its connection to PRP8 ${ }^{\mathrm{Jab}}$. Retention of BRR2 following U4 snRNP dissociation suggests BRR2 may additionally carry out other roles in the spliceosome besides ATP-dependent helicase activity. Prior to U4/U6 snRNA 
unwinding, BRR2 is kept in an inhibited state by the C-terminal region of PRP8 $8^{\mathrm{Jab}}$ [191]. In the pre- ${ }^{\text {act }}, \mathrm{BRR} 2$ is repositioned following U4/U6 snRNP unwinding and is kept in a very different position than in the $\mathrm{B}$ or $\mathrm{B}^{\text {act }}$ complex, with its $\mathrm{C}$-terminal region being rotated relative to its position in $\mathrm{B}^{\text {act }}$ (Fig. 3.6). BRR2 must be tightly regulated prior to activation to prevent premature unwinding of the U4/U6 snRNA duplex. This regulation is carried out by multiple factors and domains, including but not limited to $\mathrm{PRP}^{\mathrm{RH}}, \mathrm{PRP} 8^{\mathrm{Jab}}$, and FBP21. The trigger for BRR2 unwindase activity is unclear, but appears to be closely tied to changes in the factors that regulate BRR2. While the pre-B ${ }^{\text {act }}$ structures do not offer explicit insight into BRR2 regulation, they do indicate that factors governing BRR2 regulation are repositioned following release of U4 snRNA by BRR2. BRR2 repositioning appears to be closely tied to the dynamics of PRP8 $8^{\mathrm{Jab}}$, which is mobile in all spliceosome intermediates from the tri-snRNP to $\mathrm{B}^{\text {act }}$ (Fig. 3.5.)

$\mathrm{PRP}^{\mathrm{Jab} 1}$ contains an intrinsically disordered region that is inserted into BRR2 to prevent binding of RNA [191]. This unstructured region of PRP8 ${ }^{\mathrm{Jab} 1}$ must be removed from BRR2 to allow for it to be loaded onto the U4/U6 snRNA duplex. BRR2 is proposed to become inactivated following $\mathrm{U} 4$ release, again by the C-terminus of PRP8 ${ }^{\mathrm{Jab} 1}$ [191]. In pre$\mathrm{B}^{\text {act-1 }}$, TCERG-1 binds the N-terminal region of BRR2 (Fig. 3.13). This is a previously uncharacterized protein-protein interaction that could stabilize or regulate the activity of BRR2, or at least aid in its initial repositioning. Following dissociation of TCERG-1, BRR2 remains in a similar position in pre-B ${ }^{\text {act-2 }}$, anchored to its binding site on PRP8 $8^{\mathrm{Jab}}$. This positioning is in stark contrast to the position of BRR2 in the $\mathrm{B}^{\text {act }}$, in which BRR2 is again rotated, forming connections to SF3B3 (Fig. 3.6). This interaction appears to be important for stabilizing the U2 snRNP following its docking to PRP8 (Fig. 3.7). These repositioning events appear to be coupled to PRP8 ${ }^{\mathrm{RH}}$ and PRP8 ${ }^{\mathrm{Jab} 1}$ repositioning (Fig. 3.5). Nonetheless, it is unclear whether BRR2 repositioning is a consequence or the cause of $\mathrm{PRP} 8^{\mathrm{RH}}$ and $\mathrm{PRP} 8^{\mathrm{Jab} 1}$ dynamics, or what forces may drive these large scale rearrangements.

The pre- $\mathrm{B}^{\text {act }}$ complexes are the first snapshots of what happens to $\mathrm{PRP}{ }^{\mathrm{RH}}$ immediately following U4 snRNA dissociation. In addition to its dynamic repositioning throughout 
the assembly and function of the spliceosome, $\mathrm{PRP} 8^{\mathrm{RH}}$ exhibits several regulatory functions. $\mathrm{PRP} 8^{\mathrm{RH}}$ regulates BRR2 activity by preventing BRR2 binding to U4 snRNA [193]. Moreover, $\mathrm{PRP} 8^{\mathrm{RH}}$ contains a motif that undergoes a conformational toggle between a $\beta$-finger and a loop, and this toggle is implicated in control of splicing fidelity as well as $\mathrm{Mg}^{2+}$ binding that stimulates splicing (Fig. 1.11] [194, 288]. Furthermore, PRP8 ${ }^{\mathrm{RH}}$ was suggested to be involved in organization of the spliceosome's catalytic center [188]. Consistent with a role in catalytic center formation, $\mathrm{PRP} 8^{\mathrm{RH}}$ is positioned near the U6 ISL in both pre- ${ }^{\text {act }}$ structures. In pre- $\mathrm{B}^{\text {act- } 1}$, the $\beta$-finger is positioned above WBP11 and the U6 ISL and points outward from the core of the spliceosome. In pre- $\mathrm{B}^{\text {act-2 }}$, the $\beta$ finger is rotated farther away from the catalytic center and KIN17. In addition, the entire $\mathrm{PRP}^{\mathrm{RH}}$ appears to be more flexible in pre-B ${ }^{\text {act-1 }}$, implying that it may be destabilized prior to being drastically repositioned in $\mathrm{B}^{\text {act }}$. The proximity of $\mathrm{PRP} 8^{\mathrm{RH}}$ and its rotation in the pre- $\mathrm{B}^{\text {act-1 }}$ to pre- $\mathrm{B}^{\text {act-2 }}$ transition suggests that it may be involved in guiding the formation of U6 ISL by modulating protein-RNA interactions surrounding the nascent catalytic center.

Finally, $\mathrm{PRP} 8^{\mathrm{RH}}$ positioning and dynamics appear to facilitate docking of U2 snRNP to PRP8 (Fig. 3.7). In the B complex, the functionally important SMU1/RED proteins likely stabilize the U2 snRNP on the spliceosome by bridging it to BRR2, as U2 snRNP would otherwise be connected only via very flexible connections mediated by U2 snRNA [98. This bridge appears to be replaced by $\mathrm{PRP} 8^{\mathrm{RH}}$ in both pre-B $\mathrm{B}^{\text {act- } 1}$ and pre- $\mathrm{B}^{\text {act-2}}$, with $\mathrm{PRP}^{\mathrm{RH}}$ being situated between BRR2 and U2 snRNP (see section 4.3.1). Moreover, it is reasonable to suggest that the rotation of $\mathrm{PRP} 8^{\mathrm{RH}}$ from pre- $\mathrm{B}^{\text {act- } 1}$ to pre- $\mathrm{B}^{\text {act-2 }}$ (section 3.3 may be involved in the more stable docking of U2 snRNP, particularly SF3B1 ${ }^{\mathrm{HEAT}}$, to the latter.

Repositioning of the Switch loop (SWL) of PRP8 (PRP8 ${ }^{\mathrm{SWL}}$ ) (aa 1329-1367) also occurs during activation (Fig. 1.11 and 3.5). Prior to activation, $\mathrm{PRP} 8^{\mathrm{SWL}}$ maintains contact with the PRP8 ${ }^{\mathrm{En}}$, while in the activated and catalytic spliceosomes it undergoes approximately a $180^{\circ}$ rotation to face the $\mathrm{PRP} 8^{\mathrm{SWL}}[200$. This change is proposed to transition the PRP8 ${ }^{\mathrm{SWL}}$ from an "inactive" to an "active" conformation [200]. In the inactive confor- 
mation of the B complex, PRP8 $8^{\mathrm{SWL}}$ is contacted by a flexible strand of SNU66 (approximately aa 142-160) [98. This contact is disrupted in pre-B ${ }^{\text {act }}$, but $\mathrm{PRP} 8^{\mathrm{SWL}}$ remains in place. Thus, while SNU66 may contribute to locking PRP8 ${ }^{\mathrm{SWL}}$ in the inactive form, other factors must contribute to preventing $\mathrm{PRP} 8^{\mathrm{SWL}}$ repositioning. $\mathrm{PRP} 8^{\mathrm{SWL}}$ may remain in the inactive form due to the presence of the B-specific proteins (in particular UBL5 and MFAP1), which are bound immediately beneath the PRP8 ${ }^{\text {SWL }}$. Displacement of UBL5 and MFAP1 appears to coincide with the transition of PRP8SWL to the active conformation, in which it PRP8 $8^{\mathrm{SWL}}$ closely interacts with SRRM2 that binds to the activated complex (summarized in Fig. 4.3). This finding is consistent with a previous suggestion based on structural evidence, which stated that either SNU66 or the B-specific proteins could be responsible for regulating the repositioning of PRP8 ${ }^{\mathrm{SWL}}[200]$. PRP8 ${ }^{\mathrm{SWL}}$ should be more thoroughly investigated to understand whether its conformational dynamics play important roles in spliceosome activation and/or catalysis.

\subsection{TCERG-1 and potential links to co-transcriptional RNA splicing}

TCERG-1 (also known as CA150) is reported to bind the Pol II CTD and to regulate transcription and to interact with the spliceosome, although the exact nature by which TCERG-1 exerts its activity in the spliceosome is unknown [289 291]. Although TCERG1 was identified as a component of the spliceosome, it has not been mapped to any 3D structure of the spliceosome until now. Binding of TCERG-1 to pre-B ${ }^{\text {act-1 }}$ suggests that it may be recruited in the early stages of transitioning from the $\mathrm{B}$ to pre- $\mathrm{B}^{\text {act- } 1}$ complex, following dissociation of U4 snRNP (Fig. 3.13). The extensive contacts between TCERG1 to BRR2, PRP8, CPB80/20, and the B-specific proteins suggest that it may be involved in stabilizing or guiding the intermediate state of the pre- $\mathrm{B}^{\text {act-1 }}$ spliceosome. Contacts to $\mathrm{BRR}^{\mathrm{NC}}$ indicate that TCERG-1 may also help stabilize the repositioning of BRR2 after it unwinds the U4/U6 duplex and thereby undergoes a complex rotational movement 
(Figs. 3.13 and 3.7). Binding of TCERG-1 to pre-B ${ }^{\text {act-1 }}$ appears to represent a transient interaction with the spliceosome, as TCERG-1 is destabilized in pre-B ${ }^{\text {act-2 }}$.

In addition to TCERG-1, WBP11 in pre-B ${ }^{\text {act-1 }}$ provides another example of a potential link between the splicing and transcription machineries. Both TCERG-1 and WBP11 bind the Pol II CTD, although it is not known whether these interactions occur simultaneously. WBP11 also interacts with PQBP1 (also called NPW38) and protein phosphatase 1 (PP1) [283, 284]. PQBP1 has been reported to interact not only with the spliceosome, but also with the transcription machinery. WBP11 and PQBP1 form a complex in which PQBP1 binds the spliceosomal protein DIM1 (also called U5-15 kDa; associated with the B complex) and Pol II CTD [292 294]. It is unsurprising that PQBP1 has not been observed in the B complex, as the C-terminal region of PQBP1 that interacts with DIM1 is intrinsically disordered [295]. PQBP1 binds to the C-terminal of WBP11, while only the short N-terminal $\alpha$-helix of WBP11 was identified in pre-B ${ }^{\text {act-1 }}$. Although PQBP1 is detectable within the mass spectrometry data of the pre- $\mathrm{B}^{\text {act }}$ sample, it could not be localized within the cryo-EM reconstruction of either pre-B ${ }^{\text {act }}$ complex. WBP11 was shown to bind and weakly inhibit PP1, and the inhibition increased when WBP11 was phosphorylated [283]. The interplay between WBP11, PQBP1, PP1, and the Pol II CTD should be more thoroughly investigated to understand the role of possible crosstalk between transcription and pre-mRNA splicing, and how this may be intimately tied to association of WBP11 to the spliceosome during activation. It is noteworthy that both TCERG-1 and WBP11 dissociate from the spliceosome, or at least to become destabilized, in pre- $\mathrm{B}^{\text {act-2 }}$. These transient interactions may suggest that early steps of spliceosome activation could be closely tied with transcription, and that these two processes could be mutually affected at this stage of spliceosome assembly. Nonetheless, more experiments will be required to understand how TCERG-1 may be recruited to the spliceosome and whether it may mediate crosstalk between transcription and splicing.

Another link in pre-B ${ }^{\text {act-1 }}$ between the transcription factor TCERG-1 and the spliceosome is SRSF1 (Fig. 3.13). SRSF1 (also called ASF/SF2) is a serine-arginine (SR) protein that binds RNA and influences spliceosome assembly by aiding U1 snRNP recognition 
of the 5'ss [296]. Domains FF4 and FF5 of TCERG-1 were shown to interact with the splicing factor SRSF1, thereby assisting in its localization it to nuclear speckles where spliceosome components are thought to be concentrated [297]. The RRMs (RRM1 and RRM2) of SRSF1 were demonstrated to interact with U1-70K ${ }^{\mathrm{RRM}}$, while the RS domain in the C-terminal region of SRSF1 was shown to bind to Pol II CTD [298, 299]. As Pol II CTD is known to bind a plethora of splicing factors, it is unknown whether the binding of TCERG-1 and SRSF1 to Pol II CTD is simultaneous and/or coordinated. Nonetheless, it is noteworthy that both TCERG-1 and SRSF1 RRM2 are both localized in the pre- $\mathrm{B}^{\text {act-1 }}$ complex. While TCERG-1 clearly becomes destabilized in pre-B ${ }^{\text {act-2 }}$, it is unclear whether SRSF1 ${ }^{\mathrm{RRM} 2}$ also dissociates from the pre-mRNA, or whether it is simply not visualized due to the apparent flexibility of this region in pre- $\mathrm{B}^{\text {act-2}}$. Binding of TCERG-1 to pre-B ${ }^{\text {act-1 }}$ but not pre-B ${ }^{\text {act-2 }}$ would appear to be consistent with a model in which TCERG-1 binds transiently to the transcription machinery or the spliceosome, as previously suggested [300]. Further experiments should be conducted to understand the interplay between TCERG-1 and spliceosome activation, as well as the nature of possible feedback mechanisms between transcription and splicing and how these may be mediated by TCERG-1.

\subsection{Mutually exclusive interactions during spliceosome activation}

The pre- $\mathrm{B}^{\text {act }}$ complexes reveal previously undocumented transitions in the activation of the spliceosome, an event consisting of the dissociation of approximately 32 proteins and U4 snRNA and the association of at least 28 proteins. Many of the proteins bound to the B complex occupy binding sites that are later occupied by different factors in $\mathrm{B}^{\text {act}}$, thus being mutually exclusive interactions. 


\subsubsection{Dissociation of B-specific proteins is a prerequisite for PRP8 conformational change and integration of $B^{\text {act }}$ proteins}

Removal of B-specific proteins is necessary for the large-scale conformational change of PRP8 from an open to a closed conformation (Fig. 3.4. The B-specific proteins would furthermore clash with several $\mathrm{B}^{\text {act }}$-specific factors: CWC27 and RNF113A. It is unclear what governs the removal of the B-specific proteins to allow for conformational change of PRP8 as well as for the association of the $\mathrm{B}^{\text {act }}$-specific factors, including CWC22/CWC27 (which forms a heterodimer), RNF113A, and SRRM2.

Several B-specific proteins (SMU1, RED, and FBP21) are not visualized in pre-B ${ }^{\text {act }}$ structures, yet were localized in the B complex. SMU1 and RED together form a dimer that bridges BRR2 to SF3B3, potentially stabilizing the flexibly associated U2 snRNP [196]. SMU1 and RED were shown to be required for splicing of short introns (approximately 100 nts or fewer) [205]. SMU1 contains a WD40 domain while RED contains two short $\alpha$-helices and extensive disordered regions that crosslink to many components of the spliceosome [196, 301]. Knockdown of SMU1 and/or RED prevents activation of the spliceosome, stalling assembly at the B complex stage [205]. Progressive shortening of the sequence between the 5'ss and the BS of the intron resulted in splicing being blocked when SMU1/RED were absent, suggesting the latter could be involved in mediating structural rearrangements required for positioning of the intron [205]. Both SMU1 and RED are detected in the mass-spectrometry data of the purified pre- ${ }^{\text {act }}$, suggesting they remain present in the complex but are loosely associated and therefore undetectable in the EM densities (Table B.1). In the human B complex, bridging of BRR2 and SF3B3 of U2 snRNP may assist in the docking of U2 onto the spliceosome during activation. In the pre- $\mathrm{B}^{\text {act }}$ complex, this bridge appears to be either destabilized or not present, despite the presence of SMU1/RED in the sample. This apparently loose association between SMU1/RED and the spliceosome could suggest that SMU1/RED act transiently at the B complex stage during activation, helping to guide U2 snRNP into place, and are subsequently destabilized. Furthermore, BRR2 and SF3B3 are in a different orientation 
relative to one another in the pre- $\mathrm{B}^{\text {act }}$ structures than in the $\mathrm{B}$ complex, leading to these components being separated by a larger gap (see Fig. 3.7). The gap between BRR2 and SF3B3 is occupied by $\mathrm{PRP} 8^{\mathrm{RH}}$, which appears to be dynamic even between pre-B $\mathrm{B}^{\text {act-1 }}$ and pre-B ${ }^{\text {act-2 }}$ (Fig. 3.5). Consistent with its proposed role in activation, it is thus reasonable to hypothesize that SMU1/RED thus acts transiently and subsequently becomes destabilized from its original binding site between BRR2 and SF3B3 after guiding U2 snRNP into place, and that its bridging interactions may be replaced by the repositioned $\mathrm{PRP} 8^{\mathrm{RH}}$ in pre-B ${ }^{\text {act }}$.

While nearly all B-specific proteins are abundant in the pre-B ${ }^{\text {act }}$ sample, FBP21 was not detectable (Tables B.1 and B.2. This likely is due to the close association of FBP21 with U4/U6 snRNP-related proteins, including PRP6, DIM1, CypH, which are dissociated during activation [276]. FBP21 binds to a site that is sandwiched between BRR2 and PRP6/DIM1 in the B complex [98]. Following unwinding of U4/U6 snRNA duplex, BRR2 becomes repositioned and U4 snRNP is dissociated from the spliceosome to allow activation to occur. During this transition, the interaction between FBP21 and BRR2 appears to be disrupted, possibly suggesting that FBP21 remains associated with U4 snRNP components and is thereby displaced. Importantly, only the N-terminal Znf domain of FBP21 has been localized in the spliceosome, while FBP21 has shown to have additional interactions with numerous spliceosomal proteins, including BRR2, which it was reported to regulate by binding of an intrinsically disordered region to BRR2 $2^{\mathrm{C} \text {-term }}$ [126]. FBP21 was shown to interact with WBP11 (also known as NPW38BP/SIPP1) with its two tandem WW domains [302]. Both proteins are classified as B-specific proteins due to their characteristic association with the B complex. Although FBP21 is not abundant in the pre- $\mathrm{B}^{\text {act }}$ samples, it may nevertheless be involved in positioning of WBP11 into the nascent catalytic center of the spliceosome during the transition from the B to the pre-B ${ }^{\text {act }}$ complex.

B-specific proteins that block the conformational change of PRP8 consist of PRP38, MFAP1, SNU23, and UBL5. These proteins are deeply lodged within the cleft between $\mathrm{PRP}^{\mathrm{En}}$ and PRP8 ${ }^{\mathrm{NTD}}$. PRP38 is of particular importance in the organization of the 
B-specific proteins, as it forms direct contacts to MFAP1, SNU23, and UBL5 - consistent with its proposed role as a platform that helps coordinate multiple proteins during spliceosome activation [204]. Previous studies in yeast indicated that PRP38 is required for splicing, particularly at the activation step. Intriguingly, depletion of PRP38 did not inhibit pre-spliceosome formation, but abrogated spliceosome activation by preventing the release of U4 snRNA [303]. This still did not clarify whether PRP38 remained bound to the spliceosome after U4 snRNA dissociation. In contrast, the pre-B ${ }^{\text {act }}$ complexes provide biochemical and structural evidence that PRP38 (and associated MFAP1, SNU23, UBL5) indeed remains bound after U4 snRNA is released. Given the requirement of PRP38 in splicing, it appears that PRP38 and the remaining associated B-specific proteins could facilitate U4 snRNA release by holding PRP8 in an open conformation, which may favorably position the U4/U6 snRNA duplex for unwinding by BRR2. 


\subsubsection{Displacement of WBP11 from pre- $B^{\text {act-1 }}$ allows for rearrangements of $\mathrm{U} 6 \mathrm{ISL}$ en route to a mature catalytic center}

WBP11, bound to the nascent catalytic center only in pre- $\mathrm{B}^{\text {act-1 }}$, must be dislodged from the core of the spliceosome to allow for maturation of the active site (Fig. 3.14). The position of the WBP11 would clash with the folding of U6 snRNA, particularly the formation of helix Ia. Strikingly, WBP11 passes along the backbone of the U6 ISL and is situated above a functionally relevant nucleotide, U74, that becomes bulged out of the ISL in the $\mathrm{B}^{\text {act }}$ (Fig. 3.14). U74 becomes stacked with G46 and interacts with C55 to contribute to formation of the triple helix (Fig. 3.18). U74 appears to be stacked into the ISL in pre- $\mathrm{B}^{\text {act-1}}$. This arrangement may suggest that WBP11 could initially aid in the formation or placement of the ISL, and becomes displaced following the docking of the ISL to PRP8 ${ }^{\text {NTD }}$. How WBP11 may become displaced from the spliceosomal core is yet unclear. However, the presence of WBP11 in pre- $\mathrm{B}^{\text {act-1 }}$ is in line with a report documenting WBP11 as a part of the spliceosome [283]. Interestingly, a C-terminal region of WBP11 (aa 180-372) was documented to be the portion of this protein that stalls splicing catalysis, while the $\alpha$-helix of WBP11 mapped in the pre- ${ }^{\text {act-1 }}$ is located in the N-terminal region (aa 2-59) [283]. Thus the N-terminal $\alpha$-helix appears to play a role in spliceosome activation (or regulation), while the C-terminal region may be involved in regulation of splicing catalysis. In summary, the position of WBP11 in pre-B ${ }^{\text {act-1 implies }}$ that WBP11 exerts its regulatory activity at least in part during formation of the active site, perhaps by functioning as a checkpoint to ensure proper folding of the catalytic RNA network.

\subsubsection{Exchange of U6 LSm for IBC, SYF3, and PPIL2}

In pre-B ${ }^{\text {act-1 }}$, U6 LSm proteins are still bound to U2/U6 helix II (see section 3.5.4). In pre-B $^{\text {act-2, }}$ U6 LSm is replaced by SYF3, which cradles U2/U6 helix II and coordinates 
the protein-protein interactions stabilizing the IBC (Fig. 3.11). Of SYF3, only the Nterminal HAT repeats are stably bound to pre- $\mathrm{B}^{\text {act-2 }}$, while the C-terminal HAT repeats appear to be flexible and only integrated in $\mathrm{B}^{\text {act }}$. It is unclear whether SYF3 ${ }^{\mathrm{C} \text {-term }}$ is indispensable for splicing activation or other steps during assembly. SYF3 ${ }^{\mathrm{N}-\text { term }}$ provides not only a binding site for U2/U6 helix II, but also a platform for one of its interaction partners, PPIL2. SYF3 was shown to be important for spliceosome function in vitro, as immunodepletion resulted in inhibition of splicing [304]. In addition to providing an interface for the binding of U2/U6 helix II, PPIL2 and the IBC, SYF3 also appears to be important for stabilization of the catalytic center. In $\mathrm{B}^{\text {act }}$, SYF $3^{\mathrm{N}-\text { term }}$ becomes pushed toward the ISL and comes into contact with the flipped out C60 base of the ISL as well as with a strand of SKIP (Fig. 4.2). These contacts are not yet established in pre-B ${ }^{\text {act-2 }}$ but appear to be primed to occur following the transition of PRP8 from an open to a closed conformation.

The functional significance of the interaction between SYF3 ${ }^{\text {N-term }}$ and PPIL2 ${ }^{\text {PPIase }}$ (Fig. 3.11 is less clear, albeit consistent with a previous report revealing binding between these domains [230]. The proline isomerase activity of PPIL2 has been reported to be nonfunctional, suggesting that PPIL2 ${ }^{\text {PPIase }}$ may play more of a scaffolding role than a catalytic one in the spliceosome 305. In contrast, the U-box domain (PPIL2 ${ }^{\mathrm{U}-b o x}$ ) exhibits E3 ubiquitin ligase activity [306], although its target is unknown. PPIL2 ${ }^{\mathrm{U}-b o x}$ binds to a cleft situated on SNU114, directly opposite to the Armadillo repeats of CTNNBL1 in pre$B^{\text {act-2 }}$ (Fig. 3.9). PPIL2 appears to join the spliceosome during activation and become destabilized following Step 1 of splicing, although the significance of this transient binding remains unclear [276]. An in vitro approach showed a concentration dependent inhibition of spliceosome catalysis by PPIL2, suggesting that its dissociation may be necessary to allow splicing to occur, yet it is unknown whether this occurs similarly in vivo [307].

Binding of SYF $3^{\text {N-term }}$ to PPIL2 was found not to influence the ubiquitin ligase activity of PPIL2 [230]. Both domains of PPIL2 were reported to bind to ZNF830, which is detected in the sample by mass spectrometry but whose function in splicing is unclear and which is the only component of the IBC that has not been localized in a spliceosomal 
reconstruction (Fig. 3.1] 306]. PPIL2 thus appears to coordinate multiple proteinprotein interactions in the spliceosome prior to activation, joining first at the pre- $\mathrm{B}^{\text {act-2 }}$ and remaining bound until the complex is fully activated. Furthermore, the PPIL2 ${ }^{\mathrm{U}-b o x}$ could maintain structural and functional interactions required for activation. Probing of the ubiquitin ligase activity of PPIL2 $2^{\mathrm{U}-b o x}$ in the context of splicing will be necessary to better understand the role of PPIL2 in spliceosome assembly and activation.

\subsubsection{Destabilization of KIN17 liberates PRP2 binding site}

KIN17 is a known component of the spliceosome but was localized for the first time in the reconstruction of pre-Bact-2 reported here (Fig. 3.15) [75]. Previous in vitro studies have determined the structure of KIN17 domains and have provided some indications of its RNA binding activity and potential protein-protein interactions, but did not offer evidence as to the location or putative functions of KIN17 in the spliceosome (see section 3.6.2.

Absence of density for KIN17 in pre-B ${ }^{\text {act-1 }}$ suggests that KIN17 is recruited during transition from pre- $\mathrm{B}^{\text {act-1 }}$ to pre- $\mathrm{B}^{\text {act-2 }}$. The position of $\mathrm{KIN} 17^{\mathrm{WH}}$ is incompatible with the position of the $\mathrm{U} 6 / 5^{\prime}$ ss helix in $\mathrm{B}^{\text {act }}$, and must therefore be displaced following pre- $\mathrm{B}^{\text {act-2 }}$ formation. Dissociation of KIN17 from the spliceosome thus appears to be a prerequisite for final folding of the catalytic center, yet due to its proximity thereof, may assist in guiding maturation of the active site.

KIN17 ${ }^{\mathrm{WH}}$ does not have known RNA binding activity, and was suggested to be involved in protein-protein interactions, although its function in RNA processing is currently unknown [287]. The position of KIN17 $7^{\mathrm{WH}}$ in pre- $\mathrm{B}^{\text {act-2}}$ suggests that it may involved in positioning proteins that surround and possibly assist formation of the catalytic center. The nearby helix of KIN17, which intersects two WD40 domains of SF3B3 of the U2 snRNP, appears to coordinate protein-protein interactions that could be involved in docking of U2 snRNP onto the spliceosome. There is currently no reported function of this helix of KIN17. The C-terminal SH3 domain of KIN17 (KIN17 $\left.{ }^{\mathrm{SH} 3}\right)$ is loosely docked 
to the HEAT repeats $7-8$ of SF3B1 of the $\mathrm{U} 2$ snRNP. KIN17 ${ }^{\mathrm{SH} 3}$ binding appears to occur transiently, as it would need to be displaced to liberate the binding site for PRP2 in the $\mathrm{B}^{\text {act }}$. KIN $17^{\mathrm{SH} 3}$ binds RNA in vitro, but this activity is undocumented in the context of the spliceosome [308. Positioning of $\mathrm{KIN}_{1} 7^{\mathrm{SH} 3}$ in pre-B ${ }^{\text {act-2 }}$ would allow for access to the intron sequence, which is bound by the adjacent RES complex.

KIN17 has been characterized as a component of DNA replication machinery and is known to be upregulated following UV-induced DNA damage [285, 286]. Despite identification of KIN17 in such processes, a mechanistic understanding of its action is still lacking. Nonetheless, identification of KIN17 in the pre-B ${ }^{\text {act-2 }}$ seems to indicate that it may be shared among multiple molecular machineries in the nucleus. 


\subsection{Stabilization of proteins within the spliceosome during activation}

\subsubsection{RES proteins: BUD13, SNU17, SNIP1}

Constituents of the RES complex, required for spliceosome activation, also appear to be gradually stabilized at the periphery on an interface between the loosely docked U2 snRNP and PRP8 ${ }^{R T}$ (Fig. 3.8). Of the three proteins in the RES complex, only the FH domain of SNIP1 is observed in pre- $\mathrm{B}^{\text {act-1}}$. The binding site of SNIP1 ${ }^{\mathrm{FH}}$ at the interface between U2 and U5 snRNP components - in particular SF3B1, SF3B6, and PRP8 ${ }^{\text {RT }}$ - could likely assist in the stabilization of U2 snRNP onto the spliceosome. SNIP1 also interacts with the NTR protein, SKIP, which appears to anchor it to the complex. The two other components of the RES complex, BUD13 and SNIP1, are likely attached but not localized due to flexibility of this unstable interface. BUD13 and SNU17 are more stably integrated into the complex in pre-B ${ }^{\text {act-2 }}$, with BUD13 sandwiched between a flexible strand of SKIP and SNU17, and SNU17 accommodated in a gap between SF3B1 ${ }^{\mathrm{HEAT}}$ and BUD13 (Fig. 3.8. SNU17 ${ }^{\mathrm{RRM}}$ interacts with the intron where it emerges from SF3B1 ${ }^{\mathrm{HEAT}}$, consistent with the known RNA binding activity of SNU17 downstream of the branch site [207]. The gradual stabilization of RES binding observed in the transition from pre- $\mathrm{B}^{\text {act-1 }}$ to pre$\mathrm{B}^{\text {act-2 }}$ is consistent with its reported cooperative binding properties, becoming more tightly associated as the spliceosome becomes progressively activated [207, 208]. However, RES is not yet as stabilized in pre- $\mathrm{B}^{\text {act-2 }}$ as in the $\mathrm{B}^{\text {act }}$, indicated by the absence of the C-terminus of BUD13 as observed in $\mathrm{B}^{\text {act }}$ [99]. In the latter, a short $\alpha$-helix (aa 530-538) interacts with the $\mathrm{B}^{\text {act }}$ protein $\mathrm{CWC} 22$ 99. In addition, two short C-terminal $\alpha$-helices (aa 474$495)$ point toward the helicase PRP2, which is docked to SF3B1 ${ }^{\text {HEAT }}$ and is required for catalytic activation [99, 309]. Deletion of SNU17 greatly impairs RES complex formation, which leads to lower efficiency of spliceosome activation as well as premature disassembly of spliceosomes by PRP2 [310]. The pre-B ${ }^{\text {act }}$ structures demonstrate that RES becomes 
gradually stabilized on the spliceosome during activation, and that this event appears to precede stable PRP2 binding to its position in $\mathrm{B}^{\text {act }}$.

\subsubsection{NTC binding and stabilization of U6 snRNA}

The NTC complex (see section 3.5.2), consisting of PRP19, CDC5L, PLRG1, SPF27, CWC15, and CTNNBL1, joins the spliceosome following dissociation of the U4 snRNP. Previous studies indicated that association of NTC is associated with the loss of U6 LSm while conferring added stability to U6 snRNA within the spliceosome [214].

Intriguingly, several NTC components are already stably integrated even as U6 LSm remains bound in pre- $\mathrm{B}^{\text {act-1}}$. This observation is consistent with a previous proposal that U6 LSm dissociation is likely dependent on NTC binding [214]. Instead of a concerted or mutually exclusive effect, the NTC proteins already begin to bind before U6 LSm has left the complex (see section 3.5.2. Thus, destabilization of U6 LSm must not be a prerequisite for addition of the NTC [214] (Fig. 3.11). The stable binding of additional NTC domains in pre- $\mathrm{B}^{\text {act-2}}$, as well as the loss of U6 LSm in this structure, appear to be involved in gradual stabilization of U6 snRNA (Fig. 3.9), as the RNA network of pre-B ${ }^{\text {act-2 }}$ is more mature than in pre- $\mathrm{B}^{\text {act-1 }}$ (Fig. 3.18). This stabilization of U6 snRNA is likely in part contributed by the CDC5L $\mathrm{L}^{\mathrm{My}}$, a part of the NTC (Fig. 3.19). CDC5L ${ }^{\mathrm{Myb}}$ domains are bound near PRP8 ${ }^{\mathrm{HB}}$, at an interface between U2 and U5 snRNPs (Fig. 3.9). In pre$\mathrm{B}^{\text {act-2 }}$, CDC5 $\mathrm{L}^{\mathrm{Myb}}$ and $\mathrm{PRP} 8^{\mathrm{HB}}$ domains accommodate $\mathrm{U} 2 / \mathrm{U} 6$ helix Ib, coordinating the phosphate backbone of U2 snRNA (nts G20-U22) with multiple basic residues (Fig. 3.19. This interaction appears to be stably established at the pre- $\mathrm{B}^{\text {act-2 }}$ and is also present in $\mathrm{B}^{\text {act }}$. The absence of clear density for U2/U6 helix Ib in pre-B ${ }^{\text {act }}$ may suggest that basepairing between the constituent nucleotides (U2 nts G20-U22 and U6 nts A53-C55) has not yet been established, or that the positioning of U2/U6 helix Ib may already be formed but not yet docked to its binding site on the $\mathrm{CDC}_{5} \mathrm{~L}^{\mathrm{Myb}}$ and $\mathrm{PRP} 8^{\mathrm{HB}}$ domains. U2/U6 helix $\mathrm{Ib}$ is separated from the $\mathrm{U} 6 / 5^{\prime}$ ss helix by approximately $2.5 \mathrm{~nm}$ in pre-B ${ }^{\text {act-2}}$, but is brought into proximity with these nucleotides in $\mathrm{B}^{\text {act }}$. U6 helix Ia loop, visualized as 
a low-resolution density in pre-B $\mathrm{B}^{\text {act-2 }}$, appears to connect U6/5'ss to helix Ib (Fig. 3.18. Separation of U6/5'ss and helix Ib thus appears to result in an extended conformation of the U6 helix Ia nucleotides, preventing base-pairing thereof with U2 nts G25-C28 to form U2/U6 helix Ia (Figs. 3.19 and 3.18). In addition, CDC5L $\mathrm{L}^{\mathrm{Myb}}$ domains appear to provide an additional link between the flexible U2 snRNP (via contacts with SF3A2) and the rest of the spliceosome, possibly facilitating the stable docking of the U2 snRNP (Fig. 3.9.

PLRG1 ${ }^{\mathrm{WD} 40}$ is stably bound beneath $\mathrm{PRP} 8^{\mathrm{HB}}$ in both pre- $\mathrm{B}^{\text {act-1 }}$ and pre- $\mathrm{B}^{\text {act-2 }}$ (Fig. 3.9 . and is connected to the NTC via the C-terminal region of CDC5L, which is not visible in the pre- $\mathrm{B}^{\text {act }}$ complexes but is a connection that is required for splicing to occur [311]. PLRG1 ${ }^{\mathrm{WD} 40}$ forms multiple contacts with intrinsically disordered proteins, including SKIP and CWC15 (Fig. 3.9 and 3.10). The explicit function of PLRG1 is unclear, aside from its apparent role as a binding platform for otherwise very flexible splicing factors to bind, consistent with the proposed role of WD40 domains as organizers of protein-protein interactions [312].

Only a short $\alpha$-helix of CWC15 (aa 218-226) can be localized in pre- $\mathrm{B}^{\text {act-1}}$, whereas a larger segment connected to this helix is bound to pre- $\mathrm{B}^{\text {act-2 }}$ (Fig. 3.9). As with other members of the NTC, CWC15 appears to be gradually stabilized on the spliceosome in the later stages of activation.

PRP19 and SPF27 are not visualized in reconstructions of pre- $\mathrm{B}^{\text {act }}$. This is unsurprising, as even in the $\mathrm{B}^{\text {act }}$, densities for the PRP19 and SPF27 are not stably bound until later stages of assembly, when the NTC helical bundle ( $\mathrm{NTC}^{\mathrm{HB}}$ ) becomes visible (see Fig. 4.3.). Together with CDC5L $\mathrm{L}^{\mathrm{C}-\mathrm{term}}$, PRP19 and SPF27 form the $\mathrm{NTC}^{\mathrm{HB}}$ that is accommodated by the translocation of U5-40K and PPIL1 in B ${ }^{\text {act }}$ (state 4) [99]. Additional contacts to the IBC, which is present in pre-B $\mathrm{B}^{\text {act-2}}$, likely facilitate positioning of $\mathrm{NTC}^{\mathrm{HB}}$. Thus PRP19 and SPF27, while detectable in the sample by mass spectrometry, appear to be the last members of the NTC to stably bind the spliceosome due to rearrangements in

\footnotetext{
1 "stages of activation" refers to states $1-8$ of the $\mathrm{B}^{\text {act }}$ reported in [99], detailing structurally distinct states of $\mathrm{B}^{\text {act }}$ assembly.
} 
protein-protein contacts that occur only later in the assembly pathway (see Fig. 3.1 and Table B.1.

CTNNBL1 is an essential component of the NTC, but has not been observed in any spliceosomal structure besides pre-B ${ }^{\text {act-2 }}$. CTNNBL1 coordinates multiple protein-protein interactions between CWC15 and CDC5L and thereby contributes to stability of the NTC, but is loosely bound to these components and therefore readily dissociates at higher salt concentrations (e.g., $>150 \mathrm{mM}$ ) [215, 313]. In the pre-B ${ }^{\text {act-2 }}$, a short $\alpha$-helix containing segment (aa 147-159) of CDC5L mediates the connection of the CTNNBL ${ }^{\mathrm{ARM}}$ domain to the body of the spliceosome (Fig. 3.9. The integration of CTNNBL1 and its role in spliceosome activation is an exciting topic for new research and should be analyzed by additional experiments in more detail.

NTC binding contribution to U6 snRNA stabilization appears to be more a consequence of overall complex stabilization rather than as a result of direct interactions with U6 snRNA. However, it was previously shown that the NTC proteins are required for the removal of U6 LSm from the spliceosome [304]. The pre-B ${ }^{\text {act }}$ complexes highlight a possible role for the NTC component PLRG1 ${ }^{\mathrm{WD} 40}$ in mediating this transaction by providing a interface on which U6 LSm is initially bound and subsequently replaced with SYF3 and the IBC, offering a putative structural explanation consistent with previous biochemical studies. This interaction leads to the stabilization of U2/U6 helix II and thus could potentially contribute to the overall stability of the U6 snRNA within the spliceosome.

\subsubsection{NTR proteins (SKIP, RBM22) and PRP17}

In addition to the gradual stabilization of NTC proteins such as those comprising the $\mathrm{NTC}^{\mathrm{HB}}$ (see section 4.4.2), several spliceosomal proteins involved in spliceosome activation appear to join the spliceosome and become progressively stabilized along binding interfaces throughout the complex. SKIP, PRP17, and CWC15 - all of which are present in $\mathrm{B}^{\text {act }}-$ are partially stabilized on the pre-B ${ }^{\text {act }}$. Both SKIP and RBM22 are NTC-related (NTR) proteins. 
SKIP is an intrinsically disordered protein (IDP) that was shown to join the spliceosome during activation [223]. Furthermore, SKIP is thought to be implicated in the recruitment of PPIL1, a PPIase that is integrated into the spliceosome in later stages of activation (Fig. 4.3] 222, 314]. SKIP appears to gradually undergo a disorder-order transition as it binds throughout the spliceosome, consistent with an in vitro study showing that an Nterminal fragment of SKIP becomes ordered upon binding to PPIL1 [223]. This transition is even more pronounced in the spliceosome, with many regions of SKIP stably binding to multiple interfaces throughout the pre-B ${ }^{\text {act }}$ complexes.

The SNW domain of SKIP contains $\alpha$-helical regions as well as flexible strands (Fig. 1.142 ). Multiple $\alpha$-helices of SKIP are bound to PLRG1 ${ }^{\mathrm{WD} 40}$ and along the boundary between U2 and U5 snRNPs (Figs. 3.9 and 3.10). These $\alpha$-helices are connected by flexible strands, as in the B ${ }^{\text {act }}$ [99] (Fig. 3.10). Flexible strands of SKIP appear to be further stabilized in the transition from pre- $\mathrm{B}^{\text {act-1 }}$ to pre- $\mathrm{B}^{\text {act-2}}$.

RBM22 RRM integration appears to be facilitated by binding of the IBC (Fig. 3.11. Density for RBM $22^{\mathrm{RRM}}$ is not present in pre- $\mathrm{B}^{\text {act-1}}$, suggesting that this domain remains flexible until it is stabilized by protein-protein contacts with AQR of the IBC in pre$\mathrm{B}^{\text {act-2 }}$. RBM $22^{\text {Znf }}$ is evident in both pre- $\mathrm{B}^{\text {act }}$ structures. The N-terminus of SKIP is already bound near the PPIL1 binding site, situated on RBM22 $2^{\mathrm{Znf}}$ and PRP8 ${ }^{\mathrm{NTD}}$. An $\alpha$-helix of SKIP (aa 137-161) binds to PLRG1 ${ }^{\mathrm{WD} 40}$, opposite RBM22 ${ }^{\mathrm{Znf}}$. Another longer $\alpha$-helix of SKIP (aa 282-325) wraps around the exterior portion of PRP8 ${ }^{\mathrm{RT}}$ near the junction between U2 snRNP components and PRP8, and near SNIP1 ${ }^{\mathrm{FH}}$ (Figs. 3.8 and 3.10. In pre-B ${ }^{\text {act-2}}$, additional strands as well as a short $\alpha$-helix are resolved, linking the RBM22 ${ }^{\text {Znf }}$-bound N-terminal region to binding sites on PLRG1 ${ }^{\mathrm{WD} 40}$, sandwiched tightly between SYF3 and U2/U6 helix II and protruding into a gap between the U2 nucleotides of $\mathrm{U} 2 / \mathrm{U} 6$ helix $\mathrm{Ib}, \mathrm{CDC} 5 \mathrm{~L}^{\mathrm{Myb}}$, and $\mathrm{PRP} 8^{\mathrm{HB}}$. Finally, a flexible strand in the C-terminal region (aa 405-438) winds through a gap separating PRP8 ${ }^{\mathrm{RT}}$ and the RES protein BUD13. The apparent disorder-order transition of SKIP thus appears to be detectable even in the transition between pre- $\mathrm{B}^{\text {act-1 }}$ and pre- $\mathrm{B}^{\text {act-2}}$, with multiple regions becoming ordered across distinct domain interfaces. In addition, ordering of SKIP coincides with stabilization of 
other functionally important interactions in pre-B ${ }^{\text {act-2 }}$, including U2/U6 helix Ib and helix II, binding of SYF3, RES complex, and docking of U2 snRNP. In addition, one $\alpha$-helix of SKIP (aa 282-325) appears to serve as a binding platform for a short fragment of CDC5L that extends towards CTNNBL1 ${ }^{\mathrm{ARM}}$, perhaps mediating the interaction between CDC5L and the loosely associated CTNNBL1. It is unclear whether SKIP ordering is a prerequisite for these assembly events to occur, or whether SKIP binding is part of a concerted process in which SKIP and its interacting partners are mutually stabilized. The gradual ordering of SKIP across functionally important components of the spliceosome suggest that its disordered nature allows it to establish a dynamic network that may assist in spliceosome assembly and activation. PRP17 is a splicing factor that associates with the spliceosome during activation and has been reported to facilitate excision of introns containing 200 nts or more [315 317]. Furthermore, PRP17 was proposed to play a role in modulation of splicing efficiency, as it is not required for steps 1 and 2 of splicing but appears to exert a kinetic effect at both reactions [317]. In pre- $\mathrm{B}^{\text {act-1}}$, density for

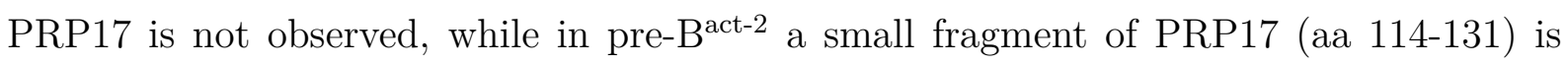
wedged between BUD31, RBM22 Znf, SKIP, and a single-stranded region of U6 snRNA (Fig. 3.12). This binding site is consistent with that of PRP17 in $\mathrm{B}^{\text {act }}$, although in the latter, PRP17 becomes more stably integrated and forms multiple connections to PPIL1, U5-40K, and RNF113A (Fig. 4.3] 99]. PPIL1 can catalyze proline isomerization in PRP17, although this reaction was shown not to be essential for the function of either component, suggesting that PPIL1 might play more of a supporting than an enzymatic role in this binary interaction [318]. The ordered domains of PRP17 ${ }^{\mathrm{WD} 40}$ and several short $\alpha$-helices are linked together by intrinsically disordered strands; similar to SKIP, PRP17 has several regions that undergo a gradual disorder-order transition upon integration to the spliceosome [220]. The presence of a short fragment of PRP17 may suggest that the cooperativity of this transition may be strongly influenced by the presence of PPIL1, which is absent in both pre- $\mathrm{B}^{\text {act }}$ structures. However, it is worth noting that both PRP17 and SKIP, two proteins that converge upon PPIL1, already begin to become ordered in the spliceosome prior to stable integration of PPIL1 (Fig. 3.10.). 


\subsubsection{PRP8 ${ }^{\mathrm{NTDL}}$, SF3B2, SF3A2, and CWC15 closely interact with U2/U6 RNA at catalytic center}

A number of proteins directly surrounding the nascent catalytic center contain domains that appear to be flexible and therefore unobserved in pre- $\mathrm{B}^{\text {act }}$, yet highly ordered in $\mathrm{B}^{\text {act }}$ (Fig. 4.1). This suggests disorder-order transitions in regions of proteins which are already docked near the nascent catalytic center in pre-B ${ }^{\text {act }}$. PRP8 ${ }^{\mathrm{NTDL}}$, a strand connecting the $\mathrm{PRP}^{\mathrm{NTD}}$ and $\mathrm{PRP} 8^{\mathrm{HB}}$ domains that wraps around U6 ISL in $\mathrm{B}^{\text {act }}$, is not visualized in either pre-B ${ }^{\text {act }}$ reconstruction (Figs. 1.11 and 4.1). This suggests that the PRP8 $8^{\text {NTL }}$ is initially flexible and becomes stably positioned in later stages of activation. Furthermore, in $\mathrm{B}^{\text {act }}, \mathrm{PRP} 8^{\mathrm{NTDL}}$ is sandwiched within a channel formed by the U6 ISL loop and PLRG1 ${ }^{\mathrm{WD} 40}$. In the open conformation of PRP8, as observed in pre$\mathrm{B}^{\text {act}}$, this channel is wider and may not allow for the same protein-protein interactions between PLRG1 $1^{\mathrm{WD} 40}$ and PRP8 ${ }^{\mathrm{NTDL}}$. Thus, conformational change may help to stabilize $\mathrm{PRP} 8^{\mathrm{NTDL}}$ by tightly compacting the channel through which it is threaded, possibly leading to stabilizing interactions with both the loop of U6 ISL as well as PLRG1 ${ }^{\mathrm{WD} 40}$.
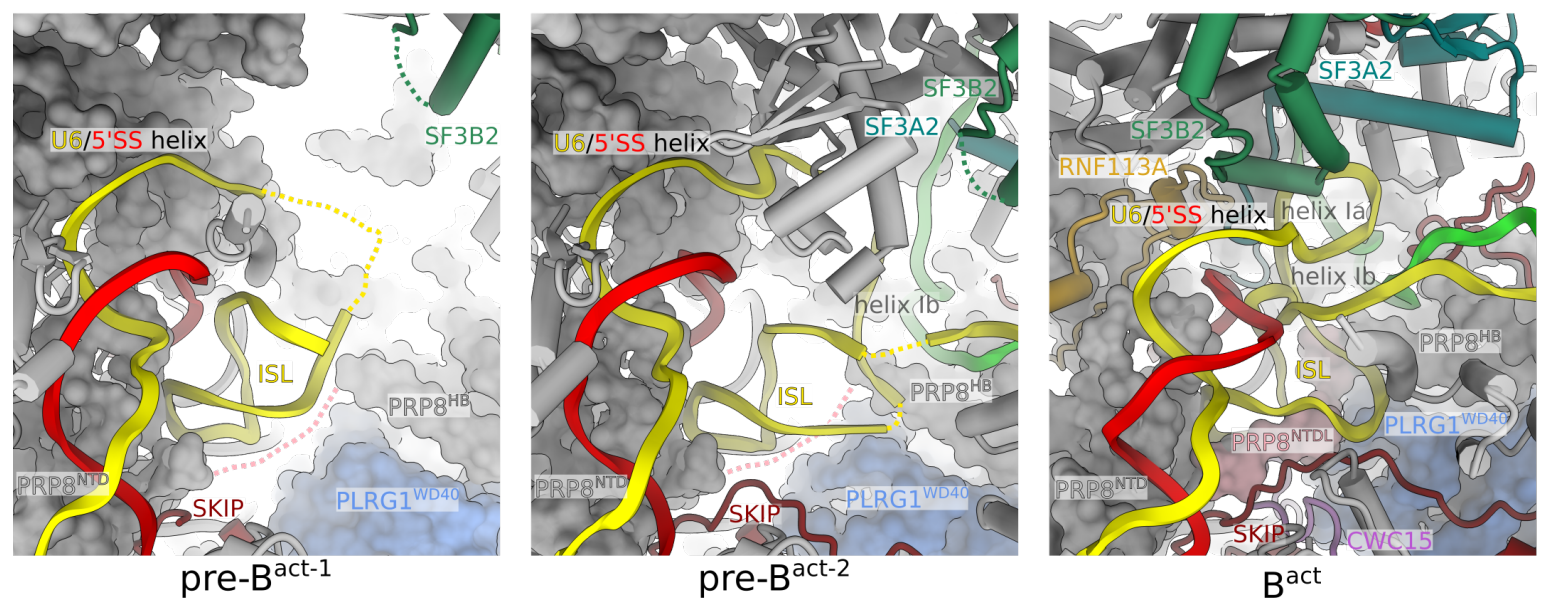

Fig. 4.1: Structuring of proteins surrounding catalytic center during activation. Proteins that surround the catalytic center in $\mathrm{B}^{\text {act }}$ (right panel; PDB $6 \mathrm{FF} 7$ ) are not yet in place in pre- $\mathrm{B}^{\text {act- }}$ and pre- $\mathrm{B}^{\text {act-2 }}$ (left, middle panels). Protein or RNA regions that are not yet stabilized in pre-B ${ }^{\text {act- } 1}$ and pre- $\mathrm{B}^{\text {act-2 }}$ are indicated with stippled lines. In $\mathrm{B}^{\text {act }}$, multiple regions of SKIP, PRP8 ${ }^{\mathrm{NTDL}}$, CWC15, and the U2 snRNP proteins SF3B2 and SF3A2 are stabilized. These same proteins or protein regions

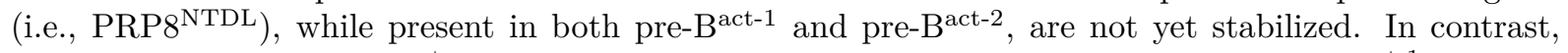
RNF113A binds only to $\mathrm{B}^{\text {act }}$ and is not detectable in the biochemical preparation of pre- ${ }^{\text {act- } 1}$ (see Fig. 3.1P). 
SF3B2 exhibits a short segment (aa 546-562) containing an $\alpha$-helix (aa 547-559) that is positioned immediately above the catalytic center in $\mathrm{B}^{\text {act }}$ (Fig. 4.1), but is not visible in pre- $\mathrm{B}^{\text {act }}$. This short segment appears to become stabilized in the compact arrangement of the catalytic center and is positioned immediately alongside the functionally important U2/U6 helix Ia. Absence of density for this $\alpha$-helix of SF3B2 may point to involvement of this domain in protein-RNA and/or protein-protein interactions involved in formation of the catalytic center.

Stabilization of SF3B2 (aa 546-562) and SF3A2 (aa 1-18) near the catalytic center may also be partially mediated by RNF113A, which is sandwiched between PRP8 ${ }^{\text {En }}$ and PRP8 ${ }^{\text {NTD }}$, where it binds immediately above the U6/5'ss and adjacent to SF3B2 (aa 546-562) and SF3A2 (aa 1-18) (Fig. 4.1). RNF113A is absent from pre-B ${ }^{\text {act }}$, as its binding site is occluded by B-specific proteins. Binding of RNF113A may thus contribute not only to stabilization of SF3B2 and SF3A2 around the catalytic center, but also to the formation of the catalytic center itself.

SF3A2 also contains an N-terminal strand (aa 1-18) that is stably incorporated into the core of the spliceosome in later stages of activation, during the pre- $\mathrm{B}^{\text {act- } 2}$ to $\mathrm{B}^{\text {act }}$ transition, as this region is visible in $\mathrm{B}^{\text {act }}$ but not resolved in pre- $\mathrm{B}^{\text {act-1 }}$ (Fig. 4.1. right panel). This strand winds along the backside of U2/U6 helix Ia and Ib, passes near the U6/5'ss, and ends near the flipped out U40 nucleotide of U5 loop I. The multiple contacts to the catalytic center suggest that SF3A2 could be involved in positioning and/or stabilization of the active site. Flexibility of the SF3A2 N-terminal region may be at least in part explained by the incomplete docking of the $5^{\prime}$ domain of $\mathrm{U} 2 \mathrm{snRNP}$ in pre-B ${ }^{\text {act-2 }}$, as SF3A2 is associated with U2 snRNP.

In $\mathrm{B}^{\text {act }}$, CWC15, a member of the NTC, has an N-terminal strand (aa 18-50) that passes between a gap between PLRG1 ${ }^{\mathrm{WD} 40}$ and U5 snRNA and contacts the loop of U6 ISL while forming multiple interactions with the nearby SKIP and RBM22 (Fig. 4.1, right panel). As only farther $\mathrm{C}$-terminal regions of $\mathrm{CWC} 15$ could be localized in pre-B ${ }^{\text {act }}$, it appears that CWC15 undergoes a gradual disorder-order transition as it associates with 
the spliceosome. CWC15 may thereby provide additional stabilization for the docking of the loop U6 ISL onto PRP8 ${ }^{\mathrm{NTD}}$, while potentially contributing to stability of other protein-protein interactions within NTC and NTR components.

\subsection{Protein-guided folding of the U2/U6 RNA-based catalytic center}

\subsubsection{Protein-RNA interactions may contribute to rearrangements of the U6 ISL stem region during activation}

The U6 ISL stem region (nts U57-U64 and A70-A78) contains three nucleotides that are bulged out of the ISL in the B ${ }^{\text {act }}$ C60, which is contacted by SKIP and SYF3 (Fig. 4.2); A73, the function of which is unclear; and U74, which coordinates two divalent metal ions and interacts with C55 of U6 snRNA and G20 of U2 snRNA to become part of the triple helix (Fig. 1.9). These three flipped out nucleotides cause distortions in the 3D structure of the ISL and likely contribute to its compact arrangement (Fig. 3.18). Despite a similar U6 ISL loop region in the pre-B ${ }^{\text {act }}$ and $\mathrm{B}^{\text {act }}$, the U6 ISL stem exhibits marked conformational differences in the pre- $\mathrm{B}^{\text {act-1 }}$, pre-B ${ }^{\text {act-2 }}$, and $\mathrm{B}^{\text {act }}$ complexes (see Fig. 3.17). These differences suggest flexibility of the U6 ISL stem region, which may be required for repositioning of nts C60, A73, and $\mathrm{U} 74$.

Rearrangements in U6 ISL are consistent with numerous studies showing alternative conformations and base-pairing within the U6 ISL stem region, in particular the nucleotides C67, A79, and U80 in yeast (C61, A73, and U74 in human) [319 321]. The mid-stem nucleotides (C67, A79, and U80) undergo conformational transitions in a pH-dependent manner in vitro; U80 is flipped out of the ISL when A79 is protonated (pH 5.7), but U80 is stacked into the ISL when A79 is deprotonated ( $\mathrm{pH}$ 7.0) [319]. Protonation of A79 and the flipping out of U80 from the ISL results in disruption of base-pairing between C67-U80 and the establishment of a wobble interaction between C67-A79 [321]. U80 (U74 
in human) is a functionally important nucleotide that binds two divalent metal ions and forms part of the catalytically essential triple helix [128]. Changes in conformation and base-pairing thus distort the mid-stem region of U6 ISL, while leaving the conformation and base-pairing of the loop region intact [320]. These studies provided insight into the dynamics of functionally important, conserved regions in the mid-stem of U6 ISL, but were all performed in vitro using isolated fragments of U6 snRNA; thus, many intermolecular interactions with U6 snRNA, as observed within the spliceosome, could not be probed in such experiments.

In the $\mathrm{B}^{\text {act}}$, which contains a fully formed catalytic center, both A79 and U80 (A73 and U74 in human) are flipped out of the ISL [99, 248]. C67 (C61 in human) is stacked into the ISL, where it base-pairs with G81 (G75 in human), while C66 (C60 in human) is flipped out of the ISL [99] [248]. C60 (human) is flipped out of the ISL in B ${ }^{\text {act }}$, but exhibits different orientations based on the surrounding protein environment. Between state 2 and state 8 of $\mathrm{B}^{\text {act }}$, repositioning of $\mathrm{C} 60$ coincides with the structuring of a flexible strand of SKIP, leading to an interaction between the SKIP residue P219 and C60 in state 8 [99]. The structuring of SKIP is also likely mediated by SYF3 and RBM22, to which SKIP forms numerous contacts. In pre- $\mathrm{B}^{\text {act-2}}$, the ISL-interacting region of SKIP is structured, but is more distant from C60 than in $\mathrm{B}^{\text {act }}$ due to the open conformation of PRP8, preventing interaction between P219 of SKIP and C60 of U6 snRNA (Fig. 4.2. While the resolution of the ISL in pre- $\mathrm{B}^{\text {act }}$ does not permit detailed analysis whether C60 is stacked into the ISL or is flipped out, it is clear that interaction C60 and SKIP cannot occur in pre-B ${ }^{\text {act-2 }}$ due to separation of SKIP and the ISL (Fig. 4.2.

Flipping out of C66 (C60 in human) and A79 (A73 in human) from the ISL was not observed in NMR structures of in vitro assembled U6 ISL, thus implying that these alternative conformations likely occur as a result of protein-RNA or additional RNA-RNA interactions formed in the spliceosome [319 321].

The mid-stem region of U6 ISL from $B^{\text {act }}$ did not fit well into the density of the pre-B ${ }^{\text {act }}$ reconstructions, although the loop region (nts C62-G74) is similar in all three complexes. 


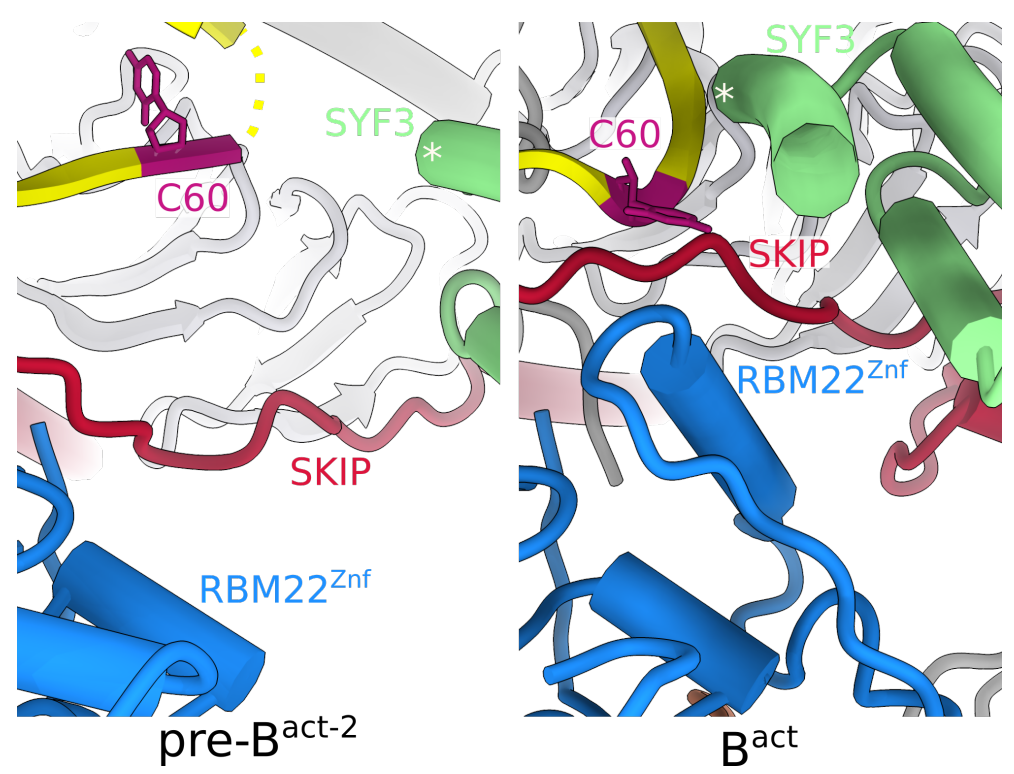

Fig. 4.2: U6-C60 interactions with SKIP, RBM22, SYF3. SKIP, RBM22, and SYF3 are

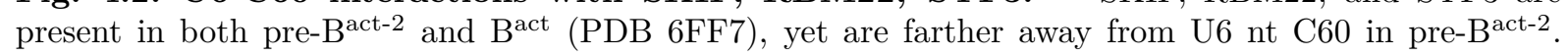
Conformational change of PRP8 is likely responsible for rearranging this protein-RNA interaction in $\mathrm{B}^{\text {act }}$, but the interaction appears to be primed in pre- $\mathrm{B}^{\text {act-2}}$. SYF3 ${ }^{\mathrm{N} 238}$ is marked with an asterisk for orientation.

In particular, the distortions to the helical structure that would be introduced by flipping out of C60, A73, and U74 from the ISL do not appear likely in pre-B ${ }^{\text {act }}$, as these conformational changes would lead to a more compact form of the ISL. The resolution of the ISL becomes progressively worse in regions farther from the loop, which is docked to $\mathrm{PRP} 8^{\mathrm{NTDL}}$. This lower resolved region corresponds to the mid-stem region, in which the alternative base-pairing and conformations have been known to occur in vitro, as well as the lower stem region, which links the ISL to the U2/U6 helix Ib and U2/U6 helix II. While the exact base-pairing patterns in the U6 ISL of pre- $\mathrm{B}^{\text {act- } 1}$ and pre- $\mathrm{B}^{\text {act-2 }}$ cannot be precisely mapped, the more elongated form of the density suggests that A73, U74, and C60 map be stacked within the ISL, likely resulting in a base-pairing arrangement that is highly similar to that observed in vitro (see Fig. 3.17] [319].

Conformational dynamics of the U6 ISL between pre- $\mathrm{B}^{\text {act-1 }}$ and pre- $\mathrm{B}^{\text {act-2 }}$ suggest that the environment surrounding the ISL may be important for modulating its structure. In both pre- $\mathrm{B}^{\text {act }}$ complexes, many proteins that surround the catalytic center in $\mathrm{B}^{\text {act }}$ are not yet in place. WBP11 (see Fig. 3.14 passes immediately along the phosphate backbone of A73 and U74 in pre-B ${ }^{\text {act-1 }}$, preventing these nucleotides from being flipped out of the 
ISL. Moreover, this positioning of WBP11 prevents potential interactions between A73 and the 5 'ss by separating the U6/5'ss helix and the ISL. However, it is conceivable that even in the absence of WBP11, A73 and U74 may not be flipped out of the helix due to the expanded arrangement of the catalytic center, which results from the open conformation of $\mathrm{PRP} 8$ that separates $\mathrm{PRP} 8^{\mathrm{HB}}$ from $\mathrm{PRP} 8^{\mathrm{NTD}}$. In pre-B ${ }^{\text {act }}$ structures, the U6/5'ss helix is positioned farther away from the ISL and U2/U6 helix Ib (pre-B ${ }^{\text {act-2 }}$ only), which would likely prevent the interaction of A73 with G44, A45, and 5'ss even in the absence of WBP11, as in pre-B $\mathrm{B}^{\text {act-2 }}$. This "expanded" configuration of U6/5'ss, the ISL, and U2/U6 helix Ib also prevents G46 and A47, located one and two nucleotides downstream of the ACAGA(GA) sequence of U6 snRNA, from interacting with helix Ib nucleotides to form the catalytic triple helix. In fact, the distance between G46 and the G54 is approximately $2.5 \mathrm{~nm}$, totally preventing Hoogsteen interactions between these nucleotides as found in the triple helix. Moreover, U74 must be flipped out of the ISL to form stacking interactions with G46 as part of the triple helix, and this interaction cannot occur when U74 and G46 are physically separated. Thus, it is reasonable to speculate that A73 and U74 are flipped out of the ISL when the RNA network of the spliceosome is brought into the compact orientation of the $\mathrm{B}^{\text {act}}$, but remain either flexible or stacked into the ISL while PRP8 is in an open conformation as in pre-B ${ }^{\text {act }}$.

\subsubsection{Conformational change of PRP8 allows for the formation of U2/U6 helix la and subsequently the triple helix}

The overall arrangement of the nascent catalytic network could be in part explained by the positioning of the RNA network onto PRP8, the central scaffold of the spliceosome (see section 1.4.1). The open conformation of PRP8 in pre-B ${ }^{\text {act }}$ separates $\mathrm{PRP} 8^{\mathrm{HB}}$ from PRP8 ${ }^{\text {NTD }}$, onto which the U6/5'ss helix and U2/U6 helix Ib are docked (Figs. 1.11 and 4.1. Formation of U2/U6 helix Ia as well as the catalytically essential triple-helix are likely facilitated by the large-scale conformational change of PRP8, in which removal of the B-specfic proteins allows for conversion from the open to closed conformation (Fig. 
3.4. The closed conformation of PRP8 respositions $\mathrm{PRP} 8^{\mathrm{HB}}$ along with $\mathrm{PRP} 8^{\mathrm{RT}}$ and $\mathrm{PRP}^{\mathrm{En}}$, such that these domains are brought into closer proximity with the PRP8 ${ }^{\mathrm{NTD}}$. $\mathrm{PRP}^{\mathrm{HB}}$ and the associated $\mathrm{CDC} 5 \mathrm{~L}^{\mathrm{Myb}}$ drive helix Ib closer to the the U6/5'ss helix, bringing them into contact to allow for the rearrangement of the U6 Ia loop to base-pair with U2 nts to form U2/U6 helix Ia (Fig. 3.18). Stabilization of U2/U6 helix Ia likely allows for the final steps in the folding of the catalytic center, namely the formation of the triple helix: U6 ISL stem is rearranged and bases A73 and U74 are flipped out of the ISL to participate in stacking interactions and triple helix formation, respectively (Fig. 3.17). Finally, the PRP8 ${ }^{\text {NTDL }}$ becomes structured, potentially contributing to stabilization of the ISL prior to catalysis (Figs. 1.11 and 4.1. Thus a large-scale conformational change of PRP8 generates a compact cavity at the core of the spliceosome, allowing for basepairing between U74 of U6 ISL, helix Ib, and G46 and A47 one and two nts downstream of the U6/5'ss helix to form the mature catalytic center of the $\mathrm{B}^{\text {act }}$ (Fig. 3.18). 
A

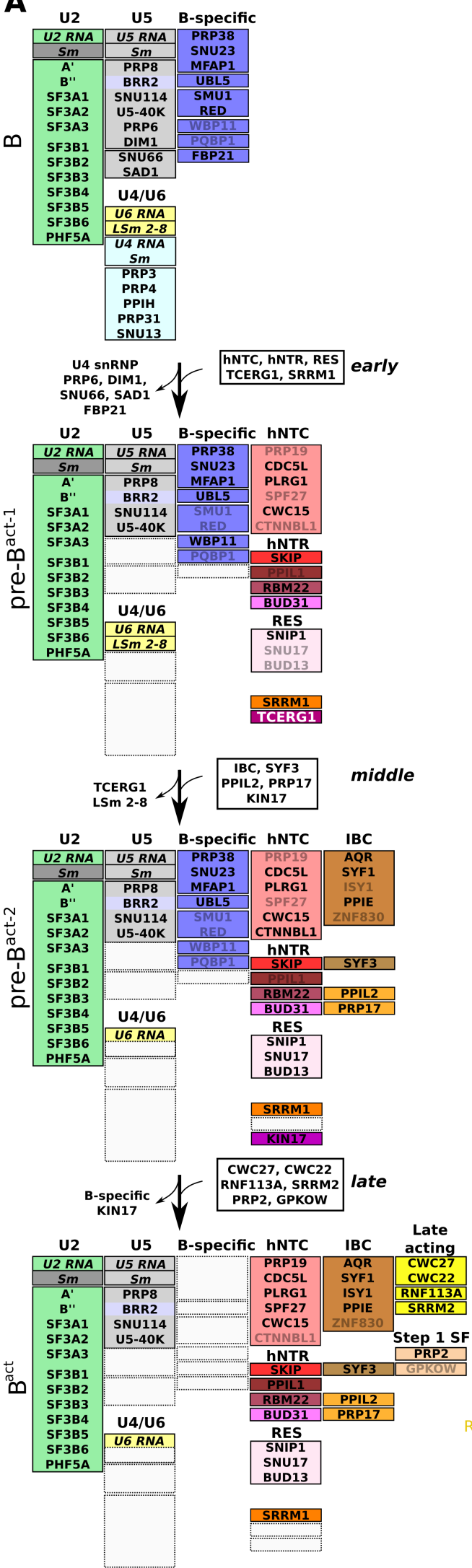

B
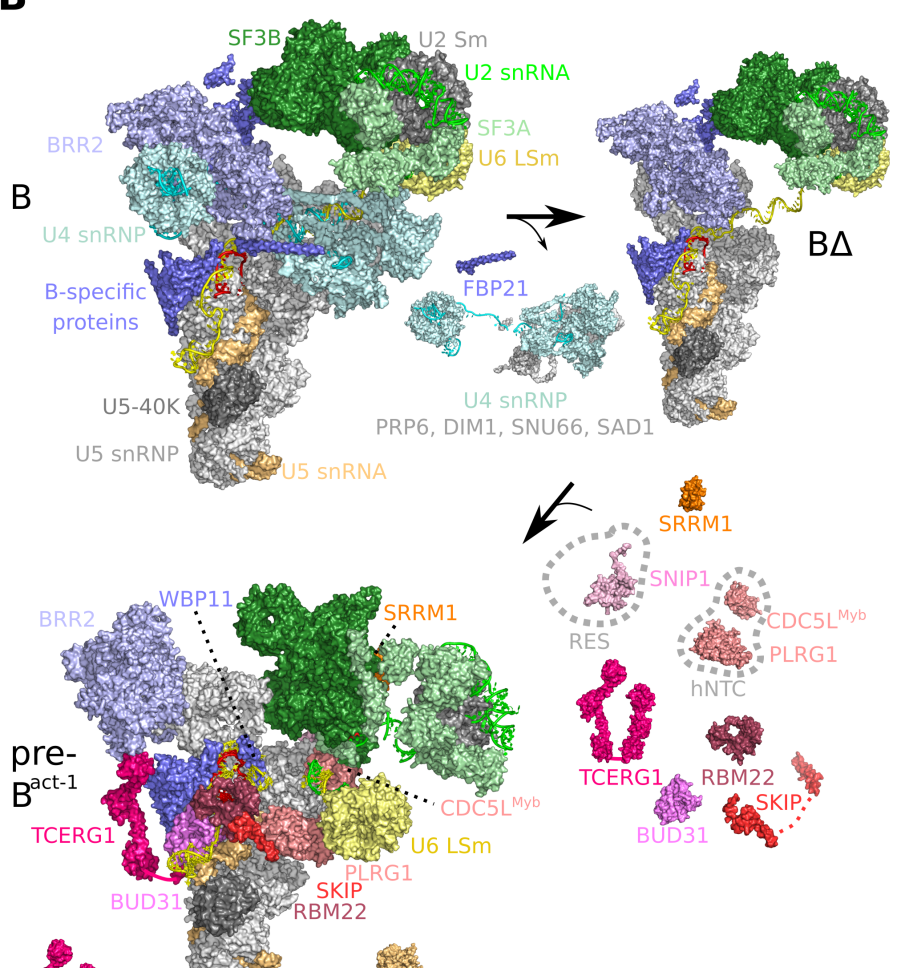

1
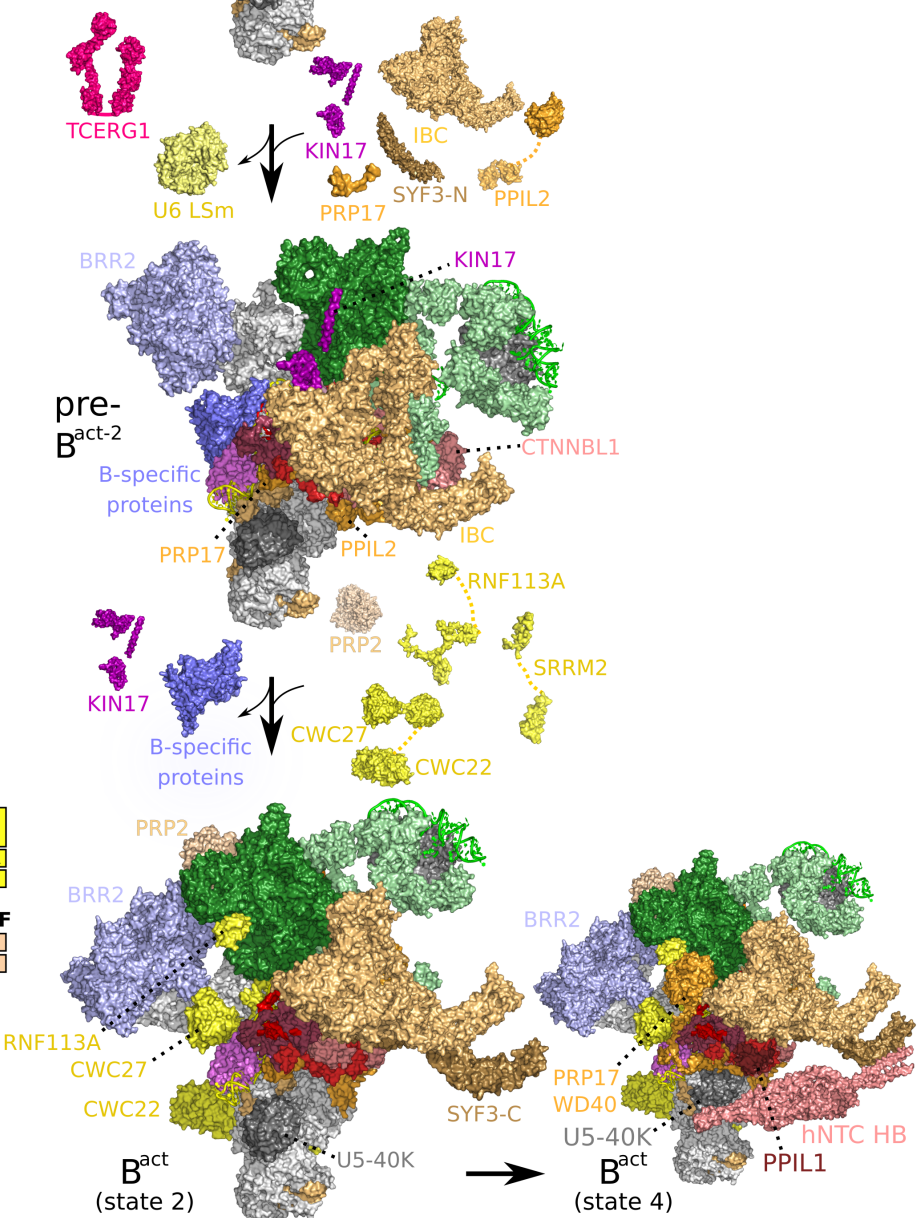

Fig. 4.3: Assembly pathway of pre- $\mathrm{B}^{\text {act-1 }}$ and pre- $\mathrm{B}^{\text {act-2 }}$ during activation. (A) Protein and RNA components of the B (PDB 5O9Z), pre-B ${ }^{\text {act-1 }}$, pre- ${ }^{\text {act-2 }}$, B $^{\text {act }}$ (unpublished PDB models) complexes are shown (top-to-bottom). Exchange of protein and RNA components is indicated by the vertical arrow, with factors that associate to the complex outlined in a box and factors that dissociate from the complex shown to the left of each arrow. (B) 3D representation of assembly pathway from $\mathrm{B}$ to $\mathrm{B}^{\text {act }}$. In the top panel, a hypothetical intermediate $\mathrm{B} \Delta$ is shown for clarity. In the bottom panel, states 2 and 4 of $\mathrm{B}^{\text {act }}$ are shown to illustrate the maturation of the $\mathrm{B}^{\text {act }}$, in which several proteins or additional domains are stably integrated (PRP17 ${ }^{\mathrm{WD} 40}$, PPIL1, NTC $\left.{ }^{\mathrm{HB}}\right)$. From [131]. Reprinted with permission from AAAS. 


\subsection{Further considerations on spliceosome activation}

Spliceosome activation constitutes one of the most drastic changes in protein and RNA inventory during the assembly of the spliceosome, with approximately 32 proteins and U4 snRNA leaving and at least 28 proteins joining the complex (Fig. 1.12. Multiple remodeling events are required to form the active site, with perhaps the most critical being the removal of U4 snRNP to allow for refolding of U6 into the catalytic network (Figs. 1.6 and 1.7 . The pre- $\mathrm{B}^{\text {act }}$ structures offer many novel insights into this massive change in spliceosome inventory and configuration, as summarized in Figure 4.3 . While DExD/Hbox ATP-dependent helicases drive many transitions from one complex to another in the splicing cycle (see section 1.3.5), the driving force between discrete transitions in the ribonucleoprotein content and architecture of the spliceosome is less well understood.

\subsubsection{Energy sources governing pre- $\mathrm{B}^{\text {act }}$ transitions}

Transitions in the spliceosome assembly pathway are driven in large part by $\mathrm{DExD} / \mathrm{H}-$ box ATP-dependent helicases. BRR2's ATPase activity catalyzes unwinding of the U4/U6 snRNA duplex, liberating from U4 snRNP from the spliceosome and allowing for largescale rearrangements and compositional changes in the spliceosome to occur that ultimately result in the formation of $\mathrm{B}^{\text {act }}$ (Fig. 1.6). ATP hydrolysis provides a "driving force" for proceeding from one complex to the next in the splicing cycle, yet it is unclear what governs transitions between pre- $\mathrm{B}^{\text {act-1 }}$ and pre- $\mathrm{B}^{\text {act-2 }}$, especially regarding the dissociation of certain factors and the recruitment of others.

An even greater degree of structural and compositional heterogeneity was documented in the $\mathrm{B}^{\text {act }}$, of which at least eight unique states were present [99]. Transitions between states were consistent with previous biochemical studies detailing the assembly of the spliceosome, suggesting that each structural state represented a "snapshot" en route to a mature $\mathrm{B}^{\text {act }}$ complex [99]. Nonetheless, transitions from one state to the next could not be explained by the energy provided from ATP hydrolysis as there is no known 
ATPase (e.g., an ATP-dependent helicase) that acts between these complexes. The high degree of heterogeneity in the sample was attributed to stochastic sampling of multiple conformations, mediated by thermal energy present in the sample prior to crosslinking and vitrification [99]. In a study of the 70S ribosome, similarly low energy barriers $(<3$ $\mathrm{k}_{\mathrm{B}} \mathrm{T}$ ) were observed in the energy landscape of the conformational changes of the complex, explaining that it can sample a variety of kinetic states independent of NTP hydrolysis 322 .

By this logic, one would expect that the pre- $\mathrm{B}^{\text {act }}$ may also be able to sample a $\mathrm{B}^{\text {act }}$ conformation even in the absence of NTP hydrolysis, as GTP and ATP are not present in the buffer upon chemical crosslinking of the sample. However, this would require at the very least the dissociation of the B-specific proteins to allow for the closed conformation of PRP8 and the recruitment of $\mathrm{B}^{\text {act }}$ specific factors. While this may indeed occur in

solution, the absence of $\mathrm{B}^{\text {act }}$ class averages in the cryo-EM dataset as well as the low concentration of factors associated with $\mathrm{B}^{\text {act }}$ formation suggests that this would at least be a minor population. Thus, one consequence of spliceosome inhibition by NSC95397 may be a kinetic trapping of the complexes, such that transitions between various states are slowed down, biasing the energy landscapes toward pre-B ${ }^{\text {act }}$-like states.

\subsubsection{Directionality of spliceosome activation phase}

Steps 1 and 2 (intron lariat formation and exon ligation) of pre-mRNA splicing are reversible, as shown biochemically [117]. It was shown that mRNA can be converted with high fidelity into pre-mRNA by changing buffer conditions of the purified sample, namely the salt concentration/type and the $\mathrm{pH}$ [117]. If splicing is reversible, what prevents "backwards" assembly of the spliceosome? Dissociation of U4 snRNP has been reported to be essentially irreversible, indicating that following disruption of U4/U6 basepairing by BRR2 in the B complex, all subsequent complexes will contain only U2, U5, and U6 snRNPs (Fig. 1.6] [323]. Re-integration of U4 snRNP into the spliceosome after activation was shown to be very unlikely [323]. For U4 snRNP to be fully reintegrated to 
the spliceosome, U6 intramolecular base-pairing and U2/U6 intermolecular base-pairing would have to be disrupted, likely by a helicase, in order to allow for U4 snRNA to base-pair with U6 snRNA as in the B complex. Although the composition and molecular architectures of the pre- $\mathrm{B}^{\text {act-1 }}$ and pre- $\mathrm{B}^{\text {act-2 }}$ strongly suggest that these intermediates are formed consecutively, it cannot be entirely ruled out that pre- $\mathrm{B}^{\text {act-1 }}$ may be a breakdown product of pre- $\mathrm{B}^{\text {act-2 }}$. Backwards assembly from pre-B $\mathrm{B}^{\text {act-2 }}$ to pre- $\mathrm{B}^{\text {act-1 }}$ would likely require the replacement of multiple mutually exclusive interactions and the destabilization of the multiple domains (i.e., such as those of NTC proteins) that become more ordered from pre- $\mathrm{B}^{\text {act-1 }}$ to pre- $\mathrm{B}^{\text {act-2}}$. NTC proteins were nonetheless shown to bind reversibly to the spliceosome, thus backwards assembly from pre- $\mathrm{B}^{\text {act-2 }}$ to pre- $\mathrm{B}^{\text {act-1 }}$ cannot be entirely ruled out [324]. The assembly pathway of the pre-B $\mathrm{B}^{\text {act }}$ could be more closely analyzed by performing a time-series in the incubation of the splicing inhibitor, NSC95397, and comparing the relative abundances of different protein markers as a function of incubation time. Another way could be to perform a similar biochemical analysis but as a function of inhibitor concentration (Fig. 3.1.

\subsubsection{Alternative spliceosome assembly pathways?}

Early spliceosome assembly has been reported to proceed not only by the sequential addition of $\mathrm{U} 1$ followed by $\mathrm{U} 2$ to the pre-mRNA, but also $\mathrm{U} 2$ followed by $\mathrm{U} 1$ binding [325]. Another report indicated that the NTC complex, which is generally thought to join the spliceosome during activation (i.e., after the integration of the tri-snRNP), was recruited to the pre-mRNA via Pol II CTD prior to the integration of the tri-snRNP [326]. Both pieces of evidence suggest that in addition to the canonical splicing cycle, there may be alternative spliceosome assembly pathways at discrete steps of assembly. This could bring into question whether pre- $\mathrm{B}^{\text {act }}$ structures assemble in a canonical or an off-pathway manner. Steps preceding the formation of the pre-B $\mathrm{B}^{\text {act-1 }}$ could be initiated with either U1 or U2 binding the pre-mRNA first, although further experiments would be required to determine whether this alternative assembly event occurs during pre-B ${ }^{\text {act }}$ 
formation. Conversely, it appears highly unlikely that the NTC is integrated into the pre$\mathrm{B}^{\text {act }}$ complex via an alternative pathway, but rather that it joins the spliceosome after trisnRNP addition. Comparison of the pre- $\mathrm{B}^{\text {act }}$ structures strongly suggests that pre- $\mathrm{B}^{\text {act-1 }}$ is a precursor to pre- $\mathrm{B}^{\text {act-2}}$, as evidenced by the gradual structuring of several proteins of the NTC (Fig. 3.9). Moreover, the temporal ordering of component addition to each structure is in line with the well established canonical pathway of spliceosome assembly, including PRP17, the RES complex, the IBC complex, NTR proteins, and other splicing factors such as SRRM1 (Figs. 3.12, 3.8, and 3.10] [116, 214, 220, 221, 223, 225, 276]. Thus while early events in prespliceosome formation could potentially proceed via an alternative pathway, it appears unlikely that downstream assembly events proceed noncanonically during pre$\mathrm{B}^{\text {act }}$ assembly. Nonetheless, it cannot be entirely ruled out that pre- $\mathrm{B}^{\text {act-1 }}$ is a breakdown product of pre- $\mathrm{B}^{\text {act-2}}$, or that there is an equilibrium between the two complexes.

\subsubsection{Possible mechanisms by which the small molecule NSC95397 may stall spliceosome assembly}

NSC95397 was identified as an inhibitor of spliceosome assembly in a small molecule screen [71]. NSC95397 was documented previously to be a CDC25 dual-specificity phosphatase inhibitor that can bind covalently to cysteine residues [327, 328]. CDC25 is involved in control of cell cycle checkpoints, but does not have a known target in the spliceosome and has not been shown to associate with the spliceosome [329]. In a previous study, several known CDC25 inhibitors were assayed in addition to NSC95397 [71]. Small molecules containing a similar organic structure of a 1,4-napthoquinone backbone were also shown to inhibit splicing, although not as potently as NSC95397. Another CDC25 inhibitor (PM-20) was structurally distinct, not bearing a 1,4-napthoquinone backbone, but did not inhibit splicing. Moreover, the $\mathrm{IC}_{50}$ of splicing inhibition by NSC95397 is much higher (50 fold) than that for CDC25 inhibition. Taken together, these results suggested that stalling of spliceosome assembly by NSC95397 is likely due to a mechanism other than CDC25 inhibition [71]. 
Additional structure activity relationship studies have analyzed quinone-based molecules in more detail. It was shown that these derivatives alter protein function in two ways: 1) covalent binding to cysteine residues; 2) production of reactive oxygen species (ROS) that interfere with protein function by oxidizing reactive side chains, including the thiol moiety of cysteines [330, 331]. In one case, NSC95397 bound covalently to cysteine residues and thereby contributed to disruption of protein-protein interactions [328]. NSC95397 was found to generate $\mathrm{H}_{2} \mathrm{O}_{2}$, a type of ROS, in the presence of dithiothreitol (DTT) [332], illustrating that it is capable of redox cycling. An in vitro study of quinone derivatives structurally similar to NSC95397 revealed that these compounds inhibited spliceosome assembly in a dose-dependent manner, but that this block could be partially removed by addition of DTT [333], which presumably prevents further oxidation of cysteine residues by ROS. An earlier study showed that the quinone-containing compound, $\beta$-lapachone, generated ROS that stalled snRNP assembly by promoting the formation of disulfide crosslinks between survival of motor neuron complexes (SMN), which are involved in the Sm core biosynthesis [334]. Likewise, it was shown that NSC95397 reduces snRNP assembly in vitro, and this effect was proposed to result from generation of ROS [71]. It was therefore suggested that the main mechanism of splicing inhibition by quinone derivatives is likely due to ROS-induced oxidation of cysteine residues of one or more proteins involved in spliceosome assembly, rather than covalent modification of cysteine residues [250, 333]. Nonetheless, it is conceivable that both of these effects may be at play. Incubation of splicing reactions with quinone derivatives resulted in accumulation of spliceosomes stalled at the B complex, suggesting that the target of these small molecules is likely a factor involved in activation [333]. This is in line with our results with the pre- $\mathrm{B}^{\text {act }}$, which is in a precatalytic state and migrates similar to a $\mathrm{B}$ complex on a native agarose gel. However, only upon closer biochemical dissection and structural analysis does it become evident that the complex has progressed past the $\mathrm{B}$ complex en route to activation.

It has previously been shown that phosphatase inhibition can stall spliceosome assembly [199]. A cocktail of phosphatase inhibitors was used to stall assembly at the pre-B/B 
complex level, suggesting that dephosphorylation of at least one unidentified target is required for spliceosome activation [199]. Stalling of spliceosome assembly prior to activation, induced by small molecule quinone derivatives, may point to a disruption of reversible protein phosphorylation, perhaps by the inhibition of one or more phosphatases. It is conceivable that NSC95397 may stall spliceosome assembly by preventing dephosphorylation of trans-acting proteins involved in spliceosome regulation, but not on spliceosome proteins directly. Alternatively, NSC95397 might exert its effect by preventing dephosphorylation of one or more yet uncharacterized target(s) bound to the spliceosome, thus blocking splicing catalysis by inhibiting dephosphorylation, or by a combination of both activities. The B-specific proteins prevent conformational change of $\mathrm{PRP} 8^{\mathrm{RT} / \mathrm{EN}}$ domains and therefore stall the spliceosome prior to activation. Although highly speculative, NSC95397 could potentially be involved in regulation of this protein exchange, possibly by influencing the post-translational modifications of the B-specific proteins directly or other proteins which govern their dissociation from the spliceosome. An examination of the post-translational modifications of the B-specific proteins before and after incubation with the NSC95397 may be warranted. Alternatively, a more general approach could be taken to profile posttranslational modifications of the global sample proteome with and without incubation with NSC95397. Potential regulators of such transitions could be purified and supplemented to the inhibited splicing reaction, to determine whether they are sufficient to relieve the assembly block induced by NSC95397. Furthermore, it would be highly interesting to test whether splicing inhibition by NSC95397 can be rescued by DTT, which would likely indicate that the mechanism by which it inhibits spliceosome assembly is at least partially through the generation of ROS. However, it may be that NSC95397 exerts its activity by nonspecific mechanisms and not by selective inhibition of a single target. Identification of the target(s) of NSC95397 may uncover novel interactions that regulate spliceosome activation. 


\subsection{Classification of dynamic complexes in single particle cryo-EM}

Using image classification techniques, it was possible to identify two distinct macromolecular complexes within a single biochemical purification detailed in this study (Figs. 3.2 and 3.3 [131]. Classification techniques used in single particle cryo-EM are able to sort for compositional and conformational heterogeneity in a dataset, as well as for fine differences in portions of a single refined complex, as recently shown for an novel intermediate state of the human $\mathrm{C}$ complex $\left(\mathrm{C}_{\mathrm{i}}\right)$ [335]. However, classification of flexible regions remains a challenging task, the success and specific outcome of which can vary largely based on many user-defined parameters. Several variables that are likely to vary from one user to the next include the use and design of masks; selection, modification, and filtering of the initial reference structure; rotational parameter specification during multibody refinement; efficiency of 2D/3D classification; choice of regularization parameter (T); number of classes; number of iterations; and angular search options. The choice of software used in image classification and map reconstruction also likely has an impact on the exact makeup of particles included in the final reconstruction, as well as their angular assignments determined during refinement steps.

It would be advantageous to have algorithms which remove user-induced bias of which parts of a macromolecule are "important," instead determining every possible region of a complex to the highest possible resolution in an unsupervised manner. Such a method would be particularly powerful if it could refine multiple regions of a flexible macromolecular complex without the need for highly variable, user-defined masks as used in multi-body refinements or focused classifications.

Shortly following publication of the manuscript corresponding to this work, a new algorithm for "nonuniform refinement" of cryo-EM reconstructions was published [336]. In refinements of macromolecular complexes, poor alignments in flexible regions often worsen the alignment of the entire reconstruction. While masked refinements partially ameliorate 
this problem, they must be manually defined and they introduce user-specific variability as well as artifacts at masks interfaces. Nonuniform refinement implements a novel strategy in which different parts of a reconstruction are filtered based on their alignment accuracy, instead of filtering the entire reconstruction in the same manner. This allows stable portions to become progressively well aligned without overfitting noisy regions in the periphery of the complex - all without the introduction of user-implemented masks. In test cases of publicly available data, nonuniform refinement was shown to improve the local resolution not only of the centrally located, stable portions of the reconstructions, but also those within peripheral regions.

A limitation of nonuniform refinement is that it is a refinement step only, and does not perform classification of the data into multiple subsets. Thus, nonuniform refinement assumes that the data has been sufficiently classified to remove compositional and conformational heterogeneity, even though this is probably not the case for most datasets. Deep learning approaches have emerged in the last year as novel ways to classify cryo-EM datasets having high structural heterogeneity, and will likely continue to bring the field closer to a complete picture of the entire array of structural states sampled by the spliceosome and other dynamic macromolecular assemblies [337, 338]. In another method to characterize structural heterogeneity, 3D principal component analysis (PCA) approaches have been used to calculate energy landscapes of large macromolecules, uncovering heterogeneous assembly intermediates in single biochemical preparations and mapping them to a Boltzmann distribution [99, 167, 339, 340]. In principle, 3D PCA approaches could also be preceded with unsupervised ab initio structure determination, possibly helping to detect classes that would otherwise not be properly classified if using only a single reference. More accurate 3D classifications will lead to reconstructions that are closer to representing the "ground truth" of a given conformation or composition of a complex, by improving the homogeneity of the final particle subset to be refined instead of refining small parts of a heterogeneous mixture of similar structures.

Although cryo-EM studies of the spliceosome and other large biological complexes have made great strides in determining the molecular architectures of very dynamic samples, 
it is clear that there is substantial room for improvement if atomic or "near atomic" (e.g., better than $2 \AA$ ) resolutions are to be reached. Such resolutions will bring cryo-EM investigations of challenging biochemical samples into the realm of chemistry, at which point chemical modifications and catalytic mechanisms can be interrogated with accuracy and precision. Better microscope hardware leading to significant boost in micrograph quality represent a clear path forward in collecting better raw data [183, 184]; likewise, more robust classification methods should be developed to deal with the compositional and conformational heterogeneity of the spliceosome and other dynamic complexes at high resolution. 


\section{Conclusion and Outlook}

The work presented herein sought to address two major questions:

- Are there structurally distinct intermediate stages of activation at which formation of the catalytic center can be observed?

- How do proteins facilitate the folding of active site U2/U6 snRNAs in the spliceosome?

By employing a small molecule inhibitor of pre-mRNA splicing, it was possible to stall spliceosome activation at a previously uncharacterized stage between a precatalytic B complex and the activated $\mathrm{B}^{\text {act }}$ complex. Analysis of the single biochemical preparation uncovered two distinct structural states of the spliceosome - pre- $\mathrm{B}^{\text {act-1 }}$ and pre- $\mathrm{B}^{\text {act-2 }}$ each lacking a mature catalytic center while bearing the U6 ISL. Importantly, our structures have implications for step-wise formation of the catalytic center's active site: the ISL forms first, followed by helix Ib, and ends with formation of helix Ia and subsequently the triple helix. The pre- $\mathrm{B}^{\text {act-1 }}$ and pre- $\mathrm{B}^{\text {act-2 }}$ provide the first structural insights into the ordering by which tertiary interactions of the spliceosome's catalytic center is assembled.

Our structures also provided new insights into conformational change, mutually exclusive protein-protein interactions, and gradual stabilization of numerous proteins which appear to facilitate the folding of active site $\mathrm{U} 2 / \mathrm{U} 6$ snRNAs in the core of the spliceosome. Both pre- $\mathrm{B}^{\text {act-1 }}$ and pre- $\mathrm{B}^{\text {act-2 }}$ surprisingly showed that the central scaffold protein of the spliceosome, PRP8, remains in an open conformation even as the catalytic center begins to form. Comparison of our structures to the $\mathrm{B}^{\text {act }}$ further suggests that the closed conformation is the final step in the formation of the catalytic center, acting as a "mold" in which $\mathrm{PRP} 8{ }^{\mathrm{HB}}$ pushes $\mathrm{U} 2 / \mathrm{U} 6$ helix Ib towards the U6/5'ss and the ISL, removing the 
spatial restraint between these regions and thus allowing helix Ia and the triple helix to form. Although mutually-exclusive protein-protein interactions are a well-documented feature of spliceosome assembly, the pre- $\mathrm{B}^{\text {act-1 }}$ and pre- $\mathrm{B}^{\text {act-2 }}$ provide the first structural evidence for several interactions, such as the concomitant exchange of LSm proteins for SYF3/IBC proteins at U2/U6 helix II; WBP11 docking and helix Ia formation; KIN17 and PRP2 binding to SF3B1. Furthermore, the pre-B ${ }^{\text {act-1 }}$ and pre- $\mathrm{B}^{\text {act-2 }}$ reveal clues as to the gradual stabilization of multiple protein subcomplexes or groups on the spliceosome, such as the NTC, NTR, RES, and the IBC. In addition, our structures indicate that several protein regions are poised for stabilization in $\mathrm{B}^{\text {act }}\left(\mathrm{PRP} 8^{\mathrm{NTDL}}\right.$, SF3B2, SF3A2, and CWC15), initially remaining flexible as a possible means of either permitting or guiding initial refolding U2/U6 snRNAs into the catalytic center. By revealing discrete structural snapshots of spliceosome activation, we were able to uncover previously uncharacterised protein-RNA interactions that appear to be part of a concerted process that ensures proper folding of the catalytic center.

While the pre- ${ }^{\text {act }}$ structures deliver novel snapshots of human spliceosome assembly, they also bring up new questions that could become topics for future investigation. First, what is/are the target(s) of the small molecule chemical inhibitor NSC95397? The answer to this question may uncover hitherto unclarified mechanisms of splicing regulation. Second, what governs the highly ordered exchange of proteins that have mutually exclusive interactions during activation? Of particular importance in this category are the B-specific proteins, whose dissociation is an obligate step to allow for PRP8 closure. Third, are the protein regions undergoing disorder-order transitions surrounding the catalytic centers necessary for splicing? Truncation or mutation of residues interacting with the U2/U6 snRNA network would be one means of examining this question in more detail. Fourth, what is responsible for the apparent structural plasticity of the U6 ISL? Addressing this question will likely help to clarify studies related to the conformational dynamics of the ISL, but in the context of the spliceosome instead of an in vitro, RNA-only system.

It will be essential to examine the formation of the spliceosome's catalytic center in more depth and at higher resolutions. Pushing the core of the pre-B ${ }^{\text {act }}$ structures and other 
complexes to sub-2 $\AA$ resolutions will likely unlock unanticipated structural modalities that the spliceosome uses to fine-tune the construction of its catalytic center. In addition, much higher resolutions would enable the modeling of the divalent metal cations coordinated by the phosphate backbone of nucleotides within the catalytic center, as well as structural water molecules. 



\section{A Abbreviations}

aa

ATP

BS

BS-A

BSL

cryo-EM

Cs

CTD

CTF

DExD/H

DNA

DTT

EM

FSC

GTP

HEAT

IBC

$\mathrm{IC}_{50}$

ISL

kDa

MDa

mRNA

MW

$\mathbf{m}_{3} \mathbf{G}$

$\mathbf{m}^{7} \mathbf{G}$

NMR amino acid

adenosine triphosphate

branch site

branch site adenosine

branch site stem-loop

cryo electron microscopy

spherical aberration coefficient

C-terminal domain

contrast transfer function

Aspartate-Glutamate-x-Aspartate/Histidine

deoxyribonucleic acid

dithiothreitol

electron microscopy

Fourier Shell Correlation

guanosine triphosphate

Huntingtin, elongation factor 3, PR/A subunit of protein phosphatase $2 \mathrm{~A}$ and the TOR lipid kinase

Intron Binding Complex

$50 \%$ inhibitory concentration

internal stem-loop

kilodalton

megadalton

messenger RNA

molecular weight

2,2,7-trimethylguanosine

7-methyl guanosine

nuclear magnetic resonance 


\begin{tabular}{|c|c|}
\hline NTC & NineTeen Complex \\
\hline nt & nucleotide \\
\hline NTD & N-terminal domain \\
\hline NTR & NineTeen Complex-Related \\
\hline PAGE & polyacrylamide gel electrophoresis \\
\hline PCA & principal component analysis \\
\hline PDB & Protein Data Bank \\
\hline Pol & polymerase \\
\hline pre-mRNA & precursor mRNA \\
\hline PSF & point spread function \\
\hline RES & Retention and Splicing \\
\hline RNA & ribonucleic acid \\
\hline RNP & ribonucleoprotein \\
\hline ROS & reactive oxygen species \\
\hline RRM & RNA recognition motif \\
\hline snRNA & small nuclear RNA \\
\hline snRNP & small nuclear ribonucleoprotein \\
\hline SNR & signal-to-noise ratio \\
\hline SR & serine-arginine rich \\
\hline ss & splice site \\
\hline XL-MS & cross-linking mass spectrometry \\
\hline $2 \mathrm{D}$ & two-dimensional \\
\hline $3 \mathrm{D}$ & three-dimensional \\
\hline
\end{tabular}




\section{B Supplementary information}

Table B.1: Intermolecular crosslinks. Crosslink analyses by pLink2.3.5 at FDR 1, 3 and 5\% are indicated. Number of CSMs (crosslinked peptide spectrum matches) and highest score are shown for each peptide. "Residue 1" and "Residue 2" denote crosslinked residues from Protein 1 and Protein 2. Crosslink analysis was performed by Dr. Olex Dybkov (Department of Cellular Biochemistry, MPI-BPC) in collaboration with Prof. Dr. Henning Urlaub (Bioanalytical Mass Spectrometry, MPI-BPC) (see 2.2 .6 .

\begin{tabular}{|c|c|c|c|c|c|c|c|c|c|c|c|}
\hline \multirow{2}{*}{ Name } & \multirow{2}{*}{$\begin{array}{l}\text { Protein } 1 \\
\text { UniProt } \\
\text { ID }\end{array}$} & \multicolumn{2}{|c|}{ Protein 2} & \multirow[b]{2}{*}{ Residue 1} & \multirow[b]{2}{*}{ Residue 2} & \multicolumn{3}{|c|}{ CSMs } & \multicolumn{3}{|c|}{ Score $_{\max }$} \\
\hline & & Name & $\begin{array}{l}\text { UniProt } \\
\text { ID }\end{array}$ & & & FDR $1 \%$ & FDR $3 \%$ & FDR $5 \%$ & F DR $1 \%$ & F DR3\% & F DR5\% \\
\hline \multirow[t]{2}{*}{ AQR } & O60306 & PRP19 & Q9UMS4 & 759 & 200 & & 4 & 9 & & 0.246 & 0.246 \\
\hline & & SYF1 & Q9HCS7 & 954 & 2 & 10 & 10 & 11 & 0.991 & 0.991 & 0.991 \\
\hline \multirow[t]{58}{*}{ BRR2 } & O75643 & CBP80 & Q09161 & 1169 & 65 & & 2 & 2 & & 0.609 & 0.609 \\
\hline & & CDC5L & Q99459 & 60 & 170 & 9 & 10 & 10 & 1.030 & 1.030 & 1.030 \\
\hline & & & & 73 & 170 & & 2 & 2 & & 0.353 & 0.353 \\
\hline & & & & 83 & 170 & & 3 & 3 & & 0.334 & 0.334 \\
\hline & & MFAP1 & P55081 & 1142 & 249 & 3 & 7 & 8 & 0.516 & 0.516 & 0.516 \\
\hline & & & & & 250 & 27 & 36 & 37 & 1.485 & 1.485 & 1.485 \\
\hline & & & & & 256 & 2 & 3 & 4 & 0.795 & 0.795 & 0.795 \\
\hline & & & & 1145 & 249 & 2 & 3 & 4 & 0.963 & 0.963 & 0.963 \\
\hline & & & & & 250 & 14 & 24 & 28 & 1.556 & 1.556 & 1.556 \\
\hline & & & & & 256 & & & 3 & & & 0.454 \\
\hline & & & & 1146 & 242 & 40 & 41 & 41 & 2.453 & 2.453 & 2.453 \\
\hline & & & & & 249 & 13 & 26 & 30 & 0.813 & 0.813 & 0.813 \\
\hline & & & & & 250 & 22 & 28 & 30 & 2.382 & 2.382 & 2.382 \\
\hline & & & & & 256 & 18 & 18 & 18 & 2.300 & 2.300 & 2.300 \\
\hline & & & & 1169 & 250 & & 2 & 3 & & 0.335 & 0.335 \\
\hline & & & & & 256 & & 2 & 3 & & 0.399 & 0.399 \\
\hline & & PQBP1 & O60828 & 60 & 87 & 2 & 3 & 3 & 1.266 & 1.266 & 1.266 \\
\hline & & & & 73 & 2 & & 3 & 3 & & 0.386 & 0.386 \\
\hline & & & & & 18 & 2 & 2 & 3 & 1.087 & 1.087 & 1.087 \\
\hline & & & & 85 & 2 & 7 & 7 & 7 & 2.323 & 2.323 & 2.323 \\
\hline & & & & & 18 & 2 & 2 & 2 & 2.851 & 2.851 & 2.851 \\
\hline & & & & 103 & 18 & 2 & 2 & 2 & 0.950 & 0.950 & 0.950 \\
\hline & & PRP8 & Q6P2Q9 & 14 & 1306 & & 2 & 3 & & 0.548 & 0.548 \\
\hline & & & & 73 & 1994 & 2 & 10 & 11 & 0.522 & 0.522 & 0.522 \\
\hline & & & & 1039 & 1636 & & 5 & 6 & & 0.535 & 0.535 \\
\hline & & & & & 1659 & 15 & 19 & 20 & 1.289 & 1.289 & 1.289 \\
\hline & & & & & 2049 & 3 & 3 & 3 & 1.131 & 1.131 & 1.131 \\
\hline & & & & & 2070 & 6 & 10 & 10 & 1.031 & 1.031 & 1.031 \\
\hline & & & & 1049 & 1866 & 13 & 13 & 13 & 3.092 & 3.092 & 3.092 \\
\hline & & & & & 1984 & 2 & 2 & 2 & 1.289 & 1.289 & 1.289 \\
\hline & & & & & 2070 & 2 & 3 & 3 & 1.655 & 1.655 & 1.655 \\
\hline & & & & 1141 & 1636 & & 2 & 2 & & 1.184 & 1.184 \\
\hline & & & & 1146 & 1636 & 9 & 9 & 9 & 1.635 & 1.635 & 1.635 \\
\hline & & & & & 1649 & 7 & 8 & 8 & 2.375 & 2.375 & 2.375 \\
\hline & & RED & Q13123 & 1874 & 520 & 6 & 6 & 6 & 1.016 & 1.016 & 1.016 \\
\hline & & SMU1 & Q2TAY7 & 971 & 308 & 14 & 17 & 18 & 1.051 & 1.051 & 1.051 \\
\hline & & & & 975 & 308 & 3 & 3 & 3 & 2.201 & 2.201 & 2.201 \\
\hline & & & & 1544 & 308 & & 2 & 2 & & 0.279 & 0.279 \\
\hline & & & & 1552 & 308 & 31 & 50 & 53 & 1.971 & 1.971 & 1.971 \\
\hline & & & & 1711 & 308 & & & 2 & & & 0.450 \\
\hline & & & & 1715 & 308 & 11 & 15 & 16 & 1.197 & 1.197 & 1.197 \\
\hline & & SNU23 & Q96NC0 & 85 & 70 & 2 & 2 & 2 & 2.348 & 2.348 & 2.348 \\
\hline & & & & 479 & 8 & 1 & 4 & 6 & 1.354 & 1.354 & 1.354 \\
\hline & & & & 557 & 8 & 12 & 21 & 24 & 1.121 & 1.121 & 1.121 \\
\hline & & & & 696 & 160 & 4 & 6 & 7 & 0.472 & 0.472 & 0.472 \\
\hline & & & & 729 & 147 & & & 2 & & & 0.568 \\
\hline & & & & 733 & 138 & 5 & 7 & 7 & 0.643 & 0.643 & 0.643 \\
\hline & & & & & 147 & 4 & 5 & 5 & 1.856 & 1.856 & 1.856 \\
\hline & & & & 804 & 8 & 4 & 4 & 4 & 0.759 & 0.759 & 0.759 \\
\hline & & & & & 15 & 6 & 8 & 8 & 1.189 & 1.189 & 1.189 \\
\hline & & & & & 18 & & & 2 & & & 0.344 \\
\hline & & TCERG1 & O14776 & 60 & 570 & 3 & 5 & 6 & 0.683 & 0.683 & 0.683 \\
\hline & & & & 440 & 634 & & 3 & 3 & & 1.936 & 1.936 \\
\hline & & WBP11 & Q9Y2W2 & 14 & 168 & 5 & 5 & 5 & 0.851 & 0.851 & 0.851 \\
\hline & & & & 60 & 168 & 2 & 4 & 4 & 0.754 & 0.754 & 0.754 \\
\hline & & & & & 169 & 4 & 5 & 6 & 1.501 & 1.501 & 1.501 \\
\hline & & & & 85 & 48 & & & 2 & & & 0.189 \\
\hline & & & & & 59 & 2 & 2 & 2 & 2.734 & 2.734 & 2.734 \\
\hline
\end{tabular}




\begin{tabular}{|c|c|c|c|c|c|c|c|c|c|c|c|}
\hline Name & $\begin{array}{l}\text { UniProt } \\
\text { ID }\end{array}$ & Name & $\begin{array}{l}\text { UniProt } \\
\text { ID }\end{array}$ & Residue 1 & Residue 2 & FDR $1 \%$ & FDR 3\% & FDR 5\% & FDR1\% & FDR3\% & FDR5\% \\
\hline & & & & & 168 & 2 & 2 & 2 & 14.531 & 14.531 & 14.531 \\
\hline \multirow[t]{7}{*}{ BUD13 } & Q9BRD0 & PRP8 & Q6P2Q9 & 65 & 1831 & 14 & 14 & 14 & 2.929 & 2.929 & 2.929 \\
\hline & & & & & 1840 & 20 & 21 & 21 & 2.421 & 2.421 & 2.421 \\
\hline & & & & 68 & 1831 & 40 & 43 & 45 & 3.605 & 3.605 & 3.605 \\
\hline & & & & & 1840 & 24 & 24 & 24 & 3.027 & 3.027 & 3.027 \\
\hline & & & & & 2108 & 3 & 3 & 3 & 2.055 & 2.055 & 2.055 \\
\hline & & & & 605 & 1505 & & 2 & 2 & & 1.180 & 1.180 \\
\hline & & SKIP & Q13573 & 605 & 441 & 22 & 23 & 23 & 3.246 & 3.246 & 3.246 \\
\hline BUD31 & P41223 & TCERG1 & O14776 & 68 & 711 & & 4 & 5 & & 0.521 & 0.521 \\
\hline CBP20 & P52298 & CBP80 & Q09161 & 7 & 17 & 8 & 11 & 14 & 0.796 & 0.796 & 0.796 \\
\hline & & & & & 37 & 3 & 6 & 6 & 0.608 & 0.608 & 0.608 \\
\hline & & & & & 67 & 6 & 9 & 14 & 1.536 & 1.536 & 1.536 \\
\hline & & & & & 327 & 7 & 13 & 14 & 0.719 & 0.719 & 0.719 \\
\hline & & & & 34 & 20 & 4 & 4 & 5 & 0.919 & 0.919 & 0.919 \\
\hline & & & & 38 & 657 & & 3 & 3 & & 0.751 & 0.751 \\
\hline & & & & 67 & 607 & 20 & 31 & 33 & 0.932 & 0.932 & 0.932 \\
\hline & & & & & 654 & 3 & 5 & 7 & 0.532 & 0.532 & 0.532 \\
\hline & & & & & 657 & & 2 & 3 & & 0.567 & 0.567 \\
\hline & & & & 68 & 607 & 124 & 127 & 127 & 3.640 & 3.640 & 3.640 \\
\hline & & & & 78 & 511 & 6 & 6 & 6 & 3.174 & 3.174 & 3.174 \\
\hline & & SRSF1 & Q07955 & 7 & 165 & 21 & 22 & 22 & 2.728 & 2.728 & 2.728 \\
\hline CBP80 & Q09161 & BRR2 & O75643 & 65 & 1169 & & 2 & 2 & & 0.609 & 0.609 \\
\hline & & CBP20 & P52298 & 17 & 7 & 8 & 11 & 14 & 0.796 & 0.796 & 0.796 \\
\hline & & & & 20 & 34 & 4 & 4 & 5 & 0.919 & 0.919 & 0.919 \\
\hline & & & & 37 & 7 & 3 & 6 & 6 & 0.608 & 0.608 & 0.608 \\
\hline & & & & 67 & 7 & 6 & 9 & 14 & 1.536 & 1.536 & 1.536 \\
\hline & & & & 327 & 7 & 7 & 13 & 14 & 0.719 & 0.719 & 0.719 \\
\hline & & & & 511 & 78 & 6 & 6 & 6 & 3.174 & 3.174 & 3.174 \\
\hline & & & & 607 & 67 & 20 & 31 & 33 & 0.932 & 0.932 & 0.932 \\
\hline & & & & & 68 & 124 & 127 & 127 & 3.640 & 3.640 & 3.640 \\
\hline & & & & 654 & 67 & 3 & 5 & 7 & 0.532 & 0.532 & 0.532 \\
\hline & & & & 657 & 38 & & 3 & 3 & & 0.751 & 0.751 \\
\hline & & & & & 67 & & 2 & 3 & & 0.567 & 0.567 \\
\hline & & SKIP & Q13573 & 607 & 503 & 4 & 4 & 4 & 2.187 & 2.187 & 2.187 \\
\hline & & & & & 509 & 2 & 3 & 3 & 1.072 & 1.072 & 1.072 \\
\hline & & SRSF1 & Q07955 & 17 & 165 & 4 & 4 & 4 & 1.131 & 1.131 & 1.131 \\
\hline & & & & 20 & 165 & 3 & 3 & 3 & 1.855 & 1.855 & 1.855 \\
\hline & & & & & 193 & 4 & 5 & 5 & 1.189 & 1.189 & 1.189 \\
\hline & & TCERG1 & O14776 & 187 & 756 & 1 & 1 & 1 & 0.510 & 0.510 & 0.510 \\
\hline & & $\mathrm{ZC} 3 \mathrm{H} 18$ & Q86VM9 & 221 & 319 & 19 & 21 & 22 & 1.585 & 1.585 & 1.585 \\
\hline CCDC12 & Q8WUD4 & MFAP1 & P55081 & 123 & 249 & 3 & 7 & 10 & 0.619 & 0.619 & 0.619 \\
\hline & & & & & 250 & 11 & 15 & 19 & 1.172 & 1.172 & 1.172 \\
\hline & & & & & 256 & 5 & 11 & 11 & 0.818 & 0.818 & 0.818 \\
\hline & & SYF1 & Q9HCS7 & 23 & 532 & 3 & 6 & 6 & 0.634 & 0.634 & 0.634 \\
\hline & & & & 42 & 532 & 5 & 5 & 5 & 1.178 & 1.178 & 1.178 \\
\hline & & & & 94 & 593 & 2 & 2 & 2 & 3.169 & 3.169 & 3.169 \\
\hline & & SYF3 & Q9BZJ0 & 117 & 607 & & 3 & 3 & & 0.700 & 0.700 \\
\hline & & & & 126 & 549 & & 3 & 4 & & 0.399 & 0.399 \\
\hline CDC5L & Q99459 & BRR2 & O75643 & 170 & 60 & 9 & 10 & 10 & 1.030 & 1.030 & 1.030 \\
\hline & & & & & 73 & & 2 & 2 & & 0.353 & 0.353 \\
\hline & & & & & 83 & & 3 & 3 & & 0.334 & 0.334 \\
\hline & & CTNNBL1 & Q8WYA6 & 28 & 56 & 8 & 9 & 9 & 1.192 & 1.192 & 1.192 \\
\hline & & & & 218 & 458 & 3 & 3 & 3 & 2.145 & 2.145 & 2.145 \\
\hline & & CWC15 & Q9P013 & 135 & 152 & & 2 & 2 & & 0.286 & 0.286 \\
\hline & & PLRG1 & O43660 & 432 & 113 & & 2 & 2 & & 0.336 & 0.336 \\
\hline & & & & 522 & 80 & 8 & 9 & 10 & 1.157 & 1.157 & 1.157 \\
\hline & & & & 598 & 31 & 5 & 6 & 7 & 2.178 & 2.178 & 2.178 \\
\hline & & & & & 41 & 3 & 3 & 3 & 1.493 & 1.493 & 1.493 \\
\hline & & & & & 68 & 2 & 3 & 3 & 1.340 & 1.340 & 1.340 \\
\hline & & & & 601 & 31 & 15 & 15 & 15 & 10.025 & 10.025 & 10.025 \\
\hline & & & & & 41 & 2 & 2 & 2 & 0.749 & 0.749 & 0.749 \\
\hline & & & & & 62 & 3 & 3 & 3 & 1.546 & 1.546 & 1.546 \\
\hline & & & & & 68 & 15 & 16 & 16 & 3.260 & 3.260 & 3.260 \\
\hline & & & & & 80 & 3 & 3 & 3 & 16.912 & 16.912 & 16.912 \\
\hline & & & & 708 & 80 & & 2 & 3 & & 0.383 & 0.383 \\
\hline & & & & 733 & 135 & & 2 & 2 & & 1.173 & 1.173 \\
\hline & & PPIL2 & Q13356 & 466 & 507 & 3 & 4 & 4 & 0.394 & 0.394 & 0.394 \\
\hline & & & & & 508 & & 4 & 4 & & 0.310 & 0.310 \\
\hline & & 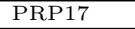 & O60508 & 200 & 333 & 6 & 12 & 12 & 0.566 & 0.566 & 0.566 \\
\hline & & $\begin{array}{l}\text { PRP19 } \\
\end{array}$ & Q9UMS4 & 380 & 244 & 2 & 2 & 2 & 0.753 & 0.753 & 0.753 \\
\hline & & & & 432 & 179 & & 2 & 2 & & 0.693 & 0.693 \\
\hline & & & & & 244 & & 2 & 2 & & 0.634 & 0.634 \\
\hline & & & & 487 & 192 & 7 & 7 & 8 & 2.404 & 2.404 & 2.404 \\
\hline & & & & 522 & 192 & 6 & 6 & 6 & 1.479 & 1.479 & 1.479 \\
\hline & & & & & 244 & 4 & 5 & 5 & 0.912 & 0.912 & 0.912 \\
\hline & & & & 570 & 122 & 9 & 23 & 27 & 0.675 & 0.675 & 0.675 \\
\hline & & & & 631 & 122 & 3 & 3 & 3 & 1.567 & 1.567 & 1.567 \\
\hline & & & & 685 & 179 & 2 & 4 & 4 & 0.741 & 0.741 & 0.741 \\
\hline & & & & & 192 & & & 3 & & & 0.257 \\
\hline & & & & & 244 & & 9 & 9 & & 0.381 & 0.381 \\
\hline & & & & & 266 & 2 & 4 & 4 & 0.412 & 0.412 & 0.412 \\
\hline & & & & 718 & 76 & 72 & 76 & 77 & 6.685 & 6.685 & 6.685 \\
\hline & & & & 771 & 244 & & 3 & 3 & & 0.508 & 0.508 \\
\hline
\end{tabular}


Table B.1 - Continued from previous page

\begin{tabular}{|c|c|c|c|c|c|c|c|c|c|c|c|}
\hline Name & $\begin{array}{l}\text { UniProt } \\
\text { ID }\end{array}$ & Name & $\begin{array}{l}\text { UniProt } \\
\text { ID }\end{array}$ & Residue 1 & Residue 2 & FDR $1 \%$ & FDR $3 \%$ & FDR 5\% & F DR1\% & FDR3\% & FDR5\% \\
\hline & & & & 782 & 179 & & 2 & 2 & & 0.306 & 0.306 \\
\hline & & & & & 192 & 5 & 6 & 6 & 1.379 & 1.379 & 1.379 \\
\hline & & & & & 244 & 28 & 33 & 34 & 1.343 & 1.343 & 1.343 \\
\hline & & & & & 261 & 3 & 3 & 3 & 1.265 & 1.265 & 1.265 \\
\hline & & & & & 266 & 10 & 11 & 14 & 0.925 & 0.925 & 0.925 \\
\hline & & & & 795 & 244 & 7 & 8 & 8 & 1.168 & 1.168 & 1.168 \\
\hline & & PRP8 & Q6P2Q9 & 7 & 892 & 37 & 51 & 52 & 1.770 & 1.770 & 1.770 \\
\hline & & & & 28 & 727 & 29 & 36 & 39 & 1.463 & 1.463 & 1.463 \\
\hline & & SF3A2 & Q15428 & 28 & 10 & 5 & 6 & 6 & 1.289 & 1.289 & 1.289 \\
\hline & & & & & 42 & 9 & 13 & 13 & 1.355 & 1.355 & 1.355 \\
\hline & & SF3B1 & O75533 & 76 & 454 & 2 & 2 & 2 & 0.887 & 0.887 & 0.887 \\
\hline & & & & 106 & 454 & 5 & 5 & 5 & 3.348 & 3.348 & 3.348 \\
\hline & & SF3B6 & Q9Y3B4 & 124 & 29 & 26 & 26 & 26 & 9.871 & 9.871 & 9.871 \\
\hline & & SKIP & Q13573 & 28 & 255 & 6 & 9 & 9 & 1.319 & 1.319 & 1.319 \\
\hline & & & & & 258 & 16 & 25 & 25 & 1.281 & 1.281 & 1.281 \\
\hline & & & & 47 & 258 & & 3 & 6 & & 0.327 & 0.327 \\
\hline & & & & 124 & 311 & 8 & 16 & 19 & 0.641 & 0.641 & 0.641 \\
\hline & & SPF27 & O75934 & 380 & 168 & 2 & 2 & 3 & 1.331 & 1.331 & 1.331 \\
\hline & & & & 685 & 97 & 6 & 17 & 22 & 0.689 & 0.689 & 0.689 \\
\hline & & & & 686 & 97 & 15 & 17 & 17 & 2.361 & 2.361 & 2.361 \\
\hline & & & & 771 & 177 & 74 & 126 & 151 & 1.449 & 1.449 & 1.449 \\
\hline & & & & 782 & 191 & 8 & 11 & 12 & 1.148 & 1.148 & 1.148 \\
\hline & & SYF1 & Q9HCS7 & 294 & 654 & 19 & 19 & 20 & 3.245 & 3.245 & 3.245 \\
\hline & & & & 522 & 532 & 8 & 8 & 8 & 1.502 & 1.502 & 1.502 \\
\hline & & SYF3 & Q9BZJ0 & 466 & 568 & & & 2 & & & 0.277 \\
\hline & & U5-40K & Q96DI7 & 795 & 270 & 2 & 3 & 5 & 0.515 & 0.515 & 0.515 \\
\hline CTNNBL1 & Q8WYA6 & CDC5L & Q99459 & 56 & 28 & 8 & 9 & 9 & 1.192 & 1.192 & 1.192 \\
\hline & & & & 458 & 218 & 3 & 3 & 3 & 2.145 & 2.145 & 2.145 \\
\hline & & CWC15 & Q9P013 & 297 & 91 & 3 & 4 & 8 & 0.539 & 0.539 & 0.539 \\
\hline & & & & 372 & 91 & 2 & 3 & 3 & 0.823 & 0.823 & 0.823 \\
\hline & & & & 527 & 18 & & & 2 & & & 0.250 \\
\hline & & PPIL2 & Q13356 & 56 & 90 & 3 & 3 & 4 & 0.545 & 0.545 & 0.545 \\
\hline & & PRP19 & Q9UMS4 & 534 & 192 & 4 & 4 & 5 & 2.205 & 2.205 & 2.205 \\
\hline & & PRP8 & Q6P2Q9 & 27 & 796 & 16 & 18 & 18 & 3.179 & 3.179 & 3.179 \\
\hline & & & & 31 & 796 & 13 & 13 & 13 & 2.264 & 2.264 & 2.264 \\
\hline & & SF3B1 & O75533 & 56 & 6 & 2 & 2 & 2 & 1.561 & 1.561 & 1.561 \\
\hline & & & & 83 & 6 & 6 & 7 & 7 & 2.140 & 2.140 & 2.140 \\
\hline & & & & 84 & 3 & & & 2 & & & 0.302 \\
\hline & & & & & 6 & 12 & 13 & 13 & 1.691 & 1.691 & 1.691 \\
\hline & & & & 91 & 3 & & 2 & 2 & & 0.376 & 0.376 \\
\hline & & & & & 6 & 6 & 14 & 20 & 2.710 & 2.710 & 2.710 \\
\hline & & & & 95 & 6 & & 3 & 3 & & 0.823 & 0.823 \\
\hline CWC15 & Q9P013 & CDC5L & Q99459 & 152 & 135 & & 2 & 2 & & 0.286 & 0.286 \\
\hline & & CTNNBL1 & Q8WYA6 & 18 & 527 & & & 2 & & & 0.250 \\
\hline & & & & 91 & 297 & 3 & 4 & 8 & 0.539 & 0.539 & 0.539 \\
\hline & & & & & 372 & 2 & 3 & 3 & 0.823 & 0.823 & 0.823 \\
\hline & & PLRG1 & O43660 & 18 & 363 & 15 & 16 & 16 & 1.804 & 1.804 & 1.804 \\
\hline & & & & 28 & 320 & 3 & 3 & 3 & 1.764 & 1.764 & 1.764 \\
\hline & & PRP8 & Q6P2Q9 & 91 & 1144 & & 2 & 2 & & 0.317 & 0.317 \\
\hline & & & & 199 & 1158 & 2 & 3 & 4 & 0.543 & 0.543 & 0.543 \\
\hline & & & & 205 & 987 & & 3 & 4 & & 0.379 & 0.379 \\
\hline & & RBM22 & Q9NW64 & 18 & 76 & 5 & 7 & 7 & 1.223 & 1.223 & 1.223 \\
\hline & & & & 28 & 40 & 3 & 4 & 4 & 0.842 & 0.842 & 0.842 \\
\hline & & SKIP & Q13573 & 18 & 153 & 16 & 16 & 16 & 2.622 & 2.622 & 2.622 \\
\hline & & & & & 217 & 1 & 5 & 5 & 0.535 & 0.535 & 0.535 \\
\hline & & & & 28 & 158 & & 2 & 2 & & 0.932 & 0.932 \\
\hline & & & & & 204 & 2 & 3 & 4 & 0.621 & 0.621 & 0.621 \\
\hline & & & & & 217 & 3 & 4 & 5 & 0.573 & 0.573 & 0.573 \\
\hline & & & & 183 & 311 & 2 & 2 & 3 & 0.816 & 0.816 & 0.816 \\
\hline & & ZNF830 & Q96NB3 & 147 & 26 & & 2 & 2 & & 0.405 & 0.405 \\
\hline HNRNPA1 & P09651 & PHF5A & Q7RTV0 & 15 & 104 & & 2 & 2 & & 0.288 & 0.288 \\
\hline ISY1 & Q9ULR0 & SF3A1 & Q15459 & 105 & 30 & 2 & 4 & 4 & 0.502 & 0.502 & 0.502 \\
\hline & & & & & 37 & 5 & 6 & 6 & 0.664 & 0.664 & 0.664 \\
\hline & & SF3B2 & Q13435 & 101 & 148 & & 2 & 2 & & 0.248 & 0.248 \\
\hline & & SYF1 & Q9HCS7 & 190 & 2 & 4 & 6 & 6 & 2.763 & 2.763 & 2.763 \\
\hline & & & & 260 & 2 & & & 2 & & & 0.660 \\
\hline & & U2-A' & P09661 & 266 & 193 & 17 & 17 & 17 & 2.627 & 2.627 & 2.627 \\
\hline & & & & & 221 & 6 & 6 & 6 & 2.095 & 2.095 & 2.095 \\
\hline KIN17 & O60870 & MFAP1 & P55081 & 231 & 67 & 3 & 3 & 3 & 0.719 & 0.719 & 0.719 \\
\hline & & PRP8 & Q6P2Q9 & 87 & 2034 & & 2 & 3 & & 0.280 & 0.280 \\
\hline & & SF3B1 & O75533 & 271 & 729 & & 2 & 2 & & 0.434 & 0.434 \\
\hline & & & & & 733 & 3 & 6 & 6 & 7.933 & 7.933 & 7.933 \\
\hline & & SF3B3 & Q15393 & 231 & 26 & 13 & 15 & 15 & 1.957 & 1.957 & 1.957 \\
\hline & & & & & 296 & 2 & 2 & 2 & 1.531 & 1.531 & 1.531 \\
\hline & & SNU23 & Q96NC0 & 120 & 70 & 3 & 3 & 3 & 2.324 & 2.324 & 2.324 \\
\hline LSm2 & Q9Y333 & $\begin{array}{l}\text { PLRG1 } \\
\end{array}$ & O43660 & 88 & 510 & 4 & 5 & 5 & 0.915 & 0.915 & 0.915 \\
\hline & & SKIP & Q13573 & 8 & 236 & 2 & 3 & 4 & 0.430 & 0.430 & 0.430 \\
\hline LSm4 & Q9Y4Z0 & LSm8 & O95777 & 1 & 28 & 14 & 14 & 14 & 2.730 & 2.730 & 2.730 \\
\hline & & & & 80 & 28 & 7 & 7 & 7 & 3.106 & 3.106 & 3.106 \\
\hline LSm6 & P62312 & PLRG1 & O43660 & 2 & 7 & 1 & 1 & 1 & 0.472 & 0.472 & 0.472 \\
\hline & & RED & Q13123 & 5 & 30 & 6 & 7 & 8 & 1.153 & 1.153 & 1.153 \\
\hline & & & & 77 & 30 & & & 2 & & & 0.456 \\
\hline & & SF3A1 & Q15459 & 5 & 131 & 4 & 4 & 4 & 1.310 & 1.310 & 1.310 \\
\hline
\end{tabular}




\begin{tabular}{|c|c|c|c|c|c|c|c|c|c|c|c|}
\hline Name & $\begin{array}{l}\text { UniProt } \\
\text { ID }\end{array}$ & Name & $\begin{array}{l}\text { UniProt } \\
\text { ID }\end{array}$ & Residue 1 & Residue 2 & FDR 1\% & FDR $3 \%$ & FDR $5 \%$ & F DR $1 \%$ & FDR3\% & F DR5\% \\
\hline \multirow[t]{2}{*}{ LSm8 } & O95777 & LSm4 & Q9Y4Z0 & 28 & 1 & 14 & 14 & 14 & 2.730 & 2.730 & 2.730 \\
\hline & & & & & 80 & 7 & 7 & 7 & 3.106 & 3.106 & 3.106 \\
\hline \multirow[t]{9}{*}{ MBPMS2 } & $\begin{array}{l}\text { P0AEX9- } \\
\text { r }\end{array}$ & PRP8 & Q6P2Q9 & 47 & 452 & & & 2 & & & 0.209 \\
\hline & & & & 438 & 2293 & 5 & 17 & 19 & 0.472 & 0.472 & 0.472 \\
\hline & & SF3B1 & O75533 & 274 & 656 & 2 & 3 & 3 & 1.690 & 1.690 & 1.690 \\
\hline & & SF3B2 & Q13435 & 47 & 448 & 2 & 3 & 3 & 0.676 & 0.676 & 0.676 \\
\hline & & SmE & P62304 & 278 & 1 & & & 2 & & & 0.661 \\
\hline & & SRRT & Q9BXP5 & 103 & 286 & 7 & 8 & 10 & 1.052 & 1.052 & 1.052 \\
\hline & & & & 180 & 286 & 25 & 31 & 40 & 1.136 & 1.136 & 1.136 \\
\hline & & & & 363 & 286 & & 4 & 6 & & 0.357 & 0.357 \\
\hline & & U2-A' & $\begin{array}{l}\text { P09661 } \\
\end{array}$ & 103 & 192 & & & 3 & & & 0.191 \\
\hline MFAP1 & P55081 & $\begin{array}{c}\text { BRR2 } \\
\end{array}$ & O75643 & 242 & 1146 & 40 & 41 & 41 & 2.453 & 2.453 & 2.453 \\
\hline & & & & 249 & 1142 & 3 & 7 & 8 & 0.516 & 0.516 & 0.516 \\
\hline & & & & & 1145 & 2 & 3 & 4 & 0.963 & 0.963 & 0.963 \\
\hline & & & & & 1146 & 13 & 26 & 30 & 0.813 & 0.813 & 0.813 \\
\hline & & & & 250 & 1142 & 27 & 36 & 37 & 1.485 & 1.485 & 1.485 \\
\hline & & & & & 1145 & 14 & 24 & 28 & 1.556 & 1.556 & 1.556 \\
\hline & & & & & 1146 & 22 & 28 & 30 & 2.382 & 2.382 & 2.382 \\
\hline & & & & & 1169 & & 2 & 3 & & 0.335 & 0.335 \\
\hline & & & & 256 & 1142 & 2 & 3 & 4 & 0.795 & 0.795 & 0.795 \\
\hline & & & & & 1145 & & & 3 & & & 0.454 \\
\hline & & & & & 1146 & 18 & 18 & 18 & 2.300 & 2.300 & 2.300 \\
\hline & & & & & 1169 & & 2 & 3 & & 0.399 & 0.399 \\
\hline & & CCDC12 & Q8WUD4 & 249 & 123 & 3 & 7 & 10 & 0.619 & 0.619 & 0.619 \\
\hline & & & & 250 & 123 & 11 & 15 & 19 & 1.172 & 1.172 & 1.172 \\
\hline & & & & 256 & 123 & 5 & 11 & 11 & 0.818 & 0.818 & 0.818 \\
\hline & & KIN17 & O60870 & 67 & 231 & 3 & 3 & 3 & 0.719 & 0.719 & 0.719 \\
\hline & & PRP8 & Q6P2Q9 & 238 & 2293 & 9 & 14 & 16 & 0.848 & 0.848 & 0.848 \\
\hline & & & & 242 & 1649 & 25 & 30 & 31 & 1.453 & 1.453 & 1.453 \\
\hline & & & & & 2293 & 46 & 53 & 54 & 2.052 & 2.052 & 2.052 \\
\hline & & & & 249 & 1649 & 41 & 43 & 46 & 2.123 & 2.123 & 2.123 \\
\hline & & & & 250 & 1649 & 17 & 17 & 17 & 2.174 & 2.174 & 2.174 \\
\hline & & & & 256 & 1636 & 2 & 2 & 2 & 0.687 & 0.687 & 0.687 \\
\hline & & & & & 1649 & & & 2 & & & 0.207 \\
\hline & & SF3B1 & O75533 & 9 & 807 & 15 & 15 & 15 & 1.516 & 1.516 & 1.516 \\
\hline & & SF3B3 & Q15393 & 67 & 26 & & 2 & 2 & & 0.248 & 0.248 \\
\hline & & SMU1 & Q2TAY7 & 67 & 308 & 17 & 21 & 21 & 1.104 & 1.104 & 1.104 \\
\hline & & SRSF1 & Q07955 & 329 & 179 & 13 & 18 & 19 & 1.295 & 1.295 & 1.295 \\
\hline & & SYF1 & Q9HCS7 & 285 & 539 & 4 & 4 & 4 & 0.809 & 0.809 & 0.809 \\
\hline & & TCERG1 & O14776 & 415 & 878 & 4 & 9 & 10 & 1.176 & 1.176 & 1.176 \\
\hline PHF5A & Q7RTV0 & HNRNPA1 & P09651 & 104 & 15 & & 2 & 2 & & 0.288 & 0.288 \\
\hline & & SF3B1 & O75533 & 108 & 946 & & 2 & 2 & & 0.289 & 0.289 \\
\hline & & SF3B3 & Q15393 & 13 & 137 & 7 & 9 & 9 & 1.464 & 1.464 & 1.464 \\
\hline & & SF3B5 & Q9BWJ5 & 13 & 17 & 6 & 7 & 7 & 1.145 & 1.145 & 1.145 \\
\hline PLRG1 & O43660 & CDC5L & Q99459 & 31 & 598 & 5 & 6 & 7 & 2.178 & 2.178 & 2.178 \\
\hline & & & & & 601 & 15 & 15 & 15 & 10.025 & 10.025 & 10.025 \\
\hline & & & & 41 & 598 & 3 & 3 & 3 & 1.493 & 1.493 & 1.493 \\
\hline & & & & & 601 & 2 & 2 & 2 & 0.749 & 0.749 & 0.749 \\
\hline & & & & 62 & 601 & 3 & 3 & 3 & 1.546 & 1.546 & 1.546 \\
\hline & & & & 68 & 598 & 2 & 3 & 3 & 1.340 & 1.340 & 1.340 \\
\hline & & & & & 601 & 15 & 16 & 16 & 3.260 & 3.260 & 3.260 \\
\hline & & & & 80 & 522 & 8 & 9 & 10 & 1.157 & 1.157 & 1.157 \\
\hline & & & & & 601 & 3 & 3 & 3 & 16.912 & 16.912 & 16.912 \\
\hline & & & & & 708 & & 2 & 3 & & 0.383 & 0.383 \\
\hline & & & & 113 & 432 & & 2 & 2 & & 0.336 & 0.336 \\
\hline & & & & 135 & 733 & & 2 & 2 & & 1.173 & 1.173 \\
\hline & & CWC15 & Q9P013 & 320 & 28 & 3 & 3 & 3 & 1.764 & 1.764 & 1.764 \\
\hline & & & & 363 & 18 & 15 & 16 & 16 & 1.804 & 1.804 & 1.804 \\
\hline & & LSm2 & Q9Y333 & 510 & 88 & 4 & 5 & 5 & 0.915 & 0.915 & 0.915 \\
\hline & & LSm6 & P62312 & 7 & 2 & 1 & 1 & 1 & 0.472 & 0.472 & 0.472 \\
\hline & & PPIL1 & Q9Y3C6 & 62 & 80 & 2 & 3 & 3 & 0.573 & 0.573 & 0.573 \\
\hline & & & & 68 & 80 & 8 & 8 & 9 & 2.608 & 2.608 & 2.608 \\
\hline & & PRP19 & Q9UMS4 & 1 & 122 & 14 & 16 & 17 & 1.899 & 1.899 & 1.899 \\
\hline & & & & & 244 & 4 & 5 & 5 & 1.546 & 1.546 & 1.546 \\
\hline & & & & 7 & 244 & 2 & 3 & 3 & 1.343 & 1.343 & 1.343 \\
\hline & & $\begin{array}{l}\text { PRP8 } \\
\end{array}$ & Q6P2Q9 & 268 & 670 & 3 & 9 & 11 & 0.584 & 0.584 & 0.584 \\
\hline & & & & & 674 & & & 2 & & & 0.442 \\
\hline & & & & 320 & 666 & 4 & 8 & 8 & 0.978 & 0.978 & 0.978 \\
\hline & & & & & 670 & 24 & 27 & 28 & 1.358 & 1.358 & 1.358 \\
\hline & & RBM22 & Q9NW64 & 363 & 40 & 3 & 3 & 3 & 0.754 & 0.754 & 0.754 \\
\hline & & RED & Q13123 & 320 & 553 & 5 & 5 & 6 & 1.345 & 1.345 & 1.345 \\
\hline & & SKIP & Q13573 & 113 & 81 & 2 & 2 & 2 & 1.745 & 1.745 & 1.745 \\
\hline & & & & & 97 & & 2 & 2 & & 0.584 & 0.584 \\
\hline & & & & 135 & 97 & 2 & 2 & 3 & 0.416 & 0.416 & 0.416 \\
\hline & & & & 173 & 81 & & 3 & 3 & & 0.710 & 0.710 \\
\hline & & & & & 97 & 2 & 4 & 4 & 0.779 & 0.779 & 0.779 \\
\hline & & & & & 110 & & 3 & 4 & & 0.397 & 0.397 \\
\hline & & & & 180 & 97 & 5 & 5 & 5 & 1.618 & 1.618 & 1.618 \\
\hline & & & & & 110 & 3 & 3 & 3 & 0.500 & 0.500 & 0.500 \\
\hline & & & & & 115 & & 3 & 3 & & 0.423 & 0.423 \\
\hline & & & & & 122 & 10 & 11 & 12 & 0.832 & 0.832 & 0.832 \\
\hline & & & & 320 & 158 & 35 & 38 & 40 & 2.214 & 2.214 & 2.214 \\
\hline
\end{tabular}


Table B.1 - Continued from previous page

\begin{tabular}{|c|c|c|c|c|c|c|c|c|c|c|c|}
\hline Name & $\begin{array}{l}\text { UniProt } \\
\text { ID }\end{array}$ & Name & $\begin{array}{l}\text { UniProt } \\
\text { ID }\end{array}$ & Residue 1 & Residue 2 & FDR $1 \%$ & FDR $3 \%$ & FDR 5\% & FDR $1 \%$ & FDR3\% & FDR5\% \\
\hline & & & & & 170 & & 2 & 2 & & 0.323 & 0.323 \\
\hline & & & & 363 & 153 & 3 & 4 & 5 & 1.083 & 1.083 & 1.083 \\
\hline & & & & & 158 & 14 & 16 & 19 & 1.286 & 1.286 & 1.286 \\
\hline & & & & 396 & 122 & 4 & 6 & 6 & 0.731 & 0.731 & 0.731 \\
\hline & & & & 510 & 110 & 2 & 6 & 7 & 0.694 & 0.694 & 0.694 \\
\hline & & & & & 236 & 2 & 2 & 6 & 0.421 & 0.421 & 0.421 \\
\hline & & & & & 240 & 5 & 6 & 6 & 0.974 & 0.974 & 0.974 \\
\hline & & SNU114 & Q15029 & 237 & 602 & 2 & 3 & 4 & 0.524 & 0.524 & 0.524 \\
\hline & & SPF27 & O75934 & 62 & 136 & 12 & 12 & 13 & 1.939 & 1.939 & 1.939 \\
\hline & & & & 68 & 136 & 11 & 12 & 12 & 3.413 & 3.413 & 3.413 \\
\hline & & & & 80 & 168 & 2 & 2 & 2 & 2.075 & 2.075 & 2.075 \\
\hline & & & & 113 & 168 & 8 & 11 & 12 & 1.103 & 1.103 & 1.103 \\
\hline & & & & 135 & 136 & 2 & 2 & 2 & 1.855 & 1.855 & 1.855 \\
\hline & & & & & 168 & 8 & 9 & 9 & 1.343 & 1.343 & 1.343 \\
\hline & & WBP11 & Q9Y2W2 & 268 & 10 & & 4 & 5 & & 0.649 & 0.649 \\
\hline PPIE & Q9UNP9 & SF3A1 & Q15459 & 104 & 210 & & & 2 & & & 0.446 \\
\hline \multirow[t]{19}{*}{ PPIL1 } & Q9Y3C6 & PLRG1 & O43660 & 80 & 62 & 2 & 3 & 3 & 0.573 & 0.573 & 0.573 \\
\hline & & & & & 68 & 8 & 8 & 9 & 2.608 & 2.608 & 2.608 \\
\hline & & SKIP & Q13573 & 58 & 81 & 27 & 27 & 27 & 2.753 & 2.753 & 2.753 \\
\hline & & & & & 97 & 2 & 4 & 4 & 1.204 & 1.204 & 1.204 \\
\hline & & & & 80 & 193 & 5 & 5 & 5 & 1.332 & 1.332 & 1.332 \\
\hline & & & & 91 & 48 & & 3 & 3 & & 0.308 & 0.308 \\
\hline & & & & 158 & 97 & 4 & 7 & 7 & 1.290 & 1.290 & 1.290 \\
\hline & & & & & 193 & & & 2 & & & 0.370 \\
\hline & & SPF27 & O75934 & 80 & 136 & 8 & 8 & 8 & 2.484 & 2.484 & 2.484 \\
\hline & & & & & 168 & 9 & 10 & 10 & 2.210 & 2.210 & 2.210 \\
\hline & & & & 158 & 168 & 2 & 2 & 3 & 0.477 & 0.477 & 0.477 \\
\hline & & U5-40K & Q96DI7 & 80 & 1 & 26 & 33 & 35 & 1.630 & 1.630 & 1.630 \\
\hline & & & & & 6 & & 6 & 7 & & 0.430 & 0.430 \\
\hline & & & & & 8 & 8 & 8 & 8 & 3.983 & 3.983 & 3.983 \\
\hline & & & & & 18 & 13 & 13 & 13 & 1.974 & 1.974 & 1.974 \\
\hline & & & & & 270 & & 2 & 2 & & 0.245 & 0.245 \\
\hline & & & & & 275 & 17 & 18 & 18 & 2.524 & 2.524 & 2.524 \\
\hline & & & & & 322 & 4 & 4 & 4 & 2.753 & 2.753 & 2.753 \\
\hline & & & & & 349 & 3 & 3 & 3 & 1.773 & 1.773 & 1.773 \\
\hline PPIL2 & Q13356 & CDC5L & Q99459 & 507 & 466 & 3 & 4 & 4 & 0.394 & 0.394 & 0.394 \\
\hline & & & & 508 & 466 & & 4 & 4 & & 0.310 & 0.310 \\
\hline & & CTNNBL1 & Q8WYA6 & 90 & 56 & 3 & 3 & 4 & 0.545 & 0.545 & 0.545 \\
\hline & & PRP8 & Q6P2Q9 & 226 & 727 & 9 & 9 & 9 & 1.925 & 1.925 & 1.925 \\
\hline & & & & 230 & 727 & 6 & 10 & 10 & 0.649 & 0.649 & 0.649 \\
\hline & & & & 231 & 727 & 9 & 10 & 10 & 1.226 & 1.226 & 1.226 \\
\hline & & & & 245 & 727 & 5 & 5 & 5 & 8.264 & 8.264 & 8.264 \\
\hline & & SF3A2 & Q15428 & 418 & 91 & 2 & 2 & 2 & 0.699 & 0.699 & 0.699 \\
\hline & & SF3B1 & O75533 & 90 & 6 & 3 & 3 & 3 & 1.154 & 1.154 & 1.154 \\
\hline & & SKIP & Q13573 & 277 & 240 & & 2 & 2 & & 0.292 & 0.292 \\
\hline & & SYF3 & Q9BZJ0 & 460 & 445 & & & 2 & & & 0.354 \\
\hline & & & & 462 & 445 & 8 & 8 & 12 & 0.858 & 0.858 & 0.858 \\
\hline & & & & & 485 & 2 & 3 & 3 & 0.483 & 0.483 & 0.483 \\
\hline & & & & 490 & 569 & & 2 & 3 & & 0.531 & 0.531 \\
\hline & & & & & 602 & 1 & 2 & 2 & 0.510 & 0.510 & 0.510 \\
\hline & & ZNF830 & Q96NB3 & 313 & 228 & 8 & 8 & 8 & 1.629 & 1.629 & 1.629 \\
\hline & & & & & 234 & 29 & 30 & 32 & 2.622 & 2.622 & 2.622 \\
\hline & & & & 450 & 228 & 5 & 7 & 9 & 1.095 & 1.095 & 1.095 \\
\hline & & & & & 234 & 5 & 5 & 5 & 2.125 & 2.125 & 2.125 \\
\hline & & & & 454 & 228 & 2 & 3 & 3 & 0.679 & 0.679 & 0.679 \\
\hline & & & & 460 & 234 & & 3 & 3 & & 0.544 & 0.544 \\
\hline & & & & 462 & 228 & 4 & 4 & 4 & 1.222 & 1.222 & 1.222 \\
\hline & & & & & 234 & & & 1 & & & 0.637 \\
\hline PPP1CA & P62136 & PPP1R8 & Q12972 & 26 & 78 & 2 & 3 & 4 & 0.545 & 0.545 & 0.545 \\
\hline & & & & & 129 & 3 & 3 & 4 & 1.094 & 1.094 & 1.094 \\
\hline & & & & & 234 & 3 & 4 & 4 & 0.882 & 0.882 & 0.882 \\
\hline & & & & 305 & 234 & 6 & 7 & 7 & 0.796 & 0.796 & 0.796 \\
\hline PPP1R8 & Q12972 & PPP1CA & P62136 & 78 & 26 & 2 & 3 & 4 & 0.545 & 0.545 & 0.545 \\
\hline & & & & 129 & 26 & 3 & 3 & 4 & 1.094 & 1.094 & 1.094 \\
\hline & & & & 234 & 26 & 3 & 4 & 4 & 0.882 & 0.882 & 0.882 \\
\hline & & & & & 305 & 6 & 7 & 7 & 0.796 & 0.796 & 0.796 \\
\hline & & SF3A1 & Q15459 & 234 & 115 & 3 & 3 & 3 & 0.758 & 0.758 & 0.758 \\
\hline & & SNU23 & Q96NC0 & 334 & 155 & 6 & 9 & 9 & 1.503 & 1.503 & 1.503 \\
\hline & & & & & 167 & 3 & 3 & 3 & 1.039 & 1.039 & 1.039 \\
\hline & & WBP11 & Q9Y2W2 & 234 & 610 & & 1 & 1 & & 0.291 & 0.291 \\
\hline PQBP1 & O60828 & BRR2 & O75643 & 2 & 73 & & 3 & 3 & & 0.386 & 0.386 \\
\hline & & & & & 85 & 7 & 7 & 7 & 2.323 & 2.323 & 2.323 \\
\hline & & & & 18 & 73 & 2 & 2 & 3 & 1.087 & 1.087 & 1.087 \\
\hline & & & & & 85 & 2 & 2 & 2 & 2.851 & 2.851 & 2.851 \\
\hline & & & & & 103 & 2 & 2 & 2 & 0.950 & 0.950 & 0.950 \\
\hline & & & & 87 & 60 & 2 & 3 & 3 & 1.266 & 1.266 & 1.266 \\
\hline & & PRP8 & Q6P2Q9 & 2 & 1831 & 36 & 45 & 49 & 2.057 & 2.057 & 2.057 \\
\hline & & WBP11 & Q9Y2W2 & 2 & 168 & 2 & 2 & 2 & 1.542 & 1.542 & 1.542 \\
\hline & & & & 18 & 168 & 4 & 4 & 4 & 9.348 & 9.348 & 9.348 \\
\hline & & & & & 169 & 2 & 2 & 2 & 1.262 & 1.262 & 1.262 \\
\hline & & & & 87 & 168 & & 3 & 3 & & 0.836 & 0.836 \\
\hline & & & & & 169 & 8 & 9 & 9 & 1.887 & 1.887 & 1.887 \\
\hline PRP17 & O60508 & CDC5L & Q99459 & 333 & 200 & 6 & 12 & 12 & 0.566 & 0.566 & 0.566 \\
\hline
\end{tabular}




\begin{tabular}{|c|c|c|c|c|c|c|c|c|c|c|c|}
\hline Name & $\begin{array}{l}\text { UniProt } \\
\text { ID }\end{array}$ & Name & $\begin{array}{l}\text { UniProt } \\
\text { ID }\end{array}$ & Residue 1 & Residue 2 & FDR $1 \%$ & FDR 3\% & FDR 5\% & FDR $1 \%$ & FDR3\% & FDR5\% \\
\hline & & PRP8 & Q6P2Q9 & 151 & 29 & 2 & 2 & 2 & 0.550 & 0.550 & 0.550 \\
\hline & & & & 551 & 2034 & 5 & 5 & 5 & 1.881 & 1.881 & 1.881 \\
\hline \multirow[t]{54}{*}{ PRP19 } & Q9UMS4 & AQR & O60306 & 200 & 759 & & 4 & 9 & & 0.246 & 0.246 \\
\hline & & CDC5L & Q99459 & 76 & 718 & 72 & 76 & 77 & 6.685 & 6.685 & 6.685 \\
\hline & & & & 122 & 570 & 9 & 23 & 27 & 0.675 & 0.675 & 0.675 \\
\hline & & & & & 631 & 3 & 3 & 3 & 1.567 & 1.567 & 1.567 \\
\hline & & & & 179 & 432 & & 2 & 2 & & 0.693 & 0.693 \\
\hline & & & & & 685 & 2 & 4 & 4 & 0.741 & 0.741 & 0.741 \\
\hline & & & & & 782 & & 2 & 2 & & 0.306 & 0.306 \\
\hline & & & & 192 & 487 & 7 & 7 & 8 & 2.404 & 2.404 & 2.404 \\
\hline & & & & & 522 & 6 & 6 & 6 & 1.479 & 1.479 & 1.479 \\
\hline & & & & & 685 & & & 3 & & & 0.257 \\
\hline & & & & & 782 & 5 & 6 & 6 & 1.379 & 1.379 & 1.379 \\
\hline & & & & 244 & 380 & 2 & 2 & 2 & 0.753 & 0.753 & 0.753 \\
\hline & & & & & 432 & & 2 & 2 & & 0.634 & 0.634 \\
\hline & & & & & 522 & 4 & 5 & 5 & 0.912 & 0.912 & 0.912 \\
\hline & & & & & 685 & & 9 & 9 & & 0.381 & 0.381 \\
\hline & & & & & 771 & & 3 & 3 & & 0.508 & 0.508 \\
\hline & & & & & 782 & 28 & 33 & 34 & 1.343 & 1.343 & 1.343 \\
\hline & & & & & 795 & 7 & 8 & 8 & 1.168 & 1.168 & 1.168 \\
\hline & & & & 261 & 782 & 3 & 3 & 3 & 1.265 & 1.265 & 1.265 \\
\hline & & & & 266 & 685 & 2 & 4 & 4 & 0.412 & 0.412 & 0.412 \\
\hline & & & & & 782 & 10 & 11 & 14 & 0.925 & 0.925 & 0.925 \\
\hline & & CTNNBL1 & Q8WYA6 & 192 & 534 & 4 & 4 & 5 & 2.205 & 2.205 & 2.205 \\
\hline & & PLRG1 & O43660 & 122 & 1 & 14 & 16 & 17 & 1.899 & 1.899 & 1.899 \\
\hline & & & & 244 & 1 & 4 & 5 & 5 & 1.546 & 1.546 & 1.546 \\
\hline & & & & & 7 & 2 & 3 & 3 & 1.343 & 1.343 & 1.343 \\
\hline & & SF3A1 & Q15459 & 266 & 2 & 2 & 2 & 2 & 1.516 & 1.516 & 1.516 \\
\hline & & SmD2 & $\begin{array}{l}\text { P62316 } \\
\end{array}$ & 192 & 51 & 3 & 9 & 9 & 1.241 & 1.241 & 1.241 \\
\hline & & SPF 27 & O75934 & 76 & 85 & 3 & 6 & 6 & 2.109 & 2.109 & 2.109 \\
\hline & & & & 122 & 168 & 8 & 8 & 8 & 1.841 & 1.841 & 1.841 \\
\hline & & & & 179 & 168 & 18 & 20 & 20 & 2.075 & 2.075 & 2.075 \\
\hline & & & & 192 & 168 & 14 & 15 & 15 & 3.667 & 3.667 & 3.667 \\
\hline & & & & & 218 & 3 & 3 & 3 & 0.664 & 0.664 & 0.664 \\
\hline & & & & 244 & 47 & 10 & 10 & 10 & 1.851 & 1.851 & 1.851 \\
\hline & & & & & 168 & 41 & 47 & 49 & 2.180 & 2.180 & 2.180 \\
\hline & & & & & 177 & 4 & 4 & 8 & 1.370 & 1.370 & 1.370 \\
\hline & & & & & 191 & 2 & 2 & 2 & 0.972 & 0.972 & 0.972 \\
\hline & & & & & 218 & 8 & 14 & 15 & 0.750 & 0.750 & 0.750 \\
\hline & & & & 265 & 168 & & 2 & 2 & & 0.335 & 0.335 \\
\hline & & & & 266 & 47 & 3 & 3 & 3 & 1.336 & 1.336 & 1.336 \\
\hline & & & & & 168 & 16 & 16 & 16 & 3.048 & 3.048 & 3.048 \\
\hline & & & & & 218 & & & 2 & & & 0.290 \\
\hline & & SYF1 & Q9HCS7 & 266 & 708 & 3 & 4 & 4 & 0.992 & 0.992 & 0.992 \\
\hline & & U5-40K & Q96DI7 & 122 & 226 & & 2 & 3 & & 0.555 & 0.555 \\
\hline & & & & & 270 & & 6 & 9 & & 0.451 & 0.451 \\
\hline & & & & & 275 & 17 & 17 & 18 & 1.655 & 1.655 & 1.655 \\
\hline & & & & 179 & 1 & 2 & 2 & 2 & 0.608 & 0.608 & 0.608 \\
\hline & & & & 192 & 270 & & 3 & 4 & & 0.457 & 0.457 \\
\hline & & & & & 275 & 21 & 22 & 22 & 3.772 & 3.772 & 3.772 \\
\hline & & & & 244 & 1 & & 2 & 2 & & 0.588 & 0.588 \\
\hline & & & & & 275 & 17 & 17 & 17 & 1.749 & 1.749 & 1.749 \\
\hline & & & & 266 & 275 & & 2 & 2 & & 0.596 & 0.596 \\
\hline & & & & 425 & 275 & & 3 & 4 & & 0.286 & 0.286 \\
\hline & & ZC3H18 & Q86VM9 & 179 & 918 & 9 & 10 & 10 & 2.517 & 2.517 & 2.517 \\
\hline & & & & & 921 & 7 & 7 & $\begin{array}{lll}7 & \\
\end{array}$ & 1.175 & 1.175 & 1.175 \\
\hline PRP38 & Q8NAV1 & RBM22 & Q9NW64 & 7 & 170 & 3 & 3 & 3 & 0.822 & 0.822 & 0.822 \\
\hline & & SNU23 & Q96NC0 & 7 & 39 & 2 & 3 & 3 & 1.154 & 1.154 & 1.154 \\
\hline & & & & & 45 & 19 & 41 & 47 & 1.083 & 1.083 & 1.083 \\
\hline & & & & & 102 & 26 & 33 & 37 & 2.767 & 2.767 & 2.767 \\
\hline & & & & 50 & 132 & & 5 & 6 & & 0.407 & 0.407 \\
\hline & & & & 92 & 123 & & 2 & 2 & & 0.843 & 0.843 \\
\hline $\begin{array}{l}\text { PRP4B } \\
\end{array}$ & Q13523 & ZC3H18 & Q86VM9 & 99 & 933 & 2 & 2 & 2 & 0.956 & 0.956 & 0.956 \\
\hline & & & & & 948 & 26 & 34 & 42 & 1.058 & 1.058 & 1.058 \\
\hline & & & & & 952 & 32 & 42 & 45 & 1.825 & 1.825 & 1.825 \\
\hline & & & & 117 & 936 & 34 & 35 & 35 & 2.034 & 2.034 & 2.034 \\
\hline PRP8 & Q6P2Q9 & $\begin{array}{l}\text { BRR2 } \\
\end{array}$ & O75643 & 1306 & 14 & & 2 & 3 & & 0.548 & 0.548 \\
\hline & & & & 1636 & 1039 & & 5 & 6 & & 0.535 & 0.535 \\
\hline & & & & & 1141 & & 2 & 2 & & 1.184 & 1.184 \\
\hline & & & & & 1146 & 9 & 9 & 9 & 1.635 & 1.635 & 1.635 \\
\hline & & & & 1649 & 1146 & 7 & 8 & 8 & 2.375 & 2.375 & 2.375 \\
\hline & & & & 1659 & 1039 & 15 & 19 & 20 & 1.289 & 1.289 & 1.289 \\
\hline & & & & 1866 & 1049 & 13 & 13 & 13 & 3.092 & 3.092 & 3.092 \\
\hline & & & & 1984 & 1049 & 2 & 2 & 2 & 1.289 & 1.289 & 1.289 \\
\hline & & & & 1994 & 73 & 2 & 10 & 11 & 0.522 & 0.522 & 0.522 \\
\hline & & & & 2049 & 1039 & 3 & 3 & 3 & 1.131 & 1.131 & 1.131 \\
\hline & & & & 2070 & 1039 & 6 & 10 & 10 & 1.031 & 1.031 & 1.031 \\
\hline & & & & & 1049 & 2 & 3 & 3 & 1.655 & 1.655 & 1.655 \\
\hline & & BUD13 & Q9BRD0 & 1505 & 605 & & 2 & 2 & & 1.180 & 1.180 \\
\hline & & & & 1831 & 65 & 14 & 14 & 14 & 2.929 & 2.929 & 2.929 \\
\hline & & & & & 68 & 40 & 43 & 45 & 3.605 & 3.605 & 3.605 \\
\hline & & & & 1840 & 65 & 20 & 21 & 21 & 2.421 & 2.421 & 2.421 \\
\hline & & & & & 68 & 24 & 24 & 24 & 3.027 & 3.027 & 3.027 \\
\hline
\end{tabular}


Table B.1 - Continued from previous page

\begin{tabular}{|c|c|c|c|c|c|c|c|c|c|c|c|}
\hline Name & $\begin{array}{l}\text { UniProt } \\
\text { ID }\end{array}$ & Name & $\begin{array}{l}\text { UniProt } \\
\text { ID }\end{array}$ & Residue 1 & Residue 2 & FDR $1 \%$ & FDR $3 \%$ & FDR 5\% & F DR $1 \%$ & F DR3\% & F DR5\% \\
\hline & & & & 2108 & 68 & 3 & 3 & 3 & 2.055 & 2.055 & 2.055 \\
\hline & & CDC5L & Q99459 & 727 & 28 & 29 & 36 & 39 & 1.463 & 1.463 & 1.463 \\
\hline & & & & 892 & 7 & 37 & 51 & 52 & 1.770 & 1.770 & 1.770 \\
\hline & & CTNNBL1 & Q8WYA6 & 796 & 27 & 16 & 18 & 18 & 3.179 & 3.179 & 3.179 \\
\hline & & & & & 31 & 13 & 13 & 13 & 2.264 & 2.264 & 2.264 \\
\hline & & CWC15 & Q9P013 & 987 & 205 & & 3 & 4 & & 0.379 & 0.379 \\
\hline & & & & 1144 & 91 & & 2 & 2 & & 0.317 & 0.317 \\
\hline & & & & 1158 & 199 & 2 & 3 & 4 & 0.543 & 0.543 & 0.543 \\
\hline & & KIN17 & O60870 & 2034 & 87 & & 2 & 3 & & 0.280 & 0.280 \\
\hline & & MBPMS2 & $\begin{array}{l}\text { P0AEX9- } \\
\text { r }\end{array}$ & 452 & 47 & & & 2 & & & 0.209 \\
\hline & & & & 2293 & 438 & 5 & 17 & 19 & 0.472 & 0.472 & 0.472 \\
\hline & & MFAP1 & P55081 & 1636 & 256 & 2 & 2 & 2 & 0.687 & 0.687 & 0.687 \\
\hline & & & & 1649 & 242 & 25 & 30 & 31 & 1.453 & 1.453 & 1.453 \\
\hline & & & & & 249 & 41 & 43 & 46 & 2.123 & 2.123 & 2.123 \\
\hline & & & & & 250 & 17 & 17 & 17 & 2.174 & 2.174 & 2.174 \\
\hline & & & & & 256 & & & 2 & & & 0.207 \\
\hline & & & & 2293 & 238 & 9 & 14 & 16 & 0.848 & 0.848 & 0.848 \\
\hline & & & & & 242 & 46 & 53 & 54 & 2.052 & 2.052 & 2.052 \\
\hline & & PLRG1 & $\mathrm{O} 43660$ & 666 & 320 & 4 & 8 & 8 & 0.978 & 0.978 & 0.978 \\
\hline & & & & 670 & 268 & 3 & 9 & 11 & 0.584 & 0.584 & 0.584 \\
\hline & & & & & 320 & 24 & 27 & 28 & 1.358 & 1.358 & 1.358 \\
\hline & & & & 674 & 268 & & & 2 & & & 0.442 \\
\hline & & PPIL2 & Q13356 & 727 & 226 & 9 & 9 & 9 & 1.925 & 1.925 & 1.925 \\
\hline & & & & & 230 & 6 & 10 & 10 & 0.649 & 0.649 & 0.649 \\
\hline & & & & & 231 & 9 & 10 & 10 & 1.226 & 1.226 & 1.226 \\
\hline & & & & & 245 & 5 & 5 & 5 & 8.264 & 8.264 & 8.264 \\
\hline & & PQBP1 & O60828 & 1831 & 2 & 36 & 45 & 49 & 2.057 & 2.057 & 2.057 \\
\hline & & PRP17 & O60508 & 29 & 151 & 2 & 2 & 2 & 0.550 & 0.550 & 0.550 \\
\hline & & & & 2034 & 551 & 5 & 5 & 5 & 1.881 & 1.881 & 1.881 \\
\hline & & RED & Q13123 & 666 & 541 & & 1 & 1 & & 0.300 & 0.300 \\
\hline & & & & 670 & 534 & 11 & 15 & 15 & 1.554 & 1.554 & 1.554 \\
\hline & & & & & 541 & 1 & 8 & 12 & 0.532 & 0.532 & 0.532 \\
\hline & & & & & 544 & & 3 & 5 & & 0.935 & 0.935 \\
\hline & & & & & 553 & & 4 & 6 & & 0.394 & 0.394 \\
\hline & & & & 674 & 534 & & 2 & 4 & & 0.402 & 0.402 \\
\hline & & & & & 553 & & & 2 & & & 0.203 \\
\hline & & & & 746 & 520 & 9 & 10 & 10 & 2.787 & 2.787 & 2.787 \\
\hline & & & & & 553 & 4 & 4 & 4 & 1.725 & 1.725 & 1.725 \\
\hline & & & & 774 & 520 & 11 & 13 & 13 & 1.085 & 1.085 & 1.085 \\
\hline & & & & 1020 & 520 & 38 & 41 & 42 & 2.572 & 2.572 & 2.572 \\
\hline & & & & 1958 & 191 & & & 2 & & & 0.321 \\
\hline & & & & & 194 & & & 3 & & & 0.527 \\
\hline & & & & & 198 & 3 & 3 & 3 & 0.919 & 0.919 & 0.919 \\
\hline & & SF3A2 & Q15428 & 769 & 10 & 13 & 17 & 19 & 1.256 & 1.256 & 1.256 \\
\hline & & & & 853 & 10 & & 2 & 2 & & 1.348 & 1.348 \\
\hline & & & & 892 & 10 & 2 & 3 & 3 & 1.144 & 1.144 & 1.144 \\
\hline & & SF3B1 & O75533 & 837 & 80 & 3 & 3 & 3 & 3.101 & 3.101 & 3.101 \\
\hline & & & & & 81 & 3 & 3 & 3 & 3.159 & 3.159 & 3.159 \\
\hline & & & & 892 & 468 & 6 & 6 & 6 & 1.909 & 1.909 & 1.909 \\
\hline & & & & 1222 & 80 & 3 & 3 & 3 & 1.957 & 1.957 & 1.957 \\
\hline & & & & 1505 & 943 & & 3 & 3 & & 0.251 & 0.251 \\
\hline & & & & 1838 & 1008 & & 2 & 2 & & 0.543 & 0.543 \\
\hline & & & & 1958 & 963 & 14 & 15 & 15 & 1.837 & 1.837 & 1.837 \\
\hline & & & & & 1008 & 2 & 2 & 2 & 3.511 & 3.511 & 3.511 \\
\hline & & & & 1978 & 1014 & 6 & 12 & 12 & 1.542 & 1.542 & 1.542 \\
\hline & & & & 1984 & 1014 & & 3 & 3 & & 0.682 & 0.682 \\
\hline & & & & 2034 & 1008 & 5 & 9 & 9 & 1.559 & 1.559 & 1.559 \\
\hline & & SF3B2 & Q13435 & 892 & 894 & & 3 & 4 & & 0.340 & 0.340 \\
\hline & & & & 1020 & 891 & & 2 & 2 & & 0.319 & 0.319 \\
\hline & & & & 1801 & 790 & 8 & 8 & 8 & 1.529 & 1.529 & 1.529 \\
\hline & & & & & 815 & 21 & 21 & 21 & 3.426 & 3.426 & 3.426 \\
\hline & & & & 1958 & 815 & 2 & 3 & 3 & 17.629 & 17.629 & 17.629 \\
\hline & & & & 1993 & 857 & 4 & 8 & 9 & 0.577 & 0.577 & 0.577 \\
\hline & & & & & 870 & & & 2 & & & 0.573 \\
\hline & & & & & 877 & & 2 & 2 & & 0.336 & 0.336 \\
\hline & & SF3B3 & Q15393 & 1958 & 1191 & & 3 & 4 & & 1.151 & 1.151 \\
\hline & & SKIP & Q13573 & 666 & 158 & 19 & 39 & 50 & 0.968 & 0.968 & 0.968 \\
\hline & & & & & 217 & 7 & 7 & 8 & 0.918 & 0.918 & 0.918 \\
\hline & & & & 670 & 158 & 2 & 5 & 5 & 0.561 & 0.561 & 0.561 \\
\hline & & & & 702 & 236 & 22 & 26 & 26 & 2.128 & 2.128 & 2.128 \\
\hline & & & & & 246 & & 2 & 2 & & 0.704 & 0.704 \\
\hline & & & & 705 & 246 & 2 & 8 & 10 & 0.481 & 0.481 & 0.481 \\
\hline & & & & & 258 & & & 2 & & & 0.292 \\
\hline & & & & 721 & 255 & & 1 & 2 & & 0.269 & 0.269 \\
\hline & & & & 727 & 258 & 2 & 2 & 2 & 2.103 & 2.103 & 2.103 \\
\hline & & & & & 266 & 2 & 4 & 10 & 0.701 & 0.701 & 0.701 \\
\hline & & & & 774 & 258 & 3 & 3 & 3 & 1.190 & 1.190 & 1.190 \\
\hline & & & & 796 & 255 & & & 2 & & & 0.352 \\
\hline & & & & & 258 & 21 & 21 & 22 & 3.650 & 3.650 & 3.650 \\
\hline & & & & & 266 & 6 & 28 & 35 & 0.592 & 0.592 & 0.592 \\
\hline & & & & 847 & 416 & 5 & 6 & 6 & 1.083 & 1.083 & 1.083 \\
\hline & & & & 892 & 266 & & 6 & 7 & & 0.687 & 0.687 \\
\hline
\end{tabular}




\begin{tabular}{|c|c|c|c|c|c|c|c|c|c|c|c|}
\hline Name & $\begin{array}{l}\text { UniProt } \\
\text { ID }\end{array}$ & Name & $\begin{array}{l}\text { UniProt } \\
\text { ID }\end{array}$ & Residue 1 & Residue 2 & FDR $1 \%$ & FDR $3 \%$ & FDR 5\% & F DR $1 \%$ & FDR3\% & F DR5\% \\
\hline & & & & 1020 & 255 & & 2 & 3 & & 0.230 & 0.230 \\
\hline & & & & & 258 & 12 & 16 & 17 & 1.700 & 1.700 & 1.700 \\
\hline & & & & 1344 & 441 & 38 & 39 & 39 & 4.538 & 4.538 & 4.538 \\
\hline & & & & & 452 & 13 & 14 & 15 & 3.912 & 3.912 & 3.912 \\
\hline & & & & & 456 & 6 & 6 & 6 & 2.045 & 2.045 & 2.045 \\
\hline & & & & 1505 & 441 & 17 & 18 & 18 & 1.648 & 1.648 & 1.648 \\
\hline & & SMU1 & Q2TAY7 & 1801 & 107 & 14 & 14 & 15 & 1.600 & 1.600 & 1.600 \\
\hline & & & & 1838 & 107 & & 2 & 3 & & 0.285 & 0.285 \\
\hline & & & & 2108 & 107 & 27 & 32 & 33 & 2.317 & 2.317 & 2.317 \\
\hline & & SNU114 & Q15029 & 218 & 405 & 23 & 27 & 28 & 3.670 & 3.670 & 3.670 \\
\hline & & & & & 409 & & 2 & 4 & & 0.623 & 0.623 \\
\hline & & & & 366 & 341 & 2 & 2 & 2 & 1.012 & 1.012 & 1.012 \\
\hline & & & & & 358 & & 2 & 4 & & 0.252 & 0.252 \\
\hline & & & & & 359 & 62 & 66 & 67 & 2.466 & 2.466 & 2.466 \\
\hline & & & & 796 & 64 & 4 & 4 & 4 & 15.455 & 15.455 & 15.455 \\
\hline & & SNU23 & Q96NC0 & 1838 & 70 & 9 & 17 & 17 & 0.683 & 0.683 & 0.683 \\
\hline & & & & 2031 & 70 & 3 & 3 & 3 & 3.525 & 3.525 & 3.525 \\
\hline & & & & 2034 & 70 & 49 & 50 & 50 & 3.906 & 3.906 & 3.906 \\
\hline & & TCERG1 & O14776 & 1210 & 1024 & 6 & 16 & 23 & 0.768 & 0.768 & 0.768 \\
\hline & & U5-40K & Q96DI7 & 29 & 286 & 22 & 22 & 22 & 2.107 & 2.107 & 2.107 \\
\hline & & & & 36 & 286 & 2 & 3 & 5 & 2.014 & 2.014 & 2.014 \\
\hline & & & & 43 & 286 & 29 & 44 & 50 & 2.810 & 2.810 & 2.810 \\
\hline & & & & 50 & 131 & & 2 & 2 & & 1.633 & 1.633 \\
\hline & & & & & 349 & 11 & 11 & 11 & 7.715 & 7.715 & 7.715 \\
\hline & & WBP11 & Q9Y2W2 & 674 & 10 & & 3 & 3 & & 0.307 & 0.307 \\
\hline & & & & & 13 & & 3 & 3 & & 0.598 & 0.598 \\
\hline & & & & 746 & 10 & 5 & 8 & 9 & 0.983 & 0.983 & 0.983 \\
\hline & & & & 774 & 10 & & 2 & 2 & & 0.351 & 0.351 \\
\hline & & & & & 13 & 2 & 4 & 4 & 1.084 & 1.084 & 1.084 \\
\hline & & & & 2031 & 51 & & 5 & 11 & & 0.570 & 0.570 \\
\hline & & & & 2034 & 48 & & 4 & 5 & & 0.343 & 0.343 \\
\hline & & & & & 51 & & 8 & 9 & & 0.360 & 0.360 \\
\hline & & ZC3H18 & Q86VM9 & 366 & 499 & 2 & 2 & 2 & 0.913 & 0.913 & 0.913 \\
\hline & & & & & 500 & 6 & 9 & 11 & 0.807 & 0.807 & 0.807 \\
\hline & & & & & 510 & & 2 & 2 & & 0.310 & 0.310 \\
\hline RBM22 & Q9NW64 & CWC15 & Q9P013 & 40 & 28 & 3 & 4 & 4 & 0.842 & 0.842 & 0.842 \\
\hline & & & & 76 & 18 & 5 & 7 & 7 & 1.223 & 1.223 & 1.223 \\
\hline & & PLRG1 & O43660 & 40 & 363 & 3 & 3 & 3 & 0.754 & 0.754 & 0.754 \\
\hline & & PRP38 & Q8NAV1 & 170 & 7 & 3 & 3 & 3 & 0.822 & 0.822 & 0.822 \\
\hline & & SKIP & Q13573 & 40 & 153 & 2 & 2 & 2 & 1.302 & 1.302 & 1.302 \\
\hline & & & & & 158 & 4 & 7 & 8 & 0.666 & 0.666 & 0.666 \\
\hline & & & & & 170 & & & 3 & & & 0.194 \\
\hline & & & & 78 & 217 & & 1 & 2 & & 0.386 & 0.386 \\
\hline & & & & 104 & 193 & 2 & 2 & 2 & 1.020 & 1.020 & 1.020 \\
\hline & & & & 109 & 193 & & 3 & 4 & & 0.883 & 0.883 \\
\hline & & & & 114 & 193 & 13 & 18 & 19 & 1.559 & 1.559 & 1.559 \\
\hline & & & & 139 & 193 & 2 & 7 & 9 & 0.799 & 0.799 & 0.799 \\
\hline & & & & 149 & 193 & 2 & 3 & 4 & 0.815 & 0.815 & 0.815 \\
\hline & & & & 286 & 81 & 7 & 8 & 8 & 3.029 & 3.029 & 3.029 \\
\hline & & SNU23 & Q96NC0 & 76 & 8 & & 2 & 2 & & 0.534 & 0.534 \\
\hline & & & & 149 & 15 & 2 & 2 & 2 & 2.532 & 2.532 & 2.532 \\
\hline & & & & 158 & 15 & 2 & 5 & 5 & 0.627 & 0.627 & 0.627 \\
\hline RED & Q13123 & BRR2 & O75643 & 520 & 1874 & 6 & 6 & 6 & 1.016 & 1.016 & 1.016 \\
\hline & & LSm6 & P62312 & 30 & 5 & 6 & 7 & 8 & 1.153 & 1.153 & 1.153 \\
\hline & & & & & 77 & & & 2 & & & 0.456 \\
\hline & & PLRG1 & O43660 & 553 & 320 & 5 & 5 & 6 & 1.345 & 1.345 & 1.345 \\
\hline & & PRP8 & Q6P2Q9 & 191 & 1958 & & & 2 & & & 0.321 \\
\hline & & & & 194 & 1958 & & & 3 & & & 0.527 \\
\hline & & & & 198 & 1958 & 3 & 3 & 3 & 0.919 & 0.919 & 0.919 \\
\hline & & & & 520 & 746 & 9 & 10 & 10 & 2.787 & 2.787 & 2.787 \\
\hline & & & & & 774 & 11 & 13 & 13 & 1.085 & 1.085 & 1.085 \\
\hline & & & & & 1020 & 38 & 41 & 42 & 2.572 & 2.572 & 2.572 \\
\hline & & & & 534 & 670 & 11 & 15 & 15 & 1.554 & 1.554 & 1.554 \\
\hline & & & & & 674 & & 2 & 4 & & 0.402 & 0.402 \\
\hline & & & & 541 & 666 & & 1 & 1 & & 0.300 & 0.300 \\
\hline & & & & & 670 & 1 & 8 & 12 & 0.532 & 0.532 & 0.532 \\
\hline & & & & 544 & 670 & & 3 & 5 & & 0.935 & 0.935 \\
\hline & & & & 553 & 670 & & 4 & 6 & & 0.394 & 0.394 \\
\hline & & & & & 674 & & & 2 & & & 0.203 \\
\hline & & & & & 746 & 4 & 4 & 4 & 1.725 & 1.725 & 1.725 \\
\hline & & SF3B1 & O75533 & 112 & 943 & & 3 & 3 & & 0.281 & 0.281 \\
\hline & & & & 137 & 816 & & 2 & 4 & & 0.285 & 0.285 \\
\hline & & SF3B2 & Q13435 & 78 & 547 & 6 & 8 & 8 & 1.041 & 1.041 & 1.041 \\
\hline & & & & & 870 & 6 & 8 & 8 & 1.860 & 1.860 & 1.860 \\
\hline & & & & 83 & 547 & 6 & 7 & 7 & 1.881 & 1.881 & 1.881 \\
\hline & & SF3B3 & Q15393 & 209 & 1191 & 4 & 5 & 5 & 0.422 & 0.422 & 0.422 \\
\hline & & SKIP & Q13573 & 520 & 258 & 2 & 2 & 2 & 0.904 & 0.904 & 0.904 \\
\hline & & & & 544 & 217 & & 2 & 2 & & 0.317 & 0.317 \\
\hline & & SMU1 & Q2TAY7 & 191 & 107 & 3 & 4 & 4 & 0.858 & 0.858 & 0.858 \\
\hline & & & & 194 & 107 & 21 & 24 & 24 & 1.402 & 1.402 & 1.402 \\
\hline & & & & 197 & 107 & 3 & 3 & 3 & 1.895 & 1.895 & 1.895 \\
\hline & & & & 198 & 107 & 10 & 12 & 12 & 2.382 & 2.382 & 2.382 \\
\hline & & & & 209 & 337 & & 2 & 2 & & 0.330 & 0.330 \\
\hline
\end{tabular}


Table B.1 - Continued from previous page

\begin{tabular}{|c|c|c|c|c|c|c|c|c|c|c|c|}
\hline Name & $\begin{array}{l}\text { UniProt } \\
\text { ID }\end{array}$ & Name & $\begin{array}{l}\text { UniProt } \\
\text { ID }\end{array}$ & Residue 1 & Residue 2 & FDR $1 \%$ & FDR 3\% & FDR 5\% & FDR1\% & FDR3\% & FDR5\% \\
\hline & & & & 331 & 107 & 3 & 3 & 3 & 0.787 & 0.787 & 0.787 \\
\hline & & WBP11 & Q9Y2W2 & 534 & 10 & 4 & 5 & 5 & 1.069 & 1.069 & 1.069 \\
\hline \multirow[t]{39}{*}{ SF3A1 } & Q15459 & ISY1 & Q9ULR0 & 30 & 105 & 2 & 4 & 4 & 0.502 & 0.502 & 0.502 \\
\hline & & & & 37 & 105 & 5 & 6 & 6 & 0.664 & 0.664 & 0.664 \\
\hline & & LSm6 & P62312 & 131 & 5 & 4 & 4 & 4 & 1.310 & 1.310 & 1.310 \\
\hline & & PPIE & Q9UNP9 & 210 & 104 & & & 2 & & & 0.446 \\
\hline & & PPP1R8 & Q12972 & 115 & 234 & 3 & 3 & 3 & 0.758 & 0.758 & 0.758 \\
\hline & & PRP19 & Q9UMS4 & 2 & 266 & 2 & 2 & 2 & 1.516 & 1.516 & 1.516 \\
\hline & & SF3A2 & Q15428 & 131 & 204 & & & 2 & & & 0.570 \\
\hline & & & & 258 & 101 & 7 & 10 & 10 & 0.661 & 0.661 & 0.661 \\
\hline & & & & 259 & 101 & 2 & 4 & 5 & 0.486 & 0.486 & 0.486 \\
\hline & & & & 264 & 101 & & 2 & 2 & & 0.517 & 0.517 \\
\hline & & & & & 190 & 5 & 8 & 8 & 0.939 & 0.939 & 0.939 \\
\hline & & SF3A3 & Q12874 & 131 & 29 & & 5 & 7 & & 0.363 & 0.363 \\
\hline & & & & & 201 & 4 & 4 & 6 & 2.130 & 2.130 & 2.130 \\
\hline & & & & & 212 & 2 & 3 & 3 & 0.657 & 0.657 & 0.657 \\
\hline & & & & & 219 & 6 & 15 & 20 & 0.790 & 0.790 & 0.790 \\
\hline & & & & 188 & 89 & 47 & 61 & 67 & 1.810 & 1.810 & 1.810 \\
\hline & & & & & 92 & 119 & 186 & 204 & 2.107 & 2.107 & 2.107 \\
\hline & & & & 251 & 29 & 11 & 18 & 19 & 2.407 & 2.407 & 2.407 \\
\hline & & & & 258 & 29 & 21 & 27 & 30 & 1.869 & 1.869 & 1.869 \\
\hline & & & & 259 & 29 & 7 & 9 & 10 & 0.631 & 0.631 & 0.631 \\
\hline & & SF3B2 & Q13435 & 210 & 275 & 3 & 5 & 7 & 0.660 & 0.660 & 0.660 \\
\hline & & & & 217 & 275 & & 3 & 5 & & 0.325 & 0.325 \\
\hline & & SRRT & Q9BXP5 & 399 & 286 & & 2 & 4 & & 0.280 & 0.280 \\
\hline & & SYF1 & Q9HCS7 & 2 & 708 & 5 & 5 & 5 & 1.492 & 1.492 & 1.492 \\
\hline & & & & 55 & 549 & 11 & 11 & 11 & 1.613 & 1.613 & 1.613 \\
\hline & & U2-A' & P09661 & 188 & 193 & 18 & 18 & 18 & 3.097 & 3.097 & 3.097 \\
\hline & & & & & 221 & 10 & 10 & 10 & 2.230 & 2.230 & 2.230 \\
\hline & & & & 223 & 205 & 2 & 8 & 11 & 0.421 & 0.421 & 0.421 \\
\hline & & WBP11 & Q9Y2W2 & 102 & 610 & 15 & 29 & 31 & 1.682 & 1.682 & 1.682 \\
\hline & & & & & 614 & 4 & 7 & 7 & 0.502 & 0.802 & 0.802 \\
\hline & & & & 105 & 610 & & 3 & 3 & & 0.478 & 0.478 \\
\hline & & & & & 614 & 9 & 11 & 11 & 1.115 & 1.115 & 1.115 \\
\hline & & & & 115 & 599 & 2 & 2 & 2 & 1.264 & 1.264 & 1.264 \\
\hline & & & & & 610 & 23 & 29 & 30 & 1.198 & 1.198 & 1.198 \\
\hline & & & & & 614 & 20 & 20 & 20 & 2.278 & 2.278 & 2.278 \\
\hline & & & & & 626 & 2 & 2 & 2 & 2.172 & 2.172 & 2.172 \\
\hline & & & & 131 & 610 & 4 & 4 & 4 & 0.781 & 0.781 & 0.781 \\
\hline & & & & & 614 & 4 & 4 & 4 & 1.208 & 1.208 & 1.208 \\
\hline & & & & & 626 & 2 & 2 & 2 & 1.057 & 1.057 & 1.057 \\
\hline SF3A2 & Q15428 & CDC5L & Q99459 & 10 & 28 & 5 & 6 & 6 & 1.289 & 1.289 & 1.289 \\
\hline & & & & 42 & 28 & 9 & 13 & 13 & 1.355 & 1.355 & 1.355 \\
\hline & & PPIL2 & Q13356 & 91 & 418 & 2 & 2 & 2 & 0.699 & 0.699 & 0.699 \\
\hline & & PRP8 & Q6P2Q9 & 10 & 769 & 13 & 17 & 19 & 1.256 & 1.256 & 1.256 \\
\hline & & & & & 853 & & 2 & 2 & & 1.348 & 1.348 \\
\hline & & & & & 892 & 2 & 3 & 3 & 1.144 & 1.144 & 1.144 \\
\hline & & SF3A1 & Q15459 & 101 & 258 & 7 & 10 & 10 & 0.661 & 0.661 & 0.661 \\
\hline & & & & & 259 & 2 & 4 & 5 & 0.486 & 0.486 & 0.486 \\
\hline & & & & & 264 & & 2 & 2 & & 0.517 & 0.517 \\
\hline & & & & 190 & 264 & 5 & 8 & 8 & 0.939 & 0.939 & 0.939 \\
\hline & & & & 204 & 131 & & & 2 & & & 0.570 \\
\hline & & SF3A3 & Q12874 & 76 & 390 & 4 & 6 & 6 & 0.918 & 0.918 & 0.918 \\
\hline & & & & 91 & 390 & 24 & 26 & 26 & 1.591 & 1.591 & 1.591 \\
\hline & & SF3B1 & O75533 & 10 & 454 & 8 & 8 & 8 & 2.086 & 2.086 & 2.086 \\
\hline & & & & & 496 & & 3 & 4 & & 0.804 & 0.804 \\
\hline & & & & & 499 & 2 & 4 & 6 & 0.473 & 0.473 & 0.473 \\
\hline & & SF3B2 & Q13435 & 10 & 543 & 6 & 8 & 9 & 0.923 & 0.923 & 0.923 \\
\hline & & & & & 547 & 5 & 5 & 5 & 0.999 & 0.999 & 0.999 \\
\hline & & & & & 556 & 6 & 8 & 11 & 0.492 & 0.492 & 0.492 \\
\hline & & & & & 560 & & & 3 & & & 0.319 \\
\hline & & & & & 563 & & 2 & 2 & & 0.784 & 0.784 \\
\hline & & & & & 604 & 2 & 2 & 3 & 0.641 & 0.641 & 0.641 \\
\hline & & & & 216 & 268 & 12 & 13 & 16 & 1.458 & 1.458 & 1.458 \\
\hline & & & & & 275 & 6 & 6 & 6 & 1.296 & 1.296 & 1.296 \\
\hline & & SF3B4 & Q15427 & 190 & 183 & & 2 & 2 & & 0.308 & 0.308 \\
\hline & & SF3B6 & Q9Y3B4 & 10 & 29 & 2 & 2 & 2 & 1.603 & 1.603 & 1.603 \\
\hline & & & & & 105 & 3 & 5 & 6 & 0.786 & 0.786 & 0.786 \\
\hline & & & & & 106 & 6 & 6 & 6 & 0.875 & 0.875 & 0.875 \\
\hline & & & & & 116 & 3 & 5 & 7 & 0.672 & 0.672 & 0.672 \\
\hline & & SKIP & Q13573 & 10 & 258 & 7 & 7 & 7 & 0.931 & 0.931 & 0.931 \\
\hline & & WBP11 & Q9Y2W2 & 118 & 556 & 27 & 36 & 38 & 2.024 & 2.024 & 2.024 \\
\hline & & & & & 557 & 32 & 53 & 59 & 0.733 & 0.733 & 0.733 \\
\hline & & & & & 565 & 31 & 79 & 103 & 0.719 & 0.719 & 0.719 \\
\hline SF3A3 & Q12874 & SF3A1 & Q15459 & 29 & 131 & & 5 & 7 & & 0.363 & 0.363 \\
\hline & & & & & 251 & 11 & 18 & 19 & 2.407 & 2.407 & 2.407 \\
\hline & & & & & 258 & 21 & 27 & 30 & 1.869 & 1.869 & 1.869 \\
\hline & & & & & 259 & 7 & 9 & 10 & 0.631 & 0.631 & 0.631 \\
\hline & & & & 89 & 188 & 47 & 61 & 67 & 1.810 & 1.810 & 1.810 \\
\hline & & & & 92 & 188 & 119 & 186 & 204 & 2.107 & 2.107 & 2.107 \\
\hline & & & & 201 & 131 & 4 & 4 & 6 & 2.130 & 2.130 & 2.130 \\
\hline & & & & 212 & 131 & 2 & 3 & 3 & 0.657 & 0.657 & 0.657 \\
\hline & & & & 219 & 131 & 6 & 15 & 20 & 0.790 & 0.790 & 0.790 \\
\hline
\end{tabular}




\begin{tabular}{|c|c|c|c|c|c|c|c|c|c|c|c|}
\hline Name & $\begin{array}{l}\text { UniProt } \\
\text { ID }\end{array}$ & Name & $\begin{array}{l}\text { UniProt } \\
\text { ID }\end{array}$ & Residue 1 & Residue 2 & FDR $1 \%$ & FDR $3 \%$ & FDR 5\% & FDR $1 \%$ & FDR3\% & FDR5\% \\
\hline & & SF3A2 & Q15428 & 390 & 76 & 4 & 6 & 6 & 0.918 & 0.918 & 0.918 \\
\hline & & & & & 91 & 24 & 26 & 26 & 1.591 & 1.591 & 1.591 \\
\hline & & SF3B2 & Q13435 & 69 & 280 & 37 & 49 & 57 & 3.963 & 3.963 & 3.963 \\
\hline & & & & 461 & 486 & 4 & 6 & 9 & 0.477 & 0.477 & 0.477 \\
\hline & & SF3B3 & Q15393 & 463 & 1074 & 35 & 81 & 100 & 0.824 & 0.824 & 0.824 \\
\hline & & & & 466 & 984 & & 3 & 3 & & 0.488 & 0.488 \\
\hline & & & & & 1074 & 32 & 59 & 66 & 0.840 & 0.840 & 0.840 \\
\hline & & & & 496 & 974 & 3 & 8 & 10 & 0.678 & 0.913 & 0.913 \\
\hline & & U2-A' & P09661 & 64 & 179 & 4 & 4 & 4 & 1.268 & 1.268 & 1.268 \\
\hline & & & & & 193 & 2 & 3 & 3 & 1.758 & 1.758 & 1.758 \\
\hline & & & & 69 & 56 & 2 & 2 & 2 & 1.204 & 1.204 & 1.204 \\
\hline & & & & & 179 & 31 & 32 & 32 & 3.188 & 3.188 & 3.188 \\
\hline & & & & 92 & 193 & 4 & 5 & 5 & 0.961 & 0.961 & 0.961 \\
\hline & & & & 97 & 30 & 95 & 101 & 103 & 25.397 & 25.397 & 25.397 \\
\hline & & U2-B" & P08579 & 97 & 57 & 3 & 5 & 5 & 0.721 & 0.721 & 0.721 \\
\hline & & & & 463 & 103 & & & 5 & & & 0.209 \\
\hline \multirow{55}{*}{ SF3B1 } & O75533 & CDC5L & Q99459 & 454 & 76 & 2 & 2 & 2 & 0.887 & 0.887 & 0.887 \\
\hline & & & & & 106 & 5 & 5 & 5 & 3.348 & 3.348 & 3.348 \\
\hline & & CTNNBL1 & Q8WYA6 & 3 & 84 & & & 2 & & 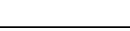 & 0.302 \\
\hline & & & & & 91 & & 2 & 2 & & 0.376 & 0.376 \\
\hline & & & & 6 & 56 & 2 & 2 & 2 & 1.561 & 1.561 & 1.561 \\
\hline & & & & & 83 & 6 & 7 & 7 & 2.140 & 2.140 & 2.140 \\
\hline & & & & & 84 & 12 & 13 & 13 & 1.691 & 1.691 & 1.691 \\
\hline & & & & & 91 & 6 & 14 & 20 & 2.710 & 2.710 & 2.710 \\
\hline & & & & & 95 & & 3 & 3 & & 0.823 & 0.823 \\
\hline & & KIN17 & O60870 & 729 & 271 & & 2 & 2 & & 0.434 & 0.434 \\
\hline & & & & 733 & 271 & 3 & 6 & 6 & 7.933 & 7.933 & 7.933 \\
\hline & & MBPMS2 & $\begin{array}{l}\text { P0AEX9- } \\
\mathrm{r}\end{array}$ & 656 & 274 & 2 & 3 & 3 & 1.690 & 1.690 & 1.690 \\
\hline & & MFAP1 & P55081 & 807 & 9 & 15 & 15 & 15 & 1.516 & 1.516 & 1.516 \\
\hline & & PHF5A & Q7RTV0 & 946 & 108 & & 2 & 2 & & 0.289 & 0.289 \\
\hline & & PPIL2 & Q13356 & 6 & 90 & 3 & 3 & 3 & 1.154 & 1.154 & 1.154 \\
\hline & & PRP8 & Q6P2Q9 & 80 & 837 & 3 & 3 & 3 & 3.101 & 3.101 & 3.101 \\
\hline & & & & & 1222 & 3 & 3 & 3 & 1.957 & 1.957 & 1.957 \\
\hline & & & & 81 & 837 & 3 & 3 & 3 & 3.159 & 3.159 & 3.159 \\
\hline & & & & 468 & 892 & 6 & 6 & 6 & 1.909 & 1.909 & 1.909 \\
\hline & & & & 943 & 1505 & & 3 & 3 & & 0.251 & 0.251 \\
\hline & & & & 963 & 1958 & 14 & 15 & 15 & 1.837 & 1.837 & 1.837 \\
\hline & & & & 1008 & 1838 & & 2 & 2 & & 0.543 & 0.543 \\
\hline & & & & & 1958 & 2 & 2 & 2 & 3.511 & 3.511 & 3.511 \\
\hline & & & & & 2034 & 5 & 9 & 9 & 1.559 & 1.559 & 1.559 \\
\hline & & & & 1014 & 1978 & 6 & 12 & 12 & 1.542 & 1.542 & 1.542 \\
\hline & & & & & 1984 & & 3 & 3 & & 0.682 & 0.682 \\
\hline & & RED & Q13123 & 816 & 137 & & 2 & 4 & & 0.285 & 0.285 \\
\hline & & & & 943 & 112 & & 3 & 3 & & 0.281 & 0.281 \\
\hline & & SF3A2 & Q15428 & 454 & 10 & 8 & 8 & 8 & 2.086 & 2.086 & 2.086 \\
\hline & & & & 496 & 10 & & 3 & 4 & & 0.804 & 0.804 \\
\hline & & & & 499 & 10 & 2 & 4 & 6 & 0.473 & 0.473 & 0.473 \\
\hline & & SF3B2 & Q13435 & 1025 & 556 & & & 2 & & & 0.375 \\
\hline & & & & & 870 & & 8 & 10 & & 0.454 & 0.454 \\
\hline & & & & 1086 & 857 & 12 & 12 & 12 & 4.584 & 4.584 & 4.584 \\
\hline & & & & 1292 & 486 & 4 & 15 & 18 & 0.739 & 0.739 & 0.739 \\
\hline & & SF3B3 & Q15393 & 513 & 137 & 8 & 9 & 9 & 1.406 & 1.406 & 1.406 \\
\hline & & SF3B5 & Q9BWJ5 & 513 & 17 & 24 & 29 & 30 & 1.205 & 1.205 & 1.205 \\
\hline & & SF3B6 & Q9Y3B4 & 80 & 7 & & 2 & 2 & & 1.369 & 1.369 \\
\hline & & & & 81 & 7 & 9 & 12 & 13 & 1.222 & 1.222 & 1.222 \\
\hline & & & & 175 & 116 & & & 3 & & & 0.204 \\
\hline & & & & 195 & 116 & & 3 & 4 & & 0.315 & 0.315 \\
\hline & & & & 413 & 29 & 4 & 4 & 4 & 1.077 & 1.077 & 1.077 \\
\hline & & & & 430 & 7 & 10 & 11 & 14 & 1.245 & 1.245 & 1.245 \\
\hline & & & & & 41 & 6 & 6 & 6 & 2.306 & 2.306 & 2.306 \\
\hline & & & & 454 & 29 & 26 & 28 & 31 & 3.790 & 3.790 & 3.790 \\
\hline & & & & 866 & 7 & 13 & 15 & 15 & 1.892 & 1.892 & 1.892 \\
\hline & & & & 943 & 41 & 17 & 31 & 32 & 0.994 & 0.994 & 0.994 \\
\hline & & & & 946 & 41 & & 6 & 6 & & 0.488 & 0.488 \\
\hline & & SKIP & Q13573 & 81 & 317 & 3 & 3 & 3 & 1.297 & 1.297 & 1.297 \\
\hline & & & & & 319 & 2 & 3 & 3 & 1.183 & 1.183 & 1.183 \\
\hline & & & & & 323 & 2 & 2 & 2 & 1.539 & 1.539 & 1.539 \\
\hline & & & & 943 & 441 & 1 & 1 & 1 & 0.414 & 0.414 & 0.414 \\
\hline & & SNIP1 & Q8TAD8 & 816 & 216 & & 1 & 1 & & 0.572 & 0.572 \\
\hline & & TCERG1 & O14776 & 1 & 794 & & & 1 & & & 0.184 \\
\hline & & U2-A' & P09661 & 175 & 193 & & 2 & 2 & & 0.263 & 0.263 \\
\hline \multirow[t]{10}{*}{ SF3B2 } & Q13435 & ISY1 & Q9ULR0 & 148 & 101 & & 2 & 2 & & 0.248 & 0.248 \\
\hline & & MBPMS2 & $\begin{array}{l}\text { P0AEX9- } \\
\text { r }\end{array}$ & 448 & 47 & 2 & 3 & 3 & 0.676 & 0.676 & 0.676 \\
\hline & & PRP8 & Q6P2Q9 & 790 & 1801 & 8 & 8 & 8 & 1.529 & 1.529 & 1.529 \\
\hline & & & & 815 & 1801 & 21 & 21 & 21 & 3.426 & 3.426 & 3.426 \\
\hline & & & & & 1958 & 2 & 3 & 3 & 17.629 & 17.629 & 17.629 \\
\hline & & & & 857 & 1993 & 4 & 8 & 9 & 0.577 & 0.577 & 0.577 \\
\hline & & & & 870 & 1993 & & & 2 & & & 0.573 \\
\hline & & & & 877 & 1993 & & 2 & 2 & & 0.336 & 0.336 \\
\hline & & & & 891 & 1020 & & 2 & 2 & & 0.319 & 0.319 \\
\hline & & & & 894 & 892 & & 3 & 4 & & 0.340 & 0.340 \\
\hline
\end{tabular}


Table B.1 - Continued from previous page

\begin{tabular}{|c|c|c|c|c|c|c|c|c|c|c|c|}
\hline Name & $\begin{array}{l}\text { UniProt } \\
\text { ID }\end{array}$ & Name & $\begin{array}{l}\text { UniProt } \\
\text { ID }\end{array}$ & Residue 1 & Residue 2 & FDR $1 \%$ & FDR 3\% & FDR 5\% & F DR1\% & F DR3\% & FDR5\% \\
\hline & & RED & Q13123 & 547 & 78 & 6 & 8 & 8 & 1.041 & 1.041 & 1.041 \\
\hline & & & & & 83 & 6 & 7 & 7 & 1.881 & 1.881 & 1.881 \\
\hline & & & & 870 & 78 & 6 & 8 & 8 & 1.860 & 1.860 & 1.860 \\
\hline & & SF3A1 & Q15459 & 275 & 210 & 3 & 5 & 7 & 0.660 & 0.660 & 0.660 \\
\hline & & & & & 217 & & 3 & 5 & & 0.325 & 0.325 \\
\hline & & SF3A2 & Q15428 & 268 & 216 & 12 & 13 & 16 & 1.458 & 1.458 & 1.458 \\
\hline & & & & 275 & 216 & 6 & 6 & 6 & 1.296 & 1.296 & 1.296 \\
\hline & & & & 543 & 10 & 6 & 8 & 9 & 0.923 & 0.923 & 0.923 \\
\hline & & & & 547 & 10 & 5 & 5 & 5 & 0.999 & 0.999 & 0.999 \\
\hline & & & & 556 & 10 & 6 & 8 & 11 & 0.492 & 0.492 & 0.492 \\
\hline & & & & 560 & 10 & & & 3 & & & 0.319 \\
\hline & & & & 563 & 10 & & 2 & 2 & & 0.784 & 0.784 \\
\hline & & & & 604 & 10 & 2 & 2 & 3 & 0.641 & 0.641 & 0.641 \\
\hline & & SF3A3 & Q12874 & 280 & 69 & 37 & 49 & 57 & 3.963 & 3.963 & 3.963 \\
\hline & & & & 486 & 461 & 4 & 6 & 9 & 0.477 & 0.477 & 0.477 \\
\hline & & SF3B1 & O75533 & 486 & 1292 & 4 & 15 & 18 & 0.739 & 0.739 & 0.739 \\
\hline & & & & 556 & 1025 & & & 2 & & & 0.375 \\
\hline & & & & 857 & 1086 & 12 & 12 & 12 & 4.584 & 4.584 & 4.584 \\
\hline & & & & 870 & 1025 & & 8 & 10 & & 0.454 & 0.454 \\
\hline & & SF3B3 & Q15393 & 604 & 1074 & 29 & 46 & 47 & 0.996 & 0.996 & 0.996 \\
\hline & & & & 605 & 1074 & 42 & 44 & 44 & 2.110 & 2.110 & 2.110 \\
\hline & & SF3B5 & Q9BWJ5 & 486 & 82 & 3 & 6 & 6 & 0.711 & 0.711 & 0.711 \\
\hline & & SmB & $\begin{array}{l}\mathrm{P} 14678 \\
\end{array}$ & 320 & 57 & 16 & 17 & 20 & 1.609 & 1.609 & 1.609 \\
\hline & & SmD2 & P62316 & 387 & 6 & & 3 & 3 & & 0.273 & 0.273 \\
\hline & & & & & 8 & 2 & 2 & 2 & 2.907 & 2.907 & 2.907 \\
\hline & & SYF3 & Q9BZJ0 & 556 & 229 & 7 & 11 & 11 & 1.150 & 1.150 & 1.150 \\
\hline & & U2-A' & P09661 & 280 & 179 & 3 & 5 & 7 & 0.628 & 0.628 & 0.628 \\
\hline & & & & & 191 & & & 2 & & & 0.203 \\
\hline & & & & & 193 & 16 & 17 & 18 & 2.415 & 2.415 & 2.415 \\
\hline & & & & 320 & 172 & 2 & 3 & 3 & 0.433 & 0.433 & 0.433 \\
\hline & & U2-B" & P08579 & 352 & 111 & 4 & 7 & 7 & 0.931 & 0.931 & 0.931 \\
\hline & & WBP11 & Q9Y2W2 & 556 & 98 & 5 & 5 & 6 & 0.484 & 0.484 & 0.484 \\
\hline & & & & & 105 & 3 & 7 & 8 & 0.643 & 0.643 & 0.643 \\
\hline SF3B3 & Q15393 & KIN17 & O60870 & 26 & 231 & 13 & 15 & 15 & 1.957 & 1.957 & 1.957 \\
\hline & & & & 296 & 231 & 2 & 2 & 2 & 1.531 & 1.531 & 1.531 \\
\hline & & MFAP1 & P55081 & 26 & 67 & & 2 & 2 & & 0.248 & 0.248 \\
\hline & & PHF5A & Q7RTV0 & 137 & 13 & 7 & 9 & 9 & 1.464 & 1.464 & 1.464 \\
\hline & & PRP8 & Q6P2Q9 & 1191 & 1958 & & 3 & 4 & & 1.151 & 1.151 \\
\hline & & RED & Q13123 & 1191 & 209 & 4 & 5 & 5 & 0.422 & 0.422 & 0.422 \\
\hline & & SF3A3 & Q12874 & 974 & 496 & 3 & 8 & 10 & 0.678 & 0.913 & 0.913 \\
\hline & & & & 984 & 466 & & 3 & 3 & & 0.488 & 0.488 \\
\hline & & & & 1074 & 463 & 35 & 81 & 100 & 0.824 & 0.824 & 0.824 \\
\hline & & & & & 466 & 32 & 59 & 66 & 0.840 & 0.840 & 0.840 \\
\hline & & SF3B1 & O75533 & 137 & 513 & 8 & 9 & 9 & 1.406 & 1.406 & 1.406 \\
\hline & & SF3B2 & Q13435 & 1074 & 604 & 29 & 46 & 47 & 0.996 & 0.996 & 0.996 \\
\hline & & & & & 605 & 42 & 44 & 44 & 2.110 & 2.110 & 2.110 \\
\hline & & SF3B5 & Q9BWJ5 & 137 & 17 & 43 & 62 & 67 & 4.104 & 4.104 & 4.104 \\
\hline SF3B4 & Q15427 & SF3A2 & Q15428 & 183 & 190 & & 2 & 2 & & 0.308 & 0.308 \\
\hline SF3B5 & Q9BWJ5 & PHF5A & Q7RTV0 & 17 & 13 & 6 & 7 & 7 & 1.145 & 1.145 & 1.145 \\
\hline & & SF3B1 & O75533 & 17 & 513 & 24 & 29 & 30 & 1.205 & 1.205 & 1.205 \\
\hline & & SF3B2 & Q13435 & 82 & 486 & 3 & 6 & 6 & 0.711 & 0.711 & 0.711 \\
\hline & & SF3B3 & Q15393 & 17 & 137 & 43 & 62 & 67 & 4.104 & 4.104 & 4.104 \\
\hline SF3B6 & Q9Y3B4 & CDC5L & Q99459 & 29 & 124 & 26 & 26 & 26 & 9.871 & 9.871 & 9.871 \\
\hline & & SF3A2 & Q15428 & 29 & 10 & 2 & 2 & 2 & 1.603 & 1.603 & 1.603 \\
\hline & & & & 105 & 10 & 3 & 5 & 6 & 0.786 & 0.786 & 0.786 \\
\hline & & & & 106 & 10 & 6 & 6 & 6 & 0.875 & 0.875 & 0.875 \\
\hline & & & & 116 & 10 & 3 & 5 & 7 & 0.672 & 0.672 & 0.672 \\
\hline & & SF3B1 & O75533 & 7 & 80 & & 2 & 2 & & 1.369 & 1.369 \\
\hline & & & & & 81 & 9 & 12 & 13 & 1.222 & 1.222 & 1.222 \\
\hline & & & & & 430 & 10 & 11 & 14 & 1.245 & 1.245 & 1.245 \\
\hline & & & & & 866 & 13 & 15 & 15 & 1.892 & 1.892 & 1.892 \\
\hline & & & & 29 & 413 & 4 & 4 & 4 & 1.077 & 1.077 & 1.077 \\
\hline & & & & & 454 & 26 & 28 & 31 & 3.790 & 3.790 & 3.790 \\
\hline & & & & 41 & 430 & 6 & 6 & 6 & 2.306 & 2.306 & 2.306 \\
\hline & & & & & 943 & 17 & 31 & 32 & 0.994 & 0.994 & 0.994 \\
\hline & & & & & 946 & & 6 & 6 & & 0.488 & 0.488 \\
\hline & & & & 116 & 175 & & & 3 & & & 0.204 \\
\hline & & & & & 195 & & 3 & 4 & & 0.315 & 0.315 \\
\hline SKIP & Q13573 & BUD13 & Q9BRD0 & 441 & 605 & 22 & 23 & 23 & 3.246 & 3.246 & 3.246 \\
\hline & & CBP80 & Q09161 & 503 & 607 & 4 & 4 & 4 & 2.187 & 2.187 & 2.187 \\
\hline & & & & 509 & 607 & 2 & 3 & 3 & 1.072 & 1.072 & 1.072 \\
\hline & & CDC5L & Q99459 & 255 & 28 & 6 & 9 & 9 & 1.319 & 1.319 & 1.319 \\
\hline & & & & 258 & 28 & 16 & 25 & 25 & 1.281 & 1.281 & 1.281 \\
\hline & & & & & 47 & & 3 & 6 & & 0.327 & 0.327 \\
\hline & & & & 311 & 124 & 8 & 16 & 19 & 0.641 & 0.641 & 0.641 \\
\hline & & CWC15 & Q9P013 & 153 & 18 & 16 & 16 & 16 & 2.622 & 2.622 & 2.622 \\
\hline & & & & 158 & 28 & & 2 & 2 & & 0.932 & 0.932 \\
\hline & & & & 204 & 28 & 2 & 3 & 4 & 0.621 & 0.621 & 0.621 \\
\hline & & & & 217 & 18 & 1 & 5 & 5 & 0.535 & 0.535 & 0.535 \\
\hline & & & & & 28 & 3 & 4 & 5 & 0.573 & 0.573 & 0.573 \\
\hline & & & & 311 & 183 & 2 & 2 & 3 & 0.816 & 0.816 & 0.816 \\
\hline & & LSm2 & Q9Y333 & 236 & 8 & 2 & 3 & 4 & 0.430 & 0.430 & 0.430 \\
\hline & & PLRG1 & O43660 & 81 & 113 & 2 & 2 & 2 & 1.745 & 1.745 & 1.745 \\
\hline
\end{tabular}




\begin{tabular}{|c|c|c|c|c|c|c|c|c|c|c|c|}
\hline Name & $\begin{array}{l}\text { UniProt } \\
\text { ID }\end{array}$ & Name & $\begin{array}{l}\text { UniProt } \\
\text { ID }\end{array}$ & Residue 1 & Residue 2 & FDR 1\% & FDR 3\% & FDR 5\% & FDR $1 \%$ & FDR3\% & FDR5\% \\
\hline & & & & & 173 & & 3 & 3 & & 0.710 & 0.710 \\
\hline & & & & 97 & 113 & & 2 & 2 & & 0.584 & 0.584 \\
\hline & & & & & 135 & 2 & 2 & 3 & 0.416 & 0.416 & 0.416 \\
\hline & & & & & 173 & 2 & 4 & 4 & 0.779 & 0.779 & 0.779 \\
\hline & & & & & 180 & 5 & 5 & 5 & 1.618 & 1.618 & 1.618 \\
\hline & & & & 110 & 173 & & 3 & 4 & & 0.397 & 0.397 \\
\hline & & & & & 180 & 3 & 3 & 3 & 0.500 & 0.500 & 0.500 \\
\hline & & & & & 510 & 2 & 6 & 7 & 0.694 & 0.694 & 0.694 \\
\hline & & & & 115 & 180 & & 3 & 3 & & 0.423 & 0.423 \\
\hline & & & & 122 & 180 & 10 & 11 & 12 & 0.832 & 0.832 & 0.832 \\
\hline & & & & & 396 & 4 & 6 & 6 & 0.731 & 0.731 & 0.731 \\
\hline & & & & 153 & 363 & 3 & 4 & 5 & 1.083 & 1.083 & 1.083 \\
\hline & & & & 158 & 320 & 35 & 38 & 40 & 2.214 & 2.214 & 2.214 \\
\hline & & & & & 363 & 14 & 16 & 19 & 1.286 & 1.286 & 1.286 \\
\hline & & & & 170 & 320 & & 2 & 2 & & 0.323 & 0.323 \\
\hline & & & & 236 & 510 & 2 & 2 & 6 & 0.421 & 0.421 & 0.421 \\
\hline & & & & 240 & 510 & 5 & 6 & 6 & 0.974 & 0.974 & 0.974 \\
\hline & & PPIL1 & Q9Y3C6 & 48 & 91 & & 3 & 3 & & 0.308 & 0.308 \\
\hline & & & & 81 & 58 & 27 & 27 & 27 & 2.753 & 2.753 & 2.753 \\
\hline & & & & 97 & 58 & 2 & 4 & 4 & 1.204 & 1.204 & 1.204 \\
\hline & & & & & 158 & 4 & 7 & 7 & 1.290 & 1.290 & 1.290 \\
\hline & & & & 193 & 80 & 5 & 5 & 5 & 1.332 & 1.332 & 1.332 \\
\hline & & & & & 158 & & & 2 & & & 0.370 \\
\hline & & PPIL2 & Q13356 & 240 & 277 & & 2 & 2 & & 0.292 & 0.292 \\
\hline & & PRP8 & Q6P2Q9 & 158 & 666 & 19 & 39 & 50 & 0.968 & 0.968 & 0.968 \\
\hline & & & & & 670 & 2 & 5 & 5 & 0.561 & 0.561 & 0.561 \\
\hline & & & & 217 & 666 & 7 & 7 & 8 & 0.918 & 0.918 & 0.918 \\
\hline & & & & 236 & 702 & 22 & 26 & 26 & 2.128 & 2.128 & 2.128 \\
\hline & & & & 246 & 702 & & 2 & 2 & & 0.704 & 0.704 \\
\hline & & & & & 705 & 2 & 8 & 10 & 0.481 & 0.481 & 0.481 \\
\hline & & & & 255 & 721 & & 1 & 2 & & 0.269 & 0.269 \\
\hline & & & & & 796 & & & 2 & & & 0.352 \\
\hline & & & & & 1020 & & 2 & 3 & & 0.230 & 0.230 \\
\hline & & & & 258 & 705 & & & 2 & & & 0.292 \\
\hline & & & & & 727 & 2 & 2 & 2 & 2.103 & 2.103 & 2.103 \\
\hline & & & & & 774 & 3 & 3 & 3 & 1.190 & 1.190 & 1.190 \\
\hline & & & & & 796 & 21 & 21 & 22 & 3.650 & 3.650 & 3.650 \\
\hline & & & & & 1020 & 12 & 16 & 17 & 1.700 & 1.700 & 1.700 \\
\hline & & & & 266 & 727 & 2 & 4 & 10 & 0.701 & 0.701 & 0.701 \\
\hline & & & & & 796 & 6 & 28 & 35 & 0.592 & 0.592 & 0.592 \\
\hline & & & & & 892 & & 6 & 7 & & 0.687 & 0.687 \\
\hline & & & & 416 & 847 & 5 & 6 & 6 & 1.083 & 1.083 & 1.083 \\
\hline & & & & 441 & 1344 & 38 & 39 & 39 & 4.538 & 4.538 & 4.538 \\
\hline & & & & & 1505 & 17 & 18 & 18 & 1.648 & 1.648 & 1.648 \\
\hline & & & & 452 & 1344 & 13 & 14 & 15 & 3.912 & 3.912 & 3.912 \\
\hline & & & & 456 & 1344 & 6 & 6 & 6 & 2.045 & 2.045 & 2.045 \\
\hline & & RBM22 & Q9NW64 & 81 & 286 & 7 & 8 & 8 & 3.029 & 3.029 & 3.029 \\
\hline & & & & 153 & 40 & 2 & 2 & 2 & 1.302 & 1.302 & 1.302 \\
\hline & & & & 158 & 40 & 4 & 7 & 8 & 0.666 & 0.666 & 0.666 \\
\hline & & & & 170 & 40 & & & 3 & & & 0.194 \\
\hline & & & & 193 & 104 & 2 & 2 & 2 & 1.020 & 1.020 & 1.020 \\
\hline & & & & & 109 & & 3 & 4 & & 0.883 & 0.883 \\
\hline & & & & & 114 & 13 & 18 & 19 & 1.559 & 1.559 & 1.559 \\
\hline & & & & & 139 & 2 & 7 & 9 & 0.799 & 0.799 & 0.799 \\
\hline & & & & & 149 & 2 & 3 & 4 & 0.815 & 0.815 & 0.815 \\
\hline & & & & 217 & 78 & & 1 & 2 & & 0.386 & 0.386 \\
\hline & & RED & Q13123 & 217 & 544 & & 2 & 2 & & 0.317 & 0.317 \\
\hline & & & & 258 & 520 & 2 & 2 & 2 & 0.904 & 0.904 & 0.904 \\
\hline & & SF3A2 & Q15428 & 258 & 10 & 7 & 7 & 7 & 0.931 & 0.931 & 0.931 \\
\hline & & SF3B1 & O75533 & 317 & 81 & 3 & 3 & 3 & 1.297 & 1.297 & 1.297 \\
\hline & & & & 319 & 81 & 2 & 3 & 3 & 1.183 & 1.183 & 1.183 \\
\hline & & & & 323 & 81 & 2 & 2 & 2 & 1.539 & 1.539 & 1.539 \\
\hline & & & & 441 & 943 & 1 & 1 & 1 & 0.414 & 0.414 & 0.414 \\
\hline & & SNIP1 & Q8TAD8 & 323 & 355 & 5 & 10 & 12 & 0.663 & 0.663 & 0.663 \\
\hline & & & & 339 & 342 & 32 & 43 & 49 & 1.789 & 1.789 & 1.789 \\
\hline & & & & & 353 & 2 & 2 & 2 & 1.637 & 1.637 & 1.637 \\
\hline & & & & & 355 & 21 & 24 & 24 & 1.528 & 1.528 & 1.528 \\
\hline & & & & 344 & 342 & 8 & 18 & 23 & 0.547 & 0.547 & 0.547 \\
\hline & & & & 379 & 342 & 5 & 11 & 12 & 0.735 & 0.735 & 0.735 \\
\hline & & SYF3 & Q9BZJ0 & 23 & 388 & 2 & 2 & 2 & 1.679 & 1.679 & 1.679 \\
\hline & & U5-40K & Q96DI7 & 48 & 275 & 4 & 4 & 4 & 2.240 & 2.240 & 2.240 \\
\hline & & & & & 322 & 5 & 6 & 6 & 1.276 & 1.276 & 1.276 \\
\hline & & & & 81 & 18 & 5 & 6 & 6 & 1.837 & 1.837 & 1.837 \\
\hline & & & & & 275 & 6 & 6 & 6 & 2.195 & 2.195 & 2.195 \\
\hline & & & & 97 & 18 & 2 & 3 & 3 & 0.691 & 0.691 & 0.691 \\
\hline & & & & & 322 & 2 & 2 & 2 & 1.031 & 1.031 & 1.031 \\
\hline & & & & 110 & 18 & 1 & 2 & 2 & 0.560 & 0.560 & 0.560 \\
\hline & & & & 153 & 349 & 6 & 7 & 10 & 1.331 & 1.331 & 1.331 \\
\hline & & & & 170 & 349 & & 2 & 3 & & 0.850 & 0.850 \\
\hline & & & & 193 & 1 & $\begin{array}{lll}7 & \end{array}$ & 9 & 9 & 2.990 & 2.990 & 2.990 \\
\hline SmB & $\begin{array}{l}\text { P14678 } \\
\end{array}$ & SF3B2 & Q13435 & 57 & 320 & 16 & 17 & 20 & 1.609 & 1.609 & 1.609 \\
\hline & & SmD1 & P62314 & 5 & 41 & & 6 & 10 & & 0.421 & 0.421 \\
\hline & & & & 8 & 41 & 14 & 16 & 16 & 1.298 & 1.298 & 1.298 \\
\hline
\end{tabular}


Table B.1 - Continued from previous page

\begin{tabular}{|c|c|c|c|c|c|c|c|c|c|c|c|}
\hline Name & $\begin{array}{l}\text { UniProt } \\
\text { ID }\end{array}$ & Name & $\begin{array}{l}\text { UniProt } \\
\text { ID }\end{array}$ & Residue 1 & Residue 2 & FDR $1 \%$ & FDR $3 \%$ & FDR 5\% & F DR $1 \%$ & FDR3\% & FDR5\% \\
\hline & & SmD3 & P62318 & 88 & 84 & 2 & 4 & 4 & 0.729 & 0.729 & 0.729 \\
\hline & & & & & 87 & & & 2 & & & 0.225 \\
\hline \multirow[t]{3}{*}{ SmD1 } & P62314 & $\mathrm{SmB}$ & P14678 & 41 & 5 & & 6 & 10 & & 0.421 & 0.421 \\
\hline & & & & & 8 & 14 & 16 & 16 & 1.298 & 1.298 & 1.298 \\
\hline & & U5-40K & Q96DI7 & 86 & 226 & 2 & 2 & 3 & 0.520 & 0.520 & 0.520 \\
\hline \multirow[t]{6}{*}{ SmD2 } & P62316 & PRP19 & Q9UMS4 & 51 & 192 & 3 & 9 & 9 & 1.241 & 1.241 & 1.241 \\
\hline & & SF3B2 & Q13435 & 6 & 387 & & 3 & 3 & & 0.273 & 0.273 \\
\hline & & & & 8 & 387 & 2 & 2 & 2 & 2.907 & 2.907 & 2.907 \\
\hline & & SmF & P62306 & 8 & 8 & 20 & 21 & 23 & 1.581 & 1.581 & 1.581 \\
\hline & & & & 18 & 8 & 7 & 7 & 7 & 1.539 & 1.539 & 1.539 \\
\hline & & SNU114 & Q15029 & 6 & 352 & 1 & 5 & 7 & 0.378 & 0.378 & 0.378 \\
\hline SmD3 & P62318 & SmB & $\begin{array}{l}\text { P14678 } \\
\end{array}$ & 84 & 88 & 2 & 4 & 4 & 0.729 & 0.729 & 0.729 \\
\hline & & & & 87 & 88 & & & 2 & & & 0.225 \\
\hline & & U2-B" & P08579 & 84 & 93 & 3 & 3 & 3 & 1.443 & 1.443 & 1.443 \\
\hline & & & & & 101 & & & 2 & & & 0.290 \\
\hline $\mathrm{SmE}$ & P62304 & MBPMS2 & $\begin{array}{l}\text { P0AEX9- } \\
\text { r }\end{array}$ & 1 & 278 & & & 2 & & & 0.661 \\
\hline & & SmG & $\begin{array}{l}\mathrm{P} 62308 \\
\end{array}$ & 12 & 10 & & 2 & 6 & & 0.351 & 0.351 \\
\hline & & & & & 15 & 2 & 4 & 4 & 0.679 & 0.679 & 0.679 \\
\hline SmF & P62306 & SmD2 & P62316 & 8 & 8 & 20 & 21 & 23 & 1.581 & 1.581 & 1.581 \\
\hline & & & & & 18 & 7 & 7 & 7 & 1.539 & 1.539 & 1.539 \\
\hline SmG & P62308 & SmE & P62304 & 10 & 12 & & 2 & 6 & & 0.351 & 0.351 \\
\hline & & & & 15 & 12 & 2 & 4 & 4 & 0.679 & 0.679 & 0.679 \\
\hline SMU1 & Q2TAY7 & BRR2 & O75643 & 308 & 971 & 14 & 17 & 18 & 1.051 & 1.051 & 1.051 \\
\hline & & & & & 975 & 3 & 3 & 3 & 2.201 & 2.201 & 2.201 \\
\hline & & & & & 1544 & & 2 & 2 & & 0.279 & 0.279 \\
\hline & & & & & 1552 & 31 & 50 & 53 & 1.971 & 1.971 & 1.971 \\
\hline & & & & & 1711 & & & 2 & & & 0.450 \\
\hline & & & & & 1715 & 11 & 15 & 16 & 1.197 & 1.197 & 1.197 \\
\hline & & MFAP1 & P55081 & 308 & 67 & 17 & 21 & 21 & 1.104 & 1.104 & 1.104 \\
\hline & & PRP8 & Q6P2Q9 & 107 & 1801 & 14 & 14 & 15 & 1.600 & 1.600 & 1.600 \\
\hline & & & & & 1838 & & 2 & 3 & & 0.285 & 0.285 \\
\hline & & & & & 2108 & 27 & 32 & 33 & 2.317 & 2.317 & 2.317 \\
\hline & & RED & Q13123 & 107 & 191 & 3 & 4 & 4 & 0.858 & 0.858 & 0.858 \\
\hline & & & & & 194 & 21 & 24 & 24 & 1.402 & 1.402 & 1.402 \\
\hline & & & & & 197 & 3 & 3 & 3 & 1.895 & 1.895 & 1.895 \\
\hline & & & & & 198 & 10 & 12 & 12 & 2.382 & 2.382 & 2.382 \\
\hline & & & & & 331 & 3 & 3 & 3 & 0.787 & 0.787 & 0.787 \\
\hline & & & & 337 & 209 & & 2 & 2 & & 0.330 & 0.330 \\
\hline SNIP1 & Q8TAD8 & SF3B1 & O75533 & 216 & 816 & & 1 & 1 & & 0.572 & 0.572 \\
\hline & & SKIP & Q13573 & 342 & 339 & 32 & 43 & 49 & 1.789 & 1.789 & 1.789 \\
\hline & & & & & 344 & 8 & 18 & 23 & 0.547 & 0.547 & 0.547 \\
\hline & & & & & 379 & 5 & 11 & 12 & 0.735 & 0.735 & 0.735 \\
\hline & & & & 353 & 339 & 2 & 2 & 2 & 1.637 & 1.637 & 1.637 \\
\hline & & & & 355 & 323 & 5 & 10 & 12 & 0.663 & 0.663 & 0.663 \\
\hline & & & & & 339 & 21 & 24 & 24 & 1.528 & 1.528 & 1.528 \\
\hline SNU114 & Q15029 & PLRG1 & $\mathrm{O} 43660$ & 602 & 237 & 2 & 3 & 4 & 0.524 & 0.524 & 0.524 \\
\hline & & PRP8 & Q6P2Q9 & 64 & 796 & 4 & 4 & 4 & 15.455 & 15.455 & 15.455 \\
\hline & & & & 341 & 366 & 2 & 2 & 2 & 1.012 & 1.012 & 1.012 \\
\hline & & & & 358 & 366 & & 2 & 4 & & 0.252 & 0.252 \\
\hline & & & & 359 & 366 & 62 & 66 & 67 & 2.466 & 2.466 & 2.466 \\
\hline & & & & 405 & 218 & 23 & 27 & 28 & 3.670 & 3.670 & 3.670 \\
\hline & & & & 409 & 218 & & 2 & 4 & & 0.623 & 0.623 \\
\hline & & SmD2 & P62316 & 352 & 6 & 1 & 5 & 7 & 0.378 & 0.378 & 0.378 \\
\hline & & TCERG1 & O14776 & 914 & 992 & 5 & 6 & 6 & 1.894 & 1.894 & 1.894 \\
\hline & & & & 963 & 992 & & & 2 & & & 0.782 \\
\hline & & ZC3H18 & Q86VM9 & 244 & 510 & 4 & 6 & 8 & 0.795 & 0.795 & 0.795 \\
\hline & & & & 359 & 500 & & 2 & 2 & & 0.547 & 0.547 \\
\hline & & & & 694 & 510 & & 2 & 3 & & 0.636 & 0.636 \\
\hline SNU23 & Q96NC0 & BRR2 & O75643 & 8 & 479 & 1 & 4 & 6 & 1.354 & 1.354 & 1.354 \\
\hline & & & & & 557 & 12 & 21 & 24 & 1.121 & 1.121 & 1.121 \\
\hline & & & & & 804 & 4 & 4 & 4 & 0.759 & 0.759 & 0.759 \\
\hline & & & & 15 & 804 & 6 & 8 & 8 & 1.189 & 1.189 & 1.189 \\
\hline & & & & 18 & 804 & & & 2 & & & 0.344 \\
\hline & & & & 70 & 85 & 2 & 2 & 2 & 2.348 & 2.348 & 2.348 \\
\hline & & & & 138 & 733 & 5 & 7 & 7 & 0.643 & 0.643 & 0.643 \\
\hline & & & & 147 & 729 & & & 2 & & & 0.568 \\
\hline & & & & & 733 & 4 & 5 & 5 & 1.856 & 1.856 & 1.856 \\
\hline & & & & 160 & 696 & 4 & 6 & 7 & 0.472 & 0.472 & 0.472 \\
\hline & & KIN17 & O60870 & 70 & 120 & 3 & 3 & 3 & 2.324 & 2.324 & 2.324 \\
\hline & & PPP1R8 & Q12972 & 155 & 334 & 6 & 9 & 9 & 1.503 & 1.503 & 1.503 \\
\hline & & & & 167 & 334 & 3 & 3 & 3 & 1.039 & 1.039 & 1.039 \\
\hline & & PRP38 & Q8NAV1 & 39 & 7 & 2 & 3 & 3 & 1.154 & 1.154 & 1.154 \\
\hline & & & & 45 & 7 & 19 & 41 & 47 & 1.083 & 1.083 & 1.083 \\
\hline & & & & 102 & 7 & 26 & 33 & 37 & 2.767 & 2.767 & 2.767 \\
\hline & & & & 123 & 92 & & 2 & 2 & & 0.843 & 0.843 \\
\hline & & & & 132 & 50 & & 5 & 6 & & 0.407 & 0.407 \\
\hline & & PRP8 & Q6P2Q9 & 70 & 1838 & 9 & 17 & 17 & 0.683 & 0.683 & 0.683 \\
\hline & & & & & 2031 & 3 & 3 & 3 & 3.525 & 3.525 & 3.525 \\
\hline & & & & & 2034 & 49 & 50 & 50 & 3.906 & 3.906 & 3.906 \\
\hline & & RBM22 & Q9NW64 & 8 & 76 & & 2 & 2 & & 0.534 & 0.534 \\
\hline & & & & 15 & 149 & 2 & 2 & 2 & 2.532 & 2.532 & 2.532 \\
\hline & & & & & 158 & 2 & 5 & 5 & 0.627 & 0.627 & 0.627 \\
\hline
\end{tabular}




\begin{tabular}{|c|c|c|c|c|c|c|c|c|c|c|c|}
\hline Name & $\begin{array}{l}\text { UniProt } \\
\text { ID }\end{array}$ & Name & $\begin{array}{l}\text { UniProt } \\
\text { ID }\end{array}$ & Residue 1 & Residue 2 & FDR $1 \%$ & FDR 3\% & FDR $5 \%$ & FDR $1 \%$ & FDR3\% & FDR5\% \\
\hline & & U5-40K & Q96DI7 & 136 & 8 & & 1 & 2 & & 0.235 & 0.235 \\
\hline & & WBP11 & Q9Y2W2 & 8 & 169 & & 2 & 2 & & 0.377 & 0.377 \\
\hline & & & & 45 & 76 & 4 & 7 & 8 & 1.092 & 1.092 & 1.092 \\
\hline & & & & 70 & 48 & & 4 & 6 & & 0.325 & 0.325 \\
\hline & & & & & 59 & 2 & 2 & 2 & 3.695 & 3.695 & 3.695 \\
\hline & & & & 102 & 76 & 18 & 23 & 23 & 2.737 & 2.737 & 2.737 \\
\hline \multirow[t]{36}{*}{ SPF 27 } & O75934 & CDC5L & Q99459 & 97 & 685 & 6 & 17 & 22 & 0.689 & 0.689 & 0.689 \\
\hline & & & & & 686 & 15 & 17 & 17 & 2.361 & 2.361 & 2.361 \\
\hline & & & & 168 & 380 & 2 & 2 & 3 & 1.331 & 1.331 & 1.331 \\
\hline & & & & 177 & 771 & 74 & 126 & 151 & 1.449 & 1.449 & 1.449 \\
\hline & & & & 191 & 782 & 8 & 11 & 12 & 1.148 & 1.148 & 1.148 \\
\hline & & $\begin{array}{l}\text { PLRG1 } \\
\end{array}$ & O43660 & 136 & 62 & 12 & 12 & 13 & 1.939 & 1.939 & 1.939 \\
\hline & & & & & 68 & 11 & 12 & 12 & 3.413 & 3.413 & 3.413 \\
\hline & & & & & 135 & 2 & 2 & 2 & 1.855 & 1.855 & 1.855 \\
\hline & & & & 168 & 80 & 2 & 2 & 2 & 2.075 & 2.075 & 2.075 \\
\hline & & & & & 113 & 8 & 11 & 12 & 1.103 & 1.103 & 1.103 \\
\hline & & & & & 135 & 8 & 9 & 9 & 1.343 & 1.343 & 1.343 \\
\hline & & PPIL1 & Q9Y3C6 & 136 & 80 & 8 & 8 & 8 & 2.484 & 2.484 & 2.484 \\
\hline & & & & 168 & 80 & 9 & 10 & 10 & 2.210 & 2.210 & 2.210 \\
\hline & & & & & 158 & 2 & 2 & 3 & 0.477 & 0.477 & 0.477 \\
\hline & & PRP19 & Q9UMS4 & 47 & 244 & 10 & 10 & 10 & 1.851 & 1.851 & 1.851 \\
\hline & & & & & 266 & 3 & 3 & 3 & 1.336 & 1.336 & 1.336 \\
\hline & & & & 85 & 76 & 3 & 6 & 6 & 2.109 & 2.109 & 2.109 \\
\hline & & & & 168 & 122 & 8 & 8 & 8 & 1.841 & 1.841 & 1.841 \\
\hline & & & & & 179 & 18 & 20 & 20 & 2.075 & 2.075 & 2.075 \\
\hline & & & & & 192 & 14 & 15 & 15 & 3.667 & 3.667 & 3.667 \\
\hline & & & & & 244 & 41 & 47 & 49 & 2.180 & 2.180 & 2.180 \\
\hline & & & & & 265 & & 2 & 2 & & 0.335 & 0.335 \\
\hline & & & & & 266 & 16 & 16 & 16 & 3.048 & 3.048 & 3.048 \\
\hline & & & & 177 & 244 & 4 & 4 & 8 & 1.370 & 1.370 & 1.370 \\
\hline & & & & 191 & 244 & 2 & 2 & 2 & 0.972 & 0.972 & 0.972 \\
\hline & & & & 218 & 192 & 3 & 3 & 3 & 0.664 & 0.664 & 0.664 \\
\hline & & & & & 244 & 8 & 14 & 15 & 0.750 & 0.750 & 0.750 \\
\hline & & & & & 266 & & & 2 & & & 0.290 \\
\hline & & SRRM1 & Q8IYB3 & 97 & 217 & & 2 & 2 & & 0.321 & 0.321 \\
\hline & & U5-40K & Q96DI7 & 168 & 8 & 5 & 5 & 5 & 2.865 & 2.865 & 2.865 \\
\hline & & & & & 18 & 3 & 4 & 4 & 0.756 & 0.756 & 0.756 \\
\hline & & & & & 226 & 6 & 8 & 8 & 1.298 & 1.298 & 1.298 \\
\hline & & & & & 270 & 6 & 9 & 10 & 0.617 & 0.617 & 0.617 \\
\hline & & & & & 275 & 43 & 46 & 47 & 3.253 & 3.253 & 3.253 \\
\hline & & & & & 322 & 3 & 3 & 3 & 1.409 & 1.409 & 1.409 \\
\hline & & & & 177 & 275 & 2 & 5 & 6 & 1.211 & 1.211 & 1.211 \\
\hline SRRM1 & Q8IYB3 & SPF 27 & O75934 & 217 & 97 & & 2 & 2 & & 0.321 & 0.321 \\
\hline \multirow[t]{7}{*}{ SRRT } & Q9BXP5 & MBPMS2 & $\begin{array}{l}\text { P0AEX9- } \\
\mathrm{r}\end{array}$ & 286 & 103 & 7 & 8 & 10 & 1.052 & 1.052 & 1.052 \\
\hline & & & & & 180 & 25 & 31 & 40 & 1.136 & 1.136 & 1.136 \\
\hline & & & & & 363 & & 4 & 6 & & 0.357 & 0.357 \\
\hline & & SF3A1 & Q15459 & 286 & 399 & & 2 & 4 & & 0.280 & 0.280 \\
\hline & & ZC3H18 & Q86VM9 & 723 & 211 & & 3 & 3 & & 0.488 & 0.488 \\
\hline & & & & 730 & 188 & & 3 & 4 & & 0.324 & 0.324 \\
\hline & & & & 744 & 188 & 2 & 3 & 3 & 1.091 & 1.091 & 1.091 \\
\hline SRSF1 & Q07955 & CBP20 & $\begin{array}{l}\text { P52298 } \\
\end{array}$ & 165 & 7 & 21 & 22 & 22 & 2.728 & 2.728 & 2.728 \\
\hline & & $\begin{array}{l}\text { CBP80 } \\
\text {. }\end{array}$ & Q09161 & 165 & 17 & 4 & 4 & 4 & 1.131 & 1.131 & 1.131 \\
\hline & & & & & 20 & 3 & 3 & 3 & 1.855 & 1.855 & 1.855 \\
\hline & & & & 193 & 20 & 4 & 5 & 5 & 1.189 & 1.189 & 1.189 \\
\hline & & MFAP1 & P55081 & 179 & 329 & 13 & 18 & 19 & 1.295 & 1.295 & 1.295 \\
\hline & & TCERG1 & O14776 & 179 & 1016 & & 4 & 6 & & 0.488 & 0.488 \\
\hline & & & & & 1024 & 2 & 3 & 3 & 0.691 & 0.691 & 0.691 \\
\hline & & UBL5 & Q9BZL1 & 179 & 1 & & & 2 & & & 1.037 \\
\hline SYF1 & Q9HCS7 & $\mathrm{AQR}$ & O60306 & 2 & 954 & 10 & 10 & 11 & 0.991 & 0.991 & 0.991 \\
\hline & & CCDC12 & Q8WUD4 & 532 & 23 & 3 & 6 & 6 & 0.634 & 0.634 & 0.634 \\
\hline & & & & & 42 & 5 & 5 & 5 & 1.178 & 1.178 & 1.178 \\
\hline & & & & 593 & 94 & 2 & 2 & 2 & 3.169 & 3.169 & 3.169 \\
\hline & & CDC5L & Q99459 & 532 & 522 & 8 & 8 & 8 & 1.502 & 1.502 & 1.502 \\
\hline & & & & 654 & 294 & 19 & 19 & 20 & 3.245 & 3.245 & 3.245 \\
\hline & & ISY1 & Q9ULR0 & 2 & 190 & 4 & 6 & 6 & 2.763 & 2.763 & 2.763 \\
\hline & & & & & 260 & & & 2 & & & 0.660 \\
\hline & & MFAP1 & P55081 & 539 & 285 & 4 & 4 & 4 & 0.809 & 0.809 & 0.809 \\
\hline & & PRP19 & Q9UMS4 & 708 & 266 & 3 & 4 & 4 & 0.992 & 0.992 & 0.992 \\
\hline & & SF3A1 & Q15459 & 549 & 55 & 11 & 11 & 11 & 1.613 & 1.613 & 1.613 \\
\hline & & & & 708 & 2 & 5 & 5 & 5 & 1.492 & 1.492 & 1.492 \\
\hline & & SYF3 & Q9BZJ0 & 482 & 388 & 25 & 30 & 34 & 1.075 & 1.075 & 1.075 \\
\hline & & & & & 427 & 2 & 3 & 4 & 0.607 & 0.607 & 0.607 \\
\hline & & & & & 475 & 2 & 3 & 4 & 0.691 & 0.691 & 0.691 \\
\hline & & U2-A' & P09661 & 2 & 205 & 5 & 6 & 6 & 1.644 & 1.644 & 1.644 \\
\hline & & & & 50 & 193 & & 5 & 6 & & 0.368 & 0.368 \\
\hline & & ZNF830 & Q96NB3 & 482 & 279 & 5 & 9 & 9 & 0.851 & 0.851 & 0.851 \\
\hline & & & & 654 & 216 & 7 & 7 & 7 & 2.416 & 2.416 & 2.416 \\
\hline SYF3 & Q9BZJ0 & CCDC12 & Q8WUD4 & 549 & 126 & & 3 & 4 & & 0.399 & 0.399 \\
\hline & & & & 607 & 117 & & 3 & 3 & & 0.700 & 0.700 \\
\hline & & CDC5L & Q99459 & 568 & 466 & & & 2 & & & 0.277 \\
\hline & & PPIL2 & Q13356 & 445 & 460 & & & 2 & & & 0.354 \\
\hline & & & & & 462 & 8 & 8 & 12 & 0.858 & 0.858 & 0.858 \\
\hline
\end{tabular}


Table B.1 - Continued from previous page

\begin{tabular}{|c|c|c|c|c|c|c|c|c|c|c|c|}
\hline Name & $\begin{array}{l}\text { UniProt } \\
\text { ID }\end{array}$ & Name & $\begin{array}{l}\text { UniProt } \\
\text { ID }\end{array}$ & Residue 1 & Residue 2 & FDR 1\% & FDR 3\% & FDR 5\% & FDR $1 \%$ & F DR3\% & F DR5\% \\
\hline & & & & 485 & 462 & 2 & 3 & 3 & 0.483 & 0.483 & 0.483 \\
\hline & & & & 569 & 490 & & 2 & 3 & & 0.531 & 0.531 \\
\hline & & & & 602 & 490 & 1 & 2 & 2 & 0.510 & 0.510 & 0.510 \\
\hline & & SF3B2 & Q13435 & 229 & 556 & 7 & 11 & 11 & 1.150 & 1.150 & 1.150 \\
\hline & & SKIP & Q13573 & 388 & 23 & 2 & 2 & 2 & 1.679 & 1.679 & 1.679 \\
\hline & & SYF1 & Q9HCS7 & 388 & 482 & 25 & 30 & 34 & 1.075 & 1.075 & 1.075 \\
\hline & & & & 427 & 482 & 2 & 3 & 4 & 0.607 & 0.607 & 0.607 \\
\hline & & & & 475 & 482 & 2 & 3 & 4 & 0.691 & 0.691 & 0.691 \\
\hline & & ZNF830 & Q96NB3 & 445 & 228 & 3 & 4 & 4 & 0.587 & 0.587 & 0.587 \\
\hline & & & & & 234 & 14 & 15 & 15 & 2.437 & 2.437 & 2.437 \\
\hline & & & & 460 & 234 & 9 & 12 & 15 & 1.573 & 1.573 & 1.573 \\
\hline & & & & 475 & 234 & 1 & 4 & 6 & 0.670 & 0.670 & 0.670 \\
\hline \multirow[t]{17}{*}{ TCERG1 } & O14776 & BRR2 & O75643 & 570 & 60 & 3 & 5 & 6 & 0.683 & 0.683 & 0.683 \\
\hline & & & & 634 & 440 & & 3 & 3 & & 1.936 & 1.936 \\
\hline & & BUD31 & P41223 & 711 & 68 & & 4 & 5 & & 0.521 & 0.521 \\
\hline & & CBP80 & Q09161 & 756 & 187 & 1 & 1 & 1 & 0.510 & 0.510 & 0.510 \\
\hline & & MFAP1 & P55081 & 878 & 415 & 4 & 9 & 10 & 1.176 & 1.176 & 1.176 \\
\hline & & PRP8 & Q6P2Q9 & 1024 & 1210 & 6 & 16 & 23 & 0.768 & 0.768 & 0.768 \\
\hline & & SF3B1 & O75533 & 794 & 1 & & & 1 & & & 0.184 \\
\hline & & SNU114 & Q15029 & 992 & 914 & 5 & 6 & 6 & 1.894 & 1.894 & 1.894 \\
\hline & & & & & 963 & & & 2 & & & 0.782 \\
\hline & & SRSF1 & Q07955 & 1016 & 179 & & 4 & 6 & & 0.488 & 0.488 \\
\hline & & & & 1024 & 179 & 2 & 3 & 3 & 0.691 & 0.691 & 0.691 \\
\hline & & U2-B" & P08579 & 495 & 109 & & 5 & 6 & & 0.271 & 0.271 \\
\hline & & & & 634 & 108 & 1 & 1 & 1 & 0.490 & 0.490 & 0.490 \\
\hline & & & & & 109 & & 2 & 2 & & 0.816 & 0.816 \\
\hline & & WBP11 & Q9Y2W2 & 570 & 169 & 2 & 2 & 2 & 2.062 & 2.062 & 2.062 \\
\hline & & ZC3H18 & Q86VM9 & 895 & 510 & & 2 & 2 & & 0.391 & 0.391 \\
\hline & & & & 1016 & 622 & 3 & 4 & 4 & 0.802 & 0.802 & 0.802 \\
\hline \multirow[t]{20}{*}{ U2-A' } & P09661 & ISY1 & Q9ULR0 & 193 & 266 & 17 & 17 & 17 & 2.627 & 2.627 & 2.627 \\
\hline & & & & 221 & 266 & 6 & 6 & 6 & 2.095 & 2.095 & 2.095 \\
\hline & & MBPMS2 & $\begin{array}{l}\text { P0AEX9- } \\
\mathrm{r}\end{array}$ & 192 & 103 & & & 3 & & & 0.191 \\
\hline & & SF3A1 & Q15459 & 193 & 188 & 18 & 18 & 18 & 3.097 & 3.097 & 3.097 \\
\hline & & & & 205 & 223 & 2 & 8 & 11 & 0.421 & 0.421 & 0.421 \\
\hline & & & & 221 & 188 & 10 & 10 & 10 & 2.230 & 2.230 & 2.230 \\
\hline & & SF3A3 & Q12874 & 30 & 97 & 95 & 101 & 103 & 25.397 & 25.397 & 25.397 \\
\hline & & & & 56 & 69 & 2 & 2 & 2 & 1.204 & 1.204 & 1.204 \\
\hline & & & & 179 & 64 & 4 & 4 & 4 & 1.268 & 1.268 & 1.268 \\
\hline & & & & & 69 & 31 & 32 & 32 & 3.188 & 3.188 & 3.188 \\
\hline & & & & 193 & 64 & 2 & 3 & 3 & 1.758 & 1.758 & 1.758 \\
\hline & & & & & 92 & 4 & 5 & 5 & 0.961 & 0.961 & 0.961 \\
\hline & & SF3B1 & O75533 & 193 & 175 & & 2 & 2 & & 0.263 & 0.263 \\
\hline & & SF3B2 & Q13435 & 172 & 320 & 2 & 3 & 3 & 0.433 & 0.433 & 0.433 \\
\hline & & & & 179 & 280 & 3 & 5 & 7 & 0.628 & 0.628 & 0.628 \\
\hline & & & & 191 & 280 & & & 2 & & & 0.203 \\
\hline & & & & 193 & 280 & 16 & 17 & 18 & 2.415 & 2.415 & 2.415 \\
\hline & & SYF1 & Q9HCS7 & 193 & 50 & & 5 & 6 & & 0.368 & 0.368 \\
\hline & & & & 205 & 2 & 5 & 6 & 6 & 1.644 & 1.644 & 1.644 \\
\hline & & U2-B" & P08579 & 30 & 111 & 2 & 2 & 2 & 1.466 & 1.466 & 1.466 \\
\hline U2-B" & $\begin{array}{l}\text { P08579 } \\
\end{array}$ & SF3A3 & Q12874 & 57 & 97 & 3 & 5 & 5 & 0.721 & 0.721 & 0.721 \\
\hline & & & & 103 & 463 & & & 5 & & & 0.209 \\
\hline & & SF3B2 & Q13435 & 111 & 352 & 4 & 7 & 7 & 0.931 & 0.931 & 0.931 \\
\hline & & SmD3 & P62318 & 93 & 84 & 3 & 3 & 3 & 1.443 & 1.443 & 1.443 \\
\hline & & & & 101 & 84 & & & 2 & & & 0.290 \\
\hline & & TCERG1 & O14776 & 108 & 634 & 1 & 1 & 1 & 0.490 & 0.490 & 0.490 \\
\hline & & & & 109 & 495 & & 5 & 6 & & 0.271 & 0.271 \\
\hline & & & & & 634 & & 2 & 2 & & 0.816 & 0.816 \\
\hline & & U2-A' & $\begin{array}{l}\text { P09661 } \\
\end{array}$ & 111 & 30 & 2 & 2 & 2 & 1.466 & 1.466 & 1.466 \\
\hline U5-40K & Q96DI7 & CDC5L & Q99459 & 270 & 795 & 2 & 3 & 5 & 0.515 & 0.515 & 0.515 \\
\hline & & $\begin{array}{l}\text { PPIL1 } \\
\end{array}$ & Q9Y3C6 & 1 & 80 & 26 & 33 & 35 & 1.630 & 1.630 & 1.630 \\
\hline & & & & 6 & 80 & & 6 & 7 & & 0.430 & 0.430 \\
\hline & & & & 8 & 80 & 8 & 8 & 8 & 3.983 & 3.983 & 3.983 \\
\hline & & & & 18 & 80 & 13 & 13 & 13 & 1.974 & 1.974 & 1.974 \\
\hline & & & & 270 & 80 & & 2 & 2 & & 0.245 & 0.245 \\
\hline & & & & 275 & 80 & 17 & 18 & 18 & 2.524 & 2.524 & 2.524 \\
\hline & & & & 322 & 80 & 4 & 4 & 4 & 2.753 & 2.753 & 2.753 \\
\hline & & & & 349 & 80 & 3 & 3 & 3 & 1.773 & 1.773 & 1.773 \\
\hline & & $\begin{array}{l}\text { PRP19 } \\
\text { PR }\end{array}$ & Q9UMS4 & 1 & 179 & 2 & 2 & 2 & 0.608 & 0.608 & 0.608 \\
\hline & & & & & 244 & & 2 & 2 & & 0.588 & 0.588 \\
\hline & & & & 226 & 122 & & 2 & 3 & & 0.555 & 0.555 \\
\hline & & & & 270 & 122 & & 6 & 9 & & 0.451 & 0.451 \\
\hline & & & & & 192 & & 3 & 4 & & 0.457 & 0.457 \\
\hline & & & & 275 & 122 & 17 & 17 & 18 & 1.655 & 1.655 & 1.655 \\
\hline & & & & & 192 & 21 & 22 & 22 & 3.772 & 3.772 & 3.772 \\
\hline & & & & & 244 & 17 & 17 & 17 & 1.749 & 1.749 & 1.749 \\
\hline & & & & & 266 & & 2 & 2 & & 0.596 & 0.596 \\
\hline & & & & & 425 & & 3 & 4 & & 0.286 & 0.286 \\
\hline & & PRP8 & Q6P2Q9 & 131 & 50 & & 2 & 2 & & 1.633 & 1.633 \\
\hline & & & & 286 & 29 & 22 & 22 & 22 & 2.107 & 2.107 & 2.107 \\
\hline & & & & & 36 & 2 & 3 & 5 & 2.014 & 2.014 & 2.014 \\
\hline & & & & & 43 & 29 & 44 & 50 & 2.810 & 2.810 & 2.810 \\
\hline & & & & 349 & 50 & 11 & 11 & 11 & 7.715 & 7.715 & 7.715 \\
\hline
\end{tabular}




\begin{tabular}{|c|c|c|c|c|c|c|c|c|c|c|c|}
\hline Name & $\begin{array}{l}\text { UniProt } \\
\text { ID }\end{array}$ & Name & $\begin{array}{l}\text { UniProt } \\
\text { ID }\end{array}$ & Residue 1 & Residue 2 & FDR $1 \%$ & FDR 3\% & FDR 5\% & FDR $1 \%$ & FDR3\% & F DR5\% \\
\hline & & SKIP & Q13573 & 1 & 193 & 7 & 9 & 9 & 2.990 & 2.990 & 2.990 \\
\hline & & & & 18 & 81 & 5 & 6 & 6 & 1.837 & 1.837 & 1.837 \\
\hline & & & & & 97 & 2 & 3 & 3 & 0.691 & 0.691 & 0.691 \\
\hline & & & & & 110 & 1 & 2 & 2 & 0.560 & 0.560 & 0.560 \\
\hline & & & & 275 & 48 & 4 & 4 & 4 & 2.240 & 2.240 & 2.240 \\
\hline & & & & & 81 & 6 & 6 & 6 & 2.195 & 2.195 & 2.195 \\
\hline & & & & 322 & 48 & 5 & 6 & 6 & 1.276 & 1.276 & 1.276 \\
\hline & & & & & 97 & 2 & 2 & 2 & 1.031 & 1.031 & 1.031 \\
\hline & & & & 349 & 153 & 6 & 7 & 10 & 1.331 & 1.331 & 1.331 \\
\hline & & & & & 170 & & 2 & 3 & & 0.850 & 0.850 \\
\hline & & SmD1 & $\mathrm{P} 62314$ & 226 & 86 & 2 & 2 & 3 & 0.520 & 0.520 & 0.520 \\
\hline & & SNU23 & Q96NC0 & 8 & 136 & & 1 & 2 & & 0.235 & 0.235 \\
\hline & & SPF27 & O75934 & 8 & 168 & 5 & 5 & 5 & 2.865 & 2.865 & 2.865 \\
\hline & & & & 18 & 168 & 3 & 4 & 4 & 0.756 & 0.756 & 0.756 \\
\hline & & & & 226 & 168 & 6 & 8 & 8 & 1.298 & 1.298 & 1.298 \\
\hline & & & & 270 & 168 & 6 & 9 & 10 & 0.617 & 0.617 & 0.617 \\
\hline & & & & 275 & 168 & 43 & 46 & 47 & 3.253 & 3.253 & 3.253 \\
\hline & & & & & 177 & 2 & 5 & 6 & 1.211 & 1.211 & 1.211 \\
\hline & & & & 322 & 168 & 3 & 3 & 3 & 1.409 & 1.409 & 1.409 \\
\hline UBL5 & Q9BZL1 & SRSF1 & Q07955 & 1 & 179 & & & 2 & & & 1.037 \\
\hline \multirow[t]{44}{*}{ WBP11 } & Q9Y2W2 & BRR2 & O75643 & 48 & 85 & & & 2 & & & 0.189 \\
\hline & & & & 59 & 85 & 2 & 2 & 2 & 2.734 & 2.734 & 2.734 \\
\hline & & & & 168 & 14 & 5 & 5 & 5 & 0.851 & 0.851 & 0.851 \\
\hline & & & & & 60 & 2 & 4 & 4 & 0.754 & 0.754 & 0.754 \\
\hline & & & & & 85 & 2 & 2 & 2 & 14.531 & 14.531 & 14.531 \\
\hline & & & & 169 & 60 & 4 & 5 & 6 & 1.501 & 1.501 & 1.501 \\
\hline & & $\begin{array}{l}\text { PLRG1 } \\
\end{array}$ & O43660 & 10 & 268 & & 4 & 5 & & 0.649 & 0.649 \\
\hline & & PPP1R8 & Q12972 & 610 & 234 & & 1 & 1 & & 0.291 & 0.291 \\
\hline & & PQBP1 & O60828 & 168 & 2 & 2 & 2 & 2 & 1.542 & 1.542 & 1.542 \\
\hline & & & & & 18 & 4 & 4 & 4 & 9.348 & 9.348 & 9.348 \\
\hline & & & & & 87 & & 3 & 3 & & 0.836 & 0.836 \\
\hline & & & & 169 & 18 & 2 & 2 & 2 & 1.262 & 1.262 & 1.262 \\
\hline & & & & & 87 & 8 & 9 & 9 & 1.887 & 1.887 & 1.887 \\
\hline & & PRP8 & Q6P2Q9 & 10 & 674 & & 3 & 3 & & 0.307 & 0.307 \\
\hline & & & & & 746 & 5 & 8 & 9 & 0.983 & 0.983 & 0.983 \\
\hline & & & & & 774 & & 2 & 2 & & 0.351 & 0.351 \\
\hline & & & & 13 & 674 & & 3 & 3 & & 0.598 & 0.598 \\
\hline & & & & & 774 & 2 & 4 & 4 & 1.084 & 1.084 & 1.084 \\
\hline & & & & 48 & 2034 & & 4 & 5 & & 0.343 & 0.343 \\
\hline & & & & 51 & 2031 & & 5 & 11 & & 0.570 & 0.570 \\
\hline & & & & & 2034 & & 8 & 9 & & 0.360 & 0.360 \\
\hline & & RED & Q13123 & 10 & 534 & 4 & 5 & 5 & 1.069 & 1.069 & 1.069 \\
\hline & & SF3A1 & Q15459 & 599 & 115 & 2 & 2 & 2 & 1.264 & 1.264 & 1.264 \\
\hline & & & & 610 & 102 & 15 & 29 & 31 & 1.682 & 1.682 & 1.682 \\
\hline & & & & & 105 & & 3 & 3 & & 0.478 & 0.478 \\
\hline & & & & & 115 & 23 & 29 & 30 & 1.198 & 1.198 & 1.198 \\
\hline & & & & & 131 & 4 & 4 & 4 & 0.781 & 0.781 & 0.781 \\
\hline & & & & 614 & 102 & 4 & 7 & 7 & 0.502 & 0.802 & 0.802 \\
\hline & & & & & 105 & 9 & 11 & 11 & 1.115 & 1.115 & 1.115 \\
\hline & & & & & 115 & 20 & 20 & 20 & 2.278 & 2.278 & 2.278 \\
\hline & & & & & 131 & 4 & 4 & 4 & 1.208 & 1.208 & 1.208 \\
\hline & & & & 626 & 115 & 2 & 2 & 2 & 2.172 & 2.172 & 2.172 \\
\hline & & & & & 131 & 2 & 2 & 2 & 1.057 & 1.057 & 1.057 \\
\hline & & SF 3A2 & Q15428 & 556 & 118 & 27 & 36 & 38 & 2.024 & 2.024 & 2.024 \\
\hline & & & & 557 & 118 & 32 & 53 & 59 & 0.733 & 0.733 & 0.733 \\
\hline & & & & 565 & 118 & 31 & 79 & 103 & 0.719 & 0.719 & 0.719 \\
\hline & & SF3B2 & Q13435 & 98 & 556 & 5 & 5 & 6 & 0.484 & 0.484 & 0.484 \\
\hline & & & & 105 & 556 & 3 & 7 & 8 & 0.643 & 0.643 & 0.643 \\
\hline & & SNU23 & Q96NC0 & 48 & 70 & & 4 & 6 & & 0.325 & 0.325 \\
\hline & & & & 59 & 70 & 2 & 2 & 2 & 3.695 & 3.695 & 3.695 \\
\hline & & & & 76 & 45 & 4 & 7 & 8 & 1.092 & 1.092 & 1.092 \\
\hline & & & & & 102 & 18 & 23 & 23 & 2.737 & 2.737 & 2.737 \\
\hline & & & & 169 & 8 & & 2 & 2 & & 0.377 & 0.377 \\
\hline & & TCERG1 & O14776 & 169 & 570 & 2 & 2 & 2 & 2.062 & 2.062 & 2.062 \\
\hline ZC3H18 & Q86VM9 & CBP80 & Q09161 & 319 & 221 & 19 & 21 & 22 & 1.585 & 1.585 & 1.585 \\
\hline & & $\begin{array}{l}\text { PRP19 } \\
\end{array}$ & Q9UMS4 & 918 & 179 & 9 & 10 & 10 & 2.517 & 2.517 & 2.517 \\
\hline & & & & 921 & 179 & 7 & 7 & 7 & 1.175 & 1.175 & 1.175 \\
\hline & & PRP4B & Q13523 & 933 & 99 & 2 & 2 & 2 & 0.956 & 0.956 & 0.956 \\
\hline & & & & 936 & 117 & 34 & 35 & 35 & 2.034 & 2.034 & 2.034 \\
\hline & & & & 948 & 99 & 26 & 34 & 42 & 1.058 & 1.058 & 1.058 \\
\hline & & & & 952 & 99 & 32 & 42 & 45 & 1.825 & 1.825 & 1.825 \\
\hline & & $\begin{array}{l}\text { PRP8 } \\
\end{array}$ & Q6P2Q9 & 499 & 366 & 2 & 2 & 2 & 0.913 & 0.913 & 0.913 \\
\hline & & & & 500 & 366 & 6 & 9 & 11 & 0.807 & 0.807 & 0.807 \\
\hline & & & & 510 & 366 & & 2 & 2 & & 0.310 & 0.310 \\
\hline & & SNU114 & Q15029 & 500 & 359 & & 2 & 2 & & 0.547 & 0.547 \\
\hline & & & & 510 & 244 & 4 & 6 & 8 & 0.795 & 0.795 & 0.795 \\
\hline & & & & & 694 & & 2 & 3 & & 0.636 & 0.636 \\
\hline & & SRRT & Q9BXP5 & 188 & 730 & & 3 & 4 & & 0.324 & 0.324 \\
\hline & & & & & 744 & 2 & 3 & 3 & 1.091 & 1.091 & 1.091 \\
\hline & & & & 211 & 723 & & 3 & 3 & & 0.488 & 0.488 \\
\hline & & TCERG1 & O14776 & 510 & 895 & & 2 & 2 & & 0.391 & 0.391 \\
\hline & & & & 622 & 1016 & 3 & 4 & 4 & 0.802 & 0.802 & 0.802 \\
\hline ZNF830 & Q96NB3 & CWC15 & Q9P013 & 26 & 147 & & 2 & 2 & & 0.405 & 0.405 \\
\hline
\end{tabular}


Table B.1 - Continued from previous page

\begin{tabular}{|c|c|c|c|c|c|c|c|c|c|c|c|}
\hline Name & $\begin{array}{l}\text { UniProt } \\
\text { ID }\end{array}$ & Name & $\begin{array}{l}\text { UniProt } \\
\text { ID }\end{array}$ & Residue 1 & Residue 2 & FDR 1\% & FDR $3 \%$ & FDR $5 \%$ & F DR1\% & F DR3\% & F DR5\% \\
\hline & & PPIL2 & Q13356 & 228 & 313 & 8 & 8 & 8 & 1.629 & 1.629 & 1.629 \\
\hline & & & & & 450 & 5 & 7 & 9 & 1.095 & 1.095 & 1.095 \\
\hline & & & & & 454 & 2 & 3 & 3 & 0.679 & 0.679 & 0.679 \\
\hline & & & & & 462 & 4 & 4 & 4 & 1.222 & 1.222 & 1.222 \\
\hline & & & & 234 & 313 & 29 & 30 & 32 & 2.622 & 2.622 & 2.622 \\
\hline & & & & & 450 & 5 & 5 & 5 & 2.125 & 2.125 & 2.125 \\
\hline & & & & & 460 & & 3 & 3 & & 0.544 & 0.544 \\
\hline & & & & & 462 & & & 1 & & & 0.637 \\
\hline & & SYF1 & Q9HCS7 & 216 & 654 & 7 & 7 & 7 & 2.416 & 2.416 & 2.416 \\
\hline & & & & 279 & 482 & 5 & 9 & 9 & 0.851 & 0.851 & 0.851 \\
\hline & & SYF3 & Q9BZJ0 & 228 & 445 & 3 & 4 & 4 & 0.587 & 0.587 & 0.587 \\
\hline & & & & 234 & 445 & 14 & 15 & 15 & 2.437 & 2.437 & 2.437 \\
\hline & & & & & 460 & 9 & 12 & 15 & 1.573 & 1.573 & 1.573 \\
\hline & & & & & 475 & 1 & 4 & 6 & 0.670 & 0.670 & 0.670 \\
\hline
\end{tabular}

Table B.2: Intramolecular crosslinks. Crosslink analyses by pLink2.3.5 at FDR 1, 3 and $5 \%$ are indicated. Number of CSMs (crosslinked peptide spectrum matches) and highest score are shown for each peptide. "Residue 1" and "Residue 2" denote crosslinked residues within a single protein. A single crosslinking experiment was performed and analyzed with mass spectrometry using three technical replicates. Crosslink analysis was performed by Dr. Olex Dybkov (Department of Cellular Biochemistry, MPI-BPC) in collaboration with Prof. Dr. Henning Urlaub (Bioanalytical Mass Spectrometry, MPI-BPC) (see 2.2.6.

CSMs

Score $_{\max }$

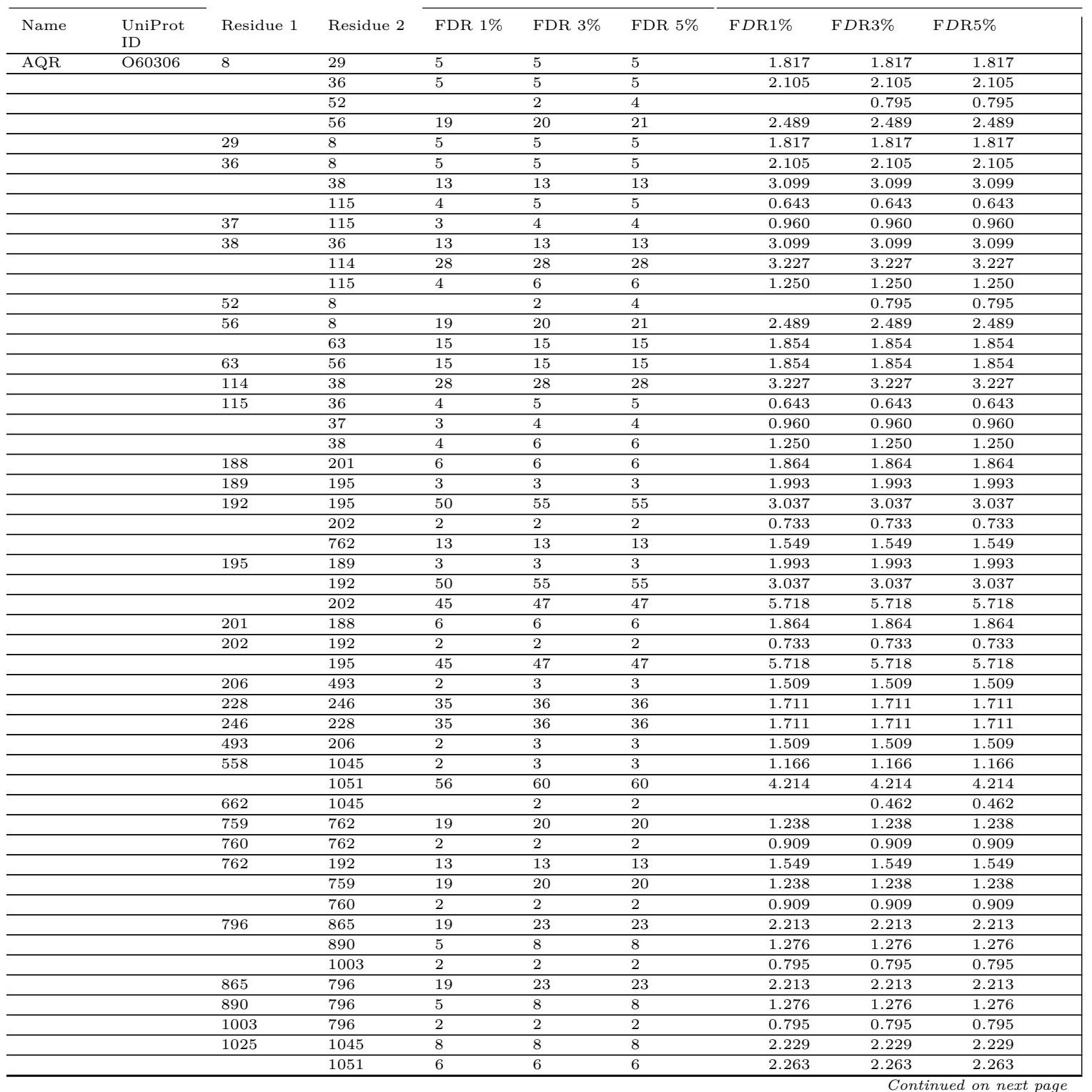


Table B.2 - Continued from previous page

\begin{tabular}{|c|c|c|c|c|c|c|c|c|c|}
\hline Name & $\begin{array}{l}\text { UniProt } \\
\text { ID }\end{array}$ & Residue 1 & Residue 2 & FDR $1 \%$ & FDR 3\% & FDR 5\% & FDR1\% & F DR3\% & FDR5\% \\
\hline & & 1045 & 558 & 2 & 3 & 3 & 1.166 & 1.166 & 1.166 \\
\hline & & & 662 & & 2 & 2 & & 0.462 & 0.462 \\
\hline & & & 1025 & 8 & 8 & 8 & 2.229 & 2.229 & 2.229 \\
\hline & & & 1051 & 75 & 77 & 77 & 2.850 & 2.850 & 2.850 \\
\hline & & 1051 & 558 & 56 & 60 & 60 & 4.214 & 4.214 & 4.214 \\
\hline & & & 1025 & 6 & 6 & 6 & 2.263 & 2.263 & 2.263 \\
\hline & & & 1045 & 75 & 77 & 77 & 2.850 & 2.850 & 2.850 \\
\hline & & 1234 & 1256 & 8 & 11 & 11 & 1.164 & 1.164 & 1.164 \\
\hline & & 1256 & 1234 & 8 & 11 & 11 & 1.164 & 1.164 & 1.164 \\
\hline \multirow[t]{74}{*}{ BRR2 } & O75643 & 1 & 1711 & & 2 & 2 & & 0.225 & 0.225 \\
\hline & & 14 & 46 & 38 & 39 & 39 & 3.985 & 3.985 & 3.985 \\
\hline & & & 55 & 30 & 34 & 34 & 1.935 & 1.935 & 1.935 \\
\hline & & & 60 & 28 & 28 & 28 & 2.908 & 2.908 & 2.908 \\
\hline & & & 73 & 9 & 10 & 10 & 1.822 & 1.822 & 1.822 \\
\hline & & & 83 & 5 & 5 & 5 & 2.027 & 2.027 & 2.027 \\
\hline & & & 85 & 5 & 5 & 5 & 2.679 & 2.679 & 2.679 \\
\hline & & & 151 & 3 & 3 & 3 & 0.635 & 0.635 & 0.635 \\
\hline & & & 349 & 2 & 2 & 2 & 2.321 & 2.321 & 2.321 \\
\hline & & & 1874 & 3 & 3 & 3 & 1.291 & 1.291 & 1.291 \\
\hline & & 46 & 14 & 38 & 39 & 39 & 3.985 & 3.985 & 3.985 \\
\hline & & & 55 & 93 & 101 & 101 & 1.853 & 1.853 & 1.853 \\
\hline & & & 60 & 129 & 137 & 137 & 3.400 & 3.400 & 3.400 \\
\hline & & & 73 & 3 & 3 & 3 & 2.184 & 2.184 & 2.184 \\
\hline & & & 1603 & 3 & 3 & 3 & 1.432 & 1.432 & 1.432 \\
\hline & & 55 & 14 & 30 & 34 & 34 & 1.935 & 1.935 & 1.935 \\
\hline & & & 46 & 93 & 101 & 101 & 1.853 & 1.853 & 1.853 \\
\hline & & & 60 & 122 & 130 & 130 & 1.998 & 1.998 & 1.998 \\
\hline & & & 70 & 7 & 9 & 10 & 0.802 & 0.802 & 0.802 \\
\hline & & & 83 & 8 & 8 & 8 & 2.137 & 2.137 & 2.137 \\
\hline & & & 103 & 9 & 9 & 9 & 1.088 & 1.088 & 1.088 \\
\hline & & & 107 & 2 & 2 & 2 & 0.893 & 0.893 & 0.893 \\
\hline & & 60 & 14 & 28 & 28 & 28 & 2.908 & 2.908 & 2.908 \\
\hline & & & 46 & 129 & 137 & 137 & 3.400 & 3.400 & 3.400 \\
\hline & & & 55 & 122 & 130 & 130 & 1.998 & 1.998 & 1.998 \\
\hline & & & 70 & 20 & 23 & 23 & 1.292 & 1.292 & 1.292 \\
\hline & & & 73 & 24 & 25 & 25 & 2.330 & 2.330 & 2.330 \\
\hline & & & 83 & 13 & 13 & 14 & 1.757 & 1.757 & 1.757 \\
\hline & & & 103 & 4 & 4 & 4 & 2.294 & 2.294 & 2.294 \\
\hline & & & 107 & 8 & 13 & 13 & 1.385 & 1.385 & 1.385 \\
\hline & & & 155 & & 2 & 2 & & 1.082 & 1.082 \\
\hline & & 70 & 55 & 7 & 9 & 10 & 0.802 & 0.802 & 0.802 \\
\hline & & & 60 & 20 & 23 & 23 & 1.292 & 1.292 & 1.292 \\
\hline & & & 83 & 2 & 2 & 2 & 0.617 & 0.617 & 0.617 \\
\hline & & & 155 & & 2 & 2 & & 0.620 & 0.620 \\
\hline & & 73 & 14 & 9 & 10 & 10 & 1.822 & 1.822 & 1.822 \\
\hline & & & 46 & 3 & 3 & 3 & 2.184 & 2.184 & 2.184 \\
\hline & & & 60 & 24 & 25 & 25 & 2.330 & 2.330 & 2.330 \\
\hline & & & 83 & 13 & 13 & 13 & 1.771 & 1.771 & 1.771 \\
\hline & & & 85 & 3 & 3 & 3 & 3.539 & 3.539 & 3.539 \\
\hline & & & 103 & 2 & 2 & 2 & 0.534 & 0.534 & 0.534 \\
\hline & & & 155 & 6 & 6 & 6 & 1.497 & 1.497 & 1.497 \\
\hline & & 83 & 14 & 5 & 5 & 5 & 2.027 & 2.027 & 2.027 \\
\hline & & & 55 & 8 & 8 & 8 & 2.137 & 2.137 & 2.137 \\
\hline & & & 60 & 13 & 13 & 14 & 1.757 & 1.757 & 1.757 \\
\hline & & & 70 & 2 & 2 & 2 & 0.617 & 0.617 & 0.617 \\
\hline & & & 73 & 13 & 13 & 13 & 1.771 & 1.771 & 1.771 \\
\hline & & & 103 & 8 & 8 & 8 & 2.288 & 2.288 & 2.288 \\
\hline & & & 107 & 12 & 14 & 15 & 2.163 & 2.163 & 2.163 \\
\hline & & & 155 & & 2 & 2 & & 0.357 & 0.357 \\
\hline & & 85 & 14 & 5 & 5 & 5 & 2.679 & 2.679 & 2.679 \\
\hline & & & 73 & 3 & 3 & 3 & 3.539 & 3.539 & 3.539 \\
\hline & & & 155 & 3 & 3 & 3 & 4.038 & 4.038 & 4.038 \\
\hline & & 103 & 55 & 9 & 9 & 9 & 1.088 & 1.088 & 1.088 \\
\hline & & & 60 & 4 & 4 & 4 & 2.294 & 2.294 & 2.294 \\
\hline & & & 73 & 2 & 2 & 2 & 0.534 & 0.534 & 0.534 \\
\hline & & & 83 & 8 & 8 & 8 & 2.288 & 2.288 & 2.288 \\
\hline & & & 107 & 3 & 3 & 3 & 1.323 & 1.323 & 1.323 \\
\hline & & 107 & 55 & 2 & 2 & 2 & 0.893 & 0.893 & 0.893 \\
\hline & & & 60 & 8 & 13 & 13 & 1.385 & 1.385 & 1.385 \\
\hline & & & 83 & 12 & 14 & 15 & 2.163 & 2.163 & 2.163 \\
\hline & & & 103 & 3 & 3 & 3 & 1.323 & 1.323 & 1.323 \\
\hline & & & 178 & 3 & 3 & 3 & 1.456 & 1.456 & 1.456 \\
\hline & & 147 & 155 & 22 & 43 & 43 & 1.076 & 1.076 & 1.076 \\
\hline & & 151 & 14 & 3 & 3 & 3 & 0.635 & 0.635 & 0.635 \\
\hline & & & 155 & 95 & 130 & 131 & 1.684 & 1.684 & 1.684 \\
\hline & & & 177 & 10 & 10 & 10 & 1.088 & 1.088 & 1.088 \\
\hline & & & 1049 & 26 & 27 & 27 & 1.171 & 1.171 & 1.171 \\
\hline & & 155 & 60 & & 2 & 2 & & 1.082 & 1.082 \\
\hline & & & 70 & & 2 & 2 & & 0.620 & 0.620 \\
\hline & & & 73 & 6 & 6 & 6 & 1.497 & 1.497 & 1.497 \\
\hline & & & 83 & & 2 & 2 & & 0.357 & 0.357 \\
\hline & & & 85 & 3 & 3 & 3 & 4.038 & 4.038 & 4.038 \\
\hline & & & 147 & 22 & 43 & 43 & 1.076 & 1.076 & 1.076 \\
\hline
\end{tabular}


Table B.2 - Continued from previous page

\begin{tabular}{|c|c|c|c|c|c|c|c|c|c|}
\hline Name & $\begin{array}{l}\text { UniProt } \\
\text { ID }\end{array}$ & Residue 1 & Residue 2 & FDR $1 \%$ & FDR 3\% & FDR $5 \%$ & F $D \mathrm{R} 1 \%$ & F DR3\% & F DR5\% \\
\hline & & & 151 & 95 & 130 & 131 & 1.684 & 1.684 & 1.684 \\
\hline & & & 1172 & & 3 & 3 & & 0.233 & 0.233 \\
\hline & & 177 & 151 & 10 & 10 & 10 & 1.088 & 1.088 & 1.088 \\
\hline & & 178 & 107 & 3 & 3 & 3 & 1.456 & 1.456 & 1.456 \\
\hline & & 254 & 256 & 31 & 32 & 32 & 3.300 & 3.300 & 3.300 \\
\hline & & & 487 & 2 & 2 & 2 & 0.556 & 0.556 & 0.556 \\
\hline & & 255 & 349 & 23 & 23 & 23 & 3.695 & 3.695 & 3.695 \\
\hline & & & 358 & 6 & 6 & 6 & 3.151 & 3.151 & 3.151 \\
\hline & & & 368 & 44 & 44 & 44 & 1.869 & 1.869 & 1.869 \\
\hline & & & 944 & 12 & 12 & 12 & 2.552 & 2.552 & 2.552 \\
\hline & & 256 & 254 & 31 & 32 & 32 & 3.300 & 3.300 & 3.300 \\
\hline & & & 319 & 5 & 7 & 7 & 2.131 & 2.131 & 2.131 \\
\hline & & & 349 & 2 & 2 & 2 & 2.677 & 2.677 & 2.677 \\
\hline & & & 358 & 11 & 12 & 12 & 3.565 & 3.565 & 3.565 \\
\hline & & & 368 & 34 & 37 & 38 & 2.197 & 2.197 & 2.197 \\
\hline & & & 487 & 2 & 3 & 3 & 1.893 & 1.893 & 1.893 \\
\hline & & & 729 & & & 2 & & & 0.180 \\
\hline & & & 944 & 11 & 11 & 11 & 1.776 & 1.776 & 1.776 \\
\hline & & & 1134 & & 2 & 2 & & 0.304 & 0.304 \\
\hline & & & 1556 & 2 & 2 & 2 & 2.590 & 2.590 & 2.590 \\
\hline & & 285 & 966 & 2 & 2 & 2 & 2.279 & 2.279 & 2.279 \\
\hline & & & 1874 & 8 & 8 & 8 & 3.147 & 3.147 & 3.147 \\
\hline & & 294 & 349 & 30 & 30 & 30 & 4.022 & 4.022 & 4.022 \\
\hline & & 319 & 256 & 5 & 7 & 7 & 2.131 & 2.131 & 2.131 \\
\hline & & 349 & 14 & 2 & 2 & 2 & 2.321 & 2.321 & 2.321 \\
\hline & & & 255 & 23 & 23 & 23 & 3.695 & 3.695 & 3.695 \\
\hline & & & 256 & 2 & 2 & 2 & 2.677 & 2.677 & 2.677 \\
\hline & & & 294 & 30 & 30 & 30 & 4.022 & 4.022 & 4.022 \\
\hline & & & 695 & 2 & 2 & 2 & 1.214 & 1.214 & 1.214 \\
\hline & & & 944 & 3 & 4 & 4 & 2.364 & 2.364 & 2.364 \\
\hline & & & 1544 & 2 & 3 & 3 & 0.379 & 0.379 & 0.379 \\
\hline & & 358 & 255 & 6 & 6 & 6 & 3.151 & 3.151 & 3.151 \\
\hline & & & 256 & 11 & 12 & 12 & 3.565 & 3.565 & 3.565 \\
\hline & & & 944 & 3 & 3 & 3 & 3.470 & 3.470 & 3.470 \\
\hline & & 368 & 255 & 44 & 44 & 44 & 1.869 & 1.869 & 1.869 \\
\hline & & & 256 & 34 & 37 & 38 & 2.197 & 2.197 & 2.197 \\
\hline & & & 426 & 8 & 8 & 8 & 1.389 & 1.389 & 1.389 \\
\hline & & & 944 & 33 & 33 & 33 & 2.410 & 2.410 & 2.410 \\
\hline & & & 975 & 3 & 3 & 3 & 1.561 & 1.561 & 1.561 \\
\hline & & & 1556 & 5 & 5 & 5 & 1.443 & 1.443 & 1.443 \\
\hline & & 426 & 368 & 8 & 8 & 8 & 1.389 & 1.389 & 1.389 \\
\hline & & & 451 & 3 & 3 & 3 & 2.538 & 2.538 & 2.538 \\
\hline & & & 944 & 51 & 52 & 52 & 2.941 & 2.941 & 2.941 \\
\hline & & 440 & 695 & 3 & 6 & 7 & 0.545 & 0.545 & 0.545 \\
\hline & & & 696 & 78 & 88 & 90 & 4.284 & 4.284 & 4.284 \\
\hline & & & 699 & 3 & 9 & 9 & 0.725 & 0.725 & 0.725 \\
\hline & & & 745 & 13 & 13 & 13 & 2.935 & 2.935 & 2.935 \\
\hline & & 451 & 426 & 3 & 3 & 3 & 2.538 & 2.538 & 2.538 \\
\hline & & & 487 & 121 & 135 & 136 & 2.465 & 2.465 & 2.465 \\
\hline & & & 745 & 7 & 8 & 8 & 2.703 & 2.703 & 2.703 \\
\hline & & & 864 & 57 & 57 & 57 & 13.644 & 13.644 & 13.644 \\
\hline & & 453 & 479 & 3 & 4 & 4 & 1.400 & 1.400 & 1.400 \\
\hline & & & 487 & 363 & 378 & 378 & 7.933 & 7.933 & 7.933 \\
\hline & & & 745 & & 2 & 2 & & 1.507 & 1.507 \\
\hline & & 466 & 479 & 174 & 177 & 178 & 13.693 & 13.693 & 13.693 \\
\hline & & & 487 & 82 & 94 & 94 & 6.448 & 6.448 & 6.448 \\
\hline & & 479 & 453 & 3 & 4 & 4 & 1.400 & 1.400 & 1.400 \\
\hline & & & 466 & 174 & 177 & 178 & 13.693 & 13.693 & 13.693 \\
\hline & & & 487 & 4 & 5 & 5 & 1.064 & 1.064 & 1.064 \\
\hline & & & 557 & 32 & 33 & 33 & 4.500 & 4.500 & 4.500 \\
\hline & & 487 & 254 & 2 & 2 & 2 & 0.556 & 0.556 & 0.556 \\
\hline & & & 256 & 2 & 3 & 3 & 1.893 & 1.893 & 1.893 \\
\hline & & & 451 & 121 & 135 & 136 & 2.465 & 2.465 & 2.465 \\
\hline & & & 453 & 363 & 378 & 378 & 7.933 & 7.933 & 7.933 \\
\hline & & & 466 & 82 & 94 & 94 & 6.448 & 6.448 & 6.448 \\
\hline & & & 479 & 4 & 5 & 5 & 1.064 & 1.064 & 1.064 \\
\hline & & & 671 & 16 & 19 & 19 & 1.901 & 1.901 & 1.901 \\
\hline & & 557 & 479 & 32 & 33 & 33 & 4.500 & 4.500 & 4.500 \\
\hline & & 577 & 599 & 26 & 30 & 31 & 1.623 & 1.623 & 1.623 \\
\hline & & & 1567 & 69 & 82 & 83 & 1.594 & 1.594 & 1.594 \\
\hline & & 592 & 599 & 74 & 93 & 95 & 2.202 & 2.202 & 2.202 \\
\hline & & 599 & 577 & 26 & 30 & 31 & 1.623 & 1.623 & 1.623 \\
\hline & & & 592 & 74 & 93 & 95 & 2.202 & 2.202 & 2.202 \\
\hline & & 671 & 487 & 16 & 19 & 19 & 1.901 & 1.901 & 1.901 \\
\hline & & 695 & 349 & 2 & 2 & 2 & 1.214 & 1.214 & 1.214 \\
\hline & & & 440 & 3 & 6 & 7 & 0.545 & 0.545 & 0.545 \\
\hline & & 696 & 440 & 78 & 88 & 90 & 4.284 & 4.284 & 4.284 \\
\hline & & 699 & 440 & 3 & 9 & 9 & 0.725 & 0.725 & 0.725 \\
\hline & & 718 & 804 & 13 & 13 & 13 & 2.953 & 2.953 & 2.953 \\
\hline & & & 864 & 19 & 19 & 19 & 12.473 & 12.473 & 12.473 \\
\hline & & 729 & 256 & & & 2 & & & 0.180 \\
\hline & & 745 & 440 & 13 & 13 & 13 & 2.935 & 2.935 & 2.935 \\
\hline & & & 451 & 7 & 8 & 8 & 2.703 & 2.703 & 2.703 \\
\hline
\end{tabular}


Table B.2 - Continued from previous page

\begin{tabular}{|c|c|c|c|c|c|c|c|c|c|}
\hline Name & $\begin{array}{l}\text { UniProt } \\
\text { ID }\end{array}$ & Residue 1 & Residue 2 & FDR $1 \%$ & FDR $3 \%$ & FDR $5 \%$ & FDR $1 \%$ & FDR3\% & FDR5\% \\
\hline & & & 453 & & 2 & 2 & & 1.507 & 1.507 \\
\hline & & 770 & 1134 & 33 & 33 & 33 & 3.247 & 3.247 & 3.247 \\
\hline & & 804 & 718 & 13 & 13 & 13 & 2.953 & 2.953 & 2.953 \\
\hline & & 838 & 1025 & 2 & 2 & 2 & 1.110 & 1.110 & 1.110 \\
\hline & & 864 & 451 & 57 & 57 & 57 & 13.644 & 13.644 & 13.644 \\
\hline & & & 718 & 19 & 19 & 19 & 12.473 & 12.473 & 12.473 \\
\hline & & 914 & 1556 & 4 & 4 & 4 & 2.544 & 2.544 & 2.544 \\
\hline & & 944 & 255 & 12 & 12 & 12 & 2.552 & 2.552 & 2.552 \\
\hline & & & 256 & 11 & 11 & 11 & 1.776 & 1.776 & 1.776 \\
\hline & & & 349 & 3 & 4 & 4 & 2.364 & 2.364 & 2.364 \\
\hline & & & 358 & 3 & 3 & 3 & 3.470 & 3.470 & 3.470 \\
\hline & & & 368 & 33 & 33 & 33 & 2.410 & 2.410 & 2.410 \\
\hline & & & 426 & 51 & 52 & 52 & 2.941 & 2.941 & 2.941 \\
\hline & & 966 & 285 & 2 & 2 & 2 & 2.279 & 2.279 & 2.279 \\
\hline & & & 974 & 13 & 27 & 28 & 1.173 & 1.173 & 1.173 \\
\hline & & & 1552 & 2 & 4 & 4 & 0.678 & 0.678 & 0.678 \\
\hline & & 971 & 975 & 57 & 60 & 61 & 2.966 & 2.966 & 2.966 \\
\hline & & & 1544 & & 2 & 2 & & 0.321 & 0.321 \\
\hline & & & 1552 & 110 & 122 & 125 & 1.868 & 1.868 & 1.868 \\
\hline & & & 1711 & 273 & 281 & 281 & 2.977 & 2.977 & 2.977 \\
\hline & & & 1715 & 2 & 2 & 2 & 0.961 & 0.961 & 0.961 \\
\hline & & 974 & 966 & 13 & 27 & 28 & 1.173 & 1.173 & 1.173 \\
\hline & & & 1552 & 2 & 2 & 2 & 1.675 & 1.675 & 1.675 \\
\hline & & & 1711 & 3 & 3 & 3 & 1.570 & 1.570 & 1.570 \\
\hline & & 975 & 368 & 3 & 3 & 3 & 1.561 & 1.561 & 1.561 \\
\hline & & & 971 & 57 & 60 & 61 & 2.966 & 2.966 & 2.966 \\
\hline & & & 1552 & 40 & 40 & 40 & 3.898 & 3.898 & 3.898 \\
\hline & & & 1556 & 13 & 13 & 13 & 3.605 & 3.605 & 3.605 \\
\hline & & 1006 & 1049 & 69 & 71 & 71 & 13.732 & 13.732 & 13.732 \\
\hline & & 1025 & 838 & 2 & 2 & 2 & 1.110 & 1.110 & 1.110 \\
\hline & & 1049 & 151 & 26 & 27 & 27 & 1.171 & 1.171 & 1.171 \\
\hline & & & 1006 & 69 & 71 & 71 & 13.732 & 13.732 & 13.732 \\
\hline & & 1120 & 1134 & 165 & 172 & 173 & 5.734 & 5.734 & 5.734 \\
\hline & & & 1176 & 3 & 4 & 4 & 0.815 & 0.815 & 0.815 \\
\hline & & 1134 & 256 & & 2 & 2 & & 0.304 & 0.304 \\
\hline & & & 770 & 33 & 33 & 33 & 3.247 & 3.247 & 3.247 \\
\hline & & & 1120 & 165 & 172 & 173 & 5.734 & 5.734 & 5.734 \\
\hline & & & 1146 & 2 & 2 & 2 & 2.993 & 2.993 & 2.993 \\
\hline & & & 1169 & 43 & 47 & 47 & 2.346 & 2.346 & 2.346 \\
\hline & & & 1172 & 29 & 31 & 31 & 2.106 & 2.106 & 2.106 \\
\hline & & & 1176 & 406 & 409 & 410 & 3.816 & 3.816 & 3.816 \\
\hline & & & 1213 & 3 & 3 & 3 & 2.295 & 2.295 & 2.295 \\
\hline & & 1141 & 1146 & 6 & 6 & 6 & 1.130 & 1.130 & 1.130 \\
\hline & & 1142 & 1169 & 10 & 11 & 11 & 2.400 & 2.400 & 2.400 \\
\hline & & 1146 & 1134 & 2 & 2 & 2 & 2.993 & 2.993 & 2.993 \\
\hline & & & 1141 & 6 & 6 & 6 & 1.130 & 1.130 & 1.130 \\
\hline & & & 1176 & 2 & 2 & 2 & 1.425 & 1.425 & 1.425 \\
\hline & & 1169 & 1134 & 43 & 47 & 47 & 2.346 & 2.346 & 2.346 \\
\hline & & & 1142 & 10 & 11 & 11 & 2.400 & 2.400 & 2.400 \\
\hline & & & 1176 & 66 & 70 & 70 & 2.027 & 2.027 & 2.027 \\
\hline & & 1172 & 155 & & 3 & 3 & & 0.233 & 0.233 \\
\hline & & & 1134 & 29 & 31 & 31 & 2.106 & 2.106 & 2.106 \\
\hline & & 1176 & 1120 & 3 & 4 & 4 & 0.815 & 0.815 & 0.815 \\
\hline & & & 1134 & 406 & 409 & 410 & 3.816 & 3.816 & 3.816 \\
\hline & & & 1146 & 2 & 2 & 2 & 1.425 & 1.425 & 1.425 \\
\hline & & & 1169 & 66 & 70 & 70 & 2.027 & 2.027 & 2.027 \\
\hline & & 1213 & 1134 & 3 & 3 & 3 & 2.295 & 2.295 & 2.295 \\
\hline & & 1242 & 1567 & 39 & 39 & 39 & 2.705 & 2.705 & 2.705 \\
\hline & & & 1883 & 2 & 2 & 2 & 2.238 & 2.238 & 2.238 \\
\hline & & 1244 & 1567 & & 2 & 2 & & 0.469 & 0.469 \\
\hline & & & 1883 & 10 & 10 & 10 & 2.049 & 2.049 & 2.049 \\
\hline & & 1294 & 1498 & 11 & 12 & 12 & 10.047 & 10.047 & 10.047 \\
\hline & & & 1716 & 43 & 44 & 44 & 14.963 & 14.963 & 14.963 \\
\hline & & 1404 & 1421 & 187 & 188 & 189 & 7.255 & 7.255 & 7.255 \\
\hline & & & 2059 & 133 & 133 & 133 & 5.372 & 5.372 & 5.372 \\
\hline & & 1417 & 2059 & 72 & 72 & 72 & 3.930 & 3.930 & 3.930 \\
\hline & & & 2080 & 2 & 2 & 2 & 1.018 & 1.018 & 1.018 \\
\hline & & 1421 & 1404 & 187 & 188 & 189 & 7.255 & 7.255 & 7.255 \\
\hline & & & 1443 & 23 & 23 & 23 & 4.403 & 4.403 & 4.403 \\
\hline & & & 2059 & 498 & 502 & 503 & 41.652 & 41.652 & 41.652 \\
\hline & & 1440 & 2080 & 22 & 26 & 26 & 1.379 & 1.379 & 1.379 \\
\hline & & 1443 & 1421 & 23 & 23 & 23 & 4.403 & 4.403 & 4.403 \\
\hline & & & 1743 & 4 & 4 & 4 & 9.326 & 9.326 & 9.326 \\
\hline & & & 2080 & 7 & 9 & 9 & 2.728 & 2.728 & 2.728 \\
\hline & & 1498 & 1294 & 11 & 12 & 12 & 10.047 & 10.047 & 10.047 \\
\hline & & 1544 & 349 & 2 & 3 & 3 & 0.379 & 0.379 & 0.379 \\
\hline & & & 971 & & 2 & 2 & & 0.321 & 0.321 \\
\hline & & & 1556 & 2 & 2 & 2 & 2.290 & 2.290 & 2.290 \\
\hline & & 1552 & 966 & 2 & 4 & 4 & 0.678 & 0.678 & 0.678 \\
\hline & & & 971 & 110 & 122 & 125 & 1.868 & 1.868 & 1.868 \\
\hline & & & 974 & 2 & 2 & 2 & 1.675 & 1.675 & 1.675 \\
\hline & & & 975 & 40 & 40 & 40 & 3.898 & 3.898 & 3.898 \\
\hline & & & 1711 & 23 & 24 & 24 & 1.896 & 1.896 & 1.896 \\
\hline
\end{tabular}


Table B. 2 - Continued from previous page

\begin{tabular}{|c|c|c|c|c|c|c|c|c|c|}
\hline Name & $\begin{array}{l}\text { UniProt } \\
\text { ID }\end{array}$ & Residue 1 & Residue 2 & FDR 1\% & FDR 3\% & FDR 5\% & FDR1\% & FDR3\% & FDR5\% \\
\hline & & 1556 & 256 & 2 & 2 & 2 & 2.590 & 2.590 & 2.590 \\
\hline & & & 368 & 5 & 5 & 5 & 1.443 & 1.443 & 1.443 \\
\hline & & & 914 & 4 & 4 & 4 & 2.544 & 2.544 & 2.544 \\
\hline & & & 975 & 13 & 13 & 13 & 3.605 & 3.605 & 3.605 \\
\hline & & & 1544 & 2 & 2 & 2 & 2.290 & 2.290 & 2.290 \\
\hline & & & 1595 & 3 & 3 & 3 & 2.428 & 2.428 & 2.428 \\
\hline & & 1567 & 577 & 69 & 82 & 83 & 1.594 & 1.594 & 1.594 \\
\hline & & & 1242 & 39 & 39 & 39 & 2.705 & 2.705 & 2.705 \\
\hline & & & 1244 & & 2 & 2 & & 0.469 & 0.469 \\
\hline & & 1595 & 1556 & 3 & 3 & 3 & 2.428 & 2.428 & 2.428 \\
\hline & & & 1610 & 60 & 62 & 62 & 3.650 & 3.650 & 3.650 \\
\hline & & 1603 & 46 & 3 & 3 & 3 & 1.432 & 1.432 & 1.432 \\
\hline & & 1610 & 1595 & 60 & 62 & 62 & 3.650 & 3.650 & 3.650 \\
\hline & & 1710 & 2059 & & 2 & 2 & & 0.469 & 0.469 \\
\hline & & 1711 & 1 & & 2 & 2 & & 0.225 & 0.225 \\
\hline & & & 971 & 273 & 281 & 281 & 2.977 & 2.977 & 2.977 \\
\hline & & & 974 & 3 & 3 & 3 & 1.570 & 1.570 & 1.570 \\
\hline & & & 1552 & 23 & 24 & 24 & 1.896 & 1.896 & 1.896 \\
\hline & & & 1716 & & 3 & 3 & & 0.335 & 0.335 \\
\hline & & 1715 & 971 & 2 & 2 & 2 & 0.961 & 0.961 & 0.961 \\
\hline & & 1716 & 1294 & 43 & 44 & 44 & 14.963 & 14.963 & 14.963 \\
\hline & & & 1711 & & 3 & 3 & & 0.335 & 0.335 \\
\hline & & 1743 & 1443 & 4 & 4 & 4 & 9.326 & 9.326 & 9.326 \\
\hline & & 1841 & 1878 & 14 & 17 & 17 & 1.477 & 1.477 & 1.477 \\
\hline & & 1874 & 14 & 3 & 3 & 3 & 1.291 & 1.291 & 1.291 \\
\hline & & & 285 & 8 & 8 & 8 & 3.147 & 3.147 & 3.147 \\
\hline & & & 1883 & 2 & 2 & 2 & 0.921 & 0.921 & 0.921 \\
\hline & & & 1961 & 17 & 17 & 17 & 1.724 & 1.724 & 1.724 \\
\hline & & 1878 & 1841 & 14 & 17 & 17 & 1.477 & 1.477 & 1.477 \\
\hline & & & 1977 & 18 & 53 & 54 & 0.654 & 0.654 & 0.654 \\
\hline & & 1883 & 1242 & 2 & 2 & 2 & 2.238 & 2.238 & 2.238 \\
\hline & & & 1244 & 10 & 10 & 10 & 2.049 & 2.049 & 2.049 \\
\hline & & & 1874 & 2 & 2 & 2 & 0.921 & 0.921 & 0.921 \\
\hline & & 1961 & 1874 & 17 & 17 & 17 & 1.724 & 1.724 & 1.724 \\
\hline & & 1977 & 1878 & 18 & 53 & 54 & 0.654 & 0.654 & 0.654 \\
\hline & & 2059 & 1404 & 133 & 133 & 133 & 5.372 & 5.372 & 5.372 \\
\hline & & & 1417 & 72 & 72 & 72 & 3.930 & 3.930 & 3.930 \\
\hline & & & 1421 & 498 & 502 & 503 & 41.652 & 41.652 & 41.652 \\
\hline & & & 1710 & & 2 & 2 & & 0.469 & 0.469 \\
\hline & & 2080 & 1417 & 2 & 2 & 2 & 1.018 & 1.018 & 1.018 \\
\hline & & & 1440 & 22 & 26 & 26 & 1.379 & 1.379 & 1.379 \\
\hline & & & 1443 & 7 & 9 & 9 & 2.728 & 2.728 & 2.728 \\
\hline & & & 2087 & 4 & 5 & 5 & 0.654 & 0.654 & 0.654 \\
\hline & & & 2089 & 44 & 58 & 59 & 1.526 & 1.526 & 1.526 \\
\hline & & & 2091 & 46 & 48 & 48 & 4.314 & 4.314 & 4.314 \\
\hline & & 2087 & 2080 & 4 & 5 & 5 & 0.654 & 0.654 & 0.654 \\
\hline & & 2089 & 2080 & 44 & 58 & 59 & 1.526 & 1.526 & 1.526 \\
\hline & & 2091 & 2080 & 46 & 48 & 48 & 4.314 & 4.314 & 4.314 \\
\hline \multirow[t]{20}{*}{ BUD13 } & Q9BRD0 & 286 & 314 & & 3 & 3 & & 0.374 & 0.374 \\
\hline & & 314 & 286 & & 3 & 3 & & 0.374 & 0.374 \\
\hline & & 330 & 339 & 2 & 3 & 3 & 1.151 & 1.151 & 1.151 \\
\hline & & 333 & 340 & 7 & 7 & 7 & 2.024 & 2.024 & 2.024 \\
\hline & & 339 & 330 & 2 & 3 & 3 & 1.151 & 1.151 & 1.151 \\
\hline & & 340 & 333 & 7 & 7 & 7 & 2.024 & 2.024 & 2.024 \\
\hline & & 417 & 427 & 3 & 3 & 3 & 1.077 & 1.077 & 1.077 \\
\hline & & 427 & 417 & 3 & 3 & 3 & 1.077 & 1.077 & 1.077 \\
\hline & & 466 & 474 & 7 & 7 & 7 & 2.766 & 2.766 & 2.766 \\
\hline & & 474 & 466 & 7 & 7 & 7 & 2.766 & 2.766 & 2.766 \\
\hline & & & 499 & 2 & 2 & 2 & 1.045 & 1.045 & 1.045 \\
\hline & & 499 & 474 & 2 & 2 & 2 & 1.045 & 1.045 & 1.045 \\
\hline & & & 520 & 3 & 3 & 3 & 1.143 & 1.143 & 1.143 \\
\hline & & 520 & 499 & 3 & 3 & 3 & 1.143 & 1.143 & 1.143 \\
\hline & & 550 & 553 & 2 & 2 & 2 & 0.761 & 0.761 & 0.761 \\
\hline & & & 555 & 5 & 5 & 5 & 1.408 & 1.408 & 1.408 \\
\hline & & 553 & 550 & 2 & 2 & 2 & 0.761 & 0.761 & 0.761 \\
\hline & & 555 & 550 & 5 & 5 & 5 & 1.408 & 1.408 & 1.408 \\
\hline & & 596 & 604 & 5 & 6 & 6 & 1.109 & 1.109 & 1.109 \\
\hline & & 604 & 596 & 5 & 6 & 6 & 1.109 & 1.109 & 1.109 \\
\hline \multirow[t]{6}{*}{ BUD31 } & $\mathrm{P} 41223$ & 9 & 86 & 3 & 3 & 3 & 1.799 & 1.799 & 1.799 \\
\hline & & 28 & 40 & 12 & 13 & 13 & 1.252 & 1.252 & 1.252 \\
\hline & & 40 & 28 & 12 & 13 & 13 & 1.252 & 1.252 & 1.252 \\
\hline & & 66 & 68 & 9 & 10 & 10 & 2.412 & 2.412 & 2.412 \\
\hline & & 68 & 66 & 9 & 10 & 10 & 2.412 & 2.412 & 2.412 \\
\hline & & 86 & 9 & 3 & 3 & 3 & 1.799 & 1.799 & 1.799 \\
\hline CBP20 & P52298 & 34 & 38 & 12 & 12 & 12 & 3.135 & 3.135 & 3.135 \\
\hline & & & 120 & 56 & 59 & 59 & 2.927 & 2.927 & 2.927 \\
\hline & & 38 & 34 & 12 & 12 & 12 & 3.135 & 3.135 & 3.135 \\
\hline & & & 120 & 8 & 8 & 8 & 3.107 & 3.107 & 3.107 \\
\hline & & 75 & 78 & 20 & 21 & 21 & 2.711 & 2.711 & 2.711 \\
\hline & & & 120 & 23 & 24 & 24 & 3.666 & 3.666 & 3.666 \\
\hline & & 78 & 75 & 20 & 21 & 21 & 2.711 & 2.711 & 2.711 \\
\hline & & 120 & 34 & 56 & 59 & 59 & 2.927 & 2.927 & 2.927 \\
\hline & & & 38 & 8 & 8 & 8 & 3.107 & 3.107 & 3.107 \\
\hline
\end{tabular}


Table B. 2 - Continued from previous page

\begin{tabular}{|c|c|c|c|c|c|c|c|c|c|}
\hline Name & $\begin{array}{l}\text { UniProt } \\
\text { ID }\end{array}$ & Residue 1 & Residue 2 & FDR $1 \%$ & FDR $3 \%$ & FDR 5\% & F $D R 1 \%$ & F DR3\% & FDR5\% \\
\hline & & & 75 & 23 & 24 & 24 & 3.666 & 3.666 & 3.666 \\
\hline \multirow[t]{68}{*}{ CBP80 } & Q09161 & 17 & 20 & 121 & 129 & 130 & 5.127 & 5.127 & 5.127 \\
\hline & & & 37 & 7 & 7 & 7 & 2.035 & 2.035 & 2.035 \\
\hline & & & 67 & 45 & 47 & 47 & 2.258 & 2.258 & 2.258 \\
\hline & & & 327 & & & 1 & & & 0.128 \\
\hline & & 20 & 17 & 121 & 129 & 130 & 5.127 & 5.127 & 5.127 \\
\hline & & & 41 & 3 & 3 & 3 & 2.556 & 2.556 & 2.556 \\
\hline & & & 67 & 97 & 102 & 102 & 2.802 & 2.802 & 2.802 \\
\hline & & & 327 & 6 & 7 & 7 & 0.921 & 0.921 & 0.921 \\
\hline & & & 671 & 2 & 2 & 3 & 0.914 & 0.914 & 0.914 \\
\hline & & 37 & 17 & 7 & 7 & 7 & 2.035 & 2.035 & 2.035 \\
\hline & & & 82 & 8 & 8 & 8 & 1.997 & 1.997 & 1.997 \\
\hline & & 41 & 20 & 3 & 3 & 3 & 2.556 & 2.556 & 2.556 \\
\hline & & & 330 & 24 & 24 & 24 & 4.070 & 4.070 & 4.070 \\
\hline & & & 342 & 27 & 28 & 28 & 2.134 & 2.134 & 2.134 \\
\hline & & 67 & 17 & 45 & 47 & 47 & 2.258 & 2.258 & 2.258 \\
\hline & & & 20 & 97 & 102 & 102 & 2.802 & 2.802 & 2.802 \\
\hline & & 82 & 37 & 8 & 8 & 8 & 1.997 & 1.997 & 1.997 \\
\hline & & 188 & 707 & & 2 & 2 & & 0.324 & 0.324 \\
\hline & & 204 & 238 & 2 & 2 & 2 & 0.530 & 0.530 & 0.530 \\
\hline & & & 240 & 3 & 3 & 3 & 0.717 & 0.717 & 0.717 \\
\hline & & & 241 & 2 & 2 & 2 & 0.688 & 0.688 & 0.688 \\
\hline & & 238 & 204 & 2 & 2 & 2 & 0.530 & 0.530 & 0.530 \\
\hline & & 240 & 204 & 3 & 3 & 3 & 0.717 & 0.717 & 0.717 \\
\hline & & 241 & 204 & 2 & 2 & 2 & 0.688 & 0.688 & 0.688 \\
\hline & & 327 & 17 & & & 1 & & & 0.128 \\
\hline & & & 20 & 6 & 7 & 7 & 0.921 & 0.921 & 0.921 \\
\hline & & 330 & 41 & 24 & 24 & 24 & 4.070 & 4.070 & 4.070 \\
\hline & & 342 & 41 & 27 & 28 & 28 & 2.134 & 2.134 & 2.134 \\
\hline & & 511 & 557 & 6 & 6 & 6 & 3.386 & 3.386 & 3.386 \\
\hline & & & 568 & 10 & 10 & 10 & 3.908 & 3.908 & 3.908 \\
\hline & & 557 & 511 & 6 & 6 & 6 & 3.386 & 3.386 & 3.386 \\
\hline & & 568 & 511 & 10 & 10 & 10 & 3.908 & 3.908 & 3.908 \\
\hline & & & 607 & 67 & 68 & 68 & 4.514 & 4.514 & 4.514 \\
\hline & & 574 & 698 & 10 & 10 & 10 & 4.147 & 4.147 & 4.147 \\
\hline & & 607 & 568 & 67 & 68 & 68 & 4.514 & 4.514 & 4.514 \\
\hline & & 647 & 654 & 10 & 14 & 14 & 1.612 & 1.612 & 1.612 \\
\hline & & & 657 & 12 & 15 & 16 & 1.599 & 1.599 & 1.599 \\
\hline & & & 698 & 39 & 40 & 40 & 2.398 & 2.398 & 2.398 \\
\hline & & 650 & 657 & 258 & 262 & 262 & 3.459 & 3.459 & 3.459 \\
\hline & & & 698 & 15 & 16 & 16 & 1.961 & 1.961 & 1.961 \\
\hline & & 654 & 647 & 10 & 14 & 14 & 1.612 & 1.612 & 1.612 \\
\hline & & & 665 & 7 & 7 & 7 & 1.876 & 1.876 & 1.876 \\
\hline & & & 698 & 146 & 150 & 150 & 4.646 & 4.646 & 4.646 \\
\hline & & 657 & 647 & 12 & 15 & 16 & 1.599 & 1.599 & 1.599 \\
\hline & & & 650 & 258 & 262 & 262 & 3.459 & 3.459 & 3.459 \\
\hline & & & 698 & 5 & 5 & 5 & 2.212 & 2.212 & 2.212 \\
\hline & & 663 & 671 & 4 & 6 & 6 & 1.021 & 1.021 & 1.021 \\
\hline & & & 684 & 25 & 29 & 29 & 2.405 & 2.405 & 2.405 \\
\hline & & 665 & 654 & 7 & 7 & 7 & 1.876 & 1.876 & 1.876 \\
\hline & & & 671 & 17 & 27 & 28 & 1.142 & 1.142 & 1.142 \\
\hline & & & 684 & 128 & 141 & 142 & 3.605 & 3.605 & 3.605 \\
\hline & & & 698 & 19 & 20 & 20 & 3.958 & 3.958 & 3.958 \\
\hline & & 671 & 20 & 2 & 2 & 3 & 0.914 & 0.914 & 0.914 \\
\hline & & & 663 & 4 & 6 & 6 & 1.021 & 1.021 & 1.021 \\
\hline & & & 665 & 17 & 27 & 28 & 1.142 & 1.142 & 1.142 \\
\hline & & & 684 & 34 & 41 & 43 & 1.329 & 1.329 & 1.329 \\
\hline & & 684 & 663 & 25 & 29 & 29 & 2.405 & 2.405 & 2.405 \\
\hline & & & 665 & 128 & 141 & 142 & 3.605 & 3.605 & 3.605 \\
\hline & & & 671 & 34 & 41 & 43 & 1.329 & 1.329 & 1.329 \\
\hline & & & 698 & 8 & 8 & 8 & 3.164 & 3.164 & 3.164 \\
\hline & & 698 & 574 & 10 & 10 & 10 & 4.147 & 4.147 & 4.147 \\
\hline & & & 647 & 39 & 40 & 40 & 2.398 & 2.398 & 2.398 \\
\hline & & & 650 & 15 & 16 & 16 & 1.961 & 1.961 & 1.961 \\
\hline & & & 654 & 146 & 150 & 150 & 4.646 & 4.646 & 4.646 \\
\hline & & & 657 & 5 & 5 & 5 & 2.212 & 2.212 & 2.212 \\
\hline & & & 665 & 19 & 20 & 20 & 3.958 & 3.958 & 3.958 \\
\hline & & & 684 & 8 & 8 & 8 & 3.164 & 3.164 & 3.164 \\
\hline & & 707 & 188 & & 2 & 2 & & 0.324 & 0.324 \\
\hline \multirow[t]{14}{*}{ CCDC12 } & Q8WUD4 & 23 & 28 & & 3 & 4 & & 0.598 & 0.598 \\
\hline & & 28 & 23 & & 3 & 4 & & 0.598 & 0.598 \\
\hline & & & 32 & 6 & 6 & 6 & 1.270 & 1.270 & 1.270 \\
\hline & & & 34 & 6 & 7 & 7 & 1.421 & 1.421 & 1.421 \\
\hline & & 32 & 28 & 6 & 6 & 6 & 1.270 & 1.270 & 1.270 \\
\hline & & 34 & 28 & 6 & 7 & 7 & 1.421 & 1.421 & 1.421 \\
\hline & & & 42 & 3 & 4 & 4 & 0.736 & 0.736 & 0.736 \\
\hline & & 42 & 34 & 3 & 4 & 4 & 0.736 & 0.736 & 0.736 \\
\hline & & & 126 & & & 2 & & & 0.143 \\
\hline & & 53 & 78 & 4 & 4 & 4 & 1.778 & 1.778 & 1.778 \\
\hline & & 70 & 78 & 38 & 40 & 40 & 2.166 & 2.166 & 2.166 \\
\hline & & 71 & 78 & 23 & 25 & 25 & 1.287 & 1.287 & 1.287 \\
\hline & & 78 & 53 & 4 & 4 & 4 & 1.778 & 1.778 & 1.778 \\
\hline & & & 70 & 38 & 40 & 40 & 2.166 & 2.166 & 2.166 \\
\hline
\end{tabular}


Table B.2 - Continued from previous page

\begin{tabular}{|c|c|c|c|c|c|c|c|c|c|}
\hline Name & $\begin{array}{l}\text { UniProt } \\
\text { ID }\end{array}$ & Residue 1 & Residue 2 & FDR 1\% & FDR 3\% & FDR $5 \%$ & FDR1\% & FDR3\% & FDR5\% \\
\hline & & & 71 & 23 & 25 & 25 & 1.287 & 1.287 & 1.287 \\
\hline & & & 87 & 8 & 8 & 8 & 13.725 & 13.725 & 13.725 \\
\hline & & & 126 & 2 & 2 & 2 & 1.054 & 1.054 & 1.054 \\
\hline & & 87 & 78 & 8 & 8 & 8 & 13.725 & 13.725 & 13.725 \\
\hline & & 122 & 126 & 13 & 13 & 13 & 1.947 & 1.947 & 1.947 \\
\hline & & 126 & 42 & & & 2 & & & 0.143 \\
\hline & & & 78 & 2 & 2 & 2 & 1.054 & 1.054 & 1.054 \\
\hline & & & 122 & 13 & 13 & 13 & 1.947 & 1.947 & 1.947 \\
\hline \multirow[t]{75}{*}{ CDC5L } & Q99459 & 7 & 47 & 2 & 4 & 4 & 0.810 & 0.810 & 0.810 \\
\hline & & & 124 & 2 & 2 & 2 & 0.553 & 0.553 & 0.553 \\
\hline & & 20 & 28 & 85 & 88 & 88 & 3.752 & 3.752 & 3.752 \\
\hline & & & 60 & 6 & 6 & 6 & 3.668 & 3.668 & 3.668 \\
\hline & & & 135 & 2 & 2 & 2 & 2.037 & 2.037 & 2.037 \\
\hline & & 28 & 20 & 85 & 88 & 88 & 3.752 & 3.752 & 3.752 \\
\hline & & & 47 & 13 & 17 & 17 & 2.737 & 2.737 & 2.737 \\
\hline & & & 60 & 22 & 22 & 22 & 2.367 & 2.367 & 2.367 \\
\hline & & & 218 & 2 & 2 & 2 & 1.670 & 1.670 & 1.670 \\
\hline & & 47 & 7 & 2 & 4 & 4 & 0.810 & 0.810 & 0.810 \\
\hline & & & 28 & 13 & 17 & 17 & 2.737 & 2.737 & 2.737 \\
\hline & & 59 & 70 & 16 & 16 & 16 & 1.544 & 1.544 & 1.544 \\
\hline & & 60 & 20 & 6 & 6 & 6 & 3.668 & 3.668 & 3.668 \\
\hline & & & 28 & 22 & 22 & 22 & 2.367 & 2.367 & 2.367 \\
\hline & & 70 & 59 & 16 & 16 & 16 & 1.544 & 1.544 & 1.544 \\
\hline & & 76 & 106 & 44 & 45 & 45 & 3.678 & 3.678 & 3.678 \\
\hline & & & 124 & 13 & 13 & 13 & 1.963 & 1.963 & 1.963 \\
\hline & & 106 & 76 & 44 & 45 & 45 & 3.678 & 3.678 & 3.678 \\
\hline & & & 122 & 7 & 7 & 7 & 6.565 & 6.565 & 6.565 \\
\hline & & & 124 & 12 & 12 & 12 & 2.927 & 2.927 & 2.927 \\
\hline & & 122 & 106 & 7 & 7 & 7 & 6.565 & 6.565 & 6.565 \\
\hline & & 124 & 7 & 2 & 2 & 2 & 0.553 & 0.553 & 0.553 \\
\hline & & & 76 & 13 & 13 & 13 & 1.963 & 1.963 & 1.963 \\
\hline & & & 106 & 12 & 12 & 12 & 2.927 & 2.927 & 2.927 \\
\hline & & 135 & 20 & 2 & 2 & 2 & 2.037 & 2.037 & 2.037 \\
\hline & & & 187 & 2 & 2 & 2 & 0.700 & 0.700 & 0.700 \\
\hline & & 170 & 174 & 5 & 5 & 5 & 1.296 & 1.296 & 1.296 \\
\hline & & 174 & 170 & 5 & 5 & 5 & 1.296 & 1.296 & 1.296 \\
\hline & & & 187 & 4 & 4 & 4 & 0.625 & 0.625 & 0.625 \\
\hline & & 187 & 135 & 2 & 2 & 2 & 0.700 & 0.700 & 0.700 \\
\hline & & & 174 & 4 & 4 & 4 & 0.625 & 0.625 & 0.625 \\
\hline & & & 200 & 15 & 18 & 18 & 3.849 & 3.849 & 3.849 \\
\hline & & & 218 & 2 & 3 & 3 & 0.494 & 0.494 & 0.494 \\
\hline & & 200 & 187 & 15 & 18 & 18 & 3.849 & 3.849 & 3.849 \\
\hline & & & 218 & 9 & 10 & 11 & 2.073 & 2.073 & 2.073 \\
\hline & & & 219 & 12 & 14 & 14 & 1.696 & 1.696 & 1.696 \\
\hline & & & 255 & 6 & 6 & 6 & 2.236 & 2.236 & 2.236 \\
\hline & & 218 & 28 & 2 & 2 & 2 & 1.670 & 1.670 & 1.670 \\
\hline & & & 187 & 2 & 3 & 3 & 0.494 & 0.494 & 0.494 \\
\hline & & & 200 & 9 & 10 & 11 & 2.073 & 2.073 & 2.073 \\
\hline & & & 255 & 8 & 9 & 9 & 1.063 & 1.063 & 1.063 \\
\hline & & & 294 & 7 & 7 & 7 & 5.084 & 5.084 & 5.084 \\
\hline & & 219 & 200 & 12 & 14 & 14 & 1.696 & 1.696 & 1.696 \\
\hline & & & 268 & 2 & 2 & 2 & 1.368 & 1.368 & 1.368 \\
\hline & & & 294 & 3 & 3 & 3 & 4.939 & 4.939 & 4.939 \\
\hline & & 255 & 200 & 6 & 6 & 6 & 2.236 & 2.236 & 2.236 \\
\hline & & & 218 & 8 & 9 & 9 & 1.063 & 1.063 & 1.063 \\
\hline & & & 264 & 3 & 3 & 3 & 1.902 & 1.902 & 1.902 \\
\hline & & & 268 & 12 & 14 & 14 & 1.432 & 1.432 & 1.432 \\
\hline & & & 271 & 14 & 16 & 16 & 1.971 & 1.971 & 1.971 \\
\hline & & & 294 & 3 & 3 & 3 & 2.125 & 2.125 & 2.125 \\
\hline & & 264 & 255 & 3 & 3 & 3 & 1.902 & 1.902 & 1.902 \\
\hline & & & 270 & 29 & 34 & 34 & 1.733 & 1.733 & 1.733 \\
\hline & & & 271 & 40 & 43 & 44 & 1.821 & 1.821 & 1.821 \\
\hline & & 268 & 219 & 2 & 2 & 2 & 1.368 & 1.368 & 1.368 \\
\hline & & & 255 & 12 & 14 & 14 & 1.432 & 1.432 & 1.432 \\
\hline & & & 271 & 57 & 60 & 61 & 1.747 & 1.747 & 1.747 \\
\hline & & 270 & 264 & 29 & 34 & 34 & 1.733 & 1.733 & 1.733 \\
\hline & & & 294 & 27 & 27 & 27 & 4.696 & 4.696 & 4.696 \\
\hline & & 271 & 255 & 14 & 16 & 16 & 1.971 & 1.971 & 1.971 \\
\hline & & & 264 & 40 & 43 & 44 & 1.821 & 1.821 & 1.821 \\
\hline & & & 268 & 57 & 60 & 61 & 1.747 & 1.747 & 1.747 \\
\hline & & & 294 & & 2 & 2 & & 3.618 & 3.618 \\
\hline & & & 312 & 2 & 2 & 2 & 1.872 & 1.872 & 1.872 \\
\hline & & 290 & 294 & 121 & 129 & 129 & 5.572 & 5.572 & 5.572 \\
\hline & & & 312 & 12 & 12 & 12 & 1.436 & 1.436 & 1.436 \\
\hline & & 291 & 294 & 13 & 15 & 15 & 2.245 & 2.245 & 2.245 \\
\hline & & 294 & 218 & 7 & 7 & 7 & 5.084 & 5.084 & 5.084 \\
\hline & & & 219 & 3 & 3 & 3 & 4.939 & 4.939 & 4.939 \\
\hline & & & 255 & 3 & 3 & 3 & 2.125 & 2.125 & 2.125 \\
\hline & & & 270 & 27 & 27 & 27 & 4.696 & 4.696 & 4.696 \\
\hline & & & 271 & & 2 & 2 & & 3.618 & 3.618 \\
\hline & & & 290 & 121 & 129 & 129 & 5.572 & 5.572 & 5.572 \\
\hline & & & 291 & 13 & 15 & 15 & 2.245 & 2.245 & 2.245 \\
\hline & & & 380 & 3 & 3 & 3 & 3.560 & 3.560 & 3.560 \\
\hline
\end{tabular}


Table B.2 - Continued from previous page

\begin{tabular}{|c|c|c|c|c|c|c|c|c|c|}
\hline Name & $\begin{array}{l}\text { UniProt } \\
\text { ID }\end{array}$ & Residue 1 & Residue 2 & FDR $1 \%$ & FDR $3 \%$ & FDR 5\% & F $D R 1 \%$ & F DR3\% & FDR5\% \\
\hline & & 312 & 271 & 2 & 2 & 2 & 1.872 & 1.872 & 1.872 \\
\hline & & & 290 & 12 & 12 & 12 & 1.436 & 1.436 & 1.436 \\
\hline & & 380 & 294 & 3 & 3 & 3 & 3.560 & 3.560 & 3.560 \\
\hline & & & 432 & 13 & 13 & 13 & 1.839 & 1.839 & 1.839 \\
\hline & & & 447 & 5 & 5 & 5 & 2.869 & 2.869 & 2.869 \\
\hline & & & 466 & 4 & 4 & 4 & 1.422 & 1.422 & 1.422 \\
\hline & & & 487 & 3 & 3 & 3 & 3.194 & 3.194 & 3.194 \\
\hline & & 432 & 380 & 13 & 13 & 13 & 1.839 & 1.839 & 1.839 \\
\hline & & & 447 & 53 & 54 & 54 & 2.707 & 2.707 & 2.707 \\
\hline & & & 466 & 16 & 16 & 16 & 2.358 & 2.358 & 2.358 \\
\hline & & & 487 & 3 & 3 & 3 & 2.080 & 2.080 & 2.080 \\
\hline & & & 522 & 12 & 16 & 16 & 1.483 & 1.483 & 1.483 \\
\hline & & 447 & 380 & 5 & 5 & 5 & 2.869 & 2.869 & 2.869 \\
\hline & & & 432 & 53 & 54 & 54 & 2.707 & 2.707 & 2.707 \\
\hline & & & 487 & 6 & 6 & 6 & 2.644 & 2.644 & 2.644 \\
\hline & & 466 & 380 & 4 & 4 & 4 & 1.422 & 1.422 & 1.422 \\
\hline & & & 432 & 16 & 16 & 16 & 2.358 & 2.358 & 2.358 \\
\hline & & & 487 & 12 & 12 & 12 & 3.477 & 3.477 & 3.477 \\
\hline & & 487 & 380 & 3 & 3 & 3 & 3.194 & 3.194 & 3.194 \\
\hline & & & 432 & 3 & 3 & 3 & 2.080 & 2.080 & 2.080 \\
\hline & & & 447 & 6 & 6 & 6 & 2.644 & 2.644 & 2.644 \\
\hline & & & 466 & 12 & 12 & 12 & 3.477 & 3.477 & 3.477 \\
\hline & & & 522 & & 2 & 2 & & 1.389 & 1.389 \\
\hline & & & 708 & 2 & 2 & 2 & 1.433 & 1.433 & 1.433 \\
\hline & & & 718 & 2 & 2 & 2 & 2.489 & 2.489 & 2.489 \\
\hline & & 500 & 522 & 2 & 2 & 2 & 1.850 & 1.850 & 1.850 \\
\hline & & 522 & 432 & 12 & 16 & 16 & 1.483 & 1.483 & 1.483 \\
\hline & & & 487 & & 2 & 2 & & 1.389 & 1.389 \\
\hline & & & 500 & 2 & 2 & 2 & 1.850 & 1.850 & 1.850 \\
\hline & & & 532 & 11 & 11 & 11 & 3.516 & 3.516 & 3.516 \\
\hline & & & 535 & 11 & 12 & 12 & 3.953 & 3.953 & 3.953 \\
\hline & & & 539 & 4 & 4 & 4 & 2.045 & 2.045 & 2.045 \\
\hline & & & 598 & 11 & 11 & 11 & 1.830 & 1.830 & 1.830 \\
\hline & & & 601 & 13 & 14 & 14 & 2.749 & 2.749 & 2.749 \\
\hline & & & 626 & 13 & 13 & 13 & 2.223 & 2.223 & 2.223 \\
\hline & & 532 & 522 & 11 & 11 & 11 & 3.516 & 3.516 & 3.516 \\
\hline & & & 539 & 12 & 12 & 12 & 1.615 & 1.615 & 1.615 \\
\hline & & & 601 & 2 & 2 & 2 & 1.411 & 1.411 & 1.411 \\
\hline & & & 623 & 8 & 27 & 28 & 1.007 & 1.007 & 1.007 \\
\hline & & & 626 & 69 & 72 & 72 & 2.116 & 2.116 & 2.116 \\
\hline & & & 630 & 7 & 7 & 7 & 1.361 & 1.361 & 1.361 \\
\hline & & 535 & 522 & 11 & 12 & 12 & 3.953 & 3.953 & 3.953 \\
\hline & & & 539 & 55 & 59 & 59 & 2.813 & 2.813 & 2.813 \\
\hline & & & 543 & 2 & 21 & 23 & 0.438 & 0.438 & 0.438 \\
\hline & & & 598 & 3 & 8 & 8 & 0.480 & 0.480 & 0.480 \\
\hline & & & 601 & 11 & 13 & 13 & 1.287 & 1.287 & 1.287 \\
\hline & & & 623 & 4 & 26 & 27 & 0.497 & 0.497 & 0.497 \\
\hline & & & 626 & 98 & 123 & 124 & 2.001 & 2.001 & 2.001 \\
\hline & & & 630 & 20 & 24 & 24 & 1.583 & 1.583 & 1.583 \\
\hline & & & 631 & 6 & 6 & 6 & 1.923 & 1.923 & 1.923 \\
\hline & & 539 & 522 & 4 & 4 & 4 & 2.045 & 2.045 & 2.045 \\
\hline & & & 532 & 12 & 12 & 12 & 1.615 & 1.615 & 1.615 \\
\hline & & & 535 & 55 & 59 & 59 & 2.813 & 2.813 & 2.813 \\
\hline & & & 623 & 44 & 116 & 127 & 1.190 & 1.190 & 1.190 \\
\hline & & & 626 & 262 & 265 & 266 & 3.657 & 3.657 & 3.657 \\
\hline & & & 630 & 49 & 49 & 49 & 3.272 & 3.272 & 3.272 \\
\hline & & 543 & 535 & 2 & 21 & 23 & 0.438 & 0.438 & 0.438 \\
\hline & & & 626 & 21 & 28 & 28 & 1.222 & 1.222 & 1.222 \\
\hline & & & 630 & & 3 & 3 & & 0.324 & 0.324 \\
\hline & & 570 & 577 & 14 & 15 & 15 & 12.094 & 12.094 & 12.094 \\
\hline & & 577 & 570 & 14 & 15 & 15 & 12.094 & 12.094 & 12.094 \\
\hline & & & 599 & & 2 & 2 & & 0.415 & 0.415 \\
\hline & & 598 & 522 & 11 & 11 & 11 & 1.830 & 1.830 & 1.830 \\
\hline & & & 535 & 3 & 8 & 8 & 0.480 & 0.480 & 0.480 \\
\hline & & & 694 & 5 & 7 & 7 & 0.577 & 0.577 & 0.577 \\
\hline & & & 708 & 17 & 19 & 21 & 1.698 & 1.698 & 1.698 \\
\hline & & & 712 & 23 & 24 & 24 & 1.415 & 1.415 & 1.415 \\
\hline & & 599 & 577 & & 2 & 2 & & 0.415 & 0.415 \\
\hline & & 601 & 522 & 13 & 14 & 14 & 2.749 & 2.749 & 2.749 \\
\hline & & & 532 & 2 & 2 & 2 & 1.411 & 1.411 & 1.411 \\
\hline & & & 535 & 11 & 13 & 13 & 1.287 & 1.287 & 1.287 \\
\hline & & & 694 & 13 & 14 & 14 & 1.862 & 1.862 & 1.862 \\
\hline & & & 708 & 45 & 48 & 48 & 2.895 & 2.895 & 2.895 \\
\hline & & & 712 & 27 & 30 & 31 & 1.537 & 1.537 & 1.537 \\
\hline & & 623 & 532 & 8 & 27 & 28 & 1.007 & 1.007 & 1.007 \\
\hline & & & 535 & 4 & 26 & 27 & 0.497 & 0.497 & 0.497 \\
\hline & & & 539 & 44 & 116 & 127 & 1.190 & 1.190 & 1.190 \\
\hline & & 626 & 522 & 13 & 13 & 13 & 2.223 & 2.223 & 2.223 \\
\hline & & & 532 & 69 & 72 & 72 & 2.116 & 2.116 & 2.116 \\
\hline & & & 535 & 98 & 123 & 124 & 2.001 & 2.001 & 2.001 \\
\hline & & & 539 & 262 & 265 & 266 & 3.657 & 3.657 & 3.657 \\
\hline & & & 543 & 21 & 28 & 28 & 1.222 & 1.222 & 1.222 \\
\hline & & & 631 & 19 & 19 & 19 & 2.288 & 2.288 & 2.288 \\
\hline
\end{tabular}


Table B.2 - Continued from previous page

\begin{tabular}{|c|c|c|c|c|c|c|c|c|c|}
\hline Name & $\begin{array}{l}\text { UniProt } \\
\text { ID }\end{array}$ & Residue 1 & Residue 2 & FDR 1\% & FDR 3\% & FDR 5\% & FDR1\% & FDR3\% & FDR5\% \\
\hline & & 630 & 532 & 7 & 7 & 7 & 1.361 & 1.361 & 1.361 \\
\hline & & & 535 & 20 & 24 & 24 & 1.583 & 1.583 & 1.583 \\
\hline & & & 539 & 49 & 49 & 49 & 3.272 & 3.272 & 3.272 \\
\hline & & & 543 & & 3 & 3 & & 0.324 & 0.324 \\
\hline & & 631 & 535 & 6 & 6 & 6 & 1.923 & 1.923 & 1.923 \\
\hline & & & 626 & 19 & 19 & 19 & 2.288 & 2.288 & 2.288 \\
\hline & & 685 & 694 & 7 & 8 & 8 & 1.451 & 1.451 & 1.451 \\
\hline & & 694 & 598 & 5 & 7 & 7 & 0.577 & 0.577 & 0.577 \\
\hline & & & 601 & 13 & 14 & 14 & 1.862 & 1.862 & 1.862 \\
\hline & & & 685 & 7 & 8 & 8 & 1.451 & 1.451 & 1.451 \\
\hline & & 708 & 487 & 2 & 2 & 2 & 1.433 & 1.433 & 1.433 \\
\hline & & & 598 & 17 & 19 & 21 & 1.698 & 1.698 & 1.698 \\
\hline & & & 601 & 45 & 48 & 48 & 2.895 & 2.895 & 2.895 \\
\hline & & & 712 & 25 & 28 & 28 & 1.352 & 1.352 & 1.352 \\
\hline & & & 718 & 11 & 11 & 11 & 2.718 & 2.718 & 2.718 \\
\hline & & 712 & 598 & 23 & 24 & 24 & 1.415 & 1.415 & 1.415 \\
\hline & & & 601 & 27 & 30 & 31 & 1.537 & 1.537 & 1.537 \\
\hline & & & 708 & 25 & 28 & 28 & 1.352 & 1.352 & 1.352 \\
\hline & & & 716 & 2 & 3 & 3 & 0.613 & 0.613 & 0.613 \\
\hline & & 716 & 712 & 2 & 3 & 3 & 0.613 & 0.613 & 0.613 \\
\hline & & 718 & 487 & 2 & 2 & 2 & 2.489 & 2.489 & 2.489 \\
\hline & & & 708 & 11 & 11 & 11 & 2.718 & 2.718 & 2.718 \\
\hline & & 771 & 782 & 39 & 39 & 39 & 2.329 & 2.329 & 2.329 \\
\hline & & 782 & 771 & 39 & 39 & 39 & 2.329 & 2.329 & 2.329 \\
\hline \multirow[t]{40}{*}{ CTNNBL1 } & Q8WYA6 & 16 & 27 & 21 & 25 & 25 & 1.161 & 1.161 & 1.161 \\
\hline & & & 31 & 11 & 15 & 15 & 4.313 & 4.313 & 4.313 \\
\hline & & 27 & 16 & 21 & 25 & 25 & 1.161 & 1.161 & 1.161 \\
\hline & & & 31 & 84 & 98 & 102 & 2.661 & 2.661 & 2.661 \\
\hline & & 31 & 16 & 11 & 15 & 15 & 4.313 & 4.313 & 4.313 \\
\hline & & & 27 & 84 & 98 & 102 & 2.661 & 2.661 & 2.661 \\
\hline & & & 56 & 25 & 27 & 27 & 2.432 & 2.432 & 2.432 \\
\hline & & & 57 & 15 & 15 & 15 & 2.433 & 2.433 & 2.433 \\
\hline & & 56 & 31 & 25 & 27 & 27 & 2.432 & 2.432 & 2.432 \\
\hline & & & 91 & 2 & 4 & 4 & 0.753 & 0.753 & 0.753 \\
\hline & & 57 & 31 & 15 & 15 & 15 & 2.433 & 2.433 & 2.433 \\
\hline & & 83 & 91 & 29 & 39 & 39 & 2.041 & 2.041 & 2.041 \\
\hline & & 84 & 95 & 13 & 13 & 13 & 2.521 & 2.521 & 2.521 \\
\hline & & 91 & 56 & 2 & 4 & 4 & 0.753 & 0.753 & 0.753 \\
\hline & & & 83 & 29 & 39 & 39 & 2.041 & 2.041 & 2.041 \\
\hline & & & 95 & 59 & 60 & 60 & 2.074 & 2.074 & 2.074 \\
\hline & & & 102 & 102 & 109 & 109 & 3.520 & 3.520 & 3.520 \\
\hline & & 95 & 84 & 13 & 13 & 13 & 2.521 & 2.521 & 2.521 \\
\hline & & & 91 & 59 & 60 & 60 & 2.074 & 2.074 & 2.074 \\
\hline & & & 102 & 136 & 144 & 144 & 4.435 & 4.435 & 4.435 \\
\hline & & 102 & 91 & 102 & 109 & 109 & 3.520 & 3.520 & 3.520 \\
\hline & & & 95 & 136 & 144 & 144 & 4.435 & 4.435 & 4.435 \\
\hline & & 247 & 250 & & 4 & 4 & & 0.207 & 0.207 \\
\hline & & & 252 & 8 & 9 & 9 & 2.481 & 2.481 & 2.481 \\
\hline & & 250 & 247 & & 4 & 4 & & 0.207 & 0.207 \\
\hline & & 252 & 247 & 8 & 9 & 9 & 2.481 & 2.481 & 2.481 \\
\hline & & 297 & 347 & 3 & 4 & 4 & 1.168 & 1.168 & 1.168 \\
\hline & & 332 & 372 & 36 & 36 & 36 & 4.008 & 4.008 & 4.008 \\
\hline & & 347 & 297 & 3 & 4 & 4 & 1.168 & 1.168 & 1.168 \\
\hline & & 372 & 332 & 36 & 36 & 36 & 4.008 & 4.008 & 4.008 \\
\hline & & 401 & 458 & 33 & 33 & 33 & 4.563 & 4.563 & 4.563 \\
\hline & & 457 & 463 & 4 & 5 & 5 & 0.763 & 0.763 & 0.763 \\
\hline & & & 527 & 13 & 14 & 14 & 1.627 & 1.627 & 1.627 \\
\hline & & 458 & 401 & 33 & 33 & 33 & 4.563 & 4.563 & 4.563 \\
\hline & & 463 & 457 & 4 & 5 & 5 & 0.763 & 0.763 & 0.763 \\
\hline & & 527 & 457 & 13 & 14 & 14 & 1.627 & 1.627 & 1.627 \\
\hline & & & 534 & 177 & 186 & 186 & 2.471 & 2.471 & 2.471 \\
\hline & & 534 & 527 & 177 & 186 & 186 & 2.471 & 2.471 & 2.471 \\
\hline & & & 554 & 45 & 63 & 63 & 1.201 & 1.201 & 1.201 \\
\hline & & 554 & 534 & 45 & 63 & 63 & 1.201 & 1.201 & 1.201 \\
\hline \multirow[t]{19}{*}{ CWC15 } & Q9P013 & 18 & 40 & 42 & 44 & 44 & 2.574 & 2.574 & 2.574 \\
\hline & & & 42 & 26 & 31 & 31 & 1.542 & 1.542 & 1.542 \\
\hline & & & 91 & 6 & 6 & 6 & 2.171 & 2.171 & 2.171 \\
\hline & & 28 & 40 & 63 & 72 & 72 & 2.169 & 2.169 & 2.169 \\
\hline & & & 42 & 54 & 59 & 59 & 1.354 & 1.354 & 1.354 \\
\hline & & & 91 & 15 & 18 & 18 & 1.742 & 1.742 & 1.742 \\
\hline & & 40 & 18 & 42 & 44 & 44 & 2.574 & 2.574 & 2.574 \\
\hline & & & 28 & 63 & 72 & 72 & 2.169 & 2.169 & 2.169 \\
\hline & & & 91 & & 1 & 1 & & 0.772 & 0.772 \\
\hline & & 42 & 18 & 26 & 31 & 31 & 1.542 & 1.542 & 1.542 \\
\hline & & & 28 & 54 & 59 & 59 & 1.354 & 1.354 & 1.354 \\
\hline & & 91 & 18 & 6 & 6 & 6 & 2.171 & 2.171 & 2.171 \\
\hline & & & 28 & 15 & 18 & 18 & 1.742 & 1.742 & 1.742 \\
\hline & & & 40 & & 1 & 1 & & 0.772 & 0.772 \\
\hline & & 183 & 195 & 6 & 7 & 7 & 3.140 & 3.140 & 3.140 \\
\hline & & 195 & 183 & 6 & 7 & 7 & 3.140 & 3.140 & 3.140 \\
\hline & & 205 & 221 & 6 & 6 & 6 & 1.671 & 1.671 & 1.671 \\
\hline & & 221 & 205 & 6 & 6 & 6 & 1.671 & 1.671 & 1.671 \\
\hline & & & 226 & 3 & 5 & 5 & 0.791 & 0.791 & 0.791 \\
\hline
\end{tabular}


Table B.2 - Continued from previous page

\begin{tabular}{|c|c|c|c|c|c|c|c|c|c|}
\hline Name & $\begin{array}{l}\text { UniProt } \\
\text { ID }\end{array}$ & Residue 1 & Residue 2 & FDR $1 \%$ & FDR 3\% & FDR 5\% & FDR1\% & F DR3\% & FDR5\% \\
\hline & & 226 & 221 & 3 & 5 & 5 & 0.791 & 0.791 & 0.791 \\
\hline \multirow[t]{54}{*}{ HNRNPA1 } & P09651 & 3 & 15 & 54 & 56 & 56 & 6.364 & 6.364 & 6.364 \\
\hline & & & 52 & 8 & 8 & 8 & 1.575 & 1.575 & 1.575 \\
\hline & & & 106 & 28 & 29 & 30 & 4.136 & 4.136 & 4.136 \\
\hline & & & 113 & 17 & 18 & 18 & 2.782 & 2.782 & 2.782 \\
\hline & & & 166 & 4 & 4 & 4 & 2.850 & 2.850 & 2.850 \\
\hline & & & 350 & 38 & 38 & 38 & 3.562 & 3.562 & 3.562 \\
\hline & & 8 & 15 & 54 & 54 & 54 & 4.418 & 4.418 & 4.418 \\
\hline & & & 106 & 12 & 12 & 12 & 1.920 & 1.920 & 1.920 \\
\hline & & & 113 & 7 & 7 & 7 & 1.253 & 1.253 & 1.253 \\
\hline & & & 166 & 4 & 4 & 4 & 0.952 & 0.952 & 0.952 \\
\hline & & & 350 & 25 & 25 & 25 & 1.632 & 1.632 & 1.632 \\
\hline & & 15 & 3 & 54 & 56 & 56 & 6.364 & 6.364 & 6.364 \\
\hline & & & 8 & 54 & 54 & 54 & 4.418 & 4.418 & 4.418 \\
\hline & & & 106 & 43 & 43 & 43 & 7.045 & 7.045 & 7.045 \\
\hline & & & 166 & 9 & 9 & 9 & 3.569 & 3.569 & 3.569 \\
\hline & & & 350 & 23 & 23 & 23 & 3.795 & 3.795 & 3.795 \\
\hline & & 52 & 3 & 8 & 8 & 8 & 1.575 & 1.575 & 1.575 \\
\hline & & & 106 & 18 & 19 & 19 & 1.460 & 1.460 & 1.460 \\
\hline & & & 113 & 5 & 5 & 5 & 0.654 & 0.654 & 0.654 \\
\hline & & & 350 & 16 & 19 & 19 & 0.917 & 0.917 & 0.917 \\
\hline & & 78 & 106 & 3 & 4 & 4 & 2.228 & 2.228 & 2.228 \\
\hline & & & 113 & 2 & 4 & 4 & 0.865 & 0.865 & 0.865 \\
\hline & & & 144 & & 2 & 2 & & 0.260 & 0.260 \\
\hline & & & 166 & 3 & 3 & 3 & 0.962 & 0.962 & 0.962 \\
\hline & & & 350 & & 2 & 2 & & 0.366 & 0.366 \\
\hline & & 105 & 350 & 6 & 7 & 7 & 1.842 & 1.842 & 1.842 \\
\hline & & 106 & 3 & 28 & 29 & 30 & 4.136 & 4.136 & 4.136 \\
\hline & & & 8 & 12 & 12 & 12 & 1.920 & 1.920 & 1.920 \\
\hline & & & 15 & 43 & 43 & 43 & 7.045 & 7.045 & 7.045 \\
\hline & & & 52 & 18 & 19 & 19 & 1.460 & 1.460 & 1.460 \\
\hline & & & 78 & 3 & 4 & 4 & 2.228 & 2.228 & 2.228 \\
\hline & & & 350 & 66 & 70 & 70 & 4.046 & 4.046 & 4.046 \\
\hline & & 113 & 3 & 17 & 18 & 18 & 2.782 & 2.782 & 2.782 \\
\hline & & & 8 & 7 & 7 & 7 & 1.253 & 1.253 & 1.253 \\
\hline & & & 52 & 5 & 5 & 5 & 0.654 & 0.654 & 0.654 \\
\hline & & & 78 & 2 & 4 & 4 & 0.865 & 0.865 & 0.865 \\
\hline & & & 144 & 5 & 6 & 7 & 1.240 & 1.240 & 1.240 \\
\hline & & & 350 & 30 & 33 & 33 & 2.402 & 2.402 & 2.402 \\
\hline & & 144 & 78 & & 2 & 2 & & 0.260 & 0.260 \\
\hline & & & 113 & 5 & 6 & 7 & 1.240 & 1.240 & 1.240 \\
\hline & & 166 & 3 & 4 & 4 & 4 & 2.850 & 2.850 & 2.850 \\
\hline & & & 8 & 4 & 4 & 4 & 0.952 & 0.952 & 0.952 \\
\hline & & & 15 & 9 & 9 & 9 & 3.569 & 3.569 & 3.569 \\
\hline & & & 78 & 3 & 3 & 3 & 0.962 & 0.962 & 0.962 \\
\hline & & & 350 & 16 & 17 & 17 & 2.505 & 2.505 & 2.505 \\
\hline & & 350 & 3 & 38 & 38 & 38 & 3.562 & 3.562 & 3.562 \\
\hline & & & 8 & 25 & 25 & 25 & 1.632 & 1.632 & 1.632 \\
\hline & & & 15 & 23 & 23 & 23 & 3.795 & 3.795 & 3.795 \\
\hline & & & 52 & 16 & 19 & 19 & 0.917 & 0.917 & 0.917 \\
\hline & & & 78 & & 2 & 2 & & 0.366 & 0.366 \\
\hline & & & 105 & 6 & 7 & 7 & 1.842 & 1.842 & 1.842 \\
\hline & & & 106 & 66 & 70 & 70 & 4.046 & 4.046 & 4.046 \\
\hline & & & 113 & 30 & 33 & 33 & 2.402 & 2.402 & 2.402 \\
\hline & & & 166 & 16 & 17 & 17 & 2.505 & 2.505 & 2.505 \\
\hline \multirow[t]{28}{*}{ ISY1 } & Q9ULR0 & 24 & 41 & 20 & 23 & 23 & 2.925 & 2.925 & 2.925 \\
\hline & & & 44 & 6 & 8 & 8 & 0.910 & 0.910 & 0.910 \\
\hline & & 26 & 41 & 7 & 8 & 8 & 1.039 & 1.039 & 1.039 \\
\hline & & & 44 & 13 & 16 & 16 & 0.690 & 0.690 & 0.690 \\
\hline & & 41 & 24 & 20 & 23 & 23 & 2.925 & 2.925 & 2.925 \\
\hline & & & 26 & 7 & 8 & 8 & 1.039 & 1.039 & 1.039 \\
\hline & & 44 & 24 & 6 & 8 & 8 & 0.910 & 0.910 & 0.910 \\
\hline & & & 26 & 13 & 16 & 16 & 0.690 & 0.690 & 0.690 \\
\hline & & & 55 & 6 & 7 & 7 & 1.307 & 1.307 & 1.307 \\
\hline & & 55 & 44 & 6 & 7 & 7 & 1.307 & 1.307 & 1.307 \\
\hline & & 84 & 92 & 24 & 24 & 24 & 2.919 & 2.919 & 2.919 \\
\hline & & & 101 & 12 & 15 & 15 & 2.055 & 2.055 & 2.055 \\
\hline & & & 105 & 70 & 70 & 70 & 4.132 & 4.132 & 4.132 \\
\hline & & & 112 & & 2 & 2 & & 0.397 & 0.397 \\
\hline & & & 121 & 125 & 127 & 127 & 4.039 & 4.039 & 4.039 \\
\hline & & 92 & 84 & 24 & 24 & 24 & 2.919 & 2.919 & 2.919 \\
\hline & & & 105 & 20 & 20 & 20 & 3.077 & 3.077 & 3.077 \\
\hline & & 101 & 84 & 12 & 15 & 15 & 2.055 & 2.055 & 2.055 \\
\hline & & & 121 & 9 & 9 & 9 & 1.669 & 1.669 & 1.669 \\
\hline & & 105 & 84 & 70 & 70 & 70 & 4.132 & 4.132 & 4.132 \\
\hline & & & 92 & 20 & 20 & 20 & 3.077 & 3.077 & 3.077 \\
\hline & & & 121 & 60 & 61 & 61 & 3.068 & 3.068 & 3.068 \\
\hline & & 112 & 84 & & 2 & 2 & & 0.397 & 0.397 \\
\hline & & 121 & 84 & 125 & 127 & 127 & 4.039 & 4.039 & 4.039 \\
\hline & & & 101 & 9 & 9 & 9 & 1.669 & 1.669 & 1.669 \\
\hline & & & 105 & 60 & 61 & 61 & 3.068 & 3.068 & 3.068 \\
\hline & & 179 & 190 & 5 & 8 & 8 & 0.857 & 0.857 & 0.857 \\
\hline & & 190 & 179 & 5 & 8 & 8 & 0.857 & 0.857 & 0.857 \\
\hline
\end{tabular}


Table B.2 - Continued from previous page

\begin{tabular}{|c|c|c|c|c|c|c|c|c|c|}
\hline Name & $\begin{array}{l}\text { UniProt } \\
\text { ID }\end{array}$ & Residue 1 & Residue 2 & FDR 1\% & FDR 3\% & FDR 5\% & FDR1\% & FDR3\% & FDR5\% \\
\hline \multirow[t]{24}{*}{ KIN17 } & O60870 & 42 & 324 & & & 1 & & & $9.921 \cdot 10^{-2}$ \\
\hline & & 231 & 242 & 3 & 3 & 3 & 2.518 & 2.518 & 2.518 \\
\hline & & 240 & 242 & 13 & 13 & 13 & 2.771 & 2.771 & 2.771 \\
\hline & & 242 & 231 & 3 & 3 & 3 & 2.518 & 2.518 & 2.518 \\
\hline & & & 240 & 13 & 13 & 13 & 2.771 & 2.771 & 2.771 \\
\hline & & 252 & 258 & 2 & 2 & 2 & 1.328 & 1.328 & 1.328 \\
\hline & & 258 & 252 & 2 & 2 & 2 & 1.328 & 1.328 & 1.328 \\
\hline & & 288 & 302 & 14 & 14 & 14 & 3.385 & 3.385 & 3.385 \\
\hline & & 292 & 300 & 8 & 10 & 10 & 1.991 & 1.991 & 1.991 \\
\hline & & 293 & 324 & 2 & 3 & 3 & 0.618 & 0.618 & 0.618 \\
\hline & & 300 & 292 & 8 & 10 & 10 & 1.991 & 1.991 & 1.991 \\
\hline & & & 302 & 5 & 5 & 5 & 2.772 & 2.772 & 2.772 \\
\hline & & 302 & 288 & 14 & 14 & 14 & 3.385 & 3.385 & 3.385 \\
\hline & & & 300 & 5 & 5 & 5 & 2.772 & 2.772 & 2.772 \\
\hline & & & 341 & 1 & 1 & 1 & 0.911 & 0.911 & 0.911 \\
\hline & & & 391 & 8 & 8 & 8 & 2.085 & 2.085 & 2.085 \\
\hline & & 317 & 391 & 4 & 4 & 4 & 2.258 & 2.258 & 2.258 \\
\hline & & 324 & 42 & & & 1 & & & $9.921 \cdot 10^{-2}$ \\
\hline & & & 293 & 2 & 3 & 3 & 0.618 & 0.618 & 0.618 \\
\hline & & 341 & 302 & 1 & 1 & 1 & 0.911 & 0.911 & 0.911 \\
\hline & & & 391 & 7 & 7 & 7 & 3.600 & 3.600 & 3.600 \\
\hline & & 391 & 302 & 8 & 8 & 8 & 2.085 & 2.085 & 2.085 \\
\hline & & & 317 & 4 & 4 & 4 & 2.258 & 2.258 & 2.258 \\
\hline & & & 341 & 7 & 7 & 7 & 3.600 & 3.600 & 3.600 \\
\hline \multirow[t]{8}{*}{ LSm2 } & Q9Y333 & 1 & 88 & 26 & 27 & 27 & 5.800 & 5.800 & 5.800 \\
\hline & & & 94 & 9 & 9 & 9 & 1.459 & 1.459 & 1.459 \\
\hline & & 8 & 88 & 21 & 21 & 21 & 2.235 & 2.235 & 2.235 \\
\hline & & & 94 & 3 & 3 & 3 & 1.605 & 1.605 & 1.605 \\
\hline & & 88 & 1 & 26 & 27 & 27 & 5.800 & 5.800 & 5.800 \\
\hline & & & 8 & 21 & 21 & 21 & 2.235 & 2.235 & 2.235 \\
\hline & & 94 & 1 & 9 & 9 & 9 & 1.459 & 1.459 & 1.459 \\
\hline & & & 8 & 3 & 3 & 3 & 1.605 & 1.605 & 1.605 \\
\hline \multirow[t]{2}{*}{$\mathrm{LSm} 4$} & Q9Y4Z0 & 1 & 80 & 48 & 49 & 50 & 4.814 & 4.814 & 4.814 \\
\hline & & 80 & 1 & 48 & 49 & 50 & 4.814 & 4.814 & 4.814 \\
\hline LSm6 & P62312 & 5 & 77 & 4 & 4 & 4 & 1.609 & 1.609 & 1.609 \\
\hline & & 13 & 77 & 5 & 5 & 5 & 2.114 & 2.114 & 2.114 \\
\hline & & 77 & 5 & 4 & 4 & 4 & 1.609 & 1.609 & 1.609 \\
\hline & & & 13 & 5 & 5 & 5 & 2.114 & 2.114 & 2.114 \\
\hline $\mathrm{LSm} 7$ & Q9UK45 & 4 & 9 & 12 & 17 & 17 & 1.772 & 1.772 & 1.772 \\
\hline & & 6 & 8 & 3 & 3 & 3 & 1.583 & 1.583 & 1.583 \\
\hline & & & 9 & 29 & 31 & 31 & 2.383 & 2.383 & 2.383 \\
\hline & & 7 & 9 & 4 & 4 & 4 & 1.299 & 1.299 & 1.299 \\
\hline & & 8 & 6 & 3 & 3 & 3 & 1.583 & 1.583 & 1.583 \\
\hline & & 9 & 4 & 12 & 17 & 17 & 1.772 & 1.772 & 1.772 \\
\hline & & & 6 & 29 & 31 & 31 & 2.383 & 2.383 & 2.383 \\
\hline & & & 7 & 4 & 4 & 4 & 1.299 & 1.299 & 1.299 \\
\hline MBPMS2 & $\begin{array}{l}\text { P0AEX9- } \\
\mathrm{r}\end{array}$ & 2 & 314 & & 2 & 2 & & 0.191 & 0.191 \\
\hline & & 7 & 26 & 6 & 7 & 7 & 3.399 & 3.399 & 3.399 \\
\hline & & & 35 & 4 & 4 & 4 & 1.156 & 1.156 & 1.156 \\
\hline & & & 47 & 5 & 5 & 5 & 2.548 & 2.548 & 2.548 \\
\hline & & 16 & 30 & & 3 & 3 & & 0.224 & 0.224 \\
\hline & & & 35 & & 2 & 2 & & 0.507 & 0.507 \\
\hline & & & 296 & 15 & 15 & 15 & 3.181 & 3.181 & 3.181 \\
\hline & & & 298 & 13 & 13 & 13 & 2.415 & 2.415 & 2.415 \\
\hline & & 26 & 7 & 6 & 7 & 7 & 3.399 & 3.399 & 3.399 \\
\hline & & & 30 & 301 & 308 & 308 & 5.173 & 5.173 & 5.173 \\
\hline & & & 35 & 124 & 140 & 140 & 4.497 & 4.497 & 4.497 \\
\hline & & & 43 & 14 & 14 & 14 & 1.925 & 1.925 & 1.925 \\
\hline & & & 47 & 5 & 5 & 5 & 3.144 & 3.144 & 3.144 \\
\hline & & & 296 & 22 & 22 & 22 & 4.393 & 4.393 & 4.393 \\
\hline & & 27 & 47 & 2 & 2 & 2 & 3.225 & 3.225 & 3.225 \\
\hline & & 30 & 16 & & 3 & 3 & & 0.224 & 0.224 \\
\hline & & & 26 & 301 & 308 & 308 & 5.173 & 5.173 & 5.173 \\
\hline & & & 43 & 11 & 15 & 15 & 2.011 & 2.011 & 2.011 \\
\hline & & & 47 & 24 & 26 & 26 & 3.153 & 3.153 & 3.153 \\
\hline & & & 120 & 7 & 9 & 10 & 2.031 & 2.031 & 2.031 \\
\hline & & & 176 & & 4 & 5 & & 0.823 & 0.823 \\
\hline & & & 296 & 3 & 3 & 3 & 1.506 & 1.506 & 1.506 \\
\hline & & 35 & 7 & 4 & 4 & 4 & 1.156 & 1.156 & 1.156 \\
\hline & & & 16 & & 2 & 2 & & 0.507 & 0.507 \\
\hline & & & 26 & 124 & 140 & 140 & 4.497 & 4.497 & 4.497 \\
\hline & & & 176 & 7 & 13 & 14 & 0.594 & 0.594 & 0.594 \\
\hline & & 43 & 26 & 14 & 14 & 14 & 1.925 & 1.925 & 1.925 \\
\hline & & & 30 & 11 & 15 & 15 & 2.011 & 2.011 & 2.011 \\
\hline & & & 47 & 6 & 6 & 6 & 0.796 & 0.796 & 0.796 \\
\hline & & & 176 & & 6 & 6 & & 0.454 & 0.454 \\
\hline & & & 298 & 2 & 2 & 2 & 2.520 & 2.520 & 2.520 \\
\hline & & & 424 & & 2 & 2 & & 0.866 & 0.866 \\
\hline & & & 438 & 4 & 7 & 7 & 2.894 & 2.894 & 2.894 \\
\hline & & & 447 & 7 & 13 & 13 & 1.127 & 1.127 & 1.127 \\
\hline & & 47 & 7 & 5 & 5 & 5 & 2.548 & 2.548 & 2.548 \\
\hline & & & 26 & 5 & 5 & 5 & 3.144 & 3.144 & 3.144 \\
\hline
\end{tabular}


Table B.2 - Continued from previous page

\begin{tabular}{|c|c|c|c|c|c|c|c|c|c|}
\hline Name & $\begin{array}{l}\text { UniProt } \\
\text { ID }\end{array}$ & Residue 1 & Residue 2 & FDR $1 \%$ & FDR $3 \%$ & FDR $5 \%$ & FDR $1 \%$ & FDR3\% & FDR5\% \\
\hline & & & 27 & 2 & 2 & 2 & 3.225 & 3.225 & 3.225 \\
\hline & & & 30 & 24 & 26 & 26 & 3.153 & 3.153 & 3.153 \\
\hline & & & 43 & 6 & 6 & 6 & 0.796 & 0.796 & 0.796 \\
\hline & & & 84 & 8 & 8 & 8 & 7.914 & 7.914 & 7.914 \\
\hline & & & 89 & 2 & 2 & 2 & 3.285 & 3.285 & 3.285 \\
\hline & & & 103 & 8 & 8 & 8 & 5.653 & 5.653 & 5.653 \\
\hline & & & 120 & 3 & 3 & 3 & 4.505 & 4.505 & 4.505 \\
\hline & & & 176 & 3 & 6 & 6 & 1.808 & 1.808 & 1.808 \\
\hline & & & 438 & 22 & 28 & 28 & 1.972 & 1.972 & 1.972 \\
\hline & & & 442 & 7 & 9 & 9 & 3.511 & 3.511 & 3.511 \\
\hline & & & 447 & 54 & 55 & 56 & 1.970 & 1.970 & 1.970 \\
\hline & & 84 & 47 & 8 & 8 & 8 & 7.914 & 7.914 & 7.914 \\
\hline & & & 84 & & 4 & 6 & & 1.858 & 1.858 \\
\hline & & & 103 & 28 & 31 & 31 & 5.437 & 5.437 & 5.437 \\
\hline & & & 128 & 24 & 29 & 29 & 1.406 & 1.406 & 1.406 \\
\hline & & & 138 & 4 & 4 & 4 & 1.373 & 1.373 & 1.373 \\
\hline & & & 176 & 2 & 4 & 4 & 0.487 & 0.487 & 0.487 \\
\hline & & & 252 & 3 & 3 & 3 & 5.156 & 5.156 & 5.156 \\
\hline & & & 296 & 2 & 2 & 2 & 1.759 & 1.759 & 1.759 \\
\hline & & & 306 & 450 & 452 & 452 & 9.343 & 9.343 & 9.343 \\
\hline & & & 314 & 7 & 8 & 8 & 1.263 & 1.263 & 1.263 \\
\hline & & & 327 & 7 & 7 & 7 & 4.788 & 4.788 & 4.788 \\
\hline & & 89 & 47 & 2 & 2 & 2 & 3.285 & 3.285 & 3.285 \\
\hline & & & 306 & 55 & 55 & 55 & 2.985 & 2.985 & 2.985 \\
\hline & & 103 & 47 & 8 & 8 & 8 & 5.653 & 5.653 & 5.653 \\
\hline & & & 84 & 28 & 31 & 31 & 5.437 & 5.437 & 5.437 \\
\hline & & & 128 & 6 & 6 & 6 & 4.145 & 4.145 & 4.145 \\
\hline & & & 138 & 9 & 11 & 11 & 2.134 & 2.134 & 2.134 \\
\hline & & & 176 & 580 & 591 & 591 & 5.504 & 5.504 & 5.504 \\
\hline & & & 252 & 3 & 3 & 3 & 4.583 & 4.583 & 4.583 \\
\hline & & & 257 & 2 & 2 & 2 & 4.072 & 4.072 & 4.072 \\
\hline & & & 274 & 127 & 128 & 128 & 27.568 & 27.568 & 27.568 \\
\hline & & & 447 & 36 & 37 & 37 & 2.076 & 2.076 & 2.076 \\
\hline & & 120 & 30 & 7 & 9 & 10 & 2.031 & 2.031 & 2.031 \\
\hline & & & 47 & 3 & 3 & 3 & 4.505 & 4.505 & 4.505 \\
\hline & & & 138 & 5 & 5 & 5 & 1.785 & 1.785 & 1.785 \\
\hline & & & 141 & 19 & 19 & 19 & 2.031 & 2.031 & 2.031 \\
\hline & & & 176 & 12 & 13 & 13 & 1.532 & 1.532 & 1.532 \\
\hline & & & 180 & 3 & 4 & 4 & 3.159 & 3.159 & 3.159 \\
\hline & & & 203 & & 2 & 2 & & 2.081 & 2.081 \\
\hline & & & 274 & 2 & 2 & 2 & 2.684 & 2.684 & 2.684 \\
\hline & & & 314 & 110 & 111 & 111 & 3.150 & 3.150 & 3.150 \\
\hline & & & 424 & 5 & 6 & 6 & 1.966 & 1.966 & 1.966 \\
\hline & & & 438 & 8 & 11 & 11 & 0.718 & 0.718 & 0.718 \\
\hline & & & 442 & & 2 & 2 & & 1.371 & 1.371 \\
\hline & & & 447 & 19 & 21 & 22 & 1.787 & 1.787 & 1.787 \\
\hline & & 128 & 84 & 24 & 29 & 29 & 1.406 & 1.406 & 1.406 \\
\hline & & & 103 & 6 & 6 & 6 & 4.145 & 4.145 & 4.145 \\
\hline & & & 145 & 3 & 3 & 3 & 3.766 & 3.766 & 3.766 \\
\hline & & & 327 & 163 & 163 & 163 & 27.444 & 27.444 & 27.444 \\
\hline & & 138 & 84 & 4 & 4 & 4 & 1.373 & 1.373 & 1.373 \\
\hline & & & 103 & 9 & 11 & 11 & 2.134 & 2.134 & 2.134 \\
\hline & & & 120 & 5 & 5 & 5 & 1.785 & 1.785 & 1.785 \\
\hline & & & 145 & 10 & 10 & 10 & 3.420 & 3.420 & 3.420 \\
\hline & & & 176 & 6 & 6 & 6 & 1.803 & 1.803 & 1.803 \\
\hline & & & 180 & 12 & 12 & 12 & 1.921 & 1.921 & 1.921 \\
\hline & & & 190 & 10 & 10 & 10 & 1.853 & 1.853 & 1.853 \\
\hline & & & 201 & 39 & 39 & 39 & 2.404 & 2.404 & 2.404 \\
\hline & & & 203 & 347 & 351 & 351 & 16.203 & 16.203 & 16.203 \\
\hline & & & 220 & 32 & 37 & 38 & 4.234 & 4.234 & 4.234 \\
\hline & & & 252 & 3 & 3 & 3 & 2.776 & 2.776 & 2.776 \\
\hline & & 141 & 120 & 19 & 19 & 19 & 2.031 & 2.031 & 2.031 \\
\hline & & & 145 & 9 & 10 & 10 & 2.669 & 2.669 & 2.669 \\
\hline & & & 203 & 30 & 31 & 31 & 10.593 & 10.593 & 10.593 \\
\hline & & 145 & 128 & 3 & 3 & 3 & 3.766 & 3.766 & 3.766 \\
\hline & & & 138 & 10 & 10 & 10 & 3.420 & 3.420 & 3.420 \\
\hline & & & 141 & 9 & 10 & 10 & 2.669 & 2.669 & 2.669 \\
\hline & & & 180 & & 2 & 2 & & 0.623 & 0.623 \\
\hline & & 171 & 180 & 12 & 12 & 12 & 2.564 & 2.564 & 2.564 \\
\hline & & 176 & 30 & & 4 & 5 & & 0.823 & 0.823 \\
\hline & & & 35 & 7 & 13 & 14 & 0.594 & 0.594 & 0.594 \\
\hline & & & 43 & & 6 & 6 & & 0.454 & 0.454 \\
\hline & & & 47 & 3 & 6 & 6 & 1.808 & 1.808 & 1.808 \\
\hline & & & 84 & 2 & 4 & 4 & 0.487 & 0.487 & 0.487 \\
\hline & & & 103 & 580 & 591 & 591 & 5.504 & 5.504 & 5.504 \\
\hline & & & 120 & 12 & 13 & 13 & 1.532 & 1.532 & 1.532 \\
\hline & & & 138 & 6 & 6 & 6 & 1.803 & 1.803 & 1.803 \\
\hline & & & 176 & & 2 & 2 & & 1.471 & 1.471 \\
\hline & & & 180 & 7 & 10 & 10 & 1.233 & 1.233 & 1.233 \\
\hline & & & 190 & 15 & 15 & 15 & 1.524 & 1.524 & 1.524 \\
\hline & & & 201 & 11 & 11 & 11 & 2.074 & 2.074 & 2.074 \\
\hline & & & 274 & & 3 & 3 & & 0.368 & 0.368 \\
\hline & & & 363 & 1 & 1 & 1 & 0.434 & 0.434 & 0.434 \\
\hline
\end{tabular}


Table B.2 - Continued from previous page

\begin{tabular}{|c|c|c|c|c|c|c|c|c|c|}
\hline Name & $\begin{array}{l}\text { UniProt } \\
\text { ID }\end{array}$ & Residue 1 & Residue 2 & FDR 1\% & FDR 3\% & FDR 5\% & FDR1\% & FDR3\% & FDR5\% \\
\hline & & & 438 & 6 & 8 & 8 & 0.667 & 0.667 & 0.667 \\
\hline & & & 447 & 19 & 24 & 24 & 1.734 & 1.734 & 1.734 \\
\hline & & & 138 & 12 & 12 & 12 & 1.921 & 1.921 & 1.921 \\
\hline & & & 145 & & 2 & 2 & & 0.623 & 0.623 \\
\hline & & & 171 & 12 & 12 & 12 & 2.564 & 2.564 & 2.564 \\
\hline & & & 438 & 5 & 5 & 5 & 1.115 & 1.115 & 1.115 \\
\hline & & & 447 & 16 & 17 & 17 & 1.716 & 1.716 & 1.716 \\
\hline & & 190 & 138 & 10 & 10 & 10 & 1.853 & 1.853 & 1.853 \\
\hline & & & 176 & 15 & 15 & 15 & 1.524 & 1.524 & 1.524 \\
\hline & & & 252 & 53 & 55 & 55 & 39.666 & 39.666 & 39.666 \\
\hline & & & 363 & 52 & 52 & 52 & 4.388 & 4.388 & 4.388 \\
\hline & & 203 & 120 & & 2 & 2 & & 2.081 & 2.081 \\
\hline & & & 138 & 347 & 351 & 351 & 16.203 & 16.203 & 16.203 \\
\hline & & & 141 & 30 & 31 & 31 & 10.593 & 10.593 & 10.593 \\
\hline & & & 240 & 3 & 3 & 3 & 4.177 & 4.177 & 4.177 \\
\hline & & & 447 & 4 & 4 & 4 & 0.885 & 0.885 & 0.885 \\
\hline & & 220 & 138 & 32 & 37 & 38 & 4.234 & 4.234 & 4.234 \\
\hline & & & 296 & 2 & 2 & 2 & 5.049 & 5.049 & 5.049 \\
\hline & & 240 & 203 & 3 & 3 & 3 & 4.177 & 4.177 & 4.177 \\
\hline & & & 296 & 7 & 7 & 7 & 20.695 & 20.695 & 20.695 \\
\hline & & 252 & 84 & 3 & 3 & 3 & 5.156 & 5.156 & 5.156 \\
\hline & & & 103 & 3 & 3 & 3 & 4.583 & 4.583 & 4.583 \\
\hline & & & 138 & 3 & 3 & 3 & 2.776 & 2.776 & 2.776 \\
\hline & & & 190 & 53 & 55 & 55 & 39.666 & 39.666 & 39.666 \\
\hline & & & 438 & 10 & 10 & 10 & 1.768 & 1.768 & 1.768 \\
\hline & & & 447 & 16 & 16 & 16 & 2.040 & 2.040 & 2.040 \\
\hline & & 296 & 16 & 15 & 15 & 15 & 3.181 & 3.181 & 3.181 \\
\hline & & & 26 & 22 & 22 & 22 & 4.393 & 4.393 & 4.393 \\
\hline & & & 30 & 3 & 3 & 3 & 1.506 & 1.506 & 1.506 \\
\hline & & & 84 & 2 & 2 & 2 & 1.759 & 1.759 & 1.759 \\
\hline & & & 220 & 2 & 2 & 2 & 5.049 & 5.049 & 5.049 \\
\hline & & & 240 & 7 & 7 & 7 & 20.695 & 20.695 & 20.695 \\
\hline & & & 274 & 7 & 8 & 8 & 2.452 & 2.452 & 2.452 \\
\hline & & & 314 & 66 & 69 & 69 & 1.839 & 1.839 & 1.839 \\
\hline & & 298 & 16 & 13 & 13 & 13 & 2.415 & 2.415 & 2.415 \\
\hline & & & 43 & 2 & 2 & 2 & 2.520 & 2.520 & 2.520 \\
\hline & & & 274 & 5 & 5 & 5 & 2.014 & 2.014 & 2.014 \\
\hline & & & 306 & 3 & 4 & 4 & 1.095 & 1.095 & 1.095 \\
\hline & & 306 & 84 & 450 & 452 & 452 & 9.343 & 9.343 & 9.343 \\
\hline & & & 89 & 55 & 55 & 55 & 2.985 & 2.985 & 2.985 \\
\hline & & & 252 & 2 & 2 & 2 & 1.746 & 1.746 & 1.746 \\
\hline & & & 274 & 2 & 3 & 3 & 0.586 & 0.586 & 0.586 \\
\hline & & & 298 & 3 & 4 & 4 & 1.095 & 1.095 & 1.095 \\
\hline & & & 314 & & 6 & 6 & & 0.648 & 0.648 \\
\hline & & 314 & 2 & & 2 & 2 & & 0.191 & 0.191 \\
\hline & & & 84 & 7 & 8 & 8 & 1.263 & 1.263 & 1.263 \\
\hline & & & 120 & 110 & 111 & 111 & 3.150 & 3.150 & 3.150 \\
\hline & & & 296 & 66 & 69 & 69 & 1.839 & 1.839 & 1.839 \\
\hline & & & 306 & & 6 & 6 & & 0.648 & 0.648 \\
\hline & & 327 & 84 & 7 & 7 & 7 & 4.788 & 4.788 & 4.788 \\
\hline
\end{tabular}


Table B. 2 - Continued from previous page

\begin{tabular}{|c|c|c|c|c|c|c|c|c|c|}
\hline Name & $\begin{array}{l}\text { UniProt } \\
\text { ID }\end{array}$ & Residue 1 & Residue 2 & FDR $1 \%$ & FDR $3 \%$ & FDR $5 \%$ & $\mathrm{~F} D \mathrm{R} 1 \%$ & F DR3\% & FDR5\% \\
\hline & & 438 & 43 & 4 & 7 & 7 & 2.894 & 2.894 & 2.894 \\
\hline & & & 47 & 22 & 28 & 28 & 1.972 & 1.972 & 1.972 \\
\hline & & & 120 & 8 & 11 & 11 & 0.718 & 0.718 & 0.718 \\
\hline & & & 176 & 6 & 8 & 8 & 0.667 & 0.667 & 0.667 \\
\hline & & & 180 & 5 & 5 & 5 & 1.115 & 1.115 & 1.115 \\
\hline & & & 190 & 1 & 2 & 2 & 0.770 & 0.770 & 0.770 \\
\hline & & & 274 & 10 & 10 & 10 & 1.768 & 1.768 & 1.768 \\
\hline & & & 363 & 4 & 5 & 5 & 1.086 & 1.086 & 1.086 \\
\hline & & & 424 & 20 & 25 & 25 & 1.912 & 1.912 & 1.912 \\
\hline & & & 438 & 4 & 6 & 6 & 0.702 & 0.702 & 0.702 \\
\hline & & & 442 & 169 & 174 & 174 & 3.223 & 3.223 & 3.223 \\
\hline & & & 447 & 18 & 18 & 18 & 2.214 & 2.214 & 2.214 \\
\hline & & 442 & 47 & 7 & 9 & 9 & 3.511 & 3.511 & 3.511 \\
\hline & & & 120 & & 2 & 2 & & 1.371 & 1.371 \\
\hline & & & 424 & 75 & 76 & 76 & 3.037 & 3.037 & 3.037 \\
\hline & & & 438 & 169 & 174 & 174 & 3.223 & 3.223 & 3.223 \\
\hline & & 447 & 43 & 7 & 13 & 13 & 1.127 & 1.127 & 1.127 \\
\hline & & & 47 & 54 & 55 & 56 & 1.970 & 1.970 & 1.970 \\
\hline & & & 103 & 36 & 37 & 37 & 2.076 & 2.076 & 2.076 \\
\hline & & & 120 & 19 & 21 & 22 & 1.787 & 1.787 & 1.787 \\
\hline & & & 176 & 19 & 24 & 24 & 1.734 & 1.734 & 1.734 \\
\hline & & & 180 & 16 & 17 & 17 & 1.716 & 1.716 & 1.716 \\
\hline & & & 190 & 10 & 10 & 10 & 1.888 & 1.888 & 1.888 \\
\hline & & & 201 & 6 & 6 & 6 & 1.779 & 1.779 & 1.779 \\
\hline & & & 203 & 4 & 4 & 4 & 0.885 & 0.885 & 0.885 \\
\hline & & & 274 & 16 & 16 & 16 & 2.040 & 2.040 & 2.040 \\
\hline & & & 363 & 16 & 16 & 16 & 1.296 & 1.296 & 1.296 \\
\hline & & & 424 & 29 & 29 & 29 & 2.512 & 2.512 & 2.512 \\
\hline & & & 438 & 18 & 18 & 18 & 2.214 & 2.214 & 2.214 \\
\hline \multirow[t]{14}{*}{ MFAP1 } & P55081 & 230 & 238 & 41 & 42 & 42 & 3.734 & 3.734 & 3.734 \\
\hline & & 238 & 230 & 41 & 42 & 42 & 3.734 & 3.734 & 3.734 \\
\hline & & & 249 & 6 & 6 & 6 & 1.866 & 1.866 & 1.866 \\
\hline & & 242 & 250 & 16 & 27 & 27 & 2.369 & 2.369 & 2.369 \\
\hline & & 249 & 238 & 6 & 6 & 6 & 1.866 & 1.866 & 1.866 \\
\hline & & 250 & 242 & 16 & 27 & 27 & 2.369 & 2.369 & 2.369 \\
\hline & & 329 & 334 & 6 & 12 & 14 & 0.644 & 0.644 & 0.644 \\
\hline & & & 357 & 17 & 17 & 17 & 1.095 & 1.095 & 1.095 \\
\hline & & 334 & 329 & 6 & 12 & 14 & 0.644 & 0.644 & 0.644 \\
\hline & & 357 & 329 & 17 & 17 & 17 & 1.095 & 1.095 & 1.095 \\
\hline & & 381 & 390 & 2 & 2 & 2 & 1.366 & 1.366 & 1.366 \\
\hline & & 390 & 381 & 2 & 2 & 2 & 1.366 & 1.366 & 1.366 \\
\hline & & 415 & 420 & 18 & 18 & 18 & 2.219 & 2.219 & 2.219 \\
\hline & & 420 & 415 & 18 & 18 & 18 & 2.219 & 2.219 & 2.219 \\
\hline \multirow[t]{2}{*}{ PHF5A } & Q7RTV0 & 3 & 95 & 2 & 2 & 2 & 1.358 & 1.358 & 1.358 \\
\hline & & 95 & 3 & 2 & 2 & 2 & 1.358 & 1.358 & 1.358 \\
\hline PLRG1 & O43660 & 41 & 62 & 7 & 8 & 8 & 1.937 & 1.937 & 1.937 \\
\hline & & & 66 & 2 & 2 & 2 & 1.472 & 1.472 & 1.472 \\
\hline & & & 68 & 2 & 2 & 2 & 2.962 & 2.962 & 2.962 \\
\hline & & 62 & 41 & 7 & 8 & 8 & 1.937 & 1.937 & 1.937 \\
\hline & & & 68 & 20 & 20 & 20 & 3.188 & 3.188 & 3.188 \\
\hline & & & 80 & 7 & 7 & 7 & 7.317 & 7.317 & 7.317 \\
\hline & & 66 & 41 & 2 & 2 & 2 & 1.472 & 1.472 & 1.472 \\
\hline & & & 113 & 4 & 5 & 5 & 1.137 & 1.137 & 1.137 \\
\hline & & 68 & 41 & 2 & 2 & 2 & 2.962 & 2.962 & 2.962 \\
\hline & & & 62 & 20 & 20 & 20 & 3.188 & 3.188 & 3.188 \\
\hline & & & 113 & 4 & 4 & 4 & 3.244 & 3.244 & 3.244 \\
\hline & & 80 & 62 & 7 & 7 & 7 & 7.317 & 7.317 & 7.317 \\
\hline & & & 135 & 2 & 2 & 2 & 3.558 & 3.558 & 3.558 \\
\hline & & 113 & 66 & 4 & 5 & 5 & 1.137 & 1.137 & 1.137 \\
\hline & & & 68 & 4 & 4 & 4 & 3.244 & 3.244 & 3.244 \\
\hline & & & 135 & 19 & 21 & 21 & 10.687 & 10.687 & 10.687 \\
\hline & & & 180 & 5 & 5 & 5 & 2.155 & 2.155 & 2.155 \\
\hline & & 135 & 80 & 2 & 2 & 2 & 3.558 & 3.558 & 3.558 \\
\hline & & & 113 & 19 & 21 & 21 & 10.687 & 10.687 & 10.687 \\
\hline & & & 173 & 10 & 10 & 10 & 1.224 & 1.224 & 1.224 \\
\hline & & & 180 & 9 & 10 & 10 & 1.819 & 1.819 & 1.819 \\
\hline & & & 510 & 4 & 4 & 4 & 1.541 & 1.541 & 1.541 \\
\hline & & 173 & 135 & 10 & 10 & 10 & 1.224 & 1.224 & 1.224 \\
\hline & & & 181 & 28 & 32 & 32 & 4.588 & 4.588 & 4.588 \\
\hline & & & 510 & 7 & 9 & 10 & 8.955 & 8.955 & 8.955 \\
\hline & & 180 & 113 & 5 & 5 & 5 & 2.155 & 2.155 & 2.155 \\
\hline & & & 135 & 9 & 10 & 10 & 1.819 & 1.819 & 1.819 \\
\hline & & & 505 & 15 & 19 & 19 & 1.478 & 1.478 & 1.478 \\
\hline & & & 510 & 48 & 54 & 54 & 2.127 & 2.127 & 2.127 \\
\hline & & 181 & 173 & 28 & 32 & 32 & 4.588 & 4.588 & 4.588 \\
\hline & & & 505 & 2 & 4 & 4 & 2.392 & 2.392 & 2.392 \\
\hline & & & 510 & 8 & 9 & 9 & 2.148 & 2.148 & 2.148 \\
\hline & & 187 & 505 & 3 & 3 & 3 & 1.526 & 1.526 & 1.526 \\
\hline & & 279 & 320 & 9 & 9 & 9 & 2.252 & 2.252 & 2.252 \\
\hline & & 320 & 279 & 9 & 9 & 9 & 2.252 & 2.252 & 2.252 \\
\hline & & 372 & 396 & 24 & 27 & 27 & 1.625 & 1.625 & 1.625 \\
\hline & & 396 & 372 & 24 & 27 & 27 & 1.625 & 1.625 & 1.625 \\
\hline & & & 510 & 4 & 4 & 4 & 1.484 & 1.484 & 1.484 \\
\hline
\end{tabular}


Table B.2 - Continued from previous page

\begin{tabular}{|c|c|c|c|c|c|c|c|c|c|}
\hline Name & $\begin{array}{l}\text { UniProt } \\
\text { ID }\end{array}$ & Residue 1 & Residue 2 & FDR 1\% & FDR $3 \%$ & FDR 5\% & FDR $1 \%$ & FDR3\% & FDR5\% \\
\hline & & 505 & 180 & 15 & 19 & 19 & 1.478 & 1.478 & 1.478 \\
\hline & & & 181 & 2 & 4 & 4 & 2.392 & 2.392 & 2.392 \\
\hline & & & 187 & 3 & 3 & 3 & 1.526 & 1.526 & 1.526 \\
\hline & & & 512 & & 3 & 4 & & 0.287 & 0.287 \\
\hline & & 510 & 135 & 4 & 4 & 4 & 1.541 & 1.541 & 1.541 \\
\hline & & & 173 & 7 & 9 & 10 & 8.955 & 8.955 & 8.955 \\
\hline & & & 180 & 48 & 54 & 54 & 2.127 & 2.127 & 2.127 \\
\hline & & & 181 & 8 & 9 & 9 & 2.148 & 2.148 & 2.148 \\
\hline & & & 396 & 4 & 4 & 4 & 1.484 & 1.484 & 1.484 \\
\hline & & 512 & 505 & & 3 & 4 & & 0.287 & 0.287 \\
\hline \multirow{28}{*}{ PPIE } & Q9UNP9 & 5 & 83 & 33 & 38 & 38 & 3.798 & 3.798 & 3.798 \\
\hline & & & 88 & 3 & 10 & 14 & 0.710 & 0.710 & 0.710 \\
\hline & & & 104 & 21 & 24 & 24 & 3.344 & 3.344 & 3.344 \\
\hline & & & 108 & 7 & 17 & 19 & 0.975 & 0.975 & 0.975 \\
\hline & & & 123 & & 3 & 3 & & 0.248 & 0.248 \\
\hline & & 20 & 104 & 2 & 3 & 3 & 1.214 & 1.214 & 1.214 \\
\hline & & 83 & 5 & 33 & 38 & 38 & 3.798 & 3.798 & 3.798 \\
\hline & & & 88 & 13 & 17 & 17 & 0.636 & 0.636 & 0.636 \\
\hline & & & 104 & 16 & 16 & 16 & 2.657 & 2.657 & 2.657 \\
\hline & & & 108 & 6 & 11 & 11 & 0.717 & 0.717 & 0.717 \\
\hline & & 88 & 5 & 3 & 10 & 14 & 0.710 & 0.710 & 0.710 \\
\hline & & & 83 & 13 & 17 & 17 & 0.636 & 0.636 & 0.636 \\
\hline & & & 104 & 2 & 2 & 2 & 0.781 & 0.781 & 0.781 \\
\hline & & 103 & 108 & 7 & 9 & 9 & 1.222 & 1.222 & 1.222 \\
\hline & & 104 & 5 & 21 & 24 & 24 & 3.344 & 3.344 & 3.344 \\
\hline & & & 20 & 2 & 3 & 3 & 1.214 & 1.214 & 1.214 \\
\hline & & & 83 & 16 & 16 & 16 & 2.657 & 2.657 & 2.657 \\
\hline & & & 88 & 2 & 2 & 2 & 0.781 & 0.781 & 0.781 \\
\hline & & & 114 & 3 & 3 & 3 & 0.461 & 0.461 & 0.461 \\
\hline & & & 134 & 5 & 5 & 5 & 0.610 & 0.610 & 0.610 \\
\hline & & 108 & 5 & $\begin{array}{lll}7 & \end{array}$ & 17 & 19 & 0.975 & 0.975 & 0.975 \\
\hline & & & 83 & 6 & 11 & 11 & 0.717 & 0.717 & 0.717 \\
\hline & & & 103 & 7 & 9 & 9 & 1.222 & 1.222 & 1.222 \\
\hline & & 114 & 104 & 3 & 3 & 3 & 0.461 & 0.461 & 0.461 \\
\hline & & 123 & 5 & & 3 & 3 & & 0.248 & 0.248 \\
\hline & & 134 & 104 & 5 & 5 & 5 & 0.610 & 0.610 & 0.610 \\
\hline & & 147 & 185 & 48 & 49 & 49 & 3.254 & 3.254 & 3.254 \\
\hline & & 185 & 147 & 48 & 49 & 49 & 3.254 & 3.254 & 3.254 \\
\hline \multirow[t]{4}{*}{ PPIL1 } & Q9Y3C6 & 34 & 80 & 5 & 5 & 5 & 1.337 & 1.337 & 1.337 \\
\hline & & 52 & 158 & 231 & 298 & 303 & 2.371 & 2.371 & 2.371 \\
\hline & & 80 & 34 & 5 & 5 & 5 & 1.337 & 1.337 & 1.337 \\
\hline & & 158 & 52 & 231 & 298 & 303 & 2.371 & 2.371 & 2.371 \\
\hline PPIL2 & Q13356 & 8 & 26 & & 9 & 16 & & 0.301 & 0.301 \\
\hline & & 26 & 8 & & 9 & 16 & & 0.301 & 0.301 \\
\hline & & 230 & 232 & 12 & 12 & 12 & 1.678 & 1.678 & 1.678 \\
\hline & & & 235 & & 3 & 3 & & 0.815 & 0.815 \\
\hline & & 231 & 454 & 2 & 2 & 2 & 1.841 & 1.841 & 1.841 \\
\hline & & & 462 & 2 & 2 & 2 & 0.687 & 0.687 & 0.687 \\
\hline & & 232 & 230 & 12 & 12 & 12 & 1.678 & 1.678 & 1.678 \\
\hline & & & 450 & 33 & 33 & 33 & 4.496 & 4.496 & 4.496 \\
\hline & & & 454 & 42 & 42 & 42 & 2.857 & 2.857 & 2.857 \\
\hline & & & 462 & & 2 & 2 & & 0.457 & 0.457 \\
\hline & & 235 & 230 & & 3 & 3 & & 0.815 & 0.815 \\
\hline & & & 277 & 3 & 3 & 3 & 0.969 & 0.969 & 0.969 \\
\hline & & & 278 & 2 & 3 & 3 & 0.556 & 0.556 & 0.556 \\
\hline & & & 450 & 7 & 7 & 7 & 1.901 & 1.901 & 1.901 \\
\hline & & & 454 & 24 & 27 & 27 & 2.274 & 2.274 & 2.274 \\
\hline & & & 460 & 3 & 7 & 7 & 0.556 & 0.556 & 0.556 \\
\hline & & & 462 & 4 & 4 & 4 & 2.318 & 2.318 & 2.318 \\
\hline & & 277 & 235 & 3 & 3 & 3 & 0.969 & 0.969 & 0.969 \\
\hline & & 278 & 235 & 2 & 3 & 3 & 0.556 & 0.556 & 0.556 \\
\hline & & 302 & 348 & 13 & 14 & 14 & 5.409 & 5.409 & 5.409 \\
\hline & & 348 & 302 & 13 & 14 & 14 & 5.409 & 5.409 & 5.409 \\
\hline & & 450 & 232 & 33 & 33 & 33 & 4.496 & 4.496 & 4.496 \\
\hline & & & 235 & 7 & 7 & 7 & 1.901 & 1.901 & 1.901 \\
\hline & & & 460 & 21 & 21 & 22 & 2.332 & 2.332 & 2.332 \\
\hline & & & 462 & 35 & 37 & 37 & 2.227 & 2.227 & 2.227 \\
\hline & & 454 & 231 & 2 & 2 & 2 & 1.841 & 1.841 & 1.841 \\
\hline & & & 232 & 42 & 42 & 42 & 2.857 & 2.857 & 2.857 \\
\hline & & & 235 & 24 & 27 & 27 & 2.274 & 2.274 & 2.274 \\
\hline & & & 462 & 46 & 50 & 50 & 3.097 & 3.097 & 3.097 \\
\hline & & 460 & 235 & 3 & 7 & 7 & 0.556 & 0.556 & 0.556 \\
\hline & & & 450 & 21 & 21 & 22 & 2.332 & 2.332 & 2.332 \\
\hline & & 462 & 231 & 2 & 2 & 2 & 0.687 & 0.687 & 0.687 \\
\hline & & & 232 & & 2 & 2 & & 0.457 & 0.457 \\
\hline & & & 235 & 4 & 4 & 4 & 2.318 & 2.318 & 2.318 \\
\hline & & & 450 & 35 & 37 & 37 & 2.227 & 2.227 & 2.227 \\
\hline & & & 454 & 46 & 50 & 50 & 3.097 & 3.097 & 3.097 \\
\hline PPP1CA & $\begin{array}{l}\text { P62136 } \\
\text {. }\end{array}$ & 26 & 305 & 52 & 53 & 53 & 3.266 & 3.266 & 3.266 \\
\hline & & & 327 & 23 & 24 & 26 & 1.661 & 1.661 & 1.661 \\
\hline & & 301 & 305 & 2 & 2 & 2 & 0.502 & 0.502 & 0.502 \\
\hline & & 305 & 26 & 52 & 53 & 53 & 3.266 & 3.266 & 3.266 \\
\hline & & & 301 & 2 & 2 & 2 & 0.502 & 0.502 & 0.502 \\
\hline
\end{tabular}


Table B.2 - Continued from previous page

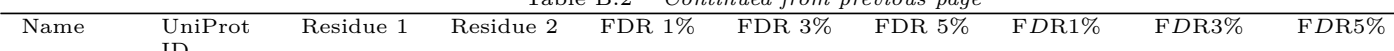

\begin{tabular}{|c|c|c|c|c|c|c|c|c|c|}
\hline & & & 327 & 14 & 14 & 14 & 1.559 & 1.559 & 1.559 \\
\hline & & 327 & 26 & 23 & 24 & 26 & 1.661 & 1.661 & 1.661 \\
\hline & & & 305 & 14 & 14 & 14 & 1.559 & 1.559 & 1.559 \\
\hline \multirow[t]{6}{*}{ PPP1CB } & P62140 & 25 & 301 & 4 & 5 & 5 & 1.588 & 1.588 & 1.588 \\
\hline & & & 303 & 15 & 17 & 17 & 1.959 & 1.959 & 1.959 \\
\hline & & & 326 & & 2 & 2 & & 0.233 & 0.233 \\
\hline & & 301 & 25 & 4 & 5 & 5 & 1.588 & 1.588 & 1.588 \\
\hline & & 303 & 25 & 15 & 17 & 17 & 1.959 & 1.959 & 1.959 \\
\hline & & 326 & 25 & & 2 & 2 & & 0.233 & 0.233 \\
\hline \multirow[t]{30}{*}{ PPP1R8 } & Q12972 & 22 & 47 & 16 & 17 & 17 & 1.952 & 1.952 & 1.952 \\
\hline & & & 78 & 28 & 29 & 29 & 2.520 & 2.520 & 2.520 \\
\hline & & 40 & 129 & 53 & 53 & 53 & 2.877 & 2.877 & 2.877 \\
\hline & & 47 & 22 & 16 & 17 & 17 & 1.952 & 1.952 & 1.952 \\
\hline & & & 78 & 258 & 259 & 259 & 4.203 & 4.203 & 4.203 \\
\hline & & & 81 & 2 & 2 & 2 & 1.053 & 1.053 & 1.053 \\
\hline & & 78 & 22 & 28 & 29 & 29 & 2.520 & 2.520 & 2.520 \\
\hline & & & 47 & 258 & 259 & 259 & 4.203 & 4.203 & 4.203 \\
\hline & & & 138 & 15 & 15 & 15 & 1.778 & 1.778 & 1.778 \\
\hline & & & 175 & 3 & 3 & 3 & 1.305 & 1.305 & 1.305 \\
\hline & & 81 & 47 & 2 & 2 & 2 & 1.053 & 1.053 & 1.053 \\
\hline & & & 129 & 2 & 2 & 2 & 0.958 & 0.958 & 0.958 \\
\hline & & & 138 & 5 & 5 & 5 & 0.947 & 0.947 & 0.947 \\
\hline & & 105 & 175 & 2 & 2 & 2 & 2.112 & 2.112 & 2.112 \\
\hline & & 129 & 40 & 53 & 53 & 53 & 2.877 & 2.877 & 2.877 \\
\hline & & & 81 & 2 & 2 & 2 & 0.958 & 0.958 & 0.958 \\
\hline & & 138 & 78 & 15 & 15 & 15 & 1.778 & 1.778 & 1.778 \\
\hline & & & 81 & 5 & 5 & 5 & 0.947 & 0.947 & 0.947 \\
\hline & & & 151 & 4 & 4 & 4 & 2.676 & 2.676 & 2.676 \\
\hline & & 151 & 138 & 4 & 4 & 4 & 2.676 & 2.676 & 2.676 \\
\hline & & 175 & 78 & 3 & 3 & 3 & 1.305 & 1.305 & 1.305 \\
\hline & & & 105 & 2 & 2 & 2 & 2.112 & 2.112 & 2.112 \\
\hline & & & 343 & 6 & 8 & 8 & 1.142 & 1.142 & 1.142 \\
\hline & & 234 & 236 & 19 & 23 & 23 & 2.849 & 2.849 & 2.849 \\
\hline & & 236 & 234 & 19 & 23 & 23 & 2.849 & 2.849 & 2.849 \\
\hline & & 334 & 344 & & 2 & 2 & & 0.537 & 0.537 \\
\hline & & 337 & 344 & & 2 & 2 & & 0.358 & 0.358 \\
\hline & & 343 & 175 & 6 & 8 & 8 & 1.142 & 1.142 & 1.142 \\
\hline & & 344 & 334 & & 2 & 2 & & 0.537 & 0.537 \\
\hline & & & 337 & & 2 & 2 & & 0.358 & 0.358 \\
\hline \multirow{44}{*}{ PQBP1 } & O60828 & 2 & 18 & 20 & 20 & 20 & 3.382 & 3.382 & 3.382 \\
\hline & & 18 & 2 & 20 & 20 & 20 & 3.382 & 3.382 & 3.382 \\
\hline & & 87 & 102 & 19 & 20 & 22 & 2.892 & 2.892 & 2.892 \\
\hline & & & 109 & 8 & 15 & 15 & 1.258 & 1.258 & 1.258 \\
\hline & & & 116 & 12 & 19 & 19 & 1.594 & 1.594 & 1.594 \\
\hline & & & 123 & 9 & 11 & 11 & 1.164 & 1.164 & 1.164 \\
\hline & & & 148 & & 2 & 2 & & 0.504 & 0.504 \\
\hline & & 90 & 102 & 17 & 22 & 23 & 1.105 & 1.105 & 1.105 \\
\hline & & & 123 & 2 & 2 & 2 & 1.427 & 1.427 & 1.427 \\
\hline & & 102 & 87 & 19 & 20 & 22 & 2.892 & 2.892 & 2.892 \\
\hline & & & 90 & 17 & 22 & 23 & 1.105 & 1.105 & 1.105 \\
\hline & & & 109 & 28 & 29 & 29 & 2.833 & 2.833 & 2.833 \\
\hline & & & 116 & 28 & 29 & 29 & 2.430 & 2.430 & 2.430 \\
\hline & & & 123 & 14 & 14 & 14 & 1.556 & 1.556 & 1.556 \\
\hline & & & 137 & & 1 & 1 & & 0.546 & 0.546 \\
\hline & & 109 & 87 & 8 & 15 & 15 & 1.258 & 1.258 & 1.258 \\
\hline & & & 102 & 28 & 29 & 29 & 2.833 & 2.833 & 2.833 \\
\hline & & & 116 & 9 & 12 & 12 & 1.997 & 1.997 & 1.997 \\
\hline & & & 123 & 18 & 20 & 20 & 2.161 & 2.161 & 2.161 \\
\hline & & & 148 & 5 & 6 & 6 & 1.609 & 1.609 & 1.609 \\
\hline & & 116 & 87 & 12 & 19 & 19 & 1.594 & 1.594 & 1.594 \\
\hline & & & 102 & 28 & 29 & 29 & 2.430 & 2.430 & 2.430 \\
\hline & & & 109 & 9 & 12 & 12 & 1.997 & 1.997 & 1.997 \\
\hline & & & 123 & 46 & 46 & 46 & 3.450 & 3.450 & 3.450 \\
\hline & & & 137 & 10 & 13 & 13 & 0.847 & 0.847 & 0.847 \\
\hline & & & 148 & 47 & 50 & 50 & 3.263 & 3.263 & 3.263 \\
\hline & & & 189 & 10 & 10 & 10 & 2.484 & 2.484 & 2.484 \\
\hline & & & 192 & 5 & 5 & 5 & 2.460 & 2.460 & 2.460 \\
\hline & & & 223 & & 3 & 3 & & 0.472 & 0.472 \\
\hline & & & 228 & 10 & 13 & 13 & 1.027 & 1.027 & 1.027 \\
\hline & & & 262 & 3 & 4 & 4 & 0.480 & 0.480 & 0.480 \\
\hline & & 123 & 87 & 9 & 11 & 11 & 1.164 & 1.164 & 1.164 \\
\hline & & & 90 & 2 & 2 & 2 & 1.427 & 1.427 & 1.427 \\
\hline & & & 102 & 14 & 14 & 14 & 1.556 & 1.556 & 1.556 \\
\hline & & & 109 & 18 & 20 & 20 & 2.161 & 2.161 & 2.161 \\
\hline & & & 116 & 46 & 46 & 46 & 3.450 & 3.450 & 3.450 \\
\hline & & & 130 & 3 & 4 & 4 & 1.720 & 1.720 & 1.720 \\
\hline & & & 137 & 12 & 14 & 14 & 0.941 & 0.941 & 0.941 \\
\hline & & & 148 & 14 & 16 & 16 & 1.518 & 1.518 & 1.518 \\
\hline & & & 167 & & 2 & 2 & & 0.399 & 0.399 \\
\hline & & & 189 & 2 & 2 & 2 & 1.076 & 1.076 & 1.076 \\
\hline & & & 228 & 3 & 3 & 3 & 1.007 & 1.007 & 1.007 \\
\hline & & 130 & 123 & 3 & 4 & 4 & 1.720 & 1.720 & 1.720 \\
\hline & & 137 & 102 & & 1 & 1 & & 0.546 & 0.546 \\
\hline
\end{tabular}


Table B.2 - Continued from previous page

\begin{tabular}{|c|c|c|c|c|c|c|c|c|c|}
\hline Name & $\begin{array}{l}\text { UniProt } \\
\text { ID }\end{array}$ & Residue 1 & Residue 2 & FDR $1 \%$ & FDR 3\% & FDR 5\% & F $D \mathrm{R} 1 \%$ & F DR3\% & F DR5\% \\
\hline & & & 116 & 10 & 13 & 13 & 0.847 & 0.847 & 0.847 \\
\hline & & & 123 & 12 & 14 & 14 & 0.941 & 0.941 & 0.941 \\
\hline & & & 148 & 37 & 42 & 42 & 1.315 & 1.315 & 1.315 \\
\hline & & 148 & 87 & & 2 & 2 & & 0.504 & 0.504 \\
\hline & & & 109 & 5 & 6 & 6 & 1.609 & 1.609 & 1.609 \\
\hline & & & 116 & 47 & 50 & 50 & 3.263 & 3.263 & 3.263 \\
\hline & & & 123 & 14 & 16 & 16 & 1.518 & 1.518 & 1.518 \\
\hline & & & 137 & 37 & 42 & 42 & 1.315 & 1.315 & 1.315 \\
\hline & & & 167 & 16 & 19 & 19 & 1.136 & 1.136 & 1.136 \\
\hline & & & 174 & 4 & 5 & 5 & 1.177 & 1.177 & 1.177 \\
\hline & & & 189 & 4 & 5 & 5 & 1.218 & 1.218 & 1.218 \\
\hline & & & 192 & 3 & 3 & 3 & 2.521 & 2.521 & 2.521 \\
\hline & & 167 & 123 & & 2 & 2 & & 0.399 & 0.399 \\
\hline & & & 148 & 16 & 19 & 19 & 1.136 & 1.136 & 1.136 \\
\hline & & & 174 & 2 & 5 & 5 & 0.480 & 0.480 & 0.480 \\
\hline & & & 189 & 23 & 24 & 24 & 2.187 & 2.187 & 2.187 \\
\hline & & & 192 & 11 & 11 & 11 & 1.776 & 1.776 & 1.776 \\
\hline & & & 197 & 2 & 2 & 2 & 1.445 & 1.445 & 1.445 \\
\hline & & & 223 & & 3 & 3 & & 0.451 & 0.451 \\
\hline & & & 228 & & 3 & 3 & & 0.642 & 0.642 \\
\hline & & 174 & 148 & 4 & 5 & 5 & 1.177 & 1.177 & 1.177 \\
\hline & & & 167 & 2 & 5 & 5 & 0.480 & 0.480 & 0.480 \\
\hline & & & 189 & 12 & 13 & 13 & 1.412 & 1.412 & 1.412 \\
\hline & & & 192 & 5 & 5 & 5 & 1.566 & 1.566 & 1.566 \\
\hline & & & 197 & 2 & 2 & 3 & 1.094 & 1.094 & 1.094 \\
\hline & & 189 & 116 & 10 & 10 & 10 & 2.484 & 2.484 & 2.484 \\
\hline & & & 123 & 2 & 2 & 2 & 1.076 & 1.076 & 1.076 \\
\hline & & & 148 & 4 & 5 & 5 & 1.218 & 1.218 & 1.218 \\
\hline & & & 167 & 23 & 24 & 24 & 2.187 & 2.187 & 2.187 \\
\hline & & & 174 & 12 & 13 & 13 & 1.412 & 1.412 & 1.412 \\
\hline & & & 192 & 116 & 117 & 117 & 3.077 & 3.077 & 3.077 \\
\hline & & & 197 & 43 & 44 & 44 & 4.653 & 4.653 & 4.653 \\
\hline & & & 223 & 17 & 20 & 20 & 2.410 & 2.410 & 2.410 \\
\hline & & & 228 & 19 & 24 & 25 & 1.962 & 1.962 & 1.962 \\
\hline & & & 262 & 3 & 3 & 4 & 0.843 & 0.843 & 0.843 \\
\hline & & 192 & 116 & 5 & 5 & 5 & 2.460 & 2.460 & 2.460 \\
\hline & & & 148 & 3 & 3 & 3 & 2.521 & 2.521 & 2.521 \\
\hline & & & 167 & 11 & 11 & 11 & 1.776 & 1.776 & 1.776 \\
\hline & & & 174 & 5 & 5 & 5 & 1.566 & 1.566 & 1.566 \\
\hline & & & 189 & 116 & 117 & 117 & 3.077 & 3.077 & 3.077 \\
\hline & & & 197 & 4 & 4 & 4 & 2.587 & 2.587 & 2.587 \\
\hline & & & 223 & 10 & 11 & 11 & 1.773 & 1.773 & 1.773 \\
\hline & & & 228 & 19 & 20 & 20 & 1.792 & 1.792 & 1.792 \\
\hline & & & 262 & 5 & 7 & 7 & 2.370 & 2.370 & 2.370 \\
\hline & & 197 & 167 & 2 & 2 & 2 & 1.445 & 1.445 & 1.445 \\
\hline & & & 174 & 2 & 2 & 3 & 1.094 & 1.094 & 1.094 \\
\hline & & & 189 & 43 & 44 & 44 & 4.653 & 4.653 & 4.653 \\
\hline & & & 192 & 4 & 4 & 4 & 2.587 & 2.587 & 2.587 \\
\hline & & & 223 & 11 & 12 & 12 & 1.362 & 1.362 & 1.362 \\
\hline & & & 228 & 12 & 12 & 12 & 16.731 & 16.731 & 16.731 \\
\hline & & 223 & 116 & & 3 & 3 & & 0.472 & 0.472 \\
\hline & & & 167 & & 3 & 3 & & 0.451 & 0.451 \\
\hline & & & 189 & 17 & 20 & 20 & 2.410 & 2.410 & 2.410 \\
\hline & & & 192 & 10 & 11 & 11 & 1.773 & 1.773 & 1.773 \\
\hline & & & 197 & 11 & 12 & 12 & 1.362 & 1.362 & 1.362 \\
\hline & & & 228 & 31 & 33 & 33 & 1.529 & 1.529 & 1.529 \\
\hline & & & 262 & 2 & 5 & 7 & 0.494 & 0.494 & 0.494 \\
\hline & & 228 & 116 & 10 & 13 & 13 & 1.027 & 1.027 & 1.027 \\
\hline & & & 123 & 3 & 3 & 3 & 1.007 & 1.007 & 1.007 \\
\hline & & & 167 & & 3 & 3 & & 0.642 & 0.642 \\
\hline & & & 189 & 19 & 24 & 25 & 1.962 & 1.962 & 1.962 \\
\hline & & & 192 & 19 & 20 & 20 & 1.792 & 1.792 & 1.792 \\
\hline & & & 197 & 12 & 12 & 12 & 16.731 & 16.731 & 16.731 \\
\hline & & & 223 & 31 & 33 & 33 & 1.529 & 1.529 & 1.529 \\
\hline & & & 262 & 25 & 34 & 35 & 1.880 & 1.880 & 1.880 \\
\hline & & 262 & 116 & 3 & 4 & 4 & 0.480 & 0.480 & 0.480 \\
\hline & & & 189 & 3 & 3 & 4 & 0.843 & 0.843 & 0.843 \\
\hline & & & 192 & 5 & 7 & 7 & 2.370 & 2.370 & 2.370 \\
\hline & & & 223 & 2 & 5 & 7 & 0.494 & 0.494 & 0.494 \\
\hline & & & 228 & 25 & 34 & 35 & 1.880 & 1.880 & 1.880 \\
\hline \multirow[t]{13}{*}{ PRP17 } & O60508 & 177 & 203 & & 2 & 3 & & 0.601 & 0.601 \\
\hline & & 203 & 177 & & 2 & 3 & & 0.601 & 0.601 \\
\hline & & 228 & 333 & 24 & 27 & 27 & 2.744 & 2.744 & 2.744 \\
\hline & & 233 & 333 & 13 & 13 & 13 & 2.236 & 2.236 & 2.236 \\
\hline & & 238 & 312 & 13 & 31 & 34 & 2.437 & 2.437 & 2.437 \\
\hline & & & 314 & $\begin{array}{ll}7 \\
\end{array}$ & 7 & 7 & 1.970 & 1.970 & 1.970 \\
\hline & & 289 & 312 & & 2 & 2 & & 0.776 & 0.776 \\
\hline & & & 333 & 85 & 93 & 93 & 2.109 & 2.109 & 2.109 \\
\hline & & & 551 & 63 & 64 & 64 & 5.238 & 5.238 & 5.238 \\
\hline & & 312 & 238 & 13 & 31 & 34 & 2.437 & 2.437 & 2.437 \\
\hline & & & 289 & & 2 & 2 & & 0.776 & 0.776 \\
\hline & & 314 & 238 & 7 & 7 & 7 & 1.970 & 1.970 & 1.970 \\
\hline & & 333 & 228 & 24 & 27 & 27 & 2.744 & 2.744 & 2.744 \\
\hline
\end{tabular}


Table B.2 - Continued from previous page

\begin{tabular}{|c|c|c|c|c|c|c|c|c|c|}
\hline Name & $\begin{array}{l}\text { UniProt } \\
\text { ID }\end{array}$ & Residue 1 & Residue 2 & FDR $1 \%$ & FDR $3 \%$ & FDR $5 \%$ & FDR $1 \%$ & FDR3\% & FDR5\% \\
\hline & & & 233 & 13 & 13 & 13 & 2.236 & 2.236 & 2.236 \\
\hline & & & 289 & 85 & 93 & 93 & 2.109 & 2.109 & 2.109 \\
\hline & & & 374 & 1 & 1 & 1 & 1.471 & 1.471 & 1.471 \\
\hline & & 374 & 333 & 1 & 1 & 1 & 1.471 & 1.471 & 1.471 \\
\hline & & & 398 & 26 & 27 & 27 & 2.362 & 2.362 & 2.362 \\
\hline & & 398 & 374 & 26 & 27 & 27 & 2.362 & 2.362 & 2.362 \\
\hline & & 531 & 547 & 6 & 6 & 6 & 1.746 & 1.746 & 1.746 \\
\hline & & 547 & 531 & 6 & 6 & 6 & 1.746 & 1.746 & 1.746 \\
\hline & & 551 & 289 & 63 & 64 & 64 & 5.238 & 5.238 & 5.238 \\
\hline \multirow[t]{74}{*}{ PRP19 } & Q9UMS4 & 1 & 425 & & 2 & 3 & & 0.353 & 0.353 \\
\hline & & 64 & 76 & 3 & 3 & 3 & 3.200 & 3.200 & 3.200 \\
\hline & & 76 & 64 & 3 & 3 & 3 & 3.200 & 3.200 & 3.200 \\
\hline & & 122 & 135 & 27 & 27 & 27 & 7.016 & 7.016 & 7.016 \\
\hline & & & 179 & 55 & 58 & 58 & 2.989 & 2.989 & 2.989 \\
\hline & & & 192 & 71 & 71 & 71 & 3.369 & 3.369 & 3.369 \\
\hline & & & 244 & 86 & 86 & 86 & 3.657 & 3.657 & 3.657 \\
\hline & & & 261 & 40 & 41 & 41 & 3.177 & 3.177 & 3.177 \\
\hline & & & 265 & 34 & 36 & 36 & 2.689 & 2.689 & 2.689 \\
\hline & & & 266 & 57 & 59 & 59 & 3.747 & 3.747 & 3.747 \\
\hline & & & 380 & 28 & 30 & 30 & 3.100 & 3.100 & 3.100 \\
\hline & & & 423 & 1 & 1 & 1 & 1.254 & 1.254 & 1.254 \\
\hline & & & 425 & 34 & 36 & 36 & 1.519 & 1.519 & 1.519 \\
\hline & & & 428 & 7 & 7 & 7 & 2.460 & 2.460 & 2.460 \\
\hline & & 135 & 122 & 27 & 27 & 27 & 7.016 & 7.016 & 7.016 \\
\hline & & & 135 & 4 & 4 & 6 & 2.352 & 2.352 & 2.352 \\
\hline & & & 179 & 14 & 14 & 14 & 2.662 & 2.662 & 2.662 \\
\hline & & & 192 & 19 & 19 & 19 & 15.970 & 15.970 & 15.970 \\
\hline & & & 244 & 50 & 51 & 51 & 12.810 & 12.810 & 12.810 \\
\hline & & & 261 & 11 & 12 & 12 & 12.714 & 12.714 & 12.714 \\
\hline & & & 265 & 5 & 10 & 10 & 0.892 & 0.892 & 0.892 \\
\hline & & & 266 & 12 & 12 & 12 & 8.408 & 8.408 & 8.408 \\
\hline & & & 380 & 4 & 7 & 7 & 1.314 & 1.314 & 1.314 \\
\hline & & & 423 & 14 & 14 & 14 & 2.329 & 2.329 & 2.329 \\
\hline & & & 425 & 19 & 25 & 25 & 5.991 & 5.991 & 5.991 \\
\hline & & 179 & 122 & 55 & 58 & 58 & 2.989 & 2.989 & 2.989 \\
\hline & & & 135 & 14 & 14 & 14 & 2.662 & 2.662 & 2.662 \\
\hline & & & 192 & 183 & 195 & 195 & 5.759 & 5.759 & 5.759 \\
\hline & & & 244 & 86 & 88 & 88 & 4.331 & 4.331 & 4.331 \\
\hline & & & 261 & 6 & 6 & 6 & 2.990 & 2.990 & 2.990 \\
\hline & & & 265 & 31 & 32 & 32 & 1.507 & 1.507 & 1.507 \\
\hline & & & 266 & 19 & 19 & 19 & 4.044 & 4.044 & 4.044 \\
\hline & & & 425 & 11 & 11 & 11 & 1.258 & 1.258 & 1.258 \\
\hline & & 189 & 200 & 72 & 143 & 154 & 1.525 & 1.525 & 1.525 \\
\hline & & & 425 & 25 & 28 & 28 & 1.460 & 1.460 & 1.460 \\
\hline & & 192 & 122 & 71 & 71 & 71 & 3.369 & 3.369 & 3.369 \\
\hline & & & 135 & 19 & 19 & 19 & 15.970 & 15.970 & 15.970 \\
\hline & & & 179 & 183 & 195 & 195 & 5.759 & 5.759 & 5.759 \\
\hline & & & 244 & 38 & 38 & 38 & 3.295 & 3.295 & 3.295 \\
\hline & & & 261 & 10 & 10 & 10 & 2.775 & 2.775 & 2.775 \\
\hline & & & 265 & 15 & 15 & 15 & 1.413 & 1.413 & 1.413 \\
\hline & & & 266 & 12 & 12 & 12 & 4.817 & 4.817 & 4.817 \\
\hline & & & 423 & 59 & 59 & 60 & 2.720 & 2.720 & 2.720 \\
\hline & & & 425 & 53 & 71 & 71 & 2.482 & 2.482 & 2.482 \\
\hline & & & 463 & 81 & 81 & 81 & 30.792 & 30.792 & 30.792 \\
\hline & & 200 & 189 & 72 & 143 & 154 & 1.525 & 1.525 & 1.525 \\
\hline & & & 463 & 15 & 15 & 15 & 5.716 & 5.716 & 5.716 \\
\hline & & 206 & 244 & & 2 & 2 & & 0.717 & 0.717 \\
\hline & & 244 & 122 & 86 & 86 & 86 & 3.657 & 3.657 & 3.657 \\
\hline & & & 135 & 50 & 51 & 51 & 12.810 & 12.810 & 12.810 \\
\hline & & & 179 & 86 & 88 & 88 & 4.331 & 4.331 & 4.331 \\
\hline & & & 192 & 38 & 38 & 38 & 3.295 & 3.295 & 3.295 \\
\hline & & & 206 & & 2 & 2 & & 0.717 & 0.717 \\
\hline & & & 244 & & & 6 & & & 0.213 \\
\hline & & & 261 & 359 & 361 & 361 & 5.387 & 5.387 & 5.387 \\
\hline & & & 265 & 802 & 874 & 877 & 10.209 & 10.209 & 10.209 \\
\hline & & & 266 & 191 & 195 & 195 & 22.729 & 22.729 & 22.729 \\
\hline & & & 380 & 8 & 8 & 8 & 1.356 & 1.356 & 1.356 \\
\hline & & & 425 & 2 & 2 & 2 & 0.509 & 0.509 & 0.509 \\
\hline & & 261 & 122 & 40 & 41 & 41 & 3.177 & 3.177 & 3.177 \\
\hline & & & 135 & 11 & 12 & 12 & 12.714 & 12.714 & 12.714 \\
\hline & & & 179 & 6 & 6 & 6 & 2.990 & 2.990 & 2.990 \\
\hline & & & 192 & 10 & 10 & 10 & 2.775 & 2.775 & 2.775 \\
\hline & & & 244 & 359 & 361 & 361 & 5.387 & 5.387 & 5.387 \\
\hline & & & 265 & & 3 & 3 & & 0.487 & 0.487 \\
\hline & & & 266 & 17 & 17 & 17 & 2.313 & 2.313 & 2.313 \\
\hline & & 265 & 122 & 34 & 36 & 36 & 2.689 & 2.689 & 2.689 \\
\hline & & & 135 & 5 & 10 & 10 & 0.892 & 0.892 & 0.892 \\
\hline & & & 179 & 31 & 32 & 32 & 1.507 & 1.507 & 1.507 \\
\hline & & & 192 & 15 & 15 & 15 & 1.413 & 1.413 & 1.413 \\
\hline & & & 244 & 802 & 874 & 877 & 10.209 & 10.209 & 10.209 \\
\hline & & & 261 & & 3 & 3 & & 0.487 & 0.487 \\
\hline & & & 266 & 31 & 36 & 37 & 1.296 & 1.296 & 1.296 \\
\hline & & 266 & 122 & 57 & 59 & 59 & 3.747 & 3.747 & 3.747 \\
\hline
\end{tabular}


Table B.2 - Continued from previous page

\begin{tabular}{|c|c|c|c|c|c|c|c|c|c|}
\hline Name & $\begin{array}{l}\text { UniProt } \\
\text { ID }\end{array}$ & Residue 1 & Residue 2 & FDR $1 \%$ & FDR $3 \%$ & FDR 5\% & F DR $1 \%$ & FDR3\% & FDR5\% \\
\hline & & & 135 & 12 & 12 & 12 & 8.408 & 8.408 & 8.408 \\
\hline & & & 179 & 19 & 19 & 19 & 4.044 & 4.044 & 4.044 \\
\hline & & & 192 & 12 & 12 & 12 & 4.817 & 4.817 & 4.817 \\
\hline & & & 244 & 191 & 195 & 195 & 22.729 & 22.729 & 22.729 \\
\hline & & & 261 & 17 & 17 & 17 & 2.313 & 2.313 & 2.313 \\
\hline & & & 265 & 31 & 36 & 37 & 1.296 & 1.296 & 1.296 \\
\hline & & & 266 & 16 & 18 & 18 & 1.357 & 1.357 & 1.357 \\
\hline & & 380 & 122 & 28 & 30 & 30 & 3.100 & 3.100 & 3.100 \\
\hline & & & 135 & 4 & 7 & 7 & 1.314 & 1.314 & 1.314 \\
\hline & & & 244 & 8 & 8 & 8 & 1.356 & 1.356 & 1.356 \\
\hline & & 417 & 423 & 2 & 2 & 2 & 0.469 & 0.469 & 0.469 \\
\hline & & & 425 & 23 & 32 & 33 & 1.162 & 1.162 & 1.162 \\
\hline & & 423 & 122 & 1 & 1 & 1 & 1.254 & 1.254 & 1.254 \\
\hline & & & 135 & 14 & 14 & 14 & 2.329 & 2.329 & 2.329 \\
\hline & & & 192 & 59 & 59 & 60 & 2.720 & 2.720 & 2.720 \\
\hline & & & 417 & 2 & 2 & 2 & 0.469 & 0.469 & 0.469 \\
\hline & & 425 & 1 & & 2 & 3 & & 0.353 & 0.353 \\
\hline & & & 122 & 34 & 36 & 36 & 1.519 & 1.519 & 1.519 \\
\hline & & & 135 & 19 & 25 & 25 & 5.991 & 5.991 & 5.991 \\
\hline & & & 179 & 11 & 11 & 11 & 1.258 & 1.258 & 1.258 \\
\hline & & & 189 & 25 & 28 & 28 & 1.460 & 1.460 & 1.460 \\
\hline & & & 192 & 53 & 71 & 71 & 2.482 & 2.482 & 2.482 \\
\hline & & & 244 & 2 & 2 & 2 & 0.509 & 0.509 & 0.509 \\
\hline & & & 417 & 23 & 32 & 33 & 1.162 & 1.162 & 1.162 \\
\hline & & & 463 & 2 & 2 & 2 & 0.717 & 0.717 & 0.717 \\
\hline & & 428 & 122 & 7 & 7 & 7 & 2.460 & 2.460 & 2.460 \\
\hline & & 463 & 192 & 81 & 81 & 81 & 30.792 & 30.792 & 30.792 \\
\hline & & & 200 & 15 & 15 & 15 & 5.716 & 5.716 & 5.716 \\
\hline & & & 425 & 2 & 2 & 2 & 0.717 & 0.717 & 0.717 \\
\hline PRP38 & Q8NAV1 & 92 & 118 & 64 & 67 & 67 & 3.744 & 3.744 & 3.744 \\
\hline & & 97 & 131 & 67 & 68 & 68 & 2.837 & 2.837 & 2.837 \\
\hline & & 118 & 92 & 64 & 67 & 67 & 3.744 & 3.744 & 3.744 \\
\hline & & 131 & 97 & 67 & 68 & 68 & 2.837 & 2.837 & 2.837 \\
\hline PRP4B & Q13523 & 111 & 117 & 4 & 4 & 4 & 3.177 & 3.177 & 3.177 \\
\hline & & 117 & 111 & 4 & 4 & 4 & 3.177 & 3.177 & 3.177 \\
\hline & & 168 & 177 & 15 & 26 & 27 & 1.127 & 1.127 & 1.127 \\
\hline & & & 181 & 3 & 6 & 6 & 1.924 & 1.924 & 1.924 \\
\hline & & 170 & 181 & 8 & 13 & 15 & 0.680 & 0.680 & 0.680 \\
\hline & & 177 & 168 & 15 & 26 & 27 & 1.127 & 1.127 & 1.127 \\
\hline & & 181 & 168 & 3 & 6 & 6 & 1.924 & 1.924 & 1.924 \\
\hline & & & 170 & 8 & 13 & 15 & 0.680 & 0.680 & 0.680 \\
\hline & & & 202 & & 2 & 2 & & 0.294 & 0.294 \\
\hline & & 198 & 200 & 2 & 3 & 3 & 1.076 & 1.076 & 1.076 \\
\hline & & & 202 & 12 & 16 & 16 & 1.837 & 1.837 & 1.837 \\
\hline & & & 209 & 4 & 5 & 5 & 0.772 & 0.772 & 0.772 \\
\hline & & 199 & 202 & 6 & 6 & 6 & 1.549 & 1.549 & 1.549 \\
\hline & & 200 & 198 & 2 & 3 & 3 & 1.076 & 1.076 & 1.076 \\
\hline & & 202 & 181 & & 2 & 2 & & 0.294 & 0.294 \\
\hline & & & 198 & 12 & 16 & 16 & 1.837 & 1.837 & 1.837 \\
\hline & & & 199 & 6 & 6 & 6 & 1.549 & 1.549 & 1.549 \\
\hline & & & 211 & 22 & 23 & 23 & 1.839 & 1.839 & 1.839 \\
\hline & & 209 & 198 & 4 & 5 & 5 & 0.772 & 0.772 & 0.772 \\
\hline & & 211 & 202 & 22 & 23 & 23 & 1.839 & 1.839 & 1.839 \\
\hline & & 254 & 268 & 4 & 4 & 4 & 1.867 & 1.867 & 1.867 \\
\hline & & 262 & 990 & & 2 & 2 & & 0.152 & 0.152 \\
\hline & & 268 & 254 & 4 & 4 & 4 & 1.867 & 1.867 & 1.867 \\
\hline & & 393 & 625 & & 2 & 2 & & 0.571 & 0.571 \\
\hline & & 551 & 915 & 6 & 6 & 6 & 1.790 & 1.790 & 1.790 \\
\hline & & 593 & 747 & 10 & 10 & 11 & 1.921 & 1.921 & 1.921 \\
\hline & & 608 & 625 & 14 & 14 & 14 & 2.239 & 2.239 & 2.239 \\
\hline & & & 626 & 19 & 20 & 20 & 3.950 & 3.950 & 3.950 \\
\hline & & & 656 & 3 & 3 & 3 & 1.611 & 1.611 & 1.611 \\
\hline & & & 780 & 61 & 67 & 67 & 2.625 & 2.625 & 2.625 \\
\hline & & & 783 & 76 & 76 & 76 & 3.752 & 3.752 & 3.752 \\
\hline & & & 790 & 13 & 13 & 13 & 2.596 & 2.596 & 2.596 \\
\hline & & & 827 & 2 & 2 & 2 & 0.659 & 0.659 & 0.659 \\
\hline & & 625 & 393 & & 2 & 2 & & 0.571 & 0.571 \\
\hline & & & 608 & 14 & 14 & 14 & 2.239 & 2.239 & 2.239 \\
\hline & & & 656 & 2 & 3 & 3 & 1.098 & 1.098 & 1.098 \\
\hline & & & 659 & & 2 & 2 & & 1.478 & 1.478 \\
\hline & & & 783 & 17 & 17 & 17 & 3.014 & 3.014 & 3.014 \\
\hline & & & 892 & 23 & 24 & 24 & 1.581 & 1.581 & 1.581 \\
\hline & & & 936 & 3 & 3 & 3 & 1.545 & 1.545 & 1.545 \\
\hline & & 626 & 608 & 19 & 20 & 20 & 3.950 & 3.950 & 3.950 \\
\hline & & & 656 & 5 & 5 & 5 & 1.217 & 1.217 & 1.217 \\
\hline & & & 659 & 6 & 6 & 6 & 1.886 & 1.886 & 1.886 \\
\hline & & & 780 & 5 & 5 & 5 & 1.856 & 1.856 & 1.856 \\
\hline & & & 783 & 23 & 23 & 23 & 3.470 & 3.470 & 3.470 \\
\hline & & & 892 & 27 & 27 & 27 & 2.783 & 2.783 & 2.783 \\
\hline & & & 936 & 4 & 4 & 4 & 3.500 & 3.500 & 3.500 \\
\hline & & 656 & 608 & 3 & 3 & 3 & 1.611 & 1.611 & 1.611 \\
\hline & & & 625 & 2 & 3 & 3 & 1.098 & 1.098 & 1.098 \\
\hline & & & 626 & 5 & 5 & 5 & 1.217 & 1.217 & 1.217 \\
\hline
\end{tabular}


Table B. 2 - Continued from previous page

\begin{tabular}{|c|c|c|c|c|c|c|c|c|c|}
\hline Name & $\begin{array}{l}\text { UniProt } \\
\text { ID }\end{array}$ & Residue 1 & Residue 2 & FDR $1 \%$ & FDR $3 \%$ & FDR $5 \%$ & FDR $1 \%$ & F DR3\% & F DR5\% \\
\hline & & & 685 & 8 & 8 & 8 & 1.271 & 1.271 & 1.271 \\
\hline & & & 717 & 25 & 27 & 27 & 2.579 & 2.579 & 2.579 \\
\hline & & & 727 & 7 & 9 & 9 & 1.073 & 1.073 & 1.073 \\
\hline & & & 760 & 6 & 6 & 6 & 1.430 & 1.430 & 1.430 \\
\hline & & & 780 & 18 & 18 & 18 & 1.609 & 1.609 & 1.609 \\
\hline & & & 783 & 25 & 25 & 25 & 2.834 & 2.834 & 2.834 \\
\hline & & & 817 & 3 & 4 & 4 & 1.275 & 1.275 & 1.275 \\
\hline & & & 892 & 44 & 45 & 45 & 2.624 & 2.624 & 2.624 \\
\hline & & & 936 & 6 & 7 & 8 & 3.261 & 3.261 & 3.261 \\
\hline & & 659 & 625 & & 2 & 2 & & 1.478 & 1.478 \\
\hline & & & 626 & 6 & 6 & 6 & 1.886 & 1.886 & 1.886 \\
\hline & & & 685 & 5 & 6 & 6 & 0.825 & 0.825 & 0.825 \\
\hline & & & 717 & 6 & 6 & 6 & 1.341 & 1.341 & 1.341 \\
\hline & & & 760 & 11 & 11 & 11 & 1.803 & 1.803 & 1.803 \\
\hline & & & 780 & 16 & 16 & 16 & 2.571 & 2.571 & 2.571 \\
\hline & & & 783 & 13 & 13 & 13 & 2.359 & 2.359 & 2.359 \\
\hline & & & 892 & 37 & 38 & 38 & 2.129 & 2.129 & 2.129 \\
\hline & & & 936 & 3 & 3 & 3 & 2.301 & 2.301 & 2.301 \\
\hline & & 685 & 656 & 8 & 8 & 8 & 1.271 & 1.271 & 1.271 \\
\hline & & & 659 & 5 & 6 & 6 & 0.825 & 0.825 & 0.825 \\
\hline & & & 737 & & 2 & 2 & & 0.341 & 0.341 \\
\hline & & & 760 & 98 & 114 & 116 & 2.944 & 2.944 & 2.944 \\
\hline & & 717 & 656 & 25 & 27 & 27 & 2.579 & 2.579 & 2.579 \\
\hline & & & 659 & 6 & 6 & 6 & 1.341 & 1.341 & 1.341 \\
\hline & & 727 & 656 & 7 & 9 & 9 & 1.073 & 1.073 & 1.073 \\
\hline & & & 737 & 3 & 3 & 3 & 1.830 & 1.830 & 1.830 \\
\hline & & & 738 & 22 & 22 & 22 & 2.495 & 2.495 & 2.495 \\
\hline & & 731 & 738 & 18 & 18 & 18 & 4.065 & 4.065 & 4.065 \\
\hline & & 737 & 685 & & 2 & 2 & & 0.341 & 0.341 \\
\hline & & & 727 & 3 & 3 & 3 & 1.830 & 1.830 & 1.830 \\
\hline & & 738 & 727 & 22 & 22 & 22 & 2.495 & 2.495 & 2.495 \\
\hline & & & 731 & 18 & 18 & 18 & 4.065 & 4.065 & 4.065 \\
\hline & & 747 & 593 & 10 & 10 & 11 & 1.921 & 1.921 & 1.921 \\
\hline & & & 804 & 44 & 47 & 47 & 2.179 & 2.179 & 2.179 \\
\hline & & 760 & 656 & 6 & 6 & 6 & 1.430 & 1.430 & 1.430 \\
\hline & & & 659 & 11 & 11 & 11 & 1.803 & 1.803 & 1.803 \\
\hline & & & 685 & 98 & 114 & 116 & 2.944 & 2.944 & 2.944 \\
\hline & & 780 & 608 & 61 & 67 & 67 & 2.625 & 2.625 & 2.625 \\
\hline & & & 626 & 5 & 5 & 5 & 1.856 & 1.856 & 1.856 \\
\hline & & & 656 & 18 & 18 & 18 & 1.609 & 1.609 & 1.609 \\
\hline & & & 659 & 16 & 16 & 16 & 2.571 & 2.571 & 2.571 \\
\hline & & & 827 & 24 & 25 & 26 & 1.344 & 1.344 & 1.344 \\
\hline & & 783 & 608 & 76 & 76 & 76 & 3.752 & 3.752 & 3.752 \\
\hline & & & 625 & 17 & 17 & 17 & 3.014 & 3.014 & 3.014 \\
\hline & & & 626 & 23 & 23 & 23 & 3.470 & 3.470 & 3.470 \\
\hline & & & 656 & 25 & 25 & 25 & 2.834 & 2.834 & 2.834 \\
\hline & & & 659 & 13 & 13 & 13 & 2.359 & 2.359 & 2.359 \\
\hline & & & 892 & 13 & 14 & 14 & 2.007 & 2.007 & 2.007 \\
\hline & & 790 & 608 & 13 & 13 & 13 & 2.596 & 2.596 & 2.596 \\
\hline & & & 827 & 7 & 8 & 8 & 2.209 & 2.209 & 2.209 \\
\hline & & & 972 & 2 & 2 & 2 & 2.587 & 2.587 & 2.587 \\
\hline & & & 1006 & 33 & 33 & 33 & 4.463 & 4.463 & 4.463 \\
\hline & & 804 & 747 & 44 & 47 & 47 & 2.179 & 2.179 & 2.179 \\
\hline & & & 817 & 6 & 7 & 7 & 2.415 & 2.415 & 2.415 \\
\hline & & & 1006 & 3 & 3 & 3 & 1.948 & 1.948 & 1.948 \\
\hline & & 817 & 656 & 3 & 4 & 4 & 1.275 & 1.275 & 1.275 \\
\hline & & & 804 & 6 & 7 & 7 & 2.415 & 2.415 & 2.415 \\
\hline & & 827 & 608 & 2 & 2 & 2 & 0.659 & 0.659 & 0.659 \\
\hline & & & 780 & 24 & 25 & 26 & 1.344 & 1.344 & 1.344 \\
\hline & & & 790 & 7 & 8 & 8 & 2.209 & 2.209 & 2.209 \\
\hline & & 864 & 911 & 2 & 2 & 2 & 1.437 & 1.437 & 1.437 \\
\hline & & & 915 & 17 & 17 & 17 & 3.640 & 3.640 & 3.640 \\
\hline & & 892 & 625 & 23 & 24 & 24 & 1.581 & 1.581 & 1.581 \\
\hline & & & 626 & 27 & 27 & 27 & 2.783 & 2.783 & 2.783 \\
\hline & & & 656 & 44 & 45 & 45 & 2.624 & 2.624 & 2.624 \\
\hline & & & 659 & 37 & 38 & 38 & 2.129 & 2.129 & 2.129 \\
\hline & & & 783 & 13 & 14 & 14 & 2.007 & 2.007 & 2.007 \\
\hline & & & 936 & 120 & 120 & 120 & 3.438 & 3.438 & 3.438 \\
\hline & & & 942 & 35 & 35 & 35 & 3.135 & 3.135 & 3.135 \\
\hline & & 905 & 911 & 3 & 3 & 3 & 1.347 & 1.347 & 1.347 \\
\hline & & 907 & 990 & 10 & 10 & 10 & 2.138 & 2.138 & 2.138 \\
\hline & & 911 & 864 & 2 & 2 & 2 & 1.437 & 1.437 & 1.437 \\
\hline & & & 905 & 3 & 3 & 3 & 1.347 & 1.347 & 1.347 \\
\hline & & & 915 & 149 & 153 & 153 & 8.649 & 8.649 & 8.649 \\
\hline & & & 990 & 140 & 151 & 152 & 2.829 & 2.829 & 2.829 \\
\hline & & 915 & 551 & 6 & 6 & 6 & 1.790 & 1.790 & 1.790 \\
\hline & & & 864 & 17 & 17 & 17 & 3.640 & 3.640 & 3.640 \\
\hline & & & 911 & 149 & 153 & 153 & 8.649 & 8.649 & 8.649 \\
\hline & & & 990 & 104 & 106 & 106 & 5.628 & 5.628 & 5.628 \\
\hline & & 936 & 625 & 3 & 3 & 3 & 1.545 & 1.545 & 1.545 \\
\hline & & & 626 & 4 & 4 & 4 & 3.500 & 3.500 & 3.500 \\
\hline & & & 656 & 6 & 7 & 8 & 3.261 & 3.261 & 3.261 \\
\hline & & & 659 & 3 & 3 & 3 & 2.301 & 2.301 & 2.301 \\
\hline
\end{tabular}


Table B.2 - Continued from previous page

\begin{tabular}{|c|c|c|c|c|c|c|c|c|c|}
\hline Name & $\begin{array}{l}\text { UniProt } \\
\text { ID }\end{array}$ & Residue 1 & Residue 2 & FDR 1\% & FDR 3\% & FDR $5 \%$ & FDR $1 \%$ & FDR3\% & FDR5\% \\
\hline & & & 892 & 120 & 120 & 120 & 3.438 & 3.438 & 3.438 \\
\hline & & & 942 & 23 & 24 & 24 & 2.688 & 2.688 & 2.688 \\
\hline & & 942 & 892 & 35 & 35 & 35 & 3.135 & 3.135 & 3.135 \\
\hline & & & 936 & 23 & 24 & 24 & 2.688 & 2.688 & 2.688 \\
\hline & & 972 & 790 & 2 & 2 & 2 & 2.587 & 2.587 & 2.587 \\
\hline & & 990 & 262 & & 2 & 2 & & 0.152 & 0.152 \\
\hline & & & 907 & 10 & 10 & 10 & 2.138 & 2.138 & 2.138 \\
\hline & & & 911 & 140 & 151 & 152 & 2.829 & 2.829 & 2.829 \\
\hline & & & 915 & 104 & 106 & 106 & 5.628 & 5.628 & 5.628 \\
\hline & & 1006 & 790 & 33 & 33 & 33 & 4.463 & 4.463 & 4.463 \\
\hline & & & 804 & 3 & 3 & 3 & 1.948 & 1.948 & 1.948 \\
\hline \multirow[t]{72}{*}{ PRP8 } & Q6P2Q9 & 29 & 36 & 77 & 77 & 77 & 4.423 & 4.423 & 4.423 \\
\hline & & 33 & 36 & 10 & 10 & 10 & 2.368 & 2.368 & 2.368 \\
\hline & & 36 & 29 & 77 & 77 & 77 & 4.423 & 4.423 & 4.423 \\
\hline & & & 33 & 10 & 10 & 10 & 2.368 & 2.368 & 2.368 \\
\hline & & 43 & 48 & 25 & 35 & 35 & 1.173 & 1.173 & 1.173 \\
\hline & & & 50 & 22 & 23 & 23 & 2.397 & 2.397 & 2.397 \\
\hline & & 48 & 43 & 25 & 35 & 35 & 1.173 & 1.173 & 1.173 \\
\hline & & & 50 & 10 & 16 & 17 & 0.907 & 0.907 & 0.907 \\
\hline & & & 58 & & 2 & 2 & & 0.374 & 0.374 \\
\hline & & 50 & 43 & 22 & 23 & 23 & 2.397 & 2.397 & 2.397 \\
\hline & & & 48 & 10 & 16 & 17 & 0.907 & 0.907 & 0.907 \\
\hline & & & 480 & 2 & 2 & 2 & 2.068 & 2.068 & 2.068 \\
\hline & & 58 & 48 & & 2 & 2 & & 0.374 & 0.374 \\
\hline & & 85 & 93 & 2 & 2 & 2 & 0.599 & 0.599 & 0.599 \\
\hline & & & 480 & & 2 & 2 & & 1.055 & 1.055 \\
\hline & & 93 & 85 & 2 & 2 & 2 & 0.599 & 0.599 & 0.599 \\
\hline & & & 666 & 13 & 14 & 14 & 1.836 & 1.836 & 1.836 \\
\hline & & 101 & 744 & & 2 & 2 & & 0.163 & 0.163 \\
\hline & & & 773 & 4 & 5 & 5 & 1.057 & 1.057 & 1.057 \\
\hline & & 278 & 449 & 3 & 3 & 3 & 1.372 & 1.372 & 1.372 \\
\hline & & 442 & 609 & 53 & 53 & 53 & 3.316 & 3.316 & 3.316 \\
\hline & & 449 & 278 & 3 & 3 & 3 & 1.372 & 1.372 & 1.372 \\
\hline & & 480 & 50 & 2 & 2 & 2 & 2.068 & 2.068 & 2.068 \\
\hline & & & 85 & & 2 & 2 & & 1.055 & 1.055 \\
\hline & & 511 & 533 & 2 & 4 & 4 & 0.780 & 0.780 & 0.780 \\
\hline & & & 666 & 27 & 29 & 29 & 2.780 & 2.780 & 2.780 \\
\hline & & & 670 & 5 & 5 & 5 & 1.443 & 1.443 & 1.443 \\
\hline & & 533 & 511 & 2 & 4 & 4 & 0.780 & 0.780 & 0.780 \\
\hline & & 606 & 1838 & & 2 & 2 & & 0.385 & 0.385 \\
\hline & & 609 & 442 & 53 & 53 & 53 & 3.316 & 3.316 & 3.316 \\
\hline & & & 623 & 4 & 4 & 4 & 2.672 & 2.672 & 2.672 \\
\hline & & 623 & 609 & 4 & 4 & 4 & 2.672 & 2.672 & 2.672 \\
\hline & & 666 & 93 & 13 & 14 & 14 & 1.836 & 1.836 & 1.836 \\
\hline & & & 511 & 27 & 29 & 29 & 2.780 & 2.780 & 2.780 \\
\hline & & & 674 & 6 & 6 & 6 & 1.973 & 1.973 & 1.973 \\
\hline & & 670 & 511 & 5 & 5 & 5 & 1.443 & 1.443 & 1.443 \\
\hline & & 674 & 666 & 6 & 6 & 6 & 1.973 & 1.973 & 1.973 \\
\hline & & & 774 & 30 & 30 & 31 & 4.083 & 4.083 & 4.083 \\
\hline & & 721 & 746 & 59 & 61 & 61 & 3.080 & 3.080 & 3.080 \\
\hline & & & 774 & 43 & 43 & 43 & 4.032 & 4.032 & 4.032 \\
\hline & & & 1020 & 10 & 10 & 10 & 2.541 & 2.541 & 2.541 \\
\hline & & 727 & 744 & 2 & 3 & 3 & 1.038 & 1.038 & 1.038 \\
\hline & & 744 & 101 & & 2 & 2 & & 0.163 & 0.163 \\
\hline & & & 727 & 2 & 3 & 3 & 1.038 & 1.038 & 1.038 \\
\hline & & 746 & 721 & 59 & 61 & 61 & 3.080 & 3.080 & 3.080 \\
\hline & & & 774 & 102 & 105 & 105 & 3.541 & 3.541 & 3.541 \\
\hline & & 769 & 774 & 15 & 16 & 17 & 2.219 & 2.219 & 2.219 \\
\hline & & & 853 & 82 & 89 & 89 & 3.821 & 3.821 & 3.821 \\
\hline & & & 1020 & 5 & 5 & 5 & 1.332 & 1.332 & 1.332 \\
\hline & & & 1262 & 89 & 90 & 90 & 2.299 & 2.299 & 2.299 \\
\hline & & & 1449 & 100 & 100 & 100 & 3.261 & 3.261 & 3.261 \\
\hline & & & 2140 & & 2 & 2 & & 0.352 & 0.352 \\
\hline & & 773 & 101 & 4 & 5 & 5 & 1.057 & 1.057 & 1.057 \\
\hline & & & 853 & 21 & 29 & 30 & 1.038 & 1.038 & 1.038 \\
\hline & & & 1020 & 46 & 52 & 52 & 1.462 & 1.462 & 1.462 \\
\hline & & & 1262 & 20 & 20 & 21 & 1.300 & 1.300 & 1.300 \\
\hline & & & 1449 & 22 & 24 & 24 & 1.537 & 1.537 & 1.537 \\
\hline & & 774 & 674 & 30 & 30 & 31 & 4.083 & 4.083 & 4.083 \\
\hline & & & 721 & 43 & 43 & 43 & 4.032 & 4.032 & 4.032 \\
\hline & & & 746 & 102 & 105 & 105 & 3.541 & 3.541 & 3.541 \\
\hline & & & 769 & 15 & 16 & 17 & 2.219 & 2.219 & 2.219 \\
\hline & & & 1020 & 134 & 139 & 140 & 3.037 & 3.037 & 3.037 \\
\hline & & 833 & 932 & 29 & 29 & 29 & 1.912 & 1.912 & 1.912 \\
\hline & & 853 & 769 & 82 & 89 & 89 & 3.821 & 3.821 & 3.821 \\
\hline & & & 773 & 21 & 29 & 30 & 1.038 & 1.038 & 1.038 \\
\hline & & & 1262 & 2 & 2 & 2 & 0.985 & 0.985 & 0.985 \\
\hline & & & 1320 & 55 & 63 & 64 & 2.393 & 2.393 & 2.393 \\
\hline & & & 1449 & 88 & 92 & 92 & 2.505 & 2.505 & 2.505 \\
\hline & & & 1463 & 37 & 44 & 44 & 2.032 & 2.032 & 2.032 \\
\hline & & & 1505 & 64 & 71 & 72 & 2.277 & 2.277 & 2.277 \\
\hline & & 882 & 892 & 26 & 27 & 27 & 3.186 & 3.186 & 3.186 \\
\hline & & 892 & 882 & 26 & 27 & 27 & 3.186 & 3.186 & 3.186 \\
\hline
\end{tabular}


Table B.2 - Continued from previous page

\begin{tabular}{|c|c|c|c|c|c|c|c|c|c|}
\hline Name & $\begin{array}{l}\text { UniProt } \\
\text { ID }\end{array}$ & Residue 1 & Residue 2 & FDR $1 \%$ & FDR $3 \%$ & FDR $5 \%$ & FDR1\% & FDR3\% & FDR5\% \\
\hline & & 932 & 833 & 29 & 29 & 29 & 1.912 & 1.912 & 1.912 \\
\hline & & 983 & 1158 & 89 & 94 & 94 & 4.846 & 4.846 & 4.846 \\
\hline & & 987 & 1158 & 99 & 105 & 105 & 3.960 & 3.960 & 3.960 \\
\hline & & 1020 & 721 & 10 & 10 & 10 & 2.541 & 2.541 & 2.541 \\
\hline & & & 769 & 5 & 5 & 5 & 1.332 & 1.332 & 1.332 \\
\hline & & & 773 & 46 & 52 & 52 & 1.462 & 1.462 & 1.462 \\
\hline & & & 774 & 134 & 139 & 140 & 3.037 & 3.037 & 3.037 \\
\hline & & 1131 & 1158 & 2 & 3 & 3 & 1.239 & 1.239 & 1.239 \\
\hline & & 1132 & 1344 & 2 & 2 & 2 & 2.554 & 2.554 & 2.554 \\
\hline & & & 1463 & & & 2 & & & 0.113 \\
\hline & & & 2113 & 3 & 7 & 7 & 0.449 & 0.449 & 0.449 \\
\hline & & 1144 & 1180 & 21 & 21 & 21 & 3.530 & 3.530 & 3.530 \\
\hline & & 1158 & 983 & 89 & 94 & 94 & 4.846 & 4.846 & 4.846 \\
\hline & & & 987 & 99 & 105 & 105 & 3.960 & 3.960 & 3.960 \\
\hline & & & 1131 & 2 & 3 & 3 & 1.239 & 1.239 & 1.239 \\
\hline & & 1180 & 1144 & 21 & 21 & 21 & 3.530 & 3.530 & 3.530 \\
\hline & & 1262 & 769 & 89 & 90 & 90 & 2.299 & 2.299 & 2.299 \\
\hline & & & 773 & 20 & 20 & 21 & 1.300 & 1.300 & 1.300 \\
\hline & & & 853 & 2 & 2 & 2 & 0.985 & 0.985 & 0.985 \\
\hline & & 1290 & 1449 & 2 & 3 & 3 & 0.567 & 0.567 & 0.567 \\
\hline & & 1320 & 853 & 55 & 63 & 64 & 2.393 & 2.393 & 2.393 \\
\hline & & & 1463 & 80 & 85 & 86 & 3.076 & 3.076 & 3.076 \\
\hline & & 1344 & 1132 & 2 & 2 & 2 & 2.554 & 2.554 & 2.554 \\
\hline & & & 1735 & 135 & 135 & 135 & 4.372 & 4.372 & 4.372 \\
\hline & & & 1838 & 9 & 10 & 10 & 1.497 & 1.497 & 1.497 \\
\hline & & 1449 & 769 & 100 & 100 & 100 & 3.261 & 3.261 & 3.261 \\
\hline & & & 773 & 22 & 24 & 24 & 1.537 & 1.537 & 1.537 \\
\hline & & & 853 & 88 & 92 & 92 & 2.505 & 2.505 & 2.505 \\
\hline & & & 1290 & 2 & 3 & 3 & 0.567 & 0.567 & 0.567 \\
\hline & & & 1463 & 42 & 43 & 43 & 3.956 & 3.956 & 3.956 \\
\hline & & 1463 & 853 & 37 & 44 & 44 & 2.032 & 2.032 & 2.032 \\
\hline & & & 1132 & & & 2 & & & 0.113 \\
\hline & & & 1320 & 80 & 85 & 86 & 3.076 & 3.076 & 3.076 \\
\hline & & & 1449 & 42 & 43 & 43 & 3.956 & 3.956 & 3.956 \\
\hline & & & 1505 & 2 & 2 & 2 & 1.369 & 1.369 & 1.369 \\
\hline & & 1505 & 853 & 64 & 71 & 72 & 2.277 & 2.277 & 2.277 \\
\hline & & & 1463 & 2 & 2 & 2 & 1.369 & 1.369 & 1.369 \\
\hline & & 1735 & 1344 & 135 & 135 & 135 & 4.372 & 4.372 & 4.372 \\
\hline & & & 2244 & 66 & 67 & 67 & 2.214 & 2.214 & 2.214 \\
\hline & & & 2249 & 61 & 62 & 62 & 3.134 & 3.134 & 3.134 \\
\hline & & 1801 & 1831 & 2 & 4 & 4 & 1.044 & 1.044 & 1.044 \\
\hline & & 1820 & 1840 & 24 & 24 & 24 & 3.458 & 3.458 & 3.458 \\
\hline & & 1831 & 1801 & 2 & 4 & 4 & 1.044 & 1.044 & 1.044 \\
\hline & & & 1838 & 20 & 21 & 21 & 1.213 & 1.213 & 1.213 \\
\hline & & & 1840 & 34 & 35 & 35 & 3.524 & 3.524 & 3.524 \\
\hline & & 1838 & 606 & & 2 & 2 & & 0.385 & 0.385 \\
\hline & & & 1344 & 9 & 10 & 10 & 1.497 & 1.497 & 1.497 \\
\hline & & & 1831 & 20 & 21 & 21 & 1.213 & 1.213 & 1.213 \\
\hline & & & 1866 & 52 & 53 & 53 & 4.246 & 4.246 & 4.246 \\
\hline & & & 2031 & 6 & 6 & 6 & 0.785 & 0.785 & 0.785 \\
\hline & & & 2034 & 34 & 34 & 34 & 2.032 & 2.032 & 2.032 \\
\hline & & & 2049 & 2 & 2 & 2 & 1.078 & 1.078 & 1.078 \\
\hline & & & 2098 & 2 & 2 & 2 & 1.503 & 1.503 & 1.503 \\
\hline & & & 2249 & 8 & 10 & 10 & 1.217 & 1.217 & 1.217 \\
\hline & & 1840 & 1820 & 24 & 24 & 24 & 3.458 & 3.458 & 3.458 \\
\hline & & & 1831 & 34 & 35 & 35 & 3.524 & 3.524 & 3.524 \\
\hline & & & 1993 & & 3 & 3 & & 0.282 & 0.282 \\
\hline & & 1859 & 1885 & 3 & 3 & 3 & 2.506 & 2.506 & 2.506 \\
\hline & & & 2034 & 1 & 2 & 2 & 1.825 & 1.825 & 1.825 \\
\hline & & 1866 & 1838 & 52 & 53 & 53 & 4.246 & 4.246 & 4.246 \\
\hline & & & 2034 & 6 & 6 & 6 & 4.164 & 4.164 & 4.164 \\
\hline & & 1885 & 1859 & 3 & 3 & 3 & 2.506 & 2.506 & 2.506 \\
\hline & & 1901 & 1955 & 1 & 1 & 1 & 1.256 & 1.256 & 1.256 \\
\hline & & 1955 & 1901 & 1 & 1 & 1 & 1.256 & 1.256 & 1.256 \\
\hline & & 1978 & 2034 & & 4 & 4 & & 1.646 & 1.646 \\
\hline & & & 2249 & 5 & 6 & 6 & 14.929 & 14.929 & 14.929 \\
\hline & & 1984 & 2034 & 5 & 5 & 5 & 2.247 & 2.247 & 2.247 \\
\hline & & & 2249 & 15 & 15 & 16 & 2.869 & 2.869 & 2.869 \\
\hline & & 1993 & 1840 & & 3 & 3 & & 0.282 & 0.282 \\
\hline & & 2031 & 1838 & 6 & 6 & 6 & 0.785 & 0.785 & 0.785 \\
\hline & & 2034 & 1838 & 34 & 34 & 34 & 2.032 & 2.032 & 2.032 \\
\hline & & & 1859 & 1 & 2 & 2 & 1.825 & 1.825 & 1.825 \\
\hline & & & 1866 & 6 & 6 & 6 & 4.164 & 4.164 & 4.164 \\
\hline & & & 1978 & & 4 & 4 & & 1.646 & 1.646 \\
\hline & & & 1984 & 5 & 5 & 5 & 2.247 & 2.247 & 2.247 \\
\hline & & & 2049 & 12 & 12 & 12 & 3.082 & 3.082 & 3.082 \\
\hline & & & 2070 & 3 & 3 & 3 & 2.736 & 2.736 & 2.736 \\
\hline & & 2049 & 1838 & 2 & 2 & 2 & 1.078 & 1.078 & 1.078 \\
\hline & & & 2034 & 12 & 12 & 12 & 3.082 & 3.082 & 3.082 \\
\hline & & & 2249 & 3 & 3 & 3 & 1.398 & 1.398 & 1.398 \\
\hline & & 2070 & 2034 & 3 & 3 & 3 & 2.736 & 2.736 & 2.736 \\
\hline & & 2098 & 1838 & 2 & 2 & 2 & 1.503 & 1.503 & 1.503 \\
\hline & & 2113 & 1132 & 3 & 7 & 7 & 0.449 & 0.449 & 0.449 \\
\hline
\end{tabular}


Table B.2 - Continued from previous page

\begin{tabular}{|c|c|c|c|c|c|c|c|c|c|}
\hline Name & $\begin{array}{l}\text { UniProt } \\
\text { ID }\end{array}$ & Residue 1 & Residue 2 & FDR 1\% & FDR 3\% & FDR 5\% & FDR1\% & FDR3\% & FDR5\% \\
\hline & & 2140 & 769 & & 2 & 2 & & 0.352 & 0.352 \\
\hline & & 2198 & 2244 & 5 & 6 & 7 & 1.346 & 1.346 & 1.346 \\
\hline & & 2244 & 1735 & 66 & 67 & 67 & 2.214 & 2.214 & 2.214 \\
\hline & & & 2198 & 5 & 6 & 7 & 1.346 & 1.346 & 1.346 \\
\hline & & 2249 & 1735 & 61 & 62 & 62 & 3.134 & 3.134 & 3.134 \\
\hline & & & 1838 & 8 & 10 & 10 & 1.217 & 1.217 & 1.217 \\
\hline & & & 1978 & 5 & 6 & 6 & 14.929 & 14.929 & 14.929 \\
\hline & & & 1984 & 15 & 15 & 16 & 2.869 & 2.869 & 2.869 \\
\hline & & & 2049 & 3 & 3 & 3 & 1.398 & 1.398 & 1.398 \\
\hline \multirow{50}{*}{ RBM22 } & Q9NW64 & 76 & 109 & 2 & 3 & 3 & 0.568 & 0.568 & 0.568 \\
\hline & & & 114 & & 2 & 2 & & 0.258 & 0.258 \\
\hline & & & 139 & 4 & 6 & 6 & 0.799 & 0.799 & 0.799 \\
\hline & & 78 & 139 & 7 & 7 & 7 & 2.730 & 2.730 & 2.730 \\
\hline & & 104 & 114 & 26 & 26 & 26 & 3.521 & 3.521 & 3.521 \\
\hline & & & 139 & 21 & 22 & 22 & 1.300 & 1.300 & 1.300 \\
\hline & & & 149 & 48 & 48 & 48 & 2.925 & 2.925 & 2.925 \\
\hline & & & 286 & 18 & 18 & 18 & 2.528 & 2.528 & 2.528 \\
\hline & & 109 & 76 & 2 & 3 & 3 & 0.568 & 0.568 & 0.568 \\
\hline & & & 139 & 12 & 14 & 14 & 2.186 & 2.186 & 2.186 \\
\hline & & & 149 & 12 & 13 & 13 & 1.352 & 1.352 & 1.352 \\
\hline & & & 286 & 7 & 8 & 9 & 2.085 & 2.085 & 2.085 \\
\hline & & 114 & 76 & & 2 & 2 & & 0.258 & 0.258 \\
\hline & & & 104 & 26 & 26 & 26 & 3.521 & 3.521 & 3.521 \\
\hline & & & 139 & 19 & 19 & 20 & 2.363 & 2.363 & 2.363 \\
\hline & & & 149 & 8 & 10 & 10 & 3.178 & 3.178 & 3.178 \\
\hline & & & 286 & 19 & 20 & 20 & 3.425 & 3.425 & 3.425 \\
\hline & & 139 & 76 & 4 & 6 & 6 & 0.799 & 0.799 & 0.799 \\
\hline & & & 78 & 7 & 7 & 7 & 2.730 & 2.730 & 2.730 \\
\hline & & & 104 & 21 & 22 & 22 & 1.300 & 1.300 & 1.300 \\
\hline & & & 109 & 12 & 14 & 14 & 2.186 & 2.186 & 2.186 \\
\hline & & & 114 & 19 & 19 & 20 & 2.363 & 2.363 & 2.363 \\
\hline & & & 229 & 3 & 4 & 4 & 2.232 & 2.232 & 2.232 \\
\hline & & & 286 & 14 & 15 & 15 & 1.531 & 1.531 & 1.531 \\
\hline & & & 290 & 3 & 4 & 4 & 1.314 & 1.314 & 1.314 \\
\hline & & 149 & 104 & 48 & 48 & 48 & 2.925 & 2.925 & 2.925 \\
\hline & & & 109 & 12 & 13 & 13 & 1.352 & 1.352 & 1.352 \\
\hline & & & 114 & 8 & 10 & 10 & 3.178 & 3.178 & 3.178 \\
\hline & & & 158 & 5 & 9 & 9 & 1.131 & 1.131 & 1.131 \\
\hline & & & 286 & 11 & 11 & 11 & 2.547 & 2.547 & 2.547 \\
\hline & & & 290 & 8 & 9 & 9 & 1.891 & 1.891 & 1.891 \\
\hline & & & 301 & 10 & 11 & 11 & 1.918 & 1.918 & 1.918 \\
\hline & & 158 & 149 & 5 & 9 & 9 & 1.131 & 1.131 & 1.131 \\
\hline & & & 212 & & 2 & 2 & & 0.782 & 0.782 \\
\hline & & & 290 & 4 & 5 & 5 & 1.299 & 1.299 & 1.299 \\
\hline & & 212 & 158 & & 2 & 2 & & 0.782 & 0.782 \\
\hline & & 229 & 139 & 3 & 4 & 4 & 2.232 & 2.232 & 2.232 \\
\hline & & 286 & 104 & 18 & 18 & 18 & 2.528 & 2.528 & 2.528 \\
\hline & & & 109 & 7 & 8 & 9 & 2.085 & 2.085 & 2.085 \\
\hline & & & 114 & 19 & 20 & 20 & 3.425 & 3.425 & 3.425 \\
\hline & & & 139 & 14 & 15 & 15 & 1.531 & 1.531 & 1.531 \\
\hline & & & 149 & 11 & 11 & 11 & 2.547 & 2.547 & 2.547 \\
\hline & & 290 & 139 & 3 & 4 & 4 & 1.314 & 1.314 & 1.314 \\
\hline & & & 149 & 8 & 9 & 9 & 1.891 & 1.891 & 1.891 \\
\hline & & & 158 & 4 & 5 & 5 & 1.299 & 1.299 & 1.299 \\
\hline & & 301 & 149 & 10 & 11 & 11 & 1.918 & 1.918 & 1.918 \\
\hline & & & 313 & 6 & 6 & 6 & 1.197 & 1.197 & 1.197 \\
\hline & & & 315 & 3 & 3 & 3 & 1.361 & 1.361 & 1.361 \\
\hline & & 313 & 301 & 6 & 6 & 6 & 1.197 & 1.197 & 1.197 \\
\hline & & 315 & 301 & 3 & 3 & 3 & 1.361 & 1.361 & 1.361 \\
\hline \multirow[t]{24}{*}{ RED } & Q13123 & 1 & 305 & & & 2 & & & 0.169 \\
\hline & & 30 & 38 & 13 & 16 & 16 & 2.303 & 2.303 & 2.303 \\
\hline & & 38 & 30 & 13 & 16 & 16 & 2.303 & 2.303 & 2.303 \\
\hline & & & 54 & 4 & 4 & 4 & 1.643 & 1.643 & 1.643 \\
\hline & & 54 & 38 & 4 & 4 & 4 & 1.643 & 1.643 & 1.643 \\
\hline & & 112 & 142 & & 2 & 2 & & 0.388 & 0.388 \\
\hline & & 137 & 151 & 5 & 9 & 9 & 0.795 & 0.795 & 0.795 \\
\hline & & 142 & 112 & & 2 & 2 & & 0.388 & 0.388 \\
\hline & & & 151 & 7 & 13 & 14 & 1.221 & 1.221 & 1.221 \\
\hline & & & 164 & 2 & 3 & 4 & 0.642 & 0.642 & 0.642 \\
\hline & & 151 & 137 & 5 & 9 & 9 & 0.795 & 0.795 & 0.795 \\
\hline & & & 142 & 7 & 13 & 14 & 1.221 & 1.221 & 1.221 \\
\hline & & & 173 & 6 & 6 & 6 & 1.732 & 1.732 & 1.732 \\
\hline & & 164 & 142 & 2 & 3 & 4 & 0.642 & 0.642 & 0.642 \\
\hline & & 173 & 151 & 6 & 6 & 6 & 1.732 & 1.732 & 1.732 \\
\hline & & & 181 & 11 & 11 & 11 & 1.881 & 1.881 & 1.881 \\
\hline & & 181 & 173 & 11 & 11 & 11 & 1.881 & 1.881 & 1.881 \\
\hline & & 191 & 197 & 25 & 27 & 27 & 2.493 & 2.493 & 2.493 \\
\hline & & & 198 & 34 & 36 & 36 & 3.358 & 3.358 & 3.358 \\
\hline & & & 205 & 25 & 26 & 26 & 2.053 & 2.053 & 2.053 \\
\hline & & & 209 & 24 & 24 & 24 & 2.261 & 2.261 & 2.261 \\
\hline & & & 222 & 2 & 7 & 7 & 0.632 & 0.632 & 0.632 \\
\hline & & 194 & 198 & 110 & 117 & 118 & 4.046 & 4.046 & 4.046 \\
\hline & & & 205 & 41 & 48 & 49 & 1.721 & 1.721 & 1.721 \\
\hline
\end{tabular}


Table B.2 - Continued from previous page

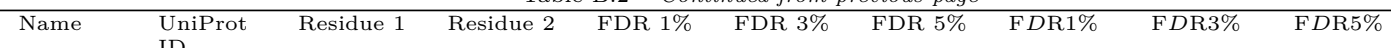

\begin{tabular}{|c|c|c|c|c|c|c|c|}
\hline & 209 & 43 & 48 & 48 & 2.397 & 2.397 & 2.397 \\
\hline & 222 & 20 & 30 & 30 & 1.775 & 1.775 & 1.775 \\
\hline & 224 & 9 & 11 & 11 & 1.588 & 1.588 & 1.588 \\
\hline & 509 & & 2 & 3 & & 0.170 & 0.170 \\
\hline 197 & 191 & 25 & 27 & 27 & 2.493 & 2.493 & 2.493 \\
\hline & 222 & 16 & 19 & 19 & 1.658 & 1.658 & 1.658 \\
\hline 198 & 191 & 34 & 36 & 36 & 3.358 & 3.358 & 3.358 \\
\hline & 194 & 110 & 117 & 118 & 4.046 & 4.046 & 4.046 \\
\hline & 222 & 72 & 75 & 75 & 2.429 & 2.429 & 2.429 \\
\hline & 224 & 22 & 23 & 23 & 1.992 & 1.992 & 1.992 \\
\hline 205 & 191 & 25 & 26 & 26 & 2.053 & 2.053 & 2.053 \\
\hline & 194 & 41 & 48 & 49 & 1.721 & 1.721 & 1.721 \\
\hline & 222 & 101 & 116 & 117 & 2.370 & 2.370 & 2.370 \\
\hline & 224 & 12 & 12 & 12 & 2.232 & 2.232 & 2.232 \\
\hline 209 & 191 & 24 & 24 & 24 & 2.261 & 2.261 & 2.261 \\
\hline & 194 & 43 & 48 & 48 & 2.397 & 2.397 & 2.397 \\
\hline & 222 & 5 & 6 & 6 & 1.110 & 1.110 & 1.110 \\
\hline 222 & 191 & 2 & 7 & 7 & 0.632 & 0.632 & 0.632 \\
\hline & 194 & 20 & 30 & 30 & 1.775 & 1.775 & 1.775 \\
\hline & 197 & 16 & 19 & 19 & 1.658 & 1.658 & 1.658 \\
\hline & 198 & 72 & 75 & 75 & 2.429 & 2.429 & 2.429 \\
\hline & 205 & 101 & 116 & 117 & 2.370 & 2.370 & 2.370 \\
\hline & 209 & 5 & 6 & 6 & 1.110 & 1.110 & 1.110 \\
\hline 224 & 194 & 9 & 11 & 11 & 1.588 & 1.588 & 1.588 \\
\hline & 198 & 22 & 23 & 23 & 1.992 & 1.992 & 1.992 \\
\hline & 205 & 12 & 12 & 12 & 2.232 & 2.232 & 2.232 \\
\hline 281 & 303 & 11 & 11 & 11 & 2.292 & 2.292 & 2.292 \\
\hline & 305 & 4 & 4 & 4 & 2.310 & 2.310 & 2.310 \\
\hline 303 & 281 & 11 & 11 & 11 & 2.292 & 2.292 & 2.292 \\
\hline 305 & 1 & & & 2 & & & 0.169 \\
\hline & 281 & 4 & 4 & 4 & 2.310 & 2.310 & 2.310 \\
\hline 378 & 386 & 25 & 28 & 28 & 1.988 & 1.988 & 1.988 \\
\hline & 388 & 4 & 4 & 4 & 0.942 & 0.942 & 0.942 \\
\hline & 404 & 4 & 4 & 4 & 1.206 & 1.206 & 1.206 \\
\hline 379 & 386 & 10 & 19 & 19 & 1.173 & 1.173 & 1.173 \\
\hline & 388 & 4 & 4 & 5 & 1.238 & 1.238 & 1.238 \\
\hline & 398 & 5 & 6 & 6 & 0.832 & 0.832 & 0.832 \\
\hline 386 & 378 & 25 & 28 & 28 & 1.988 & 1.988 & 1.988 \\
\hline & 379 & 10 & 19 & 19 & 1.173 & 1.173 & 1.173 \\
\hline & 398 & 14 & 18 & 18 & 1.775 & 1.775 & 1.775 \\
\hline & 404 & 17 & 17 & 17 & 1.992 & 1.992 & 1.992 \\
\hline & 408 & 7 & 7 & 8 & 1.890 & 1.890 & 1.890 \\
\hline 388 & 378 & 4 & 4 & 4 & 0.942 & 0.942 & 0.942 \\
\hline & 379 & 4 & 4 & 5 & 1.238 & 1.238 & 1.238 \\
\hline 398 & 379 & 5 & 6 & 6 & 0.832 & 0.832 & 0.832 \\
\hline & 386 & 14 & 18 & 18 & 1.775 & 1.775 & 1.775 \\
\hline & 408 & 23 & 24 & 24 & 2.835 & 2.835 & 2.835 \\
\hline & 413 & 27 & 27 & 27 & 14.887 & 14.887 & 14.887 \\
\hline & 427 & 6 & 6 & 6 & 1.698 & 1.698 & 1.698 \\
\hline & 432 & 4 & 4 & 4 & 1.492 & 1.492 & 1.492 \\
\hline 404 & 378 & 4 & 4 & 4 & 1.206 & 1.206 & 1.206 \\
\hline & 386 & 17 & 17 & 17 & 1.992 & 1.992 & 1.992 \\
\hline & 413 & 29 & 30 & 30 & 4.196 & 4.196 & 4.196 \\
\hline & 427 & 13 & 13 & 13 & 1.929 & 1.929 & 1.929 \\
\hline & 432 & 4 & 4 & 4 & 2.410 & 2.410 & 2.410 \\
\hline & 534 & 2 & 2 & 2 & 0.476 & 0.476 & 0.476 \\
\hline 408 & 386 & 7 & 7 & 8 & 1.890 & 1.890 & 1.890 \\
\hline & 398 & 23 & 24 & 24 & 2.835 & 2.835 & 2.835 \\
\hline & 427 & 13 & 13 & 13 & 1.124 & 1.124 & 1.124 \\
\hline & 428 & 2 & 3 & 3 & 0.890 & 0.890 & 0.890 \\
\hline & 432 & 7 & 10 & 10 & 1.322 & 1.322 & 1.322 \\
\hline 413 & 398 & 27 & 27 & 27 & 14.887 & 14.887 & 14.887 \\
\hline & 404 & 29 & 30 & 30 & 4.196 & 4.196 & 4.196 \\
\hline 427 & 398 & 6 & 6 & 6 & 1.698 & 1.698 & 1.698 \\
\hline & 404 & 13 & 13 & 13 & 1.929 & 1.929 & 1.929 \\
\hline & 408 & 13 & 13 & 13 & 1.124 & 1.124 & 1.124 \\
\hline 428 & 408 & 2 & 3 & 3 & 0.890 & 0.890 & 0.890 \\
\hline 432 & 398 & 4 & 4 & 4 & 1.492 & 1.492 & 1.492 \\
\hline & 404 & 4 & 4 & 4 & 2.410 & 2.410 & 2.410 \\
\hline & 408 & 7 & 10 & 10 & 1.322 & 1.322 & 1.322 \\
\hline 474 & 496 & 20 & 20 & 20 & 1.813 & 1.813 & 1.813 \\
\hline & 509 & 13 & 13 & 13 & 1.519 & 1.519 & 1.519 \\
\hline & 520 & 10 & 10 & 11 & 1.233 & 1.233 & 1.233 \\
\hline 475 & 496 & 23 & 23 & 23 & 1.931 & 1.931 & 1.931 \\
\hline & 501 & 16 & 16 & 16 & 3.532 & 3.532 & 3.532 \\
\hline & 509 & 20 & 21 & 21 & 2.142 & 2.142 & 2.142 \\
\hline & 520 & 8 & 8 & 8 & 3.436 & 3.436 & 3.436 \\
\hline & 525 & 2 & 2 & 2 & 1.241 & 1.241 & 1.241 \\
\hline & 534 & 2 & 2 & 2 & 3.476 & 3.476 & 3.476 \\
\hline 496 & 474 & 20 & 20 & 20 & 1.813 & 1.813 & 1.813 \\
\hline & 475 & 23 & 23 & 23 & 1.931 & 1.931 & 1.931 \\
\hline & 509 & 7 & 7 & 7 & 1.299 & 1.299 & 1.299 \\
\hline 501 & 475 & 16 & 16 & 16 & 3.532 & 3.532 & 3.532 \\
\hline
\end{tabular}


Table B.2 - Continued from previous page

\begin{tabular}{|c|c|c|c|c|c|c|c|c|c|}
\hline Name & $\begin{array}{l}\text { UniProt } \\
\text { ID }\end{array}$ & Residue 1 & Residue 2 & FDR $1 \%$ & FDR 3\% & FDR 5\% & FDR $1 \%$ & FDR3\% & FDR5\% \\
\hline & & 509 & 194 & & 2 & 3 & & 0.170 & 0.170 \\
\hline & & & 474 & 13 & 13 & 13 & 1.519 & 1.519 & 1.519 \\
\hline & & & 475 & 20 & 21 & 21 & 2.142 & 2.142 & 2.142 \\
\hline & & & 496 & 7 & 7 & 7 & 1.299 & 1.299 & 1.299 \\
\hline & & & 520 & 34 & 34 & 34 & 2.936 & 2.936 & 2.936 \\
\hline & & & 534 & 3 & 3 & 3 & 2.471 & 2.471 & 2.471 \\
\hline & & 520 & 474 & 10 & 10 & 11 & 1.233 & 1.233 & 1.233 \\
\hline & & & 475 & 8 & 8 & 8 & 3.436 & 3.436 & 3.436 \\
\hline & & & 509 & 34 & 34 & 34 & 2.936 & 2.936 & 2.936 \\
\hline & & & 533 & 2 & 3 & 3 & 0.958 & 0.958 & 0.958 \\
\hline & & & 534 & 29 & 29 & 29 & 4.860 & 4.860 & 4.860 \\
\hline & & 525 & 475 & 2 & 2 & 2 & 1.241 & 1.241 & 1.241 \\
\hline & & & 533 & 7 & 10 & 11 & 0.716 & 0.716 & 0.716 \\
\hline & & 533 & 520 & 2 & 3 & 3 & 0.958 & 0.958 & 0.958 \\
\hline & & & 525 & 7 & 10 & 11 & 0.716 & 0.716 & 0.716 \\
\hline & & & 541 & 22 & 23 & 23 & 1.751 & 1.751 & 1.751 \\
\hline & & 534 & 404 & 2 & 2 & 2 & 0.476 & 0.476 & 0.476 \\
\hline & & & 475 & 2 & 2 & 2 & 3.476 & 3.476 & 3.476 \\
\hline & & & 509 & 3 & 3 & 3 & 2.471 & 2.471 & 2.471 \\
\hline & & & 520 & 29 & 29 & 29 & 4.860 & 4.860 & 4.860 \\
\hline & & & 544 & 6 & 6 & 6 & 3.360 & 3.360 & 3.360 \\
\hline & & & 556 & 3 & 3 & 3 & 1.192 & 1.192 & 1.192 \\
\hline & & 541 & 533 & 22 & 23 & 23 & 1.751 & 1.751 & 1.751 \\
\hline & & & 544 & 42 & 43 & 43 & 4.222 & 4.222 & 4.222 \\
\hline & & & 553 & 28 & 30 & 30 & 3.682 & 3.682 & 3.682 \\
\hline & & & 556 & 7 & 9 & 9 & 0.691 & 0.691 & 0.691 \\
\hline & & 544 & 534 & 6 & 6 & 6 & 3.360 & 3.360 & 3.360 \\
\hline & & & 541 & 42 & 43 & 43 & 4.222 & 4.222 & 4.222 \\
\hline & & & 556 & 7 & 8 & 8 & 1.184 & 1.184 & 1.184 \\
\hline & & 553 & 541 & 28 & 30 & 30 & 3.682 & 3.682 & 3.682 \\
\hline & & 556 & 534 & 3 & 3 & 3 & 1.192 & 1.192 & 1.192 \\
\hline & & & 541 & 7 & 9 & 9 & 0.691 & 0.691 & 0.691 \\
\hline & & & 544 & 7 & 8 & 8 & 1.184 & 1.184 & 1.184 \\
\hline SF3A1 & Q15459 & 1 & 424 & & 2 & 2 & & 0.219 & 0.219 \\
\hline & & 2 & 30 & 6 & 7 & 7 & 3.171 & 3.171 & 3.171 \\
\hline & & & 55 & 3 & 3 & 3 & 2.757 & 2.757 & 2.757 \\
\hline & & & 102 & 15 & 15 & 15 & 2.253 & 2.253 & 2.253 \\
\hline & & & 105 & 2 & 2 & 2 & 1.161 & 1.161 & 1.161 \\
\hline & & & 115 & 16 & 16 & 16 & 2.956 & 2.956 & 2.956 \\
\hline & & & 131 & 5 & 5 & 5 & 2.351 & 2.351 & 2.351 \\
\hline & & 20 & 102 & 2 & 2 & 2 & 2.436 & 2.436 & 2.436 \\
\hline & & 30 & 2 & 6 & 7 & 7 & 3.171 & 3.171 & 3.171 \\
\hline & & & 97 & 15 & 17 & 17 & 1.213 & 1.213 & 1.213 \\
\hline & & & 102 & 22 & 26 & 27 & 1.037 & 1.037 & 1.037 \\
\hline & & & 105 & 14 & 21 & 21 & 2.030 & 2.030 & 2.030 \\
\hline & & & 115 & 10 & 10 & 10 & 5.294 & 5.294 & 5.294 \\
\hline & & 37 & 97 & 14 & 14 & 14 & 1.298 & 1.298 & 1.298 \\
\hline & & & 102 & 14 & 17 & 17 & 1.368 & 1.368 & 1.368 \\
\hline & & & 105 & 3 & 3 & 3 & 1.637 & 1.637 & 1.637 \\
\hline & & & 115 & 12 & 12 & 12 & 2.817 & 2.817 & 2.817 \\
\hline & & 55 & 2 & 3 & 3 & 3 & 2.757 & 2.757 & 2.757 \\
\hline & & & 80 & 153 & 157 & 158 & 6.078 & 6.078 & 6.078 \\
\hline & & & 97 & 84 & 85 & 85 & 3.144 & 3.144 & 3.144 \\
\hline & & & 102 & 4 & 4 & 4 & 3.018 & 3.018 & 3.018 \\
\hline & & & 105 & 127 & 129 & 129 & 3.230 & 3.230 & 3.230 \\
\hline & & & 115 & 38 & 38 & 38 & 2.722 & 2.722 & 2.722 \\
\hline & & & 131 & 7 & 7 & 7 & 2.132 & 2.132 & 2.132 \\
\hline & & 80 & 55 & 153 & 157 & 158 & 6.078 & 6.078 & 6.078 \\
\hline & & & 115 & 31 & 31 & 31 & 5.066 & 5.066 & 5.066 \\
\hline & & & 131 & 3 & 3 & 3 & 3.311 & 3.311 & 3.311 \\
\hline & & 97 & 30 & 15 & 17 & 17 & 1.213 & 1.213 & 1.213 \\
\hline & & & 37 & 14 & 14 & 14 & 1.298 & 1.298 & 1.298 \\
\hline & & & 55 & 84 & 85 & 85 & 3.144 & 3.144 & 3.144 \\
\hline & & & 105 & 56 & 65 & 65 & 2.942 & 2.942 & 2.942 \\
\hline & & & 115 & 8 & 11 & 12 & 1.638 & 1.638 & 1.638 \\
\hline & & 102 & 2 & 15 & 15 & 15 & 2.253 & 2.253 & 2.253 \\
\hline & & & 20 & 2 & 2 & 2 & 2.436 & 2.436 & 2.436 \\
\hline & & & 30 & 22 & 26 & 27 & 1.037 & 1.037 & 1.037 \\
\hline & & & 37 & 14 & 17 & 17 & 1.368 & 1.368 & 1.368 \\
\hline & & & 55 & 4 & 4 & 4 & 3.018 & 3.018 & 3.018 \\
\hline & & & 115 & 44 & 45 & 45 & 1.993 & 1.993 & 1.993 \\
\hline & & & 131 & 14 & 15 & 15 & 1.539 & 1.539 & 1.539 \\
\hline & & & 188 & & 3 & 4 & & 0.353 & 0.353 \\
\hline & & 105 & 2 & 2 & 2 & 2 & 1.161 & 1.161 & 1.161 \\
\hline & & & 30 & 14 & 21 & 21 & 2.030 & 2.030 & 2.030 \\
\hline & & & 37 & 3 & 3 & 3 & 1.637 & 1.637 & 1.637 \\
\hline & & & 55 & 127 & 129 & 129 & 3.230 & 3.230 & 3.230 \\
\hline & & & 97 & 56 & 65 & 65 & 2.942 & 2.942 & 2.942 \\
\hline & & & 131 & 3 & 3 & 3 & 1.509 & 1.509 & 1.509 \\
\hline & & 115 & 2 & 16 & 16 & 16 & 2.956 & 2.956 & 2.956 \\
\hline & & & 30 & 10 & 10 & 10 & 5.294 & 5.294 & 5.294 \\
\hline & & & 37 & 12 & 12 & 12 & 2.817 & 2.817 & 2.817 \\
\hline & & & 55 & 38 & 38 & 38 & 2.722 & 2.722 & 2.722 \\
\hline
\end{tabular}


Table B.2 - Continued from previous page

\begin{tabular}{|c|c|c|c|c|c|c|c|c|c|}
\hline Name & $\begin{array}{l}\text { UniProt } \\
\text { ID }\end{array}$ & Residue 1 & Residue 2 & FDR $1 \%$ & FDR $3 \%$ & FDR $5 \%$ & F $D R 1 \%$ & FDR3\% & FDR5\% \\
\hline & & & 80 & 31 & 31 & 31 & 5.066 & 5.066 & 5.066 \\
\hline & & & 97 & 8 & 11 & 12 & 1.638 & 1.638 & 1.638 \\
\hline & & & 102 & 44 & 45 & 45 & 1.993 & 1.993 & 1.993 \\
\hline & & 131 & 2 & 5 & 5 & 5 & 2.351 & 2.351 & 2.351 \\
\hline & & & 55 & 7 & 7 & 7 & 2.132 & 2.132 & 2.132 \\
\hline & & & 80 & 3 & 3 & 3 & 3.311 & 3.311 & 3.311 \\
\hline & & & 102 & 14 & 15 & 15 & 1.539 & 1.539 & 1.539 \\
\hline & & & 105 & 3 & 3 & 3 & 1.509 & 1.509 & 1.509 \\
\hline & & & 223 & 2 & 3 & 3 & 1.193 & 1.193 & 1.193 \\
\hline & & & 230 & 3 & 5 & 5 & 1.588 & 1.588 & 1.588 \\
\hline & & & 231 & 25 & 25 & 25 & 2.504 & 2.504 & 2.504 \\
\hline & & & 251 & 10 & 10 & 10 & 1.271 & 1.271 & 1.271 \\
\hline & & & 258 & 4 & 4 & 4 & 1.632 & 1.632 & 1.632 \\
\hline & & & 259 & 32 & 32 & 32 & 2.027 & 2.027 & 2.027 \\
\hline & & 144 & 251 & 30 & 32 & 32 & 1.678 & 1.678 & 1.678 \\
\hline & & & 259 & 26 & 27 & 27 & 9.195 & 9.195 & 9.195 \\
\hline & & 188 & 102 & & 3 & 4 & & 0.353 & 0.353 \\
\hline & & 223 & 131 & 2 & 3 & 3 & 1.193 & 1.193 & 1.193 \\
\hline & & & 230 & 32 & 34 & 34 & 1.705 & 1.705 & 1.705 \\
\hline & & & 231 & 268 & 277 & 277 & 2.541 & 2.541 & 2.541 \\
\hline & & 228 & 231 & 47 & 47 & 47 & 2.972 & 2.972 & 2.972 \\
\hline & & 230 & 131 & 3 & 5 & 5 & 1.588 & 1.588 & 1.588 \\
\hline & & & 223 & 32 & 34 & 34 & 1.705 & 1.705 & 1.705 \\
\hline & & 231 & 131 & 25 & 25 & 25 & 2.504 & 2.504 & 2.504 \\
\hline & & & 223 & 268 & 277 & 277 & 2.541 & 2.541 & 2.541 \\
\hline & & & 228 & 47 & 47 & 47 & 2.972 & 2.972 & 2.972 \\
\hline & & 251 & 131 & 10 & 10 & 10 & 1.271 & 1.271 & 1.271 \\
\hline & & & 144 & 30 & 32 & 32 & 1.678 & 1.678 & 1.678 \\
\hline & & & 258 & 146 & 146 & 146 & 4.306 & 4.306 & 4.306 \\
\hline & & & 259 & 40 & 46 & 46 & 3.412 & 3.412 & 3.412 \\
\hline & & 258 & 131 & 4 & 4 & 4 & 1.632 & 1.632 & 1.632 \\
\hline & & & 251 & 146 & 146 & 146 & 4.306 & 4.306 & 4.306 \\
\hline & & 259 & 131 & 32 & 32 & 32 & 2.027 & 2.027 & 2.027 \\
\hline & & & 144 & 26 & 27 & 27 & 9.195 & 9.195 & 9.195 \\
\hline & & & 251 & 40 & 46 & 46 & 3.412 & 3.412 & 3.412 \\
\hline & & 391 & 399 & 30 & 30 & 30 & 2.674 & 2.674 & 2.674 \\
\hline & & & 419 & 45 & 52 & 53 & 2.195 & 2.195 & 2.195 \\
\hline & & & 424 & 44 & 49 & 49 & 2.083 & 2.083 & 2.083 \\
\hline & & & 449 & 6 & 6 & 6 & 2.085 & 2.085 & 2.085 \\
\hline & & 396 & 424 & 27 & 30 & 30 & 2.102 & 2.102 & 2.102 \\
\hline & & & 449 & 2 & 2 & 2 & 2.132 & 2.132 & 2.132 \\
\hline & & 399 & 391 & 30 & 30 & 30 & 2.674 & 2.674 & 2.674 \\
\hline & & & 424 & 76 & 80 & 80 & 3.466 & 3.466 & 3.466 \\
\hline & & & 449 & 13 & 14 & 14 & 4.494 & 4.494 & 4.494 \\
\hline & & & 499 & 2 & 2 & 2 & 4.232 & 4.232 & 4.232 \\
\hline & & 419 & 391 & 45 & 52 & 53 & 2.195 & 2.195 & 2.195 \\
\hline & & & 449 & 9 & 9 & 9 & 2.412 & 2.412 & 2.412 \\
\hline & & & 467 & 13 & 13 & 13 & 2.742 & 2.742 & 2.742 \\
\hline & & & 486 & 5 & 6 & 6 & 2.719 & 2.719 & 2.719 \\
\hline & & & 487 & 9 & 9 & 9 & 2.325 & 2.325 & 2.325 \\
\hline & & & 499 & 2 & 2 & 2 & 2.809 & 2.809 & 2.809 \\
\hline & & & 533 & 5 & 5 & 6 & 1.129 & 1.129 & 1.129 \\
\hline & & 424 & 1 & & 2 & 2 & & 0.219 & 0.219 \\
\hline & & & 391 & 44 & 49 & 49 & 2.083 & 2.083 & 2.083 \\
\hline & & & 396 & 27 & 30 & 30 & 2.102 & 2.102 & 2.102 \\
\hline & & & 399 & 76 & 80 & 80 & 3.466 & 3.466 & 3.466 \\
\hline & & & 449 & 52 & 52 & 52 & 3.223 & 3.223 & 3.223 \\
\hline & & & 467 & 5 & 5 & 5 & 2.034 & 2.034 & 2.034 \\
\hline & & & 486 & 7 & 7 & 7 & 2.528 & 2.528 & 2.528 \\
\hline & & & 487 & 26 & 26 & 26 & 3.960 & 3.960 & 3.960 \\
\hline & & & 499 & 20 & 20 & 20 & 2.961 & 2.961 & 2.961 \\
\hline & & & 533 & 18 & 18 & 18 & 2.729 & 2.729 & 2.729 \\
\hline & & 449 & 391 & 6 & 6 & 6 & 2.085 & 2.085 & 2.085 \\
\hline & & & 396 & 2 & 2 & 2 & 2.132 & 2.132 & 2.132 \\
\hline & & & 399 & 13 & 14 & 14 & 4.494 & 4.494 & 4.494 \\
\hline & & & 419 & 9 & 9 & 9 & 2.412 & 2.412 & 2.412 \\
\hline & & & 424 & 52 & 52 & 52 & 3.223 & 3.223 & 3.223 \\
\hline & & & 486 & 4 & 4 & 4 & 2.920 & 2.920 & 2.920 \\
\hline & & & 487 & 8 & 8 & 8 & 2.604 & 2.604 & 2.604 \\
\hline & & & 499 & 2 & 2 & 2 & 3.064 & 3.064 & 3.064 \\
\hline & & & 533 & 7 & 7 & 7 & 1.635 & 1.635 & 1.635 \\
\hline & & 467 & 419 & 13 & 13 & 13 & 2.742 & 2.742 & 2.742 \\
\hline & & & 424 & 5 & 5 & 5 & 2.034 & 2.034 & 2.034 \\
\hline & & & 486 & 20 & 22 & 22 & 4.000 & 4.000 & 4.000 \\
\hline & & & 487 & 11 & 11 & 11 & 4.564 & 4.564 & 4.564 \\
\hline & & 486 & 419 & 5 & 6 & 6 & 2.719 & 2.719 & 2.719 \\
\hline & & & 424 & 7 & 7 & 7 & 2.528 & 2.528 & 2.528 \\
\hline & & & 449 & 4 & 4 & 4 & 2.920 & 2.920 & 2.920 \\
\hline & & & 467 & 20 & 22 & 22 & 4.000 & 4.000 & 4.000 \\
\hline & & & 495 & 46 & 48 & 48 & 3.431 & 3.431 & 3.431 \\
\hline & & & 499 & 50 & 51 & 51 & 23.334 & 23.334 & 23.334 \\
\hline & & & 533 & 16 & 16 & 16 & 2.957 & 2.957 & 2.957 \\
\hline & & 487 & 419 & 9 & 9 & 9 & 2.325 & 2.325 & 2.325 \\
\hline
\end{tabular}


Table B.2 - Continued from previous page

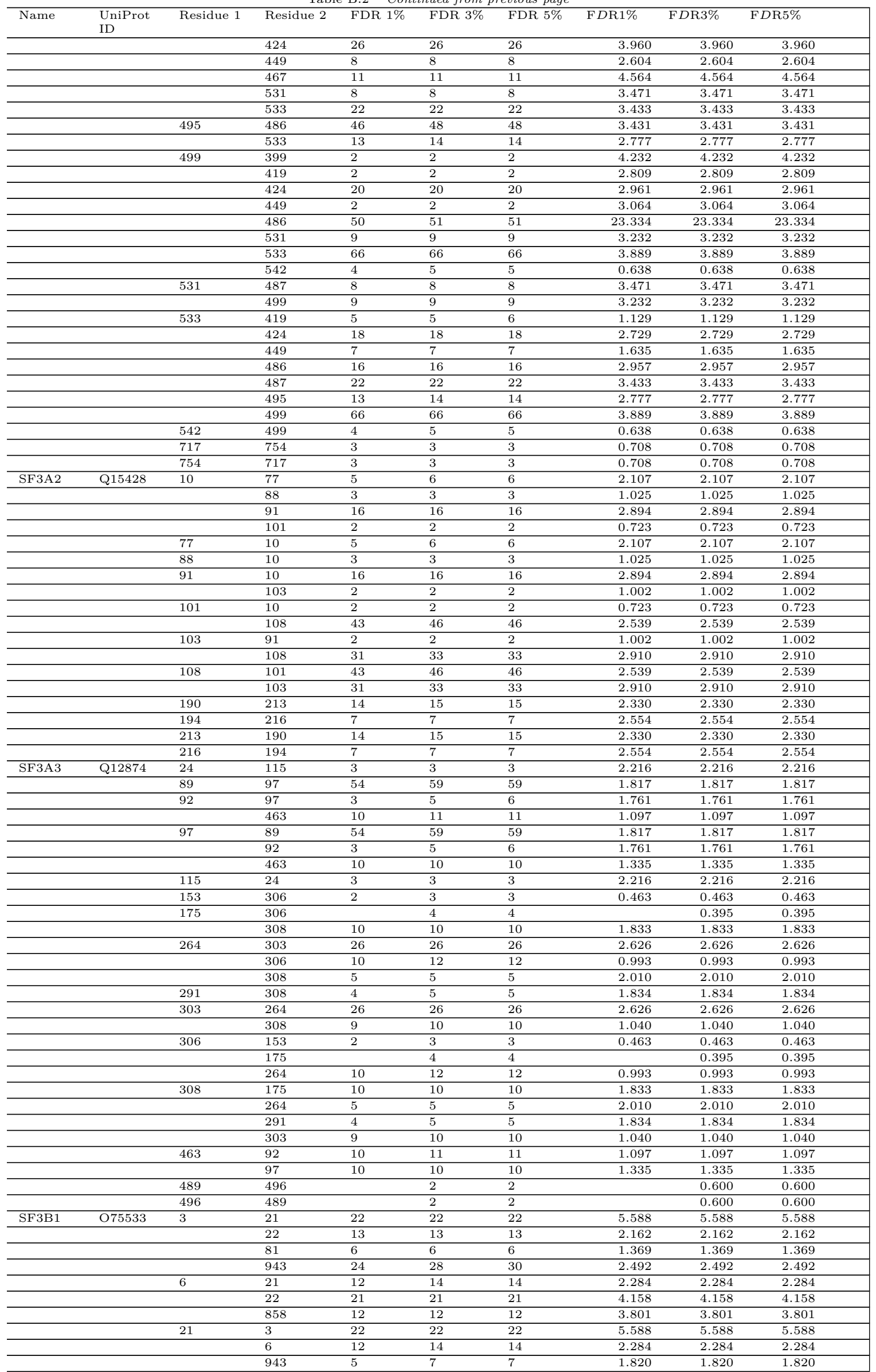


Table B.2 - Continued from previous page

\begin{tabular}{|c|c|c|c|c|c|c|c|c|c|}
\hline Name & $\begin{array}{l}\text { UniProt } \\
\text { ID }\end{array}$ & Residue 1 & Residue 2 & FDR 1\% & FDR $3 \%$ & FDR 5\% & FDR $1 \%$ & FDR3\% & FDR5\% \\
\hline & & 22 & 3 & 13 & 13 & 13 & 2.162 & 2.162 & 2.162 \\
\hline & & & 6 & 21 & 21 & 21 & 4.158 & 4.158 & 4.158 \\
\hline & & & 943 & 13 & 13 & 13 & 1.526 & 1.526 & 1.526 \\
\hline & & 81 & 3 & 6 & 6 & 6 & 1.369 & 1.369 & 1.369 \\
\hline & & & 120 & 7 & 8 & 8 & 1.596 & 1.596 & 1.596 \\
\hline & & & 163 & 2 & 2 & 2 & 1.268 & 1.268 & 1.268 \\
\hline & & 111 & 866 & 8 & 8 & 8 & 12.335 & 12.335 & 12.335 \\
\hline & & 120 & 81 & 7 & 8 & 8 & 1.596 & 1.596 & 1.596 \\
\hline & & & 163 & 13 & 14 & 14 & 2.885 & 2.885 & 2.885 \\
\hline & & & 177 & 7 & 7 & 7 & 1.725 & 1.725 & 1.725 \\
\hline & & 141 & 163 & 5 & 8 & 8 & 0.769 & 0.769 & 0.769 \\
\hline & & & 175 & & 3 & 3 & & 0.384 & 0.384 \\
\hline & & & 177 & 2 & 2 & 2 & 0.665 & 0.665 & 0.665 \\
\hline & & 146 & 163 & 2 & 2 & 2 & 1.995 & 1.995 & 1.995 \\
\hline & & & 175 & 5 & 8 & 8 & 1.073 & 1.073 & 1.073 \\
\hline & & & 177 & 5 & 5 & 5 & 2.185 & 2.185 & 2.185 \\
\hline & & 163 & 81 & 2 & 2 & 2 & 1.268 & 1.268 & 1.268 \\
\hline & & & 120 & 13 & 14 & 14 & 2.885 & 2.885 & 2.885 \\
\hline & & & 141 & 5 & 8 & 8 & 0.769 & 0.769 & 0.769 \\
\hline & & & 146 & 2 & 2 & 2 & 1.995 & 1.995 & 1.995 \\
\hline & & & 175 & 15 & 16 & 17 & 1.881 & 1.881 & 1.881 \\
\hline & & & 177 & 7 & 8 & 8 & 1.210 & 1.210 & 1.210 \\
\hline & & & 195 & 5 & 6 & 6 & 0.927 & 0.927 & 0.927 \\
\hline & & & 214 & 11 & 11 & 11 & 3.388 & 3.388 & 3.388 \\
\hline & & & 656 & 31 & 31 & 31 & 2.540 & 2.540 & 2.540 \\
\hline & & 175 & 141 & & 3 & 3 & & 0.384 & 0.384 \\
\hline & & & 146 & 5 & 8 & 8 & 1.073 & 1.073 & 1.073 \\
\hline & & & 163 & 15 & 16 & 17 & 1.881 & 1.881 & 1.881 \\
\hline & & & 182 & 71 & 75 & 75 & 2.555 & 2.555 & 2.555 \\
\hline & & & 195 & 9 & 11 & 11 & 1.966 & 1.966 & 1.966 \\
\hline & & & 214 & 10 & 12 & 12 & 1.698 & 1.698 & 1.698 \\
\hline & & 177 & 120 & 7 & 7 & 7 & 1.725 & 1.725 & 1.725 \\
\hline & & & 141 & 2 & 2 & 2 & 0.665 & 0.665 & 0.665 \\
\hline & & & 146 & 5 & 5 & 5 & 2.185 & 2.185 & 2.185 \\
\hline & & & 163 & 7 & 8 & 8 & 1.210 & 1.210 & 1.210 \\
\hline & & & 195 & 11 & 13 & 13 & 1.283 & 1.283 & 1.283 \\
\hline & & & 213 & 2 & 2 & 2 & 0.768 & 0.768 & 0.768 \\
\hline & & & 214 & 4 & 4 & 4 & 2.467 & 2.467 & 2.467 \\
\hline & & 182 & 175 & 71 & 75 & 75 & 2.555 & 2.555 & 2.555 \\
\hline & & 195 & 163 & 5 & 6 & 6 & 0.927 & 0.927 & 0.927 \\
\hline & & & 175 & 9 & 11 & 11 & 1.966 & 1.966 & 1.966 \\
\hline & & & 177 & 11 & 13 & 13 & 1.283 & 1.283 & 1.283 \\
\hline & & & 213 & 11 & 11 & 11 & 2.419 & 2.419 & 2.419 \\
\hline & & & 214 & 31 & 36 & 36 & 2.641 & 2.641 & 2.641 \\
\hline & & & 240 & 15 & 15 & 15 & 3.087 & 3.087 & 3.087 \\
\hline & & & 252 & 2 & 2 & 2 & 0.920 & 0.920 & 0.920 \\
\hline & & 213 & 177 & 2 & 2 & 2 & 0.768 & 0.768 & 0.768 \\
\hline & & & 195 & 11 & 11 & 11 & 2.419 & 2.419 & 2.419 \\
\hline & & & 240 & 29 & 29 & 29 & 2.548 & 2.548 & 2.548 \\
\hline & & 214 & 163 & 11 & 11 & 11 & 3.388 & 3.388 & 3.388 \\
\hline & & & 175 & 10 & 12 & 12 & 1.698 & 1.698 & 1.698 \\
\hline & & & 177 & 4 & 4 & 4 & 2.467 & 2.467 & 2.467 \\
\hline & & & 195 & 31 & 36 & 36 & 2.641 & 2.641 & 2.641 \\
\hline & & & 240 & 40 & 42 & 42 & 4.631 & 4.631 & 4.631 \\
\hline & & & 252 & 19 & 19 & 19 & 7.320 & 7.320 & 7.320 \\
\hline & & 240 & 195 & 15 & 15 & 15 & 3.087 & 3.087 & 3.087 \\
\hline & & & 213 & 29 & 29 & 29 & 2.548 & 2.548 & 2.548 \\
\hline & & & 214 & 40 & 42 & 42 & 4.631 & 4.631 & 4.631 \\
\hline & & & 499 & & 4 & 4 & & 0.467 & 0.467 \\
\hline & & 252 & 195 & 2 & 2 & 2 & 0.920 & 0.920 & 0.920 \\
\hline & & & 214 & 19 & 19 & 19 & 7.320 & 7.320 & 7.320 \\
\hline & & 298 & 333 & 2 & 2 & 2 & 1.038 & 1.038 & 1.038 \\
\hline & & 333 & 298 & 2 & 2 & 2 & 1.038 & 1.038 & 1.038 \\
\hline & & 493 & 496 & 20 & 26 & 26 & 1.866 & 1.866 & 1.866 \\
\hline & & & 522 & 8 & 8 & 8 & 1.076 & 1.076 & 1.076 \\
\hline & & 496 & 493 & 20 & 26 & 26 & 1.866 & 1.866 & 1.866 \\
\hline & & & 503 & 67 & 71 & 72 & 3.720 & 3.720 & 3.720 \\
\hline & & 499 & 240 & & 4 & 4 & & 0.467 & 0.467 \\
\hline & & 503 & 496 & 67 & 71 & 72 & 3.720 & 3.720 & 3.720 \\
\hline & & & 522 & 38 & 48 & 48 & 2.326 & 2.326 & 2.326 \\
\hline & & 513 & 554 & 13 & 13 & 13 & 1.986 & 1.986 & 1.986 \\
\hline & & 522 & 493 & 8 & 8 & 8 & 1.076 & 1.076 & 1.076 \\
\hline & & & 503 & 38 & 48 & 48 & 2.326 & 2.326 & 2.326 \\
\hline & & 554 & 513 & 13 & 13 & 13 & 1.986 & 1.986 & 1.986 \\
\hline & & 649 & 656 & 8 & 8 & 8 & 2.903 & 2.903 & 2.903 \\
\hline & & 653 & 656 & 28 & 28 & 28 & 3.546 & 3.546 & 3.546 \\
\hline & & 656 & 163 & 31 & 31 & 31 & 2.540 & 2.540 & 2.540 \\
\hline & & & 649 & 8 & 8 & 8 & 2.903 & 2.903 & 2.903 \\
\hline & & & 653 & 28 & 28 & 28 & 3.546 & 3.546 & 3.546 \\
\hline & & 666 & 700 & 6 & 6 & 6 & 2.829 & 2.829 & 2.829 \\
\hline & & & 741 & 3 & 3 & 3 & 3.423 & 3.423 & 3.423 \\
\hline & & 700 & 666 & 6 & 6 & 6 & 2.829 & 2.829 & 2.829 \\
\hline & & & 741 & 16 & 16 & 16 & 2.126 & 2.126 & 2.126 \\
\hline
\end{tabular}


Table B.2 - Continued from previous page

\begin{tabular}{|c|c|c|c|c|c|c|c|c|c|}
\hline Name & $\begin{array}{l}\text { UniProt } \\
\text { ID }\end{array}$ & Residue 1 & Residue 2 & FDR 1\% & FDR 3\% & FDR 5\% & FDR1\% & FDR3\% & FDR5\% \\
\hline & & 741 & 666 & 3 & 3 & 3 & 3.423 & 3.423 & 3.423 \\
\hline & & & 700 & 16 & 16 & 16 & 2.126 & 2.126 & 2.126 \\
\hline & & & 786 & 27 & 27 & 27 & 2.559 & 2.559 & 2.559 \\
\hline & & & 790 & 30 & 30 & 30 & 2.765 & 2.765 & 2.765 \\
\hline & & 786 & 741 & 27 & 27 & 27 & 2.559 & 2.559 & 2.559 \\
\hline & & & 793 & 15 & 16 & 16 & 1.329 & 1.329 & 1.329 \\
\hline & & 790 & 741 & 30 & 30 & 30 & 2.765 & 2.765 & 2.765 \\
\hline & & 793 & 786 & 15 & 16 & 16 & 1.329 & 1.329 & 1.329 \\
\hline & & 858 & 6 & 12 & 12 & 12 & 3.801 & 3.801 & 3.801 \\
\hline & & 866 & 111 & 8 & 8 & 8 & 12.335 & 12.335 & 12.335 \\
\hline & & 923 & 963 & 4 & 5 & 5 & 2.137 & 2.137 & 2.137 \\
\hline & & 943 & 3 & 24 & 28 & 30 & 2.492 & 2.492 & 2.492 \\
\hline & & & 21 & 5 & 7 & 7 & 1.820 & 1.820 & 1.820 \\
\hline & & & 22 & 13 & 13 & 13 & 1.526 & 1.526 & 1.526 \\
\hline & & 963 & 923 & 4 & 5 & 5 & 2.137 & 2.137 & 2.137 \\
\hline & & & 1008 & 43 & 43 & 43 & 3.170 & 3.170 & 3.170 \\
\hline & & 1008 & 963 & 43 & 43 & 43 & 3.170 & 3.170 & 3.170 \\
\hline \multirow[t]{66}{*}{ SF3B2 } & Q13435 & 10 & 77 & 2 & 2 & 2 & 4.448 & 4.448 & 4.448 \\
\hline & & 35 & 77 & 11 & 11 & 11 & 12.052 & 12.052 & 12.052 \\
\hline & & 77 & 10 & 2 & 2 & 2 & 4.448 & 4.448 & 4.448 \\
\hline & & & 35 & 11 & 11 & 11 & 12.052 & 12.052 & 12.052 \\
\hline & & 148 & 165 & 25 & 26 & 26 & 3.804 & 3.804 & 3.804 \\
\hline & & & 173 & 10 & 12 & 12 & 2.163 & 2.163 & 2.163 \\
\hline & & & 182 & 2 & 4 & 4 & 0.660 & 0.660 & 0.660 \\
\hline & & & 198 & 3 & 3 & 3 & 2.014 & 2.014 & 2.014 \\
\hline & & 165 & 148 & 25 & 26 & 26 & 3.804 & 3.804 & 3.804 \\
\hline & & & 182 & 15 & 16 & 16 & 2.839 & 2.839 & 2.839 \\
\hline & & 173 & 148 & 10 & 12 & 12 & 2.163 & 2.163 & 2.163 \\
\hline & & 182 & 148 & 2 & 4 & 4 & 0.660 & 0.660 & 0.660 \\
\hline & & & 165 & 15 & 16 & 16 & 2.839 & 2.839 & 2.839 \\
\hline & & 198 & 148 & 3 & 3 & 3 & 2.014 & 2.014 & 2.014 \\
\hline & & 268 & 280 & 94 & 127 & 129 & 1.725 & 1.725 & 1.725 \\
\hline & & 280 & 268 & 94 & 127 & 129 & 1.725 & 1.725 & 1.725 \\
\hline & & 323 & 335 & & & 2 & & & 0.148 \\
\hline & & 335 & 323 & & & 2 & & & 0.148 \\
\hline & & 387 & 394 & 17 & 17 & 17 & 3.629 & 3.629 & 3.629 \\
\hline & & & 400 & 9 & 9 & 9 & 1.496 & 1.496 & 1.496 \\
\hline & & & 403 & 9 & 9 & 9 & 2.562 & 2.562 & 2.562 \\
\hline & & & 405 & 3 & 3 & 3 & 2.506 & 2.506 & 2.506 \\
\hline & & 394 & 387 & 17 & 17 & 17 & 3.629 & 3.629 & 3.629 \\
\hline & & & 405 & 5 & 6 & 6 & 1.098 & 1.098 & 1.098 \\
\hline & & 400 & 387 & 9 & 9 & 9 & 1.496 & 1.496 & 1.496 \\
\hline & & & 405 & 31 & 33 & 34 & 2.359 & 2.359 & 2.359 \\
\hline & & & 409 & 9 & 10 & 10 & 1.332 & 1.332 & 1.332 \\
\hline & & & 412 & 2 & 2 & 2 & 0.856 & 0.856 & 0.856 \\
\hline & & 401 & 405 & 26 & 26 & 26 & 1.480 & 1.480 & 1.480 \\
\hline & & & 409 & 3 & 4 & 4 & 0.683 & 0.683 & 0.683 \\
\hline & & 403 & 387 & 9 & 9 & 9 & 2.562 & 2.562 & 2.562 \\
\hline & & & 409 & 4 & 5 & 5 & 1.680 & 1.680 & 1.680 \\
\hline & & & 420 & 3 & 3 & 3 & 2.064 & 2.064 & 2.064 \\
\hline & & 405 & 387 & 3 & 3 & 3 & 2.506 & 2.506 & 2.506 \\
\hline & & & 394 & 5 & 6 & 6 & 1.098 & 1.098 & 1.098 \\
\hline & & & 400 & 31 & 33 & 34 & 2.359 & 2.359 & 2.359 \\
\hline & & & 401 & 26 & 26 & 26 & 1.480 & 1.480 & 1.480 \\
\hline & & & 412 & 47 & 73 & 74 & 1.028 & 1.028 & 1.028 \\
\hline & & & 420 & 6 & 8 & 8 & 2.450 & 2.450 & 2.450 \\
\hline & & 409 & 400 & 9 & 10 & 10 & 1.332 & 1.332 & 1.332 \\
\hline & & & 401 & 3 & 4 & 4 & 0.683 & 0.683 & 0.683 \\
\hline & & & 403 & 4 & 5 & 5 & 1.680 & 1.680 & 1.680 \\
\hline & & & 412 & & 3 & 3 & & 0.226 & 0.226 \\
\hline & & & 420 & 6 & 25 & 27 & 0.560 & 0.560 & 0.560 \\
\hline & & & 556 & & & 2 & & & 0.242 \\
\hline & & 412 & 400 & 2 & 2 & 2 & 0.856 & 0.856 & 0.856 \\
\hline & & & 405 & 47 & 73 & 74 & 1.028 & 1.028 & 1.028 \\
\hline & & & 409 & & 3 & 3 & & 0.226 & 0.226 \\
\hline & & & 605 & & 2 & 2 & & 0.419 & 0.419 \\
\hline & & & 891 & & 4 & 4 & & 0.310 & 0.310 \\
\hline & & & 894 & & 5 & 5 & & 0.558 & 0.558 \\
\hline & & 420 & 403 & 3 & 3 & 3 & 2.064 & 2.064 & 2.064 \\
\hline & & & 405 & 6 & 8 & 8 & 2.450 & 2.450 & 2.450 \\
\hline & & & 409 & 6 & 25 & 27 & 0.560 & 0.560 & 0.560 \\
\hline & & 529 & 570 & 38 & 38 & 38 & 3.544 & 3.544 & 3.544 \\
\hline & & 543 & 550 & 17 & 21 & 21 & 2.362 & 2.362 & 2.362 \\
\hline & & & 552 & 7 & 9 & 9 & 1.562 & 1.562 & 1.562 \\
\hline & & & 556 & 25 & 26 & 26 & 2.484 & 2.484 & 2.484 \\
\hline & & & 560 & 45 & 45 & 45 & 1.869 & 1.869 & 1.869 \\
\hline & & & 563 & 34 & 34 & 34 & 3.236 & 3.236 & 3.236 \\
\hline & & & 570 & 6 & 6 & 6 & 0.982 & 0.982 & 0.982 \\
\hline & & & 857 & 10 & 10 & 10 & 1.930 & 1.930 & 1.930 \\
\hline & & 547 & 552 & 19 & 23 & 24 & 1.522 & 1.522 & 1.522 \\
\hline & & & 556 & 26 & 26 & 26 & 2.044 & 2.044 & 2.044 \\
\hline & & & 560 & 25 & 26 & 26 & 1.293 & 1.293 & 1.293 \\
\hline & & & 563 & 10 & 10 & 10 & 2.241 & 2.241 & 2.241 \\
\hline
\end{tabular}


Table B.2 - Continued from previous page

\begin{tabular}{|c|c|c|c|c|c|c|c|c|c|}
\hline Name & $\begin{array}{l}\text { UniProt } \\
\text { ID }\end{array}$ & Residue 1 & Residue 2 & FDR 1\% & FDR $3 \%$ & FDR 5\% & FDR1\% & FDR3\% & FDR5\% \\
\hline & & & 857 & 6 & 6 & 6 & 2.462 & 2.462 & 2.462 \\
\hline & & & 870 & 11 & 11 & 11 & 2.145 & 2.145 & 2.145 \\
\hline & & 550 & 543 & 17 & 21 & 21 & 2.362 & 2.362 & 2.362 \\
\hline & & & 556 & 14 & 14 & 14 & 2.139 & 2.139 & 2.139 \\
\hline & & & 560 & 13 & 14 & 14 & 1.476 & 1.476 & 1.476 \\
\hline & & & 870 & 32 & 33 & 33 & 1.286 & 1.286 & 1.286 \\
\hline & & & 877 & 8 & 11 & 11 & 1.671 & 1.671 & 1.671 \\
\hline & & 552 & 543 & 7 & 9 & 9 & 1.562 & 1.562 & 1.562 \\
\hline & & & 547 & 19 & 23 & 24 & 1.522 & 1.522 & 1.522 \\
\hline & & & 556 & 15 & 16 & 17 & 1.227 & 1.227 & 1.227 \\
\hline & & & 560 & 6 & 6 & 6 & 1.425 & 1.425 & 1.425 \\
\hline & & & 563 & & 2 & 2 & & 0.468 & 0.468 \\
\hline & & & 570 & 2 & 2 & 2 & 0.601 & 0.601 & 0.601 \\
\hline & & & 870 & 11 & 12 & 12 & 0.921 & 0.921 & 0.921 \\
\hline & & 556 & 409 & & & 2 & & & 0.242 \\
\hline & & & 543 & 25 & 26 & 26 & 2.484 & 2.484 & 2.484 \\
\hline & & & 547 & 26 & 26 & 26 & 2.044 & 2.044 & 2.044 \\
\hline & & & 550 & 14 & 14 & 14 & 2.139 & 2.139 & 2.139 \\
\hline & & & 552 & 15 & 16 & 17 & 1.227 & 1.227 & 1.227 \\
\hline & & & 563 & 23 & 23 & 23 & 2.517 & 2.517 & 2.517 \\
\hline & & & 570 & 14 & 14 & 14 & 1.786 & 1.786 & 1.786 \\
\hline & & & 870 & 11 & 11 & 11 & 1.866 & 1.866 & 1.866 \\
\hline & & 560 & 543 & 45 & 45 & 45 & 1.869 & 1.869 & 1.869 \\
\hline & & & 547 & 25 & 26 & 26 & 1.293 & 1.293 & 1.293 \\
\hline & & & 550 & 13 & 14 & 14 & 1.476 & 1.476 & 1.476 \\
\hline & & & 552 & 6 & 6 & 6 & 1.425 & 1.425 & 1.425 \\
\hline & & & 570 & 46 & 46 & 46 & 1.889 & 1.889 & 1.889 \\
\hline & & & 857 & 8 & 8 & 8 & 1.035 & 1.035 & 1.035 \\
\hline & & 563 & 543 & 34 & 34 & 34 & 3.236 & 3.236 & 3.236 \\
\hline & & & 547 & 10 & 10 & 10 & 2.241 & 2.241 & 2.241 \\
\hline & & & 552 & & 2 & 2 & & 0.468 & 0.468 \\
\hline & & & 556 & 23 & 23 & 23 & 2.517 & 2.517 & 2.517 \\
\hline & & 570 & 529 & 38 & 38 & 38 & 3.544 & 3.544 & 3.544 \\
\hline & & & 543 & 6 & 6 & 6 & 0.982 & 0.982 & 0.982 \\
\hline & & & 552 & 2 & 2 & 2 & 0.601 & 0.601 & 0.601 \\
\hline & & & 556 & 14 & 14 & 14 & 1.786 & 1.786 & 1.786 \\
\hline & & & 560 & 46 & 46 & 46 & 1.889 & 1.889 & 1.889 \\
\hline & & & 815 & 3 & 3 & 3 & 2.907 & 2.907 & 2.907 \\
\hline & & 581 & 604 & 25 & 25 & 25 & 2.941 & 2.941 & 2.941 \\
\hline & & & 605 & 15 & 15 & 15 & 3.408 & 3.408 & 3.408 \\
\hline & & 583 & 604 & 46 & 46 & 46 & 3.021 & 3.021 & 3.021 \\
\hline & & & 605 & 32 & 32 & 32 & 3.580 & 3.580 & 3.580 \\
\hline & & 604 & 581 & 25 & 25 & 25 & 2.941 & 2.941 & 2.941 \\
\hline & & & 583 & 46 & 46 & 46 & 3.021 & 3.021 & 3.021 \\
\hline & & 605 & 412 & & 2 & 2 & & 0.419 & 0.419 \\
\hline & & & 581 & 15 & 15 & 15 & 3.408 & 3.408 & 3.408 \\
\hline & & & 583 & 32 & 32 & 32 & 3.580 & 3.580 & 3.580 \\
\hline & & & 649 & 17 & 17 & 17 & 18.369 & 18.369 & 18.369 \\
\hline & & 627 & 672 & 2 & 2 & 2 & 12.700 & 12.700 & 12.700 \\
\hline & & 649 & 605 & 17 & 17 & 17 & 18.369 & 18.369 & 18.369 \\
\hline & & 672 & 627 & 2 & 2 & 2 & 12.700 & 12.700 & 12.700 \\
\hline & & 770 & 815 & 6 & 6 & 6 & 10.981 & 10.981 & 10.981 \\
\hline & & 790 & 815 & 26 & 27 & 27 & 15.919 & 15.919 & 15.919 \\
\hline & & 815 & 570 & 3 & 3 & 3 & 2.907 & 2.907 & 2.907 \\
\hline & & & 770 & 6 & 6 & 6 & 10.981 & 10.981 & 10.981 \\
\hline & & & 790 & 26 & 27 & 27 & 15.919 & 15.919 & 15.919 \\
\hline & & & 857 & 6 & 6 & 6 & 7.208 & 7.208 & 7.208 \\
\hline & & & 870 & 4 & 4 & 4 & 2.008 & 2.008 & 2.008 \\
\hline & & & 877 & 3 & 3 & 3 & 2.612 & 2.612 & 2.612 \\
\hline & & 843 & 877 & 6 & 7 & 7 & 2.947 & 2.947 & 2.947 \\
\hline & & 857 & 543 & 10 & 10 & 10 & 1.930 & 1.930 & 1.930 \\
\hline & & & 547 & 6 & 6 & 6 & 2.462 & 2.462 & 2.462 \\
\hline & & & 560 & 8 & 8 & 8 & 1.035 & 1.035 & 1.035 \\
\hline & & & 815 & 6 & 6 & 6 & 7.208 & 7.208 & 7.208 \\
\hline & & & 874 & & & 2 & & & 0.179 \\
\hline & & & 875 & & 5 & 6 & & 0.372 & 0.372 \\
\hline & & & 877 & 18 & 18 & 18 & 4.163 & 4.163 & 4.163 \\
\hline & & 870 & 547 & 11 & 11 & 11 & 2.145 & 2.145 & 2.145 \\
\hline & & & 550 & 32 & 33 & 33 & 1.286 & 1.286 & 1.286 \\
\hline & & & 552 & 11 & 12 & 12 & 0.921 & 0.921 & 0.921 \\
\hline & & & 556 & 11 & 11 & 11 & 1.866 & 1.866 & 1.866 \\
\hline & & & 815 & 4 & 4 & 4 & 2.008 & 2.008 & 2.008 \\
\hline & & & 874 & & 2 & 2 & & 0.401 & 0.401 \\
\hline & & & 877 & 98 & 105 & 105 & 3.362 & 3.362 & 3.362 \\
\hline & & & 891 & 6 & 7 & 7 & 1.176 & 1.176 & 1.176 \\
\hline & & 874 & 857 & & & 2 & & & 0.179 \\
\hline & & & 870 & & 2 & 2 & & 0.401 & 0.401 \\
\hline & & 875 & 857 & & 5 & 6 & & 0.372 & 0.372 \\
\hline & & 877 & 550 & 8 & 11 & 11 & 1.671 & 1.671 & 1.671 \\
\hline & & & 815 & 3 & 3 & 3 & 2.612 & 2.612 & 2.612 \\
\hline & & & 843 & 6 & 7 & 7 & 2.947 & 2.947 & 2.947 \\
\hline & & & 857 & 18 & 18 & 18 & 4.163 & 4.163 & 4.163 \\
\hline & & & 870 & 98 & 105 & 105 & 3.362 & 3.362 & 3.362 \\
\hline
\end{tabular}


Table B.2 - Continued from previous page

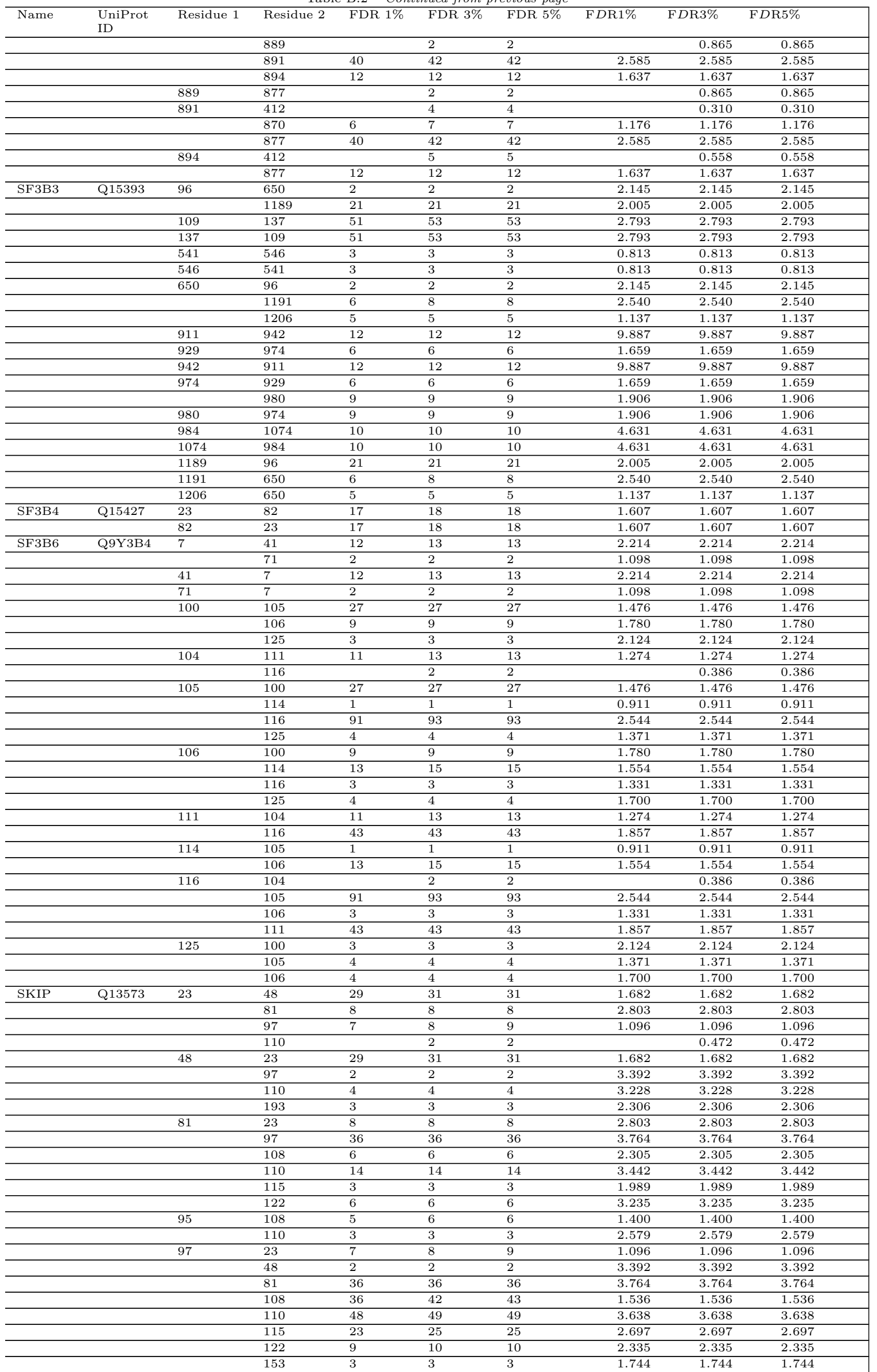


Table B.2 - Continued from previous page

\begin{tabular}{|c|c|c|c|c|c|c|c|c|c|}
\hline Name & $\begin{array}{l}\text { UniProt } \\
\text { ID }\end{array}$ & Residue 1 & Residue 2 & FDR 1\% & FDR 3\% & FDR 5\% & F DR1\% & F DR3\% & FDR5\% \\
\hline & & & 193 & 7 & 7 & 7 & 1.757 & 1.757 & 1.757 \\
\hline & & 108 & 81 & 6 & 6 & 6 & 2.305 & 2.305 & 2.305 \\
\hline & & & 95 & 5 & 6 & 6 & 1.400 & 1.400 & 1.400 \\
\hline & & & 97 & 36 & 42 & 43 & 1.536 & 1.536 & 1.536 \\
\hline & & & 115 & 15 & 16 & 16 & 1.332 & 1.332 & 1.332 \\
\hline & & & 122 & 3 & 5 & 5 & 0.935 & 0.935 & 0.935 \\
\hline & & & 153 & 2 & 2 & 2 & 0.810 & 0.810 & 0.810 \\
\hline & & 110 & 23 & & 2 & 2 & & 0.472 & 0.472 \\
\hline & & & 48 & 4 & 4 & 4 & 3.228 & 3.228 & 3.228 \\
\hline & & & 81 & 14 & 14 & 14 & 3.442 & 3.442 & 3.442 \\
\hline & & & 95 & 3 & 3 & 3 & 2.579 & 2.579 & 2.579 \\
\hline & & & 97 & 48 & 49 & 49 & 3.638 & 3.638 & 3.638 \\
\hline & & & 122 & 33 & 34 & 34 & 2.778 & 2.778 & 2.778 \\
\hline & & 115 & 81 & 3 & 3 & 3 & 1.989 & 1.989 & 1.989 \\
\hline & & & 97 & 23 & 25 & 25 & 2.697 & 2.697 & 2.697 \\
\hline & & & 108 & 15 & 16 & 16 & 1.332 & 1.332 & 1.332 \\
\hline & & 122 & 81 & 6 & 6 & 6 & 3.235 & 3.235 & 3.235 \\
\hline & & & 97 & 9 & 10 & 10 & 2.335 & 2.335 & 2.335 \\
\hline & & & 108 & 3 & 5 & 5 & 0.935 & 0.935 & 0.935 \\
\hline & & & 110 & 33 & 34 & 34 & 2.778 & 2.778 & 2.778 \\
\hline & & 141 & 153 & 5 & 5 & 5 & 1.189 & 1.189 & 1.189 \\
\hline & & 146 & 153 & 102 & 110 & 111 & 2.059 & 2.059 & 2.059 \\
\hline & & & 158 & 2 & 2 & 2 & 0.793 & 0.793 & 0.793 \\
\hline & & & 170 & 4 & 4 & 4 & 1.105 & 1.105 & 1.105 \\
\hline & & 153 & 97 & 3 & 3 & 3 & 1.744 & 1.744 & 1.744 \\
\hline & & & 108 & 2 & 2 & 2 & 0.810 & 0.810 & 0.810 \\
\hline & & & 141 & 5 & 5 & 5 & 1.189 & 1.189 & 1.189 \\
\hline & & & 146 & 102 & 110 & 111 & 2.059 & 2.059 & 2.059 \\
\hline & & & 170 & 49 & 49 & 49 & 2.362 & 2.362 & 2.362 \\
\hline & & & 193 & 26 & 26 & 26 & 3.090 & 3.090 & 3.090 \\
\hline & & & 217 & 12 & 12 & 12 & 1.847 & 1.847 & 1.847 \\
\hline & & 158 & 146 & 2 & 2 & 2 & 0.793 & 0.793 & 0.793 \\
\hline & & & 170 & 11 & 11 & 11 & 2.027 & 2.027 & 2.027 \\
\hline & & & 193 & 18 & 20 & 20 & 1.676 & 1.676 & 1.676 \\
\hline & & & 204 & & 3 & 3 & & 0.457 & 0.457 \\
\hline & & & 216 & 25 & 28 & 28 & 1.471 & 1.471 & 1.471 \\
\hline & & & 217 & 34 & 36 & 36 & 2.345 & 2.345 & 2.345 \\
\hline & & 170 & 146 & 4 & 4 & 4 & 1.105 & 1.105 & 1.105 \\
\hline & & & 153 & 49 & 49 & 49 & 2.362 & 2.362 & 2.362 \\
\hline & & & 158 & 11 & 11 & 11 & 2.027 & 2.027 & 2.027 \\
\hline & & & 193 & 27 & 28 & 28 & 3.005 & 3.005 & 3.005 \\
\hline & & & 204 & 4 & 4 & 4 & 1.509 & 1.509 & 1.509 \\
\hline & & & 213 & 2 & 2 & 2 & 0.611 & 0.611 & 0.611 \\
\hline & & & 217 & 8 & 8 & 8 & 1.243 & 1.243 & 1.243 \\
\hline & & 193 & 48 & 3 & 3 & 3 & 2.306 & 2.306 & 2.306 \\
\hline & & & 97 & 7 & 7 & 7 & 1.757 & 1.757 & 1.757 \\
\hline & & & 153 & 26 & 26 & 26 & 3.090 & 3.090 & 3.090 \\
\hline & & & 158 & 18 & 20 & 20 & 1.676 & 1.676 & 1.676 \\
\hline & & & 170 & 27 & 28 & 28 & 3.005 & 3.005 & 3.005 \\
\hline & & & 204 & 3 & 3 & 3 & 0.661 & 0.661 & 0.661 \\
\hline & & & 217 & 2 & 4 & 4 & 1.649 & 1.649 & 1.649 \\
\hline & & 204 & 158 & & 3 & 3 & & 0.457 & 0.457 \\
\hline & & & 170 & 4 & 4 & 4 & 1.509 & 1.509 & 1.509 \\
\hline & & & 193 & 3 & 3 & 3 & 0.661 & 0.661 & 0.661 \\
\hline & & & 217 & 4 & 4 & 4 & 1.439 & 1.439 & 1.439 \\
\hline & & 213 & 170 & 2 & 2 & 2 & 0.611 & 0.611 & 0.611 \\
\hline & & & 217 & 26 & 26 & 26 & 4.954 & 4.954 & 4.954 \\
\hline & & 216 & 158 & 25 & 28 & 28 & 1.471 & 1.471 & 1.471 \\
\hline & & 217 & 153 & 12 & 12 & 12 & 1.847 & 1.847 & 1.847 \\
\hline & & & 158 & 34 & 36 & 36 & 2.345 & 2.345 & 2.345 \\
\hline & & & 170 & 8 & 8 & 8 & 1.243 & 1.243 & 1.243 \\
\hline & & & 193 & 2 & 4 & 4 & 1.649 & 1.649 & 1.649 \\
\hline & & & 204 & 4 & 4 & 4 & 1.439 & 1.439 & 1.439 \\
\hline & & & 213 & 26 & 26 & 26 & 4.954 & 4.954 & 4.954 \\
\hline & & 246 & 266 & 18 & 26 & 26 & 0.966 & 0.966 & 0.966 \\
\hline & & 255 & 266 & 6 & 7 & 7 & 0.784 & 0.784 & 0.784 \\
\hline & & 266 & 246 & 18 & 26 & 26 & 0.966 & 0.966 & 0.966 \\
\hline & & & 255 & 6 & 7 & 7 & 0.784 & 0.784 & 0.784 \\
\hline & & 311 & 317 & 3 & 3 & 3 & 2.150 & 2.150 & 2.150 \\
\hline & & & 319 & 42 & 46 & 46 & 2.686 & 2.686 & 2.686 \\
\hline & & 315 & 323 & 16 & 16 & 16 & 1.485 & 1.485 & 1.485 \\
\hline & & 317 & 311 & 3 & 3 & 3 & 2.150 & 2.150 & 2.150 \\
\hline & & & 323 & 3 & 3 & 3 & 0.785 & 0.785 & 0.785 \\
\hline & & 319 & 311 & 42 & 46 & 46 & 2.686 & 2.686 & 2.686 \\
\hline & & & 330 & 3 & 3 & 3 & 1.643 & 1.643 & 1.643 \\
\hline & & 323 & 315 & 16 & 16 & 16 & 1.485 & 1.485 & 1.485 \\
\hline & & & 317 & 3 & 3 & 3 & 0.785 & 0.785 & 0.785 \\
\hline & & & 330 & 43 & 48 & 48 & 2.505 & 2.505 & 2.505 \\
\hline & & & 339 & 2 & 4 & 4 & 0.702 & 0.702 & 0.702 \\
\hline & & 330 & 319 & 3 & 3 & 3 & 1.643 & 1.643 & 1.643 \\
\hline & & & 323 & 43 & 48 & 48 & 2.505 & 2.505 & 2.505 \\
\hline & & & 339 & 42 & 51 & 51 & 1.574 & 1.574 & 1.574 \\
\hline & & & 344 & 12 & 18 & 18 & 1.533 & 1.533 & 1.533 \\
\hline
\end{tabular}


Table B.2 - Continued from previous page

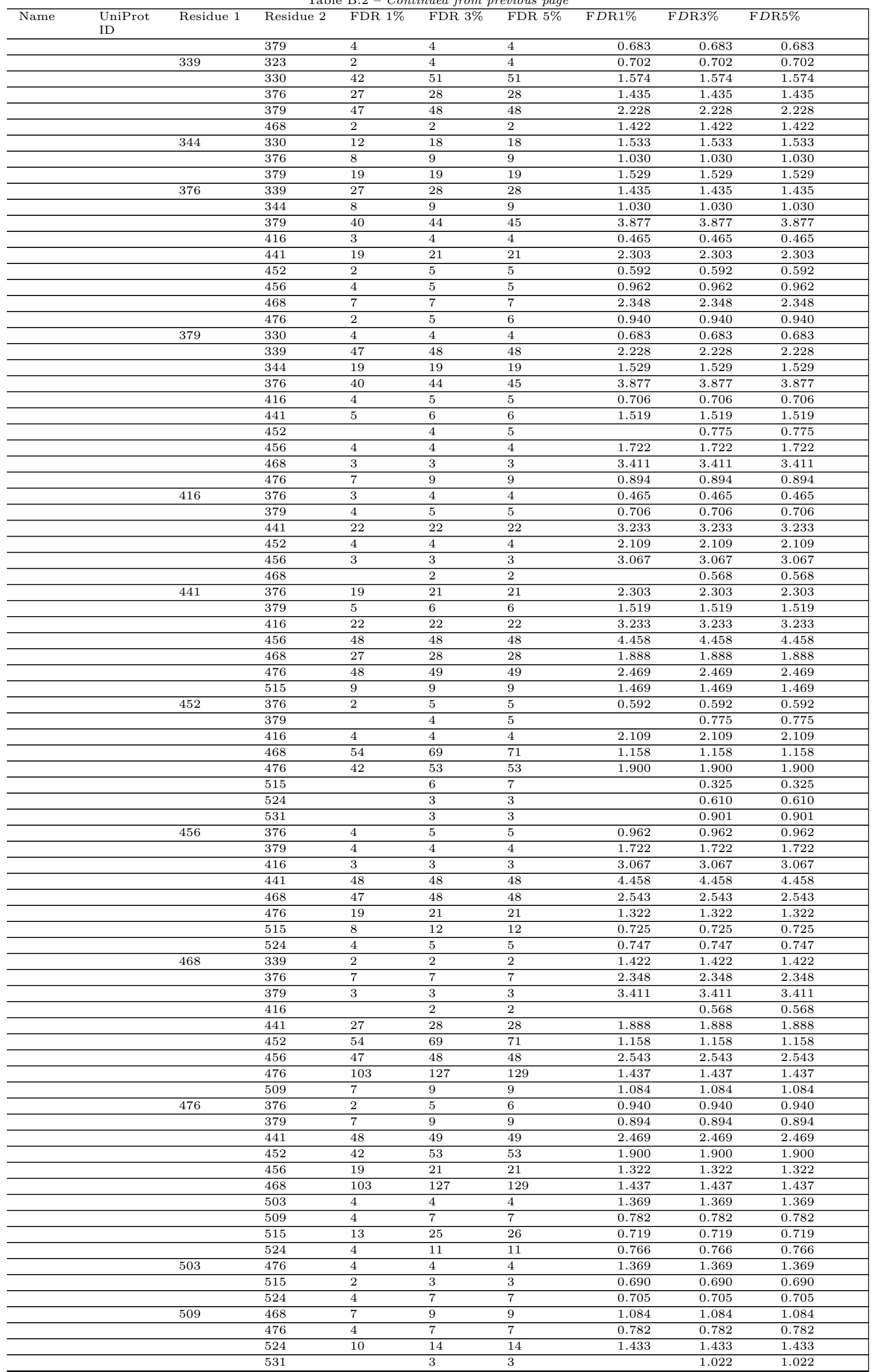


Table B.2 - Continued from previous page

\begin{tabular}{|c|c|c|c|c|c|c|c|c|c|}
\hline Name & $\begin{array}{l}\text { UniProt } \\
\text { ID }\end{array}$ & Residue 1 & Residue 2 & FDR $1 \%$ & FDR 3\% & FDR $5 \%$ & FDR1\% & FDR3\% & FDR5\% \\
\hline & & 515 & 441 & 9 & 9 & 9 & 1.469 & 1.469 & 1.469 \\
\hline & & & 452 & & 6 & 7 & & 0.325 & 0.325 \\
\hline & & & 456 & 8 & 12 & 12 & 0.725 & 0.725 & 0.725 \\
\hline & & & 476 & 13 & 25 & 26 & 0.719 & 0.719 & 0.719 \\
\hline & & & 503 & 2 & 3 & 3 & 0.690 & 0.690 & 0.690 \\
\hline & & & 531 & 2 & 3 & 5 & 0.896 & 0.896 & 0.896 \\
\hline & & 524 & 452 & & 3 & 3 & & 0.610 & 0.610 \\
\hline & & & 456 & 4 & 5 & 5 & 0.747 & 0.747 & 0.747 \\
\hline & & & 476 & 4 & 11 & 11 & 0.766 & 0.766 & 0.766 \\
\hline & & & 503 & 4 & 7 & 7 & 0.705 & 0.705 & 0.705 \\
\hline & & & 509 & 10 & 14 & 14 & 1.433 & 1.433 & 1.433 \\
\hline & & 531 & 452 & & 3 & 3 & & 0.901 & 0.901 \\
\hline & & & 509 & & 3 & 3 & & 1.022 & 1.022 \\
\hline & & & 515 & 2 & 3 & 5 & 0.896 & 0.896 & 0.896 \\
\hline \multirow[t]{10}{*}{$\mathrm{SmB}$} & $\begin{array}{l}\text { P14678 } \\
\end{array}$ & 50 & 57 & 3 & 3 & 3 & 1.471 & 1.471 & 1.471 \\
\hline & & & 64 & & 3 & 3 & & 0.373 & 0.373 \\
\hline & & & 88 & 2 & 3 & 3 & 0.568 & 0.568 & 0.568 \\
\hline & & 52 & 57 & 2 & 3 & 3 & 0.600 & 0.600 & 0.600 \\
\hline & & 57 & 50 & 3 & 3 & 3 & 1.471 & 1.471 & 1.471 \\
\hline & & & 52 & 2 & 3 & 3 & 0.600 & 0.600 & 0.600 \\
\hline & & & 64 & & 3 & 5 & & 0.210 & 0.210 \\
\hline & & 64 & 50 & & 3 & 3 & & 0.373 & 0.373 \\
\hline & & & 57 & & 3 & 5 & & 0.210 & 0.210 \\
\hline & & 88 & 50 & 2 & 3 & 3 & 0.568 & 0.568 & 0.568 \\
\hline \multirow[t]{2}{*}{ SmD1 } & $\begin{array}{l}\text { P62314 } \\
\end{array}$ & 1 & 86 & 3 & 3 & 3 & 1.855 & 1.855 & 1.855 \\
\hline & & 86 & 1 & 3 & 3 & 3 & 1.855 & 1.855 & 1.855 \\
\hline $\begin{array}{l}\text { SmD2 } \\
\end{array}$ & P62316 & 6 & 18 & 55 & 60 & 61 & 2.169 & 2.169 & 2.169 \\
\hline & & 18 & 6 & 55 & 60 & 61 & 2.169 & 2.169 & 2.169 \\
\hline & & 51 & 118 & 24 & 25 & 25 & 8.665 & 8.665 & 8.665 \\
\hline & & 71 & 118 & 13 & 13 & 13 & 1.690 & 1.690 & 1.690 \\
\hline & & 86 & 98 & & 3 & 3 & & 0.260 & 0.260 \\
\hline & & 88 & 98 & & 6 & 8 & & 0.344 & 0.344 \\
\hline & & & 118 & 8 & 10 & 10 & 2.687 & 2.687 & 2.687 \\
\hline & & 92 & 98 & & 17 & 20 & & 0.391 & 0.391 \\
\hline & & 98 & 86 & & 3 & 3 & & 0.260 & 0.260 \\
\hline & & & 88 & & 6 & 8 & & 0.344 & 0.344 \\
\hline & & & 92 & & 17 & 20 & & 0.391 & 0.391 \\
\hline & & 118 & 51 & 24 & 25 & 25 & 8.665 & 8.665 & 8.665 \\
\hline & & & 71 & 13 & 13 & 13 & 1.690 & 1.690 & 1.690 \\
\hline & & & 88 & 8 & 10 & 10 & 2.687 & 2.687 & 2.687 \\
\hline SmD3 & P62318 & 8 & 84 & & 2 & 2 & & 0.416 & 0.416 \\
\hline & & & 87 & & 2 & 2 & & 0.201 & 0.201 \\
\hline & & 84 & 8 & & 2 & 2 & & 0.416 & 0.416 \\
\hline & & 87 & 8 & & 2 & 2 & & 0.201 & 0.201 \\
\hline SmE & P62304 & 67 & 72 & 4 & 4 & 4 & 1.916 & 1.916 & 1.916 \\
\hline & & 72 & 67 & 4 & 4 & 4 & 1.916 & 1.916 & 1.916 \\
\hline SmG & P62308 & 3 & 11 & & 2 & 2 & & 0.253 & 0.253 \\
\hline & & 10 & 15 & 9 & 10 & 10 & 0.937 & 0.937 & 0.937 \\
\hline & & 11 & 3 & & 2 & 2 & & 0.253 & 0.253 \\
\hline & & 15 & 10 & 9 & 10 & 10 & 0.937 & 0.937 & 0.937 \\
\hline SMU1 & Q2TAY7 & 107 & 247 & 6 & 6 & 6 & 2.758 & 2.758 & 2.758 \\
\hline & & 170 & 190 & 55 & 57 & 57 & 2.869 & 2.869 & 2.869 \\
\hline & & & 194 & 23 & 23 & 23 & 4.209 & 4.209 & 4.209 \\
\hline & & & 512 & & 3 & 3 & & 0.360 & 0.360 \\
\hline & & 190 & 170 & 55 & 57 & 57 & 2.869 & 2.869 & 2.869 \\
\hline & & & 200 & 13 & 13 & 13 & 1.573 & 1.573 & 1.573 \\
\hline & & & 247 & 5 & 5 & 5 & 1.777 & 1.777 & 1.777 \\
\hline & & & 292 & 6 & 6 & 6 & 1.689 & 1.689 & 1.689 \\
\hline & & & 317 & 7 & 7 & 7 & 2.212 & 2.212 & 2.212 \\
\hline & & & 337 & 13 & 13 & 13 & 2.112 & 2.112 & 2.112 \\
\hline & & & 408 & 6 & 6 & 6 & 1.286 & 1.286 & 1.286 \\
\hline & & & 512 & 25 & 28 & 29 & 3.322 & 3.322 & 3.322 \\
\hline & & 194 & 170 & 23 & 23 & 23 & 4.209 & 4.209 & 4.209 \\
\hline & & & 292 & & 2 & 2 & & 2.341 & 2.341 \\
\hline & & & 337 & 9 & 9 & 9 & 1.401 & 1.401 & 1.401 \\
\hline & & & 408 & 10 & 10 & 10 & 3.445 & 3.445 & 3.445 \\
\hline & & & 512 & 41 & 53 & 53 & 1.436 & 1.436 & 1.436 \\
\hline & & 200 & 190 & 13 & 13 & 13 & 1.573 & 1.573 & 1.573 \\
\hline & & & 512 & 30 & 43 & 44 & 0.970 & 0.970 & 0.970 \\
\hline & & 210 & 247 & 77 & 78 & 78 & 2.806 & 2.806 & 2.806 \\
\hline & & & 485 & 135 & 148 & 150 & 15.323 & 15.323 & 15.323 \\
\hline & & & 509 & 119 & 125 & 126 & 6.908 & 6.908 & 6.908 \\
\hline & & 214 & 485 & 74 & 77 & 79 & 22.768 & 22.768 & 22.768 \\
\hline & & 247 & 107 & 6 & 6 & 6 & 2.758 & 2.758 & 2.758 \\
\hline & & & 190 & 5 & 5 & 5 & 1.777 & 1.777 & 1.777 \\
\hline & & & 210 & 77 & 78 & 78 & 2.806 & 2.806 & 2.806 \\
\hline & & & 289 & 3 & 5 & 6 & 0.601 & 0.601 & 0.601 \\
\hline & & & 308 & 2 & 2 & 2 & 0.980 & 0.980 & 0.980 \\
\hline & & 289 & 247 & 3 & 5 & 6 & 0.601 & 0.601 & 0.601 \\
\hline & & & 512 & 4 & 4 & 4 & 2.380 & 2.380 & 2.380 \\
\hline & & 292 & 190 & 6 & 6 & 6 & 1.689 & 1.689 & 1.689 \\
\hline & & & 194 & & 2 & 2 & & 2.341 & 2.341 \\
\hline & & & 337 & 171 & 176 & 176 & 2.799 & 2.799 & 2.799 \\
\hline
\end{tabular}


Table B.2 - Continued from previous page

\begin{tabular}{|c|c|c|c|c|c|c|c|c|c|}
\hline Name & $\begin{array}{l}\text { UniProt } \\
\text { ID }\end{array}$ & Residue 1 & Residue 2 & FDR $1 \%$ & FDR 3\% & FDR $5 \%$ & F DR $1 \%$ & FDR3\% & FDR5\% \\
\hline & & 308 & 247 & 2 & 2 & 2 & 0.980 & 0.980 & 0.980 \\
\hline & & 317 & 190 & 7 & 7 & 7 & 2.212 & 2.212 & 2.212 \\
\hline & & & 343 & 11 & 11 & 11 & 0.988 & 0.988 & 0.988 \\
\hline & & & 379 & 21 & 22 & 22 & 3.645 & 3.645 & 3.645 \\
\hline & & & 408 & 5 & 5 & 5 & 2.928 & 2.928 & 2.928 \\
\hline & & 337 & 190 & 13 & 13 & 13 & 2.112 & 2.112 & 2.112 \\
\hline & & & 194 & 9 & 9 & 9 & 1.401 & 1.401 & 1.401 \\
\hline & & & 292 & 171 & 176 & 176 & 2.799 & 2.799 & 2.799 \\
\hline & & & 343 & 12 & 12 & 12 & 1.826 & 1.826 & 1.826 \\
\hline & & 340 & 379 & 3 & 3 & 3 & 1.711 & 1.711 & 1.711 \\
\hline & & 343 & 317 & 11 & 11 & 11 & 0.988 & 0.988 & 0.988 \\
\hline & & & 337 & 12 & 12 & 12 & 1.826 & 1.826 & 1.826 \\
\hline & & & 379 & 54 & 54 & 54 & 2.546 & 2.546 & 2.546 \\
\hline & & 374 & 388 & 10 & 11 & 11 & 1.491 & 1.491 & 1.491 \\
\hline & & 379 & 317 & 21 & 22 & 22 & 3.645 & 3.645 & 3.645 \\
\hline & & & 340 & 3 & 3 & 3 & 1.711 & 1.711 & 1.711 \\
\hline & & & 343 & 54 & 54 & 54 & 2.546 & 2.546 & 2.546 \\
\hline & & 388 & 374 & 10 & 11 & 11 & 1.491 & 1.491 & 1.491 \\
\hline & & 408 & 190 & 6 & 6 & 6 & 1.286 & 1.286 & 1.286 \\
\hline & & & 194 & 10 & 10 & 10 & 3.445 & 3.445 & 3.445 \\
\hline & & & 317 & 5 & 5 & 5 & 2.928 & 2.928 & 2.928 \\
\hline & & 485 & 210 & 135 & 148 & 150 & 15.323 & 15.323 & 15.323 \\
\hline & & & 214 & 74 & 77 & 79 & 22.768 & 22.768 & 22.768 \\
\hline & & 509 & 210 & 119 & 125 & 126 & 6.908 & 6.908 & 6.908 \\
\hline & & 512 & 170 & & 3 & 3 & & 0.360 & 0.360 \\
\hline & & & 190 & 25 & 28 & 29 & 3.322 & 3.322 & 3.322 \\
\hline & & & 194 & 41 & 53 & 53 & 1.436 & 1.436 & 1.436 \\
\hline & & & 200 & 30 & 43 & 44 & 0.970 & 0.970 & 0.970 \\
\hline & & & 289 & 4 & 4 & 4 & 2.380 & 2.380 & 2.380 \\
\hline SNIP1 & Q8TAD8 & 214 & 223 & 2 & 2 & 2 & 1.081 & 1.081 & 1.081 \\
\hline & & & 301 & & 2 & 2 & & 0.486 & 0.486 \\
\hline & & 216 & 223 & 18 & 18 & 18 & 2.358 & 2.358 & 2.358 \\
\hline & & & 301 & 15 & 15 & 15 & 1.335 & 1.335 & 1.335 \\
\hline & & 221 & 301 & 10 & 10 & 10 & 1.982 & 1.982 & 1.982 \\
\hline & & 223 & 214 & 2 & 2 & 2 & 1.081 & 1.081 & 1.081 \\
\hline & & & 216 & 18 & 18 & 18 & 2.358 & 2.358 & 2.358 \\
\hline & & & 265 & 3 & 3 & 3 & 2.697 & 2.697 & 2.697 \\
\hline & & & 301 & 4 & 6 & 6 & 2.554 & 2.554 & 2.554 \\
\hline & & 245 & 301 & 106 & 108 & 109 & 3.723 & 3.723 & 3.723 \\
\hline & & 265 & 223 & 3 & 3 & 3 & 2.697 & 2.697 & 2.697 \\
\hline & & 301 & 214 & & 2 & 2 & & 0.486 & 0.486 \\
\hline & & & 216 & 15 & 15 & 15 & 1.335 & 1.335 & 1.335 \\
\hline & & & 221 & 10 & 10 & 10 & 1.982 & 1.982 & 1.982 \\
\hline & & & 223 & 4 & 6 & 6 & 2.554 & 2.554 & 2.554 \\
\hline & & & 245 & 106 & 108 & 109 & 3.723 & 3.723 & 3.723 \\
\hline & & 325 & 353 & & 2 & 2 & & 2.426 & 2.426 \\
\hline & & 342 & 353 & 11 & 15 & 15 & 1.635 & 1.635 & 1.635 \\
\hline & & 353 & 325 & & 2 & 2 & & 2.426 & 2.426 \\
\hline & & & 342 & 11 & 15 & 15 & 1.635 & 1.635 & 1.635 \\
\hline SNU114 & Q15029 & 95 & 602 & 3 & 3 & 3 & 2.589 & 2.589 & 2.589 \\
\hline & & 98 & 602 & 4 & 4 & 4 & 2.106 & 2.106 & 2.106 \\
\hline & & & 646 & 7 & 7 & 7 & 3.103 & 3.103 & 3.103 \\
\hline & & 244 & 963 & & & 2 & & & 0.241 \\
\hline & & 352 & 355 & 66 & 68 & 68 & 2.340 & 2.340 & 2.340 \\
\hline & & & 359 & 191 & 194 & 194 & 4.313 & 4.313 & 4.313 \\
\hline & & 355 & 352 & 66 & 68 & 68 & 2.340 & 2.340 & 2.340 \\
\hline & & & 359 & 7 & 7 & 7 & 2.261 & 2.261 & 2.261 \\
\hline & & 359 & 352 & 191 & 194 & 194 & 4.313 & 4.313 & 4.313 \\
\hline & & & 355 & 7 & 7 & 7 & 2.261 & 2.261 & 2.261 \\
\hline & & 602 & 95 & 3 & 3 & 3 & 2.589 & 2.589 & 2.589 \\
\hline & & & 98 & 4 & 4 & 4 & 2.106 & 2.106 & 2.106 \\
\hline & & & 609 & 3 & 3 & 3 & 1.931 & 1.931 & 1.931 \\
\hline & & 609 & 602 & 3 & 3 & 3 & 1.931 & 1.931 & 1.931 \\
\hline & & 646 & 98 & 7 & 7 & 7 & 3.103 & 3.103 & 3.103 \\
\hline & & 673 & 684 & 21 & 21 & 21 & 1.789 & 1.789 & 1.789 \\
\hline & & & 790 & 30 & 30 & 30 & 4.858 & 4.858 & 4.858 \\
\hline & & 684 & 673 & 21 & 21 & 21 & 1.789 & 1.789 & 1.789 \\
\hline & & & 963 & 3 & 3 & 3 & 2.055 & 2.055 & 2.055 \\
\hline & & 790 & 673 & 30 & 30 & 30 & 4.858 & 4.858 & 4.858 \\
\hline & & 914 & 951 & 11 & 11 & 11 & 11.140 & 11.140 & 11.140 \\
\hline & & & 963 & 9 & 9 & 9 & 14.354 & 14.354 & 14.354 \\
\hline & & 951 & 914 & 11 & 11 & 11 & 11.140 & 11.140 & 11.140 \\
\hline & & 963 & 244 & & & 2 & & & 0.241 \\
\hline & & & 684 & 3 & 3 & 3 & 2.055 & 2.055 & 2.055 \\
\hline & & & 914 & 9 & 9 & 9 & 14.354 & 14.354 & 14.354 \\
\hline SNU17 & Q9Y388 & 203 & 208 & 3 & 3 & 3 & 3.171 & 3.171 & 3.171 \\
\hline & & 208 & 203 & 3 & 3 & 3 & 3.171 & 3.171 & 3.171 \\
\hline & & & 215 & 2 & 2 & 2 & 1.891 & 1.891 & 1.891 \\
\hline & & 215 & 208 & 2 & 2 & 2 & 1.891 & 1.891 & 1.891 \\
\hline & & & 223 & 5 & 5 & 5 & 1.486 & 1.486 & 1.486 \\
\hline & & & 226 & 6 & 7 & 7 & 1.560 & 1.560 & 1.560 \\
\hline & & 223 & 215 & 5 & 5 & 5 & 1.486 & 1.486 & 1.486 \\
\hline & & 226 & 215 & 6 & 7 & 7 & 1.560 & 1.560 & 1.560 \\
\hline
\end{tabular}


Table B.2 - Continued from previous page

\begin{tabular}{|c|c|c|c|c|c|c|c|c|c|}
\hline Name & $\begin{array}{l}\text { UniProt } \\
\text { ID }\end{array}$ & Residue 1 & Residue 2 & FDR 1\% & FDR 3\% & FDR 5\% & FDR1\% & FDR3\% & FDR5\% \\
\hline \multirow[t]{42}{*}{ SNU23 } & Q96NC0 & 8 & 15 & 86 & 88 & 88 & 3.718 & 3.718 & 3.718 \\
\hline & & & 18 & 49 & 50 & 50 & 1.701 & 1.701 & 1.701 \\
\hline & & & 23 & 5 & 6 & 6 & 1.681 & 1.681 & 1.681 \\
\hline & & & 27 & 20 & 25 & 25 & 1.647 & 1.647 & 1.647 \\
\hline & & & 36 & 11 & 11 & 11 & 2.247 & 2.247 & 2.247 \\
\hline & & & 45 & 2 & 2 & 2 & 0.691 & 0.691 & 0.691 \\
\hline & & 15 & 8 & 86 & 88 & 88 & 3.718 & 3.718 & 3.718 \\
\hline & & & 23 & 2 & 4 & 4 & 0.683 & 0.683 & 0.683 \\
\hline & & & 27 & 22 & 25 & 25 & 2.874 & 2.874 & 2.874 \\
\hline & & 18 & 8 & 49 & 50 & 50 & 1.701 & 1.701 & 1.701 \\
\hline & & & 27 & 8 & 13 & 13 & 1.471 & 1.471 & 1.471 \\
\hline & & 23 & 8 & 5 & 6 & 6 & 1.681 & 1.681 & 1.681 \\
\hline & & & 15 & 2 & 4 & 4 & 0.683 & 0.683 & 0.683 \\
\hline & & 27 & 8 & 20 & 25 & 25 & 1.647 & 1.647 & 1.647 \\
\hline & & & 15 & 22 & 25 & 25 & 2.874 & 2.874 & 2.874 \\
\hline & & & 18 & 8 & 13 & 13 & 1.471 & 1.471 & 1.471 \\
\hline & & & 36 & 8 & 10 & 11 & 1.165 & 1.165 & 1.165 \\
\hline & & & 39 & 6 & 6 & 6 & 1.372 & 1.372 & 1.372 \\
\hline & & 36 & 8 & 11 & 11 & 11 & 2.247 & 2.247 & 2.247 \\
\hline & & & 27 & 8 & 10 & 11 & 1.165 & 1.165 & 1.165 \\
\hline & & 39 & 27 & 6 & 6 & 6 & 1.372 & 1.372 & 1.372 \\
\hline & & 45 & 8 & 2 & 2 & 2 & 0.691 & 0.691 & 0.691 \\
\hline & & & 55 & 4 & 4 & 4 & 1.056 & 1.056 & 1.056 \\
\hline & & 55 & 45 & 4 & 4 & 4 & 1.056 & 1.056 & 1.056 \\
\hline & & & 64 & 2 & 3 & 3 & 1.092 & 1.092 & 1.092 \\
\hline & & & 132 & 6 & 6 & 6 & 2.294 & 2.294 & 2.294 \\
\hline & & & 136 & 25 & 27 & 27 & 1.402 & 1.402 & 1.402 \\
\hline & & 64 & 55 & 2 & 3 & 3 & 1.092 & 1.092 & 1.092 \\
\hline & & 123 & 130 & 9 & 10 & 10 & 2.168 & 2.168 & 2.168 \\
\hline & & 124 & 132 & 3 & 3 & 3 & 1.117 & 1.117 & 1.117 \\
\hline & & 130 & 123 & 9 & 10 & 10 & 2.168 & 2.168 & 2.168 \\
\hline & & 132 & 55 & 6 & 6 & 6 & 2.294 & 2.294 & 2.294 \\
\hline & & & 124 & 3 & 3 & 3 & 1.117 & 1.117 & 1.117 \\
\hline & & & 138 & 8 & 8 & 8 & 2.432 & 2.432 & 2.432 \\
\hline & & 136 & 55 & 25 & 27 & 27 & 1.402 & 1.402 & 1.402 \\
\hline & & 138 & 132 & 8 & 8 & 8 & 2.432 & 2.432 & 2.432 \\
\hline & & 155 & 160 & 1 & 2 & 3 & 0.418 & 0.418 & 0.418 \\
\hline & & 157 & 163 & 3 & 3 & 3 & 1.303 & 1.303 & 1.303 \\
\hline & & 160 & 155 & 1 & 2 & 3 & 0.418 & 0.418 & 0.418 \\
\hline & & & 167 & & 2 & 2 & & 0.995 & 0.995 \\
\hline & & 163 & 157 & 3 & 3 & 3 & 1.303 & 1.303 & 1.303 \\
\hline & & 167 & 160 & & 2 & 2 & & 0.995 & 0.995 \\
\hline \multirow[t]{4}{*}{ SPF27 } & O75934 & 151 & 158 & 14 & 14 & 14 & 2.693 & 2.693 & 2.693 \\
\hline & & 158 & 151 & 14 & 14 & 14 & 2.693 & 2.693 & 2.693 \\
\hline & & & 168 & 18 & 18 & 18 & 4.826 & 4.826 & 4.826 \\
\hline & & 168 & 158 & 18 & 18 & 18 & 4.826 & 4.826 & 4.826 \\
\hline SRRM1 & Q8IYB3 & 36 & 128 & 4 & 4 & 4 & 1.321 & 1.321 & 1.321 \\
\hline & & 37 & 78 & 3 & 3 & 3 & 2.236 & 2.236 & 2.236 \\
\hline & & 42 & 890 & 4 & 6 & 6 & 0.709 & 0.709 & 0.709 \\
\hline & & 54 & 128 & 90 & 100 & 101 & 2.301 & 2.301 & 2.301 \\
\hline & & & 132 & 13 & 21 & 21 & 0.879 & 0.879 & 0.879 \\
\hline & & 78 & 37 & 3 & 3 & 3 & 2.236 & 2.236 & 2.236 \\
\hline & & 128 & 36 & 4 & 4 & 4 & 1.321 & 1.321 & 1.321 \\
\hline & & & 54 & 90 & 100 & 101 & 2.301 & 2.301 & 2.301 \\
\hline & & & 140 & 17 & 18 & 18 & 2.017 & 2.017 & 2.017 \\
\hline & & 132 & 54 & 13 & 21 & 21 & 0.879 & 0.879 & 0.879 \\
\hline & & & 140 & 93 & 101 & 102 & 1.814 & 1.814 & 1.814 \\
\hline & & & 145 & 4 & 4 & 4 & 1.835 & 1.835 & 1.835 \\
\hline & & 140 & 128 & 17 & 18 & 18 & 2.017 & 2.017 & 2.017 \\
\hline & & & 132 & 93 & 101 & 102 & 1.814 & 1.814 & 1.814 \\
\hline & & & 146 & 64 & 64 & 64 & 3.401 & 3.401 & 3.401 \\
\hline & & & 151 & 26 & 28 & 28 & 1.849 & 1.849 & 1.849 \\
\hline & & & 153 & 5 & 5 & 5 & 1.529 & 1.529 & 1.529 \\
\hline & & & 156 & 3 & 3 & 3 & 1.677 & 1.677 & 1.677 \\
\hline & & & 159 & 2 & 2 & 2 & 1.257 & 1.257 & 1.257 \\
\hline & & 145 & 132 & 4 & 4 & 4 & 1.835 & 1.835 & 1.835 \\
\hline & & & 151 & 13 & 13 & 13 & 1.602 & 1.602 & 1.602 \\
\hline & & & 153 & 4 & 4 & 4 & 1.210 & 1.210 & 1.210 \\
\hline & & & 156 & 6 & 7 & 8 & 1.286 & 1.286 & 1.286 \\
\hline & & & 159 & 9 & 11 & 11 & 2.275 & 2.275 & 2.275 \\
\hline & & 146 & 140 & 64 & 64 & 64 & 3.401 & 3.401 & 3.401 \\
\hline & & & 159 & 4 & 4 & 4 & 2.304 & 2.304 & 2.304 \\
\hline & & 151 & 140 & 26 & 28 & 28 & 1.849 & 1.849 & 1.849 \\
\hline & & & 145 & 13 & 13 & 13 & 1.602 & 1.602 & 1.602 \\
\hline & & & 159 & 4 & 5 & 5 & 1.941 & 1.941 & 1.941 \\
\hline & & 153 & 140 & 5 & 5 & 5 & 1.529 & 1.529 & 1.529 \\
\hline & & & 145 & 4 & 4 & 4 & 1.210 & 1.210 & 1.210 \\
\hline & & 156 & 140 & 3 & 3 & 3 & 1.677 & 1.677 & 1.677 \\
\hline & & & 145 & 6 & 7 & 8 & 1.286 & 1.286 & 1.286 \\
\hline & & 159 & 140 & 2 & 2 & 2 & 1.257 & 1.257 & 1.257 \\
\hline & & & 145 & 9 & 11 & 11 & 2.275 & 2.275 & 2.275 \\
\hline & & & 146 & 4 & 4 & 4 & 2.304 & 2.304 & 2.304 \\
\hline & & & 151 & 4 & 5 & 5 & 1.941 & 1.941 & 1.941 \\
\hline
\end{tabular}


Table B.2 - Continued from previous page

\begin{tabular}{|c|c|c|c|c|c|c|c|c|c|}
\hline Name & $\begin{array}{l}\text { UniProt } \\
\text { ID }\end{array}$ & Residue 1 & Residue 2 & FDR 1\% & FDR 3\% & FDR 5\% & FDR1\% & FDR3\% & FDR5\% \\
\hline & & 231 & 249 & 20 & 20 & 20 & 2.398 & 2.398 & 2.398 \\
\hline & & 249 & 231 & 20 & 20 & 20 & 2.398 & 2.398 & 2.398 \\
\hline & & 438 & 443 & 19 & 21 & 21 & 2.152 & 2.152 & 2.152 \\
\hline & & 443 & 438 & 19 & 21 & 21 & 2.152 & 2.152 & 2.152 \\
\hline & & 885 & 890 & 5 & 5 & 5 & 1.544 & 1.544 & 1.544 \\
\hline & & 890 & 42 & 4 & 6 & 6 & 0.709 & 0.709 & 0.709 \\
\hline & & & 885 & 5 & 5 & 5 & 1.544 & 1.544 & 1.544 \\
\hline & & & 897 & 35 & 36 & 36 & 2.308 & 2.308 & 2.308 \\
\hline & & 897 & 890 & 35 & 36 & 36 & 2.308 & 2.308 & 2.308 \\
\hline \multirow[t]{74}{*}{ SRRT } & Q9BXP5 & 153 & 176 & 22 & 22 & 22 & 2.796 & 2.796 & 2.796 \\
\hline & & & 442 & 2 & 2 & 2 & 1.503 & 1.503 & 1.503 \\
\hline & & 170 & 687 & 13 & 13 & 13 & 1.767 & 1.767 & 1.767 \\
\hline & & 176 & 153 & 22 & 22 & 22 & 2.796 & 2.796 & 2.796 \\
\hline & & & 200 & 2 & 6 & 6 & 0.888 & 0.888 & 0.888 \\
\hline & & & 442 & & 2 & 2 & & 0.342 & 0.342 \\
\hline & & & 476 & 3 & 3 & 3 & 1.759 & 1.759 & 1.759 \\
\hline & & & 687 & 216 & 228 & 229 & 3.940 & 3.940 & 3.940 \\
\hline & & 192 & 739 & 3 & 3 & 3 & 0.909 & 0.909 & 0.909 \\
\hline & & 200 & 176 & 2 & 6 & 6 & 0.888 & 0.888 & 0.888 \\
\hline & & & 413 & 7 & 7 & 7 & 2.381 & 2.381 & 2.381 \\
\hline & & & 419 & 7 & 9 & 9 & 3.023 & 3.023 & 3.023 \\
\hline & & & 442 & 77 & 80 & 80 & 3.005 & 3.005 & 3.005 \\
\hline & & & 476 & 30 & 30 & 30 & 3.627 & 3.627 & 3.627 \\
\hline & & 208 & 442 & 7 & 7 & 7 & 0.754 & 0.754 & 0.754 \\
\hline & & 256 & 285 & 3 & 3 & 3 & 2.418 & 2.418 & 2.418 \\
\hline & & 279 & 286 & 17 & 18 & 18 & 3.152 & 3.152 & 3.152 \\
\hline & & & 301 & 18 & 18 & 18 & 2.243 & 2.243 & 2.243 \\
\hline & & & 403 & & & 2 & & & 0.334 \\
\hline & & 285 & 256 & 3 & 3 & 3 & 2.418 & 2.418 & 2.418 \\
\hline & & & 301 & 57 & 62 & 65 & 2.956 & 2.956 & 2.956 \\
\hline & & & 305 & 10 & 12 & 12 & 1.467 & 1.467 & 1.467 \\
\hline & & & 308 & 2 & 4 & 4 & 0.685 & 0.685 & 0.685 \\
\hline & & & 313 & 6 & 6 & 6 & 2.787 & 2.787 & 2.787 \\
\hline & & & 327 & 2 & 3 & 3 & 0.734 & 0.734 & 0.734 \\
\hline & & & 395 & 7 & 9 & 9 & 1.107 & 1.107 & 1.107 \\
\hline & & & 397 & & 2 & 2 & & 0.671 & 0.671 \\
\hline & & & 413 & 5 & 5 & 5 & 1.043 & 1.043 & 1.043 \\
\hline & & & 442 & 4 & 7 & 7 & 0.827 & 0.827 & 0.827 \\
\hline & & & 476 & 3 & 3 & 3 & 1.250 & 1.250 & 1.250 \\
\hline & & & 607 & 4 & 7 & 7 & 0.661 & 0.661 & 0.661 \\
\hline & & 286 & 279 & 17 & 18 & 18 & 3.152 & 3.152 & 3.152 \\
\hline & & & 301 & 30 & 30 & 30 & 2.641 & 2.641 & 2.641 \\
\hline & & & 305 & 15 & 16 & 16 & 1.337 & 1.337 & 1.337 \\
\hline & & & 309 & 3 & 4 & 4 & 1.273 & 1.273 & 1.273 \\
\hline & & & 313 & 2 & 3 & 4 & 0.596 & 0.596 & 0.596 \\
\hline & & & 413 & & 2 & 2 & & 0.585 & 0.585 \\
\hline & & & 442 & 2 & 3 & 3 & 0.654 & 0.654 & 0.654 \\
\hline & & & 476 & 2 & 2 & 2 & 0.892 & 0.892 & 0.892 \\
\hline & & & 607 & 6 & 6 & 6 & 0.940 & 0.940 & 0.940 \\
\hline & & 301 & 279 & 18 & 18 & 18 & 2.243 & 2.243 & 2.243 \\
\hline & & & 285 & 57 & 62 & 65 & 2.956 & 2.956 & 2.956 \\
\hline & & & 286 & 30 & 30 & 30 & 2.641 & 2.641 & 2.641 \\
\hline & & & 309 & 5 & 5 & 5 & 1.978 & 1.978 & 1.978 \\
\hline & & & 313 & 3 & 3 & 3 & 1.399 & 1.399 & 1.399 \\
\hline & & 305 & 285 & 10 & 12 & 12 & 1.467 & 1.467 & 1.467 \\
\hline & & & 286 & 15 & 16 & 16 & 1.337 & 1.337 & 1.337 \\
\hline & & & 309 & 10 & 12 & 12 & 1.412 & 1.412 & 1.412 \\
\hline & & 308 & 285 & 2 & 4 & 4 & 0.685 & 0.685 & 0.685 \\
\hline & & & 313 & 10 & 14 & 14 & 1.507 & 1.507 & 1.507 \\
\hline & & 309 & 286 & 3 & 4 & 4 & 1.273 & 1.273 & 1.273 \\
\hline & & & 301 & 5 & 5 & 5 & 1.978 & 1.978 & 1.978 \\
\hline & & & 305 & 10 & 12 & 12 & 1.412 & 1.412 & 1.412 \\
\hline & & 313 & 285 & 6 & 6 & 6 & 2.787 & 2.787 & 2.787 \\
\hline & & & 286 & 2 & 3 & 4 & 0.596 & 0.596 & 0.596 \\
\hline & & & 301 & 3 & 3 & 3 & 1.399 & 1.399 & 1.399 \\
\hline & & & 308 & 10 & 14 & 14 & 1.507 & 1.507 & 1.507 \\
\hline & & 327 & 285 & 2 & 3 & 3 & 0.734 & 0.734 & 0.734 \\
\hline & & & 338 & 2 & 2 & 2 & 1.686 & 1.686 & 1.686 \\
\hline & & 334 & 338 & 15 & 21 & 21 & 2.045 & 2.045 & 2.045 \\
\hline & & & 343 & 3 & 4 & 4 & 0.447 & 0.447 & 0.447 \\
\hline & & 337 & 338 & 15 & 15 & 15 & 1.628 & 1.628 & 1.628 \\
\hline & & & 343 & 17 & 21 & 22 & 1.040 & 1.040 & 1.040 \\
\hline & & & 346 & & 2 & 2 & & 0.535 & 0.535 \\
\hline & & 338 & 327 & 2 & 2 & 2 & 1.686 & 1.686 & 1.686 \\
\hline & & & 334 & 15 & 21 & 21 & 2.045 & 2.045 & 2.045 \\
\hline & & & 337 & 15 & 15 & 15 & 1.628 & 1.628 & 1.628 \\
\hline & & 343 & 334 & 3 & 4 & 4 & 0.447 & 0.447 & 0.447 \\
\hline & & & 337 & 17 & 21 & 22 & 1.040 & 1.040 & 1.040 \\
\hline & & 346 & 337 & & 2 & 2 & & 0.535 & 0.535 \\
\hline & & 395 & 285 & 7 & 9 & 9 & 1.107 & 1.107 & 1.107 \\
\hline & & & 413 & 5 & 5 & 5 & 1.479 & 1.479 & 1.479 \\
\hline & & 397 & 285 & & 2 & 2 & & 0.671 & 0.671 \\
\hline & & & 413 & 2 & 5 & 5 & 1.106 & 1.106 & 1.106 \\
\hline
\end{tabular}


Table B.2 - Continued from previous page

\begin{tabular}{|c|c|c|c|c|c|c|c|c|c|}
\hline Name & $\begin{array}{l}\text { UniProt } \\
\text { ID }\end{array}$ & Residue 1 & Residue 2 & FDR $1 \%$ & FDR $3 \%$ & FDR 5\% & FDR1\% & F DR3\% & FDR5\% \\
\hline & & & 419 & & 2 & 2 & & 0.315 & 0.315 \\
\hline & & & 442 & 2 & 4 & 4 & 0.500 & 0.500 & 0.500 \\
\hline & & 403 & 279 & & & 2 & & & 0.334 \\
\hline & & & 413 & 11 & 25 & 25 & 1.112 & 1.112 & 1.112 \\
\hline & & & 419 & & 5 & 6 & & 0.399 & 0.399 \\
\hline & & & 442 & & 3 & 3 & & 0.302 & 0.302 \\
\hline & & 413 & 200 & 7 & 7 & 7 & 2.381 & 2.381 & 2.381 \\
\hline & & & 285 & 5 & 5 & 5 & 1.043 & 1.043 & 1.043 \\
\hline & & & 286 & & 2 & 2 & & 0.585 & 0.585 \\
\hline & & & 395 & 5 & 5 & 5 & 1.479 & 1.479 & 1.479 \\
\hline & & & 397 & 2 & 5 & 5 & 1.106 & 1.106 & 1.106 \\
\hline & & & 403 & 11 & 25 & 25 & 1.112 & 1.112 & 1.112 \\
\hline & & & 442 & 19 & 21 & 22 & 1.713 & 1.713 & 1.713 \\
\hline & & 419 & 200 & 7 & 9 & 9 & 3.023 & 3.023 & 3.023 \\
\hline & & & 397 & & 2 & 2 & & 0.315 & 0.315 \\
\hline & & & 403 & & 5 & 6 & & 0.399 & 0.399 \\
\hline & & & 442 & 4 & 12 & 12 & 0.676 & 0.676 & 0.676 \\
\hline & & 442 & 153 & 2 & 2 & 2 & 1.503 & 1.503 & 1.503 \\
\hline & & & 176 & & 2 & 2 & & 0.342 & 0.342 \\
\hline & & & 200 & 77 & 80 & 80 & 3.005 & 3.005 & 3.005 \\
\hline & & & 208 & 7 & 7 & 7 & 0.754 & 0.754 & 0.754 \\
\hline & & & 285 & 4 & 7 & 7 & 0.827 & 0.827 & 0.827 \\
\hline & & & 286 & 2 & 3 & 3 & 0.654 & 0.654 & 0.654 \\
\hline & & & 397 & 2 & 4 & 4 & 0.500 & 0.500 & 0.500 \\
\hline & & & 403 & & 3 & 3 & & 0.302 & 0.302 \\
\hline & & & 413 & 19 & 21 & 22 & 1.713 & 1.713 & 1.713 \\
\hline & & & 419 & 4 & 12 & 12 & 0.676 & 0.676 & 0.676 \\
\hline & & & 476 & 15 & 16 & 16 & 2.498 & 2.498 & 2.498 \\
\hline & & & 669 & 3 & 3 & 3 & 0.853 & 0.853 & 0.853 \\
\hline & & 476 & 176 & 3 & 3 & 3 & 1.759 & 1.759 & 1.759 \\
\hline & & & 200 & 30 & 30 & 30 & 3.627 & 3.627 & 3.627 \\
\hline & & & 285 & 3 & 3 & 3 & 1.250 & 1.250 & 1.250 \\
\hline & & & 286 & 2 & 2 & 2 & 0.892 & 0.892 & 0.892 \\
\hline & & & 442 & 15 & 16 & 16 & 2.498 & 2.498 & 2.498 \\
\hline & & & 669 & 2 & 2 & 2 & 2.341 & 2.341 & 2.341 \\
\hline & & 514 & 522 & 2 & 2 & 2 & 1.880 & 1.880 & 1.880 \\
\hline & & & 590 & 2 & 3 & 4 & 1.729 & 1.729 & 1.729 \\
\hline & & & 604 & 4 & 4 & 4 & 12.406 & 12.406 & 12.406 \\
\hline & & 522 & 514 & 2 & 2 & 2 & 1.880 & 1.880 & 1.880 \\
\hline & & 590 & 514 & 2 & 3 & 4 & 1.729 & 1.729 & 1.729 \\
\hline & & & 611 & & 2 & 2 & & 1.180 & 1.180 \\
\hline & & & 664 & 3 & 3 & 3 & 4.173 & 4.173 & 4.173 \\
\hline & & 604 & 514 & 4 & 4 & 4 & 12.406 & 12.406 & 12.406 \\
\hline & & & 611 & 108 & 110 & 110 & 5.223 & 5.223 & 5.223 \\
\hline & & 607 & 285 & 4 & 7 & 7 & 0.661 & 0.661 & 0.661 \\
\hline & & & 286 & 6 & 6 & 6 & 0.940 & 0.940 & 0.940 \\
\hline & & 611 & 590 & & 2 & 2 & & 1.180 & 1.180 \\
\hline & & & 604 & 108 & 110 & 110 & 5.223 & 5.223 & 5.223 \\
\hline & & 664 & 590 & 3 & 3 & 3 & 4.173 & 4.173 & 4.173 \\
\hline & & 669 & 442 & 3 & 3 & 3 & 0.853 & 0.853 & 0.853 \\
\hline & & & 476 & 2 & 2 & 2 & 2.341 & 2.341 & 2.341 \\
\hline & & 687 & 170 & 13 & 13 & 13 & 1.767 & 1.767 & 1.767 \\
\hline & & & 176 & 216 & 228 & 229 & 3.940 & 3.940 & 3.940 \\
\hline & & & 691 & 4 & 4 & 4 & 2.670 & 2.670 & 2.670 \\
\hline & & 691 & 687 & 4 & 4 & 4 & 2.670 & 2.670 & 2.670 \\
\hline & & & 739 & 5 & 5 & 5 & 3.119 & 3.119 & 3.119 \\
\hline & & 699 & 739 & 4 & 5 & 5 & 1.982 & 1.982 & 1.982 \\
\hline & & 710 & 720 & 4 & 4 & 4 & 1.041 & 1.041 & 1.041 \\
\hline & & & 721 & 20 & 22 & 22 & 2.275 & 2.275 & 2.275 \\
\hline & & & 723 & 11 & 12 & 12 & 1.493 & 1.493 & 1.493 \\
\hline & & 712 & 721 & 43 & 43 & 43 & 3.574 & 3.574 & 3.574 \\
\hline & & & 723 & 25 & 26 & 26 & 2.838 & 2.838 & 2.838 \\
\hline & & 720 & 710 & 4 & 4 & 4 & 1.041 & 1.041 & 1.041 \\
\hline & & & 723 & 15 & 15 & 15 & 2.025 & 2.025 & 2.025 \\
\hline & & & 730 & 4 & 4 & 4 & 1.994 & 1.994 & 1.994 \\
\hline & & & 735 & 18 & 19 & 19 & 1.431 & 1.431 & 1.431 \\
\hline & & 721 & 710 & 20 & 22 & 22 & 2.275 & 2.275 & 2.275 \\
\hline & & & 712 & 43 & 43 & 43 & 3.574 & 3.574 & 3.574 \\
\hline & & & 730 & 12 & 12 & 12 & 4.077 & 4.077 & 4.077 \\
\hline & & 723 & 710 & 11 & 12 & 12 & 1.493 & 1.493 & 1.493 \\
\hline & & & 712 & 25 & 26 & 26 & 2.838 & 2.838 & 2.838 \\
\hline & & & 720 & 15 & 15 & 15 & 2.025 & 2.025 & 2.025 \\
\hline & & & 730 & 81 & 81 & 81 & 4.256 & 4.256 & 4.256 \\
\hline & & 730 & 720 & 4 & 4 & 4 & 1.994 & 1.994 & 1.994 \\
\hline & & & 721 & 12 & 12 & 12 & 4.077 & 4.077 & 4.077 \\
\hline & & & 723 & 81 & 81 & 81 & 4.256 & 4.256 & 4.256 \\
\hline & & & 739 & 1 & 5 & 5 & 0.487 & 0.487 & 0.487 \\
\hline & & & 744 & 27 & 27 & 27 & 3.625 & 3.625 & 3.625 \\
\hline & & 735 & 720 & 18 & 19 & 19 & 1.431 & 1.431 & 1.431 \\
\hline & & 739 & 192 & 3 & 3 & 3 & 0.909 & 0.909 & 0.909 \\
\hline & & & 691 & 5 & 5 & 5 & 3.119 & 3.119 & 3.119 \\
\hline & & & 699 & 4 & 5 & 5 & 1.982 & 1.982 & 1.982 \\
\hline & & & 730 & 1 & 5 & 5 & 0.487 & 0.487 & 0.487 \\
\hline
\end{tabular}


Table B.2 - Continued from previous page

\begin{tabular}{|c|c|c|c|c|c|c|c|c|c|}
\hline Name & $\begin{array}{l}\text { UniProt } \\
\text { ID }\end{array}$ & Residue 1 & Residue 2 & FDR 1\% & FDR $3 \%$ & FDR 5\% & FDR1\% & FDR3\% & FDR5\% \\
\hline & & 744 & 730 & 27 & 27 & 27 & 3.625 & 3.625 & 3.625 \\
\hline \multirow[t]{30}{*}{ SRSF1 } & Q07955 & 30 & 138 & 43 & 43 & 43 & 2.567 & 2.567 & 2.567 \\
\hline & & & 165 & 4 & 4 & 4 & 3.719 & 3.719 & 3.719 \\
\hline & & & 174 & 15 & 15 & 15 & 3.320 & 3.320 & 3.320 \\
\hline & & & 179 & 6 & 6 & 6 & 2.591 & 2.591 & 2.591 \\
\hline & & 38 & 138 & 330 & 333 & 333 & 2.918 & 2.918 & 2.918 \\
\hline & & & 165 & 18 & 18 & 18 & 4.208 & 4.208 & 4.208 \\
\hline & & & 174 & 22 & 22 & 22 & 3.275 & 3.275 & 3.275 \\
\hline & & & 179 & 6 & 7 & 7 & 0.860 & 0.860 & 0.860 \\
\hline & & & 193 & 27 & 27 & 27 & 2.226 & 2.226 & 2.226 \\
\hline & & 48 & 138 & 326 & 390 & 390 & 1.592 & 1.592 & 1.592 \\
\hline & & & 165 & 55 & 60 & 61 & 1.994 & 1.994 & 1.994 \\
\hline & & & 174 & 61 & 68 & 69 & 2.234 & 2.234 & 2.234 \\
\hline & & & 179 & 21 & 30 & 30 & 1.116 & 1.116 & 1.116 \\
\hline & & & 193 & 17 & 19 & 19 & 1.433 & 1.433 & 1.433 \\
\hline & & 138 & 30 & 43 & 43 & 43 & 2.567 & 2.567 & 2.567 \\
\hline & & & 38 & 330 & 333 & 333 & 2.918 & 2.918 & 2.918 \\
\hline & & & 48 & 326 & 390 & 390 & 1.592 & 1.592 & 1.592 \\
\hline & & 165 & 30 & 4 & 4 & 4 & 3.719 & 3.719 & 3.719 \\
\hline & & & 38 & 18 & 18 & 18 & 4.208 & 4.208 & 4.208 \\
\hline & & & 48 & 55 & 60 & 61 & 1.994 & 1.994 & 1.994 \\
\hline & & & 174 & 39 & 40 & 40 & 3.200 & 3.200 & 3.200 \\
\hline & & 174 & 30 & 15 & 15 & 15 & 3.320 & 3.320 & 3.320 \\
\hline & & & 38 & 22 & 22 & 22 & 3.275 & 3.275 & 3.275 \\
\hline & & & 48 & 61 & 68 & 69 & 2.234 & 2.234 & 2.234 \\
\hline & & & 165 & 39 & 40 & 40 & 3.200 & 3.200 & 3.200 \\
\hline & & 179 & 30 & 6 & 6 & 6 & 2.591 & 2.591 & 2.591 \\
\hline & & & 38 & 6 & 7 & 7 & 0.860 & 0.860 & 0.860 \\
\hline & & & 48 & 21 & 30 & 30 & 1.116 & 1.116 & 1.116 \\
\hline & & 193 & 38 & 27 & 27 & 27 & 2.226 & 2.226 & 2.226 \\
\hline & & & 48 & 17 & 19 & 19 & 1.433 & 1.433 & 1.433 \\
\hline \multirow[t]{36}{*}{ SYF1 } & Q9HCS7 & 2 & 45 & 4 & 4 & 4 & 2.027 & 2.027 & 2.027 \\
\hline & & & 50 & 3 & 3 & 3 & 1.546 & 1.546 & 1.546 \\
\hline & & & 62 & 3 & 3 & 3 & 2.048 & 2.048 & 2.048 \\
\hline & & 45 & 2 & 4 & 4 & 4 & 2.027 & 2.027 & 2.027 \\
\hline & & 50 & 2 & 3 & 3 & 3 & 1.546 & 1.546 & 1.546 \\
\hline & & & 76 & 12 & 15 & 16 & 1.659 & 1.659 & 1.659 \\
\hline & & & 83 & 31 & 34 & 34 & 2.236 & 2.236 & 2.236 \\
\hline & & 62 & 2 & 3 & 3 & 3 & 2.048 & 2.048 & 2.048 \\
\hline & & 76 & 50 & 12 & 15 & 16 & 1.659 & 1.659 & 1.659 \\
\hline & & & 83 & 37 & 40 & 40 & 3.540 & 3.540 & 3.540 \\
\hline & & 83 & 50 & 31 & 34 & 34 & 2.236 & 2.236 & 2.236 \\
\hline & & & 76 & 37 & 40 & 40 & 3.540 & 3.540 & 3.540 \\
\hline & & 393 & 485 & 5 & 5 & 5 & 4.014 & 4.014 & 4.014 \\
\hline & & 420 & 458 & 26 & 27 & 27 & 3.136 & 3.136 & 3.136 \\
\hline & & 423 & 458 & 41 & 42 & 42 & 3.135 & 3.135 & 3.135 \\
\hline & & 458 & 420 & 26 & 27 & 27 & 3.136 & 3.136 & 3.136 \\
\hline & & & 423 & 41 & 42 & 42 & 3.135 & 3.135 & 3.135 \\
\hline & & 485 & 393 & 5 & 5 & 5 & 4.014 & 4.014 & 4.014 \\
\hline & & 549 & 590 & 5 & 6 & 6 & 2.881 & 2.881 & 2.881 \\
\hline & & 590 & 549 & 5 & 6 & 6 & 2.881 & 2.881 & 2.881 \\
\hline & & 708 & 721 & 106 & 107 & 107 & 4.241 & 4.241 & 4.241 \\
\hline & & & 747 & 6 & 6 & 6 & 1.744 & 1.744 & 1.744 \\
\hline & & & 770 & 22 & 22 & 22 & 3.485 & 3.485 & 3.485 \\
\hline & & & 794 & 57 & 64 & 64 & 4.822 & 4.822 & 4.822 \\
\hline & & & 853 & 3 & 3 & 3 & 1.379 & 1.379 & 1.379 \\
\hline & & 721 & 708 & 106 & 107 & 107 & 4.241 & 4.241 & 4.241 \\
\hline & & & 794 & 3 & 4 & 4 & 1.799 & 1.799 & 1.799 \\
\hline & & 747 & 708 & 6 & 6 & 6 & 1.744 & 1.744 & 1.744 \\
\hline & & & 794 & 2 & 2 & 2 & 1.013 & 1.013 & 1.013 \\
\hline & & 770 & 708 & 22 & 22 & 22 & 3.485 & 3.485 & 3.485 \\
\hline & & & 794 & 2 & 2 & 2 & 1.555 & 1.555 & 1.555 \\
\hline & & 794 & 708 & 57 & 64 & 64 & 4.822 & 4.822 & 4.822 \\
\hline & & & 721 & 3 & 4 & 4 & 1.799 & 1.799 & 1.799 \\
\hline & & & 747 & 2 & 2 & 2 & 1.013 & 1.013 & 1.013 \\
\hline & & & 770 & 2 & 2 & 2 & 1.555 & 1.555 & 1.555 \\
\hline & & 853 & 708 & 3 & 3 & 3 & 1.379 & 1.379 & 1.379 \\
\hline SYF3 & Q9BZJ0 & 175 & 180 & 3 & 4 & 4 & 1.230 & 1.230 & 1.230 \\
\hline & & & 182 & 10 & 11 & 11 & 1.950 & 1.950 & 1.950 \\
\hline & & 180 & 175 & 3 & 4 & 4 & 1.230 & 1.230 & 1.230 \\
\hline & & 182 & 175 & 10 & 11 & 11 & 1.950 & 1.950 & 1.950 \\
\hline & & 213 & 256 & 3 & 3 & 3 & 2.205 & 2.205 & 2.205 \\
\hline & & 224 & 256 & 16 & 17 & 17 & 3.349 & 3.349 & 3.349 \\
\hline & & 256 & 213 & 3 & 3 & 3 & 2.205 & 2.205 & 2.205 \\
\hline & & & 224 & 16 & 17 & 17 & 3.349 & 3.349 & 3.349 \\
\hline & & 288 & 315 & 2 & 2 & 2 & 1.175 & 1.175 & 1.175 \\
\hline & & 315 & 288 & 2 & 2 & 2 & 1.175 & 1.175 & 1.175 \\
\hline & & 357 & 388 & 122 & 124 & 124 & 3.196 & 3.196 & 3.196 \\
\hline & & 381 & 388 & 10 & 11 & 11 & 1.890 & 1.890 & 1.890 \\
\hline & & 388 & 357 & 122 & 124 & 124 & 3.196 & 3.196 & 3.196 \\
\hline & & & 381 & 10 & 11 & 11 & 1.890 & 1.890 & 1.890 \\
\hline & & 427 & 461 & 6 & 7 & 7 & 0.851 & 0.851 & 0.851 \\
\hline & & 437 & 445 & 32 & 34 & 34 & 3.452 & 3.452 & 3.452 \\
\hline
\end{tabular}


Table B.2 - Continued from previous page

\begin{tabular}{|c|c|c|c|c|c|c|c|c|c|}
\hline Name & $\begin{array}{l}\text { UniProt } \\
\text { ID }\end{array}$ & Residue 1 & Residue 2 & FDR $1 \%$ & FDR $3 \%$ & FDR 5\% & F $D R 1 \%$ & F DR3\% & FDR5\% \\
\hline & & 445 & 437 & 32 & 34 & 34 & 3.452 & 3.452 & 3.452 \\
\hline & & & 460 & 9 & 10 & 10 & 1.142 & 1.142 & 1.142 \\
\hline & & & 475 & 28 & 29 & 29 & 4.229 & 4.229 & 4.229 \\
\hline & & & 485 & 2 & 2 & 2 & 0.982 & 0.982 & 0.982 \\
\hline & & 460 & 445 & 9 & 10 & 10 & 1.142 & 1.142 & 1.142 \\
\hline & & & 475 & 62 & 64 & 64 & 4.822 & 4.822 & 4.822 \\
\hline & & 461 & 427 & 6 & 7 & 7 & 0.851 & 0.851 & 0.851 \\
\hline & & 475 & 445 & 28 & 29 & 29 & 4.229 & 4.229 & 4.229 \\
\hline & & & 460 & 62 & 64 & 64 & 4.822 & 4.822 & 4.822 \\
\hline & & 485 & 445 & 2 & 2 & 2 & 0.982 & 0.982 & 0.982 \\
\hline & & 527 & 531 & 2 & 2 & 2 & 0.736 & 0.736 & 0.736 \\
\hline & & 531 & 527 & 2 & 2 & 2 & 0.736 & 0.736 & 0.736 \\
\hline & & 568 & 602 & 21 & 23 & 23 & 2.060 & 2.060 & 2.060 \\
\hline & & 602 & 568 & 21 & 23 & 23 & 2.060 & 2.060 & 2.060 \\
\hline & & 730 & 740 & 19 & 20 & 20 & 1.210 & 1.210 & 1.210 \\
\hline & & & 823 & 2 & 2 & 2 & 1.616 & 1.616 & 1.616 \\
\hline & & & 827 & & 2 & 3 & & 0.237 & 0.237 \\
\hline & & 740 & 730 & 19 & 20 & 20 & 1.210 & 1.210 & 1.210 \\
\hline & & & 776 & 10 & 10 & 10 & 1.814 & 1.814 & 1.814 \\
\hline & & & 823 & 15 & 15 & 15 & 1.475 & 1.475 & 1.475 \\
\hline & & & 827 & 2 & 3 & 3 & 0.474 & 0.474 & 0.474 \\
\hline & & 776 & 740 & 10 & 10 & 10 & 1.814 & 1.814 & 1.814 \\
\hline & & & 832 & & 3 & 3 & & 0.190 & 0.190 \\
\hline & & 823 & 730 & 2 & 2 & 2 & 1.616 & 1.616 & 1.616 \\
\hline & & & 740 & 15 & 15 & 15 & 1.475 & 1.475 & 1.475 \\
\hline & & & 827 & 30 & 38 & 39 & 2.343 & 2.343 & 2.343 \\
\hline & & & 832 & 6 & 27 & 31 & 1.064 & 1.064 & 1.064 \\
\hline & & 826 & 832 & & 3 & 4 & & 0.166 & 0.166 \\
\hline & & 827 & 730 & & 2 & 3 & & 0.237 & 0.237 \\
\hline & & & 740 & 2 & 3 & 3 & 0.474 & 0.474 & 0.474 \\
\hline & & & 823 & 30 & 38 & 39 & 2.343 & 2.343 & 2.343 \\
\hline & & 832 & 776 & & 3 & 3 & & 0.190 & 0.190 \\
\hline & & & 823 & 6 & 27 & 31 & 1.064 & 1.064 & 1.064 \\
\hline & & & 826 & & 3 & 4 & & 0.166 & 0.166 \\
\hline TCERG1 & O14776 & 1 & 734 & & & 5 & & & 0.107 \\
\hline & & 146 & 161 & 10 & 10 & 10 & 2.214 & 2.214 & 2.214 \\
\hline & & & 444 & 4 & 4 & 4 & 1.816 & 1.816 & 1.816 \\
\hline & & 161 & 146 & 10 & 10 & 10 & 2.214 & 2.214 & 2.214 \\
\hline & & 444 & 146 & 4 & 4 & 4 & 1.816 & 1.816 & 1.816 \\
\hline & & & 459 & 5 & 5 & 5 & 1.907 & 1.907 & 1.907 \\
\hline & & 459 & 444 & 5 & 5 & 5 & 1.907 & 1.907 & 1.907 \\
\hline & & & 468 & 15 & 15 & 15 & 1.126 & 1.126 & 1.126 \\
\hline & & 464 & 468 & 5 & 6 & 6 & 0.804 & 0.804 & 0.804 \\
\hline & & & 478 & 3 & 3 & 3 & 2.232 & 2.232 & 2.232 \\
\hline & & 466 & 474 & 2 & 2 & 2 & 1.635 & 1.635 & 1.635 \\
\hline & & & 478 & 6 & 7 & 7 & 1.514 & 1.514 & 1.514 \\
\hline & & 468 & 459 & 15 & 15 & 15 & 1.126 & 1.126 & 1.126 \\
\hline & & & 464 & 5 & 6 & 6 & 0.804 & 0.804 & 0.804 \\
\hline & & & 474 & 15 & 15 & 15 & 1.662 & 1.662 & 1.662 \\
\hline & & & 478 & 20 & 28 & 29 & 4.811 & 4.811 & 4.811 \\
\hline & & 472 & 478 & 76 & 91 & 95 & 6.493 & 6.493 & 6.493 \\
\hline & & 474 & 466 & 2 & 2 & 2 & 1.635 & 1.635 & 1.635 \\
\hline & & & 468 & 15 & 15 & 15 & 1.662 & 1.662 & 1.662 \\
\hline & & 478 & 464 & 3 & 3 & 3 & 2.232 & 2.232 & 2.232 \\
\hline & & & 466 & 6 & 7 & 7 & 1.514 & 1.514 & 1.514 \\
\hline & & & 468 & 20 & 28 & 29 & 4.811 & 4.811 & 4.811 \\
\hline & & & 472 & 76 & 91 & 95 & 6.493 & 6.493 & 6.493 \\
\hline & & & 500 & & 7 & 8 & & 0.493 & 0.493 \\
\hline & & & 503 & & 2 & 2 & & 0.209 & 0.209 \\
\hline & & & 507 & & 2 & 2 & & 0.127 & 0.127 \\
\hline & & & 985 & 19 & 66 & 73 & 0.974 & 0.974 & 0.974 \\
\hline & & 495 & 503 & 284 & 387 & 395 & 14.529 & 14.529 & 14.529 \\
\hline & & & 507 & 7 & 17 & 18 & 1.273 & 1.273 & 1.273 \\
\hline & & & 516 & 6 & 16 & 16 & 2.580 & 2.580 & 2.580 \\
\hline & & & 729 & & & 2 & & & 0.169 \\
\hline & & & 734 & & & 2 & & & 0.163 \\
\hline & & 500 & 478 & & 7 & 8 & & 0.493 & 0.493 \\
\hline & & 503 & 478 & & 2 & 2 & & 0.209 & 0.209 \\
\hline & & & 495 & 284 & 387 & 395 & 14.529 & 14.529 & 14.529 \\
\hline & & 507 & 478 & & 2 & 2 & & 0.127 & 0.127 \\
\hline & & & 495 & 7 & 17 & 18 & 1.273 & 1.273 & 1.273 \\
\hline & & 516 & 495 & 6 & 16 & 16 & 2.580 & 2.580 & 2.580 \\
\hline & & & 522 & 6 & 6 & 6 & 1.743 & 1.743 & 1.743 \\
\hline & & & 585 & & 2 & 2 & & 1.099 & 1.099 \\
\hline & & 520 & 578 & 39 & 41 & 41 & 4.055 & 4.055 & 4.055 \\
\hline & & & 585 & 8 & 9 & 9 & 1.681 & 1.681 & 1.681 \\
\hline & & 522 & 516 & 6 & 6 & 6 & 1.743 & 1.743 & 1.743 \\
\hline & & & 578 & 5 & 5 & 5 & 3.265 & 3.265 & 3.265 \\
\hline & & & 585 & 5 & 5 & 5 & 1.524 & 1.524 & 1.524 \\
\hline & & & 724 & 2 & 2 & 2 & 0.709 & 0.709 & 0.709 \\
\hline & & 570 & 579 & 92 & 92 & 93 & 3.492 & 3.492 & 3.492 \\
\hline & & & 585 & 23 & 25 & 25 & 1.848 & 1.848 & 1.848 \\
\hline & & 578 & 520 & 39 & 41 & 41 & 4.055 & 4.055 & 4.055 \\
\hline
\end{tabular}


Table B.2 - Continued from previous page

\begin{tabular}{|c|c|c|c|c|c|c|c|c|c|}
\hline Name & $\begin{array}{l}\text { UniProt } \\
\text { ID }\end{array}$ & Residue 1 & Residue 2 & FDR $1 \%$ & FDR 3\% & FDR 5\% & FDR1\% & FDR3\% & FDR5\% \\
\hline & & & 522 & 5 & 5 & 5 & 3.265 & 3.265 & 3.265 \\
\hline & & & 585 & 34 & 35 & 35 & 1.798 & 1.798 & 1.798 \\
\hline & & 579 & 570 & 92 & 92 & 93 & 3.492 & 3.492 & 3.492 \\
\hline & & & 640 & & 4 & 7 & & 0.275 & 0.275 \\
\hline & & 585 & 516 & & 2 & 2 & & 1.099 & 1.099 \\
\hline & & & 520 & 8 & 9 & 9 & 1.681 & 1.681 & 1.681 \\
\hline & & & 522 & 5 & 5 & 5 & 1.524 & 1.524 & 1.524 \\
\hline & & & 570 & 23 & 25 & 25 & 1.848 & 1.848 & 1.848 \\
\hline & & & 578 & 34 & 35 & 35 & 1.798 & 1.798 & 1.798 \\
\hline & & & 599 & 4 & 4 & 4 & 1.354 & 1.354 & 1.354 \\
\hline & & 599 & 585 & 4 & 4 & 4 & 1.354 & 1.354 & 1.354 \\
\hline & & 626 & 634 & 2 & 2 & 2 & 0.977 & 0.977 & 0.977 \\
\hline & & 627 & 634 & 4 & 4 & 4 & 1.555 & 1.555 & 1.555 \\
\hline & & 634 & 626 & 2 & 2 & 2 & 0.977 & 0.977 & 0.977 \\
\hline & & & 627 & 4 & 4 & 4 & 1.555 & 1.555 & 1.555 \\
\hline & & & 649 & 2 & 2 & 2 & 0.682 & 0.682 & 0.682 \\
\hline & & & 700 & & 5 & 5 & & 0.645 & 0.645 \\
\hline & & 640 & 579 & & 4 & 7 & & 0.275 & 0.275 \\
\hline & & 649 & 634 & 2 & 2 & 2 & 0.682 & 0.682 & 0.682 \\
\hline & & 667 & 711 & 155 & 160 & 160 & 3.302 & 3.302 & 3.302 \\
\hline & & 700 & 634 & & 5 & 5 & & 0.645 & 0.645 \\
\hline & & & 703 & 103 & 103 & 103 & 4.908 & 4.908 & 4.908 \\
\hline & & & 711 & 5 & 5 & 5 & 0.737 & 0.737 & 0.737 \\
\hline & & 703 & 700 & 103 & 103 & 103 & 4.908 & 4.908 & 4.908 \\
\hline & & 711 & 667 & 155 & 160 & 160 & 3.302 & 3.302 & 3.302 \\
\hline & & & 700 & 5 & 5 & 5 & 0.737 & 0.737 & 0.737 \\
\hline & & 721 & 729 & 5 & 6 & 6 & 1.682 & 1.682 & 1.682 \\
\hline & & 722 & 729 & 11 & 17 & 18 & 1.207 & 1.207 & 1.207 \\
\hline & & & 740 & & & 2 & & & 0.113 \\
\hline & & & 761 & 1 & 1 & 1 & 0.863 & 0.863 & 0.863 \\
\hline & & & 796 & 1 & 1 & 1 & 0.388 & 0.388 & 0.388 \\
\hline & & 724 & 522 & 2 & 2 & 2 & 0.709 & 0.709 & 0.709 \\
\hline & & & 761 & 17 & 18 & 18 & 2.113 & 2.113 & 2.113 \\
\hline & & & 796 & & 8 & 9 & & 1.473 & 1.473 \\
\hline & & 729 & 495 & & & 2 & & & 0.169 \\
\hline & & & 721 & 5 & 6 & 6 & 1.682 & 1.682 & 1.682 \\
\hline & & & 722 & 11 & 17 & 18 & 1.207 & 1.207 & 1.207 \\
\hline & & & 765 & 9 & 10 & 10 & 0.899 & 0.899 & 0.899 \\
\hline & & & 767 & 75 & 91 & 92 & 2.446 & 2.446 & 2.446 \\
\hline & & 734 & 1 & & & 5 & & & 0.107 \\
\hline & & & 495 & & & 2 & & & 0.163 \\
\hline & & 740 & 722 & & & 2 & & & 0.113 \\
\hline & & & 753 & 104 & 107 & 107 & 3.540 & 3.540 & 3.540 \\
\hline & & & 756 & 23 & 25 & 25 & 2.935 & 2.935 & 2.935 \\
\hline & & & 783 & 5 & 5 & 5 & 2.262 & 2.262 & 2.262 \\
\hline & & & 785 & 20 & 20 & 20 & 2.421 & 2.421 & 2.421 \\
\hline & & 753 & 740 & 104 & 107 & 107 & 3.540 & 3.540 & 3.540 \\
\hline & & & 761 & 15 & 16 & 16 & 1.610 & 1.610 & 1.610 \\
\hline & & 756 & 740 & 23 & 25 & 25 & 2.935 & 2.935 & 2.935 \\
\hline & & & 761 & 60 & 61 & 61 & 4.649 & 4.649 & 4.649 \\
\hline & & & 1016 & & 2 & 2 & & 0.312 & 0.312 \\
\hline & & 761 & 722 & 1 & 1 & 1 & 0.863 & 0.863 & 0.863 \\
\hline & & & 724 & 17 & 18 & 18 & 2.113 & 2.113 & 2.113 \\
\hline & & & 753 & 15 & 16 & 16 & 1.610 & 1.610 & 1.610 \\
\hline & & & 756 & 60 & 61 & 61 & 4.649 & 4.649 & 4.649 \\
\hline & & 765 & 729 & 9 & 10 & 10 & 0.899 & 0.899 & 0.899 \\
\hline & & 767 & 729 & 75 & 91 & 92 & 2.446 & 2.446 & 2.446 \\
\hline & & & 895 & & & 2 & & & 0.130 \\
\hline & & 782 & 796 & 2 & 2 & 2 & 1.734 & 1.734 & 1.734 \\
\hline & & 783 & 740 & 5 & 5 & 5 & 2.262 & 2.262 & 2.262 \\
\hline & & & 796 & 7 & 7 & 7 & 1.694 & 1.694 & 1.694 \\
\hline & & 785 & 740 & 20 & 20 & 20 & 2.421 & 2.421 & 2.421 \\
\hline & & & 796 & 174 & 183 & 184 & 3.265 & 3.265 & 3.265 \\
\hline & & & 828 & 3 & 3 & 3 & 1.908 & 1.908 & 1.908 \\
\hline & & & 846 & 20 & 20 & 20 & 1.626 & 1.626 & 1.626 \\
\hline & & & 1016 & & 2 & 2 & & 0.519 & 0.519 \\
\hline & & 789 & 796 & 84 & 92 & 92 & 1.572 & 1.572 & 1.572 \\
\hline & & & 846 & 6 & 8 & 8 & 1.165 & 1.165 & 1.165 \\
\hline & & 796 & 722 & 1 & 1 & 1 & 0.388 & 0.388 & 0.388 \\
\hline & & & 724 & & 8 & 9 & & 1.473 & 1.473 \\
\hline & & & 782 & 2 & 2 & 2 & 1.734 & 1.734 & 1.734 \\
\hline & & & 783 & 7 & 7 & 7 & 1.694 & 1.694 & 1.694 \\
\hline & & & 785 & 174 & 183 & 184 & 3.265 & 3.265 & 3.265 \\
\hline & & & 789 & 84 & 92 & 92 & 1.572 & 1.572 & 1.572 \\
\hline & & & 841 & 2 & 2 & 2 & 1.526 & 1.526 & 1.526 \\
\hline & & & 846 & 88 & 89 & 89 & 2.672 & 2.672 & 2.672 \\
\hline & & & 849 & 92 & 93 & 93 & 5.860 & 5.860 & 5.860 \\
\hline & & & 855 & 3 & 3 & 3 & 2.033 & 2.033 & 2.033 \\
\hline & & 816 & 820 & 17 & 26 & 26 & 0.930 & 0.930 & 0.930 \\
\hline & & & 857 & & 2 & 2 & & 0.781 & 0.781 \\
\hline & & & 1016 & & 3 & 3 & & 0.319 & 0.319 \\
\hline & & 818 & 828 & 57 & 61 & 61 & 1.650 & 1.650 & 1.650 \\
\hline & & & 841 & 7 & 7 & 7 & 1.621 & 1.621 & 1.621 \\
\hline
\end{tabular}


Table B.2 - Continued from previous page

\begin{tabular}{|c|c|c|c|c|c|c|c|c|c|}
\hline Name & $\begin{array}{l}\text { UniProt } \\
\text { ID }\end{array}$ & Residue 1 & Residue 2 & FDR $1 \%$ & FDR $3 \%$ & FDR $5 \%$ & FDR $1 \%$ & FDR3\% & FDR5\% \\
\hline & & & 857 & 2 & 2 & 2 & 0.705 & 0.705 & 0.705 \\
\hline & & 820 & 816 & 17 & 26 & 26 & 0.930 & 0.930 & 0.930 \\
\hline & & & 828 & 4 & 4 & 4 & 1.240 & 1.240 & 1.240 \\
\hline & & & 855 & 6 & 7 & 7 & 0.481 & 0.481 & 0.481 \\
\hline & & & 857 & 5 & 8 & 8 & 1.027 & 1.027 & 1.027 \\
\hline & & 828 & 785 & 3 & 3 & 3 & 1.908 & 1.908 & 1.908 \\
\hline & & & 818 & 57 & 61 & 61 & 1.650 & 1.650 & 1.650 \\
\hline & & & 820 & 4 & 4 & 4 & 1.240 & 1.240 & 1.240 \\
\hline & & & 927 & 8 & 8 & 8 & 1.035 & 1.035 & 1.035 \\
\hline & & 841 & 796 & 2 & 2 & 2 & 1.526 & 1.526 & 1.526 \\
\hline & & & 818 & 7 & 7 & 7 & 1.621 & 1.621 & 1.621 \\
\hline & & & 849 & 24 & 24 & 24 & 2.873 & 2.873 & 2.873 \\
\hline & & 846 & 785 & 20 & 20 & 20 & 1.626 & 1.626 & 1.626 \\
\hline & & & 789 & 6 & 8 & 8 & 1.165 & 1.165 & 1.165 \\
\hline & & & 796 & 88 & 89 & 89 & 2.672 & 2.672 & 2.672 \\
\hline & & & 855 & 9 & 9 & 9 & 1.673 & 1.673 & 1.673 \\
\hline & & & 857 & 15 & 15 & 15 & 3.179 & 3.179 & 3.179 \\
\hline & & & 927 & 2 & 2 & 2 & 1.069 & 1.069 & 1.069 \\
\hline & & 849 & 796 & 92 & 93 & 93 & 5.860 & 5.860 & 5.860 \\
\hline & & & 841 & 24 & 24 & 24 & 2.873 & 2.873 & 2.873 \\
\hline & & 855 & 796 & 3 & 3 & 3 & 2.033 & 2.033 & 2.033 \\
\hline & & & 820 & 6 & 7 & 7 & 0.481 & 0.481 & 0.481 \\
\hline & & & 846 & 9 & 9 & 9 & 1.673 & 1.673 & 1.673 \\
\hline & & & 927 & 11 & 12 & 12 & 1.171 & 1.171 & 1.171 \\
\hline & & 857 & 816 & & 2 & 2 & & 0.781 & 0.781 \\
\hline & & & 818 & 2 & 2 & 2 & 0.705 & 0.705 & 0.705 \\
\hline & & & 820 & 5 & 8 & 8 & 1.027 & 1.027 & 1.027 \\
\hline & & & 846 & 15 & 15 & 15 & 3.179 & 3.179 & 3.179 \\
\hline & & 878 & 885 & 110 & 124 & 124 & 2.531 & 2.531 & 2.531 \\
\hline & & & 895 & 3 & 4 & 4 & 1.211 & 1.211 & 1.211 \\
\hline & & & 927 & 2 & 2 & 2 & 1.296 & 1.296 & 1.296 \\
\hline & & & 942 & 7 & 14 & 15 & 0.706 & 0.706 & 0.706 \\
\hline & & 885 & 878 & 110 & 124 & 124 & 2.531 & 2.531 & 2.531 \\
\hline & & & 895 & 10 & 14 & 14 & 1.182 & 1.182 & 1.182 \\
\hline & & & 942 & 13 & 15 & 15 & 1.297 & 1.297 & 1.297 \\
\hline & & 895 & 767 & & & 2 & & & 0.130 \\
\hline & & & 878 & 3 & 4 & 4 & 1.211 & 1.211 & 1.211 \\
\hline & & & 885 & 10 & 14 & 14 & 1.182 & 1.182 & 1.182 \\
\hline & & 904 & 944 & 19 & 19 & 19 & 3.138 & 3.138 & 3.138 \\
\hline & & & 955 & 20 & 20 & 20 & 2.726 & 2.726 & 2.726 \\
\hline & & 927 & 828 & 8 & 8 & 8 & 1.035 & 1.035 & 1.035 \\
\hline & & & 846 & 2 & 2 & 2 & 1.069 & 1.069 & 1.069 \\
\hline & & & 855 & 11 & 12 & 12 & 1.171 & 1.171 & 1.171 \\
\hline & & & 878 & 2 & 2 & 2 & 1.296 & 1.296 & 1.296 \\
\hline & & & 942 & 20 & 34 & 35 & 1.217 & 1.217 & 1.217 \\
\hline & & 942 & 878 & 7 & 14 & 15 & 0.706 & 0.706 & 0.706 \\
\hline & & & 885 & 13 & 15 & 15 & 1.297 & 1.297 & 1.297 \\
\hline & & & 927 & 20 & 34 & 35 & 1.217 & 1.217 & 1.217 \\
\hline & & 944 & 904 & 19 & 19 & 19 & 3.138 & 3.138 & 3.138 \\
\hline & & 955 & 904 & 20 & 20 & 20 & 2.726 & 2.726 & 2.726 \\
\hline & & 957 & 992 & 8 & 8 & 8 & 2.017 & 2.017 & 2.017 \\
\hline & & 978 & 982 & 22 & 29 & 29 & 1.323 & 1.323 & 1.323 \\
\hline & & & 985 & 36 & 44 & 44 & 1.231 & 1.231 & 1.231 \\
\hline & & 982 & 978 & 22 & 29 & 29 & 1.323 & 1.323 & 1.323 \\
\hline & & 985 & 478 & 19 & 66 & 73 & 0.974 & 0.974 & 0.974 \\
\hline & & & 978 & 36 & 44 & 44 & 1.231 & 1.231 & 1.231 \\
\hline & & 992 & 957 & 8 & 8 & 8 & 2.017 & 2.017 & 2.017 \\
\hline & & 1016 & 756 & & 2 & 2 & & 0.312 & 0.312 \\
\hline & & & 785 & & 2 & 2 & & 0.519 & 0.519 \\
\hline & & & 816 & & 3 & 3 & & 0.319 & 0.319 \\
\hline & & & 1024 & 203 & 211 & 211 & 2.913 & 2.913 & 2.913 \\
\hline & & & 1069 & 346 & 358 & 358 & 4.508 & 4.508 & 4.508 \\
\hline & & 1024 & 1016 & 203 & 211 & 211 & 2.913 & 2.913 & 2.913 \\
\hline & & 1027 & 1049 & 10 & 10 & 10 & 2.308 & 2.308 & 2.308 \\
\hline & & 1035 & 1049 & 2 & 2 & 2 & 0.918 & 0.918 & 0.918 \\
\hline & & 1049 & 1027 & 10 & 10 & 10 & 2.308 & 2.308 & 2.308 \\
\hline & & & 1035 & 2 & 2 & 2 & 0.918 & 0.918 & 0.918 \\
\hline & & 1069 & 1016 & 346 & 358 & 358 & 4.508 & 4.508 & 4.508 \\
\hline U2-A' & $\begin{array}{l}\text { P09661 } \\
\end{array}$ & 3 & 56 & 14 & 14 & 14 & 3.777 & 3.777 & 3.777 \\
\hline & & 56 & 3 & 14 & 14 & 14 & 3.777 & 3.777 & 3.777 \\
\hline & & & 179 & 17 & 19 & 19 & 3.533 & 3.533 & 3.533 \\
\hline & & 128 & 172 & 11 & 12 & 12 & 3.018 & 3.018 & 3.018 \\
\hline & & & 179 & 60 & 61 & 61 & 1.970 & 1.970 & 1.970 \\
\hline & & & 193 & 8 & 8 & 8 & 2.056 & 2.056 & 2.056 \\
\hline & & & 221 & 1 & 1 & 1 & 1.036 & 1.036 & 1.036 \\
\hline & & 129 & 160 & 58 & 58 & 58 & 2.972 & 2.972 & 2.972 \\
\hline & & & 163 & 15 & 15 & 15 & 5.315 & 5.315 & 5.315 \\
\hline & & & 172 & 90 & 99 & 99 & 2.816 & 2.816 & 2.816 \\
\hline & & & 179 & 188 & 189 & 190 & 4.380 & 4.380 & 4.380 \\
\hline & & & 191 & 70 & 73 & 73 & 3.528 & 3.528 & 3.528 \\
\hline & & & 192 & 12 & 12 & 12 & 2.323 & 2.323 & 2.323 \\
\hline & & & 193 & 53 & 54 & 54 & 2.962 & 2.962 & 2.962 \\
\hline & & & 205 & 53 & 54 & 54 & 2.256 & 2.256 & 2.256 \\
\hline
\end{tabular}


Table B.2 - Continued from previous page

\begin{tabular}{|c|c|c|c|c|c|c|c|c|c|}
\hline Name & $\begin{array}{l}\text { UniProt } \\
\text { ID }\end{array}$ & Residue 1 & Residue 2 & FDR $1 \%$ & FDR $3 \%$ & FDR $5 \%$ & F DR $1 \%$ & FDR3\% & FDR5\% \\
\hline & & & 221 & 80 & 81 & 81 & 3.263 & 3.263 & 3.263 \\
\hline & & 138 & 163 & 10 & 10 & 10 & 0.966 & 0.966 & 0.966 \\
\hline & & & 172 & 73 & 77 & 77 & 3.973 & 3.973 & 3.973 \\
\hline & & & 179 & 8 & 8 & 8 & 1.893 & 1.893 & 1.893 \\
\hline & & 160 & 129 & 58 & 58 & 58 & 2.972 & 2.972 & 2.972 \\
\hline & & & 172 & 12 & 13 & 13 & 1.262 & 1.262 & 1.262 \\
\hline & & & 179 & & 2 & 2 & & 0.872 & 0.872 \\
\hline & & & 221 & 4 & 5 & 5 & 1.496 & 1.496 & 1.496 \\
\hline & & 163 & 129 & 15 & 15 & 15 & 5.315 & 5.315 & 5.315 \\
\hline & & & 138 & 10 & 10 & 10 & 0.966 & 0.966 & 0.966 \\
\hline & & & 172 & 30 & 34 & 35 & 2.004 & 2.004 & 2.004 \\
\hline & & & 205 & & 2 & 2 & & 0.676 & 0.676 \\
\hline & & 172 & 128 & 11 & 12 & 12 & 3.018 & 3.018 & 3.018 \\
\hline & & & 129 & 90 & 99 & 99 & 2.816 & 2.816 & 2.816 \\
\hline & & & 138 & 73 & 77 & 77 & 3.973 & 3.973 & 3.973 \\
\hline & & & 160 & 12 & 13 & 13 & 1.262 & 1.262 & 1.262 \\
\hline & & & 163 & 30 & 34 & 35 & 2.004 & 2.004 & 2.004 \\
\hline & & & 179 & 430 & 443 & 445 & 5.234 & 5.234 & 5.234 \\
\hline & & & 191 & 62 & 67 & 67 & 2.368 & 2.368 & 2.368 \\
\hline & & & 192 & 6 & 6 & 6 & 1.674 & 1.674 & 1.674 \\
\hline & & & 193 & 45 & 45 & 45 & 3.941 & 3.941 & 3.941 \\
\hline & & & 205 & 32 & 32 & 32 & 2.055 & 2.055 & 2.055 \\
\hline & & & 221 & 55 & 55 & 55 & 4.443 & 4.443 & 4.443 \\
\hline & & 179 & 56 & 17 & 19 & 19 & 3.533 & 3.533 & 3.533 \\
\hline & & & 128 & 60 & 61 & 61 & 1.970 & 1.970 & 1.970 \\
\hline & & & 129 & 188 & 189 & 190 & 4.380 & 4.380 & 4.380 \\
\hline & & & 138 & 8 & 8 & 8 & 1.893 & 1.893 & 1.893 \\
\hline & & & 160 & & 2 & 2 & & 0.872 & 0.872 \\
\hline & & & 172 & 430 & 443 & 445 & 5.234 & 5.234 & 5.234 \\
\hline & & & 193 & 107 & 109 & 109 & 4.249 & 4.249 & 4.249 \\
\hline & & & 205 & 30 & 30 & 30 & 5.153 & 5.153 & 5.153 \\
\hline & & & 221 & 51 & 53 & 53 & 5.324 & 5.324 & 5.324 \\
\hline & & 191 & 129 & 70 & 73 & 73 & 3.528 & 3.528 & 3.528 \\
\hline & & & 172 & 62 & 67 & 67 & 2.368 & 2.368 & 2.368 \\
\hline & & & 193 & 72 & 73 & 73 & 3.849 & 3.849 & 3.849 \\
\hline & & & 205 & 74 & 78 & 78 & 1.932 & 1.932 & 1.932 \\
\hline & & & 221 & 23 & 26 & 26 & 1.553 & 1.553 & 1.553 \\
\hline & & 192 & 129 & 12 & 12 & 12 & 2.323 & 2.323 & 2.323 \\
\hline & & & 172 & 6 & 6 & 6 & 1.674 & 1.674 & 1.674 \\
\hline & & & 205 & 11 & 12 & 12 & 0.922 & 0.922 & 0.922 \\
\hline & & & 221 & 1 & 1 & 1 & 0.516 & 0.516 & 0.516 \\
\hline & & 193 & 128 & 8 & 8 & 8 & 2.056 & 2.056 & 2.056 \\
\hline & & & 129 & 53 & 54 & 54 & 2.962 & 2.962 & 2.962 \\
\hline & & & 172 & 45 & 45 & 45 & 3.941 & 3.941 & 3.941 \\
\hline & & & 179 & 107 & 109 & 109 & 4.249 & 4.249 & 4.249 \\
\hline & & & 191 & 72 & 73 & 73 & 3.849 & 3.849 & 3.849 \\
\hline & & & 205 & 2 & 4 & 4 & 0.833 & 0.833 & 0.833 \\
\hline & & & 221 & 47 & 47 & 47 & 4.631 & 4.631 & 4.631 \\
\hline & & 205 & 129 & 53 & 54 & 54 & 2.256 & 2.256 & 2.256 \\
\hline & & & 163 & & 2 & 2 & & 0.676 & 0.676 \\
\hline & & & 172 & 32 & 32 & 32 & 2.055 & 2.055 & 2.055 \\
\hline & & & 179 & 30 & 30 & 30 & 5.153 & 5.153 & 5.153 \\
\hline & & & 191 & 74 & 78 & 78 & 1.932 & 1.932 & 1.932 \\
\hline & & & 192 & 11 & 12 & 12 & 0.922 & 0.922 & 0.922 \\
\hline & & & 193 & 2 & 4 & 4 & 0.833 & 0.833 & 0.833 \\
\hline & & & 221 & 148 & 151 & 151 & 4.753 & 4.753 & 4.753 \\
\hline & & 221 & 128 & 1 & 1 & 1 & 1.036 & 1.036 & 1.036 \\
\hline & & & 129 & 80 & 81 & 81 & 3.263 & 3.263 & 3.263 \\
\hline & & & 160 & 4 & 5 & 5 & 1.496 & 1.496 & 1.496 \\
\hline & & & 172 & 55 & 55 & 55 & 4.443 & 4.443 & 4.443 \\
\hline & & & 179 & 51 & 53 & 53 & 5.324 & 5.324 & 5.324 \\
\hline & & & 191 & 23 & 26 & 26 & 1.553 & 1.553 & 1.553 \\
\hline & & & 192 & 1 & 1 & 1 & 0.516 & 0.516 & 0.516 \\
\hline & & & 193 & 47 & 47 & 47 & 4.631 & 4.631 & 4.631 \\
\hline & & & 205 & 148 & 151 & 151 & 4.753 & 4.753 & 4.753 \\
\hline \multirow[t]{18}{*}{ U2-B" } & $\mathrm{P} 08579$ & 1 & 111 & & 2 & 2 & & 0.206 & 0.206 \\
\hline & & 17 & 20 & & & 2 & & & 0.350 \\
\hline & & 20 & 17 & & & 2 & & & 0.350 \\
\hline & & 47 & 111 & 3 & 3 & 3 & 1.070 & 1.070 & 1.070 \\
\hline & & 57 & 93 & 72 & 75 & 75 & 3.367 & 3.367 & 3.367 \\
\hline & & & 101 & 73 & 78 & 78 & 1.924 & 1.924 & 1.924 \\
\hline & & 85 & 111 & 6 & 6 & 6 & 3.160 & 3.160 & 3.160 \\
\hline & & 93 & 57 & 72 & 75 & 75 & 3.367 & 3.367 & 3.367 \\
\hline & & & 101 & 44 & 48 & 48 & 3.933 & 3.933 & 3.933 \\
\hline & & & 103 & 14 & 15 & 15 & 1.880 & 1.880 & 1.880 \\
\hline & & 101 & 57 & 73 & 78 & 78 & 1.924 & 1.924 & 1.924 \\
\hline & & & 93 & 44 & 48 & 48 & 3.933 & 3.933 & 3.933 \\
\hline & & & 108 & 2 & 2 & 2 & 0.877 & 0.877 & 0.877 \\
\hline & & & 109 & & 2 & 2 & & 0.576 & 0.576 \\
\hline & & & 111 & 4 & 5 & 5 & 1.321 & 1.321 & 1.321 \\
\hline & & 103 & 93 & 14 & 15 & 15 & 1.880 & 1.880 & 1.880 \\
\hline & & & 109 & 2 & 2 & 2 & 0.848 & 0.848 & 0.848 \\
\hline & & & 111 & 23 & 23 & 23 & 2.990 & 2.990 & 2.990 \\
\hline
\end{tabular}




\begin{tabular}{|c|c|c|c|c|c|c|c|c|c|}
\hline Name & $\begin{array}{l}\text { UniProt } \\
\text { ID }\end{array}$ & Residue 1 & Residue 2 & FDR $1 \%$ & FDR $3 \%$ & FDR $5 \%$ & FDR1\% & FDR3\% & FDR5\% \\
\hline & & 108 & 101 & 2 & 2 & 2 & 0.877 & 0.877 & 0.877 \\
\hline & & 109 & 101 & & 2 & 2 & & 0.576 & 0.576 \\
\hline & & & 103 & 2 & 2 & 2 & 0.848 & 0.848 & 0.848 \\
\hline & & 111 & 1 & & 2 & 2 & & 0.206 & 0.206 \\
\hline & & & 47 & 3 & 3 & 3 & 1.070 & 1.070 & 1.070 \\
\hline & & & 85 & 6 & 6 & 6 & 3.160 & 3.160 & 3.160 \\
\hline & & & 101 & 4 & 5 & 5 & 1.321 & 1.321 & 1.321 \\
\hline & & & 103 & 23 & 23 & 23 & 2.990 & 2.990 & 2.990 \\
\hline \multirow[t]{44}{*}{ U5-40K } & Q96DI7 & 1 & 8 & 91 & 94 & 94 & 4.834 & 4.834 & 4.834 \\
\hline & & & 18 & 46 & 51 & 52 & 3.907 & 3.907 & 3.907 \\
\hline & & & 270 & 6 & 6 & 6 & 1.658 & 1.658 & 1.658 \\
\hline & & & 275 & 86 & 87 & 87 & 3.528 & 3.528 & 3.528 \\
\hline & & & 322 & 23 & 24 & 24 & 2.990 & 2.990 & 2.990 \\
\hline & & 6 & 8 & 137 & 140 & 142 & 2.844 & 2.844 & 2.844 \\
\hline & & & 18 & 36 & 48 & 51 & 1.750 & 1.750 & 1.750 \\
\hline & & & 270 & 9 & 11 & 11 & 0.931 & 0.931 & 0.931 \\
\hline & & & 275 & 62 & 71 & 71 & 2.777 & 2.777 & 2.777 \\
\hline & & & 322 & 9 & 14 & 15 & 1.663 & 1.663 & 1.663 \\
\hline & & 8 & 1 & 91 & 94 & 94 & 4.834 & 4.834 & 4.834 \\
\hline & & & 6 & 137 & 140 & 142 & 2.844 & 2.844 & 2.844 \\
\hline & & & 270 & 6 & 6 & 6 & 1.809 & 1.809 & 1.809 \\
\hline & & & 275 & 66 & 66 & 66 & 4.785 & 4.785 & 4.785 \\
\hline & & & 322 & 2 & 2 & 2 & 2.667 & 2.667 & 2.667 \\
\hline & & & 349 & 4 & 4 & 4 & 3.251 & 3.251 & 3.251 \\
\hline & & 18 & 1 & 46 & 51 & 52 & 3.907 & 3.907 & 3.907 \\
\hline & & & 6 & 36 & 48 & 51 & 1.750 & 1.750 & 1.750 \\
\hline & & & 270 & 8 & 9 & 10 & 0.674 & 0.674 & 0.674 \\
\hline & & & 275 & 126 & 135 & 136 & 4.576 & 4.576 & 4.576 \\
\hline & & & 322 & 45 & 46 & 46 & 15.244 & 15.244 & 15.244 \\
\hline & & & 349 & 8 & 12 & 12 & 2.838 & 2.838 & 2.838 \\
\hline & & 131 & 148 & 82 & 90 & 90 & 11.321 & 11.321 & 11.321 \\
\hline & & 148 & 131 & 82 & 90 & 90 & 11.321 & 11.321 & 11.321 \\
\hline & & & 184 & 74 & 74 & 74 & 14.767 & 14.767 & 14.767 \\
\hline & & 184 & 148 & 74 & 74 & 74 & 14.767 & 14.767 & 14.767 \\
\hline & & 226 & 270 & 119 & 133 & 133 & 2.234 & 2.234 & 2.234 \\
\hline & & 270 & 1 & 6 & 6 & 6 & 1.658 & 1.658 & 1.658 \\
\hline & & & 6 & 9 & 11 & 11 & 0.931 & 0.931 & 0.931 \\
\hline & & & 8 & 6 & 6 & 6 & 1.809 & 1.809 & 1.809 \\
\hline & & & 18 & 8 & 9 & 10 & 0.674 & 0.674 & 0.674 \\
\hline & & & 226 & 119 & 133 & 133 & 2.234 & 2.234 & 2.234 \\
\hline & & & 275 & 14 & 21 & 21 & 1.905 & 1.905 & 1.905 \\
\hline & & 275 & 1 & 86 & 87 & 87 & 3.528 & 3.528 & 3.528 \\
\hline & & & 6 & 62 & 71 & 71 & 2.777 & 2.777 & 2.777 \\
\hline & & & 8 & 66 & 66 & 66 & 4.785 & 4.785 & 4.785 \\
\hline & & & 18 & 126 & 135 & 136 & 4.576 & 4.576 & 4.576 \\
\hline & & & 270 & 14 & 21 & 21 & 1.905 & 1.905 & 1.905 \\
\hline & & 322 & 1 & 23 & 24 & 24 & 2.990 & 2.990 & 2.990 \\
\hline & & & 6 & 9 & 14 & 15 & 1.663 & 1.663 & 1.663 \\
\hline & & & 8 & 2 & 2 & 2 & 2.667 & 2.667 & 2.667 \\
\hline & & & 18 & 45 & 46 & 46 & 15.244 & 15.244 & 15.244 \\
\hline & & 349 & 8 & 4 & 4 & 4 & 3.251 & 3.251 & 3.251 \\
\hline & & & 18 & 8 & 12 & 12 & 2.838 & 2.838 & 2.838 \\
\hline \multirow[t]{31}{*}{ WBP11 } & Q9Y2W2 & 46 & 98 & 33 & 33 & 33 & 1.999 & 1.999 & 1.999 \\
\hline & & 48 & 59 & & 2 & 2 & & 0.937 & 0.937 \\
\hline & & & 81 & 5 & 5 & 5 & 0.958 & 0.958 & 0.958 \\
\hline & & 51 & 109 & 66 & 66 & 66 & 3.015 & 3.015 & 3.015 \\
\hline & & & 117 & 6 & 6 & 6 & 0.924 & 0.924 & 0.924 \\
\hline & & 59 & 48 & & 2 & 2 & & 0.937 & 0.937 \\
\hline & & & 81 & 2 & 2 & 2 & 0.966 & 0.966 & 0.966 \\
\hline & & & 117 & 2 & 2 & 2 & 2.937 & 2.937 & 2.937 \\
\hline & & 81 & 48 & 5 & 5 & 5 & 0.958 & 0.958 & 0.958 \\
\hline & & & 59 & 2 & 2 & 2 & 0.966 & 0.966 & 0.966 \\
\hline & & 98 & 46 & 33 & 33 & 33 & 1.999 & 1.999 & 1.999 \\
\hline & & 105 & 109 & 175 & 180 & 180 & 4.827 & 4.827 & 4.827 \\
\hline & & & 599 & & 2 & 2 & & 0.281 & 0.281 \\
\hline & & 109 & 51 & 66 & 66 & 66 & 3.015 & 3.015 & 3.015 \\
\hline & & & 105 & 175 & 180 & 180 & 4.827 & 4.827 & 4.827 \\
\hline & & & 372 & & 2 & 2 & & 0.213 & 0.213 \\
\hline & & 117 & 51 & 6 & 6 & 6 & 0.924 & 0.924 & 0.924 \\
\hline & & & 59 & 2 & 2 & 2 & 2.937 & 2.937 & 2.937 \\
\hline & & 168 & 218 & 7 & 7 & 7 & 3.202 & 3.202 & 3.202 \\
\hline & & 218 & 168 & 7 & 7 & 7 & 3.202 & 3.202 & 3.202 \\
\hline & & 302 & 316 & 12 & 19 & 19 & 1.328 & 1.328 & 1.328 \\
\hline & & 303 & 316 & 27 & 28 & 28 & 3.472 & 3.472 & 3.472 \\
\hline & & & 322 & & 3 & 4 & & 1.111 & 1.111 \\
\hline & & 316 & 302 & 12 & 19 & 19 & 1.328 & 1.328 & 1.328 \\
\hline & & & 303 & 27 & 28 & 28 & 3.472 & 3.472 & 3.472 \\
\hline & & & 322 & 24 & 27 & 27 & 2.292 & 2.292 & 2.292 \\
\hline & & & 325 & 14 & 14 & 14 & 1.621 & 1.621 & 1.621 \\
\hline & & 322 & 303 & & 3 & 4 & & 1.111 & 1.111 \\
\hline & & & 316 & 24 & 27 & 27 & 2.292 & 2.292 & 2.292 \\
\hline & & 325 & 316 & 14 & 14 & 14 & 1.621 & 1.621 & 1.621 \\
\hline & & 372 & 109 & & 2 & 2 & & 0.213 & 0.213 \\
\hline
\end{tabular}


Table B.2 - Continued from previous page

\begin{tabular}{|c|c|c|c|c|c|c|c|c|c|}
\hline Name & $\begin{array}{l}\text { UniProt } \\
\text { ID }\end{array}$ & Residue 1 & Residue 2 & FDR $1 \%$ & FDR $3 \%$ & FDR 5\% & FDR $1 \%$ & FDR3\% & FDR5\% \\
\hline & & 556 & 565 & 43 & 45 & 45 & 2.552 & 2.552 & 2.552 \\
\hline & & & 572 & 16 & 17 & 17 & 2.536 & 2.536 & 2.536 \\
\hline & & & 590 & 2 & 2 & 2 & 0.917 & 0.917 & 0.917 \\
\hline & & 557 & 590 & 9 & 9 & 9 & 2.093 & 2.093 & 2.093 \\
\hline & & & 614 & 2 & 2 & 2 & 1.442 & 1.442 & 1.442 \\
\hline & & 565 & 556 & 43 & 45 & 45 & 2.552 & 2.552 & 2.552 \\
\hline & & & 590 & 11 & 13 & 13 & 2.100 & 2.100 & 2.100 \\
\hline & & & 614 & 4 & 4 & 4 & 2.175 & 2.175 & 2.175 \\
\hline & & 572 & 556 & 16 & 17 & 17 & 2.536 & 2.536 & 2.536 \\
\hline & & & 590 & 42 & 48 & 48 & 2.624 & 2.624 & 2.624 \\
\hline & & & 599 & 4 & 4 & 4 & 2.865 & 2.865 & 2.865 \\
\hline & & & 610 & 4 & 4 & 4 & 2.320 & 2.320 & 2.320 \\
\hline & & & 614 & 9 & 9 & 9 & 2.576 & 2.576 & 2.576 \\
\hline & & 590 & 556 & 2 & 2 & 2 & 0.917 & 0.917 & 0.917 \\
\hline & & & 557 & 9 & 9 & 9 & 2.093 & 2.093 & 2.093 \\
\hline & & & 565 & 11 & 13 & 13 & 2.100 & 2.100 & 2.100 \\
\hline & & & 572 & 42 & 48 & 48 & 2.624 & 2.624 & 2.624 \\
\hline & & & 599 & 95 & 95 & 95 & 4.091 & 4.091 & 4.091 \\
\hline & & & 610 & 98 & 104 & 104 & 2.992 & 2.992 & 2.992 \\
\hline & & & 614 & 36 & 36 & 36 & 2.399 & 2.399 & 2.399 \\
\hline & & 599 & 105 & & 2 & 2 & & 0.281 & 0.281 \\
\hline & & & 572 & 4 & 4 & 4 & 2.865 & 2.865 & 2.865 \\
\hline & & & 590 & 95 & 95 & 95 & 4.091 & 4.091 & 4.091 \\
\hline & & & 614 & 48 & 49 & 49 & 3.666 & 3.666 & 3.666 \\
\hline & & 610 & 572 & 4 & 4 & 4 & 2.320 & 2.320 & 2.320 \\
\hline & & & 590 & 98 & 104 & 104 & 2.992 & 2.992 & 2.992 \\
\hline & & & 626 & 11 & 11 & 11 & 2.141 & 2.141 & 2.141 \\
\hline & & 614 & 557 & 2 & 2 & 2 & 1.442 & 1.442 & 1.442 \\
\hline & & & 565 & 4 & 4 & 4 & 2.175 & 2.175 & 2.175 \\
\hline & & & 572 & 9 & 9 & 9 & 2.576 & 2.576 & 2.576 \\
\hline & & & 590 & 36 & 36 & 36 & 2.399 & 2.399 & 2.399 \\
\hline & & & 599 & 48 & 49 & 49 & 3.666 & 3.666 & 3.666 \\
\hline & & & 635 & 3 & 3 & 3 & 1.626 & 1.626 & 1.626 \\
\hline & & 626 & 610 & 11 & 11 & 11 & 2.141 & 2.141 & 2.141 \\
\hline & & 635 & 614 & 3 & 3 & 3 & 1.626 & 1.626 & 1.626 \\
\hline \multirow[t]{8}{*}{ YBOX1 } & P67809 & 26 & 53 & 13 & 20 & 22 & 1.686 & 1.686 & 1.686 \\
\hline & & & 58 & & 2 & 2 & & 0.896 & 0.896 \\
\hline & & & 301 & & 4 & 4 & & 1.629 & 1.629 \\
\hline & & 53 & 26 & 13 & 20 & 22 & 1.686 & 1.686 & 1.686 \\
\hline & & 58 & 26 & & 2 & 2 & & 0.896 & 0.896 \\
\hline & & 296 & 304 & 25 & 30 & 31 & 1.220 & 1.220 & 1.220 \\
\hline & & 301 & 26 & & 4 & 4 & & 1.629 & 1.629 \\
\hline & & 304 & 296 & 25 & 30 & 31 & 1.220 & 1.220 & 1.220 \\
\hline \multirow[t]{40}{*}{ ZC3H18 } & Q86VM9 & 149 & 168 & 21 & 22 & 22 & 16.541 & 16.541 & 16.541 \\
\hline & & & 177 & 7 & 8 & 8 & 6.115 & 6.115 & 6.115 \\
\hline & & 158 & 168 & 113 & 123 & 124 & 11.070 & 11.070 & 11.070 \\
\hline & & & 177 & 5 & 5 & 5 & 2.437 & 2.437 & 2.437 \\
\hline & & & 184 & 2 & 3 & 3 & 0.537 & 0.537 & 0.537 \\
\hline & & & 211 & & 2 & 13 & & 0.234 & 0.234 \\
\hline & & & 500 & & 2 & 3 & & 0.153 & 0.153 \\
\hline & & 168 & 149 & 21 & 22 & 22 & 16.541 & 16.541 & 16.541 \\
\hline & & & 158 & 113 & 123 & 124 & 11.070 & 11.070 & 11.070 \\
\hline & & & 184 & 21 & 21 & 21 & 2.761 & 2.761 & 2.761 \\
\hline & & & 188 & 13 & 13 & 13 & 2.775 & 2.775 & 2.775 \\
\hline & & 177 & 149 & 7 & 8 & 8 & 6.115 & 6.115 & 6.115 \\
\hline & & & 158 & 5 & 5 & 5 & 2.437 & 2.437 & 2.437 \\
\hline & & & 187 & 3 & 3 & 3 & 2.201 & 2.201 & 2.201 \\
\hline & & & 188 & 42 & 51 & 52 & 3.867 & 3.867 & 3.867 \\
\hline & & 184 & 158 & 2 & 3 & 3 & 0.537 & 0.537 & 0.537 \\
\hline & & & 168 & 21 & 21 & 21 & 2.761 & 2.761 & 2.761 \\
\hline & & & 188 & 180 & 212 & 219 & 5.190 & 5.190 & 5.190 \\
\hline & & 186 & 188 & 47 & 53 & 55 & 6.102 & 6.102 & 6.102 \\
\hline & & 187 & 177 & 3 & 3 & 3 & 2.201 & 2.201 & 2.201 \\
\hline & & 188 & 168 & 13 & 13 & 13 & 2.775 & 2.775 & 2.775 \\
\hline & & & 177 & 42 & 51 & 52 & 3.867 & 3.867 & 3.867 \\
\hline & & & 184 & 180 & 212 & 219 & 5.190 & 5.190 & 5.190 \\
\hline & & & 186 & 47 & 53 & 55 & 6.102 & 6.102 & 6.102 \\
\hline & & 211 & 158 & & 2 & 13 & & 0.234 & 0.234 \\
\hline & & & 217 & & 16 & 17 & & 0.749 & 0.749 \\
\hline & & 217 & 211 & & 16 & 17 & & 0.749 & 0.749 \\
\hline & & 309 & 314 & 26 & 27 & 27 & 2.917 & 2.917 & 2.917 \\
\hline & & & 319 & 9 & 9 & 9 & 2.449 & 2.449 & 2.449 \\
\hline & & 314 & 309 & 26 & 27 & 27 & 2.917 & 2.917 & 2.917 \\
\hline & & & 319 & 26 & 26 & 26 & 2.899 & 2.899 & 2.899 \\
\hline & & & 328 & 1 & 1 & 1 & 0.498 & 0.498 & 0.498 \\
\hline & & 319 & 309 & 9 & 9 & 9 & 2.449 & 2.449 & 2.449 \\
\hline & & & 314 & 26 & 26 & 26 & 2.899 & 2.899 & 2.899 \\
\hline & & & 343 & & 4 & 4 & & 0.392 & 0.392 \\
\hline & & 328 & 314 & 1 & 1 & 1 & 0.498 & 0.498 & 0.498 \\
\hline & & 343 & 319 & & 4 & 4 & & 0.392 & 0.392 \\
\hline & & & 838 & & 3 & 3 & & 0.175 & 0.175 \\
\hline & & 450 & 510 & 2 & 2 & 2 & 1.017 & 1.017 & 1.017 \\
\hline & & 463 & 499 & 2 & 4 & 4 & 0.550 & 0.550 & 0.550 \\
\hline
\end{tabular}


Table B.2 - Continued from previous page

\begin{tabular}{|c|c|c|c|c|c|c|c|c|c|}
\hline Name & $\begin{array}{l}\text { UniProt } \\
\text { ID }\end{array}$ & Residue 1 & Residue 2 & FDR $1 \%$ & FDR $3 \%$ & FDR $5 \%$ & FDR $1 \%$ & FDR3\% & FDR5\% \\
\hline & & 480 & 622 & 11 & 11 & 11 & 1.434 & 1.434 & 1.434 \\
\hline & & 484 & 499 & & 2 & 2 & & 0.338 & 0.338 \\
\hline & & & 500 & & 2 & 2 & & 0.184 & 0.184 \\
\hline & & 499 & 463 & 2 & 4 & 4 & 0.550 & 0.550 & 0.550 \\
\hline & & & 484 & & 2 & 2 & & 0.338 & 0.338 \\
\hline & & & 510 & 3 & 4 & 4 & 0.941 & 0.941 & 0.941 \\
\hline & & & 516 & 4 & 5 & 5 & 0.841 & 0.841 & 0.841 \\
\hline & & & 622 & & 3 & 3 & & 0.203 & 0.203 \\
\hline & & 500 & 158 & & 2 & 3 & & 0.153 & 0.153 \\
\hline & & & 484 & & 2 & 2 & & 0.184 & 0.184 \\
\hline & & & 651 & 2 & 2 & 2 & 1.225 & 1.225 & 1.225 \\
\hline & & 510 & 450 & 2 & 2 & 2 & 1.017 & 1.017 & 1.017 \\
\hline & & & 499 & 3 & 4 & 4 & 0.941 & 0.941 & 0.941 \\
\hline & & & 516 & 42 & 46 & 46 & 1.636 & 1.636 & 1.636 \\
\hline & & 516 & 499 & 4 & 5 & 5 & 0.841 & 0.841 & 0.841 \\
\hline & & & 510 & 42 & 46 & 46 & 1.636 & 1.636 & 1.636 \\
\hline & & 622 & 480 & 11 & 11 & 11 & 1.434 & 1.434 & 1.434 \\
\hline & & & 499 & & 3 & 3 & & 0.203 & 0.203 \\
\hline & & & 635 & 25 & 26 & 26 & 2.308 & 2.308 & 2.308 \\
\hline & & & 638 & 67 & 68 & 68 & 3.345 & 3.345 & 3.345 \\
\hline & & & 639 & 80 & 80 & 80 & 3.614 & 3.614 & 3.614 \\
\hline & & & 651 & 62 & 64 & 64 & 2.503 & 2.503 & 2.503 \\
\hline & & & 661 & 18 & 18 & 18 & 1.188 & 1.188 & 1.188 \\
\hline & & & 827 & 4 & 4 & 4 & 0.731 & 0.731 & 0.731 \\
\hline & & 631 & 635 & 24 & 42 & 46 & 0.839 & 0.839 & 0.839 \\
\hline & & & 638 & 109 & 115 & 115 & 3.333 & 3.333 & 3.333 \\
\hline & & & 639 & 154 & 158 & 161 & 3.956 & 3.956 & 3.956 \\
\hline & & & 651 & 35 & 50 & 53 & 1.517 & 1.517 & 1.517 \\
\hline & & & 661 & 6 & 7 & 7 & 1.230 & 1.230 & 1.230 \\
\hline & & 635 & 622 & 25 & 26 & 26 & 2.308 & 2.308 & 2.308 \\
\hline & & & 631 & 24 & 42 & 46 & 0.839 & 0.839 & 0.839 \\
\hline & & & 639 & 12 & 14 & 14 & 1.925 & 1.925 & 1.925 \\
\hline & & & 651 & 23 & 37 & 38 & 0.761 & 0.761 & 0.761 \\
\hline & & & 661 & 8 & 11 & 11 & 0.701 & 0.701 & 0.701 \\
\hline & & 638 & 622 & 67 & 68 & 68 & 3.345 & 3.345 & 3.345 \\
\hline & & & 631 & 109 & 115 & 115 & 3.333 & 3.333 & 3.333 \\
\hline & & & 661 & 38 & 38 & 38 & 1.975 & 1.975 & 1.975 \\
\hline & & & 880 & & 2 & 2 & & 0.364 & 0.364 \\
\hline & & & 921 & 2 & 2 & 2 & 1.120 & 1.120 & 1.120 \\
\hline & & 639 & 622 & 80 & 80 & 80 & 3.614 & 3.614 & 3.614 \\
\hline & & & 631 & 154 & 158 & 161 & 3.956 & 3.956 & 3.956 \\
\hline & & & 635 & 12 & 14 & 14 & 1.925 & 1.925 & 1.925 \\
\hline & & & 661 & 24 & 29 & 29 & 1.549 & 1.549 & 1.549 \\
\hline & & 651 & 500 & 2 & 2 & 2 & 1.225 & 1.225 & 1.225 \\
\hline & & & 622 & 62 & 64 & 64 & 2.503 & 2.503 & 2.503 \\
\hline & & & 631 & 35 & 50 & 53 & 1.517 & 1.517 & 1.517 \\
\hline & & & 635 & 23 & 37 & 38 & 0.761 & 0.761 & 0.761 \\
\hline & & & 780 & 9 & 9 & 9 & 0.732 & 0.732 & 0.732 \\
\hline & & & 827 & & 6 & 6 & & 0.246 & 0.246 \\
\hline & & & 880 & 9 & 9 & 9 & 1.608 & 1.608 & 1.608 \\
\hline & & & 901 & 7 & 7 & 7 & 0.926 & 0.926 & 0.926 \\
\hline & & & 908 & 4 & 6 & 6 & 1.105 & 1.105 & 1.105 \\
\hline & & & 918 & 2 & 2 & 2 & 0.762 & 0.762 & 0.762 \\
\hline & & & 921 & 2 & 3 & 3 & 0.717 & 0.717 & 0.717 \\
\hline & & & 948 & & 4 & 4 & & 0.614 & 0.614 \\
\hline & & 661 & 622 & 18 & 18 & 18 & 1.188 & 1.188 & 1.188 \\
\hline & & & 631 & 6 & 7 & 7 & 1.230 & 1.230 & 1.230 \\
\hline & & & 635 & 8 & 11 & 11 & 0.701 & 0.701 & 0.701 \\
\hline & & & 638 & 38 & 38 & 38 & 1.975 & 1.975 & 1.975 \\
\hline & & & 639 & 24 & 29 & 29 & 1.549 & 1.549 & 1.549 \\
\hline & & & 671 & 2 & 6 & 7 & 0.558 & 0.558 & 0.558 \\
\hline & & & 880 & 2 & 2 & 2 & 0.602 & 0.602 & 0.602 \\
\hline & & 671 & 661 & 2 & 6 & 7 & 0.558 & 0.558 & 0.558 \\
\hline & & 758 & 761 & 4 & 5 & 5 & 1.375 & 1.375 & 1.375 \\
\hline & & 761 & 758 & 4 & 5 & 5 & 1.375 & 1.375 & 1.375 \\
\hline & & 766 & 780 & 5 & 12 & 12 & 0.966 & 0.966 & 0.966 \\
\hline & & 769 & 780 & 11 & 13 & 13 & 2.064 & 2.064 & 2.064 \\
\hline & & 780 & 651 & 9 & 9 & 9 & 0.732 & 0.732 & 0.732 \\
\hline & & & 766 & 5 & 12 & 12 & 0.966 & 0.966 & 0.966 \\
\hline & & & 769 & 11 & 13 & 13 & 2.064 & 2.064 & 2.064 \\
\hline & & & 814 & 14 & 15 & 15 & 1.278 & 1.278 & 1.278 \\
\hline & & & 817 & 9 & 12 & 12 & 1.649 & 1.649 & 1.649 \\
\hline & & 783 & 814 & 15 & 19 & 19 & 1.225 & 1.225 & 1.225 \\
\hline & & & 817 & 2 & 2 & 2 & 1.391 & 1.391 & 1.391 \\
\hline & & & 823 & 2 & 3 & 3 & 0.684 & $\begin{array}{l}0.684 \\
\end{array}$ & 0.684 \\
\hline & & & 827 & & 2 & 2 & & 0.707 & 0.707 \\
\hline & & & 880 & 2 & 2 & 2 & 0.794 & 0.794 & 0.794 \\
\hline & & 789 & 817 & 32 & 32 & 32 & 2.229 & 2.229 & 2.229 \\
\hline & & & 823 & 10 & 11 & 11 & 0.990 & 0.990 & 0.990 \\
\hline & & & 827 & 10 & 11 & 11 & 0.985 & 0.985 & 0.985 \\
\hline & & & 880 & 2 & 2 & 2 & 0.894 & 0.894 & 0.894 \\
\hline & & 814 & 780 & 14 & 15 & 15 & 1.278 & 1.278 & 1.278 \\
\hline & & & 783 & 15 & 19 & 19 & 1.225 & 1.225 & 1.225 \\
\hline
\end{tabular}


Table B.2 - Continued from previous page

\begin{tabular}{|c|c|c|c|c|c|c|c|c|c|}
\hline Name & $\begin{array}{l}\text { UniProt } \\
\text { ID }\end{array}$ & Residue 1 & Residue 2 & FDR $1 \%$ & FDR $3 \%$ & FDR $5 \%$ & FDR1\% & FDR3\% & FDR5\% \\
\hline & & & 823 & 16 & 20 & 20 & 0.894 & 0.894 & 0.894 \\
\hline & & & 827 & 26 & 33 & 34 & 1.388 & 1.388 & 1.388 \\
\hline & & & 861 & 2 & 3 & 4 & 1.111 & 1.111 & 1.111 \\
\hline & & & 880 & 5 & 5 & 5 & 1.287 & 1.287 & 1.287 \\
\hline & & 817 & 780 & 9 & 12 & 12 & 1.649 & 1.649 & 1.649 \\
\hline & & & 783 & 2 & 2 & 2 & 1.391 & 1.391 & 1.391 \\
\hline & & & 789 & 32 & 32 & 32 & 2.229 & 2.229 & 2.229 \\
\hline & & & 827 & 72 & 78 & 80 & 2.225 & 2.225 & 2.225 \\
\hline & & & 861 & 3 & 3 & 3 & 1.821 & 1.821 & 1.821 \\
\hline & & 823 & 783 & 2 & 3 & 3 & 0.684 & 0.684 & 0.684 \\
\hline & & & 789 & 10 & 11 & 11 & 0.990 & 0.990 & 0.990 \\
\hline & & & 814 & 16 & 20 & 20 & 0.894 & 0.894 & 0.894 \\
\hline & & & 861 & 3 & 3 & 3 & 2.393 & 2.393 & 2.393 \\
\hline & & 827 & 622 & 4 & 4 & 4 & 0.731 & 0.731 & 0.731 \\
\hline & & & 651 & & 6 & 6 & & 0.246 & 0.246 \\
\hline & & & 783 & & 2 & 2 & & 0.707 & 0.707 \\
\hline & & & 789 & 10 & 11 & 11 & 0.985 & 0.985 & 0.985 \\
\hline & & & 814 & 26 & 33 & 34 & 1.388 & 1.388 & 1.388 \\
\hline & & & 817 & 72 & 78 & 80 & 2.225 & 2.225 & 2.225 \\
\hline & & & 880 & 12 & 14 & 14 & 1.460 & 1.460 & 1.460 \\
\hline & & 838 & 343 & & 3 & 3 & & 0.175 & 0.175 \\
\hline & & 861 & 814 & 2 & 3 & 4 & 1.111 & 1.111 & 1.111 \\
\hline & & & 817 & 3 & 3 & 3 & 1.821 & 1.821 & 1.821 \\
\hline & & & 823 & 3 & 3 & 3 & 2.393 & 2.393 & 2.393 \\
\hline & & & 880 & 36 & 39 & 39 & 3.341 & 3.341 & 3.341 \\
\hline & & & 901 & 3 & 3 & 3 & 2.078 & 2.078 & 2.078 \\
\hline & & & 908 & 7 & 7 & 7 & 1.893 & 1.893 & 1.893 \\
\hline & & & 933 & 4 & 5 & 5 & 0.949 & 0.949 & 0.949 \\
\hline & & 880 & 638 & & 2 & 2 & & 0.364 & 0.364 \\
\hline & & & 651 & 9 & 9 & 9 & 1.608 & 1.608 & 1.608 \\
\hline & & & 661 & 2 & 2 & 2 & 0.602 & 0.602 & 0.602 \\
\hline & & & 783 & 2 & 2 & 2 & 0.794 & 0.794 & 0.794 \\
\hline & & & 789 & 2 & 2 & 2 & 0.894 & 0.894 & 0.894 \\
\hline & & & 814 & 5 & 5 & 5 & 1.287 & 1.287 & 1.287 \\
\hline & & & 827 & 12 & 14 & 14 & 1.460 & 1.460 & 1.460 \\
\hline & & & 861 & 36 & 39 & 39 & 3.341 & 3.341 & 3.341 \\
\hline & & & 901 & 47 & 48 & 48 & 2.242 & 2.242 & 2.242 \\
\hline & & & 908 & 27 & 28 & 28 & 2.256 & 2.256 & 2.256 \\
\hline & & & 918 & 17 & 20 & 20 & 2.766 & 2.766 & 2.766 \\
\hline & & & 921 & 21 & 21 & 21 & 2.008 & 2.008 & 2.008 \\
\hline & & & 933 & 22 & 23 & 23 & 1.685 & 1.685 & 1.685 \\
\hline & & & 936 & 4 & 4 & 4 & 1.142 & 1.142 & 1.142 \\
\hline & & & 948 & 6 & 6 & 6 & 1.585 & 1.585 & 1.585 \\
\hline & & 901 & 651 & 7 & 7 & 7 & 0.926 & 0.926 & 0.926 \\
\hline & & & 861 & 3 & 3 & 3 & 2.078 & 2.078 & 2.078 \\
\hline & & & 880 & 47 & 48 & 48 & 2.242 & 2.242 & 2.242 \\
\hline & & & 918 & 41 & 42 & 42 & 2.204 & 2.204 & 2.204 \\
\hline & & & 921 & 50 & 50 & 50 & 2.203 & 2.203 & 2.203 \\
\hline & & & 933 & 26 & 28 & 28 & 1.629 & 1.629 & 1.629 \\
\hline & & & 936 & 12 & 12 & 12 & 1.897 & 1.897 & 1.897 \\
\hline & & & 948 & 8 & 8 & 8 & 1.292 & 1.292 & 1.292 \\
\hline & & 908 & 651 & 4 & 6 & 6 & 1.105 & 1.105 & 1.105 \\
\hline & & & 861 & 7 & 7 & 7 & 1.893 & 1.893 & 1.893 \\
\hline & & & 880 & 27 & 28 & 28 & 2.256 & 2.256 & 2.256 \\
\hline & & & 921 & 51 & 51 & 51 & 3.438 & 3.438 & 3.438 \\
\hline & & & 933 & 16 & 18 & 18 & 1.770 & 1.770 & 1.770 \\
\hline & & & 936 & 10 & 11 & 11 & 1.755 & 1.755 & 1.755 \\
\hline & & & 948 & 15 & 15 & 15 & 1.597 & 1.597 & 1.597 \\
\hline & & 918 & 651 & 2 & 2 & 2 & 0.762 & 0.762 & 0.762 \\
\hline & & & 880 & 17 & 20 & 20 & 2.766 & 2.766 & 2.766 \\
\hline & & & 901 & 41 & 42 & 42 & 2.204 & 2.204 & 2.204 \\
\hline & & & 933 & 77 & 78 & 78 & 2.935 & 2.935 & 2.935 \\
\hline & & & 936 & 20 & 20 & 20 & 2.685 & 2.685 & 2.685 \\
\hline & & 921 & 638 & 2 & 2 & 2 & 1.120 & 1.120 & 1.120 \\
\hline & & & 651 & 2 & 3 & 3 & 0.717 & 0.717 & 0.717 \\
\hline & & & 880 & 21 & 21 & 21 & 2.008 & 2.008 & 2.008 \\
\hline & & & 901 & 50 & 50 & 50 & 2.203 & 2.203 & 2.203 \\
\hline & & & 908 & 51 & 51 & 51 & 3.438 & 3.438 & 3.438 \\
\hline & & & 933 & 114 & 118 & 118 & 3.182 & 3.182 & 3.182 \\
\hline & & & 936 & 25 & 25 & 25 & 2.277 & 2.277 & 2.277 \\
\hline & & 933 & 861 & 4 & 5 & 5 & 0.949 & 0.949 & 0.949 \\
\hline & & & 880 & 22 & 23 & 23 & 1.685 & 1.685 & 1.685 \\
\hline & & & 901 & 26 & 28 & 28 & 1.629 & 1.629 & 1.629 \\
\hline & & & 908 & 16 & 18 & 18 & 1.770 & 1.770 & 1.770 \\
\hline & & & 918 & 77 & 78 & 78 & 2.935 & 2.935 & 2.935 \\
\hline & & & 921 & 114 & 118 & 118 & 3.182 & 3.182 & 3.182 \\
\hline & & & 948 & 3 & 5 & 5 & 1.294 & 1.294 & 1.294 \\
\hline & & 936 & 880 & 4 & 4 & 4 & 1.142 & 1.142 & 1.142 \\
\hline & & & 901 & 12 & 12 & 12 & 1.897 & 1.897 & 1.897 \\
\hline & & & 908 & 10 & 11 & 11 & 1.755 & 1.755 & 1.755 \\
\hline & & & 918 & 20 & 20 & 20 & 2.685 & 2.685 & 2.685 \\
\hline & & & 921 & 25 & 25 & 25 & 2.277 & 2.277 & 2.277 \\
\hline & & & 948 & 13 & 13 & 13 & 1.626 & 1.626 & 1.626 \\
\hline
\end{tabular}


Table B.2 - Continued from previous page

\begin{tabular}{|c|c|c|c|c|c|c|c|c|c|}
\hline Name & $\begin{array}{l}\text { UniProt } \\
\text { ID }\end{array}$ & Residue 1 & Residue 2 & FDR 1\% & FDR $3 \%$ & FDR $5 \%$ & F DR1\% & FDR3\% & FDR5\% \\
\hline & & 948 & 651 & & 4 & 4 & & 0.614 & 0.614 \\
\hline & & & 880 & 6 & 6 & 6 & 1.585 & 1.585 & 1.585 \\
\hline & & & 901 & 8 & 8 & 8 & 1.292 & 1.292 & 1.292 \\
\hline & & & 908 & 15 & 15 & 15 & 1.597 & 1.597 & 1.597 \\
\hline & & & 933 & 3 & 5 & 5 & 1.294 & 1.294 & 1.294 \\
\hline & & & 936 & 13 & 13 & 13 & 1.626 & 1.626 & 1.626 \\
\hline \multirow[t]{32}{*}{ ZNF830 } & Q96NB3 & 2 & 13 & & & 2 & & & 0.109 \\
\hline & & 13 & 2 & & & 2 & & & 0.109 \\
\hline & & 44 & 86 & 4 & 5 & 5 & 1.062 & 1.062 & 1.062 \\
\hline & & 86 & 44 & 4 & 5 & 5 & 1.062 & 1.062 & 1.062 \\
\hline & & 102 & 104 & 20 & 21 & 21 & 2.669 & 2.669 & 2.669 \\
\hline & & & 114 & & 3 & 3 & & 1.735 & 1.735 \\
\hline & & & 117 & 8 & 8 & 8 & 2.097 & 2.097 & 2.097 \\
\hline & & 104 & 102 & 20 & 21 & 21 & 2.669 & 2.669 & 2.669 \\
\hline & & & 117 & 20 & 20 & 20 & 4.242 & 4.242 & 4.242 \\
\hline & & & 149 & 2 & 2 & 2 & 1.245 & 1.245 & 1.245 \\
\hline & & 114 & 102 & & 3 & 3 & & 1.735 & 1.735 \\
\hline & & & 117 & 45 & 52 & 53 & 2.534 & 2.534 & 2.534 \\
\hline & & & 140 & 5 & 6 & 6 & 1.236 & 1.236 & 1.236 \\
\hline & & 117 & 102 & 8 & 8 & 8 & 2.097 & 2.097 & 2.097 \\
\hline & & & 104 & 20 & 20 & 20 & 4.242 & 4.242 & 4.242 \\
\hline & & & 114 & 45 & 52 & 53 & 2.534 & 2.534 & 2.534 \\
\hline & & & 140 & 4 & 5 & 5 & 0.872 & 0.872 & 0.872 \\
\hline & & 137 & 149 & 2 & 2 & 2 & 0.805 & 0.805 & 0.805 \\
\hline & & 140 & 114 & 5 & 6 & 6 & 1.236 & 1.236 & 1.236 \\
\hline & & & 117 & 4 & 5 & 5 & 0.872 & 0.872 & 0.872 \\
\hline & & 149 & 104 & 2 & 2 & 2 & 1.245 & 1.245 & 1.245 \\
\hline & & & 137 & 2 & 2 & 2 & 0.805 & 0.805 & 0.805 \\
\hline & & & 190 & 3 & 3 & 3 & 1.733 & 1.733 & 1.733 \\
\hline & & 190 & 149 & 3 & 3 & 3 & 1.733 & 1.733 & 1.733 \\
\hline & & & 216 & 4 & 4 & 4 & 1.077 & 1.077 & 1.077 \\
\hline & & 216 & 190 & 4 & 4 & 4 & 1.077 & 1.077 & 1.077 \\
\hline & & & 234 & 5 & 6 & 6 & 1.232 & 1.232 & 1.232 \\
\hline & & 234 & 216 & 5 & 6 & 6 & 1.232 & 1.232 & 1.232 \\
\hline & & 329 & 333 & 9 & 10 & 10 & 1.841 & 1.841 & 1.841 \\
\hline & & 331 & 339 & 12 & 14 & 15 & 0.996 & 0.996 & 0.996 \\
\hline & & 333 & 329 & 9 & 10 & 10 & 1.841 & 1.841 & 1.841 \\
\hline & & 339 & 331 & 12 & 14 & 15 & 0.996 & 0.996 & 0.996 \\
\hline
\end{tabular}




\section{Data collection}

Microscope model

Voltage (kV)

Additional hardware

Detector

Image acquisition mode

Data collection software

Magnification

Pixel size $(\AA)$

Electron exposure $\left(\mathrm{e} / \AA^{2}\right)$

Frames per micrograph

Electron exposure/micrograph (e-/ $\left./ \AA^{2}\right)$

Exposure time per micrograph (sec)

Defocus range $(\mu \mathrm{m})$

Number of datasets

Total number of micrographs collected

Micrographs used for processing

Reconstruction

EMDB map entry

PDB coordinate entry

\section{Image processing}

Symmetry imposed

Initial particle images

Final particle images

Resolution $(\AA)$

- FSC 0.143

Local resolution range $(\AA)$

Map sharpening $B$ factor $\left(\AA^{2}\right)$

\section{Model refinement statistics}

Model resolution $(\AA)$

Model composition

Non-hydrogen atoms

Protein residues

Nucleic acid residues

Ligands

Refinement method (PHENIX)

RMS deviations

Bond lengths $(\AA)$

Bond angles $(\AA)$

Validation

MolProbity score

Clashscore

Poor rotamers (\%)

C-beta deviations

Ramachandran plot

Outliers (\%)

Allowed (\%)

Favored (\%)

RNA validation

Correct sugar puckers (\%)

Good backbone conformations (\%)

CaBLAM outliers (\%)

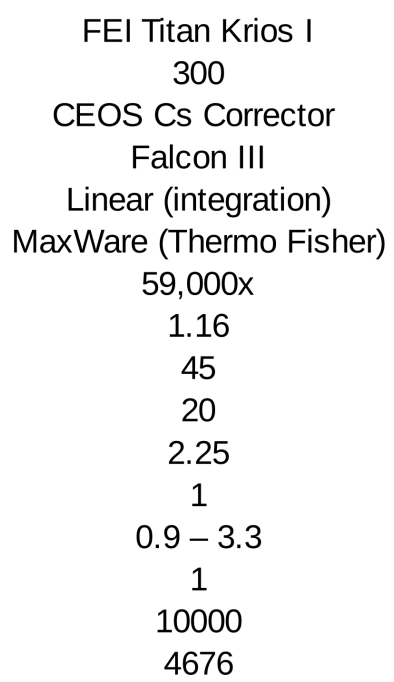

pre-B ${ }^{\text {act-1 }}$ (core)

11694

$7 \mathrm{ABF}$
pre-B ${ }^{\text {act-2 }}$ (core) pre-B ${ }^{\text {act-2 }}$ (SF3B)
$11693 \quad 11696$
7AAV 7ABH

C1

C1

C1

503208

503208

503208

39336

39336

3.9

4.2

4.5

$3.4-6.3$

$-177$

$3.5-6.3$

$-186$

$3.9-9.2$

$-219$

4.2

4.2

4.6

25835

25798

17922

4151

3353

152

3

Real-space

3

65

0

Real-space

Real-space

0.005

0.004

0.005

1.075

1.094

1.101

1.86

1.59

1.4

2.84

2.09

0.19

0.18

0

0

0

0.08

0.05

0.25

11.82

8.88

6.02

88.10

91.07

93.73

99.34

97.24

95.38

67.76

59.67

67.69

6.4

6.7

1.19

Fig. B.1: Data collection and model refinement statistics. From [131]. Reprinted with permission from AAAS. Data acquisition was carried out by Dr. Karl Bertram (Department of Structural Dynamics, MPI-BPC). 


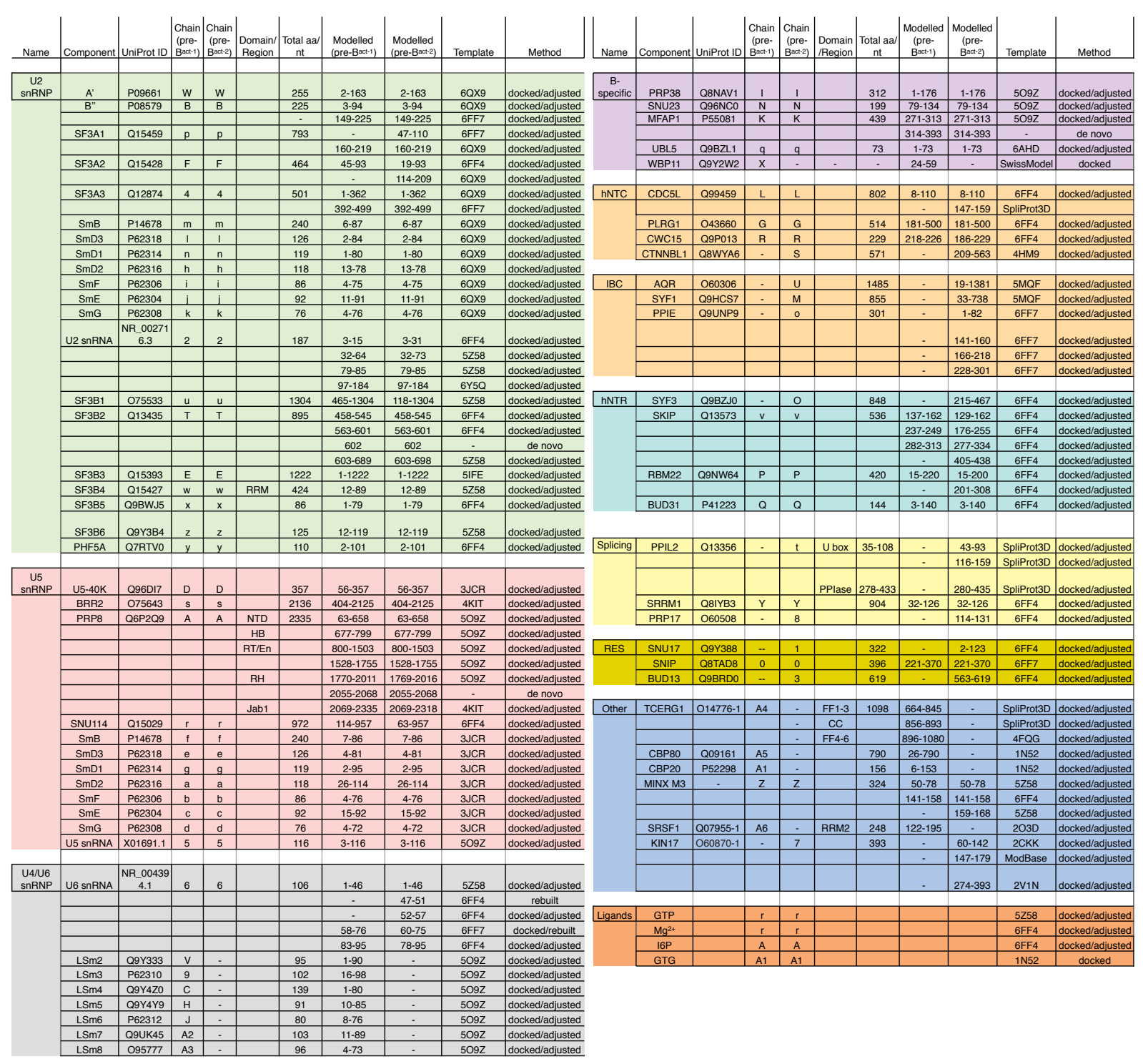

Fig. B.2: Modeling summary for pre-- ${ }^{\text {act-1 }}$ and pre-B ${ }^{\text {act-2 }}$. From [131. Reprinted with permission from AAAS. Model building was overseen by Dr. Berthold Kastner (Department of Cellular Biochemistry, MPI-BPC) (see 2.2.10. 


\section{References}

[1] Crick, F. Central dogma of molecular biology. Nature 227, 561-563 (1970). http: //doi.org/10.1038/227561a0.

[2] Sharp, P. A. The discovery of split genes and RNA splicing. In Trends in Biochemical Sciences, vol. 30, 279-281 (Elsevier, 2005). http://doi.org/10.1016/j.tibs.2005.04. 002 .

[3] Gilbert, W. Why genes in pieces? (1978). http://doi.org/10.1038/271501a0.

[4] Chow, L. T., Gelinas, R. E., Broker, T. R. \& Roberts, R. J. An amazing sequence arrangement at the 5 ends of adenovirus 2 messenger RNA. Cell (1977). http://doi. org/10.1016/0092-8674(77)90180-5.

[5] Berget, S. M., Moore, C. \& Sharp, P. A. Spliced segments at the 5' terminus of adenovirus 2 late mRNA* (adenovirus 2 mRNA processing/5' tails on mRNAs/electron microscopy of mRNA-DNA hybrids). Biochemistry 74, 3171-3175 (1977).

[6] Crick, F. Split genes and RNA splicing (1979). http://doi.org/10.1126/science. 373120 .

[7] Ruis, B. L., Kivens, W. J. \& Siliciano, P. G. The interaction between the first and last intron nucleotides in the second step of pre-mrna splicing is independent of other conserved intron nucleotides. Nucleic Acids Research 22, 5190-5195 (1994). http://doi.org/10. 1093/nar/22.24.5190.

[8] Burset, M., Seledtsov, I. A. \& Solovyev, V. V. Analysis of canonical and non-canonical splice sites in mammalian genomes. Nucleic Acids Research 28, 4364-4375 (2000). http: //doi.org/10.1093/nar/28.21.4364.

[9] Tarn, W. Y. \& Steitz, J. A. A novel spliceosome containing U11, U12, and U5 snRNPs excises a minor class (AT-AC) intron in vitro. Cell 84, 801-811 (1996). http://doi.org/ 10.1016/S0092-8674(00)81057-0.

[10] Tarn, W. Y. \& Steitz, J. A. Highly diverged U4 and U6 small nuclear RNAs required for splicing rare AT-AC introns. Science 273, 1824-1832 (1996). http://doi.org/10.1126/ science.273.5283.1824.

[11] Domdey, H. et al. Lariat structures are in vivo intermediates in yeast pre-mRNA splicing. Cell 39, 611-621 (1984). http://doi.org/10.1016/0092-8674(84)90468-9.

[12] Padgett, R. A., Konarska, M. M., Grabowski, P. J., Hardy, S. F. \& Sharp, P. A. Lariat RNA's as intermediates and products in the splicing of messenger RNA precursors. Science 225, 898-903 (1984). http://doi.org/10.1126/science.6206566

[13] Ruskin, B., Krainer, A. R., Maniatis, T. \& Green, M. R. Excision of an intact intron as a novel lariat structure during pre-mRNA splicing in vitro. Cell 38, 317-331 (1984). http://doi.org/10.1016/0092-8674(84)90553-1. 
[14] Rodriguez, J. R., Pikielny, C. W. \& Rosbash, M. In vivo characterization of yeast mRNA processing intermediates. Cell 39, 603-610 (1984). http://doi.org/10.1016/ 0092-8674(84)90467-7.

[15] Ohrt, T. et al. Molecular dissection of step 2 catalysis of yeast pre-mRNA splicing investigated in a purified system. RNA 19, 902-915 (2013). http://doi.org/10.1261/rna. 039024.113

[16] Costa, M., Walbott, H., Monachello, D., Westhof, E. \& Michel, F. Crystal structures of a group II intron lariat primed for reverse splicing. Science 354, aaf9258-aaf9258 (2016). http://doi.org/10.1126/science.aaf9258

[17] Heath, C. G., Viphakone, N. \& Wilson, S. A. The role of TREX in gene expression and disease (2016). http://doi.org/10.1042/BCJ20160010

[18] Alt, F. W. et al. Synthesis of secreted and membrane-bound immunoglobulin mu heavy chains is directed by mRNAs that differ at their 3 ends. Cell 20, 293-301 (1980). http: //doi.org/10.1016/0092-8674(80)90615-7.

[19] Early, P. et al. Two mRNAs can be produced from a single immunoglobulin $\mu$ gene by alternative RNA processing pathways. Cell 20, 313-319 (1980). http://doi.org/10. 1016/0092-8674(80)90617-0

[20] Schmucker, D. et al. Drosophila Dscam is an axon guidance receptor exhibiting extraordinary molecular diversity. Cell 101, 671-684 (2000). http://doi.org/10.1016/ S0092-8674(00)80878-8.

[21] Nilsen, T. W. \& Graveley, B. R. Expansion of the eukaryotic proteome by alternative splicing (2010). http://doi.org/10.1038/nature08909.

[22] Black, D. L. Mechanisms of Alternative Pre-Messenger RNA Splicing. Annual Review of Biochemistry 72, 291-336 (2003). http://doi.org/10.1146/annurev.biochem.72. 121801.161720 .

[23] Wang, Z. \& Burge, C. B. Splicing regulation: From a parts list of regulatory elements to an integrated splicing code (2008). http://doi.org/10.1261/rna.876308.

[24] Juneau, K., Nislow, C. \& Davis, R. W. Alternative splicing of PTC7 in Saccharomyces cerevisiae determines protein localization. Genetics 183, 185-194 (2009). http://doi. org/10.1534/genetics.109.105155.

[25] Meyer, M., Plass, M., Pérez-Valle, J., Eyras, E. \& Vilardell, J. Deciphering 3'ss Selection in the Yeast Genome Reveals an RNA Thermosensor that Mediates Alternative Splicing. Molecular Cell 43, 1033-1039 (2011). http://doi.org/10.1016/j.molcel.2011. 07.030 .

[26] Mishra, S. K. et al. Role of the ubiquitin-like protein Hub1 in splice-site usage and alternative splicing. Nature 474, 173-180 (2011). http://doi.org/10.1038/nature10143.

[27] Aslanzadeh, V., Huang, Y., Sanguinetti, G. \& Beggs, J. D. Transcription rate strongly affects splicing fidelity and cotranscriptionality in budding yeast. Genome Research 28, 203-213 (2018). http://doi.org/10.1101/gr.225615.117.

[28] Pertea, M. et al. CHESS: A new human gene catalog curated from thousands of large-scale RNA sequencing experiments reveals extensive transcriptional noise. Genome Biology 19, 1-14 (2018). http://doi.org/10.1186/s13059-018-1590-2. 
[29] Chen, J. \& Weiss, W. A. Alternative splicing in cancer: Implications for biology and therapy. Oncogene 34, 1-14 (2015). http://doi.org/10.1038/onc.2013.570.

[30] Chen, M. \& Manley, J. L. Mechanisms of alternative splicing regulation: Insights from molecular and genomics approaches. Nature Reviews Molecular Cell Biology 10, 741-754 (2009). http://doi.org/10.1038/nrm2777.

[31] Scotti, M. M. \& Swanson, M. S. RNA mis-splicing in disease. Nature Reviews Genetics 17, 19-32 (2016). http://doi.org/10.1038/nrg.2015.3.

[32] Dreyfuss, G. hnRNP proteins and the biogenesis of mRNA. In Annu. Rev. Biochem. (1993). http://doi.org/10.1109/IEEM.2013.6962450

[33] Zahler, A. M., Lane, W. S., Stolk, J. A. \& Roth, M. B. SR proteins: A conserved family of pre-mRNA splicing factors. Genes and Development 6, 837-847 (1992). http: //doi.org/10.1101/gad.6.5.837.

[34] Lee, Y. \& Rio, D. C. Mechanisms and regulation of alternative Pre-mRNA splicing (2015). http://doi.org/10.1146/annurev-biochem-060614-034316.

[35] Busch, A. \& Hertel, K. J. Evolution of SR protein and hnRNP splicing regulatory factors (2012). http://doi.org/10.1002/wrna.100.

[36] Martinez-Contreras, R. et al. hnRNP proteins and splicing control. (2007). http://doi. org/10.1007/978-0-387-77374-2_8.

[37] Conway, G., Wooley, J., Bibring, T. \& LESTOURGEONl, W. M. Ribonucleoproteins Package 700 Nucleotides of Pre-mRNA into a Repeating Array of Regular Particles. Tech. Rep. 7 (1988).

[38] Choi, Y. D., Grabowski, P. J., Sharp, P. A. \& Dreyfuss, G. Heterogeneous nuclear ribonucleoproteins: Role in RNA splicing. Science 231, 1534-1539 (1986). http: //doi.org/10.1126/science.3952495.

[39] Wang, Y., Ma, M., Xiao, X. \& Wang, Z. Intronic splicing enhancers, cognate splicing factors and context-dependent regulation rules. Nature Structural and Molecular Biology 19, 1044-1053 (2012). http://doi.org/10.1038/nsmb.2377.

[40] Lührmann, R., Kastner, B. \& Bach, M. Structure of spliceosomal snRNPs and their role in pre-mRNA splicing (1990). http://doi.org/10.1016/0167-4781(90)90001-I.

[41] Will, C. L. \& Lührmann, R. Spliceosome structure and function. Cold Spring Harbor perspectives in biology 3, a003707 (2011). http://doi.org/10.1101/cshperspect.a003707.

[42] Kunkel, G. R., Maser, R. L., Calvet, J. P. \& Pederson, T. U6 small nuclear RNA is transcribed by RNA polymerase III. Proceedings of the National Academy of Sciences of the United States of America 83, 8575-8579 (1986). http://doi.org/10.1073/pnas.83. 22.8575 .

[43] Hamm, J. \& Mattaj, I. W. Monomethylated cap structures facilitate RNA export from the nucleus. Cell 63, 109-118 (1990). http://doi.org/10.1016/0092-8674(90)90292-M.

[44] Colgan, D. F. \& Manley, J. L. Mechanism and regulation of mRNA polyadenylation (1997). http://doi.org/10.1101/gad.11.21.2755.

[45] Vankan, P., Mcguigan, C. \& Mattaj, W. Domains of U4 and U6 snRNAs required for snRNP assembly and splicing complementation in Xenopus oocytes. Tech. Rep. 10 (1990). 
[46] Pessa, H. K. et al. Minor spliceosome components are predominantly localized in the nucleus. Proceedings of the National Academy of Sciences of the United States of America 105, 8655-8660 (2008). http://doi.org/10.1073/pnas.0803646105.

[47] Branlant, C. et al. U2 RNA shares a structural domain with U1, U4, and U5 RNAs. The EMBO Journal 1, 1259-1265 (1982). http://doi.org/10.1002/j .1460-2075.1982. tb00022.x.

[48] Raker, V. A., Plessel, G. \& Luhrmann1, R. The snRNP core assembly pathway: identification of stable core protein heteromeric complexes and an snRNP subcore particle in vitro. Tech. Rep. 9 (1996).

[49] Urlaub, H., Raker, V. A., Kostka, S. \& Lührmann, R. Sm protein-Sm site RNA interactions within the inner ring of the spliceosomal snRNP core structure. EMBO Journal 20, 187196 (2001). http://doi.org/10.1093/emboj/20.1.187.

[50] Yuo, C.-Y., Ares, M. \& Weiner, A. M. Sequences Required for 3' End Formation of Human U2 Small Nuclear RNA. Tech. Rep. (1985).

[51] Mattaj, I. W. Cap trimethylation of U snRNA is cytoplasmic and dependent on U snRNP protein binding. Cell 46, 905-911 (1986). http://doi.org/10.1016/0092-8674(86) 90072-3.

[52] Neuman de Vegvar, H. E. \& Dahlberg, J. E. Nucleocytoplasmic transport and processing of small nuclear RNA precursors. Molecular and Cellular Biology 10, 3365-3375 (1990). http://doi.org/10.1128/mcb.10.7.3365.

[53] Hamm, J., Darzynkiewicz, E., Tahara, S. M. \& Mattaj, I. W. The trimethylguanosine cap structure of U1 snRNA is a component of a bipartite nuclear targeting signal. Cell 62, 569-577 (1990). http://doi.org/10.1016/0092-8674(90)90021-6.

[54] Fischer, U. \& Lührmann, R. An essential signaling role for the m3G cap in the transport of U1 snRNP to the nucleus. Science 249, 786-790 (1990). http://doi.org/10.1126/ science.2143847

[55] Hetzer, M. \& Mattaj, I. W. An ATP-dependent, Ran-independent mechanism for nuclear import of the U1A and U2B' spliceosome proteins. Journal of Cell Biology 148, 293-303 (2000). http://doi.org/10.1083/jcb.148.2.293

[56] Patel, S. B. \& Bellini, M. The assembly of a spliceosomal small nuclear ribonucleoprotein particle. Nucleic Acids Research 36, 6482-6493 (2008). http://doi.org/10.1093/nar/ gkn658.

[57] Achsel, T. et al. A doughnut-shaped heteromer of human Sm-like proteins binds to the 3'-end of U6 snRNA, thereby facilitating U4/U6 duplex formation in vitro. EMBO Journal 18, 5789-5802 (1999). http://doi.org/10.1093/emboj/18.20.5789.

[58] Reddy, R. \& Busch, H. Small Nuclear RNAs: RNA Sequences, Structure, and Modifications. In Structure and Function of Major and Minor Small Nuclear Ribonucleoprotein Particles, 1-37 (Springer Berlin Heidelberg, 1988). http://doi.org/10.1007/ 978-3-642-73020-7_1.

[59] Bohnsack, M. T. \& Sloan, K. E. Modifications in small nuclear RNAs and their roles in spliceosome assembly and function. Biological Chemistry 399, 1265-1276 (2018). http: //doi.org/10.1515/hsz-2018-0205 
[60] Yu, Y. T., Shu, M. D. \& Steitz, J. A. Modifications of U2 snRNA are required for snRNP assembly and pre-mRNA splicing. EMBO Journal 17, 5783-5795 (1998). http: //doi.org/10.1093/emboj/17.19.5783

[61] Newby, M. I. \& Greenbaum, N. L. Sculpting of the spliceosomal branch site recognition motif by a conserved pseudouridine. Nature Structural Biology 9, 958-965 (2002). http: //doi.org/10.1038/nsb873.

[62] Rueter, S. M., Dawson, T. R. \& Emeson, R. B. Regulation of alternative splicing by RNA editing. Nature 399, 75-80 (1999). http://doi.org/10.1038/19992.

[63] Melcher, T. et al. A mammalian RNA editing enzyme. Nature 379, 460-464 (1996). http://doi.org/10.1038/379460a0.

[64] Tarn, W. Y. Site-specific substitution of inosine at the terminal positions of a pre-mRNA intron: Implications for the configuration of the terminal base interaction. Biochimie 78, 1057-1065 (1996). http://doi.org/10.1016/S0300-9084(97)86730-2.

[65] Gerber, A., O'Connell, M. A. \& Keller, W. Two forms of human double-stranded RNAspecific editase 1 (hRED1) generated by the insertion of an Alu cassette. RNA 3, 453-463 (1997).

[66] Mendel, M. et al. Article Splice site m 6 A methylation prevents binding of U2AF35 to inhibit RNA splicing Splice site m 6 A methylation prevents binding of U2AF35 to inhibit RNA splicing 1-18 (2021). http://doi.org/10.1016/j.cell.2021.03.062.

[67] McCracken, S. et al. The C-terminal domain of RNA polymerase II couples mRNA processing to transcription. Nature 385, 357-360 (1997). http://doi.org/10.1038/385357a0.

[68] Carrillo Oesterreich, F. et al. Splicing of Nascent RNA Coincides with Intron Exit from RNA Polymerase II. Cell 165, 372-381 (2016). http://doi.org/10.1016/j . cell.2016. 02.045 .

[69] Zhuang, Y. \& Weiner, A. M. A compensatory base change in U1 snRNA suppresses a 5 splice site mutation. Cell 46, 827-835 (1986). http://doi.org/10.1016/0092-8674(86) 90064-4.

[70] Ruskin, B., Zamore, P. D. \& Green, M. R. A factor, U2AF, is required for U2 snRNP binding and splicing complex assembly. Cell 52, 207-219 (1988). http://doi.org/10. 1016/0092-8674(88)90509-0.

[71] Berg, M. G. et al. U1 snRNP determines mRNA length and regulates isoform expression. Cell (2012). http://doi.org/10.1016/j.cell.2012.05.029.

[72] Venters, C. C., Oh, J.-m., Di, C., So, B. R. \& Dreyfuss, G. U1 snRNP Telescripting : Suppression of Premature Transcription Termination in Introns as a New Layer of Gene Regulation (2019). http://doi.org/10.1101/cshperspect.a032235.

[73] Zhang, S. et al. Structure of a transcribing RNA polymerase II-U1 snRNP complex. Science 371, 305-309 (2021). http://doi.org/10.1126/science.abf 1870

[74] Zhou, Z., Licklider, L. J., Gygi, S. P. \& Reed, R. Comprehensive proteomic analysis of the human spliceosome. Nature 419, 182-185 (2002). http://doi.org/10.1038/ nature01031.

[75] Rappsilber, J., Ryder, U., Lamond, A. I. \& Mann, M. Large-scale proteomic analysis of the human spliceosome. Genome Research 12, 1231-1245 (2002). http://doi.org/10. $1101 / \mathrm{gr} .473902$. 
[76] Kornblihtt, A. R., De La Mata, M., Fededa, J. P., Muñoz, M. J. \& Nogués, G. Multiple links between transcription and splicing (2004). http://doi.org/10.1261/rna.7100104.

[77] McKay, S. L. \& Johnson, T. L. A bird's-eye view of post-translational modifications in the spliceosome and their roles in spliceosome dynamics (2010). http://doi.org/10.1039/ $\mathrm{c} 002828 \mathrm{~b}$

[78] Mermoud, J. E., Cohen, P. T. \& Lamond, A. I. Regulation of mammalian spliceosome assembly by a protein phosphorylation mechanism. EMBO Journal 13, 5679-5688 (1994). http://doi.org/10.1002/j.1460-2075.1994.tb06906.x.

[79] Duncan, P. I., Stojdl, D. F., Marius, R. M. \& Bell, J. C. In vivo regulation of alternative pre-mRNA splicing by the Clk1 protein kinase. Molecular and Cellular Biology 17, 59966001 (1997). http://doi.org/10.1128/mcb.17.10.5996.

[80] Manley, J. L. \& Tacke, R. SR proteins and splicing control (1996). http://doi.org/10. $1101 / \mathrm{gad} .10 .13 .1569$

[81] Wang, C. et al. Phosphorylation of spliceosomal protein SAP 155 coupled with splicing catalysis. Genes and Development 12, 1409-1414 (1998). http://doi.org/10.1101/gad. 12.10.1409.

[82] Choudhary, C. et al. Lysine acetylation targets protein complexes and co-regulates major cellular functions. Science 325, 834-840 (2009). http://doi.org/10.1126/science. 1175371 .

[83] Kuhn, A. N., Van Santen, M. A., Schwienhorst, A., Urlaub, H. \& Lührmann, R. Stalling of spliceosome assembly at distinct stages by small-molecule inhibitors of protein acetylation and deacetylation. RNA 15, 153-175 (2009). http://doi.org/10.1261/rna.1332609.

[84] Bellare, P. et al. A role for ubiquitin in the spliceosome assembly pathway. Nature Structural and Molecular Biology 15, 444-451 (2008). http://doi.org/10.1038/nsmb. 1401 .

[85] Michaud, S. \& Reed, R. An ATP-independent complex commits pre-mRNA to the mammalian spliceosome assembly pathway. Genes and Development 5, 2534-2546 (1991). http://doi.org/10.1101/gad.5.12b.2534.

[86] Mount, S. M., Pettersson, L., Hinterberger, M., Karmas, A. \& Steitz, J. A. The Ul Small Nuclear RNA-Protein Complex Selectively Binds a 5' Splice Site In Vitro. Tech. Rep. (1983).

[87] Query, C. C., McCaw, P. S. \& Sharp, P. A. A minimal spliceosomal complex A recognizes the branch site and polypyrimidine tract. Molecular and Cellular Biology 17, 2944-2953 (1997). http://doi.org/10.1128/mcb.17.5.2944.

[88] Pruzan, R., Furneaux, H., Lassota, P., Hong, G. Y. \& Hurwitz, J. Assemblage of the prespliceosome complex with separated fractions isolated from HeLa cells. Journal of Biological Chemistry 265, 2804-2813 (1990).

[89] Boesler, C. et al. ARTICLE A spliceosome intermediate with loosely associated tri-snRNP accumulates in the absence of Prp28 ATPase activity. Nature Communications 7 (2016). http://doi.org/10.1038/ncomms11997.

[90] Staley, J. P. \& Guthrie, C. An RNA switch at the 5' splice site requires ATP and the DEAD box protein Prp28p. Molecular Cell 3, 55-64 (1999). http://doi.org/10.1016/ S1097-2765(00)80174-4. 
[91] Fortner, D. M., Troy, R. G. \& Brow^, D. A. A stem/loop in U6 RNA defines a conformational switch required for pre-mRNA splicing. Tech. Rep. (1994).

[92] Raghunathan, P. L. \& Guthrie, C. RNA unwinding in U4/U6 snRNPs requires ATP hydrolysis and the DEIH-box splicing factor Brr2. Current Biology 8, 847-855 (1998). http://doi.org/10.1016/S0960-9822(07)00345-4.

[93] Mefford, M. A. \& Staley, J. P. Evidence that U2/U6 helix I promotes both catalytic steps of pre-mRNA splicing and rearranges in between these steps. RNA 15, 1386-1397 (2009). http://doi.org/10.1261/rna.1582609.

[94] Agafonov, D. E. et al. Semiquantitative Proteomic Analysis of the Human Spliceosome via a Novel Two-Dimensional Gel Electrophoresis Method. Molecular and Cellular Biology 31, 2667-2682 (2011). http://doi.org/10.1128/MCB.05266-11.

[95] Sidarovich, A. et al. Identification of a small molecule inhibitor that stalls splicing at an early step of spliceosome activation. eLife 6 (2017). http://doi.org/10.7554/eLife. 23533.

[96] Warkocki, Z. et al. Reconstitution of both steps of Saccharomyces cerevisiae splicing with purified spliceosomal components. Nature Structural \& Molecular Biology 16 (2009). http://doi.org/10.1038/nsmb.1729.

[97] Makarov, E. M. et al. Small nuclear ribonucleoprotein remodeling during catalytic activation of the spliceosome. Science 298, 2205-2208 (2002). http://doi.org/10.1126/ science.1077783.

[98] Bertram, K. et al. Cryo-EM Structure of a Pre-catalytic Human Spliceosome Primed for Activation. Cell 170, 701-713.e11 (2017). http://doi.org/10.1016/j.cell.2017.07. 011.

[99] Haselbach, D. et al. Structure and Conformational Dynamics of the Human Spliceosomal B Structure and Conformational Dynamics of the Human Spliceosomal B act Complex. Cell 172, 1-11 (2018). http://doi.org/10.1016/j.cell.2018.01.010.

[100] Wan, R., Bai, R., Yan, C., Lei, J. \& Shi, Y. Structures of the Catalytically Activated Yeast Spliceosome Reveal the Mechanism of Branching. Cell 177, 339-351.e13 (2019). http://doi.org/10.1016/j.cell.2019.02.006

[101] Bessonov, S., Anokhina, M., Will, C. L., Urlaub, H. \& Lührmann, R. Isolation of an active step I spliceosome and composition of its RNP core. Nature 452, 846-850 (2008). http://doi.org/10.1038/nature06842.

[102] Schwer, B. \& Guthrie, C. PRP16 is an RNA-dependent ATPase that interacts transiently with the spliceosome. Nature 349, 494-499 (1991). http://doi.org/10.1038/349494a0.

[103] Company, M., Arenas, J. \& Abelson, J. Requirement of the RNA helicase-like protein PRP22 for release of messenger RNA from spliceosomes. Nature 349, 487-493 (1991). http://doi.org/10.1038/349487a0.

[104] Arenas, J. E. \& Abelson, J. N. Prp43: An RNA helicase-like factor involved in spliceosome disassembly. Proceedings of the National Academy of Sciences of the United States of America 94, 11798-802 (1997).

[105] Yan, C., Wan, R. \& Shi, Y. Molecular Mechanisms of pre-mRNA Splicing through Structural Biology of the Spliceosome. Cold Spring Harbor Perspectives in Biology 11, a032409 (2019). http://doi.org/10.1101/cshperspect.a032409. 
[106] Pyle, A. M. Group II Intron Self-Splicing. Annual Review of Biophysics 45, 183-205 (2016). http://doi.org/10.1146/annurev-biophys-062215-011149.

[107] Linder, P. et al. Birth of the D-E-A-D box (1989). http://doi.org/10.1038/337121a0.

[108] Cordin, O., Banroques, J., Tanner, N. K. \& Linder, P. The DEAD-box protein family of RNA helicases (2006). http://doi.org/10.1016/j.gene.2005.10.019.

[109] Liu, Y. C. \& Cheng, S. C. Functional roles of DExD/H-box RNA helicases in Pre-mRNA splicing (2015). http://doi.org/10.1186/s12929-015-0161-z.

[110] O'Day, C. L., Dalbadie-McFarland, G. \& Abelson, J. The Saccharomyces cerevisiae Prp5 protein has RNA-dependent ATPase activity with specificity for U2 small nuclear RNA. Journal of Biological Chemistry 271, 33261-33267 (1996). http://doi.org/10.1074/ jbc.271.52.33261.

[111] Fleckner, J., Zhang, M., Valcárcel, J. \& Green, M. R. U2AF65 recruits a novel human DEAD box protein required for the U2 snRNP-branchpoint interaction. Genes and Development 11, 1864-1872 (1997). http://doi.org/10.1101/gad.11.14.1864.

[112] Zhang, Z. et al. Molecular architecture of the human 17S U2 snRNP. Nature 583, 310-313 (2020). http://doi.org/10.1038/s41586-020-2344-3.

[113] Boesler, C. et al. A spliceosome intermediate with loosely associated tri-snRNP accumulates in the absence of Prp28 ATPase activity. Nature Communications 7, 1-12 (2016). http://doi.org/10.1038/ncomms11997.

[114] Teigelkamp, S., Mundt, C., Achsel, T., Will, C. L. \& Lührmann, R. The human U5 snRNP-specific 100-kD protein is an RS domain-containing, putative RNA helicase with significant homology to the yeast splicing factor Prp28p. RNA 3, 1313-1326 (1997).

[115] Madhani, H. D. \& Guthrie, C. A novel base-pairing interaction between U2 and U6 snRNAs suggests a mechanism for the catalytic activation of the spliceosome. Cell 71, 803-817 (1992). http://doi.org/10.1016/0092-8674(92)90556-R.

[116] Bao, P., Höbartner, C., Hartmuth, K. \& Lührmann, R. Yeast Prp2 liberates the 5 splice site and the branch site adenosine for catalysis of pre-mRNA splicing. RNA 23, 1770-1779 (2017). http://doi.org/10.1261/rna.063115.117.

[117] Tseng, C. K. \& Cheng, S. C. Both catalytic steps of nuclear pre-mRNA splicing are reversible. Science 320, 1782-1784 (2008). http://doi.org/10.1126/science.1158993.

[118] Bertram, K. et al. Structural Insights into the Roles of Metazoan-Specific Splicing Factors in the Human Step 1 Spliceosome. Molecular Cell 80, 127-139.e6 (2020). http://doi. org $/ 10.1016 / j . m o l c e l .2020 .09 .012$

[119] Fica, S. M., Oubridge, C., Wilkinson, M. E., Newman, A. J. \& Nagai, K. A human postcatalytic spliceosome structure reveals essential roles of metazoan factors for exon ligation. Science 363, 710-714 (2019). http://doi.org/10.1126/science.aaw5569.

[120] Zhang, X. et al. Structures of the human spliceosomes before and after release of the ligated exon. Cell Research 29, 274-285 (2019). http://doi.org/10.1038/s41422-019-0143-x.

[121] Arenas, J. E. \& Abelson, J. N. Prp43: An RNA helicase-like factor involved in spliceosome dissassembly. Proceedings of the National Academy of Sciences of the United States of America 94, 11798-11802 (1997). http://doi.org/10.1073/pnas.94.22.11798. 
[122] Kastner, B., Will, C. L., Stark, H. \& Lührmann, R. Structural Insights into Nuclear premRNA Splicing in Higher Eukaryotes (2019). http://doi.org/10.1101/cshperspect. a032417.

[123] Mozaffari-Jovin, S. et al. The Prp8 RNase H-like domain inhibits Brr2-mediated U4/U6 snRNA unwinding by blocking Brr2 loading onto the U4 snRNA. Genes and Development 26, 2422-2434 (2012). http://doi.org/10.1101/gad.200949.112.

[124] Nguyen, T. H. D. et al. Structural basis of Brr2-Prp8 interactions and implications for U5 snRNP biogenesis and the spliceosome active site. Structure 21, 910-919 (2013). http://doi.org/10.1016/j.str.2013.04.017.

[125] Mozaffari-Jovin, S. et al. Inhibition of RNA helicase Brr2 by the C-terminal tail of the spliceosomal protein Prp8. Science 341, 80-84 (2013). http://doi.org/10.1126/ science.1237515.

[126] Henning, L. M. et al. A new role for FBP21 as regulator of Brr2 helicase activity. Nucleic Acids Research 45, 7922-7937 (2017). http://doi.org/10.1093/nar/gkx535

[127] Fica, S. M. et al. RNA catalyses nuclear pre-mRNA splicing. Nature 503, 229-234 (2013). http://doi.org/10.1038/nature12734.

[128] Fica, S. M., Mefford, M. A., Piccirilli, J. A. \& Staley, J. P. Evidence for a group II intron-like catalytic triplex in the spliceosome. Nature Structural and Molecular Biology 21, 464-471 (2014). http://doi.org/10.1038/nsmb.2815

[129] Anokhina, M. et al. RNA structure analysis of human spliceosomes reveals a compact 3D arrangement of snRNAs at the catalytic core. EMBO Journal 32, 2804-2818 (2013). http://doi.org/10.1038/emboj.2013.198.

[130] Steitz, T. A. \& Steitz, J. A. A general two-metal-ion mechanism for catalytic RNA (phosphoryl transfer mechanism/ribozyme/group I splicing/spliceosome/group H splicing). Tech. Rep. (1993).

[131] Townsend, C. et al. Mechanism of protein-guided folding of the active site U2/U6 RNA during spliceosome activation. Science 370 (2020). http://doi.org/10.1126/science. abc3753.

[132] Chan, R. T. et al. Structural basis for the second step of group II intron splicing. Nature Communications 9, 1-10 (2018). http://doi.org/10.1038/s41467-018-06678-0.

[133] Lambowitz, A. M. \& Zimmerly, S. Group II introns: Mobile ribozymes that invade DNA. Cold Spring Harbor Perspectives in Biology 3, 1-19 (2011). http://doi.org/10.1101/ cshperspect.a003616.

[134] Lambowitz, A. M. \& Belfort, M. Mobile Bacterial Group II Introns at the Crux of Eukaryotic Evolution. In Mobile DNA III, vol. 3, 1209-1236 (American Society of Microbiology, 2015). http://doi.org/10.1128/microbiolspec.mdna3-0050-2014.

[135] Michel, F., Kazuhiko, U. \& Haruo, O. Comparative and functional anatomy of group II catalytic introns - a review. Gene 82, 5-30 (1989). http://doi.org/10.1016/ 0378-1119(89) 90026-7.

[136] Pyle, A. M. The tertiary structure of group II introns: Implications for biological function and evolution (2010). http://doi.org/10.3109/10409231003796523. 
[137] Jacquier, A. \& Michel, F. Base-pairing interactions involving the 5 and 3-terminal nucleotides of group II self-splicing introns. Journal of Molecular Biology 213, 437-447 (1990). http://doi.org/10.1016/S0022-2836(05)80206-2.

[138] De Lencastre, A. \& Pyle, A. M. Three essential and conserved regions of the group II intron are proximal to the 5-splice site. RNA 14, 11-24 (2008). http://doi.org/10. $1261 /$ rna. 774008

[139] Madhani, H. D. snRNA catalysts in the spliceosome's ancient core. Cell 155, 1213-1215 (2013). http://doi.org/10.1016/j.cell.2013.11.022.

[140] Nguyen, T. H. D. et al. CryoEM structures of two spliceosomal complexes: Starter and dessert at the spliceosome feast. Current Opinion in Structural Biology 36, 48-57 (2016). http://doi.org/10.1016/j.sbi.2015.12.005

[141] Gordon, P. M., Fong, R. \& Piccirilli, J. A. A Second Divalent Metal Ion in the Group II Intron Reaction Center. Chemistry and Biology 14, 607-612 (2007). http://doi.org/ 10.1016/j.chembiol.2007.05.008

[142] Marcia, M. \& Pyle, A. M. Visualizing group II intron catalysis through the stages of splicing. Cell 151, 497-507 (2012). http://doi.org/10.1016/j.cell.2012.09.033.

[143] Keating, K. S., Toor, N., Perlman, P. S. \& Pyle, A. M. A structural analysis of the group II intron active site and implications for the spliceosome (2010). http://doi.org/10. $1261 /$ rna. 1791310

[144] Toor, N., Keating, K. S., Taylor, S. D. \& Pyle, A. M. Crystal structure of a self-spliced group II intron. Science 320, 77-82 (2008). http://doi.org/10.1126/science.1153803.

[145] Grainger, R. J. \& Beggs, J. D. Prp8 protein: At the heart of the spliceosome (2005). http://doi.org/10.1261/rna.2220705.

[146] Hoddges, P. E., Jackson, S. P., Brown, J. D. \& Beggs, J. D. Extraordinary sequence conservation of the PRP8 splicing factor. Yeast 11, 337-342 (1995). http://doi.org/ $10.1002 /$ yea.320110406.

[147] Fabrizio, P. et al. The Evolutionarily Conserved Core Design of the Catalytic Activation Step of the Yeast Spliceosome. Molecular Cell 36, 593-608 (2009). http://doi.org/10. 1016/j.molcel.2009.09.040.

[148] Dubochet, J. \& McDowall, A. VITRIFICATION OF PURE WATER FOR ELECTRON MICROSCOPY. Journal of Microscopy 124, 3-4 (1981). http://doi.org/10.1111/j. 1365-2818.1981.tb02483.x.

[149] Adrian, M., Dubochet, J., Lepault, J. \& McDowall, A. W. Cryo-electron microscopy of viruses. Nature (1984). http://doi.org/10.1038/308032a0.

[150] Unwin, P. N. T. Phase contrast and interference microscopy with the electron microscope. Phil. Trans. Roy. Soc. Lond. B 261, 95-104 (1971).

[151] Van Heel, M. et al. Single-particle electron cryo-microscopy: Towards atomic resolution. Quarterly Reviews of Biophysics 33, 307-369 (2000). http://doi.org/10.1017/ S0033583500003644.

[152] Frank, J. J. Three-dimensional electron microscopy of macromolecular assemblies : visualization of biological molecules in their native state (Oxford University Press, 2006).

[153] Wade, R. A brief look at imaging and contrast transfer. Ultramicroscopy 46, 145-156 (1992). http://doi.org/10.1016/0304-3991(92)90011-8. 
[154] Henderson, R. The potential and limitations of neutrons, electrons and X-rays for atomic resolution microscopy of unstained biological molecules. Quarterly Reviews of Biophysics 28, 171-193 (1995).

[155] Brilot, A. F. et al. Beam-induced motion of vitrified specimen on holey carbon film. Journal of Structural Biology 177, 630-637 (2012). http://doi.org/10.1016/J. JSB. 2012.02 .003 .

[156] Naydenova, K., Jia, P. \& Russo, C. J. Cryo-EM with sub-1 Å specimen movement. Science 370, 223-226 (2020). http://doi.org/10.1126/science.abb7927

[157] Li, X. et al. Electron counting and beam-induced motion correction enable near-atomicresolution single-particle cryo-EM. Nature Methods 10, 584-590 (2013). http://doi.org/ $10.1038 /$ nmeth. 2472

[158] Stark, H., Zemlin, F. \& Boettcher, C. Electron radiation damage to protein crystals of bacteriorhodopsin at different temperatures. Ultramicroscopy 63, 75-79 (1996). http: //doi.org/10.1016/0304-3991(96)00045-9.

[159] Grant, T. \& Grigorieff, N. Measuring the optimal exposure for single particle cryo-EM using a $2.6 \AA$ reconstruction of rotavirus VP6. eLife 4, e06980 (2015). http://doi.org/ 10.7554/eLife.06980.

[160] Zheng, S. Q. et al. MotionCor2: anisotropic correction of beam-induced motion for improved cryo-electron microscopy. Nature Publishing Group 14 (2017). http://doi.org/ $10.1038 /$ nmeth. 4193 .

[161] van Heel, M. \& Frank, J. Use of multivariate statistics in analysing the images of biological macromolecules. Ultramicroscopy 6, 187-94 (1981).

[162] DE ROSIER, D. J. \& KLUG, A. Reconstruction of Three Dimensional Structures from Electron Micrographs. Nature 217, 130-134 (1968). http://doi.org/10.1038/217130a0.

[163] Van Heel, M. Angular reconstitution: A posteriori assignment of projection directions for 3D reconstruction. Ultramicroscopy 21, 111-123 (1987). http://doi.org/10.1016/ 0304-3991(87)90078-7.

[164] Sigworth, F. J. Principles of cryo-EM single-particle image processing. Microscopy (Oxford, England) 65, 57-67 (2016). http://doi.org/10.1093/jmicro/dfv370.

[165] Bai, X. C., Rajendra, E., Yang, G., Shi, Y. \& Scheres, S. H. Sampling the conformational space of the catalytic subunit of human g-secretase. eLife 4, e11182 (2015). http://doi. org/10.7554/eLife.11182.

[166] Nakane, T., Kimanius, D., Lindahl, E. \& Scheres, S. H. Characterisation of molecular motions in cryo-EM single-particle data by multi-body refinement in RELION. eLife 7, 1-18 (2018). http://doi.org/10.7554/eLife.36861.

[167] Haselbach, D. et al. Long-range allosteric regulation of the human 26S proteasome by 20S proteasome-targeting cancer drugs. Nature Communications 8, 1-8 (2017). http: //doi.org/10.1038/ncomms15578.

[168] W Scheres, S. H. \& Chen, S. Prevention of overfitting in cryo-EM structure determination. Nature Methods 9 (2012). http://doi.org/10.1038/nmeth.2115.

[169] Rosenthal, P. B. \& Henderson, R. Optimal Determination of Particle Orientation, Absolute Hand, and Contrast Loss in Single-particle Electron Cryomicroscopy. Journal of Molecular Biology 333, 721-745 (2003). http://doi.org/10.1016/J.JMB.2003.07.013. 
[170] van Heel, M. \& Schatz, M. Fourier shell correlation threshold criteria. Journal of Structural Biology 151, 250-262 (2005). http://doi.org/10.1016/J.JSB.2005.05.009.

[171] Kucukelbir, A., Sigworth, F. J. \& Tagare, H. D. Quantifying the local resolution of cryoEM density maps. Nature Methods 11, 63-65 (2014). http://doi.org/10.1038/nmeth. 2727.

[172] Topf, M. \& Sali, A. Combining electron microscopy and comparative protein structure modeling. Current Opinion in Structural Biology 15, 578-585 (2005). http://doi.org/ $10.1016 / \mathrm{j} . \mathrm{sbi} .2005 .08 .001$.

[173] Urlaub, H., Hartmuth, K. \& Lührmann, R. A two-tracked approach to analyze RNAprotein crosslinking sites in native, nonlabeled small nuclear ribonucleoprotein particles. Methods 26, 170-181 (2002). http://doi.org/10.1016/S1046-2023(02)00020-8.

[174] Schmidt, C. \& Urlaub, H. Combining cryo-electron microscopy (cryo-EM) and crosslinking mass spectrometry (CX-MS) for structural elucidation of large protein assemblies. Current Opinion in Structural Biology 46, 157-168 (2017). http://doi.org/10.1016/j . sbi.2017.10.005

[175] Murshudov, G. Refinement of Atomic Structures Against cryo-EM Maps. Methods in Enzymology 579, 277-305 (2016). http://doi.org/10.1016/BS.MIE. 2016.05.033.

[176] Beckers, M., Mann, D. \& Sachse, C. Structural interpretation of cryo-EM image reconstructions. Progress in Biophysics and Molecular Biology 160, 26-36 (2021). http: //doi.org/10.1016/j.pbiomolbio.2020.07.004.

[177] Pomeranz Krummel, D. A., Oubridge, C., Leung, A. K., Li, J. \& Nagai, K. Crystal structure of human spliceosomal U1 snRNP at 5.5 resolution. Nature 458, 475-480 (2009). http://doi.org/10.1038/nature07851.

[178] Weber, G., Trowitzsch, S., Kastner, B., Lührmann, R. \& Wahl, M. C. Functional organization of the Sm core in the crystal structure of human U1 snRNP. EMBO Journal 29, 4172-4184 (2010). http://doi.org/10.1038/emboj.2010.295.

[179] Kondo, Y., Oubridge, C., van Roon, A. M. M. \& Nagai, K. Crystal structure of human U1 snRNP, a small nuclear ribonucleoprotein particle, reveals the mechanism of 5' splice site recognition. eLife 4, 1-19 (2015). http://doi.org/10.7554/eLife.04986.

[180] Galej, W. P., Oubridge, C., Newman, A. J. \& Nagai, K. Crystal structure of Prp8 reveals active site cavity of the spliceosome. Nature 493, 638-643 (2013). http://doi.org/10. $1038 /$ nature 11843

[181] Santos, K. F. et al. Structural basis for functional cooperation between tandem helicase cassettes in Brr2-mediated remodeling of the spliceosome. Proceedings of the National Academy of Sciences of the United States of America 109, 17418-17423 (2012). http: //doi.org/10.1073/pnas.1208098109.

[182] Kühlbrandt, W. The resolution revolution (2014). http://doi.org/10.1126/science. 1251652 .

[183] Nakane, T. et al. Single-particle cryo-EM at atomic resolution. Nature 587, 152-156 (2020). http://doi.org/10.1038/s41586-020-2829-0.

[184] Yip, K. M., Fischer, N., Paknia, E., Chari, A. \& Stark, H. Atomic-resolution protein structure determination by cryo-EM. Nature 587, 157-161 (2020). http://doi.org/10. 1038/s41586-020-2833-4. 
[185] Li, X. et al. A unified mechanism for intron and exon definition and back-splicing. Nature 573, 375-380 (2019). http://doi.org/10.1038/s41586-019-1523-6

[186] Plaschka, C., Newman, A. J. \& Nagai, K. Structural Basis of Nuclear pre-mRNA Splicing : Lessons from Yeast 1-21 (2019). http://doi.org/10.1101/cshperspect.a032391.

[187] Brown, J. D. \& Beggs, J. D. Roles of PRP8 protein in the assembly of splicing complexes. EMBO Journal 11, 3721-3729 (1992). http://doi.org/10.1002/j.1460-2075.1992. tb05457.x.

[188] Pena, V., Rozov, A., Fabrizio, P., Lührmann, R. \& Wahl, M. C. Structure and function of an RNase H domain at the heart of the spliceosome. EMBO Journal 27, 2929-2940 (2008). http://doi.org/10.1038/emboj.2008.209.

[189] Whittaker, E. \& Beggs, J. D. The yeast PRP8 protein interacts directly with pre-mRNA. Nucleic Acids Research 19, 5483-5489 (1991). http://doi.org/10.1093/nar/19.20. 5483 .

[190] Maeder, C., Kutach, A. K. \& Guthrie, C. ATP-dependent unwinding of U4/U6 snRNAs by the Brr2 helicase requires the C terminus of Prp8. Nature Structural and Molecular Biology 16, 42-48 (2009). http://doi.org/10.1038/nsmb.1535.

[191] Mozaffari-Jovin, S. et al. Inhibition of RNA helicase Brr2 by the C-terminal tail of the spliceosomal protein Prp8. Science 341, 80-84 (2013). http://doi.org/10.1126/ science.1237515.

[192] Ritchie, D. B. et al. Structural elucidation of a PRP8 core domain from the heart of the spliceosome. Nature Structural and Molecular Biology 15, 1199-1205 (2008). http: //doi.org/10.1038/nsmb.1505.

[193] Mozaffari-Jovin, S. et al. The Prp8 RNase H-like domain inhibits Brr2-mediated U4/U6 snRNA unwinding by blocking Brr2 loading onto the U4 snRNA. Genes and Development 26, 2422-2434 (2012). http://doi.org/10.1101/gad.200949.112.

[194] Mayerle, M. et al. Structural toggle in the RNaseH domain of Prp8 helps balance splicing fidelity and catalytic efficiency. Proceedings of the National Academy of Sciences of the United States of America 114, 4739-4744 (2017). http://doi.org/10.1073/pnas. 1701462114 .

[195] Agafonov, D. E. et al. Molecular architecture of the human U4/U6.U5 tri-snRNP. Science 351 (2016). http://doi.org/10.1126/science.aad2085.

[196] Bertram, K. et al. Cryo-EM Structure of a Pre-catalytic Human Spliceosome Primed for Activation. Cell 170, 701-713.e11 (2017). http://doi.org/10.1016/J.CELL.2017.07. 011.

[197] Bertram, K. et al. Cryo-EM structure of a human spliceosome activated for step 2 of splicing. Nature Publishing Group 542 (2017). http://doi.org/10.1038/nature21079.

[198] Haselbach, D. et al. Structure and Conformational Dynamics of the Human Spliceosomal Bact Complex. Cell 172, 454-464.e11 (2018). http://doi.org/10.1016/j.cell.2018. 01.010 .

[199] Zhan, X., Yan, C., Zhang, X., Lei, J. \& Shi, Y. Structure of a human catalytic step I spliceosome. Science 359, 537-545 (2018). http://doi.org/10.1126/science.aar6401. 
[200] Jia, X. \& Sun, C. SURVEY AND SUMMARY Structural dynamics of the N-terminal domain and the Switch loop of Prp8 during spliceosome assembly and activation. Nucleic Acids Research 46, 3833-3840 (2018). http://doi.org/10.1093/nar/gky242.

[201] Charenton, C., Wilkinson, M. E. \& Nagai, K. Mechanism of 5' splice site transfer for human spliceosome activation. Science 364, 362-367 (2019). http://doi.org/10.1126/ science.aax3289.

[202] Agafonov, D. E. et al. Semiquantitative Proteomic Analysis of the Human Spliceosome via a Novel Two-Dimensional Gel Electrophoresis Method. Molecular and Cellular Biology 31, 2667-2682 (2011). http://doi.org/10.1128/mcb.05266-11.

[203] Zhan, X., Yan, C., Zhang, X., Lei, J. \& Shi, Y. Structures of the human pre-catalytic spliceosome and its precursor spliceosome. Cell Research (2018). http://doi.org/10. 1038/s41422-018-0094-7.

[204] Schütze, T. et al. Multiple protein-protein interactions converging on the Prp38 protein during activation of the human spliceosome. Rna 22, 265-277 (2016). http://doi.org/ 10.1261/rna.054296.115.

[205] Keiper, S. et al. Smu1 and RED are required for activation of spliceosomal B complexes assembled on short introns. Nature Communications 10, 1-15 (2019). http://doi.org/ 10.1038/s41467-019-11293-8.

[206] Dziembowski, A. et al. Proteomic analysis identifies a new complex required for nuclear pre-mRNA retention and splicing. EMBO Journal 23, 4847-4856 (2004). http://doi. org $/ 10.1038 / \mathrm{sj}$.emboj.7600482.

[207] Wysoczanski, P. et al. Cooperative structure of the heterotrimeric pre-mRNA retention and splicing complex. Nature Structural and Molecular Biology 21, 911-918 (2014). http: //doi.org/10.1038/nsmb.2889.

[208] Ohrt, T. et al. Prp2-mediated protein rearrangements at the catalytic core of the spliceosome as revealed by dcFCCS. RNA 18, 1244-1256 (2012). http://doi.org/10.1261/ rna.033316.112.

[209] Schneider, C. et al. Dynamic Contacts of U2, RES, Cwc25, Prp8 and Prp45 Proteins with the Pre-mRNA Branch-Site and 3' Splice Site during Catalytic Activation and Step 1 Catalysis in Yeast Spliceosomes. PLoS Genetics 11, 1-27 (2015). http://doi.org/10. 1371/journal.pgen.1005539.

[210] Gottschalk, A., Bartels, C., Neubauer, G., Lührmann, R. \& Fabrizio, P. A Novel Yeast U2 snRNP Protein, Snu17p, Is Required for the First Catalytic Step of Splicing and for Progression of Spliceosome Assembly. Molecular and Cellular Biology 21, 3037-3046 (2001). http://doi.org/10.1128/mcb.21.9.3037-3046.2001.

[211] Bao, P., Höbartner, C., Hartmuth, K. \& Lührmann, R. Yeast Prp2 liberates the 5 splice site and the branch site adenosine for catalysis of pre-mRNA splicing. RNA 23, 1770-1779 (2017). http://doi.org/10.1261/rna.063115.117

[212] Tarn, W. Y. et al. Functional association of essential splicing factor(s) with PRP19 in a protein complex. EMBO Journal 13, 2421-2431 (1994). http://doi.org/10.1002/j. 1460-2075.1994.tb06527.x.

[213] Makarova, O. V. et al. A subset of human 35S U5 proteins, including Prp19, function prior to catalytic step 1 of splicing. EMBO Journal 23, 2381-2391 (2004). http://doi. org/10.1038/sj.emboj.7600241. 
[214] Chan, S. P., Kao, D. I., Tsai, W. Y. \& Cheng, S. C. The Prp19p-associated complex in spliceosome activation. Science 302, 279-282 (2003). http://doi.org/10.1126/science. 1086602

[215] Grote, M. et al. Molecular Architecture of the Human Prp19/CDC5L Complex. Molecular and Cellular Biology 30, 2105-2119 (2010). http://doi.org/10.1128/mcb.01505-09

[216] Burns, C. G., Ohi, R., Krainer, A. R. \& Gould, K. L. Evidence that Myb-related CDC5 proteins are required for pre-mRNA splicing. Proceedings of the National Academy of Sciences of the United States of America 96, 13789-13794 (1999). http://doi.org/10. 1073/pnas.96.24.13789.

[217] Huang, X., Wang, G., Wu, Y. \& Du, Z. The structure of full-length human CTNNBL1 reveals a distinct member of the armadillo-repeat protein family. Acta Crystallographica Section D: Biological Crystallography 69, 1598-1608 (2013). http://doi.org/10.1107/ S0907444913011360.

[218] Ahn, J. W., Kim, S., Kim, E. J., Kim, Y. J. \& Kim, K. J. Structural insights into the novel ARM-repeat protein CTNNBL1 and its association with the hPrp19-CDC5L complex. Acta Crystallographica Section D: Biological Crystallography 70, 780-788 (2014). http://doi.org/10.1107/S139900471303318X.

[219] Hogg, R., McGrail, J. C. \& O'Keefe, R. T. The function of the NineTeen Complex (NTC) in regulating spliceosome conformations and fidelity during pre-mRNA splicing. Biochemical Society Transactions 38, 1110-1115 (2010). http://doi.org/10.1042/BST0381110.

[220] Zhang, X. et al. An Atomic Structure of the Human Spliceosome. Cell 169, 918-929.e14 (2017). http://doi.org/10.1016/J.CELL.2017.04.033

[221] McGrail, J. C., Krause, A. \& O'Keefe, R. T. The RNA binding protein Cwc2 interacts directly with the U6 snRNA to link the nineteen complex to the spliceosome during premRNA splicing. Nucleic Acids Research 37, 4205-4217 (2009). http://doi.org/10. 1093/nar/gkp341.

[222] Xu, C. et al. Solution structure of human peptidyl prolyl isomerase-like protein 1 and insights into its interaction with SKIP. Journal of Biological Chemistry 281, 15900-15908 (2006). http://doi.org/10.1074/jbc.M511155200.

[223] Wang, X. et al. A large intrinsically disordered region in SKIP and its disorder-order transition induced by PPIL1 binding revealed by NMR. Journal of Biological Chemistry 285, 4951-4963 (2010). http://doi.org/10.1074/jbc.M109.087528.

[224] Li, Y. et al. The SNW Domain of SKIP Is Required for Its Integration into the Spliceosome and Its Interaction with the Paf1 Complex in Arabidopsis. Molecular Plant 9, 1040-1050 (2016). http://doi.org/10.1016/j.molp.2016.04.011.

[225] De, I. et al. The RNA helicase Aquarius exhibits structural adaptations mediating its recruitment to spliceosomes. Nature Structural \& Molecular Biology 22, 138-144 (2015). http://doi.org/10.1038/nsmb.2951.

[226] Nakatsu, Y. et al. XAB2, a novel tetratricopeptide repeat protein involved in transcriptioncoupled DNA repair and transcription. Journal of Biological Chemistry 275, 34931-34937 (2000). http://doi.org/10.1074/jbc.M004936200.

[227] Chen, C. H. et al. Functional and physical interactions between components of the Prp19passociated complex. Nucleic Acids Research 30, 1029-1037 (2002). http://doi.org/10. 1093/nar/30.4.1029. 
[228] Mi, H., Kops, O., Zimmermann, E., Jäschke, A. \& Tropschug, M. A nuclear RNA-binding cyclophilin in human T cells. FEBS Letters 398, 201-205 (1996). http://doi.org/10. 1016/S0014-5793(96)01248-3.

[229] Villa, T. \& Guthrie, C. The Isy1p component of the NineTeen Complex interacts with the ATPase Prp16p to regulate the fidelity of pre-mRNA splicing. Genes and Development 19, 1894-1904 (2005). http://doi.org/10.1101/gad.1336305

[230] Hatakeyama, S., Matsumoto, M., Yada, M. \& Nakayama, K. I. Interaction of U-boxtype ubiquitin-protein ligases (E3s) with molecular chaperones. Genes to Cells 9, 533-548 (2004). http://doi.org/10.1111/j.1356-9597.2004.00742.x.

[231] Hatakeyama, S., Yada, M., Matsumoto, M., Ishida, N. \& Nakayama, K. I. U Box Proteins as a New Family of Ubiquitin-Protein Ligases. Journal of Biological Chemistry 276, 3311133120 (2001). http://doi.org/10.1074/jbc.M102755200.

[232] Busetto, V. et al. Structural and functional insights into CWC27/CWC22 heterodimer linking the exon junction complex to spliceosomes. Nucleic acids research 48, 5670-5683 (2020). http://doi.org/10.1093/nar/gkaa267.

[233] Brickner, J. R. et al. A ubiquitin-dependent signalling axis specific for ALKBH-mediated DNA dealkylation repair. Nature 551, 389-393 (2017). http://doi.org/10.1038/ nature24484.

[234] Blencowe, B. J., Issner, R., Nickerson, J. A. \& Sharp, P. A. A coactivator of pre-mRNA splicing. Genes and Development 12, 996-1009 (1998). http://doi.org/10.1101/gad. 12.7.996.

[235] Eldridge, A. G., Li, Y., Sharp, P. A. \& Blencowe, B. J. The SRm160/300 splicing coactivator is required for exon-enhancer function. Proceedings of the National Academy of Sciences of the United States of America 96, 6125-6130 (1999). http://doi.org/10. 1073/pnas.96.11.6125.

[236] Tomsic, J. et al. A germline mutation in SRRM2, a splicing factor gene, is implicated in papillary thyroid carcinoma predisposition. Scientific reports 5, 10566 (2015). http: //doi.org/10.1038/srep10566.

[237] Zhang, X. et al. Structure of the human activated spliceosome in three conformational states. Cell Research 28, 307-322 (2018). http://doi.org/10.1038/cr.2018.14.

[238] Bai, R. et al. Mechanism of spliceosome remodeling by the ATPase/helicase Prp2 and its coactivator Spp2. Science 371 (2021). http://doi.org/10.1126/science.abe8863

[239] Valadkhan, S. \& Manley, J. L. Splicing-related catalysis by protein-free snRNAS. Nature 413, 701-707 (2001). http://doi.org/10.1038/35099500.

[240] Valadkhan, S. \& Manley, J. L. Characterization of the catalytic activity of U2 and U6 snRNAs. RNA 9, 892-904 (2003). http://doi.org/10.1261/rna.5440303

[241] Valadkhan, S., Mohammadi, A., Wachtel, C. \& Manley, J. L. Protein-free spliceosomal snRNAs catalyze a reaction that resembles the first step of splicing. RNA 13, 2300-2311 (2007). http://doi.org/10.1261/rna.626207.

[242] Valadkhan, S., Mohammadi, A., Jaladat, Y. \& Geisler, S. Protein-free small nuclear RNAs catalyze a two-step splicing reaction. Proceedings of the National Academy of Sciences of the United States of America 106, 11901-11906 (2009). http://doi.org/10.1073/pnas. 0902020106 . 
[243] Saha, D., Khandelia, P., O'Keefe, R. T. \& Vijayraghavan, U. Saccharomyces cerevisiae NineTeen Complex (NTC)-associated factor Bud31/Ycr063w assembles on precatalytic spliceosomes and improves first and second step pre-mRNA splicing efficiency. Journal of Biological Chemistry 287, 5390-5399 (2012). http://doi.org/10.1074/jbc.M111. 298547.

[244] Bao, P., Will, C. L., Urlaub, H., Boon, K. L. \& Lührmann, R. The RES complex is required for efficient transformation of the precatalytic B spliceosome into an activated Bactcomplex. Genes and Development 31, 2416-2429 (2017). http://doi.org/10.1101/ gad.308163.117.

[245] Tarn, W. Y., Lee, K. R. \& Cheng, S. C. The yeast PRP19 protein is not tightly associated with small nuclear RNAs, but appears to associate with the spliceosome after binding of U2 to the pre-mRNA and prior to formation of the functional spliceosome. Molecular and Cellular Biology 13, 1883-1891 (1993). http://doi.org/10.1128/mcb.13.3.1883.

[246] Shi, Y. The Spliceosome: A Protein-Directed Metalloribozyme (2017). http://doi.org/ $10.1016 / j \cdot j \mathrm{mb} .2017 .07 .010$

[247] Plaschka, C., Lin, P.-C. \& Nagai, K. Structure of a pre-catalytic spliceosome. Nature 546, 617 (2017). http://doi.org/10.1038/nature22799.

[248] Yan, C., Wan, R., Bai, R., Huang, G. \& Shi, Y. Structure of a yeast activated spliceosome at 3.5 Åresolution. Science (New York, N.Y.) 353, 904-911 (2016). http://doi.org/10. 1126/science.aag0291.

[249] Alsafadi, S. et al. Cancer-associated SF3B1 mutations affect alternative splicing by promoting alternative branchpoint usage. Nature Communications 7 (2016). http: //doi.org/10.1038/ncomms10615.

[250] Effenberger, K. A., Urabe, V. K. \& Jurica, M. S. Modulating splicing with small molecular inhibitors of the spliceosome. Wiley Interdisciplinary Reviews: RNA 8 (2017). http: //doi.org/10.1002/wrna.1381.

[251] Bonnal, S., Vigevani, L. \& Valcárcel, J. The spliceosome as a target of novel antitumour drugs. Nature Reviews Drug Discovery 11, 847-859 (2012). http://doi.org/10.1038/ nrd3823.

[252] Cretu, C. et al. Structural Basis of Splicing Modulation by Antitumor Macrolide Compounds. Molecular Cell 70, 265-273.e8 (2018). http://doi.org/10.1016/j.molcel. 2018.03.011.

[253] Mermoud, J. E., Cohen, P. \& Lamond, A. I. Ser/thr-specific protein phosphatases are required for both catalytic steps of pre-mRNA splicing. Nucleic Acids Research 20, 52635269 (1992). http://doi.org/10.1093/nar/20.20.5263.

[254] Pilch, B. et al. Specific inhibition of serine- and arginine-rich splicing factors phosphorylation, spliceosome assembly, and splicing by the antitumor drug NB-506. Cancer Research 61, 6876-6884 (2001).

[255] Berg, M. G. et al. A Quantitative High-Throughput In Vitro Splicing Assay Identifies Inhibitors of Spliceosome Catalysis. Molecular and Cellular Biology 32, 1271-1283 (2012). http://doi.org/10.1128/mcb.05788-11.

[256] Sidarovich, A. Identification and characterization of small molecule inhibitors of premRNA splicing that block spliceosome assembly at novel stages (2015). 
[257] Emsley, P. \& Cowtan, K. Coot: Model-building tools for molecular graphics. Acta Crystallographica Section D: Biological Crystallography 60, 2126-2132 (2004). http: //doi.org/10.1107/S0907444904019158.

[258] Afonine, P. V. et al. New tools for the analysis and validation of cryo-EM maps and atomic models. Acta Crystallographica Section D: Structural Biology 74, 814-840 (2018). http://doi.org/10.1107/S2059798318009324

[259] Punjani, A., Rubinstein, J. L., Fleet, D. J. \& Brubaker, M. A. cryoSPARC: algorithms for rapid unsupervised cryo-EM structure determination. Nature Methods 14, 290-296 (2017). http://doi.org/10.1038/nmeth.4169.

[260] Zhang, K. Gctf: Real-time CTF determination and correction. Journal of Structural Biology 193, 1-12 (2016). http://doi.org/10.1016/J.JSB.2015.11.003

[261] Perkins, D. N., Pappin, D. J., Creasy, D. M. \& Cottrell, J. S. Probability-based protein identification by searching sequence databases using mass spectrometry data. Electrophoresis 20, 3551-3567 (1999). http://doi.org/10.1002/(SICI) 1522-2683(19991201) 20: 18<3551: : AID-ELPS3551>3.0.CO;2-2.

[262] Chen, V. B. et al. MolProbity: All-atom structure validation for macromolecular crystallography. Acta Crystallographica Section D: Biological Crystallography 66, 12-21 (2010). http://doi.org/10.1107/S0907444909042073.

[263] Afonine, P. V. et al. Real-space refinement in PHENIX for cryo-EM and crystallography. Acta Crystallographica Section D: Structural Biology 74, 531-544 (2018). http://doi. org/10.1107/S2059798318006551.

[264] Yang, B. et al. Identification of cross-linked peptides from complex samples. Nature Methods 9, 904-906 (2012). http://doi.org/10.1038/nmeth.2099.

[265] Chen, Z. L. et al. A high-speed search engine pLink 2 with systematic evaluation for proteome-scale identification of cross-linked peptides. Nature Communications 10, 1-12 (2019). http://doi.org/10.1038/s41467-019-11337-z.

[266] Burley, S. K. et al. Protein Data Bank: The single global archive for 3D macromolecular structure data. Nucleic Acids Research 47, D520-D528 (2019). http://doi.org/10. 1093/nar/gky949.

[267] Kimanius, D., Forsberg, B. O., Scheres, S. H. \& Lindahl, E. Accelerated cryo-EM structure determination with parallelisation using GPUS in RELION-2. eLife 5 (2016). http: //doi.org/10.7554/eLife.18722.

[268] Zivanov, J. et al. New tools for automated high-resolution cryo-EM structure determination in RELION-3. eLife 7 (2018). http://doi.org/10.7554/eLife.42166.

[269] Korneta, I., Magnus, M. \& Bujnicki, J. M. Structural bioinformatics of the human spliceosomal proteome. Nucleic Acids Research 40, 7046-7065 (2012). http://doi.org/10. 1093/nar/gks347

[270] Waterhouse, A. et al. SWISS-MODEL: Homology modelling of protein structures and complexes. Nucleic Acids Research 46, W296-W303 (2018). http://doi.org/10.1093/ nar/gky427

[271] Pettersen, E. F. et al. UCSF Chimera-A visualization system for exploratory research and analysis. Journal of Computational Chemistry 25, 1605-1612 (2004). http://doi. org/10.1002/jcc.20084. 
[272] Goddard, T. D. et al. UCSF ChimeraX: Meeting modern challenges in visualization and analysis. Protein Science 27, 14-25 (2018). http://doi.org/10.1002/pro.3235.

[273] The UniProt Consortium. UniProt: A worldwide hub of protein knowledge. Nucleic Acids Research 47, D506-D515 (2019). http://doi.org/10.1093/nar/gky1049.

[274] Dignam, J. D., Lebovitz, R. M. \& Roeder, R. G. Accurate transcription initiation by RNA polymerase II in a soluble extract from isolated mammalian nuclei. Nucleic Acids Research 11, 1475-1489 (1983). http://doi.org/10.1093/nar/11.5.1475

[275] Das, R. \& Reed, R. Resolution of the mammalian E complex and the ATP-dependent spliceosomal complexes on native agarose mini-gels. RNA 5, 1504-1508 (1999). http: //doi.org/10.1017/S1355838299991501.

[276] Agafonov, D. E. et al. Semiquantitative Proteomic Analysis of the Human Spliceosome via a Novel Two-Dimensional Gel Electrophoresis Method. Molecular and Cellular Biology (2011). http://doi.org/10.1128/MCB.05266-11.

[277] Fabrizio, P., Laggerbauer, B., Lauber, J., Lane, W. S. \& Lührmann, R. An evolutionarily conserved U5 snRNP-specific protein is a GTP-binding factor closely related to the ribosomal translocase EF-2. EMBO Journal 16, $4092-4106$ (1997). http: //doi.org/10.1093/emboj/16.13.4092

[278] Kastner, B. et al. GraFix: sample preparation for single-particle electron cryomicroscopy. Nature Methods 5, 53-55 (2008). http://doi.org/10.1038/nmeth1139.

[279] Lu, M. et al. Crystal Structure of the Three Tandem FF Domains of the Transcription Elongation Regulator CA150. Journal of Molecular Biology 393, 397-408 (2009). http: //doi.org/10.1016/j.jmb.2009.07.086.

[280] Izaurralde, E. et al. A nuclear cap binding protein complex involved in pre-mRNA splicing. Cell 78, 657-668 (1994). http://doi.org/10.1016/0092-8674(94)90530-4.

[281] Görnemann, J., Kotovic, K. M., Hujer, K. \& Neugebauer, K. M. Cotranscriptional spliceosome assembly occurs in a stepwise fashion and requires the cap binding complex. Molecular Cell 19, 53-63 (2005). http://doi.org/10.1016/j.molcel.2005.05.007.

[282] Pabis, M. et al. The nuclear cap-binding complex interacts with the U4/U6 - U5 trisnRNP and promotes spliceosome assembly in mammalian cells. Rna 19, 1054-1063 (2013). http://doi.org/10.1261/rna.037069.112.

[283] Llorian, M., Beullens, M., Andrés, I., Ortiz, J. M. \& Bollen, M. SIPP1, a novel pre-mRNa splicing factor and interactor of protein phosphatase-1. Biochemical Journal 378, 229-238 (2004). http://doi.org/10.1042/BJ20030950.

[284] Komuro, A., Saeki, M. \& Kato, S. Association of two nuclear proteins, Npw38 and NpwBP, via the interaction between the WW domain and a novel proline-rich motif containing glycine and arginine. Journal of Biological Chemistry 274, 36513-36519 (1999). http: //doi.org/10.1074/jbc.274.51.36513

[285] Masson, C. et al. Global genome repair is required to activate KIN17, a UVC-responsive gene involved in DNA replication. Proceedings of the National Academy of Sciences of the United States of America 100, 616-621 (2003). http://doi.org/10.1073/pnas. 0236176100 . 
[286] Miccoli, L. et al. The Human Stress-Activated Protein kin17 Belongs to the Multiprotein DNA Replication Complex and Associates In Vivo with Mammalian Replication Origins. Molecular and Cellular Biology 25, 3814-3830 (2005). http://doi.org/10.1128/mcb. $25.9 .3814-3830.2005$

[287] Carlier, L. et al. Solution structure of the region 51-160 of human KIN17 reveals an atypical winged helix domain. Protein Science 16, 2750-2755 (2007). http://doi.org/ $10.1110 /$ ps.073079107.

[288] Schellenberg, M. J. et al. A conformational switch in PRP8 mediates metal ion coordination that promotes pre-mRNA exon ligation. Nature Structural and Molecular Biology 20, 728-734 (2013). http://doi.org/10.1038/nsmb.2556.

[289] Carty, S. M., Goldstrohm, A. C., Suñé, C., Garcia-Blanco, M. A. \& Greenleaf, A. L. Protein-interaction modules that organize nuclear function: FF domains of CA150 bind the phosphoCTD of RNA polymerase II. Proceedings of the National Academy of Sciences of the United States of America 97, 9015-9020 (2000). http://doi.org/10.1073/pnas. 160266597.

[290] Goldstrohm, A. C., Albrecht, T. R., Suñé, C., Bedford, M. T. \& Garcia-Blanco, M. A. The Transcription Elongation Factor CA150 Interacts with RNA Polymerase II and the Pre-mRNA Splicing Factor SF1. Molecular and Cellular Biology 21, 7617-7628 (2001). http://doi.org/10.1128/mcb.21.22.7617-7628.2001

[291] Sánchez-Álvarez, M., Goldstrohm, A. C., Garcia-Blanco, M. A. \& Suñé, C. Human Transcription Elongation Factor CA150 Localizes to Splicing Factor-Rich Nuclear Speckles and Assembles Transcription and Splicing Components into Complexes through Its Amino and Carboxyl Regions. Molecular and Cellular Biology 26, 4998-5014 (2006). http://doi.org/10.1128/mcb.01991-05.

[292] Waragai, M. et al. PQBP-1/Npw38, a nuclear protein binding to the polyglutamine tract, interacts with U5-15kD/dim1p via the carboxyl-terminal domain. Biochemical and Biophysical Research Communications 273, 592-595 (2000). http://doi.org/10.1006/ bbrc.2000.2992.

[293] Zhang, Y. Z. et al. Evidence that Dim1 associates with proteins involved in pre-mRNA splicing, and delineation of residues essential for Dim1 interactions with hnRNP F and Npw38/PQBP-1. Gene 257, 33-43 (2000). http://doi.org/10.1016/S0378-1119(00) 00372-3.

[294] Okazawa, H. et al. Interaction between mutant ataxin-1 and PQBP-1 affects transcription and cell death. Neuron 34, 701-713 (2002). http://doi.org/10.1016/S0896-6273(02) 00697-9.

[295] Nabeshima, Y., Mizuguchi, M., Kajiyama, A. \& Okazawa, H. Segmental isotope-labeling of the intrinsically disordered protein PQBP1. FEBS Letters 588, 4583-4589 (2014). http://doi.org/10.1016/j.febslet.2014.10.028.

[296] Kohtz, J. D. et al. Protein-protein interactions and 5'-splice-site recognition in mammalian mRNA precursors. Nature 368, 119-124 (1994). http://doi.org/10.1038/368119a0.

[297] Sánchez-Hernández, N. et al. The FF4 and FF5 domains of transcription elongation regulator 1 (TCERG1) target proteins to the periphery of speckles. Journal of Biological Chemistry 287, 17789-17800 (2012). http://doi.org/10.1074/jbc.M111.304782.

[298] Misteli, T. RNA splicing: What has phosphorylation got to do with it? (1999). http: //doi.org/10.1016/S0960-9822(99)80128-6. 
[299] Cho, S. et al. Interaction between the RNA binding domains of Ser-Arg splicing factor 1 and U1-70K snRNP protein determines early spliceosome assembly. Proceedings of the National Academy of Sciences of the United States of America 108, 8233-8238 (2011). http://doi.org/10.1073/pnas.1017700108.

[300] Sánchez-Hernández, N. et al. The in vivo dynamics of TCERG1, a factor that couples transcriptional elongation with splicing. Rna 22, 571-582 (2016). http://doi.org/10. 1261/rna.052795.115.

[301] Ulrich, A. K., Schulz, J. F., Kamprad, A., Schütze, T. \& Wahl, M. C. Structural Basis for the Functional Coupling of the Alternative Splicing Factors Smu1 and RED. Structure 24, 762-773 (2016). http://doi.org/10.1016/j.str.2016.03.016

[302] Huang, X. et al. Structure and function of the two tandem WW domains of the pre-mRNA splicing factor FBP21 (Formin-binding protein 21). Journal of Biological Chemistry 284, 25375-25382 (2009). http://doi.org/10.1074/jbc.M109.024828.

[303] Xie, J., Beickman, K., Otte, E. \& Rymond, B. C. Progression through the spliceosome cycle requires Prp38p function for U4/U6 snRNA dissociation. EMBO Journal 17, 29382946 (1998). http://doi.org/10.1093/emboj/17.10.2938.

[304] Chung, S. et al. Crooked neck is a component of the human spliceosome and implicated in the splicing process. Biochimica et Biophysica Acta - Gene Structure and Expression 1576, 287-297 (2002). http://doi.org/10.1016/S0167-4781(02)00368-8.

[305] Davis, T. L. et al. Structural and biochemical characterization of the human cyclophilin family of peptidyl-prolyl isomerases. PLoS Biology 8 (2010). http://doi.org/10.1371/ journal.pbio.1000439.

[306] Rajiv, C. \& Davis, T. L. Structural and functional insights into human nuclear cyclophilins (2018). http://doi.org/10.3390/biom8040161.

[307] Adams, B. M., Coates, M. N., Jackson, S. R. E., Jurica, M. S. \& Davis, T. L. Nuclear cyclophilins affect spliceosome assembly and function in vitro. Biochemical Journal 469, 223-233 (2015). http://doi.org/10.1042/BJ20150396.

[308] le Maire, A. et al. A Tandem of SH3-like Domains Participates in RNA Binding in KIN17, a Human Protein Activated in Response to Genotoxics. Journal of Molecular Biology 364, 764-776 (2006). http://doi.org/10.1016/j.jmb.2006.09.033.

[309] Kim, S. H. \& Lin, R. J. Spliceosome activation by PRP2 ATPase prior to the first transesterification reaction of pre-mRNA splicing. Molecular and Cellular Biology 16, 6810-6819 (1996). http://doi.org/10.1128/mcb.16.12.6810.

[310] Bao, P., Will, C. L., Urlaub, H., Boon, K. L. \& Lührmann, R. The RES complex is required for efficient transformation of the precatalytic B spliceosome into an activated Bact complex. Genes and Development 31, 2416-2429 (2017). http://doi.org/10.1101/ gad.308163.117.

[311] Ajuh, P., Sleeman, J., Chusainow, J. \& Lamond, A. I. A Direct Interaction between the Carboxyl-terminal Region of CDC5L and the WD40 Domain of PLRG1 Is Essential for Pre-mRNA Splicing. Journal of Biological Chemistry 276, 42370-42381 (2001). http: //doi.org/10.1074/jbc.M105453200.

[312] Smith, T. F., Gaitatzes, C., Saxena, K. \& Neer, E. J. The WD repeat: A common architecture for diverse functions. Trends in Biochemical Sciences 24, 181-185 (1999). http://doi.org/10.1016/S0968-0004(99)01384-5. 
[313] Van Maldegem, F. et al. CTNNBL1 facilitates the association of CWC15 with CDC5L and is required to maintain the abundance of the Prp19 spliceosomal complex. Nucleic Acids Research 43, 7058-7069 (2015). http://doi.org/10.1093/nar/gkv643.

[314] Stegmann, C. M., Lührmann, R. \& Wahl, M. C. The crystal structure of PPIL1 bound to cyclosporine a suggests a binding mode for a linear epitope of the SKIP protein. PLoS ONE 5 (2010). http://doi.org/10.1371/journal.pone.0010013.

[315] Lindsey, L. A. \& Garcia-Blanco, M. A. Functional conservation of the human homolog of the yeast pre-mRNA splicing factor Prp17p. Journal of Biological Chemistry 273, 3277132775 (1998). http://doi.org/10.1074/jbc.273.49.32771.

[316] Sapra, A. K., Arava, Y., Khandelia, P. \& Vijayraghavan, U. Genome-wide analysis of Pre-mRNA splicing: Intron features govern the requirement for the second-step factor, Prp17 in Saccharomyces cerevisiae and Schizosaccharomyces pombe. Journal of Biological Chemistry 279, 52437-52446 (2004). http://doi.org/10.1074/jbc.M408815200.

[317] Yehuda, S. B. et al. Identification and functional analysis of hPRP17, the human homologue of the PRP17/CDC40 yeast gene involved in splicing and cell cycle control. Rna 4, 1304-1312 (1998). http://doi.org/10.1017/S1355838298980712.

[318] Chai, G. et al. Mutations in Spliceosomal Genes PPIL1 and PRP17 Cause Neurodegenerative Pontocerebellar Hypoplasia with Microcephaly. Neuron 109, 241-256.e9 (2021). http://doi.org/10.1016/j.neuron.2020.10.035.

[319] Reiter, N. J., Blad, H., Abildgaard, F. \& Butcher, S. E. Dynamics in the U6 RNA intramolecular stem-loop: A base flipping conformational change. Biochemistry 43, 1373913747 (2004). http://doi.org/10.1021/bi048815y.

[320] Blad, H., Reiter, N. J., Abildgaard, F., Markley, J. L. \& Butcher, S. E. Dynamics and metal ion binding in the U6 RNA intramolecular stem-loop as analyzed by NMR. Journal of Molecular Biology 353, 540-555 (2005). http://doi.org/10.1016/j·jmb.2005.08.030.

[321] Venditti, V., Clos, L., Niccolai, N. \& Butcher, S. E. Minimum-Energy Path for a U6 RNA Conformational Change Involving Protonation, Base-Pair Rearrangement and Base Flipping. Journal of Molecular Biology 391, 894-905 (2009). http://doi.org/10.1016/ j.jmb.2009.07.003.

[322] Fischer, N., Konevega, A. L., Wintermeyer, W., Rodnina, M. V. \& Stark, H. Ribosome dynamics and tRNA movement by time-resolved electron cryomicroscopy. Nature 466, 329-333 (2010). http://doi.org/10.1038/nature09206

[323] Hoskins, A. A., Rodgers, M. L., Friedman, L. J., Gelles, J. \& Moore, M. J. Single molecule analysis reveals reversible and irreversible steps during spliceosome activation. eLife 5 (2016). http://doi.org/10.7554/eLife.14166.

[324] Hoskins, A. a. et al. Ordered and Dynamic Assembly of Single Spliceosomes. Science 331, 1289-1296 (2011).

[325] Shcherbakova, I. et al. Alternative Spliceosome Assembly Pathways Revealed by SingleMolecule Fluorescence Microscopy. Cell Reports 5, 151-165 (2013). http://doi.org/10. 1016/j.celrep.2013.08.026.

[326] David, C. J., Boyne, A. R., Millhouse, S. R. \& Manley, J. L. The RNA polymerase II C-terminal domain promotes splicing activation through recruitment of a U2AF65-Prp19 complex. Genes and Development 25, 972-982 (2011). http://doi.org/10.1101/gad. 2038011 . 
[327] Lazo, J. S. et al. Identification of a potent and selective pharmacophore for Cdc25 dual specificity phosphatase inhibitors. Molecular Pharmacology 61, 720-728 (2002). http: //doi.org/10.1124/mol.61.4.720.

[328] Dulyaninova, N. G. et al. Cysteine 81 is critical for the interaction of s100a4 and myosinIIA. Biochemistry 50, 7218-7227 (2011). http://doi.org/10.1021/bi200853y.

[329] Nilsson, I. \& Hoffmann, I. Cell cycle regulation by the Cdc25 phosphatase family. Progress in cell cycle research 4, 107-114 (2000). http://doi.org/10.1007/978-1-4615-4253-7_ 10 .

[330] You, Y. J., Zheng, X. G., Yong, K. \& Ahn, B. Z. Naphthazarin derivatives: Synthesis, cytotoxic mechanism and evaluation of antitumor activity. Archives of Pharmacal Research 21, 595-598 (1998). http://doi.org/10.1007/BF02975381.

[331] Valente, C. et al. The 1,4-naphthoquinone scaffold in the design of cysteine protease inhibitors. Bioorganic and Medicinal Chemistry 15, 5340-5350 (2007). http://doi.org/ $10.1016 /$ j.bmc.2007.04.068

[332] Soares, K. M. et al. Profiling the NIH small molecule repository for compounds that generate $\mathrm{H} 2 \mathrm{O} 2$ by redox cycling in reducing environments. Assay and Drug Development Technologies 8, 152-174 (2010). http://doi.org/10.1089/adt.2009.0247.

[333] Effenberger, K. A. et al. A high-throughput splicing assay identifies new classes of inhibitors of human and yeast spliceosomes. Journal of Biomolecular Screening 18, 11101120 (2013). http://doi.org/10.1177/1087057113493117

[334] Wan, L., Ottinger, E., Cho, S. \& Dreyfuss, G. Inactivation of the SMN Complex by Oxidative Stress. Molecular Cell 31, 244-254 (2008). http://doi.org/10.1016/j.molcel. 2008.06 .004 .

[335] Wilkinson, M. E., Fica, S. M., Galej, W. P. \& Nagai, K. Structural basis for conformational equilibrium of the catalytic spliceosome. Molecular Cell 81, 1439-1452.e9 (2021). http: //doi.org/10.1016/j.molcel.2021.02.021.

[336] Punjani, A., Zhang, H. \& Fleet, D. J. Non-uniform refinement: adaptive regularization improves single-particle cryo-EM reconstruction. Nature Methods 17, 1214-1221 (2020). http://doi.org/10.1038/s41592-020-00990-8.

[337] Wu, Z. et al. Deep manifold learning reveals hidden dynamics of proteasome autoregulation. arXiv (2020).

[338] Zhong, E. D., Bepler, T., Berger, B. \& Davis, J. H. CryoDRGN: reconstruction of heterogeneous cryo-EM structures using neural networks. Nature Methods 18, 176-185 (2021). http://doi.org/10.1038/s41592-020-01049-4.

[339] Schliep, J. E. Structural Characterization of the Eukaryotic Translation Initiation by Electron submitted by Electron Cryo-Microscopy. Ph.D. thesis (2018).

[340] Singh, K. et al. Discovery of a Regulatory Subunit of the Yeast Fatty Acid Synthase. Cell 180, 1130-1143.e20 (2020). http://doi.org/10.1016/j.cell.2020.02.034. 
